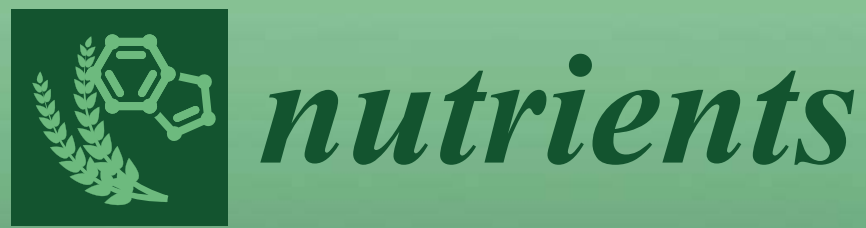

\title{
Nutrition in Inflammatory Bowel Disease (IBD)
}

Edited by Maitreyi Raman Printed Edition of the Special Issue Published in Nutrients 


\section{Nutrition in Inflammatory Bowel Disease (IBD)}





\section{Nutrition in Inflammatory Bowel Disease (IBD)}

Special Issue Editor

Maitreyi Raman

MDPI • Basel • Beijing • Wuhan • Barcelona • Belgrade

$$
\text { MDPI }
$$


Special Issue Editor

Maitreyi Raman

University of Calgary

Canada

Editorial Office

MDPI

St. Alban-Anlage 66

4052 Basel, Switzerland

This is a reprint of articles from the Special Issue published online in the open access journal Nutrients (ISSN 2072-6643) from 2018 to 2019 (available at: https://www.mdpi.com/journal/nutrients/ special_issues/nutrition_IBD)

For citation purposes, cite each article independently as indicated on the article page online and as indicated below:

LastName, A.A.; LastName, B.B.; LastName, C.C. Article Title. Journal Name Year, Article Number, Page Range.

ISBN 978-3-03921-439-6 (Pbk)

ISBN 978-3-03921-440-2 (PDF)

(C) 2019 by the authors. Articles in this book are Open Access and distributed under the Creative Commons Attribution (CC BY) license, which allows users to download, copy and build upon published articles, as long as the author and publisher are properly credited, which ensures maximum dissemination and a wider impact of our publications.

The book as a whole is distributed by MDPI under the terms and conditions of the Creative Commons license CC BY-NC-ND. 


\section{Contents}

About the Special Issue Editor $\ldots \ldots \ldots \ldots \ldots \ldots \ldots \ldots \ldots \ldots \ldots \ldots$

Maitreyi Raman and Subrata Ghosh

Diet and Nutrition in IBD—Progress and Gaps

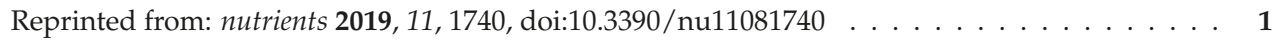

Robert Venning Bryant, Christopher G. Schultz, Soong Ooi, Charlotte Goess, Samuel Paul Costello, Andrew D. Vincent, Scott N. Schoeman, Amanda Lim, Francis Dylan Bartholomeusz, Simon P.L. Travis and Jane Mary Andrews

Obesity in Inflammatory Bowel Disease: Gains in Adiposity despite High Prevalence of Myopenia and Osteopenia

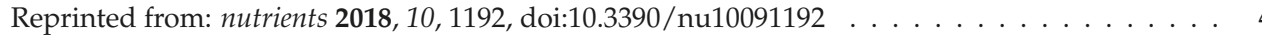

Lorian Taylor, Abdulelah Almutairdi, Nusrat Shommu, Richard Fedorak, Subrata Ghosh, Raylene A. Reimer, Remo Panaccione and Maitreyi Raman

Cross-Sectional Analysis of Overall Dietary Intake and Mediterranean Dietary Pattern in Patients with Crohn's Disease

Reprinted from: nutrients 2018, 11, 1761, doi:10.3390/nu10111761 . . . . . . . . . . . .

Helena Rolandsdotter, Kerstin Jönsson-Videsäter, Ulrika L. Fagerberg, Yigael Finkel and Michael Eberhardson

Exclusive Enteral Nutrition: Clinical Effects and Changes in Mucosal Cytokine Profile in Pediatric New Inflammatory Bowel Disease

Reprinted from: nutrients 2019, 11, 414, doi:10.3390/nu11020414 . . . . . . . . . . . . .

Sandra Vidal-Lletjós, Mireille Andriamihaja, Anne Blais, Marta Grauso, Patricia Lepage, Anne-Marie Davila, Roselyne Viel, Claire Gaudichon, Marion Leclerc, François Blachier and Annaïg Lan

Dietary Protein Intake Level Modulates Mucosal Healing and Mucosa-Adherent Microbiota in Mouse Model of Colitis

Reprinted from: nutrients $\mathbf{2 0 1 9}, 11,514$, doi:10.3390/nu11030514 . . . . . . . . . . . . . .

Gian Carlo Tenore, Ester Pagano, Stefania Lama, Daniela Vanacore, Salvatore Di Maro, Maria Maisto, Raffaele Capasso, Francesco Merlino, Francesca Borrelli, Paola Stiuso and Ettore Novellino

Intestinal Anti-Inflammatory Effect of a Peptide Derived from Gastrointestinal Digestion of Buffalo (Bubalus bubalis) Mozzarella Cheese

Reprinted from: nutrients 2019, 11, 610, doi:10.3390/nu11030610 . . . . . . . . . . . . .

Tanvi Shinde, Agampodi Promoda Perera, Ravichandra Vemuri, Shakuntla V. Gondalia, Avinash V. Karpe, David J. Beale, Sonia Shastri, Benjamin Southam, Rajaraman Eri and Roger Stanley

Synbiotic Supplementation Containing Whole Plant Sugar Cane Fibre and Probiotic Spores Potentiates Protective Synergistic Effects in Mouse Model of IBD

Reprinted from: nutrients 2019, 11, 818, doi:10.3390/nu11040818

Levi M. Teigen, Zhuo Geng, Michael J. Sadowsky, Byron P. Vaughn, Matthew J. Hamilton and Alexander Khoruts

Dietary Factors in Sulfur Metabolism and Pathogenesis of Ulcerative Colitis

Reprinted from: nutrients 2019, 11,931, doi:10.3390/nu11040931 . . . . . . . . . . . . . . . . 102 
Nicole Green, Talya Miller, David Suskind and Dale Lee

A Review of Dietary Therapy for IBD and a Vision for the Future

Reprinted from: nutrients 2019, 11, 947, doi:10.3390/nu11050947

Sing-Chung Li, Wei-Fang Hsu, Jung-Su Chang and Chun-Kuang Shih

Combination of Lactobacillus acidophilus and Bifidobacterium animalis subsp. lactis Shows a

Stronger Anti-Inflammatory Effect than Individual Strains in HT-29 Cells

Reprinted from: nutrients 2019, 11, 969, doi:10.3390/nu11050969

Jane Fletcher, Sheldon C. Cooper, Subrata Ghosh and Martin Hewison

The Role of Vitamin D in Inflammatory Bowel Disease: Mechanism to Management

Reprinted from: nutrients 2019, 11, 1019, doi:10.3390/nu11051019 . . . . . . . . . . . 152

Fernando Rizzello, Enzo Spisni, Elisabetta Giovanardi, Veronica Imbesi, Marco Salice, Patrizia Alvisi, Maria Chiara Valerii and Paolo Gionchetti

Implications of the Westernized Diet in the Onset and Progression of IBD

Reprinted from: nutrients 2019, 11, 1033, doi:10.3390/nu11051033 . . . . . . . . . . . 168

Pedro López-Muñoz, Belén Beltrán, Esteban Sáez-González, Amparo Alba, Pilar Nos and Marisa Iborra

Influence of Vitamin D Deficiency on Inflammatory Markers and Clinical Disease Activity in IBD Patients

Reprinted from: nutrients 2019, 11, 1059, doi:10.3390/nu11051059 _ . . . . . . . . . . . 192

Esteban Sáez-González, Beatriz Mateos, Pedro López-Muñoz, Marisa Iborra, Inés Moret, Pilar Nos and Belén Beltrán

Bases for the Adequate Development of Nutritional Recommendations for Patients with Inflammatory Bowel Disease

Reprinted from: nutrients 2019, 11, 1062, doi:10.3390/nu11051062 . . . . . . . . . . . . 208

Rachel Marion-Letellier, Asma Amamou, Guillaume Savoye and Subrata Ghosh

Inflammatory Bowel Diseases and Food Additives:

To Add Fuel on the Flames!

Reprinted from: nutrients 2019, 11, 1111, doi:10.3390/nu11051111 . . . . . . . . . . . . . . 219

Ilse Molendijk, Sander van der Marel and P.W. Jeroen Maljaars

Towards a Food Pharmacy: Immunologic Modulation through Diet

Reprinted from: nutrients 2019, 11, 1239, doi:10.3390/nu11061239

\section{Tong Chen, Ni Shi and Anita Afzali}

Chemopreventive Effects of Strawberry and Black Raspberry on Colorectal Cancer in

Inflammatory Bowel Disease

Reprinted from: nutrients 2019, 11, 1261, doi:10.3390/nu11061261

Kyle Kilby, Holly Mathias, Lindsay Boisvenue, Courtney Heisler and Jennifer L. Jones

Micronutrient Absorption and Related Outcomes in People with Inflammatory Bowel Disease:

A Review

Reprinted from: nutrients 2019, 11, 1388, doi:10.3390/nu11061388

Fernando Castro and Heitor S. P. de Souza

Dietary Composition and Effects in Inflammatory Bowel Disease

Reprinted from: nutrients 2019, 11, 1398, doi:10.3390/nu11061398 
Piotr Eder, Alina Niezgódka, Iwona Krela-Ká́mierczak, Kamila Stawczyk-Eder, Estera Banasik and Agnieszka Dobrowolska

Dietary Support in Elderly Patients with Inflammatory Bowel Disease

Reprinted from: nutrients 2019, 11, 1421, doi:10.3390/nu11061421 . . . . . . . . . . . . 302

Ammar Hassanzadeh Keshteli, Karen L. Madsen and Levinus A. Dieleman

Diet in the Pathogenesis and Management of Ulcerative Colitis; A Review of Randomized Controlled Dietary Interventions

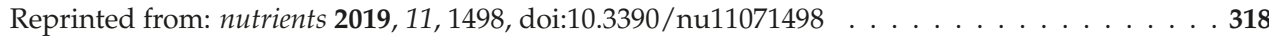

Bobbi B Laing, Anecita Gigi Lim and Lynnette R Ferguson

A Personalised Dietary Approach-A Way Forward to Manage Nutrient Deficiency, Effects of the Western Diet, and Food Intolerances in Inflammatory Bowel Disease

Reprinted from: nutrients 2019, 11, 1532, doi:10.3390/nu11071532 . . . . . . . . . . . . . 330 



\section{About the Special Issue Editor}

Maitreyi Raman is a gastroenterologist, physician nutrition specialist, and clinical associate professor at the University of Calgary, Calgary, $\mathrm{AB}$, in Canada. Dr. Raman is the medical director for Nutrition Services, for Southern Alberta; the director for Ascend (Alberta's Collaboration of Excellence for Nutrition in Digestive Diseases); and the director for the Clinical Investigator Program at the University of Calgary. Dr. Raman's research program explores the relationship between dietary patterns; nutrients; and their effects on the microbiome, metabolome, and inflammation in Inflammatory Bowel Disease. In addition, research interests include identifying and validating the optimal nutrition screening and assessment tools to diagnose malnutrition in patients with IBD. Clinical interests include short bowel syndrome, dietary therapy as a primary therapy for IBD, and malnutrition treatment 

Editorial

\title{
Diet and Nutrition in IBD-Progress and Gaps
}

\author{
Maitreyi Raman ${ }^{1, *}$ and Subrata Ghosh ${ }^{2}$ \\ 1 Division of Gastroenterology, University of Calgary, 6D33 TRW Building, 3280 Hospital Drive NW, Calgary, \\ AB T2N 4N1, Canada \\ 2 Institute of Translational Medicine, NIHR Biomedical Research Centre, University of Birmingham, \\ Birmingham B15 2TT, UK \\ * Correspondence: mkothand@ucalgary.ca
}

Received: 24 July 2019; Accepted: 25 July 2019; Published: 27 July 2019

The incidence and prevalence of Inflammatory Bowel Disease (IBD) has rapidly increased worldwide and now is a global disease with some of the highest rates observed in North America [1]. IBD is believed to arise from a shared interaction between genetic and environmental influences, resulting in an imbalance between pro-inflammatory and anti-inflammatory signaling, however the etiology of this imbalance and of IBD itself is incompletely understood [2]. Environmental risk factors for both disease onset and risk for disease exacerbation are attractive to explore, as these may be modifiable, and have the potential to represent a therapeutic opportunity with few adverse side effects. Patients identify associations between diet and disease flare and the topic of diet and IBD is among the frequently asked questions by patients to their gastroenterologist. However, guidelines for dietary recommendations as primary therapy are lacking [3]. Additionally, IBD is associated with malnutrition, sarcopenia, and micronutrient deficiencies, all of which can reduce quality of life, increase morbidity, and contribute to symptoms [4,5]. The recently published literature assessing relationships between diet, nutrition, and IBD has increased substantially in the past 2 years, reflecting the urgency to find therapeutic solutions to meet patient needs and reduce disease related complications.

Specifically, epidemiologic data often implicate dietary lipids and fatty acids imbalance, in addition to animal proteins, as increasing predisposition to IBD, while vitamin D and fiber intake may be protective [6]. Some of these are dietary components are traditionally considered part of a healthy diet, such as vegetables, fruits, dietary fibers, n-3 polyunsaturated fatty acids (n-3 PUFA), n-9 PUFA, and vitamin D, while others, such as meats, n-6 PUFA, and refined sugars are often considered part of the 'unhealthy' Western diet, but it is unclear to what extent any of these are specific for IBD. Considerable evidence has emerged that diet targets innate and adaptive immunity in the intestinal barrier and gut microbiome in addition to correcting 'malnutrition' inherent in IBD $[7,8]$. With the tremendous amount of research in the gut microbiome in IBD, it is inevitable that interest in many aspects of diet and nutrition in IBD will increase exponentially, as highlighted in this issue. However, it is imperative that studying nutritional intervention in IBD evolves new paradigms and does not simply follow drug development paths. Intervening in early disease and in IBD sufferers in remission to prevent relapse is especially attractive.

This Special Issue of Nutrients, "Nutrition in Inflammatory Bowel Disease", includes a collection of 21 manuscripts that richly describe the vibrant and diverse state of nutrition research in IBD. Close to half of the contributions are review articles that provide perspectives on mechanisms through which diet could play an important role in the pathogenesis of IBD, while another important review summarizes the state of randomized clinical trials in Ulcerative Colitis (UC), highlighting gaps and areas for research [9]. The high number of review articles on the topic of dietary mechanisms and IBD truly reflects the acute interest in this growing field. The stark lack of clinical trials exploring dietary relationships and gut inflammation is also very revealing and is a further call to action for IBD researchers. 
Eight original articles, ranging from in vitro to animal models and human clinical research, explore various nutrition-focused themes that include probiotics, symbiotics, vitamin D, dietary intake and composition, protein dosing, obesity, myopenia, and exclusive enteral nutrition on diverse IBD outcomes [4,10-16].

Vidal-Lletjos et al. identified that moderately high protein dosing modulated mucosal healing, however high the protein intake, had deleterious consequences in animal models [14]. These results are undoubtedly of interest for developing nutrition prescriptions in the clinical setting and require further study in human populations. Taylor et al. described suboptimal dietary intake and low adherence with Mediterranean diet guidelines as not keeping with anti-inflammatory dietary patterns [4]. Shinde et al. demonstrated that symbiotic supplementation ameliorated disease activity index and histological scores in animal models, warranting human trials [12]. Of interest, Bryant et al. determined that, over 24 months of longitudinal follow-up, the body composition of patients with IBD changed, with gains of fat mass but decreases in lean muscle mass [16]. The morbidity that may be associated with these findings deserves further attention.

These valuable manuscripts contribute toward advancing knowledge about current dietary intake and composition in patients with IBD, describe existing nutritional deficiencies including myopenia, and provide a platform of nutritional supplements with biologic plausibility to be subjected to further trials.

We believe this compendium is a useful summary of the nutrition progress in IBD. The need for further nutrition intervention trials with diets that are mechanistically sensible and address the industrialization of food remains as a loud unspoken statement.

Conflicts of Interest: The authors declare no conflict of interest.

\section{References}

1. Ng, S.C.; Shi, H.Y.; Hamidi, N.; Underwood, F.E.; Tang, W.; Benchimol, E.I.; Panaccione, R.; Ghosh, S.; Wu, J.C.Y.; Chan, F.K.L.; et al. The worldwide incidence and prevalence of inflammatory bowel disease in the $21^{\text {st }}$ century: A systematic review of population-based studies. Lancet 2018, 390, 2769-2778. [CrossRef]

2. Lee, S.H.; Kwon, J.E.; Cho, M.L. Immunological pathogenesis of inflammatory bowel disease. Intest. Res. 2018, 16, 26-42. [CrossRef] [PubMed]

3. Zallot, C.; Quilliot, D.; Chevaux, J.B.; Peyrin-Biroulet, C.; Gueant-Rodriguez, R.M.; Freling, E.; Collet-Fenetrier, B.; Williet, N.; Ziegler, O.; Bigard, M.A.; et al. Dietary beliefs and behavior among inflammatory bowel disease patients. Inflamm. Bowel Dis. 2013, 19, 66-72. [CrossRef] [PubMed]

4. Taylor, L.; Almutairdi, A.; Shommu, N.; Fedorak, R.; Ghosh, S.; Reimer, R.A.; Panaccione, R.; Raman, M. Cross-Sectional analysis of overall dietary intake and Mediterranean dietary pattern in patients with Crohn's disease. Nutrients 2018, 10, 1761. [CrossRef] [PubMed]

5. Ryan, E.; McNicholas, D.; Creavin, B.; Kelly, M.E.; Walsh, T.; Beddy, D. Sarcopenia and Inflammatory Bowel Disease: A Systematic Review. Inflamm. Bowel Dis. 2019, 25, 67-73. [CrossRef] [PubMed]

6. Ananthakrishnan, A.N. Epidemiology and risk factors for IBD. Nat. Rev. Gastroenterol. Hepatol. 2015, 12, 205-217. [CrossRef] [PubMed]

7. Levine, A.; Wine, E.; Assa, A.; Boneh, R.S.; Shaoul, R.; Kori, M.; Cohen, S.; Peleg, S.; Shamali, H.; On, A.; et al. Crohn's Disease exclusion diet plus partial enteral nutrition induces sustained remission in a randomized controlled trial. Gastroenterology 2019, 157, 440-450. [CrossRef] [PubMed]

8. Smith, M.I.; Yatsunenko, T.; Manary, M.J.; Trehan, I.; Mkakosya, R.; Cheng, J.; Kau, A.L.; Rich, S.S.; Concannon, P.; Mychaleckyj, J.C.; et al. Gut microbiomes of Malawian twin pairs discordant for kwashiorkor. Science 2013, 339, 548-554. [CrossRef] [PubMed]

9. Keshteli, A.H.; Madsen, K.L.; Dieleman, L.A. Diet in the pathogenesis and management of ulcerative colitis; A review of randomized controlled dietary interventions. Nutrients 2019, 11, 1498. [CrossRef] [PubMed]

10. Lopez-Munoz, P.; Beltran, B.; Saez-Gonzalez, E.; Alba, A.; Nos, P.; Iborra, M. Influence of vitamin D deficiency on inflammatory markers and clinical disease activity in IBD patients. Nutrients 2019, 11, 1059. [CrossRef] [PubMed] 
11. Li, S.C.; Hsu, W.F.; Chang, J.S.; Shih, C.K. Combination of a Lactobacillus acidophilus and Bifidobacterium animalis subsp. Lactis shows a stronger anti-inflammatory effect than individual strains in HT-20 cells. Nutrients 2019, 11, 969. [CrossRef] [PubMed]

12. Shinde, T.; Perera, A.P.; Vemuri, R.; Gondalia, S.V.; Karpe, A.V.; Beale, D.J.; Shastri, S.; Southam, B.; Eri, R.; Stanley, R. Synbiotic supplementation containing whole plant sugar cane fibre and probiotic spores potentiates protective synergistic effects in mouse model of IBD. Nutrients 2019, 11, 818. [CrossRef] [PubMed]

13. Tenore, G.C.; Pagano, E.; Lama, S.; Vanacore, D.; di Maro, S.; Maisto, M.; Capasso, R.; Merlino, F.; Stiuso, P.; Novellino, E. Intestinal anti-inflammatory effect of a peptide derived from gastrointestinal digestion of buffalo (Bulbalus bubalis) Mozzarella cheese. Nutrients 2019, 11, 610. [CrossRef] [PubMed]

14. Vidal-Lletjos, S.; Andriamihaja, M.; Blais, A.; Grauso, M.; Lepage, P.; Davila, A.M.; Viel, R.; Gaudichon, C.; Leclerc, M.; Blachier, F.; et al. Dietary protein intake level modulates mucosal healing and mucosa-adherent microbiota in mouse model of colitis. Nutrients 2019, 11, 514. [CrossRef]

15. Rolandsdotter, H.; Jonsson-Videsater, K.; Fagerberg, U.; Finkel, Y.; Eberhardson, M. Exclusive enteral nutrition: Clinical effects and changes in mucosal cytokine profile in pediatric new inflammatory bowel disease. Nutrients 2019, 11, 414. [CrossRef]

16. Bryant, R.V.; Schulz, C.G.; Ooi, S.; Goess, C.; Costello, S.P.; Vincent, A.D.; Schoeman, S.N.; Lim, A.; Bartholomeusz, F.D.; Travis, S.P.L.; et al. Obesity in inflammatory bowel disease: Gains in adiposity despite high prevalence of myopenia and psteopenia. Nutrients 2018, 10, 1192. [CrossRef] [PubMed]

(C) 2019 by the authors. Licensee MDPI, Basel, Switzerland. This article is an open access article distributed under the terms and conditions of the Creative Commons Attribution (CC BY) license (http://creativecommons.org/licenses/by/4.0/). 


\title{
Obesity in Inflammatory Bowel Disease: Gains in Adiposity despite High Prevalence of Myopenia and Osteopenia
}

\author{
Robert Venning Bryant ${ }^{1,2, *}$, Christopher G. Schultz ${ }^{3}$, Soong Ooi ${ }^{4}$, Charlotte Goess ${ }^{4}$, \\ Samuel Paul Costello ${ }^{1,2}$, Andrew D. Vincent ${ }^{5}$, Scott N. Schoeman ${ }^{1}$, Amanda Lim ${ }^{1}$, \\ Francis Dylan Bartholomeusz ${ }^{3}$, Simon P.L. Travis ${ }^{6}$ and Jane Mary Andrews ${ }^{2,4}$ \\ 1 IBD Service, Department of Gastroenterology and Hepatology, The Queen Elizabeth Hospital, \\ 28 Woodville Road, Woodville 5011, Australia; sam.costello@sa.gov.au (S.P.C.); \\ snschoeman@gmail.com (S.N.S.); Amanda.Lim@sa.gov.au (A.L.) \\ 2 School of Medicine, Faculty of Health Sciences, University of Adelaide, Adelaide 5000, Australia; \\ jane.andrews@sa.gov.au \\ 3 Department of Nuclear Medicine, PET and Bone Densitometry, Royal Adelaide Hospital, Port Road, \\ Adelaide 5000, Australia; chris.schultz@sa.gov.au (C.G.S.); Dylan.Bartholomeusz@sa.gov.au (F.D.B.) \\ 4 IBD Service, Department of Gastroenterology and Hepatology, Royal Adelaide Hospital, Port Road, \\ Adelaide 5000, Australia; soongyuan.ooi@uq.edu.au (S.O.); charlotte.goess@sa.gov.au (C.G.) \\ 5 Freemasons Foundation Centre for Men's Health, School of Medicine, University of Adelaide, North Terrace, \\ Adelaide 5000, Australia; andrew.vincent@adelaide.edu.au \\ 6 Translational Gastroenterology Unit, John Radcliffe Hospital, Headley Way, Headington, Oxford OX3 9DU, \\ UK; simon.travis@ndm.ox.ac.uk \\ * Correspondence: Robert.Bryant@sa.gov.au; Tel.: +61-8-8222-6000
}

Received: 28 July 2018; Accepted: 21 August 2018; Published: 1 September 2018

\begin{abstract}
Background: Rising rates of obesity have been reported in patients with inflammatory bowel disease (IBD); however, prospective data is lacking. The aim of this study is to prospectively evaluate body composition in adults with IBD over 24 months. Methods: Whole body dual energy X-ray absorptiometry (DXA) data was performed at 0 months, 12 months, and 24 months. Bone mineral density (BMD), fat mass index (FMI $(\mathrm{kg}) /$ height $\left(\mathrm{m}^{2}\right)$ ), appendicular skeletal muscle index (ASMI $(\mathrm{kg}) /$ height $\left(\mathrm{m}^{2}\right)$ ), visceral adipose tissue and the visceral adipose height index $\left(\mathrm{VHI}, \mathrm{VAT}\right.$ area $\left(\mathrm{cm}^{3}\right) /$ height $\left.\left(\mathrm{m}^{2}\right)\right)$, and clinical and anthropometric assessments were performed at each time point. Multivariable linear mixed effects regression analyses were performed. Results: Initially, 154 participants were assessed at baseline (70\% Crohn's disease, 55\% male, median age 31 years), of whom 129 underwent repeated DXA at 12 months, and 110 underwent repeated DXA at 24 months. Amongst those undergoing repeated DXA, their body mass index (BMI) significantly increased over time, such that by 24 months, $62 \%$ of patients were overweight or obese (annual change BMI $\beta=0.43,95 \% \mathrm{CI}=[0.18,0.67], p=0.0006$ ). Gains in BMI related to increases in both FMI and VHI $(\beta=0.33,95 \% \mathrm{CI}=[0.14,0.53], p=0.0007 ; \beta=0.08,95 \% \mathrm{CI}=[0.02,0.13]$, $p=0.001$; respectively), whereas ASMI decreased $(\beta=-0.07,95 \% \mathrm{CI}=[-0.12,-0.01], p=0.01)$ with a concordant rise in rates of myopenia $(\mathrm{OR}=3.195 \% \mathrm{CI}=[1.2,7.7] ; p=0.01)$. Rates of osteopenia and osteoporosis were high $(37 \%)$, but remained unchanged over time $(p=0.23)$. Conclusion: Increasing rates of obesity in patients with IBD coincide with decreases in lean muscle mass over time, while high rates of osteopenia remain stable. These previously undocumented issues warrant attention in routine care to prevent avoidable morbidity.
\end{abstract}

Keywords: body composition; obesity; visceral adipose tissue; fat; osteoporosis; osteopenia; sarcopenia; inflammatory bowel disease 


\section{Introduction}

Body composition refers to proportions of bone, fat, and fat-free (lean) mass in the body and may be abnormal in many patients with inflammatory bowel disease (IBD) [1]. Despite the potential negative effects of disturbances in body composition on IBD-related outcomes, response to therapy, cardiovascular disease, and quality of life (QoL), there is a paucity of prospective data on body composition in patients with IBD [1,2].

Rates of obesity are rising in patients with IBD, as in the general population; $15-40 \%$ of adults with IBD are obese, and 20-40\% are overweight [3]. IBD may be an independent risk factor for obesity that is driven by dysbiosis and aberrations in intestinal microbial metabolism [4,5]. Treatments used for IBD, in particular corticosteroids and anti-tumor necrosis factor alpha therapies, may also play a role in obesity [3]. On the other hand, adipose tissue is metabolically active, and could plausibly contribute to a pro-inflammatory susceptibility to IBD $[4,6]$. However, existing data on the impact of obesity on IBD susceptibility and disease course are conflicting. This is partly due to the use of the body mass index (BMI) as a blunt instrument for measuring adiposity, which is unable to distinguish between adiposity compartments with distinct metabolic profiles [3,6-10]. Visceral adipose tissue (VAT) is increased in many patients with Crohn's disease (CD) and has been associated with a complicated CD phenotype $[3,4,6,11-14]$. There are no longitudinal prospective data on direct measures of adiposity in patients with IBD.

There are emerging reports of high rates of both myopenia, which is defined as low lean mass, and sarcopenia, which is defined as a low lean mass coupled with loss of strength, in patients with IBD [1,15-17]. This matters, because low lean mass has been associated with an increased need for surgery and poor surgical outcomes in IBD, as well as with osteopenia [18-20]. Lean mass deficits may be difficult to detect in clinical practice, where BMI can be falsely reassuring $[17,18]$. Current data on sarcopenia in IBD are limited by small sample sizes and retrospective study designs, as well as a lack of an appropriate incorporation of a functional assessment [1].

A deficit in bone mineral density (BMD) (osteoporosis/osteopenia) is one of the most common complications of IBD, and has been reported in $20 \%$ to $50 \%$ of patients [21-23]. Reduced BMD is associated with an increased risk of pathological fractures and associated morbidity [22,24]. The pathogenesis of metabolic bone disease in IBD is likely multifactorial; however, clinical data are lacking, and the influence of IBD-related factors beyond conventional risk factors remains poorly explored [25,26].

Dual-energy X-ray absorptiometry (DXA) is in routine use in patients with IBD for the measurement of BMD. Whole body DXA adds little in terms of time and radiation, yet gleans accurate and regional body composition information and is considered a gold-standard tool for the evaluation of body composition as compared to cross-sectional imaging [1,27].

Therefore, the aims of this study were:

1. To evaluate body composition in patients with IBD, with serial prospective measurements over time;

2. To explore the influence of clinical factors on body composition in patients with IBD;

3. To explore whether standard anthropometric testing can detect aberrations in body composition.

\section{Materials and Methods}

\subsection{Subjects}

Consecutive patients with IBD (aged 18-50 years and pre-menopausal if female) managed by a tertiary IBD service were invited to participate in a prospective study between April 2012 and September 2013 (Figure 1). Those with significant medical or surgical comorbidity other than IBD, current pregnancy, or steroid use other than that required for IBD, were excluded. 


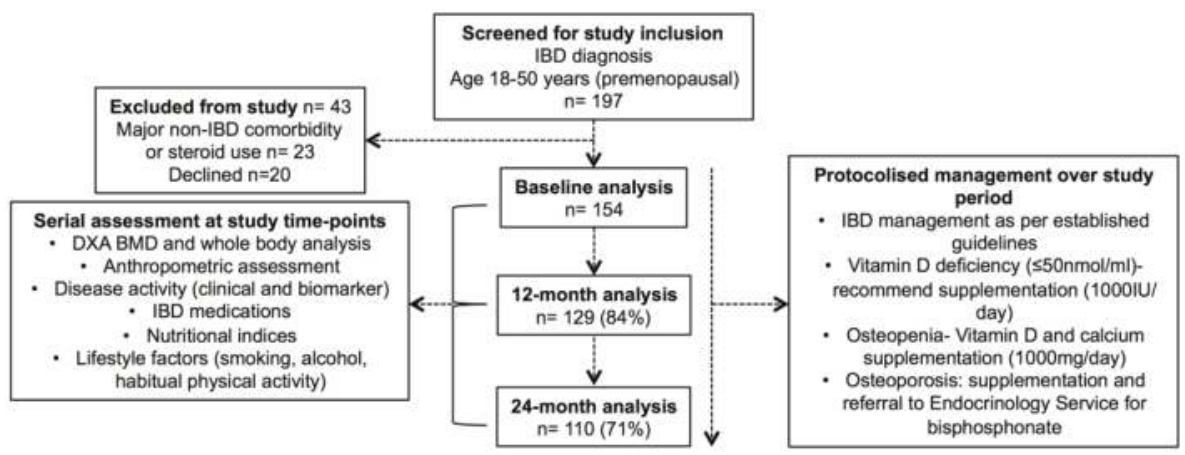

Figure 1. CONSORT diagram-inflammatory bowel disease (IBD) cohort. Legend: DXA, dual energy X-ray absorptiometry, BMD, bone mineral density.

\subsection{Subject Data Collection}

Prospective data were captured at 0 months, 12 months, and 24 months. At each study time-point case note, an IBD database and medical prescription review was undertaken. Data capture included demographics, Montreal classification at baseline, current IBD therapy, cumulative lifetime corticosteroid use (equivalent to prednisolone $\geq 10 \mathrm{mg} /$ day) and IBD-related surgery [28]. IBD disease activity was assessed at each study time point using clinical indices and biomarkers of inflammation (C-reactive protein (CRP) and faecal calprotectin (FC) using a CALPRO ${ }^{\circledR}$ ELISA test). CRP $\geq 5 \mathrm{mg} / \mathrm{L}$ and $/$ or FC $\geq 100 \mu \mathrm{g} / \mathrm{g}$ were considered consistent with active disease as a composite biomarker assessment.

Lifestyle factors were assessed at 0 months, 12 months, and 24 months. Average alcohol consumption was dichotomised into $<20 \mathrm{~g} /$ day versus $\geq 20 \mathrm{~g} /$ day. Habitual physical activity was assessed using a validated self-administered Short International Physical Activity Questionnaire (IPAQ), which approximates metabolic equivalents MET-minutes per week and allows stratification into low, medium, and high activity groups [29].

\subsection{Body Composition, Anthropometric Assessment, and Nutritional Assessment}

Dual energy X-ray absorptiometry (DXA, General Electric Lunar Prodigy Vision bone densitometer (system DF + 13727; Encore version 13.60, Madison WI, USA)) of the lumbar spine, total femur, and whole body was used to evaluate BMD and body composition at 0 months, 12 months, and 24 months using standard protocols [30].

Standard calculations of fat and muscle body components were made from DXA data. Appendicular skeletal muscle (ASM) mass, which is considered functionally relevant lean mass, was calculated as the sum of the lean mass of the arms and legs (kg) [31]. The ASM index (ASMI) is the ASM mass in $\mathrm{kg}$ divided by the height in metres, squared [32]. Fat mass (FM) is the sum of the body fat mass (kg). The fat mass index (FMI) is calculated as FM in kg divided by height in metres, squared [32]. VAT was measured using CoreScan ${ }^{\circledR}$ analysis software, which correlates well with CT scan measurements [33]. VAT was measured in terms of volume $\left(\mathrm{cm}^{3}\right)$, mass (grams), visceral adipose tissue/height index (VHI, VAT volume $\left(\mathrm{cm}^{3}\right) /$ height in metres, squared), and VAT: subcutaneous adipose tissue (SAT) ratio. Height $(\mathrm{m})$, weight $(\mathrm{kg})$, waist circumference $(\mathrm{cm})$, and hip circumference $(\mathrm{cm})$ were measured at the time of the DXA and the waist-hip ratio (WHR) was calculated.

World Health Organisation (WHO) standard categories for BMI were used: $<18.5 \mathrm{~kg} / \mathrm{m}^{2}$ (underweight), $18.5-24.9 \mathrm{~kg} / \mathrm{m}^{2}$ (normal weight), $25-29.9 \mathrm{~kg} / \mathrm{m}^{2}$ (overweight), $\geq 30 \mathrm{~kg} / \mathrm{m}^{2}$ (obese) [34]. Data from the Australian Bureau of Statistics National Health Survey 2014-2015 provided numerical comparisons with age and sex-matched Australian population for BMI and waist circumference [35]. Population-based, age and gender-matched normative data and standard deviation (SD) values from the National Health and Nutritional Examination Survey (NHANES) were used to calculate the 
$z$-scores for ASMI and the fat mass index (FMI) [17,32]. WHO population-based, age and sex-matched normative data of the lumbar spine and femur BMD were used to calculate BMD $t$-scores and $z$-scores [36]. The lowest BMD $t$-score at either site was used to stratify patients as normal $(>-1)$, osteopenic $(\leq-1>-2.5)$, or osteoporotic $(\leq-2.5)$.

Isometric handgrip strength was measured using a Jamar ${ }^{\circledR}$ Digital Hand Dynamometer, representing a robust surrogate measure of whole body strength [31]. Grip strength $z$-scores were calculated from population-based age and sex-matched normative data derived from healthy adult controls [17].

Myopenia was defined as an ASMI $\geq 1$ SD below the age and gender-matched ASMI mean [31]. Sarcopenia was defined using combined anatomical and functional criteria, as both ASMI and grip strength $>1$ SD below the age and gender-matched means [31].

Nutritional assessment was performed at each study time point using clinical and laboratory criteria, including albumin, vitamin D (automated chemiluminescent assay), calcium, haemoglobin, iron studies, vitamin B12, and folate. Low vitamin D was defined as serum $25(\mathrm{OH})$ vitamin D level $<50 \mathrm{nmol} / \mathrm{L}$.

\subsection{Management during Prospective Study Period}

The routine IBD care of enrolled subjects within the tertiary IBD service was consistent with international guidelines (Figure 1) $[37,38]$. The protocolised management of bone health and nutrition was undertaken during the study period. Oral vitamin D supplementation was recommended to those with low levels $(<50 \mathrm{nmol} / \mathrm{L})$. All of the patients were advised to follow a calcium-rich diet and engage in regular exercise. Patients who were found to be osteopenic (or at risk of osteopenia with current corticosteroid use) were advised to take calcium supplementation (1000 mg/day) and oral vitamin D supplementation ( $\geq 1000 \mathrm{IU} /$ day). Patients found to be osteoporotic were referred to the Endocrinology service for consideration of bisphosphonate therapy.

\subsection{Ethical Considerations}

The study was approved by the Royal Adelaide Hospital Research Ethics Committee (\#120304). Whole-body DXA does not confer significant additional radiation to standard BMD assessment. Radiation safety reported that the total radiation dose per DXA study visit was $2.56 \mu \mathrm{Sv}$.

\subsection{Statistical Methods}

Continuous outcomes are presented using means, standard deviations, medians, and interquartile ranges (IQR), with categorical outcomes as counts and percentages, unless otherwise stated. The lack of existing prospective data on body composition in IBD did not allow a formal power calculation. The enrolment of 150 patients was considered sufficient to provide adequate power for multivariable analysis, allowing for a dropout rate of $30 \%$ over the 24 months.

Change over time. Changes in disease activity, treatment, nutrition, and anthropometric and body composition variables in individual patients who underwent repeated measures testing over the 24-month study period were assessed using either linear or logistic mixed effects models, where appropriate. The $\beta$ coefficient describes the change in the body composition variable per unit of time in the study (12 months). In all of the models, time since baseline assessment (years) was the linear fixed effect, with random intercepts per individual. Compound symmetric and unstructured variance-covariance were assumed for the linear and logistic models respectively. In all of the models, residuals and random effect estimates were examined to ensure that the model distributional assumptions appeared satisfied. As a consequence, VHI was log transformed. Missing data values were not included in the analysis.

Assessment of factors associated with body composition. Linear mixed-effects models were constructed to explore prospective clinical associations with serial assessments of BMI, FMI, VHI, ASMI, and BMD (lumbar spine t-score) at 0 months, 12 months, and 24 months. Univariable models 
were constructed, followed by full multivariable models. We report $\beta$ coefficients that describe the change in the dependent variable per unit change in a covariate. Missing data were imputed with cohort means. Significance was set at the 5\% alpha level (two-sided). Analyses were performed on R software v3.4.3 (R Studio, Boston, MA 02210, USA) using the nlme and lme4 packages.

\section{Results}

\subsection{Subject Characteristics}

Overall, 197 patients with IBD were assessed for eligibility during the study enrolment period (April 2012-September 2013), 43 of whom were excluded (Figure 1). Initially, 154 patients were enrolled in the study at baseline, of whom $129(84 \%)$ completed the 12 -month and $110(71 \%)$ completed the 24-month study assessment. Cross-sectional analysis of the cohort at baseline has been previously published (Supplementary Table S1) [17]. Only those patients who underwent repeated measures $(n=129)$ were included in the primary analysis (Table 1$)$.

In brief, $95(74 \%)$ patients had CD, and 34 (26\%) had ulcerative colitis (UC) (Table 1). The median age of the cohort was 31 years (IQR 25-40), with a median IBD disease duration of 7.7 years (IQR 4.5-12.3). The cohort was predominantly Caucasian $(n=121,94 \%)$. Active disease, defined by composite biomarker assessment, was present in 65 patients (50\%) at baseline. Prior to enrolment, 38/95 $(40 \%)$ patients with CD and 1/34 (1\%) patient with UC had undergone abdominal surgery. At baseline, $53(41 \%)$ of patients were prescribed biologic therapy, the use of which increased over the study period (59 $(54 \%)$ patients were prescribed biologic therapy at 24 months, $p=0.004)$. Oral corticosteroid therapy was currently prescribed for $38(29 \%)$ patients, which significantly decreased over time $(p<0.0001)$. Vitamin D levels were low in $52(40 \%)$ of the patients at baseline, 50/52 (96\%) of whom were prescribed replacement therapy. Accordingly, there was a significant increase in vitamin D levels over the study period $(p=0.01)$.

\subsection{Baseline Body Composition}

A detailed cross-sectional analysis of the body composition of this cohort at baseline $(n=137$, as enrolled until July 2013) has been previously published [17].

In brief, of those who underwent repeated measures testing $(n=129)$, the mean BMI at baseline was $26.5 \pm 5.1$; with $33(26 \%)$ patients classified as overweight and $30(23 \%)$ patients classified as obese (Table 2). Myopenia was evident in 24 (19\%) patients, and functional sarcopenia was evident in $12(9 \%)$ patients. Low BMD was prevalent at baseline; with 45 (35\%) patients classified as osteopenic and 3 $(2 \%)$ patients classified as osteoporotic (48 (37\%), with either osteopenia or osteoporosis).

\subsection{Changes in Body Composition over 24 Months}

Anthropometrics. BMI increased over the study period (annual change $\beta=0.43,95 \% \mathrm{CI}=[0.18$, $0.67], p=0.0006$ ), as did the proportion of patients categorised as overweight and obese (at 24 months $31 \%$ overweight and $31 \%$ obese) (Table 2, Figure 2). Waist circumference increased over time ( $\beta=1.4$, $95 \% \mathrm{CI}=[0.4,2.3], p=0.003)$, although no significant change in WHR was observed. Mean BMI, waist circumference, and the proportions of people characterised as overweight or obese, were each numerically higher in the IBD cohort compared to the Australian population stratified by age and gender (Supplementary Table S2). 


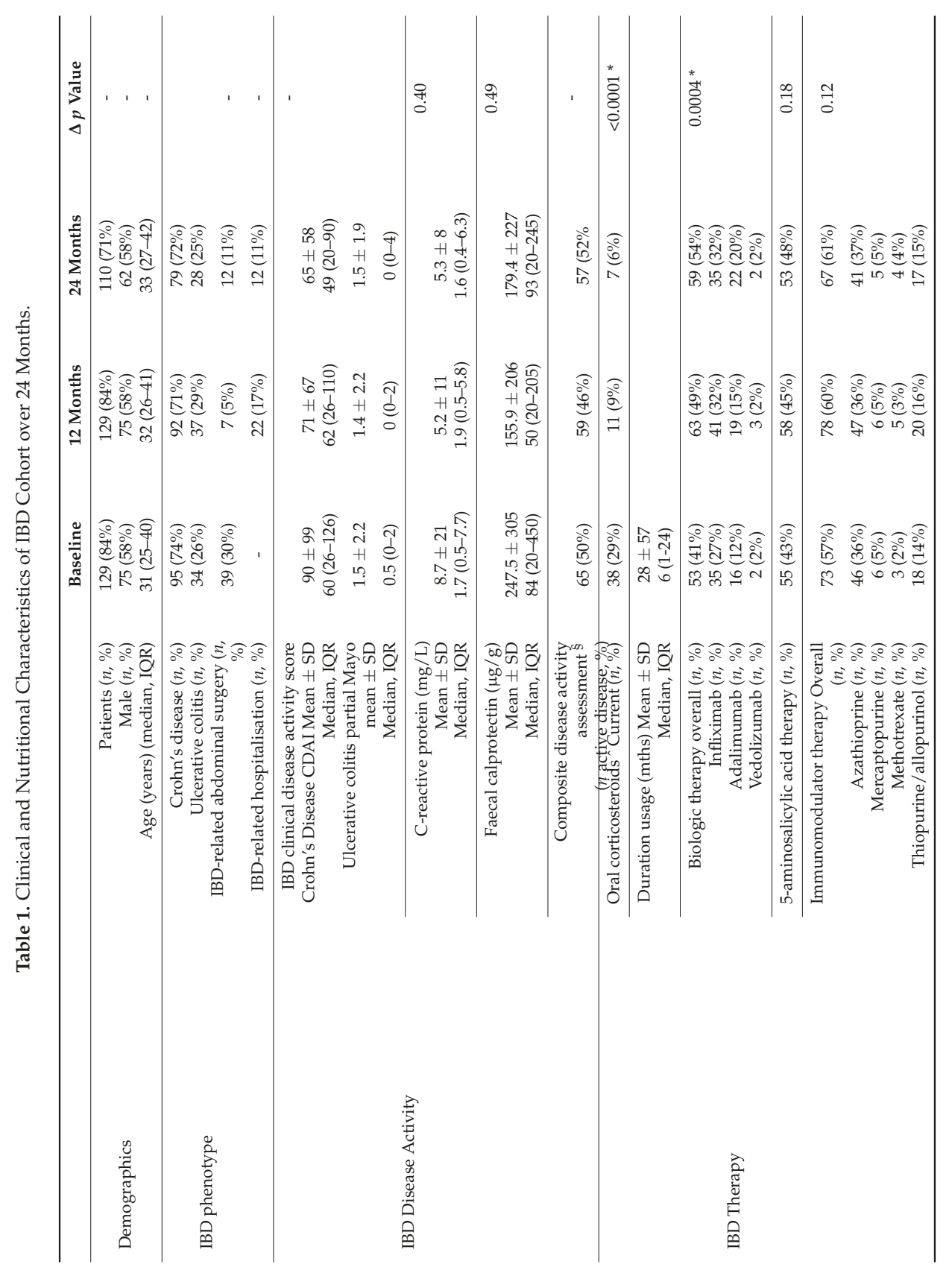




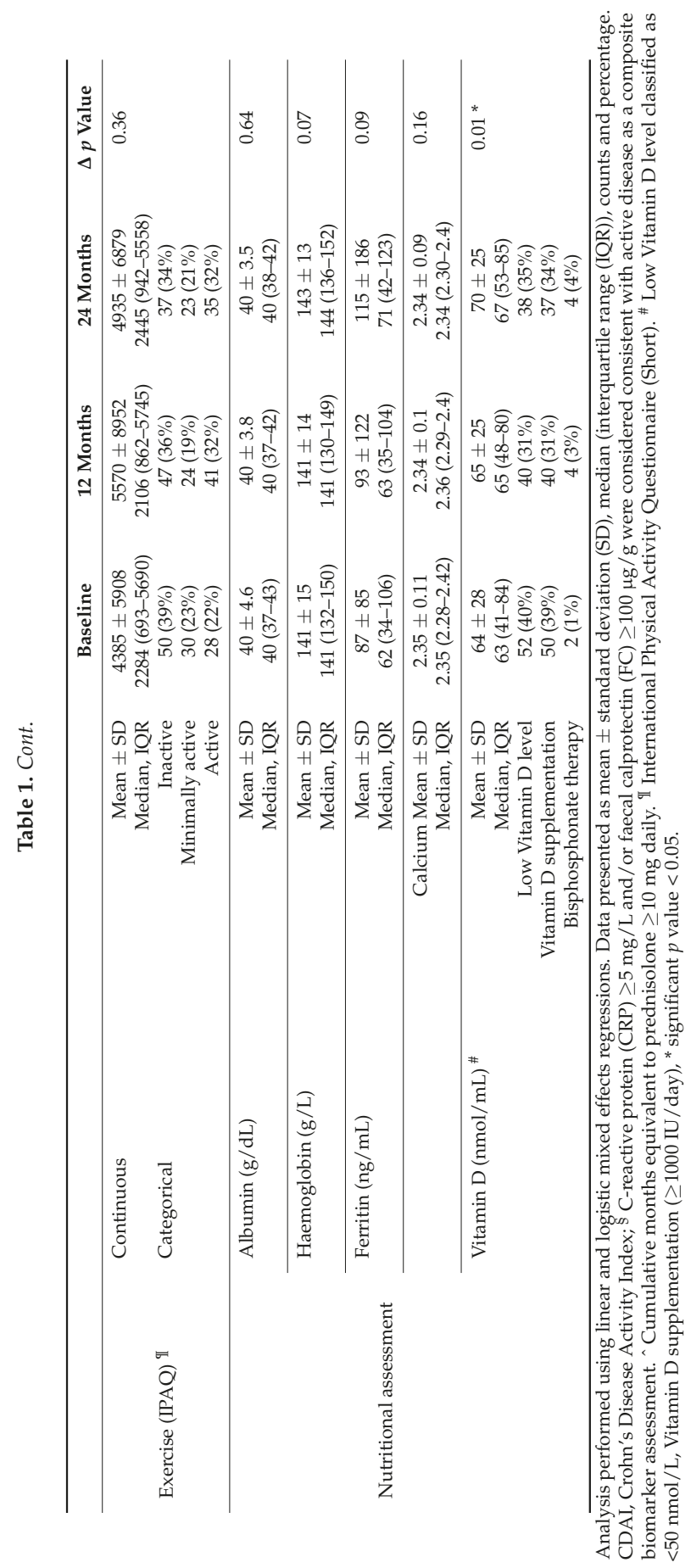




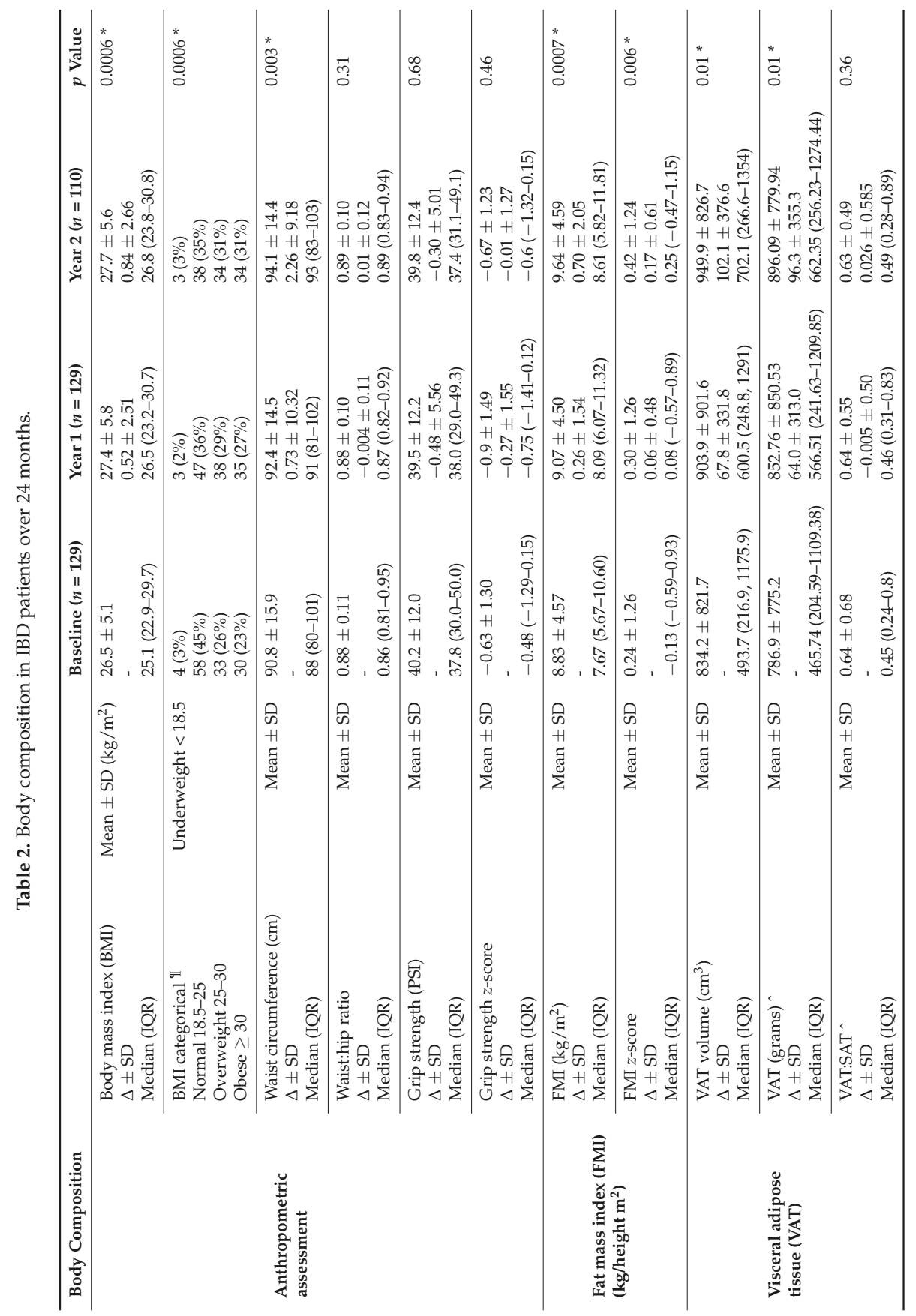




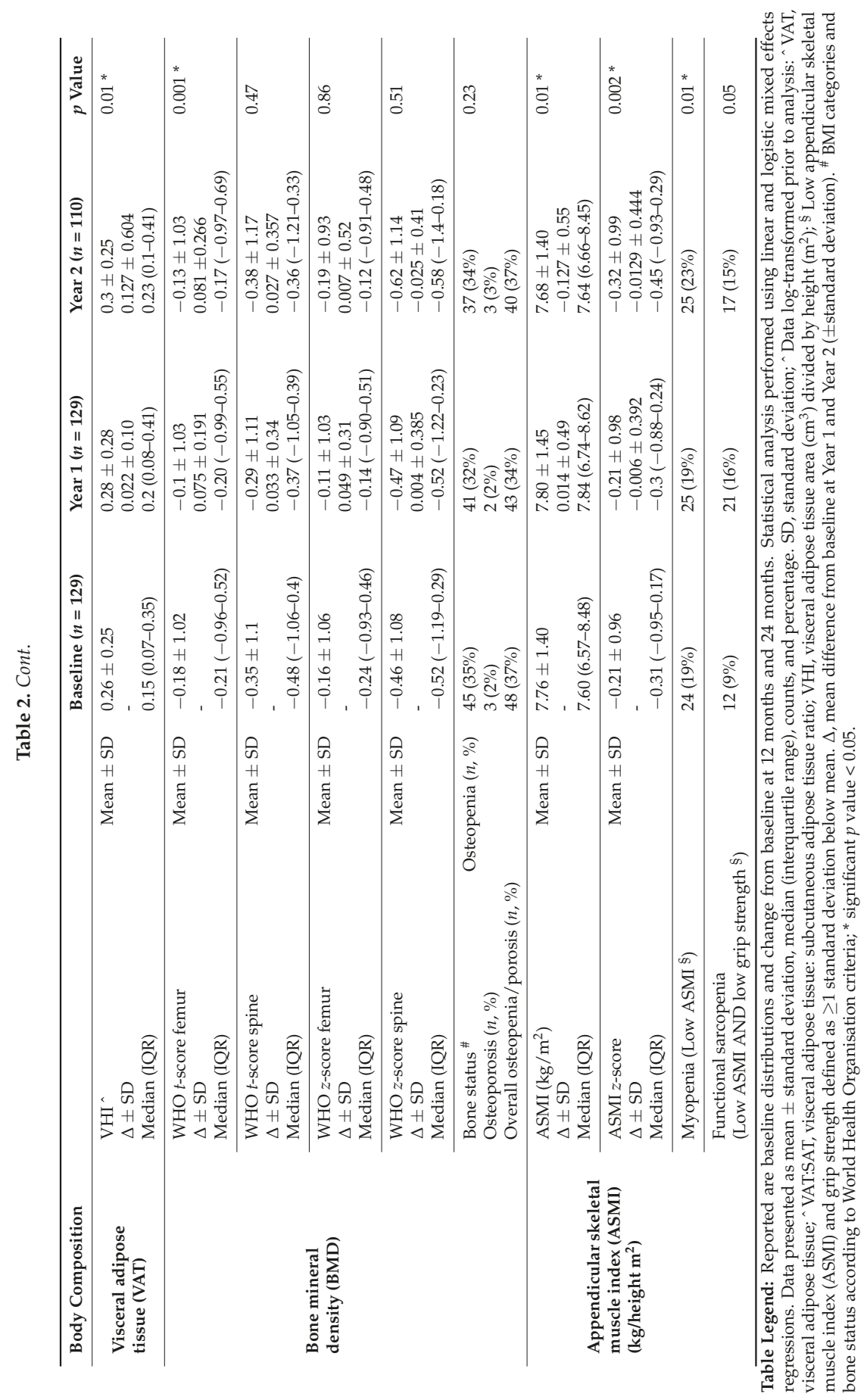



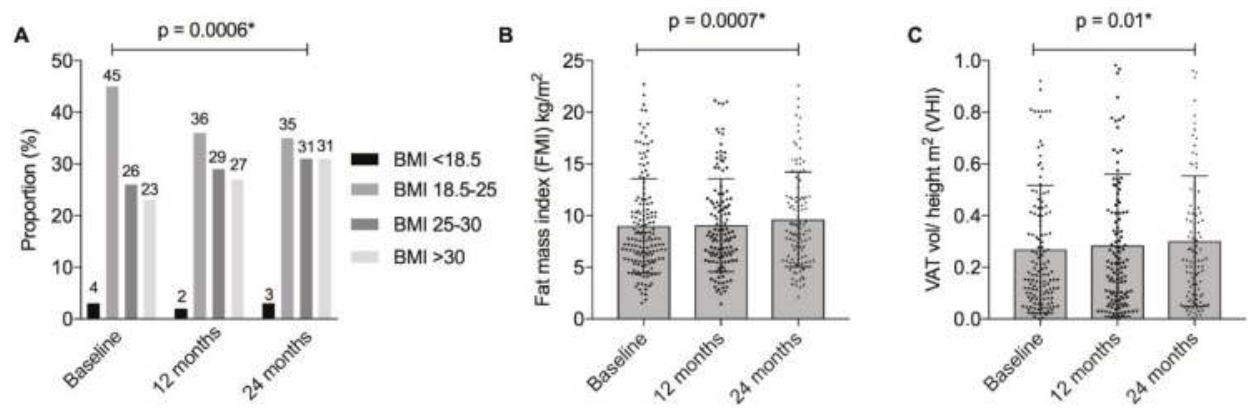

Figure 2. BMI and measures of adiposity in patients with IBD over 24 months. Figure Legend: (A) Body mass index (BMI) categories according to World Health Organisation criteria; (B) Fat mass index $\left(\mathrm{kg} /\right.$ height $\left.\mathrm{m}^{2}\right)$. Mean and standard deviation presented; (C) Visceral adipose tissue (VAT) height index (VAT volume $\mathrm{cm}^{3} /$ height $\mathrm{m}^{2}$ ). Mean and standard deviation presented. ${ }^{*} p<0.05$, denoting statistical significance.

Adiposity. FMI increased significantly over the study period $(\beta=0.33,95 \% \mathrm{CI}=[0.14,0.53]$, $p=0.0007)$, with a concordant increase in FMI $z$-score $(\beta=0.08,95 \% \mathrm{CI}=[0.02,0.14], p=0.006)$. (Table 2, Figures 2 and 3) Similarly, VAT volume increased $(\beta=0.08,95 \% \mathrm{CI}=[0.02,0.14], p=0.01)$, which resulted in an increase in VHI $(\beta=0.08,95 \% \mathrm{CI}=[0.02,0.13], p=0.01)$, but not VAT:SAT $(\beta=0.027,95 \% \mathrm{CI}=[-0.032,0.087], p=0.36)$ (Table 2$)$.

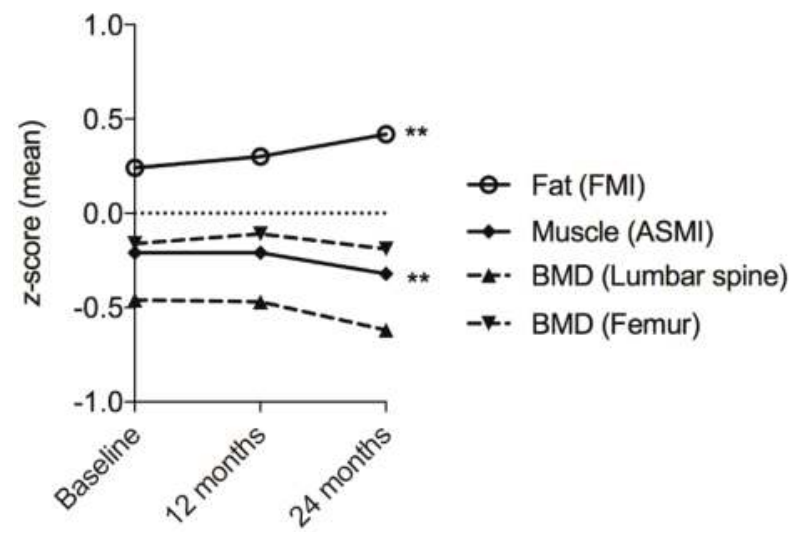

Figure 3. Changes in bone, muscle, and fat in patients with IBD over 24 months. Figure Legend: Summary of mean z-scores for fat mass index (FMI), appendicular skeletal muscle mass index (ASMI), and bone mineral density (BMD), at the femur and lumbar spine. ${ }^{* *} p<0.01$, denoting statistical significance.

Muscle. ASMI decreased significantly over the study period (ASMI $\beta=-0.07,95 \% \mathrm{CI}=[-0.12,-0.01]$, $p=0.01$; ASMI $z$-score $\beta=-0.07,95 \% \mathrm{CI}=[-0.11,-0.02], p=0.002$ ) (Table 2). There was a significant increase in myopenia over the study period (19\%, 19\%, and $24 \%$ at 0 months, 12 months, and 24 months, respectively; $\mathrm{OR}=3.195 \% \mathrm{CI}=[1.2,7.7] ; p=0.01$ ) (Figure 4 ). There was a trend towards an increase in the proportion of patients classified as sarcopenic; however, this did not reach statistical significance $(\mathrm{OR}=2.4$, $95 \% \mathrm{CI}=[1.0,6.0] ; p=0.05)$. 
A

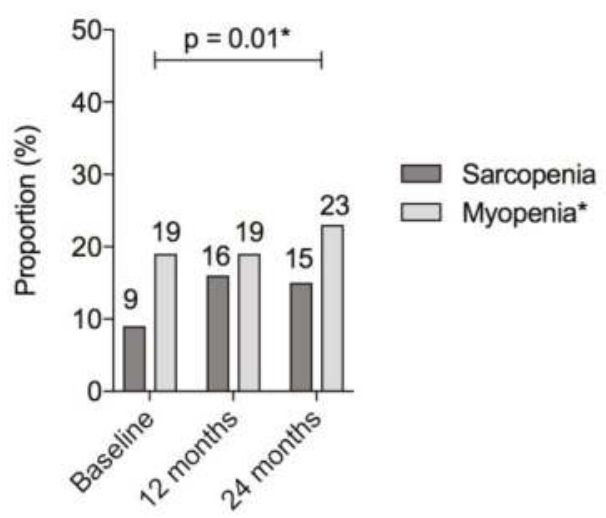

B

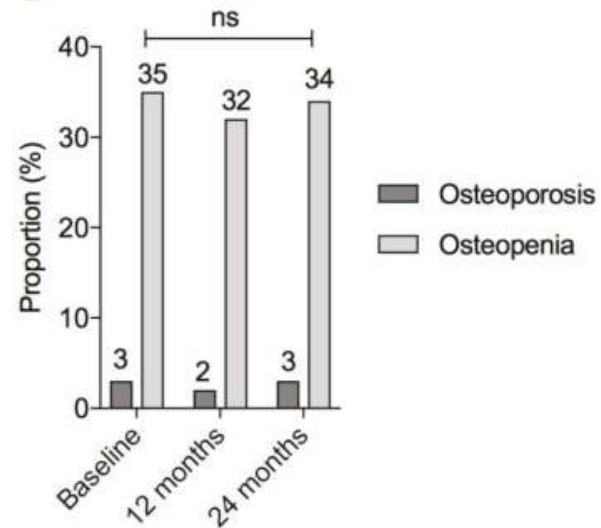

Figure 4. Myopenia, sarcopenia, and metabolic bone disease in patients with IBD over 24 months. Figure Legend: (A) Sarcopenia defined as BOTH appendicular skeletal muscle index (ASMI) and grip strength $<1$ standard deviation below gender and age-matched mean. Myopenia defined as appendicular skeletal muscle index (ASMI) $<1$ standard deviation below gender and age-matched mean; (B) osteopenia and osteoporosis according to World Health Organisation criteria. Osteopenia defined as bone mineral density (BMD) $1-2.5$ standard deviations and osteoporosis as $\geq 2.5$ standard deviations below the young adult mean. ${ }^{*} p<0.05$, denoting statistical significance; ns, non-significant $p$ value $>0.05$.

Bone. Femur BMD $t$-score increased significantly over the study period, but there was no change in lumbar spine BMD $t$-score $(\beta=0.041,95 \% C I=[0.016,0.066], p=0.001 ; \beta=0.013$, $95 \% \mathrm{CI}=[-0.022,0.048], p=0.47$, respectively). No difference in BMD $z$-scores at either site was detected over time (Table 2). Overall, there was no significant change in the proportion of patients classified with osteopenia $37 / 110(34 \%)$ or osteoporosis $3 / 110(3 \%)(p=0.23)$, despite the proactive management of bone health over the study period (Figure 4).

\subsection{Clinical Associations with Serial BMI Measurements}

BMI was positively associated with vitamin D levels $(\beta=0.006,95 \% \mathrm{CI}=[0.00,0.0 .11], p=0.03)$. FMI, ASMI, and grip strength were also positively associated with BMI over time $(\beta=1.0,95 \% \mathrm{CI}=[1.0,1.1]$, $p<0.0001 ; \beta=1.3,95 \% \mathrm{CI}=[1.1,1.5], p<0.0001 ; \beta=0.028,95 \% \mathrm{CI}=[0.008,0.049], p=0.006$, respectively) (Table 3).

\subsection{Clinical Associations with Serial FM and VAT Measurements}

Older age was positively associated with VHI $(\beta=0.040,95 \% \mathrm{CI}=[0.023,0.057], p<0.0001)$ (Supplementary Tables S3 and S4). Male gender was positively associated with VHI, but negatively associated with FMI $(\beta=0.67,95 \% \mathrm{CI}=[0.39,0.95], p<0.0001 ; \beta=-2.2,95 \% \mathrm{CI}=[-2.8,-1.6]$, $p<0.0001$, respectively). Serum vitamin D was negatively associated with FMI $(\beta=-0.007$, $95 \% \mathrm{CI}=[-0.013,-0.001], p=0.02, p=0.02)$.

Anthropometric measures (BMI and waist circumference) were associated with a higher FMI $(\beta=0.52,95 \% \mathrm{CI}=[0.45,0.59], p<0.0001 ; \beta=0.07,95 \% \mathrm{CI}=[0.04,0.10], p<0.0001$, respectively $)$ and VHI $(\beta=0.08,95 \% C I=[0.06,0.11], p<0.0001 ; \beta=0.015,95 \% C I=[0.005,0.026], p=0.003$ respectively $)$. In contrast, grip strength was negatively associated with FMI $(\beta=-0.044,95 \% \mathrm{CI}=[-0.066,-0.021]$, $p<0.0001)$. 


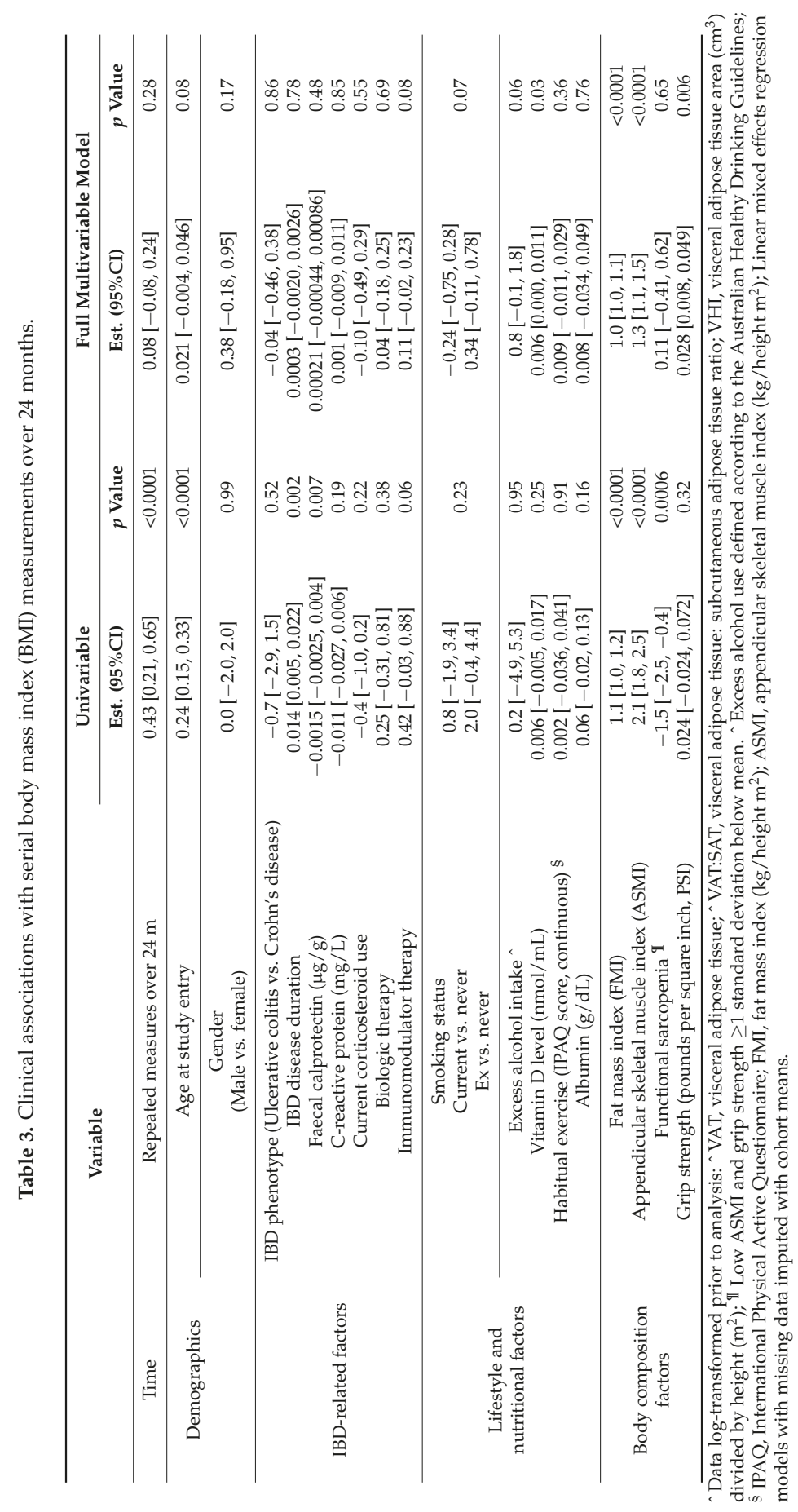




\subsection{Clinical Associations with Serial ASMI Measurements}

Male gender was associated with a higher ASMI $(\beta=1.2,95 \% \mathrm{CI}=[0.9,1.4], p<0.0001)$ (Supplementary Table S5). FC was negatively associated with ASMI $(\beta=-0.00043,95 \% \mathrm{CI}=[-0.00067,-0.00018]$, $p=0.0004)$, whereas anthropometric measures, BMI, and grip strength were positively associated with $\operatorname{ASMI}(\beta=0.13,95 \% \mathrm{CI}=[0.11,0.15], p<0.0001 ; \beta=0.020,95 \% \mathrm{CI}=[0.011,0.028], p<0.0001$, respectively $)$.

\subsection{Clinical Associations with Serial BMD Measurements}

IBD disease duration was negatively associated with lumbar spine $t$-score $(\beta=0.0031$, $95 \% \mathrm{CI}=[-0.0057,-0.0005], p=0.01)$, as was grip strength $(\beta=0.009,95 \% \mathrm{CI}=[0.002,0.017], p=0.01)$ (Supplementary Table S6). Both biologic therapy and corticosteroids (cumulative use) were associated with increase lumbar spine $t$-scores $(\beta=0.08,95 \% \mathrm{CI}=[0.01,0.16], p=0.02 ; \beta=0.10,95 \% \mathrm{CI}=[0.01,0.18]$, $p=0.02$; respectively). Neither IBD phenotype nor vitamin D level was associated with changes in the lumbar spine $t$-score.

\section{Discussion}

This prospective study has demonstrated persistent and progressive disturbances in body composition in people with IBD over a relatively short time frame. The most striking finding was a significant increase in the proportion of patients classified as overweight and obese, driven by gains in adiposity. Conversely, muscle mass decreased, with a concordant rise in rates of myopenia. Despite the proactive management of bone health, rates of osteopenia remained high and unchanged. Abnormal body composition may go frequently unrecognised in clinical practice using BMI alone, leaving patients with IBD at risk of potentially avoidable morbidity.

After 24 months of follow-up, 62\% of patients with IBD were overweight or obese despite a median age of just 33 years, which is numerically higher than age and gender- matched Australian population data [35]. Earlier studies of obesity in IBD have failed to take into account direct measures of adiposity $[3,10]$. This study demonstrates that gains in BMI were associated with significant increases in both overall FM and VAT. VAT is a strong independent predictor of incident cardiovascular disease after adjustment for clinical risk factors, including BMI [39,40]. Similar to to other chronic inflammatory conditions, there is emerging evidence to suggest that patients with IBD are at increased risk of cardiovascular disease, which is now recognised as a prime cause of morbidity in this young demographic [2,41].

How can a link between IBD and obesity be explained? In contrast to retrospective reports, we found no association between measures of adiposity and IBD phenotype, inflammatory burden, or medications over time [3,6-10]. The lack of correlation between obesity and IBD therapy, in particular cumulative corticosteroid use is surprising, yet may be in part accounted for by the significant decrease in rates of corticosteroid use over the study period. Interestingly, serum vitamin D levels were negatively associated with FMI, which may be related to the sequestration of vitamin D in adipose tissue, coupled with lifestyle factors associated with obesity (less outdoor activity) [42]. The majority of patients were classified as either inactive or minimally active, which is likely reflective of the burden of chronic illness in this cohort, yet this was not shown to be associated with BMI. It is possible that dysbiosis in IBD, which is characterised by reduced microbial diversity and an altered microbial metabolic profile, is a predisposing factor to obesity [43-45], but this is speculation despite gut metabolomic associations with post-prandial glucose [43-46].

While adiposity increased, muscle mass (ASMI) decreased. FC, as a measure of luminal inflammation in IBD, was negatively associated with ASMI, which is consistent with the known catabolic effects of chronic inflammation $[18,20,47]$. Lean mass is important in patients with IBD, and has been shown to have a bearing on response to therapy, surgical outcomes, and quality of life [15,18,20,48,49]. Grip strength proved to be a simple anthropometric test that was independently and positively associated with ASMI, while also negatively associated with FMI. 
IBD disease duration was shown to be negatively associated with BMD; however, inflammation was not associated with BMD over time, which is consistent with existing data showing a minimal impact of IBD-related inflammation on longitudinal bone loss [21,23,25,26,50]. Likewise, vitamin D levels were not associated with BMD, which is similar to other studies showing an uncertain benefit of vitamin D supplementation on BMD in IBD patients $[21,23,25,26,50]$. Biologic therapy was found to be positively associated with BMD over time, which is in line with current data $[50,51]$. The positive association between corticosteroid use and BMD is surprising, and may be a spurious result due to multiple comparisons. Nevertheless, rates of osteopenia remained high in this cohort, despite not only protocolised management of bone health, but also a warm climate and readily accessible dairy products.

The limitations of this study are the relatively small sample size, which may have limited statistical power. The study was neither structured nor powered to evaluate the influence of body composition on hospitalisation or surgery over time, nor could it evaluate the impact of these factors on body composition. It did not account for dietary intake, nor were other factors associated with cardiometabolic risk measured (cholesterol, family history, blood pressure). Although a control group was not included, the findings were compared to population-based data [35]. The predominantly Caucasian cohort, which was derived from a single tertiary IBD referral centre, may also limit the generalisability of the findings. On the other hand, it is the first prospective study that has incorporated serial measures of body composition in IBD. Furthermore, DXA, in routine use for measuring BMD, is an affordable tool for monitoring body composition, adding little to the time or radiation of the test $[21,33]$.

This study illustrates the rising rates of obesity in patents with IBD over time, driven by gains in fat mass, while lean mass decreases and metabolic bone disease remains unchanged. Apart from raising concerns about the cardiometabolic risk profile of patients with IBD, it is important that clinicians recognise that an increase in BMI may obscure a decrease in muscle mass (myopenia). The study provides a rationale for measuring body composition, as well as a means to do so with DXA, and supports recommendations for the use of grip strength as a discriminatory anthropometric test in clinical practice.

Supplementary Materials: The following are available online at http:/ /www.mdpi.com/2072-6643/10/9/1192/s1. Supplementary Table S1: Baseline clinical and nutritional characteristics of IBD Cohort; Supplementary Table S2: Body mass index and waist circumference by age as compared to data from the Australian Bureau of Statistic National Health Survey First Results 2014, 2015; Supplementary Table S3: Clinical associations with serial visceral adipose tissue (VHI)^ measurements over 24 months; Supplementary Table S4: Clinical associations with serial fat mass index (FMI) measurements over 24 months; Supplementary Table S5: Clinical associations with serial appendicular skeletal muscle index (ASMI) measurements over 24 months; Supplementary Table S6: Clinical associations with serial bone mineral density measurements (lumbar spine $t$-score) over 24 months.

Author Contributions: R.V.B.: C.G.S., F.D.B., S.P.L.T. and J.M.A. were involved in the conception and design of the project. R.V.B., C.G.S., S.O., C.G., S.N.S., A.D.V., A.L., F.D.B., and J.M.A. were involved in data acquisition and management. All authors were involved in analysis and interpretation of research data, and drafting and revision of the article. All authors approved the final version of the manuscript.

Funding: No funding was received for the study. R.V.B. received research support from the Royal Adelaide Hospital Research Foundation through the AR Clarkson Scholarship Guarantor of article: Robert Bryant.

Acknowledgments: Thank you to the Royal Adelaide Hospital IBD Nurses, Rachel Grafton and Julie Hughes for their assistance of this project. Thank you to the Royal Adelaide Hospital, Hospital Research Foundation for providing the salary scholarship to R.V.B. (AR Clarkson Award) during 2016.

Conflicts of Interest: There are no conflicts of interests to declare. Below is list of declarations for each author, none of which had a role in the design of the study; in the collection, analyses, or interpretation of data; in the writing of the manuscript, and in the decision to publish the results. R.V.B.: Employed by CALHN/TQEH. Grant/Research support/Speaker fees (all paid to employer for research support): AbbVie, Ferring, Janssen, Shire, Takeda. S.P.C.: Employed by CALHN/TQEH. Grant/Research support/Speaker fees (all paid to employer for research support): Pfizer, Merck Sharp \& Dohme, Janssen, Shire. Consultancy with Microbiotica. S.P.L.T.: Employed by Oxford University Hospitals NHS Foundation Trust and the University of Oxford. Grants/Research Support: AbbVie, IOIBD, Lilly, UCB, Vifor, and Norman Collisson Foundation. Consulting Fees: AbbVie, Allergan, Amgen, Asahi, Biogen, Boehringer Ingelheim, Bristol-Myers Squibb, Celgene, Chemocentryx, Cosmo, Enterome, Ferring, Giuliani SpA, GSK, Genentech/Roche, Immunocore, Immunometabolism, Janssen, Lilly, MSD, Neovacs, NovoNordisk, Novartis, NPS Pharmaceuticals, Pfizer, Proximagen, Receptos, Shire, Sigmoid Pharma, Takeda, 
Topivert, UCB, VHsquared, Vifor, Zeria. Speaker fees: AbbVie, Amgen, Biogen, Ferring, Takeda. No stocks or share options. J.M.A.: Employed by CALHN/RAH. Grants/Research support/Speaker \& Consultancy Fees (all paid to employer for research support): Abbot, AbbVie, Allergan, Bayer, Celgene, Ferring, Hospira, Janssen, MSD, Orphan, Pfizer, Shire, Takeda. No other declarations were provided by co-authors.

\section{References}

1. Bryant, R.V.; Trott, M.J.; Bartholomeusz, F.D.; Andrews, J.M. Systematic review: Body composition in adults with inflammatory bowel disease. Aliment. Pharmacol. Ther. 2013, 38, 213-225. [CrossRef] [PubMed]

2. Kirchgesner, J.; Beaugerie, L.; Carrat, F.; Andersen, N.N.; Jess, T.; Schwarzinger, M. Increased risk of acute arterial events in young patients and severely active IBD: A nationwide French cohort study. Gut 2017. [CrossRef] [PubMed]

3. Singh, S.; Dulai, P.S.; Zarrinpar, A.; Ramamoorthy, S.; Sandborn, W.J. Obesity in IBD: Epidemiology, pathogenesis, disease course and treatment outcomes. Nat. Rev. Gastroenterol. Hepatol. 2017, 14, 110-121. [CrossRef] [PubMed]

4. Winer, D.A.; Luck, H.; Tsai, S.; Winer, S. The intestinal immune system in obesity and insulin resistance. Cell Metab. 2016, 23, 413-426. [CrossRef] [PubMed]

5. Karmiris, K.; Koutroubakis, I.E.; Xidakis, C.; Polychronaki, M.; Voudouri, T.; Kouroumalis, E.A. Circulating levels of leptin, adiponectin, resistin, and ghrelin in inflammatory bowel disease. Inflamm. Bowel Dis. 2006, 12, 100-105. [CrossRef] [PubMed]

6. Fink, C.; Karagiannides, I.; Bakirtzi, K.; Pothoulakis, C. Adipose tissue and inflammatory bowel disease pathogenesis. Inflamm. Bowel Dis. 2012, 18, 1550-1557. [CrossRef] [PubMed]

7. Flores, A.; Burstein, E.; Cipher, D.J.; Feagins, L.A. Obesity in inflammatory bowel disease: A marker of less severe disease. Dig. Dis. Sci. 2015, 60, 2436-2445. [CrossRef] [PubMed]

8. Seminerio, J.L.; Koutroubakis, I.E.; Ramos-Rivers, C.; Hashash, J.G.; Dudekula, A.; Regueiro, M.; Baidoo, L.; Barrie, A.; Swoger, J.; Schwartz, M.; et al. Impact of obesity on the management and clinical course of patients with inflammatory bowel disease. Inflamm. Bowel Dis. 2015, 21, 2857-2863. [CrossRef] [PubMed]

9. Hass, D.J.; Brensinger, C.M.; Lewis, J.D.; Lichtenstein, G.R. The impact of increased body mass index on the clinical course of crohn's disease. Clin. Gastroenterol. Hepatol. Off. Clin. Pract. J. Am. Gastroenterol. Assoc. 2006, 4, 482-488. [CrossRef] [PubMed]

10. Chan, S.S.; Luben, R.; Olsen, A.; Tjonneland, A.; Kaaks, R.; Teucher, B.; Lindgren, S.; Grip, O.; Key, T.; Crowe, F.L.; et al. Body mass index and the risk for crohn's disease and ulcerative colitis: Data from a european prospective cohort study (the IBD in EPIC study). Am. J. Gastroenterol. 2013, 108, 575-582. [CrossRef] [PubMed]

11. Buning, C.; von Kraft, C.; Hermsdorf, M.; Gentz, E.; Wirth, E.K.; Valentini, L.; Haas, V. Visceral adipose tissue in patients with crohn's disease correlates with disease activity, inflammatory markers, and outcome. Inflamm. Bowel Dis. 2015, 21, 2590-2597. [CrossRef] [PubMed]

12. Erhayiem, B.; Dhingsa, R.; Hawkey, C.J.; Subramanian, V. Ratio of visceral to subcutaneous fat area is a biomarker of complicated crohn's disease. Clin. Gastroenterol. Hepatol. Off. Clin. Pract. J. Am. Gastroenterol. Assoc. 2011, 9 , 684-687.e681.[CrossRef] [PubMed]

13. Holt, D.Q.; Moore, G.T.; Strauss, B.J.; Hamilton, A.L.; De Cruz, P.; Kamm, M.A. Visceral adiposity predicts post-operative crohn's disease recurrence. Aliment. Pharmacol. Ther. 2017, 45, 1255-1264. [CrossRef] [PubMed]

14. Liu, G.; Wu, X.; Li, Y.; Rui, Y.; Stocchi, L.; Remzi, F.H.; Shen, B. Postoperative excessive gain in visceral adipose tissue as well as body mass index are associated with adverse outcomes of an ileal pouch. Gastroenterol. Rep. 2016, 5, 29-35. [CrossRef] [PubMed]

15. Van Langenberg, D.R.; Gatta, P.D.; Hill, B.; Zacharewicz, E.; Gibson, P.R.; Russell, A.P. Delving into disability in crohn's disease: Dysregulation of molecular pathways may explain skeletal muscle loss in crohn's disease. J. Crohn Colitis 2013, 8, 626-634. [CrossRef] [PubMed]

16. Schneider, S.M.; Al-Jaouni, R.; Filippi, J.; Wiroth, J.B.; Zeanandin, G.; Arab, K.; Hebuterne, X. Sarcopenia is prevalent in patients with crohn's disease in clinical remission. Inflamm. Bowel Dis. 2008, 14, 1562-1568. [CrossRef] [PubMed]

17. Bryant, R.V.; Ooi, S.; Schultz, C.G.; Goess, C.; Grafton, R.; Hughes, J.; Lim, A.; Bartholomeusz, F.D.; Andrews, J.M. Low muscle mass and sarcopenia: Common and predictive of osteopenia in inflammatory bowel disease. Aliment. Pharmacol. Ther. 2015, 41, 895-906. [CrossRef] [PubMed] 
18. Adams, D.W.; Gurwara, S.; Silver, H.J.; Horst, S.N.; Beaulieu, D.B.; Schwartz, D.A.; Seidner, D.L. Sarcopenia is common in overweight patients with inflammatory bowel disease and may predict need for surgery. Inflamm. Bowel Dis. 2017, 23, 1182-1186. [CrossRef] [PubMed]

19. Pedersen, M.; Cromwell, J.; Nau, P. Sarcopenia is a predictor of surgical morbidity in inflammatory bowel disease. Inflamm. Bowel Dis. 2017, 23, 1867-1872. [CrossRef] [PubMed]

20. Bamba, S.; Sasaki, M.; Takaoka, A.; Takahashi, K.; Imaeda, H.; Nishida, A.; Inatomi, O.; Sugimoto, M.; Andoh, A. Sarcopenia is a predictive factor for intestinal resection in admitted patients with crohn's disease. PLoS ONE 2017, 12, e0180036. [CrossRef] [PubMed]

21. Harbord, M.; Annese, V.; Vavricka, S.R.; Allez, M.; Barreiro-de Acosta, M.; Boberg, K.M.; Burisch, J.; De Vos, M.; De Vries, A.M.; Dick, A.D.; et al. The first european evidence-based consensus on extra-intestinal manifestations in inflammatory bowel disease. J. Crohn Colitis 2016, 10, 239-254. [CrossRef] [PubMed]

22. Targownik, L.E.; Bernstein, C.N.; Nugent, Z.; Johansson, H.; Oden, A.; McCloskey, E.; Kanis, J.A.; Leslie, W.D. Inflammatory bowel disease and the risk of fracture after controlling for frax. J. Bone Miner. Res. 2013, 28, 1007-1013. [CrossRef] [PubMed]

23. Targownik, L.E.; Bernstein, C.N.; Nugent, Z.; Leslie, W.D. Inflammatory bowel disease has a small effect on bone mineral density and risk for osteoporosis. Clin. Gastroenterol. Hepatol. Off. Clin. Pract. J. Am. Gastroenterol. Assoc. 2013, 11, 278-285. [CrossRef] [PubMed]

24. Bernstein, C.N.; Blanchard, J.F.; Leslie, W.; Wajda, A.; Yu, B.N. The incidence of fracture among patients with inflammatory bowel disease. A population-based cohort study. Ann. Intern. Med. 2000, 133, 795-799. [CrossRef] [PubMed]

25. Casals-Seoane, F.; Chaparro, M.; Mate, J.; Gisbert, J.P. Clinical course of bone metabolism disorders in patients with inflammatory bowel disease: A 5-year prospective study. Inflamm. Bowel Dis. 2016, 22, 1929-1936. [CrossRef] [PubMed]

26. Targownik, L.E.; Leslie, W.D.; Carr, R.; Clara, I.; Miller, N.; Rogala, L.; Graff, L.A.; Walker, J.R.; Bernstein, C.N Longitudinal change in bone mineral density in a population-based cohort of patients with inflammatory bowel disease. Calcif. Tissue Int. 2012, 91, 356-363. [CrossRef] [PubMed]

27. Tosato, M.; Marzetti, E.; Cesari, M.; Savera, G.; Miller, R.R.; Bernabei, R.; Landi, F.; Calvani, R. Measurement of muscle mass in sarcopenia: From imaging to biochemical markers. Aging Clin. Exp. Res. 2017, 29, 19-27. [CrossRef] [PubMed]

28. Satsangi, J.; Silverberg, M.S.; Vermeire, S.; Colombel, J.F. The montreal classification of inflammatory bowel disease: Controversies, consensus, and implications. Gut 2006, 55, 749-753. [CrossRef] [PubMed]

29. Ainsworth, B.E.; Macera, C.A.; Jones, D.A.; Reis, J.P.; Addy, C.L.; Bowles, H.R.; Kohl, H.W. Comparison of the 2001 BRFSS and the IPAQ physical activity questionnaires. Med. Sci. Sports Exerc. 2006, 38, 1584-1592. [CrossRef] [PubMed]

30. Hans, D.B.; Shepherd, J.A.; Schwartz, E.N.; Reid, D.M.; Blake, G.M.; Fordham, J.N.; Fuerst, T.; Hadji, P.; Itabashi, A.; Krieg, M.A.; et al. Peripheral dual-energy X-ray absorptiometry in the management of osteoporosis: The 2007 ISCD official positions. J. Clin. Densitom. Off. J. Int. Soc. Clin. Densitom. 2008, 11, 188-206. [CrossRef] [PubMed]

31. Cruz-Jentoft, A.J.; Baeyens, J.P.; Bauer, J.M.; Boirie, Y.; Cederholm, T.; Landi, F.; Martin, F.C.; Michel, J.P.; Rolland, Y.; Schneider, S.M.; et al. Sarcopenia: European consensus on definition and diagnosis: Report of the european working group on sarcopenia in older people. Age Ageing 2010, 39, 412-423. [CrossRef] [PubMed]

32. Kelly, T.L.; Wilson, K.E.; Heymsfield, S.B. Dual energy X-ray absorptiometry body composition reference values from NHANES. PLoS ONE 2009, 4, e7038. [CrossRef] [PubMed]

33. Kaul, S.; Rothney, M.P.; Peters, D.M.; Wacker, W.K.; Davis, C.E.; Shapiro, M.D.; Ergun, D.L. Dual-energy X-ray absorptiometry for quantification of visceral fat. Obesity 2012, 20, 1313-1318. [CrossRef] [PubMed]

34. Clinical Guidelines on the Identification; Evaluation; Treatment of Overweight and Obesity in Adults: Executive Summary. Expert panel on the identification, evaluation, and treatment of overweight in adults. Am. J. Clin. Nutr. 1998, 68, 899-917. [CrossRef] [PubMed]

35. Australian Bureau of Statistics. National Health Survey: First Results, 2014-2015, 8 December 2015 ed.; Australian Bureau of Statistics: Canberra, Australia, 2015.

36. World Health Organization. Assessment of fracture risk and its application to screening for postmenopausal osteoporosis. Report of a WHO study group. World Health Organ. Tech. Rep. Ser. 1994, 843, 1-129. 
37. Dignass, A.; Lindsay, J.O.; Sturm, A.; Windsor, A.; Colombel, J.F.; Allez, M.; D’Haens, G.; D’Hoore, A.; Mantzaris, G.; Novacek, G.; et al. Second european evidence-based consensus on the diagnosis and management of ulcerative colitis part 2: Current management. J. Crohn Colitis 2012, 6, 991-1030. [CrossRef] [PubMed]

38. Dignass, A.; Van Assche, G.; Lindsay, J.O.; Lemann, M.; Soderholm, J.; Colombel, J.F.; Danese, S.; D’Hoore, A.; Gassull, M.; Gomollon, F.; et al. The second european evidence-based consensus on the diagnosis and management of crohn's disease: Current management. J. Crohn Colitis 2010, 4, 28-62. [CrossRef] [PubMed]

39. Britton, K.A.; Massaro, J.M.; Murabito, J.M.; Kreger, B.E.; Hoffmann, U.; Fox, C.S. Body fat distribution, incident cardiovascular disease, cancer, and all-cause mortality. J. Am. Coll. Cardiol. 2013, 62, 921-925. [CrossRef] [PubMed]

40. Preis, S.R.; Massaro, J.M.; Robins, S.J.; Hoffmann, U.; Vasan, R.S.; Irlbeck, T.; Meigs, J.B.; Sutherland, P.; D'Agostino, R.B., Sr.; O’Donnell, C.J.; et al. Abdominal subcutaneous and visceral adipose tissue and insulin resistance in the framingham heart study. Obesity 2010, 18, 2191-2198. [CrossRef] [PubMed]

41. Singh, S.; Singh, H.; Loftus, E.V., Jr.; Pardi, D.S. Risk of cerebrovascular accidents and ischemic heart disease in patients with inflammatory bowel disease: A systematic review and meta-analysis. Clin. Gastroenterol. Hepatol. Off. Clin. Pract. J. Am. Gastroenterol. Assoc. 2014, 12, 382-393. [CrossRef] [PubMed]

42. Cheng, S.; Massaro, J.M.; Fox, C.S.; Larson, M.G.; Keyes, M.J.; McCabe, E.L.; Robins, S.J.; O’Donnell, C.J.; Hoffmann, U.; Jacques, P.F.; et al. Adiposity, cardiometabolic risk, and vitamin d status: The framingham heart study. Diabetes 2010, 59, 242-248. [CrossRef] [PubMed]

43. Pedersen, H.K.; Gudmundsdottir, V.; Nielsen, H.B.; Hyotylainen, T.; Nielsen, T.; Jensen, B.A.; Forslund, K.; Hildebrand, F.; Prifti, E.; Falony, G.; et al. Human gut microbes impact host serum metabolome and insulin sensitivity. Nature 2016, 535, 376-381. [CrossRef] [PubMed]

44. Qin, J.; Li, Y.; Cai, Z.; Li, S.; Zhu, J.; Zhang, F.; Liang, S.; Zhang, W.; Guan, Y.; Shen, D.; et al. A metagenome-wide association study of gut microbiota in type 2 diabetes. Nature 2012, 490, 55-60. [CrossRef] [PubMed]

45. Le Chatelier, E.; Nielsen, T.; Qin, J.; Prifti, E.; Hildebrand, F.; Falony, G.; Almeida, M.; Arumugam, M.; Batto, J.M.; Kennedy, S.; et al. Richness of human gut microbiome correlates with metabolic markers. Nature 2013, 500, 541-546. [CrossRef] [PubMed]

46. Zeevi, D.; Korem, T.; Zmora, N.; Israeli, D.; Rothschild, D.; Weinberger, A.; Ben-Yacov, O.; Lador, D.; Avnit-Sagi, T.; Lotan-Pompan, M.; et al. Personalized nutrition by prediction of glycemic responses. Cell 2015, 163, 1079-1094. [CrossRef] [PubMed]

47. Subramaniam, K.; Fallon, K.; Ruut, T.; Lane, D.; McKay, R.; Shadbolt, B.; Ang, S.; Cook, M.; Platten, J.; Pavli, P.; et al. Infliximab reverses inflammatory muscle wasting (sarcopenia) in crohn's disease. Aliment. Pharmacol. Ther. 2015, 41, 419-428. [CrossRef] [PubMed]

48. Ding, N.S.; Malietzis, G.; Lung, P.F.C.; Penez, L.; Yip, W.M.; Gabe, S.; Jenkins, J.T.; Hart, A. The body composition profile is associated with response to anti-TNF therapy in crohn's disease and may offer an alternative dosing paradigm. Aliment. Pharmacol. Ther. 2017, 46, 883-891. [CrossRef] [PubMed]

49. Holt, D.Q.; Varma, P.; Strauss, B.J.G.; Rajadurai, A.S.; Moore, G.T. Low muscle mass at initiation of anti-TNF therapy for inflammatory bowel disease is associated with early treatment failure: A retrospective analysis. Eur. J. Clin. Nutr. 2017, 71, 773-777. [CrossRef] [PubMed]

50. Krajcovicova, A.; Hlavaty, T.; Killinger, Z.; Miznerova, E.; Toth, J.; Letkovsky, J.; Nevidanska, M.; Cierny, D.; Koller, T.; Zelinkova, Z.; et al. Combination therapy with an immunomodulator and anti-TNF $\alpha$ agent improves bone mineral density in ibd patients. J. Crohn Colitis 2014, 8, 1693-1701. [CrossRef] [PubMed]

51. Peyrin-Biroulet, L.; Van Assche, G.; Gomez-Ulloa, D.; Garcia-Alvarez, L.; Lara, N.; Black, C.M.; Kachroo, S. Systematic review of tumor necrosis factor antagonists in extraintestinal manifestations in inflammatory bowel disease. Clin. Gastroenterol. Hepatol. Off. Clin. Pract. J. Am. Gastroenterol. Assoc. 2017, 15, $25-36$. [CrossRef] [PubMed]

(c) 2018 by the authors. Licensee MDPI, Basel, Switzerland. This article is an open access article distributed under the terms and conditions of the Creative Commons Attribution (CC BY) license (http:/ / creativecommons.org/licenses/by/4.0/). 


\title{
Cross-Sectional Analysis of Overall Dietary Intake and Mediterranean Dietary Pattern in Patients with Crohn's Disease
}

\author{
Lorian Taylor ${ }^{1}$, Abdulelah Almutairdi ${ }^{1}$, Nusrat Shommu ${ }^{1}$, Richard Fedorak ${ }^{2}$, Subrata Ghosh ${ }^{3}$, \\ Raylene A. Reimer ${ }^{4}$, Remo Panaccione ${ }^{1}$ and Maitreyi Raman ${ }^{1, *}$ \\ 1 Department of Medicine, University of Calgary, Calgary, AB T2N 4N1, Canada; \\ lorian.taylor@ucalgary.ca (L.T.); abdulelah.almutairdi@ucalgary.ca (A.A.); nsshommu@ucalgary.ca (N.S.); \\ rpanacci@ucalgary.ca (R.P.) \\ 2 Faculty of Medicine \& Dentistry, University of Alberta, Edmonton, AB T6G 2R7, Canada; \\ richard.fedorak@ualberta.ca \\ 3 Institute of Translational Medicine, NIHR Biomedical Research Centre, University of Birmingham and \\ Birmingham University Hospitals, Birmingham B15 2TT, UK; s.ghosh@bham.ac.uk \\ 4 Faculty of Kinesiology, University of Calgary, Calgary, AB T2N 4N1, Canada; reimer@ucalgary.ca \\ * Correspondence: mkothand@ucalgary.ca; Tel.: +403-592-5020
}

Received: 17 October 2018; Accepted: 10 November 2018; Published: 14 November 2018

\begin{abstract}
The primary objective of this study was to explore the macro- and micro-nutrient intakes and dietary patterns of patients with Crohn's disease (CD). Secondary objectives were to (a) compare the micronutrient intakes of $\mathrm{CD}$ patients with a representative sample of individuals, (b) describe the macro- and micronutrient intakes of male and female CD patients, and (c) describe Mediterranean diet scores (P-MDS) of male and female CD patients in remission that were recruited from an inflammatory bowel disease (IBD) clinic in Calgary, AB. Consecutive patients with ileal and/or colonic CD in endoscopic remission were recruited for participation in this cross-sectional study. Sixty-seven patients were enrolled with a mean age of 45 , and a Body Mass Index (BMI) $\geq 25$. Compared with the representative sample, patients with $C D$ had similar energy, protein, carbohydrate, and total fat intake. However, polyunsaturated fats (PUFA), omega-6 and 3, and monounsaturated fats (MUFA) were lower in $\mathrm{CD}$ patients and dietary fiber intake was higher $(p<0.05)$. Vitamins $\mathrm{C}, \mathrm{D}$, thiamin, niacin, magnesium, phosphorus, zinc, and potassium were all significantly lower in all CD patients when compared to the representative sample $(p<0.05)$. Few patients with CD met the P-MDS criteria and overall scores were low (mean 4.5, Standard Deviation (SD) $=1.1$ in males and 4.7, SD $=1.8$ in females). The CD patients in this study had suboptimal dietary intakes and patterns and these data may be used to inform future dietary interventions in this population to improve intake.
\end{abstract}

Keywords: Crohn's disease; dietary intake; malnutrition; Mediterranean diet

\section{Introduction}

There is a compelling argument for environmental factors such as diet to play a role in the course of inflammatory bowel disease (IBD) [1]. Given the mounting evidence for the role of diet and gut microbial composition and function in IBD development and exacerbation, a richer understanding of the role of diet in disease pathogenesis is warranted. Perturbations related to dietary intake are thought to relate to the consumption of a dietary pattern that negatively alters gut microbiota composition [2,3], leading to functional changes in short chain fatty acid (SCFA) profiles affecting the inflammation process [4] and stimulating an inappropriate immune activation of the gut mucosa.

There are limited published data describing the dietary patterns and macro- and micronutrient intakes in patients with Crohn's disease (CD); in particular, the differences between males and females. 
Nearly $50 \%$ of CD patients perceive diet to be an initiating factor in their disease, and over half of patients have reported diet exacerbates disease severity [5]. In IBD, deficiencies of micro- and macronutrients are observed and arise through multifactorial etiologies including disruptions in digestion, malabsorption, and disease activity, resulting in increased energy and nutrient requirements, anorexia, consumption of a nondiversified diet with food avoidance due to symptoms, and cachexia arising as a consequence of pro-inflammatory cytokines [6]. The reported prevalence of malnutrition is variable (12-85\%) [7-9] and depends on disease activity and the definitions used to define malnutrition. Nutrient deficiencies usually develop over time and are linked to duration of illness. Macronutrient deficiencies leading to protein-energy malnutrition are less common in the biologic era, however, they are still of significant prevalence in patients admitted to hospital. Overt malnutrition may lead to complications such as frequent infections, poor immunity, and increased hospitalizations [10]. Micronutrient deficiencies occur for multiple reasons including chronic blood loss leading to iron deficiency; chronic diarrhea resulting in hypomagnesemia or zinc deficiency; malabsorption leading to B12, folate, and vitamin D deficiencies; and other deficiencies such as antioxidants [11,12]. No previous study has reported the prevalence of macro- and micronutrient deficiencies in Canadian CD patients compared to a representative population.

While single nutrients may play a role in the natural history of IBD $[13,14]$, single nutrients are rarely consumed in isolation. Specific to IBD, epidemiological studies have described associations of increased risk of IBD and high dietary intake of refined sugar, grains, calories, animal fat from meat and dairy, and regular intake of processed foods, while a diet high in fruit, vegetables, and dietary fiber decreases risk $[15,16]$. A recent meta-analysis showed an inverse association between the intake of vegetables and fruit and $\mathrm{CD}$, respectively (pooled odds ratio for the highest versus lowest consumption of fruit $=0.57 ; 95 \%$ Confidence Interval (CI) $0.44-0.74$ ) [17]. Patients consuming a pro-inflammatory diet (e.g., high in animal protein, low in fruit and vegetables) have demonstrated a higher risk of ulcerative colitis [18]. Recently, the association between food intake and course of disease in patients with IBD was explored using fecal calprotectin and a protective role of legumes and potato, with a detrimental influence of meat in maintaining clinical remission in IBD was identified [19].

There is a large body of evidence showing Mediterranean dietary patterns regulate inflammation in chronic disease [20-22]. A Mediterranean dietary pattern is high in extra-virgin olive oil, vegetables, fruit, legumes, nuts, and seeds with a moderate consumption of fish, poultry, and milk products and is low in processed foods, baked goods, and red and processed meat. This dietary pattern is high in monounsaturated fats (MUFA), omega-3 polyunsaturated fats (PUFA), fermentable fibers and polyphenols, and adherence to a Mediterranean dietary pattern is associated with lower levels of inflammation biomarkers. A Mediterranean dietary pattern has shown positive effects in chronic disease [23], although it may impact men and women differently [24], and has been understudied in IBD.

The primary objective of this study was to examine the macro- and micro-nutrient intakes and dietary patterns of patients with $\mathrm{CD}$ to inform future dietary interventions. Secondary objectives were to (a) compare micronutrient intakes of $\mathrm{CD}$ patients to a representative sample of individuals, (b) describe macro- and micronutrient intakes of male and female CD patients, and (c) describe the Mediterranean diet scores (P-MDS) of male and female CD patients in a Canadian ambulatory care gastroenterology clinical setting.

\section{Methods}

\subsection{Study Design and Recruitment}

A single center cross-sectional study was undertaken at the University of Calgary, Foothills Medical Center, in Calgary, AB, Canada. Eligible patients were recruited consecutively from ambulatory gastrointestinal clinics at this site. 


\subsection{Eligibility Criteria}

To be eligible for the study, patients were required to: (a) be $\geq 18$ years of age; (b) have a documented diagnosis of ileal and/or colonic CD based on clinical, radiological and endoscopic criteria; (c) be in clinical and endoscopic remission, defined by a Harvey Bradshaw Index (HBI) $<5$ with evidence of mucosal healing by endoscopy defined by no large ulcers, inflammation, or strictures, within three months of recruitment; (d) been in steroid-free remission for three months prior to study entry; (e) achieved induction of remission either through anti-tumor necrosis factor (anti-TNF) agents or corticosteroids; and (f) provide informed consent. Patients were excluded if: (a) HBI >5, (b) had evidence of active endoscopic mucosal disease, (c) used corticosteroids within the preceding three months, (d) had $>1$ bowel resection, (d) presence of ostomy, (e) used laxatives in the past 3 months, (f) used prebiotic fiber supplements in the past three months, (f) used probiotic supplements in the past three months, and (g) used antibiotics in the past six months.

\subsection{Ethics and Consent}

The study protocol was approved by the University of Calgary Conjoint Health Research Ethics Board REB15-1805. All participants provided informed written consent prior to participating in the study.

\subsection{Data Collection}

Patient characteristics, medications, and symptoms were extracted from the patients' medical record and confirmed with the patient during their first study visit. The following data were collected: age, sex, HBI, recent endoscopic findings, current medication, previous IBD surgery, last use of antibiotics and/or probiotics, corticosteroids, and laxative use.

Dietary intake was collected using prospective 3-day weighed food records. Eligible patients met with the study coordinator to receive training on how to record an accurate 3-day weighed food record. Patients were instructed to document all food and drinks consumed and preparation method on two representative weekdays, and one weekend day. The food records were then reviewed by the study dietitian (RD) with the patient to identify any missing food or drink items.

Three-day dietary intake data were subsequently entered and analyzed using ESHA Food Analysis Software (Version 11.3 X, ESHA Food Processor Nutrition Analysis Software, Salem OR, USA) [25]. Intake data was reviewed for acceptable ranges of macro- and micronutrients. Adherence to a Mediterranean diet was measured using an adapted 13-item PREDIMED Mediterranean Diet Score (P-MDS) [26]; points were not given for red wine consumption considering the impact alcohol consumption may have on symptoms related to IBD. Each item in the P-MDS received a score of 0 or 1 , and items were evenly weighted. Total P-MDS scores ranged from 0-13.

Macro- and micronutrient intakes of $\mathrm{CD}$ patients were compared to population usual nutrient intakes from the Canadian Community Health Survey (CCHS; Cycle 2.2) [27]. A complete description of the sampling frame is provided in the following documentation by Statistics Canada (www.statcan.ca/cgi-bin/imdb/p2SV.pl?FunctioN=getSurvey\&SDDS=5049\&lang=en\&db= $\mathrm{IMDB} \& \mathrm{dbg}=\mathrm{f} \& \mathrm{adm}=8 \& \mathrm{dis}=2 \# \mathrm{mb}$ ). The sampling strategy was designed to provide a representative sample based on age, sex, geography, and socioeconomic status [28]. The CCHS used interviews to collect 24-h recall data with a subsample of respondents completing a second recall. To transfer daily intake into usual intake, a measurement error model was used to reduce the effect of within-individual variance and measure between-individual variance [27]. Data were compared for the following parameters: (a) total energy intake in kilocalories (kcal); (b) protein, carbohydrate, fiber, total fat, and PUFA including omega-6 and 3, MUFA and saturated fat (SFA) as proportions of total energy intake; and (c) select micronutrients (vitamins and minerals) reported as a percentage of daily recommended intakes (DRI) and mean daily/usual intakes. 


\subsection{Statistical Analysis}

Statistical analysis was carried out using SPSS (IBM Corp. Version 24.0, Armonk, NY, USA). Data were expressed as means with standard deviation (SD) or standard errors (SE) where it facilitated a comparison with normative population data. Median and interquartile ranges (IQR) were presented for P-MDS due to the variability of the data. Comparisons were done using independent sample $t$-tests to identify mean differences between males and females and between CD patients' and population usual intakes. Chi-squared tests were conducted to identify gender differences in P-MDS for categorical variables and independent sample t-tests were used for mean P-MDS scores. Statistical significance was established at $p<0.05$.

\section{Results}

\subsection{Demographic and Health}

Eighty-seven patients were originally recruited to participate, but complete dietary data were only completed by sixty-seven patients. These 67 patients had a mean age of 45 , and a mean BMI characterized as overweight (Table 1). Most patients (female $=88 \%$, male $=73 \%$ ) were receiving anti-TNF maintenance therapy. Patients who were not receiving anti-TNF therapies were not on other IBD specific therapies including dietary therapies. Less than half of the study participants received immunomodulators. Close to one quarter of patients had one previous small bowel resection, none had colonic resections, and no patients had an ileostomy.

Table 1. Patient demographics and health information.

\begin{tabular}{ccc}
\hline & Female $N=\mathbf{3 4}$ & Male $N=\mathbf{3 3}$ \\
\hline Age in years (mean, SD) & $44.7(14.4)$ & $49.7(12.7)$ \\
BMI (kg/m ${ }^{2}$; mean, SD) & $27.8(6.1)$ & $26.7(3.9)$ \\
Anti-TNF $\boldsymbol{n}, \mathbf{( \% )}$ & $30(88.2 \%)$ & $24(72.7 \%)$ \\
IMM $n \mathbf{~ ( \% )}$ & $15(44.1 \%)$ & $14(42.4 \%)$ \\
Previous bowel surgery $\boldsymbol{n}, \mathbf{( \% )}$ & $7(20.6 \%)$ & $11(33.3 \%)$ \\
\hline
\end{tabular}

Abbreviations: SD: Standard Deviation, BMI: body mass index, Anti-TNF: Anti-tumor necrosis factor, IMM: Receiving immunomodulators.

\subsection{Macronutrient Intake}

Compared with the representative sample, male and female patients with CD had similar energy, protein, carbohydrate, and total fat intake (Table 2). However, fat intake from PUFA, omega-6, omega-3 in females only, and MUFA were significantly lower in patients with CD when compared to the representative sample of Albertans (Table 2). Dietary fiber intake was significantly higher in patients with $\mathrm{CD}$ when compared to the representative sample. 


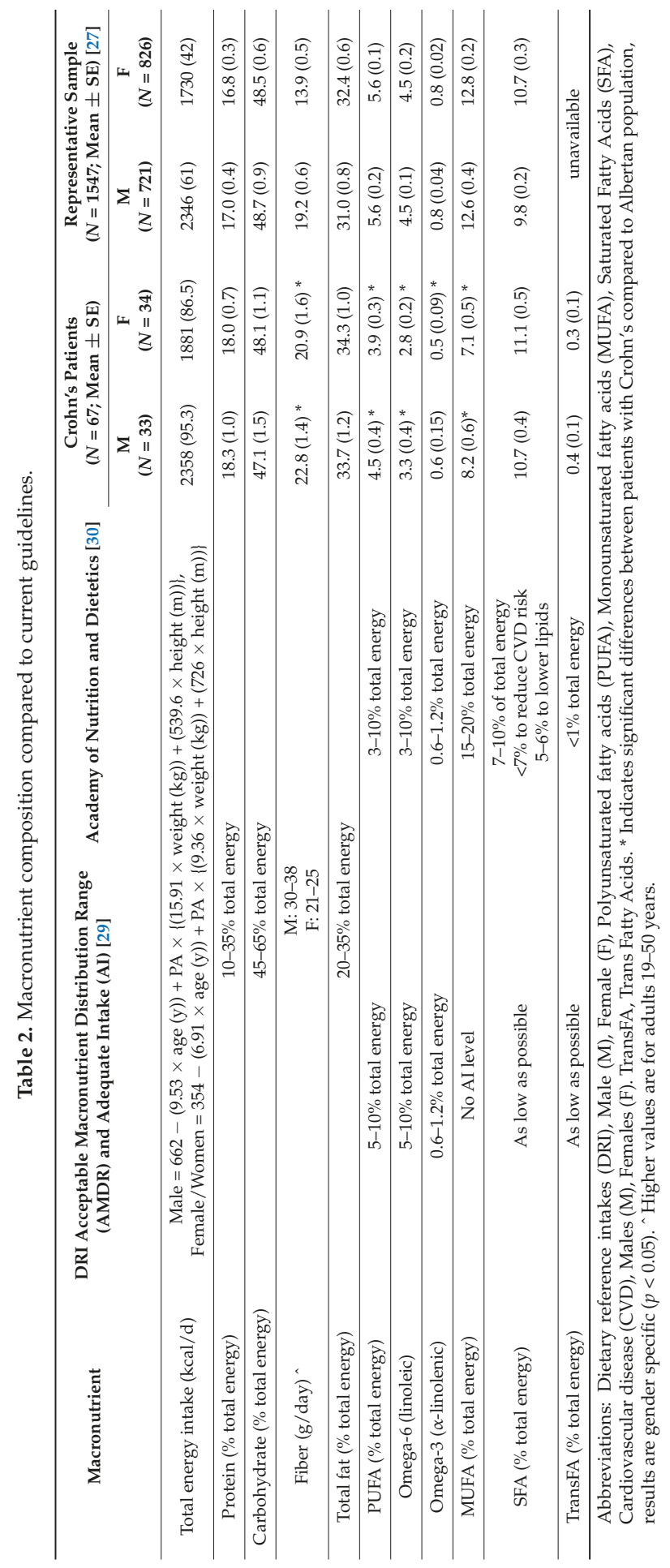




\subsection{Vitamin Intake}

Vitamin comparisons are all listed in Table 3. Compared to the representative sample, vitamins $\mathrm{C}, \mathrm{D}$, thiamin, and niacin were significantly lower in male and female patients with $C D$, whereas a lower intake of riboflavin and pyridoxine was observed only in female patients with $C D$, and lower folate intake was noted only in males with $\mathrm{CD}$. Males compared to female patients with CD had a significantly lower intake of vitamin $\mathrm{K}$, and higher thiamin, niacin, pyridoxine, and biotin intakes. Compared to the DRIs, both male and female patients had inadequate intakes of vitamins A, D, E, pantothenic acid, biotin, folate, and choline, although there was a high level of variation around the mean scores. Representative sample data for some vitamins (E, K, pantothenic acid, biotin, and choline) and minerals (chromium, copper, manganese, and selenium) were not available for comparison.

\subsection{Mineral Intake}

Compared to the representative sample, intakes of magnesium, phosphorus, zinc, and potassium was lower for both male and female CD patients, whereas sodium intake was higher in female patients (Table 4). Compared to male patients with CD, female patients had lower intakes of iron, phosphorus, and sodium. Both male and female patients with CD did not meet the DRI adequacy for calcium, chromium, magnesium, zinc, or potassium.

\subsection{P-MDS Adherence}

Few patients with CD met the P-MDS criteria for olive oil, vegetable, legume intake or consumption of sofrito sauce (made from olive oil, onions, garlic, and tomatoes, and is similar to spaghetti sauce; Table 5). One quarter of male patients and one fifth of female patients met the P-MDS criteria for fruit servings, while $36 \%$ of males and $24 \%$ of females met the P-MDS criteria for nuts. Fish and/or shellfish intake had a median intake of 0 per week and $21 \%$ of males and $32 \%$ of females ate more than three or more servings a week. Over $70 \%$ of patients met the serving recommendations for limiting butter, sugar sweetened beverages, and red or processed meat, and approximately half of the patients limited baked goods and sweets to less than three times a week. Although patients reported limiting red meat according to the P-MDS criteria ( $<6$ oz or $175 \mathrm{~g} /$ day), few chose poultry over red meat on a regular basis. 

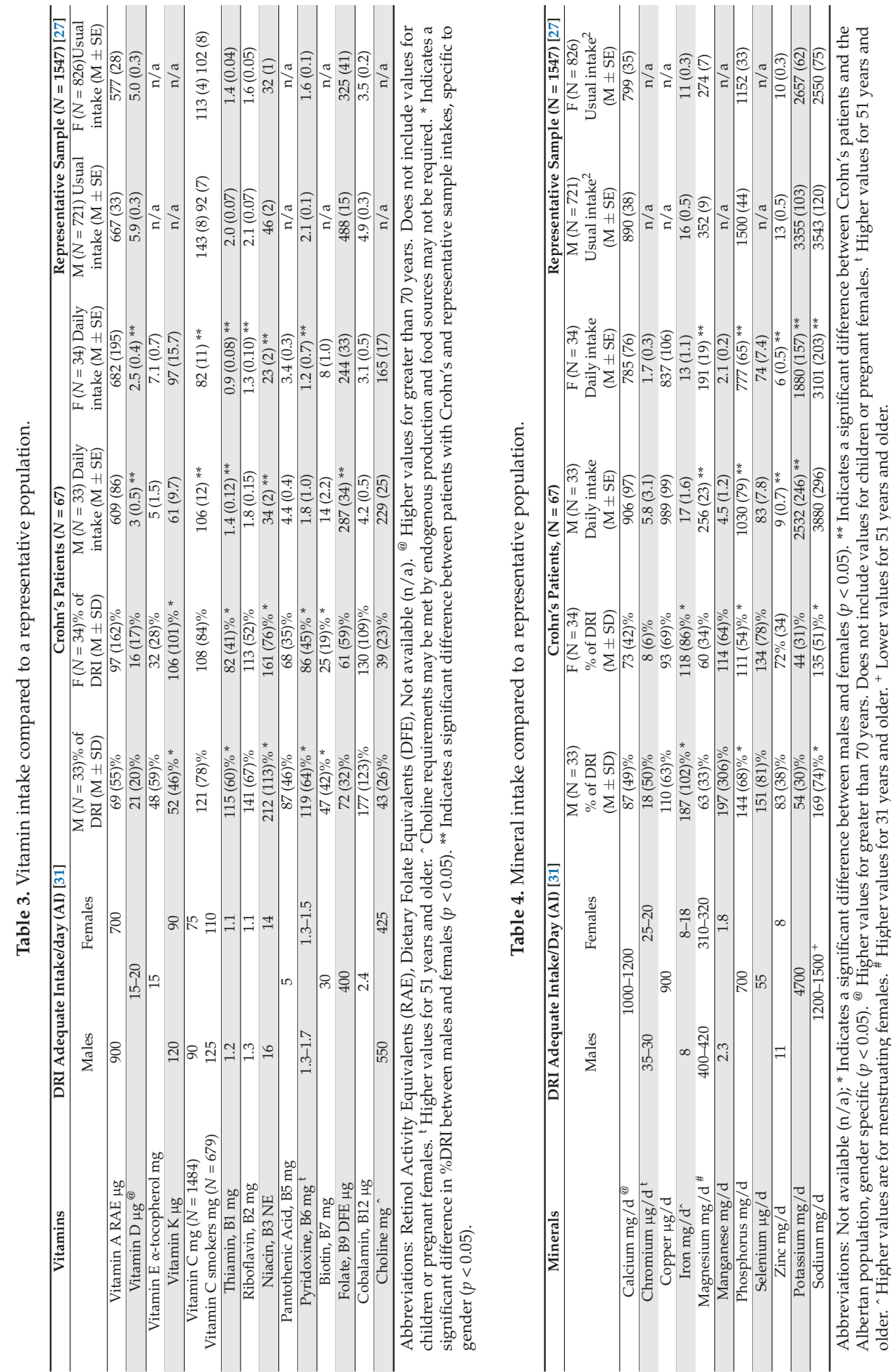


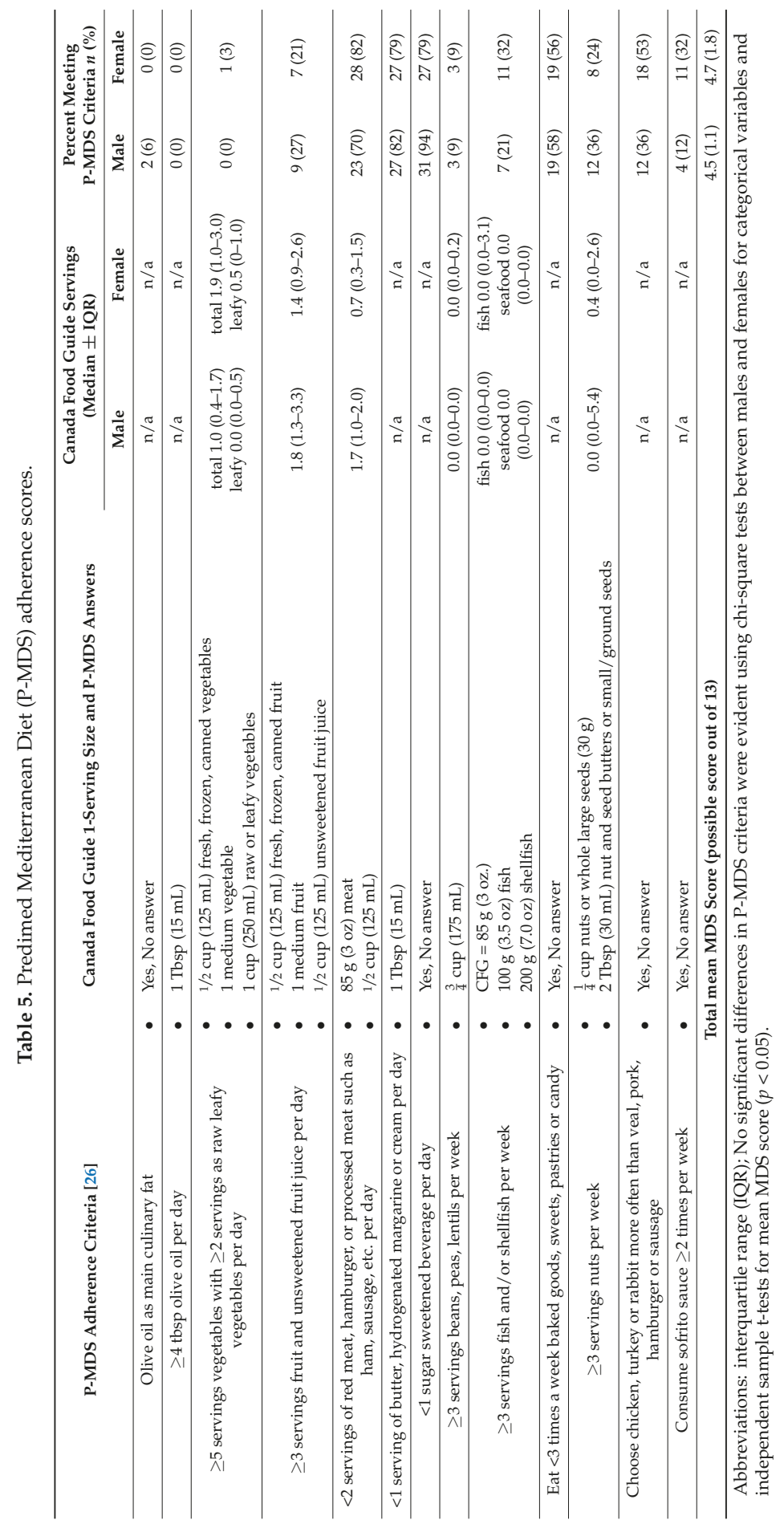




\section{Discussion}

The present study is a description of the dietary patterns and food intake at one time point at a single center, in adult patients in remission with CD. To our knowledge, this was the first study to report on P-MDS in an adult cohort of patients with CD, and to examine the population data and gender differences in nutrient intakes. Major study findings identified significantly different (a) micronutrient intakes between CD patients and a representative sample of individuals; (b) micronutrient intakes between male and female CD patients; and (c) dietary patterns compared to the P-MDS recommendations.

Few published studies have identified dietary predictors of relapse. One such study in $103 \mathrm{CD}$ and ulcerative colitis (UC) patients used a cross-sectional design to evaluate the association between food intake and course of disease; half with active disease [19]. Intake of legumes and potato were inversely associated with the risk of active disease, defined as fecal calprotectin $>150$, with close to $80 \%$ of the highest quartile consumers of legumes at a lower risk of active disease (adjusted Odds Ratio (OR) $0.21,95 \%$ CI 0.57-0.81). Similarly, patients consuming the highest intake of meat coincided with a higher risk of active disease (unadjusted OR 3.6). In the Tasson study [19], significant effects for fruits and vegetables on disease relapse were not identified. The duration of active inflammation before data collection prevented the authors from inferring the causality of diet determinants of relapse. It is compelling to observe that in our current study, only three individuals consumed three or more servings of legumes, and $30 \%$ of male patients demonstrated meat overconsumption.

Similar to our study, significant micronutrient deficits were observed including suboptimal intakes of vitamins A, E, C, folate, and zinc. Vitamins C and E are antioxidants, and supplementation has previously been shown to result in a reduction of oxidative stress [32]. Zinc plays a pivotal role in wound repair, tissue regeneration, and the immune system; zinc deficiency in IBD has been associated with an increased risk of hospitalizations, surgeries, and disease related complications. Normalization of zinc was associated with improvement of these adverse clinical outcomes [33]. Limited dietary diversity including the avoidance of food groups may offer one possible explanation for the deficiency in micronutrient intake. Optimizing dietary diversity through focused nutrition counseling may lead toward an improvement in micronutrient intake.

Compared to the representative sample, dietary MUFA intake from foods like olive and canola oils, avocado, and nuts were low in patients with $\mathrm{CD}$. One recently published study showed that dietary lipid and overall energy intake in a mixed IBD sample was higher than that of the healthy control subjects, however, no differences were observed in protein and carbohydrate intake [34]. Dietary lipid composition, unfortunately, was not reported in this study, rendering the proportion of MUFA and PUFA unknown. An older study in patients stratified by disease severity using the CD Activity Index threshold of 150, showed the mean energy and macronutrient intakes were close to the recommended levels in both patients with active disease and in remission, and reported MUFA intakes of $12 \%$ [35]; the MUFA intakes in our CD patients were significantly lower than this. In rheumatoid arthritis, a high intake of MUFAs, was recently demonstrated to be an independent predictor of remission [36].

Gender differences in micronutrient intakes were observed although there was a large degree of variability in the sample. Females consumed less thiamin compared to males, however, they still met $80 \%$ of the DRI. Given the adequacy of intake, the clinical relevance of this finding is likely not significant. Males only consumed half of the recommended potassium intake, and had lower intakes than females. Both males and females had low intakes of biotin compared to DRI recommendations; however, females were significantly lower than males at $25 \%$. The consequence and etiology for these findings are not readily apparent, although likely result from the extremely low intakes of legumes, fruit and vegetables, and nuts and seeds. Although both males and females exceeded the DRI recommendation for sodium, males had a significantly greater intake, possibly a surrogate for a greater intake of processed foods. Both males and females exceeded DRIs for sodium in the representative sample, however, to a lesser extent than our sample of patients with CD. While the dietary intake 
of sodium is under-reported in IBD, there are signs that show a diet enriched in sodium chloride enhances inflammatory cytokine production and exacerbates colitis in animal models [37].

The dietary composition in our patient sample differed from the P-MDS criteria. Olive oil was seldom used as the primary culinary fat of choice, and few patients in our sample met the P-MDS criteria for intake of vegetables or legumes and fruit including fruit juice, and median intakes were $50 \%$ below the recommended three or more fruit servings a day. For fruit and vegetable intake, the P-MDS criteria are similar to the North American dietary recommendations. Thirty percent of males exceeded the recommendations for red or processed meats per P-MDS criteria. Over $80 \%$ of patients reported inadequate intake of fish and nuts.

Data evaluating the relationship between Mediterranean diets (MD) and disease characteristics (severity and relapse) in IBD are limited. Drawing from the cardiovascular literature, the incidence of major cardiovascular events was lower among participants assigned to an energy-unrestricted MD, and MD supplemented with extra-virgin olive oil or nuts when compared to those assigned to a reduced-fat diet [23]. A subsequent study identified significant reductions in plasma concentrations of high-sensitivity C-reactive protein, interleukin-6, and tumor necrosis factor in patients following a MD supplemented with extra-virgin olive oil or nuts compared to a low fat diet, identifying anti-inflammatory signals to explain the observed clinical findings [38]. Recently, Arpon et al. identified that specific components of the MD, particularly nuts and extra virgin olive oil, were able to induce methylation changes in several peripheral white blood cells, showing a role for specific fatty acids on epigenetic modulation [39]. To our knowledge, studies assessing the gut microbiome composition in long term MD consumers compared to habitual diet in humans have not yet been completed. However, a very recent study identified significant microbiome differences in non-human primates consuming MD when compared to a Western diet [40].

The results of our study provoke further curiosity into the relationships between dietary patterns, nutrition adequacy, and disease pathogenesis in IBD. Protective mechanistic roles for MD patterns in IBD are plausible, mediated through anti-oxidant effects, microbiome changes, and anti-inflammatory effects. Interventional studies are required to test the efficacy of MD patterns on disease severity. Promising effects on disease activity have been observed with elimination diets such as the specific carbohydrate diet [41] and the autoimmune protocol diet [42], among other test diets in the recent literature, highlighting the growing interest in undertaking intervention studies in this population of patients.

The P-MDS was useful in identifying low intakes of foods rich in the deficient micronutrients like olive oil, legumes, nuts, and fruits and vegetables. In patients with suboptimal intakes of these foods, early referral to effective nutrition intervention programs and/or supplementation should be considered. At the present time, in our center, patients are not routinely referred for dietary assessment and counseling, nor do they receive nutrition risk screening. Gastroenterologists and nurse practitioners providing care to patients with IBD at our site generally refrain from offering diet-focused therapies as a solution for disease management in the absence of overt malnutrition. We recognize that the lack of dietary focus as therapy may differ from other centers. We also recognize that patients with IBD assessed at our center are interested in dietary therapies, although the source for nutrition education is not readily apparent. Patients with IBD had a higher dietary fiber intake compared to the representative sample, and $70 \%$ met the serving recommendations to limit butter, sugar sweetened beverages, and processed meat.

There are study limitations that should be noted. This study was conducted at a single Canadian center with a small sample, and therefore, the generalizability of the study findings may be limited. While there are limitations to the use of the 3-day food record to capture dietary intake such as biased estimates of intake due to underreporting or changes in normal dietary patterns, this validated tool has been used extensively to measure food intake in a variety of populations. Deficient dietary intake may not be synonymous with deficient total body stores of micronutrients. The current study design was unable to infer a relationship between intake and body stores. In addition, the representative 
sample data did not report on several micronutrients, so comparisons between patients with IBD and this population were not possible for all micronutrients. This study was underpowered to detect a medium difference between males and females and produced point estimates with a large degree of variability; therefore, results should be interpreted with caution. In addition, multiple analyses were conducted, increasing the risk of type 1 error; however, due to the exploratory nature of this study and limited sample size, a statistical correction was not made and results should be interpreted with this in mind. Additional studies with larger numbers are recommended to confirm this study's conclusions.

Studies such as this one are necessary to identify the need for dietary intervention and offers potential dietary targets for intervention in patients with $\mathrm{CD}$ in an ambulatory care setting. Future directions include a further description of dietary patterns in patients with $\mathrm{CD}$ and dietary intervention studies to test the feasibility, acceptability, compliance, and efficacy of a MD pattern on disease related outcomes. Dietary intervention studies may be regarded as complex interventions, and should be developed based on established frameworks that may include a needs assessment of the population, rationale for intervention development, and pilot studies to inform the details of a subsequent robust clinical trial [43].

\section{Conclusions}

Differences in dietary quality and micronutrient intakes were observed between a representative population and male and female patients with $\mathrm{CD}$. Compared with a MD pattern shown to have anti-inflammatory properties, patients with $\mathrm{CD}$ reported food choices that promote a more restricted nutrient intake. These results suggest patients with CD should be screened for nutrient deficiencies and inadequacy of dietary patterns. Using a simple tool such as the P-MDS may be a practical and effective way to identify pro-inflammatory dietary patterns at an individual level. These data may be used to inform future dietary interventions in this population to optimize dietary intake. Further investigation into the efficacy of nutrition interventions to improve MD quality and the effect this has on clinical outcomes and nutrient deficiency is warranted.

Author Contributions: L.T. made substantial contributions to the analyses and interpretation of data, drafted the article, revised the article critically for important intellectual content, and provided final approval of the version to be submitted. A.A. made substantial contributions to the acquisition of data, drafted the article, and provided final approval of the version to be submitted. N.S. made substantial contributions to the acquisition of data, revised the article critically for important intellectual content, and provided final approval of the version to be submitted. R.F. made substantial contributions to the conception and design of the study, revised the article critically for important intellectual content, and provided final approval of the version to be submitted. S.G. made substantial contributions to the conception and design of the study, data interpretation, revised the article critically for important intellectual content, and provided final approval of the version to be submitted. R.A.R. made substantial contributions to the conception and design of the study, revised the article critically for important intellectual content, and provided final approval of the version to be submitted. R.P. made substantial contribution to the analysis and interpretation of data, revised the article critically for important intellectual content, and provided final approval of the version to be submitted. M.R. made substantial contributions to the conception and design of the study, data interpretation, drafted the article, and provided final approval of the version to be submitted.

Funding: Funding for this study was provided by Broad Foundation.

Acknowledgments: I would like to acknowledge the IBD group at the University of Calgary for referring patients, and Nutrition Services, Calgary Zone for supporting this work through contributing Registered Dietitian expertise. Subrata Ghosh is supported by the NIHR, Biomedical Research Centre, Birmingham.

Conflicts of Interest: The authors declare no conflict of interest.

\section{References}

1. Dutta, A.K.; Chacko, A. Influence of environmental factors on the onset and course of inflammatory bowel disease. World J. Gastroenterol. 2016, 22, 1088-1100. [CrossRef] [PubMed]

2. David, L.A.; Maurice, C.F.; Carmody, R.N.; Gootenberg, D.B.; Button, J.E.; Wolfe, B.E.; Ling, A.V.; Devlin, A.S.; Varma, Y.; Fischbach, M.A.; et al. Diet rapidly and reproducibly alters the human gut microbiome. Nature 2014, 505, 559-563. [CrossRef] [PubMed] 
3. Wu, G.D.; Chen, J.; Hoffmann, C.; Bittinger, K.; Chen, Y.Y.; Keilbaugh, S.A.; Bewtra, M.; Knights, D.; Walters, W.A.; Knight, R.; et al. Linking long-term dietary patterns with gut microbial enterotypes. Science 2011, 334, 105-108. [CrossRef] [PubMed]

4. Bull, M.J.; Plummer, N.T. Part 1: The human gut microbiome in health and disease. Integr. Med. 2014, 13, $17-22$.

5. Limdi, J.K.; Aggarwal, D.; McLaughlin, J.T. Dietary practices and beliefs in patients with inflammatory bowel disease. Inflamm. Bowel Dis. 2016, 22, 164-170. [CrossRef] [PubMed]

6. Hartman, C.; Eliakim, R.; Shamir, R. Nutritional status and nutritional therapy in inflammatory bowel diseases. World J. Gastroenterol. 2009, 15, 2570-2578. [CrossRef] [PubMed]

7. Vadan, R.; Gheorghe, L.S.; Constantinescu, A.; Gheorghe, C. The prevalence of malnutrition and the evolution of nutritional status in patients with moderate to severe forms of Crohn's disease treated with infliximab. Clin. Nutr. 2011, 30, 86-91. [CrossRef] [PubMed]

8. Lomer, M.C. Dietary and nutritional considerations for inflammatory bowel disease. Proc. Nutr. Soc. 2011, 70, 329-335. [CrossRef] [PubMed]

9. Benjamin, J.; Makharia, G.K.; Kalaivani, M.; Joshi, Y.K. Nutritional status of patients with Crohn's disease. Indian J. Gastroenterol. 2008, 27, 195-200. [PubMed]

10. Weisshof, R.; Chermesh, I. Micronutrient deficiencies in inflammatory bowel disease. Curr. Opin. Clin. Nutr. Metab. Care 2015, 18, 576-581. [CrossRef] [PubMed]

11. Lucendo, A.J.; De Rezende, L.C. Importance of nutrition in inflammatory bowel disease. World J. Gastroenterol. 2009, 15, 2081-2088. [CrossRef] [PubMed]

12. Fabisiak, N.; Fabisiak, A.; Watala, C.; Fichna, J. Fat-soluble vitamin deficiencies and inflammatory bowel disease: Systematic review and meta-analysis. J. Clin. Gastroenterol. 2017, 51, 878-889. [CrossRef] [PubMed]

13. Guglielmetti, S.; Fracassetti, D.; Taverniti, V.; Del Bo', C.; Vendrame, S.; Klimis-Zacas, D.; Arioli, S.; Riso, P.; Porrini, M. Differential modulation of human intestinal bifidobacterium populations after consumption of a wild blueberry (Vaccinium angustifolium) drink. J. Agric. Food Chem. 2013, 61, 8134-8140. [CrossRef] [PubMed]

14. Clifford, M.N. Diet-derived phenols in plasma and tissues and their implications for health. Planta Med. 2004, 70, 1103-1114. [CrossRef] [PubMed]

15. Rajendran, N.; Kumar, D. Role of diet in the management of inflammatory bowel disease. World J. Gastroenterol. 2010, 16, 1442-1448. [CrossRef] [PubMed]

16. Riordan, A.M.; Ruxton, C.H.; Hunter, J.O. A review of associations between Crohn's disease and consumption of sugars. Eur. J. Clin. Nutr. 1998, 52, 229-238. [CrossRef] [PubMed]

17. Li, F.; Liu, X.; Wang, W.; Zhang, D. Consumption of vegetables and fruit and the risk of inflammatory bowel disease: A meta-analysis. Eur. J. Gastroenterol. Hepatol. 2015, 27, 623-630. [CrossRef] [PubMed]

18. Shivappa, N.; Hebert, J.R.; Rashvand, S.; Rashidkhani, B.; Hekmatdoost, A. Inflammatory potential of diet and risk of ulcerative colitis in a case-control study from Iran. Nutr. Cancer 2016, 68, 404-409. [CrossRef] [PubMed]

19. Tasson, L.; Canova, C.; Vettorato, M.G.; Savarino, E.; Zanotti, R. Influence of diet on the course of inflammatory bowel disease. Dig. Dis. Sci. 2017, 62, 2087-2094. [CrossRef] [PubMed]

20. Estruch, R. Anti-inflammatory effects of the Mediterranean diet: The experience of the PREDIMED study. Proc. Nutr. Soc. 2010, 69, 333-340. [CrossRef] [PubMed]

21. Steck, S.; Shivappa, N.; Tabung, F.; Harmon, B.E.; Wirth, M.D.; Hurley, T.G.; Hebert, J.R. The dietary inflammatory index: A new tool for assessing diet quality based on inflammatory potential. Digest 2014, 49, $1-9$.

22. Giugliano, D.; Ceriello, A.; Esposito, K. The effects of diet on inflammation: Emphasis on the metabolic syndrome. J. Am. Coll. Cardiol. 2006, 48, 677-685. [CrossRef] [PubMed]

23. Estruch, R.; Ros, E.; Salas-Salvado, J.; Covas, M.I.; Corella, D.; Aros, F.; Gomez-Gracia, E.; Ruiz-Gutierrez, V.; Fiol, M.; Lapetra, J.; et al. Primary prevention of cardiovascular disease with a mediterranean diet supplemented with extra-virgin olive oil or nuts. N. Engl. J. Med. 2018, 378, e34. [CrossRef] [PubMed]

24. Leblanc, V.; Begin, C.; Hudon, A.M.; Royer, M.M.; Corneau, L.; Dodin, S.; Lemieux, S. Gender differences in the long-term effects of a nutritional intervention program promoting the Mediterranean diet: Changes in dietary intakes, eating behaviors, anthropometric and metabolic variables. Nutr. J. 2014, 13, 107. [CrossRef] [PubMed] 
25. ESHA Research. Food Processor Nutrition Analysis Software 11.3 X; ESHA Research: Salem, Oregon, 2018.

26. Schroder, H.; Fito, M.; Estruch, R.; Martinez-Gonzalez, M.A.; Corella, D.; Salas-Salvado, J.; Lamuela-Raventos, R.M.; Ros, E.; Salaverria, I.; Fiol, M.; et al. A short screener is valid for assessing Mediterranean diet adherence among older spanish men and women. J. Nutr. 2011, 141, 1140-1145. [CrossRef] [PubMed]

27. Health Canda; Statistics Canada. Canadian Community Health Survey, Cycle 2.2, Nutrition (2004)—Nutrient Intakes from Food, Provincial, Regional and National Summary Data Tables, Volume 1, 2 and 3. Available online: https:/ / www.canada.ca/en/health-canada/services/food-nutrition/food-nutrition-surveillance/ health-nutrition-surveys/canadian-community-health-survey-cchs/canadian-community-health-surveycycle-2-2-nutrition-focus-food-nutrition-surveillance-health-canada.html\#p1 (accessed on 3 January 2018).

28. Health Canada, Office of Nutrition Policy and Promotion Health Products and Food Branch. Canadian Community Health Survey 2.2, Nutrition (2004): A Guide to Accessing and Interpreting the Data 2006. Available online: https://www.canada.ca/en/health-canada/services/food-nutrition/food-nutritionsurveillance/health-nutrition-surveys / canadian-community-health-survey-cchs / canadian-communityhealth-survey-cycle-2-2-nutrition-2004-guide-accessing-interpreting-data-health-canada-2006.html (accessed on 12 November 2018).

29. Food and Nutrition Board: Institute of Medicine. Dietary Reference Intakes for Energy, Carbohydrate, Fiber, Fat, Fatty Acids, Cholesterol, Protein, and Amino Acids. Available online: http:/ / www.nap.edu/openbook. php?record_id=10490 (accessed on 12 November 2018).

30. Academy of Nutrition and Dietetics. Position of the academy of nutrition and dietetics: dietary fatty acids for healthy adults. J. Acad. Nutr. Dietetics. 2014, 114, 136-153. [CrossRef] [PubMed]

31. Food and Nutrition Board: Institute of Medicine, Health Canada. Dietary Reference Intakes Tables. 2010. Available online: https://www.canada.ca/en/health-canada/services/food-nutrition/healthy-eating/ dietary-reference-intakes/tables.html (accessed on 12 November 2018).

32. Aghdassi, E.; Wendland, B.E.; Steinhart, A.H.; Wolman, S.L.; Jeejeebhoy, K.; Allard, J.P. Antioxidant vitamin supplementation in Crohn's disease decreases oxidative stress. A randomized controlled trial. Am. J. Gastroenterol. 2003, 98, 348-353. [PubMed]

33. Siva, S.; Rubin, D.T.; Gulotta, G.; Wroblewski, K.; Pekow, J. Zinc deficiency is associated with poor clinical outcomes in patients with inflammatory bowel disease. Inflamm. Bowel Dis. 2017, 23, 152-157. [CrossRef] [PubMed]

34. Principi, M.; Losurdo, G.; Iannone, A.; Contaldo, A.; Deflorio, V.; Ranaldo, N.; Pisani, A.; Ierardi, E.; Di Leo, A.; Barone, M. Differences in dietary habits between patients with inflammatory bowel disease in clinical remission and a healthy population. Ann. Gastroenterol. 2018, 31, 469-473. [CrossRef] [PubMed]

35. Aghdassi, E.; Wendland, B.E.; Stapleton, M.; Raman, M.; Allard, J.P. Adequacy of nutritional intake in a canadian population of patients with Crohn's disease. J. Am. Diet. Assoc. 2007, 107, 1575-1580. [CrossRef] [PubMed]

36. Matsumoto, Y.; Sugioka, Y.; Tada, M.; Okano, T.; Mamoto, K.; Inui, K.; Habu, D.; Koike, T. Monounsaturated fatty acids might be key factors in the Mediterranean diet that suppress rheumatoid arthritis disease activity: The TOMORROW study. Clin. Nutr. 2018, 37, 675-680. [CrossRef] [PubMed]

37. Monteleone, I.; Marafini, I.; Dinallo, V.; Di Fusco, D.; Troncone, E.; Zorzi, F.; Laudisi, F.; Monteleone, G. Sodium chloride-enriched diet enhanced inflammatory cytokine production and exacerbated experimental colitis in mice. J. Crohn's Colitis 2017, 11, 237-245. [CrossRef] [PubMed]

38. Casas, R.; Sacanella, E.; Urpi-Sarda, M.; Corella, D.; Castaner, O.; Lamuela-Raventos, R.M.; Salas-Salvado, J.; Martinez-Gonzalez, M.A.; Ros, E.; Estruch, R. Long-term immunomodulatory effects of a Mediterranean diet in adults at high risk of cardiovascular disease in the PREvencion con DIeta MEDiterranea (PREDIMED) randomized controlled trial. J. Nutr. 2016, 146, 1684-1693. [CrossRef] [PubMed]

39. Arpon, A.; Milagro, F.I.; Razquin, C.; Corella, D.; Estruch, R.; Fito, M.; Marti, A.; Martinez-Gonzalez, M.A.; Ros, E.; Salas-Salvado, J.; et al. Impact of consuming extra-virgin olive oil or nuts within a Mediterranean diet on DNA methylation in peripheral white blood cells within the PREDIMED-Navarra randomized controlled trial: A role for dietary lipids. Nutrients 2017, 10, 15. [CrossRef] [PubMed]

40. Nagpal, R.; Shively, C.A.; Appt, S.A.; Register, T.C.; Michalson, K.T.; Vitolins, M.Z.; Yadav, H. Gut microbiome composition in non-human primates consuming a western or Mediterranean diet. Front. Nutr. 2018, 5, 28. [CrossRef] [PubMed] 
41. Suskind, D.L.; Cohen, S.A.; Brittnacher, M.J.; Wahbeh, G.; Lee, D.; Shaffer, M.L.; Braly, K.; Hayden, H.S.; Klein, J.; Gold, B.; et al. Clinical and fecal microbial changes with diet therapy in active inflammatory bowel disease. J. Clin. Gastroenterol. 2018, 52, 155-163. [CrossRef] [PubMed]

42. Konijeti, G.G.; Kim, N.; Lewis, J.D.; Groven, S.; Chandrasekaran, A.; Grandhe, S.; Diamant, C.; Singh, E.; Oliveira, G.; Wang, X.; et al. Efficacy of the autoimmune protocol diet for Inflammatory Bowel Disease. Inflamm. Bowel Dis. 2017, 23, 2054-2060. [CrossRef] [PubMed]

43. Craig, P.; Dieppe, P.; Macintryre, S.; Michie, S.; Nazareth, I.; Petticrew, M. Developing and evaluating complex interventions: The new Medical Research Council guidance. BMJ 2008, 337, a1655. 
Article

\title{
Exclusive Enteral Nutrition: Clinical Effects and Changes in Mucosal Cytokine Profile in Pediatric New Inflammatory Bowel Disease
}

\author{
Helena Rolandsdotter ${ }^{1,2, *}$, Kerstin Jönsson-Videsäter ${ }^{3,4}$, Ulrika L. Fagerberg ${ }^{5,6}$, Yigael Finkel ${ }^{1,2}$ \\ and Michael Eberhardson 7,8 \\ 1 Department of Clinical Science and Education, Södersjukhuset, Karolinska Institutet, 11883 Stockholm, \\ Sweden; Yigael.finkel@sll.se \\ 2 Department of Gastroenterology, Sachs' Children and Youth Hospital, Södersjukhuset, 11883 Stockholm, \\ Sweden \\ 3 Clinical Immunology and Transfusion Medicine, Karolinska University Hospital, 14157 Huddinge, Sweden; \\ kerstin.jonsson-videsater@sll.se \\ 4 Department of Medicine, Huddinge, Karolinska Institutet, 14157 Huddinge, Sweden \\ 5 Department of Women's and Children's Health, Karolinska Institutet, 17177 Stockholm, Sweden; \\ ulrika.fagerberg@ki.se \\ 6 Center for Clinical Research, Västmanland Hospital, Uppsala University, 72189 Västerås, Sweden \\ 7 Department of Gastroenterology, Karolinska University Hospital, 17177 Stockholm, Sweden; \\ Michael.eberhardson@ki.se \\ 8 Department of Medicine Solna, Karolinska Institutet, 17176 Stockholm, Sweden \\ * Correspondence: helena.jonsson.rolandsdotter@ki.se; Tel.: +46-8616-4693
}

Received: 27 December 2018; Accepted: 12 February 2019; Published: 15 February 2019

\begin{abstract}
Exclusive Enteral Nutrition (EEN) is the first-line treatment in children with Crohn's disease (CD) for induction of remission. However, the mode of action remains conjectural. The aim of this study was to investigate whether the effect of EEN is paralleled by changes in the mucosal cytokine profiles (MCP). Twelve children with new onset inflammatory bowel disease (IBD) received induction treatment with a polymeric EEN. We assessed clinical, endoscopic and histologic scoring before and after EEN. Twelve colonic cytokines were analyzed by Polymerase Chain Reaction (PCR) in six of the IBD patients at onset and after EEN as well as in six non-IBD control children at the diagnostic colonoscopy. Twelve children completed 6 weeks of EEN, except from one child who completed 4 weeks. At the control colonoscopy, $83 \%$ were in complete clinical remission. Changes were found in the MCPs of individual patients after EEN. In particular, children with IBD showed significantly higher values of Interleukin (IL)-12 $\beta(p=0.008)$ and IL-23 $\alpha(p=0.02)$ compared to non-IBD controls at the diagnostic colonoscopy. Furthermore, an overall change in proinflammatory cytokines was noted in the IBD-group after treatment. Further studies are warranted to understand the role of EEN in MCP.
\end{abstract}

Keywords: exclusive enteral nutrition; children; IBD; remission; mucosal cytokines

\section{Introduction}

Crohn's disease (CD) and Ulcerative Colitis (UC), the major entities of pediatric IBD, are chronic inflammatory conditions of the gastrointestinal tract. Childhood IBD is characterized by more severe symptoms and wider disease extension at diagnosis compared to adults [1]. Abdominal pain, nausea and bloody diarrhea are common symptoms in both children and adults while growth retardation is frequently seen in pediatric patients [2]. EEN is the first-line treatment for remission induction of CD in pediatric patients. The clinical remission rate is $73-80 \%$ and it effectively induces mucosal healing 
$(\mathrm{MH})$ at a rate that is superior to corticosteroids [3-7]. EEN consists of high energy liquid formulas, which are either elemental, semi-elemental or polymeric based. EEN is recommended to all children with luminal disease, including those with colonic involvement [3].

The immunological effects driven by EEN that contribute to mucosal healing $(\mathrm{MH})$ are not yet fully understood [7]. The intestine hosts a majority of the body's immune cells (70-80\%) [8] and the interactions between a disrupted microbial composition, an impaired intestinal mucosal barrier and the mucosal immune system are considered to play an important role in the IBD development and its chronicity [7]. Some studies suggest that a deficiency of host immunity also contributes to the pathogenesis of IBD [9]. The cytokines, small immune-regulating messenger proteins, play a crucial part in the inflammatory response by regulating cell differentiation, producing proinflammatory cytokines and recruiting white blood cells from the bone marrow [10]. There are only a few studies that show that EEN seems to affect the expression of cytokines in the mucosa [11-13].

The aim of this study was to investigate the clinical effect of EEN as induction therapy and whether the effect of EEN is paralleled by changes in the mucosal cytokine profiles (MCP).

\section{Materials and Methods}

We conducted a prospective cohort study of children with new onset symptoms of possible IBD between August 2013 and September 2016. The inclusion criterion was previously healthy children up to 18 years of age with signs of IBD and the exclusion criterion was any use of immunosuppressive drugs within 6 months prior to inclusion. Thirteen children who fulfilled the ECCO/ESPGHAN consensus criteria for pediatric CD (14) entered the EEN study but one patient immediately left the study after the diagnostic endoscopy. One child received concomitant medication with mesalazine (53 mg/kg/day). Six patients who did not receive any IBD diagnosis were included as non-IBD controls. Demographic data are presented in Table 1.

Table 1. Demography and Paris classification of 13 IBD patients at inclusion.

\begin{tabular}{cc}
\hline Sex (Girls/Boys) & $6 / 7$ \\
\hline Age in years, median (IQR) range & $12.5(10.5-14.5) 7.8-16.4$ \\
Symptom duration in months, median (IQR) range & $6(3.1-11.1) 1.5-12$ \\
Paris classification & No (=13) \\
A1aL1B1G1 & 1 \\
A1bL1B1G0 & 4 \\
A1bL2B1G0 & 4 \\
A1bL2L4bB3G1 & 1 \\
A1bL3L4aB1G0 & 1 \\
A1bL2L4aB1G0 & 1 \\
A1bL3L4aB1G0p & 1 \\
\hline
\end{tabular}

\subsection{Examinations and Tests}

The patients were investigated according to the ECCO/ESPGHAN guidelines for pediatric IBD with initial laboratory investigations: peripheral blood count, liver enzymes, albumin, erythrocyte sedimentation rate (ESR), iron status, C-reactive protein (CRP), fecal calprotectin and stool cultures to exclude infectious etiology. In line with the guidelines, all patients were investigated with upper endoscopy and ileocolonoscopy under general anesthesia with multiple mucosal biopsies for histopathological evaluation [14]. For the study, four mucosal biopsies were obtained from the most inflamed site in the ileum or colon. The endoscopies were documented by a still picture system integrated in the computerized medical record program (Picsara, Mawell, Solna, Sweden). A control upper and lower endoscopy with biopsy harvesting at the same site as the diagnostic endoscopy was performed shortly after completion of EEN remission treatment in children with CD. No CC was performed in the six non-IBD controls. The body weight of study patients was obtained before and after the EEN treatment. 


\subsection{Clinical Assessment of Disease Activity and Disease Extension}

We used the validated disease activity scoring PCDAI (Pediatric CD Activity Index) with $<10$ points for remission, 10-27.5 for mild disease, $>27.5-37.5$ moderate disease and $>37.5-100$ for severe disease. A PCDAI decrease of $>12.5$ points was regarded as a clinically significant response to treatment [15]. PCDAI score was assessed at inclusion and at CC. Disease extension was assessed by Paris classification [16].

\subsection{Endoscopic Scoring}

Simple Endoscopic Score for Crohn's disease (SES-CD) was used to assess the mucosal inflammation in the CD patients [17] and was evaluated either by the endoscopist or by the investigator from the charts (the endoscopy history and endoscopy imaging). SES-CD describes the presence and size of ulcers, extent of the ulcerated surface, extent of the affected surface and the presence and type of narrowing scored with 0-3 points in rectum, sigmoid and left colon, transverse colon, right colon and ileum. Remission is equal to $0-2$ points, mild disease to 3-6 points, moderate to 7-15 points and severe disease to $\geq 16$ points.

\subsection{Histopathology Scoring}

The mucosal biopsies were reviewed by the validated histological Geboes scoring [18]. Grades $<3$ were regarded as the absence of IBD inflammation, while grades $\geq 3$ were regarded as the presence of IBD inflammation of varying degrees (3:1-3:7) with the following subgroups: a = basal plasmacytosis, $\mathrm{b}=$ lymphoid nodules, $\mathrm{c}=$ Paneth cell hyperplasia, $\mathrm{d}=$ eosinophils in the lamina propria, $\mathrm{e}=$ histiocytic cell proliferation, $\mathrm{f}=$ giant cells, $\mathrm{g}=$ granuloma, $\mathrm{h}=$ vasculitis and $\mathrm{i}=$ dysplasia.

\subsection{Exclusive Enteral Nutrition (EEN)}

Fortimel Energy ${ }^{\circledR}$, a polymeric formula, was prescribed to meet a daily energy intake estimated at $120 \%$ of recommended daily allowance (RDA). Fortimel Energy ${ }^{\circledR}$ contains $625 \mathrm{~kJ}, 5.8 \mathrm{~g}$ of fat and $5.9 \mathrm{~g}$ of protein/100 mL (Nutricia Advanced Medical Nutrition, Solna, Sweden). The EEN treatment was introduced by a daily step-wise increase by $25 \%$ of full daily intake for three days, which was followed by six weeks of EEN and rounded off by a step-wise decrease by $25 \%$ of full daily intake for 3 days. The children were allowed to drink clear beverages, eat a few popsicles and a few hard mints per day during the EEN treatment. Patients were allowed additional formula at their own discretion for daily satiation. At de-escalation, the patients were advised to avoid large amounts of dairy products, spicy food and large amount of legumes. The children and caregivers had one introductory session with a dietitian. Thereafter, the dietitian had weekly phone calls to monitor intake and motivate the patients and caregivers to complete the whole EEN treatment period. Scheduled clinical assessments were arranged after two weeks of EEN.

\subsection{Cytokine-Selection}

A panel of cytokines that are known to participate in chronic intestinal inflammation were selected for testing. We investigated Colony Stimulating Factor(CSF)-2, Interferon(IFN)- $\gamma$, Tumor Necrosis Factor(TNF)- $\alpha$, Interleukin(IL)-1 $\beta$, IL-4, IL-6, IL-10, IL-12 $\beta$, IL-22, IL-23 $\alpha$, IL-36 $\gamma$, Tumor Growth factor(TGF)- $\beta 1$ and a control gene ABL. The amount of messenger-RNA (m-RNA) in the different proinflammatory mediators was compared to the signal from the ABL-gene and the result is presented as the cytokine/ABL ratio. We used TaqMan ${ }^{\circledR}$ Gene Expression Assays, applied biosystems (Thermo Fischer Scientific ${ }^{\circledR}$, Waltham, MA, USA)

\subsection{RNA Extraction and Gene Expression by Quantitative Real-Time PCR}

Biopsies for MCP were put into the RNA-later (Invitrogen, Waltham, MA USA) and kept at $+6{ }^{\circ} \mathrm{C}$ for $24 \mathrm{~h}$ and then frozen $\left(-20^{\circ} \mathrm{C}\right)$ until analysis. Total RNA was isolated by utilizing the 
Fibrous tissue kit (Qiagen, Hilden, Germany) with slight modification. Defrosted and minced biopsies ( $3 \mathrm{~mm}$ ) were homogenized with a pestle motor (WVR ${ }^{\circledR}$, Radnor, PA, USA) for 30-60 s in $350 \mu \mathrm{L}$ of RLT-buffer and $350 \mu \mathrm{L}$ of $70 \%$ ethanol was added. The homogenates were loaded into spin columns and centrifuged before the columns were washed with $350 \mu \mathrm{L}$ of RW1-buffer. Samples in the columns were treated by DNase I and washed with $350 \mu \mathrm{L}$ of RW1-buffer, with the remainder handled according to the manufacturer protocol. cDNA was obtained by reverse transcription. Quantitative real-time PCR (qPCR) was performed using the 7500 Fast Real Time PCR System (Applied Biosystems, Foster City, CA, USA) for quantification. Probes were obtained from Applied Biosystems, TaqMan ${ }^{\circledR}$ MGB probes, FAM ${ }^{\mathrm{TM}}$ dye-labeled: IL-1ß (Hs00174097_m1), IL-4 (Hs00174122_m1), IL-6 (Hs00985639_m1), IL-10 (Hs00961622_m1), IL-12ß (Hs001011518_m1), IL-22 (Hs01574154_m1), IL-23 (Hs00900828_g1),

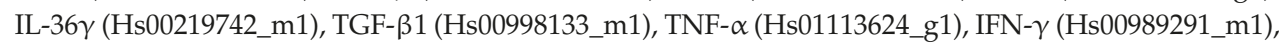
GM-CSF-2 (Hs00929873_m1) and ABL1 (Hs01104728_m1) according to the manufacturer protocol. Fold increases of mRNA transcripts were calculated as follows: $\Delta \mathrm{Ct}=\mathrm{Ct}$ (gene of interest) $-\mathrm{Ct}$ (ABL1), $\Delta \Delta \mathrm{Ct}=\Delta \mathrm{Ct}$ sample - average $\Delta \mathrm{Ct}$ control group and fold difference $=2-\Delta \Delta \mathrm{Ct}$. For technical reasons, $\mathrm{CMCP}$ was not analyzed in all children with IBD both at diagnosis and at CC. No active selection was performed.

\subsection{Ethical Approval}

The study was approved by the local Ethics Committee in Stockholm, Sweden (No. 2010/1252-31/1). Informed written consents were obtained from legal guardians for patients and also from all patients over 15 years of age before any study-related procedures were initiated in accordance with the Helsinki II Declaration.

\subsection{Statistics}

The comparisons between clinical chemistry values, SES-CD and disease activity index (PCDAI) before and after treatment were conducted with paired sample t-tests (parametric data) or Wilcoxon paired t-tests (non-parametric data) depending on the normal distribution (tested with Shapiro-Wilks test). Paired comparisons between cytokines before and after treatment were conducted with Wilcoxon paired t-tests while the comparisons between groups were conducted with Mann-Whitney U test after control of normality with Shapiro-Wilks test. All analyses were performed with the IBM SPSS Statistics Data Editor, version $23^{\circledR}$ (Armonk, NY, USA). Statistical significance was set at $p<0.05$.

\section{Results}

Thirteen children were included with a CD diagnosis according to the ECCO/ESPGHAN guidelines. One $\mathrm{CD}$ patient left the study after the first colonoscopy due to social circumstances. Out of 12 patients who completed EEN treatment, two patients were re-diagnosed with UC and one with IBD-unclassified (IBD-U) at the time of histopathological review after study completion (Table 1 for Paris classification at inclusion).

\subsection{EEN Treatment}

Eleven children completed six weeks of EEN and one CD patient completed only four weeks of EEN due to a lack of motivation, but fulfilled participation in all other aspects of the study. All patients accepted the prescribed EEN by oral intake without the use of nasogastric tube.

\subsection{Clinical Activity Scoring}

Clinical scoring of the children diagnosed with CD was measured by PCDAI, which was also used for the children who had their diagnoses changed to UC $(n=2)$ or IBD-U $(n=1)$. At inclusion, six children were considered to have mild disease (PCDAI 10-27.5) and seven patients showed moderate to severe disease (PCDAI $>27.5)$. There was a significant decrease in PCDAI at CC ( $p=0.02)$. 
The median PCDAI was 26.5 (IQR 20.0-36.6, range 20-40) at inclusion and 5 (IQR 0-5, range 0-15) after EEN treatment. Ten of twelve patients $(83 \%)$ showed clinical remission $(\mathrm{PCDAI}<10)$ after EEN induction treatment.

\subsection{Laboratory Values}

ESR, CRP, hemoglobin, plasma-albumin and F-calprotectin were monitored in all patients. There were significant decreases in $\operatorname{ESR}(p=0.005), \operatorname{CRP}(p=0.016)$ and f-calprotectin $(p=0.033)$ and significant increases in hemoglobin $(p=0.001)$ and p-albumin $(p=0.003)$ after EEN treatment (Table 2$)$.

Table 2. Laboratory values and weight in 12 patients at inclusion and at control colonoscopy.

\begin{tabular}{ccc}
\hline Value & At Inclusion Median (IQR) Range & After EEN Treatment Median (IQR) Range \\
\hline Weight $(\mathrm{kg})$ & $37.9(31.5-52.6) 27.5-59.7$ & $40.5(32.8-55.4) 29.8-59.1$ \\
ESR $(\mathrm{mm} / \mathrm{h})$ & $28(17.3-27.5) 8-53$ & $9(8-11) 2-21$ \\
CRP $(\mathrm{mg} / \mathrm{L})$ & $14(2-49.3) 1-86$ & $2.5(1-8.3) 1-20$ \\
Albumin $(\mathrm{g} / \mathrm{L})$ & $33(25-36) 24-41$ & $36.3(33.5-40.5) 31-44$ \\
Hemoglobin $(\mathrm{g} / \mathrm{L})$ & $113(101-119.5) 83-142$ & $129(121-135) 116-144$ \\
F-calprotectin $(\mathrm{mg} / \mathrm{kg})$ & $2640(1191-3169) 580-6360$ & $620(198-1556) 5-2256$ \\
\hline
\end{tabular}

\subsection{Anthropometric Data}

There was a significant gain in weight $(p=0.003)$ after EEN treatment (Table 2).

\subsection{Endoscopic Healing}

All patients were examined with a control upper and lower endoscopy at a median of 13 days (range 1-45 days) after completion of EEN. A significant decrease $(p=0.008)$ was seen in SES-CD after EEN (median SES-CD 9.5, IQR 4.5-14.3, range 4-28 at inclusion compared to median 3.5, IQR 1-10, range $0-16$ at $\mathrm{CC})$.

\subsection{Clinical Outcome of the Two UC Patients}

One UC patient showed a reduction in PCDAI ( 23 to 5 points) and ESR ( 28 to $11 \mathrm{~mm} / \mathrm{h}$ ) and an increased hemoglobin value (110 to $116 \mathrm{~g} / \mathrm{L}$ ). However, no considerable effect was seen in SES-CD, CRP and F-calprotectin. The other UC patient showed a decrease in PCDAI (40 to 15 points), ESR (53 to $2 \mathrm{~mm} / \mathrm{h}$ ), CRP (47 to $11 \mathrm{mg} / \mathrm{L}$ ), F-calprotectin (3169 to $1430 \mathrm{mg} / \mathrm{kg}$ ) and SES-CD (21 to 16 points) and a raise in albumin (28 to $31 \mathrm{~g} / \mathrm{L})$ and $\mathrm{Hb}(83$ to $128 \mathrm{~g} / \mathrm{L})$.

\subsection{Mucosal Healing}

The histopathological scoring using the Geboes score showed an improvement in ten patients, worsening in one patient and resolution of inflammation in one patient after EEN treatment (Tables A1 and A2).

\subsection{Adverse Events}

Two patients reported initial mild abdominal pain related to the intake of nutritional drinks. The treatment was tolerated without any additional side effects in all 12 patients.

\subsection{Mucosal Cytokine Profile}

In six of twelve EEN-treated children, the cytokine profile in biopsies were analyzed both at diagnosis and at CC. In the remaining seven patients, mucosal cytokines in biopsies were analyzed either at diagnosis or at CC, but could not be analyzed both at diagnosis and CC due to technical circumstances. 


\subsubsection{Cytokine Profiles in Six Patients Pre- and Post EEN}

No significant differences were found in any cytokine profile in six patients (five with CD and one with IBD-U) who completed six weeks of EEN. Furthermore, there were no associations in the cytokine profile in relation to gender, age or disease extension. In individual patients, both reductions and increases in the cytokine expression were seen. With regards to the key proinflammatory cytokine IL-12 $\beta$, only one patient demonstrated a decrease while four patients showed an increase after EEN (one sample missing). In addition, the antiinflammatory IL-10 decreased in three patients and increased in three patients, while two patients had a reduction of the regulatory TGF- $\beta 1$, one patient had no change and three patients showed increased expression after EEN treatment. The regulatory cytokine CSF-2 was downregulated in four out of five patients after EEN treatment (Table 3 and Figure 1). Table A3 for real-time PCR values per patient pre- and post-EEN.

Table 3. MCP in biopsies from six children at IBD onset and after treatment harvested from colon (in some patients, it was not possible to analyze all MCPs).

\begin{tabular}{cccc}
\hline CYTOKINE & Decreased & Unchanged & Increased \\
\hline CSF-2 & 4 & & 1 \\
IFN- $\gamma$ & 3 & & 2 \\
TNF- $\alpha$ & 4 & & 2 \\
IL-1 $\beta$ & 4 & & 2 \\
IL-4 & 3 & 2 & 2 \\
IL-6 & 2 & & 3 \\
IL-10 & 3 & & 4 \\
IL-12 $\beta$ & 1 & & 1 \\
IL-22 & 2 & & 1 \\
IL-23 $\alpha$ & 5 & 1 & 3 \\
IL-36 $\gamma$ & 4 & & \\
TGF- $\beta 1$ & 2 & &
\end{tabular}

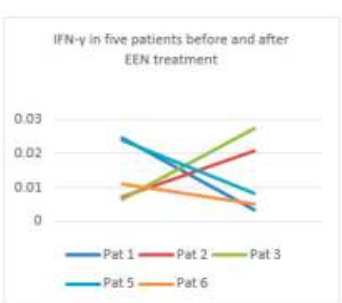

A

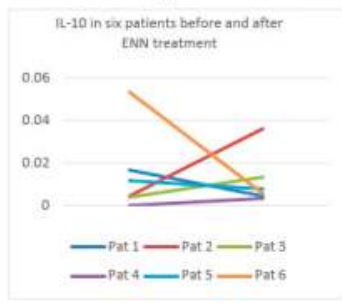

D

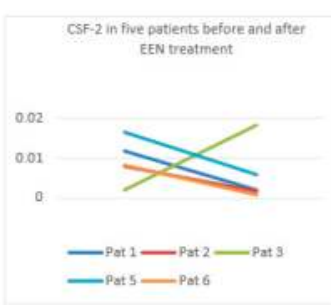

B

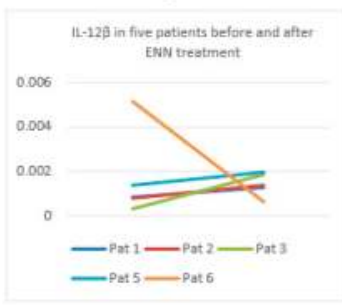

E

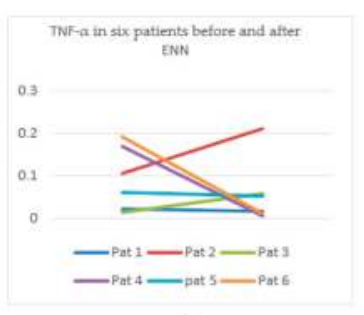

C

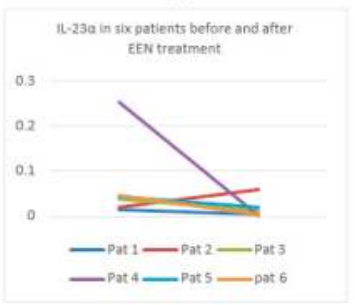

F

Figure 1. Six cytokine profiles in mucosal biopsies according to mRNA levels measured by q-PCR at disease onset and after EEN treatment. (A). IFN- $\gamma$ at onset and after EEN treatment. (B). CSF-2 at onset and after EEN treatment. (C). TNF- $\alpha$ at onset and after EEN treatment. (D). IL-10 at onset and after EEN treatment. (E). IL-12 $\beta$ at onset and after EEN treatment. (F). IL-23 $\alpha$ at onset and after EEN treatment. 


\subsubsection{Cytokine Comparison between IBD Patients and Non-IBD Controls}

When 12 MCPs in eight IBD-patients were compared to six non-IBD patients at inclusion, significantly higher values of IL-12 $\beta(p=0.007)$ and IL-23 $\alpha(p=0.025)$ were measured in the IBD patients compared to non-IBD controls.

\section{Discussion}

In the present study, we aimed to investigate the effect of EEN treatment on symptoms, biochemistry, endoscopy, histopathology and mucosal cytokine profile in children with new onset IBD.

We found a significant reduction of PCDAI, CRP, ESR and F-calprotectin; a significant increase in hemoglobin and albumin levels; and a significant gain in weight between the baseline and after completion of the EEN treatment. Mucosal healing (MH), measured by SES-CD, was seen in the majority of the patients at CC compared to diagnosis. Our results are consistent with other reports, which have showed that EEN is effective for clinical and biochemical remission in up to $80 \%$ in children with newly diagnosed CD [19,20].

Interestingly, in the two patients with a subsequent change of diagnosis from CD to UC, EEN also had an antiinflammatory effect. To the best of our knowledge, the literature on the evaluation of EEN in UC is limited [21,22].

MCP

In this pilot study, no significant differences in MCP were found after EEN treatment in children with IBD. However, we found significant higher levels of baseline IL-12 $\beta$ and IL-23 $\alpha$ in the IBD-patients compared to non-IBD controls. These cytokines are known to play an important role in the pathogenesis of CD [23].

Our intention was to investigate the effects of EEN on the mucosal cytokine profile in addition to its clinical effects. It is likely that MCP mirrors the inflammatory activity more adequately than cytokines analyzed in peripheral blood [24]. Even though the reduced expression of proinflammatory cytokines was seen after EEN treatment, no consistent effect of EEN variations was identified. This is probably due to individual variations and a limited number of patients, which is an important limitation of the study.

The expression of TGF- $\beta 1$, CSF-2 and IL-10, which are known as regulatory cytokines, demonstrated conflicting changes in the patient group after treatment. There are only a few reports published on mucosal cytokines after EEN. Yamamoto et al. investigated IL-1 $\beta$, IL-1 receptor antagonist (IL-1ra), IL-6, IL-8 and TNF- $\alpha$ with enzyme-linked immunosorbent assay (ELISA) before and after four weeks of elemental diet in 28 adult patients. After treatment, a reduction in the mucosal expression of IL-1 $\beta$, IL-1ra, IL-6, IL-8 and TNF- $\alpha$ was noticed. Furthermore, when they compared IBD patients to healthy controls, significantly higher levels of all cytokines were observed in the IBD patients [12].

Fell et al. investigated clinical efficacy and mucosal healing after eight weeks of CT3211 (a polymeric nutritional diet) in 29 children with active CD. In addition, IL- $1 \beta$, IL-8, IL-10, TGF- $\beta 1$ and IFN- $\gamma$ were analyzed with PCR in ileal- and colonic biopsies from 18 children [11]. Complete clinical remission was seen in $79 \%$ of the patients. Furthermore, macroscopic and histological healing was associated with a significant downregulation of mucosal proinflammatory cytokines in ileum or/and colon. Elevated mucosal cytokines were observed in CD patients before EEN treatment compared to non-IBD controls and ileal TGF- $\beta 1$ increased during treatment.

Thus, the reports on MCP and EEN show contradictory results and one explanation may be the diversity in methodology, but also in the types and length of EEN treatment. The individual expression of the mucosal cytokines may also reflect the diversity in patients and their immunological status since CD probably comprises several pathogenic mechanisms that differ between patients $[25,26]$. Nevertheless, earlier reports show aberrant gene expression in patients with CD, which involves cytokine expression. In 2006, Carr et al. found a strong association between an aberrant gene-expression 
of the proinflammatory cytokine IL23R and CD while Waschke et al. connected various single nucleotide polymorphisms in the TNF receptors to different CD phenotypes [27-29]. In our report, the expression of regulatory IL-10, TGF- $\beta 1$ and CSF- 2 was shown to decrease and increase in individual patients after EEN treatment. This may reflect the temporal aspect, i.e., the same cytokine may be protective during acute inflammation but harmful and perpetuate the chronic inflammation [30]. Thus, cytokines may exert variable functions during different immunological conditions [31-33].

\section{Conclusions}

Complete remission after 6 weeks of EEN was achieved in a majority of children with new onset IBD. We found a higher level of expression of IL-12 $\beta$ and IL-23 $\alpha$ in these children compared to controls at inclusion. There were individual changes in the mucosal cytokine profiles after EEN-treatment but there were no consistent trends. Further understanding of the role of both antiinflammatory and regulatory cytokines as well as the aspects of temporal changes in the cytokine expression from diagnosis to remission requires larger studies.

Author Contributions: Conceptulization and methodology: H.R., Y.F., M.E. and U.L.F. Software and formal analysis: H.R. Validation: H.R., Y.F., M.E. and U.L.F. Investigation: H.R., Y.F. and K.J.-V. Supervision: Y.F., M.E. and U.L.F. Writing-Original Draft preparation H.R. Writing-Review and Editing: H.R., Y.F., M.E., U.L.F. and K.J.-V. Project administration: H.R.

Funding: This study has been supported with an unrestricted research grant from Nutricia Sweden and with a research grant from the Kempe-Carlgrenska research foundation and from Thre research foundation.

Conflicts of Interest: Eberhardson has received honoraria for lectures and consultancy from AbbVie, Merck (MSD), Takeda, Ferring, Orion Pharma, Otsuka, Tillotts, ITH, Novartis, Pfizer and Janssen and received research funding from AbbVie and MSD. Fagerberg has recived honoraria for consultancy from InDex Pharmaceuticals A.B, Tillotts Pharma AB and AbbVie AB and financial support to institution (congress fee and travel) from Otsuka Pharma Scandinavia AB.

\section{Appendix A}

Table A1. Geboes scoring: Grades < 3 were regarded as absence of IBD inflammation, while grades $\geq 3$ were regarded as presence of IBD inflammation of varying degree (3:1-3:7) with the following subgroups: $\mathrm{a}=$ basal plasmacytosis, $\mathrm{b}=$ lymphoid nodules, $\mathrm{c}=$ Paneth cell hyperplasia, $\mathrm{d}=$ eosinophils in the lamina propria, $\mathrm{e}=$ histiocytic cell proliferation, $\mathrm{f}=$ giant cells, $\mathrm{g}=$ granuloma, $\mathrm{h}=$ vasculitis and $\mathrm{i}=$ dysplasia. The table shows 11 segments (segment $1=$ ileum, segment $11=$ rectum) before and after EEN treatment.

\begin{tabular}{|c|c|c|c|c|c|c|c|c|c|c|c|}
\hline & Segm. 1 & Segm. 2 & Segm. 3 & Segm. 4 & Segm. 5 & Segm. 6 & Segm. 7 & Segm. 8 & Segm. 9 & Segm. 10 & Segm. 11 \\
\hline $\begin{array}{c}\text { Patient } \\
\text { Endoscopy } 1 \text { Endos }\end{array}$ & & & & & & & & & & & \\
\hline Pat $1 \mathrm{CD}$ & mult.g $\mid \mathrm{g}$ & $<3 \mid<3$ & $<3 \mid<3$ & $<3 \mid<3$ & $<3 \mid<3$ & $<3 \mid<3$ & $<3 \mid<3$ & $<3 \mid<3$ & $<3 \mid<3$ & $<3 \mid<3$ & $<3 \mid<3$ \\
\hline Pat 2 UC & $<3 \mid 3: 3^{\circ}$ & $5 \mid 3: 7$ & $3: 7 \mid 3: 7$ & $3: 3 \mid 3: 3$ & $3: 3 \mid 3: 7$ & $5 \mid 3: 7$ & $5 \mid 3: 3$ & $4 \mid 3: 7$ & $3: 7 \mid 3: 7$ & & $<3 \mid 3: 7$ \\
\hline Pat 3 CD & $<3 \mid<3$ & $<3+g \mid<3$ & $5+\mathrm{b} \mid<3+\mathrm{e}$ & $<3 \mid$ mult.g & $<3 \mid<3+\mathrm{e}$ & $<3 \mid<3+e$ & $<3 \mid<3$ & $<3 \mid<3+g$ & $<3 \mid<3$ & $<3 \mid<3$ & $<3 \mid<3$ \\
\hline Pat $4 \mathrm{CD}$ & $3: 3+b+g \mid 3: 1$ & $3: 3+b+g \mid 3: 1$ & $3: 3+b+g \mid 3: 1$ & $<3 \mid<3$ & $<3 \mid<3$ & $<3 \mid<3$ & $<3 \mid<3$ & $3: 3 \mid<3$ & $<3 \mid<3$ & $<3 \mid<3$ & $<3 \mid<3$ \\
\hline Pat 5 CD & $<3 \mid<3$ & $<3 \mid<3$ & $<3 \mid<3$ & $<3 \mid<3$ & $<3 \mid<3$ & $<3 \mid<3$ & $<3 \mid<3$ & $<3 \mid<3$ & $<3 \mid<3$ & $<3 \mid<3$ & $<3 \mid<3$ \\
\hline Pat 6 CD & $3: 3+\mathrm{e} \mid<3$ & $<3 \mid<3$ & $<3 \mid<3$ & $<3 \mid<3$ & $<3 \mid<3$ & $<3 \mid<3$ & $<3 \mid<3$ & $<3 \mid<3$ & $<3 \mid<3$ & $<3 \mid<3$ & $4+g \mid<3$ \\
\hline Pat 7 UC & $<3 \mid<3$ & $3: 7 \mid 3: 3$ & $3: 7+\mathrm{e} \mid 3: 3$ & $3: 7 \mid 3: 3$ & $3: 7 \mid 3: 3$ & $3: 3 \mid 3: 3$ & $3: 713: 7$ & $3: 7 \mid 3: 3$ & $4+\mathrm{e} \mid 3: 7$ & $3: 7+\mathrm{c} \mid 3: 3$ & $3: 7 \mid 3: 3$ \\
\hline Pat $8 \mathrm{CD}$ & infl.polyp | 3:7 & $<3 \mid<3+g$ & $<3+g \mid<3$ & $<3+\mathrm{e} \mid 3: 3$ & $<3+g \mid 3: 3$ & $3: 2 \mid<3$ & $3: 2+\mathrm{g} \mid 3: 1$ & $<3 \mid<3$ & $<3+g \mid<3$ & $<3+g \mid<3$ & $<3+g \mid<3$ \\
\hline Pat 9 I-BDU & $<3 \mid<3$ & $<3 \mid<3$ & $<3 \mid<3$ & $3: 2 \mid<3$ & $<3 \mid<3$ & $<3 \mid<3$ & $3: 1 \mid<3$ & $3: 1 \mid<3$ & $3: 1 \mid<3$ & $<3 \mid<3$ & $<3 \mid<3$ \\
\hline Pat $10 \mathrm{CD}$ & $<3+g \mid<3+g$ & $<3+g \mid<3+g$ & $<3 \mid<3+g$ & $<3 \mid<3$ & $<3+g \mid<3$ & $<3 \mid<3$ & $<3 \mid<3+g$ & $<3+g \mid<3$ & $<3 \mid<3$ & $<3+g \mid<3$ & $<3+g \mid<3$ \\
\hline Pat 11 & $<3 \mid<3$ & $<3 \mid<3$ & $3: 4 \mid<3$ & $3: 7+\mathrm{e} \mid<3$ & $3: 7+\mathrm{e} \mid<3$ & $4+\mathrm{e} \mid<3$ & $4 \mid<3: 4^{\circ}$ & $4 \mid<3$ & $4 \mid 3: 4$ & $3: 7+\mathrm{e} \mid<3$ & $4 \mid<3$ \\
\hline Pat 12 & $5+\mathrm{e} \mid 3: 2$ & $3: 3 \mid 3: 2$ & $3: 3 \mid 3: 2$ & $<3 \mid<3$ & $<3 \mid 3: 7$ & $<3 \mid 3: 7$ & $<3 \mid<3$ & $<3 \mid<3$ & $<3 \mid<3$ & $<3 \mid<3$ & $3: 7 \mid<3$ \\
\hline
\end{tabular}


Table A2. Geboes results summarized (every patient, 11 segments) as improvement, no change, aggravated inflammation or no inflammation after EEN.

\begin{tabular}{|c|c|c|c|c|}
\hline Patient & Improvement & No Change & Aggravated Inflammation & No Inflammation \\
\hline 1 & 1 & & & 10 \\
\hline 2 & 4 & 4 & 3 & \\
\hline 3 & 2 & & 4 & 5 \\
\hline 4 & 4 & & & 7 \\
\hline 5 & & & & 11 \\
\hline 6 & 2 & 1 & & 8 \\
\hline 7 & 8 & & 3 & \\
\hline 8 & 7 & 2 & 1 & 1 \\
\hline 9 & 4 & & & 7 \\
\hline 10 & 4 & 5 & 2 & \\
\hline 11 & 9 & & & 2 \\
\hline 12 & 4 & & 2 & 5 \\
\hline
\end{tabular}

Table A3. Real-time PCR values per patient pre- and post-EEN and mean values and SD for group per cytokine.

\begin{tabular}{|c|c|c|c|c|c|c|}
\hline Patient & $\begin{array}{c}\text { CSF-2 } \\
\text { RQ pre-post } \\
\text { EEN }\end{array}$ & $\begin{array}{c}\text { IFN- } \gamma \\
\text { RQ pre-post } \\
\text { EEN }\end{array}$ & $\begin{array}{c}\text { TNF- } \alpha \\
\text { RQ pre-post } \\
\text { EEN }\end{array}$ & $\begin{array}{c}\text { IL1- } \beta: 1 \\
\text { RQ pre-post } \\
\text { EEN }\end{array}$ & $\begin{array}{c}\text { IL-4:1 } \\
\text { RQ pre-post } \\
\text { EEN }\end{array}$ & $\begin{array}{c}\text { IL-6:1 } \\
\text { RQ pre-post } \\
\text { EEN }\end{array}$ \\
\hline 1 & $\begin{array}{l}0.011651- \\
0.001997\end{array}$ & $\begin{array}{c}0.024588- \\
0.003389\end{array}$ & $\begin{array}{l}0.024577- \\
0.016915\end{array}$ & $\begin{array}{c}0.573893- \\
0.020431\end{array}$ & $\begin{array}{c}0.000275- \\
4.72 \times 10^{-5}\end{array}$ & $\begin{array}{l}0.001001- \\
0.001825\end{array}$ \\
\hline 2 & $\begin{array}{l}0.007851- \\
0.001629\end{array}$ & $\begin{array}{c}0.007228- \\
0.020651\end{array}$ & $\begin{array}{l}0.106451- \\
0.21017\end{array}$ & $\begin{array}{l}0.051899- \\
0.924653\end{array}$ & $\begin{array}{l}0.001325- \\
\# \# \# \# \# \#\end{array}$ & $\begin{array}{l}0.020791- \\
0.172768\end{array}$ \\
\hline 3 & $\begin{array}{l}0.001862- \\
0.018126\end{array}$ & $\begin{array}{l}0.00664- \\
0.02729\end{array}$ & $\begin{array}{c}0.014612- \\
0.059869\end{array}$ & $\begin{array}{c}0.218129- \\
0.408354\end{array}$ & $\begin{array}{c}0.000282- \\
8.68 \times 10^{-5}\end{array}$ & $\begin{array}{l}0.008237- \\
0.029142\end{array}$ \\
\hline 4 & $\begin{array}{l}\text { \#\#\#\#\#\#\#- } \\
\text { \#\#\#\#\#\#\# }\end{array}$ & $\begin{array}{c}\text { \#\#\#\#\#\#\#- } \\
0.00048\end{array}$ & $\begin{array}{c}0.168562- \\
0.007131\end{array}$ & $\begin{array}{l}1.021516- \\
0.010524\end{array}$ & $\begin{array}{l}\text { \#\#\#\#\#\#\#- } \\
\text { \#\#\#\#\#\#\# }\end{array}$ & $\begin{array}{c}\text { \#\#\#\#\#\#\#- } \\
0.003238\end{array}$ \\
\hline 5 & $\begin{array}{l}0.016589- \\
0.005784\end{array}$ & $\begin{array}{c}0.023729- \\
0.008329\end{array}$ & $\begin{array}{l}0.06296- \\
0.054617\end{array}$ & $\begin{array}{c}2.175898- \\
0.061581\end{array}$ & $\begin{array}{c}0.000737- \\
0.000148\end{array}$ & $\begin{array}{c}0.042764- \\
0.022326\end{array}$ \\
\hline 6 & $\begin{array}{c}0.008264- \\
0.000895\end{array}$ & $\begin{array}{c}0.010918- \\
0.00516\end{array}$ & $\begin{array}{c}0.190799- \\
0.012544\end{array}$ & $\begin{array}{l}3.225579- \\
0.108797\end{array}$ & $\begin{array}{l}\text { \#\#\#\#\#\#\#- } \\
\text { \#\#\#\#\#\#\# }\end{array}$ & $\begin{array}{l}0.32355- \\
0.00282\end{array}$ \\
\hline $\begin{array}{l}\text { Mean for group } \\
\text { pre-post EEN }\end{array}$ & $\begin{array}{c}0.0092434- \\
0.005686\end{array}$ & $\begin{array}{c}0.014621- \\
0.010883\end{array}$ & $\begin{array}{l}0.09466- \\
0.060208\end{array}$ & $\begin{array}{l}1.211152- \\
0.255723\end{array}$ & $\begin{array}{c}0.0006548- \\
9.39959 \times 10^{-5}\end{array}$ & $\begin{array}{l}0.158537- \\
0.038687\end{array}$ \\
\hline $\begin{array}{l}\text { SD for group } \\
\text { pre-post EEN }\end{array}$ & $\begin{array}{c}0.0048404- \\
0.006446\end{array}$ & $\begin{array}{c}0.007929 \\
0.009732\end{array}$ & $\begin{array}{l}0.09466- \\
0.060208\end{array}$ & $\begin{array}{l}1.137297- \\
0.328006\end{array}$ & $\begin{array}{c}0.0004296- \\
4.14339 \times 10^{-5}\end{array}$ & $\begin{array}{c}0.122961- \\
0.06088\end{array}$ \\
\hline Patient & $\begin{array}{c}\text { IL-10:1 } \\
\text { RQ pre-post } \\
\text { EEN }\end{array}$ & $\begin{array}{c}\text { IL-12 } \beta: 1 \\
\text { RQ pre-post } \\
\text { EEN }\end{array}$ & $\begin{array}{c}\text { IL-22:1 } \\
\text { RQ pre-post } \\
\text { EEN }\end{array}$ & $\begin{array}{c}\text { IL-23 } \alpha: 1 \\
\text { RQ pre-post } \\
\text { EEN }\end{array}$ & $\begin{array}{c}\text { IL-36 } \gamma: 1 \\
\text { RQ pre-post } \\
\text { EEN }\end{array}$ & $\begin{array}{c}\text { TGF } \beta 1: 1 \\
\text { RQ pre-post } \\
\text { EEN }\end{array}$ \\
\hline 1 & $\begin{array}{l}0.016987- \\
0.004407\end{array}$ & $\begin{array}{c}0.000851- \\
0.001291\end{array}$ & $\begin{array}{l}\text { \#\#\#\#\#\#- } \\
0.002077\end{array}$ & $\begin{array}{l}0.015346- \\
0.005568\end{array}$ & $\begin{array}{l}0.000435- \\
0.000911\end{array}$ & $\begin{array}{l}0.653298- \\
0.657096\end{array}$ \\
\hline 2 & $\begin{array}{c}0.004295- \\
0.03603\end{array}$ & $\begin{array}{c}0.000767- \\
0.001354\end{array}$ & $\begin{array}{c}\text { \#\#\#\#\#\#\#- } \\
0.016029\end{array}$ & $\begin{array}{l}0.020307- \\
0.060188\end{array}$ & $\begin{array}{l}0.002836- \\
0.002475\end{array}$ & $\begin{array}{l}1.013262- \\
4.188852\end{array}$ \\
\hline 3 & $\begin{array}{c}0.003799- \\
0.013653\end{array}$ & $\begin{array}{c}0.000296- \\
0.001842\end{array}$ & $\begin{array}{c}0.000458- \\
0.004207\end{array}$ & $\begin{array}{c}0.037239- \\
0.012223\end{array}$ & $\begin{array}{l}0.002853- \\
0.002125\end{array}$ & $\begin{array}{l}0.425452- \\
0.726292\end{array}$ \\
\hline 4 & $\begin{array}{c}\text { \#\#\#\#\#\#\#- } \\
0.003523\end{array}$ & $\begin{array}{l}\text { \#\#\#\#\#\#\#- } \\
\text { \#\#\#\#\#\#\# }\end{array}$ & $\begin{array}{c}\text { \#\#\#\#\#\#\#- } \\
\text { \#\#\#\#\#\# }\end{array}$ & $\begin{array}{c}0.254504- \\
0.003021\end{array}$ & $\begin{array}{l}\text { \#\#\#\#\#\#\#- } \\
\text { \#\#\#\#\#\#\# }\end{array}$ & $\begin{array}{l}1.497149- \\
0.271375\end{array}$ \\
\hline 5 & $\begin{array}{l}0.01185- \\
0.007582\end{array}$ & $\begin{array}{c}0.001391- \\
0.001984\end{array}$ & $\begin{array}{c}0.014448- \\
0.008729\end{array}$ & $\begin{array}{c}0.044249- \\
0.019889\end{array}$ & $\begin{array}{l}0.00407- \\
0.000911\end{array}$ & $\begin{array}{l}0.988215- \\
1.261974\end{array}$ \\
\hline 6 & $\begin{array}{l}0.053329 \\
0.005778\end{array}$ & $\begin{array}{c}0.005143- \\
0.000639\end{array}$ & $\begin{array}{c}0.008147- \\
8.46 \times 10^{-5}\end{array}$ & $\begin{array}{l}0.046621- \\
0.004837\end{array}$ & $\begin{array}{l}0.001272- \\
0.000563\end{array}$ & $\begin{array}{l}0.83872- \\
0.52586\end{array}$ \\
\hline $\begin{array}{l}\text { Mean for group } \\
\text { pre-post EEN }\end{array}$ & $\begin{array}{l}0.018052- \\
0.011829\end{array}$ & $\begin{array}{l}0.00169- \\
0.001422\end{array}$ & $\begin{array}{l}0.007684- \\
0.006225\end{array}$ & $\begin{array}{l}0.069711- \\
0.017621\end{array}$ & $\begin{array}{l}0.002293- \\
0.001397\end{array}$ & $\begin{array}{l}0.902683- \\
1.271908\end{array}$ \\
\hline $\begin{array}{l}\text { SD for group } \\
\text { pre-post EEN }\end{array}$ & $\begin{array}{c}0.018311- \\
0.011312\end{array}$ & $\begin{array}{c}0.001761- \\
0.000475\end{array}$ & $\begin{array}{l}0.005721- \\
0.005681\end{array}$ & $\begin{array}{c}0.083447- \\
0.019867\end{array}$ & $\begin{array}{l}0.001285- \\
0.000756\end{array}$ & $\begin{array}{l}0.333408- \\
1.338034\end{array}$ \\
\hline
\end{tabular}

\section{References}

1. Van Limbergen, J.; Russell, R.K.; Drummond, H.E.; Aldhous, M.C.; Round, N.K.; Nimmo, E.R.; Smith, L.; Gillett, P.M.; McGrogan, P.; Weaver, L.T.; et al. Definition of phenotypic characteristics of childhood-onset inflammatory bowel disease. Gastroenterology 2008, 135, 1114-1122. [CrossRef] [PubMed]

2. Baumgart, D.C.; Sandborn, W.J. Crohn's disease. Lancet 2012, 380, 1590-1605. [CrossRef] 
3. Ruemmele, F.M.; Veres, G.; Kolho, K.L.; Griffiths, A.; Levine, A.; Escher, J.C.; Amil Dias, J.; Barabino, A.; Braegger, C.P.; Bronsky, J.; et al. Consensus guidelines of ECCO/ESPGHAN on the medical management of pediatric Crohn's disease. J. Crohns Colitis 2014, 8, 1179-1207. [CrossRef] [PubMed]

4. Zachos, M.; Tondeur, M.; Griffiths, A.M. Enteral nutritional therapy for induction of remission in Crohn's disease. Cochrane Database Syst. Rev. 2008, 4, Cd000542.

5. Dziechciarz, P.; Horvath, A.; Shamir, R.; Szajewska, H. Meta-analysis: Enteral nutrition in active Crohn's disease in children. Aliment. Pharmacol. Ther. 2007, 26, 795-806. [CrossRef] [PubMed]

6. Day, A.S.; Whitten, K.E.; Lemberg, D.A.; Clarkson, C.; Vitug-Sales, M.; Jackson, R.; Bohane, T.D. Exclusive enteral feeding as primary therapy for Crohn's disease in Australian children and adolescents: A feasible and effective approach. J. Gastroenterol. Hepatol. 2006, 21, 1609-1614. [CrossRef] [PubMed]

7. Cuiv, P.O.; Begun, J.; Keely, S.; Lewindon, P.J.; Morrison, M. Towards an integrated understanding of the therapeutic utility of exclusive enteral nutrition in the treatment of Crohn's disease. Food Funct. 2016, 7, 1741-1751. [CrossRef] [PubMed]

8. Furness, J.B.; Kunze, W.A.; Clerc, N. Nutrient tasting and signaling mechanisms in the gut. II. The intestine as a sensory organ: Neural, endocrine and immune responses. Am. J. Physiol. 1999, 277 Pt 1, G922-G928. [PubMed]

9. Korzenik, J.R.; Dieckgraefe, B.K. Is Crohn's disease an immunodeficiency? A hypothesis suggesting possible early events in the pathogenesis of Crohn's disease. Dig. Dis. Sci. 2000, 45, 1121-1129. [CrossRef]

10. Neurath, M.F. Cytokines in inflammatory bowel disease. Nat. Rev. Immunol. 2014, 14, 329-342. [CrossRef]

11. Fell, J.M.; Paintin, M.; Arnaud-Battandier, F.; Beattie, R.M.; Hollis, A.; Kitching, P.; Donnet-Hughes, A.; MacDonald, T.T.; Walker-Smith, J.A. Mucosal healing and a fall in mucosal proinflammatory cytokine mRNA induced by a specific oral polymeric diet in paediatric Crohn's disease. Aliment. Pharmacol. Ther. 2000, 14, 281-289. [CrossRef] [PubMed]

12. Yamamoto, T.; Nakahigashi, M.; Umegae, S.; Kitagawa, T.; Matsumoto, K. Impact of elemental diet on mucosal inflammation in patients with active Crohn's disease: Cytokine production and endoscopic and histological findings. Inflamm. Bowel Dis. 2005, 11, 580-588. [CrossRef] [PubMed]

13. Schwerd, T.; Frivolt, K.; Clavel, T.; Lagkouvardos, I.; Katona, G.; Mayr, D.; Uhlig, H.H.; Haller, D.; Koletzko, S.; Bufler, P. Exclusive enteral nutrition in active pediatric Crohn disease: Effects on intestinal microbiota and immune regulation. J. Allergy Clin. Immunol. 2016, 138, 592-596. [CrossRef] [PubMed]

14. Levine, A.; Koletzko, S.; Turner, D.; Escher, J.C.; Cucchiara, S.; de Ridder, L.; Kolho, K.L.; Veres, G.; Russell, R.K.; Paerregaard, A.; et al. ESPGHAN revised porto criteria for the diagnosis of inflammatory bowel disease in children and adolescents. J. Pediatr. Gastroenterol. Nutr. 2014, 58, 795-806. [CrossRef] [PubMed]

15. Turner, D.; Griffiths, A.M.; Walters, T.D.; Seah, T.; Markowitz, J.; Pfefferkorn, M.; Keljo, D.; Waxman, J.; Otley, A.; LeLeiko, N.S.; et al. Mathematical weighting of the pediatric Crohn's disease activity index (PCDAI) and comparison with its other short versions. Inflamm. Bowel Dis. 2012, 18, 55-62. [CrossRef] [PubMed]

16. Levine, A.; Griffiths, A.; Markowitz, J.; Wilson, D.C.; Turner, D.; Russell, R.K.; Fell, J.; Ruemmele, F.M.; Walters, T.; Sherlock, M.; et al. Pediatric modification of the Montreal classification for inflammatory bowel disease: The Paris classification. Inflamm. Bowel Dis. 2011, 17, 1314-1321. [CrossRef]

17. Daperno, M.; D’Haens, G.; Van Assche, G.; Baert, F.; Bulois, P.; Maunoury, V.; Sostegni, R.; Rocca, R.; Pera, A.; Gevers, A.; et al. Development and validation of a new, simplified endoscopic activity score for Crohn's disease: The SES-CD. Gastrointest. Endosc. 2004, 60, 505-512. [CrossRef]

18. Geboes, K.; Riddell, R.; Ost, A.; Jensfelt, B.; Persson, T.; Lofberg, R. A reproducible grading scale for histological assessment of inflammation in ulcerative colitis. Gut 2000, 47, 404-409. [CrossRef]

19. Day, A.S.; Lopez, R.N. Exclusive enteral nutrition in children with Crohn's disease. World J. Gastroenterol. 2015, 21, 6809-6816. [CrossRef]

20. Bannerjee, K.; Camacho-Hubner, C.; Babinska, K.; Dryhurst, K.M.; Edwards, R.; Savage, M.O.; Sanderson, I.R.; Croft, N.M. Antiinflammatory and growth-stimulating effects precede nutritional restitution during enteral feeding in Crohn disease. J. Pediatr. Gastroenterol. Nutr. 2004, 38, 270-275. [CrossRef]

21. Gonzalez-Huix, F.; Fernandez-Banares, F.; Esteve-Comas, M.; Abad-Lacruz, A.; Cabre, E.; Acero, D.; Sanderson, I.R.; Croft, N.M. Enteral versus parenteral nutrition as adjunct therapy in acute ulcerative colitis. Am. J. Gastroenterol. 1993, 88, 227-232. [PubMed] 
22. Shaoul, R.; Brown, S.; Day, A.S. Reasoning Beyond the Potential Use of Exclusive Enteral Nutrition and Other Specified Diets in Children with Ulcerative Colitis. J. Pediatr. Gastroenterol. Nutr. 2018, 66, 378-382. [CrossRef]

23. Guan, Q.; Zhang, J. Recent Advances: The Imbalance of Cytokines in the Pathogenesis of Inflammatory Bowel Disease. Mediators Inflamm. 2017, 2017, 4810258. [CrossRef] [PubMed]

24. Dock, J.; Ramirez, C.M.; Hultin, L.; Hausner, M.A.; Hultin, P.; Elliott, J.; Yang, O.O.; Anton, P.A.; Jamieson, B.D.; Effros, R.B. Distinct aging profiles of CD8+ T cells in blood versus gastrointestinal mucosal compartments. PLoS ONE 2017, 12, e0182498. [CrossRef]

25. Roggenbuck, D.; Reinhold, D.; Baumgart, D.C.; Schierack, P.; Conrad, K.; Laass, M.W. Autoimmunity in Crohn's Disease-A Putative Stratification Factor of the Clinical Phenotype. Adv. Clin. Chem. 2016, 77, 77-101. [PubMed]

26. Cleynen, I.; Boucher, G.; Jostins, L.; Schumm, L.P.; Zeissig, S.; Ahmad, T.; Andersen, V.; Andrews, J.M.; Annese, V.; Brand, S.; et al. Inherited determinants of Crohn's disease and ulcerative colitis phenotypes: A genetic association study. Lancet 2016, 387, 156-167. [CrossRef]

27. Ek, W.E.; D'Amato, M.; Halfvarson, J. The history of genetics in inflammatory bowel disease. Ann. Gastroenterol. 2014, 27, 294-303. [PubMed]

28. Duerr, R.H.; Taylor, K.D.; Brant, S.R.; Rioux, J.D.; Silverberg, M.S.; Daly, M.J.; Steinhart, A.H.; Abraham, C.; Regueiro, M.; Griffiths, A.; et al. A genome-wide association study identifies IL23R as an inflammatory bowel disease gene. Science 2006, 314, 1461-1463. [CrossRef]

29. Waschke, K.A.; Villani, A.C.; Vermeire, S.; Dufresne, L.; Chen, T.C.; Bitton, A.; Cohen, A.; Thomson, A.B.; Wild, G.E. Tumor necrosis factor receptor gene polymorphisms in Crohn's disease: Association with clinical phenotypes. Am. J. Gastroenterol. 2005, 100, 1126-1133. [CrossRef]

30. Bamias, G.; Cominelli, F. Cytokines and intestinal inflammation. Curr. Opin. Gastroenterol. 2016, 32, 437-442. [CrossRef]

31. Sanjabi, S.; Zenewicz, L.A.; Kamanaka, M.; Flavell, R.A. Antiinflammatory and proinflammatory roles of TGF-beta, IL-10 and IL-22 in immunity and autoimmunity. Curr. Opin. Pharmacol. 2009, 9, 447-453. [CrossRef] [PubMed]

32. Egea, L.; Hirata, Y.; Kagnoff, M.F. GM-CSF: A role in immune and inflammatory reactions in the intestine. Expert Rev. Gastroenterol. Hepatol. 2010, 4, 723-731. [CrossRef] [PubMed]

33. Dabritz, J. Granulocyte macrophage colony-stimulating factor and the intestinal innate immune cell homeostasis in Crohn's disease. Am. J. Physiol. Gastrointest. Liver Physiol. 2014, 306, G455-G465. [CrossRef] [PubMed] 


\title{
Dietary Protein Intake Level Modulates Mucosal Healing and Mucosa-Adherent Microbiota in Mouse Model of Colitis
}

\author{
Sandra Vidal-Lletjós ${ }^{1}$, Mireille Andriamihaja ${ }^{1}$, Anne Blais ${ }^{1}$, Marta Grauso ${ }^{1}$, Patricia Lepage ${ }^{2}$, \\ Anne-Marie Davila ${ }^{1}$, Roselyne Viel ${ }^{3}$, Claire Gaudichon ${ }^{1}$, Marion Leclerc ${ }^{2}$, François Blachier ${ }^{1}$ \\ and Annaïg Lan ${ }^{1, *}$ \\ 1 UMR PNCA, AgroParisTech, INRA, Université Paris-Saclay, 75005 Paris, France; \\ sandravidalll@gmail.com (S.V.-L.); mireille.andriamihaja@agroparistech.fr (M.A.); \\ anne.blais@agroparistech.fr (A.B.); marta.grausoculetto@agroparistech.fr (M.G.); \\ anne-marie.davila-gay@agroparistech.fr (A.-M.D.); claire.gaudichon@agroparistech.fr (C.G.); \\ francois.blachier@agroparistech.fr (F.B.) \\ 2 UMR MICALIS, AgroParisTech, INRA, Université Paris-Saclay, 78350 Jouy-en-Josas, France; \\ patricia.lepage@inra.fr (P.L.); marion.leclerc@inra.fr (M.L.) \\ 3 H2P2, Biosit-Biogenouest, Université de Rennes 1, 35005 Rennes, France; roselyne.viel@univ-rennes1.fr \\ * Correspondence: annaig.lan@agroparistech.fr; Tel.: +33-(0)1-44087242; Fax: +33-(0)1-44081858
}

Received: 11 February 2019; Accepted: 24 February 2019; Published: 28 February 2019

\begin{abstract}
Mucosal healing after an inflammatory flare is associated with lasting clinical remission. The aim of the present work was to evaluate the impact of the amount of dietary protein on epithelial repair after an acute inflammatory episode. C57BL/6 DSS-treated mice received isocaloric diets with different levels of dietary protein: 14\% (P14), 30\% (P30) and 53\% (P53) for 3 (day 10), 6 (day 13) and 21 (day 28) days after the time of colitis maximal intensity. While the P53 diet worsened the DSS- induced inflammation both in intensity and duration, the P30 diet, when compared to the P14 diet, showed a beneficial effect during the epithelial repair process by accelerating inflammation resolution, reducing colonic permeability and increasing epithelial repair together with epithelial hyperproliferation. Dietary protein intake also impacted mucosa-adherent microbiota composition after inflammation since P30 fed mice showed increased colonization of butyrate-producing genera throughout the resolution phase. This study revealed that in our colitis model, the amount of protein in the diet modulated mucosal healing, with beneficial effects of a moderately high-protein diet, while very high-protein diet displayed deleterious effects on this process.
\end{abstract}

Keywords: dietary protein level; colitis; epithelial repair; mucosa-adherent microbiota

\section{Introduction}

In recent years, mucosal healing $(\mathrm{MH})$ has become a therapeutic goal for the prevention of the complications of inflammatory bowel diseases (IBD) [1-4]. MH relies on concomitant cellular events that provide epithelial repair to restore the tissue integrity and the physiological functions of the colon such as barrier function and water absorption $[4,5]$, these events being presumably protein and energy consuming processes. In addition, the nutritional status is known to be impaired during and after an intestinal inflammatory episode due in particular to the catabolic action of the pro-inflammatory cytokines [6]. Although there is some evidence that the daily protein needs of IBD patients may differ from those of healthy controls, further experimental and clinical data are required to fully validate this concept. The European Society for Clinical Nutrition and Metabolism recommends to increase the protein intake in active IBD to $1.2-1.5 \mathrm{~g} / \mathrm{kg}$ body weight/day in adults and to maintain a similar level as it is recommended for the general population during remission 
(about $1 \mathrm{~g} / \mathrm{kg}$ body weight/day) [7]. However, the beneficial effects of increasing dietary protein level have been barely studied. Notwithstanding, majoring dietary protein intake may impact the colonic mucosa in the healthy gut as recently reviewed [8] by altering colonocyte biology $[9,10]$ and colonic luminal environment [11-14]. Furthermore, we showed that a high protein (HP, 53\% of total energy provided by protein) intake exacerbated acute colonic inflammation when simultaneously given with colitis induction [15]. Interestingly, the same study showed that this protein level in the diet was rather beneficial after the acute episode of colitis as it enhanced colonic epithelium restoration in surviving animals, thus indicating a potential effect of the dietary protein level on colonic crypt repair after acute inflammation [15]. Actually, supplementation with some AA, alone or in combination, improved parameters related to mucosal healing in colitis model such as epithelial regeneration and colonic barrier function (for review, see Reference [16]). Moreover, published data suggest that the availability of some amino acids, such as threonine or cysteine, is limiting under conditions of colonic inflammation $[17,18]$ and that dietary supplementation of protein or amino acids is needed to promote favorably epithelial repair. However, AA supplementation may also exert deleterious impact as it has been recently shown with threonine during colitis [19]. To date, the positive effect of any AA supplementation on colitis has never been demonstrated in clinical studies.

We then hypothesized that increasing dietary protein intake might influence colon epithelial repair by a direct effect of additional AA blood supply or via microbiota-derived effects. The present study thus aims to evaluate the impact of different level of dietary proteins (normoproteic (14\% of the total energy provided by protein, P14)), moderately HP diet (30\%, P30) and an elevated HP diet (53\%, P53) on some relevant barrier function parameters after the acute inflammatory episode in the DSS-mouse model of colitis.

\section{Materials and Methods}

\subsection{Animals and Diets}

Seven-week-old C57BL/ 6 male mice were obtained from ENVIGO, France (144, weight 18-23 g). Animals were housed in a 12:12-h light-dark circle at $23^{\circ} \mathrm{C}$ and controlled humidity $(55 \pm 10 \%)$ and acclimated for one week with free access to standard mouse chow and tap water. Each mouse was maintained in an individual cage with a grid and was allowed to acclimate to the P14 diet after three days of a standard mouse chow / fresh P14 diet (Table 1). The study was performed according to the European directive for the use and care of laboratory animals (2010/63/UE) and received the agreement of the local animal ethics committee and of the ministerial committee for animal experimentation (registration number: APAFIS\#3987-2016012214388658).

Table 1. Composition of the experimental diets.

\begin{tabular}{cccc}
\hline & P14 & P30 & P53 \\
\hline Weight content (g/kg) & & & \\
Acid casein (Armor Protéines ${ }^{\circledR}$, ref. \#139860) & 112 & 232 & 424 \\
Whey protein (Armor Protéines ${ }^{\circledR}$, Protarmor 80, ref. \#139805) & 28 & 58 & 96 \\
Corn starch $_{\text {Sucrose }}$ & 622.4 & 493.2 & 287 \\
Cellulose & 100.3 & 79.5 & 45.7 \\
Soybean oil & 50 & 50 & 50 \\
Mineral Mixture (AIN 93-M) & 40 & 40 & 40 \\
Vitamin Mixture (AIN 93-V) & 35 & 35 & 35 \\
Choline & 10 & 10 & 10 \\
Energy content (\%) & 2.3 & 2.3 & 2.3 \\
Protein & & & \\
Carbohydrate & 14 & 30 & 55 \\
Fat & 76 & 60 & 35 \\
Energy density (kJ/g) & 10 & 10 & 10 \\
$\quad 14.5$ & 14.5 & 14.5 \\
\hline
\end{tabular}




\subsection{Experimental Design}

Three types of experimental isocaloric diets $(14.5 \mathrm{~kJ} / \mathrm{g})$ were used in this study: a normoproteic diet (P14, $140 \mathrm{~g} / \mathrm{kg}$ milk protein), a moderately HP diet (P30, $300 \mathrm{~g} / \mathrm{kg}$ of milk protein) and an elevated HP diet (P53, $530 \mathrm{~g} / \mathrm{kg}$ of milk protein) isocellulose diet with similar energy content (Table 1). Healthy controls (non-treated mice at day $0, n=12$ ) received fresh tap water. DSS-treated mice $(n=132)$ were given 3.5\% (wt/vol) DSS (36,000-50,000 MW, MP Biomedicals Illkirch-Graffenstaden, France) in the drinking water for 5 days, from day 1 to day 5 (fresh DSS solution being prepared every two days) to induce an acute colitis episode (Figure 1). At day 7, corresponding to the maximal intensity of colon inflammation, mice were divided into three groups with the same mean body weight (BW) and were fed either the P14, the P30 or the P53 diet during 3, 6 or 21 days. Food and drink were given ad libitum and measured daily. Disease activity was scored based on stool consistency, rectal bleeding and percentage of body weight loss, as previously described [15]. For each parameter, a score of 0 to 3 was attributed: loss of weight $(0$, none; $1,0 \%-10 \% ; 2,10 \%-20 \% ; 3,>20 \%)$, stool consistency $(0$, normal pellets; 1 , slightly loose feces; 2 , loose feces; 3 , watery diarrhea) and visible fecal blood ( 0 , negative; 1 , slightly bloody; 2 , bloody; 3 , blood in the whole colon). Mice were euthanized to evaluate the impact of diet on epithelial repair kinetics at days $10(n=12), 13(n=12)$ and $28(n=16)$ per diet group (Figure 1). Body fat and lean body mass of mice belonging to day 28 group, were measured every nine days with dual-energy $x$-ray absorptiometry (DEXA) using a PIXImus imager (GE Lunar PIXImus, GE Healthcare, Fitchburg, WI, USA). Mice were euthanized if they lost more than $20 \%$ BW, as per approved animal protocol guidelines, to meet the end point criteria.

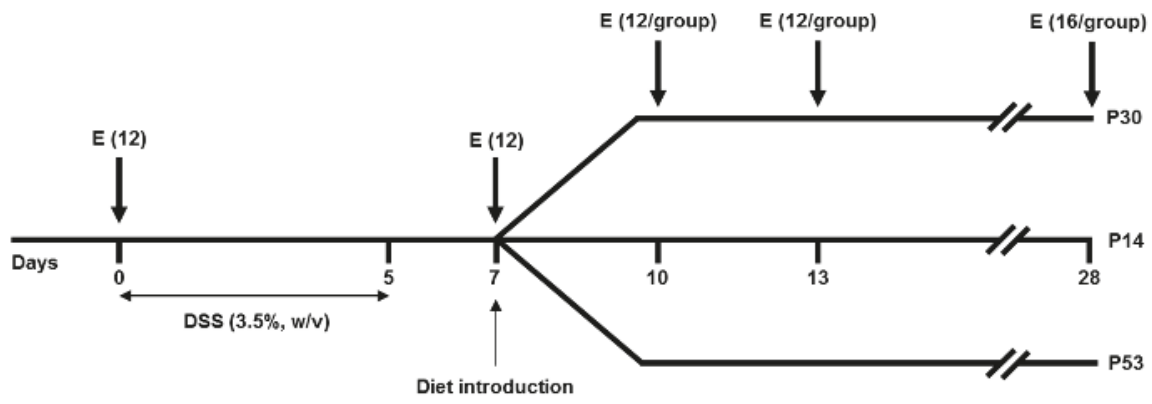

Figure 1. Schematic representation of the experimental design. Mice received either P14 (14\% of proteins), P30 (30\% of proteins) or P53 (53\% of proteins) diet from D7 to D28. Mice were euthanized (E) at day 0,7 , and during the resolution phase at days 10,13 and 28.

\subsection{Intestinal Permeability Assessment}

Firstly, epithelial barrier integrity was assessed in vivo with the $4 \mathrm{kDa}$ paracellular marker fluorescein isothiocyanate (FITC)-labeled dextran (FD4, Sigma-Aldrich, St Quentin Fallavier, France) at days 9, 12 and 18. Food was withdrawn for $4 \mathrm{~h}$ before gavage ( $600 \mathrm{mg} / \mathrm{kg}$ BW of FD4) and plasma was collected from the tail $4 \mathrm{~h}$ later $(n=8 /$ diet group). Fluorescence intensity of each sample was later used to calculate permeability using standard curves generated by serial dilution of FD4. Secondly, at euthanasia, the proximal colon section was mounted in EasyMount Ussing chambers (Physiologic Instrument Inc, San Diego, CA, USA), within $15 \mathrm{~min}$ from dissection as previously described [13]. Paracellular permeability was assessed by measuring the mucosal-to-serosal flux of FD4 at a final concentration of $0.25 \mathrm{mg} / \mathrm{mL}$, and fluorescence units (FU) were measured 90 min later with the Infinite ${ }^{\circledR} 200$ Pro spectrofluorimeter (TECAN, Männedorf, Switzerland). Tissue viability was assessed at the end of each recording by adding the cholinergic drug carbachol $\left(10^{-4} \mathrm{M}\right)$ on the serosal side. 


\subsection{Tissue Collection}

Mice were euthanized by an intracardiac puncture after sedation by isoflurane. Plasma was frozen and kept at $-80^{\circ} \mathrm{C}$ for later measurement of cytokines and lipopolysaccharide binding protein (LBP) concentrations. Colon was resected, measured, weighted and the proximal colon section mounted in Ussing chamber. Proximal colon mucosa was scraped for latter mucosa-adherent bacterial DNA extraction procedure. Colon samples were harvested for RNA analysis, myeloperoxidase (MPO) activity and protein expression assays, immediately frozen in liquid nitrogen and stored at $-80^{\circ} \mathrm{C}$ after resection. Histological analysis was performed with distal colon fixed in $4 \%$ buffered formaldehyde.

\subsubsection{Determination of Local and Systemic Inflammatory Markers}

Colon inflammation was assayed with MPO assay measurement as described in Reference [20] and colonic IL- $1 \beta$ and IL- 6 concentrations were measured by Luminex technology in total colon protein lysate by using Bio-Plex kits (Bio-Rad, Marnes-La- Coquette, France). Plasma concentration of LBP was determined with a commercial solid-phase sandwich ELISA (PikoKine ELISA Kit Mouse, Set EK1274; Boechout, Belgium).

\subsubsection{Histological Analysis}

Histological and re-epithelization scores on hematoxylin-and-eosin (HE) stained colonic sections were calculated as previously described (Vidal-Lletjós, et al., submitted) after blind microscopic assessment performed by the histological platform Histalim (Montpellier, France). Quantitative evaluation of well-oriented crypts and cell numeration in periodic acid-schiff (PAS) stained 4- $\mu \mathrm{m}$ transversal colon sections was determined using the image analysis software Pannoramic Viewer v. 1.15.4 (3DHISTECH, Budapest, Hungary). Paraffin-embedded distal colon samples were cut into $4 \mu \mathrm{m}$-thick sections, mounted on positively charged slides and dried. Immunohistochemical stainings of Ki67 and Caspase 3 were performed on the Discovery XT Automated IHC stainer using either Ventana DAB MAP detection kit (Ventana Medical Systems, Tucson, AZ, USA) or the Ventana CHROMO MAP detection kit (Ventana Medical Systems, Tucson, AZ, USA). Following deparaffination with Discovery wash solution (Ventana), antigen retrieval was performed using a Tris-based buffer solution. Later, endogen peroxidase was blocked and slides rinsed before incubation with primary antibodies either rabbit anti-ki67 (NB600-1252 Novusbio, Centennial, CO, USA) diluted at 1/100 or rabbit anti-Casp3 (9661, Cell Signaling, Danvers, MA, USA) diluted at 1/250. For Ki67 staining, signal enhancement was performed using Goat anti-Rabbit biotinylated secondary antibody (Vector laboratory, Burlingame, CA, USA) and DAB MAP detection kit. An anti-rabbit HRP (Ventana Medical Systems, Tucson, AZ, USA) secondary antibody and the CHROMO MAP detection kit (Ventana Medical Systems, Tucson, AZ, USA) was used for the caspase 3 staining. Slides were then counterstained with hematoxylin and rinsed. Slides were manually dehydrated and coverslipped. Ki67 labelling index was calculated as the percentage of Ki67 positive cells relative to the total number of cells within the same crypts.

\subsubsection{RNA Isolation and Quantitative Real-Time PCR}

Total RNA was extracted after tissue homogenization in TRIzol ${ }^{\circledR}$ Reagent (Invitrogen, Cergy Pontoise, France). Purification was performed with the RNeasy Mini Kit (Qiagen, Courtaboeuf, France) and a DNase step (Qiagen, Courtaboeuf, France). After cDNA synthesis from mRNA using a High Capacity cDNA Reverse Transcription Kit (Applied Biosystems, Fisher Scientific, Illkirch, France), quantitative real-time polymerase chain reaction (qRT-PCR) was performed (primer sequences available on demand) with the Fast SYBR Green MasterMix (Applied Biosystems, Fisher Scientific, Illkirch, France) and StepOne Real-Time PCR system (Applied Biosystems, Fisher Scientific, Illkirch, France). Gene expression level was normalized relative to the normalizing gene HPRT and normalized to day 7 group with $2^{-\Delta \Delta C t}$ calculation. 


\subsubsection{Western-Blot Analysis}

Frozen colonic tissue was homogenized in a lysis buffer [15], and $25 \mu \mathrm{g}$ of total protein lysates were loaded onto 4\%-12\% Criterion XT gel (Bio-Rad, Marnes-La-Coquette, France) before electrophoresis in MOPS buffer (Bio-Rad, Marnes-La-Coquette, France). After transfer onto nitrocellulose membrane and incubation in blocking solution (TBS pH 7.5, 0.05\% Tween 20, and 5\% (wt/vol) non-fat dry milk), membranes were incubated overnight $\left(4{ }^{\circ} \mathrm{C}\right)$ with rabbit ZO-1 antibody $(1 / 250,617300$, Invitrogen, Cergy Pontoise, France) or with mouse claudin-1 antibody (1/250, 374900, Thermo-Fisher Scientific, Bedford, MA, USA) diluted in blocking solution. After three washes, blots were incubated for $2 \mathrm{~h}$ at room temperature with an anti-rabbit or anti-mouse HRP-linked secondary antibody. Revealing was performed using enhanced chemiluminescence (ECL system, Pierce Biotechnology, Courtabœuf, France), and bands were quantified by densitometry using the FluorChem FC2 device and the AlphaView software (Cell Biosciences, Santa Clara, CA, USA). GAPDH expression (Ab9484, mouse monoclonal antibody, Abcam, Cambridge, UK) was used to ensure consistent protein loading and transferring.

\subsubsection{Evaluation of Adherent Mucosal Microbiota Composition}

From the scrapped adherent-mucosa, bacterial DNA extracts were obtained by using the PowerFecal DNA Isolation kit (MoBio Laboratories, Carlsbad, CA, USA) according to the manufacturer's protocol. Specific regions of the bacterial 16S rDNA gene were amplified using real-time qPCR. Eubacteria were quantified by real-time qPCR using specific primers (HAD-1: 5'-TGGCTCAGGACGAACGCTGGCGGC-3' and HAD-2: 5'-CCTACTGCTGCCTCCCGTAGGAGT-3'), annealing at $59{ }^{\circ} \mathrm{C}$ for total bacteria. In addition, the V3-V4 region amplification of the $16 \mathrm{~S}$ rDNA gene was performed and sent to the GenoToul platform (Castanet-Tolosan, France) for MiSeq Illumina sequencing using the MiSeq kit V2 $2 \times 250 \mathrm{bp}$. Data quality check and analysis were performed using the INRA-Migale server, as previously published [21]. In parallel, sulphate-reducing bacteria group were quantified by real-time qPCR using specific primers targeting Dissimilatory Sulfite Reductase A (DSR-1F: 5'-ACSCACTGGAAGCACGGCGG-3' and DSR-R: 5'-GTGGMRCCGTGCAKRTTGG-3'), Fast SYBR Green MasterMix (Applied Biosystems, Fisher Scientific, Illkirch, France) and StepOne Real-Time PCR system (Applied Biosystems, Fisher Scientific, Illkirch, France).

\subsection{Statistical Analysis}

All statistical analysis were performed with R software version 1.0.143 (Boston, MA, USA). For follow-up data, a mixed-model was used with mice as random effect while diet and time repeated factor were used as fixed effects. From day 7 , the mean of values for each diet were compared using Bonferonni post-hoc tests. For parameters measured at euthanasia, Anova with diet as a fixed effect was used and the means were compared using Bonferonni post-hoc tests. For all statistical tests, the level of significance was set to $p<0.05$. Principal component analysis (PCA) was performed on family-level taxonomy to assess the influence of diet on the mucosa-adherent microbiota composition at days 10, 13 and 28 as well as all times combined. The analysis of similarity (Anosim) test was used to assess the correlation between the ecological distance (based on family composition) and diet groups where an R-value of 0 indicates the highest dissimilarity possible and 1 indicates the highest similarity possible.

\section{Results}

\subsection{Dietary Protein Level Influences Colon Inflammation and Body Weight Recovery}

High protein diets, which were introduced the day of the maximal inflammatory score (at day 7), did not influence mortality, which was similar between the three groups (12.5\% per diet) from day 3 to day 14. After an average of $14 \%$ BW loss, mild to severe diarrhoea and rectal bleeding, disease activity index (DAI) started to decline after day 8 for P14 and P30 diets (Figure 2A), while a 
two-day delay was observed for P53 mice. In this latter group of animals, the DAI was systematically two-point higher from day 9 to day 19 when compared to the other two diets (Figure 2A) due mainly to higher stool consistency score. This was confirmed by the area under the curve (AUC) values, even though P14 and P30 were not different between them (P14: 72.6 and P30: 71.03 AUC of DAI measured between 0 and 28 days, NS), both were statistically different from P53 (45.8 AUC of DAI for 28 days, vs P14 and P30, $p<0.01$ ). In addition, P30 mice recovered their fat mass more rapidly than with the two other diets (P14: 33.9, P30: 49.2 and P53: 23.0 AUC of fat percentage evolution between days 7 and 26, $p<0.001$ ) (Figure 2B). These differences were not related to the global energy intake level, since diet intake per day was similar whatever the diet (data not shown). P14 and P30 fed-mice presented a similar level of inflammation based on colon MPO activity (Figure 2C) and IL-6 and IL-1 $\beta$ concentrations (Figure 2D) but these inflammatory biomarkers were higher in P53-fed mice. Colonic MPO activity was indeed significantly increased at day 28 for P53 mice compared to P30 (Figure 2C), as well as the pro-inflammatory cytokines at day 10 and at day 28 for IL-1 $\beta$ (Figure 2D). However, the overall histological score (based on crypt damage, ulceration and erosion, goblet cell depletion and cell infiltration) which showed high inter-individual variability, was not different between groups whatever the time (Figure $3 \mathrm{~A}$ ). Although the histological score was significantly reduced for all the groups when compared to day $7(9.3 \pm 1.4, p<0.05)$, some histological abnormalities persisted for all dietary groups at day 28 .

A

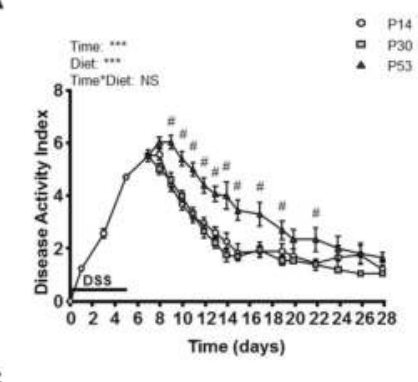

C

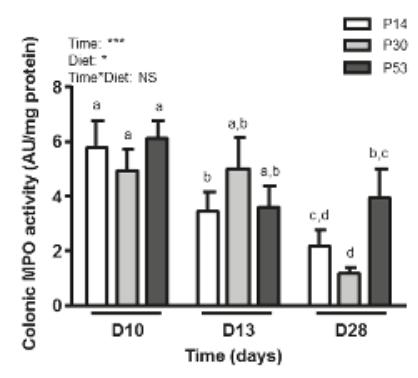

\section{B}

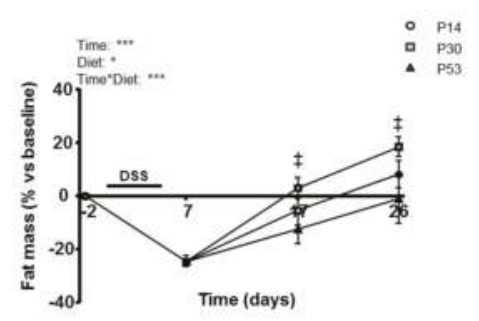

D

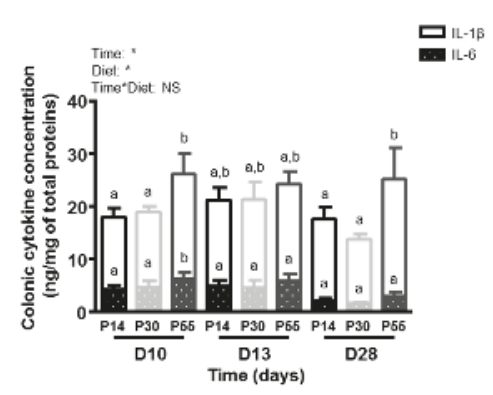

Figure 2. Effects of dietary protein intake level on inflammatory parameters at after 3 (D10), 6 (D13) or 21 days (D28) of dietary intervention with P14, P30 or P53 diet. (A): Disease activity index scoring evolution during time. \#: $p<0.05$ DSS P53 vs P14 and P30 at the same time point. (B): Evolution of fat mass (percentage vs baseline). $\ddagger: p<0.05$ DSS P53 vs P30 at the same time point. (C): Colonic MPO activity. (D): Colonic concentrations of the pro-inflammatory cytokines IL-6 and IL-1 $\beta$. Values are means $\pm \operatorname{SEM}(n=9-12)$. Means that are significantly different $(p<0.05)$ according to the post-hoc test have different letters (a or b or $\mathrm{c}$ or $\mathrm{d}$ ). * $: p<0.05 ;{ }^{* * *}: p<0.001$; NS: non-significant difference. 
A

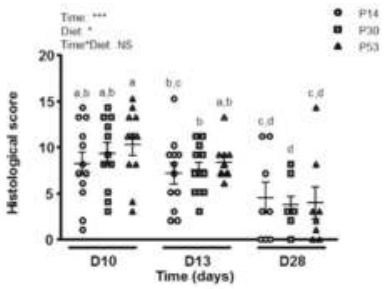

B

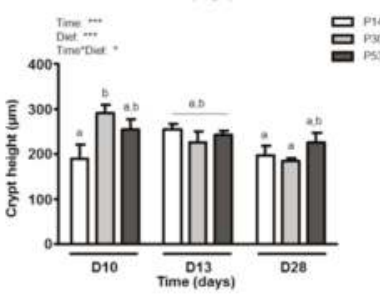

c

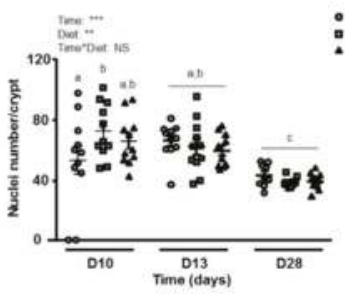

D
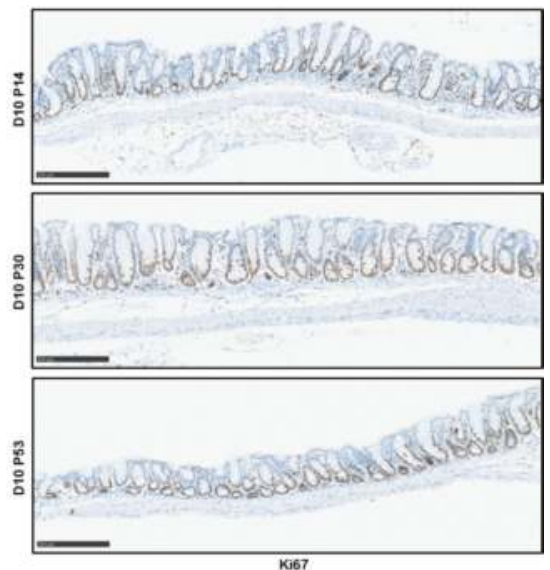

E

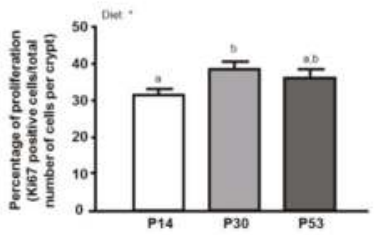

Figure 3. Effects of dietary protein intake on colon histomorphology after 3 (D10), 6 (D13) or 21 days (D28) of dietary intervention with P14, P30 or P53 diet. (A): Histological score. (B): Colon crypt length. (C): Cell numeration. (D). Representative illustrations of longitudinal colonic sections immunostained with an anti-Ki67 antibody. (E): Percentage of proliferation (Ki67-positive cells/total number of cells per crypt) at day 10. Values are means $\pm \operatorname{SEM}(n=9-12)$. Means that are significantly different $(p<0.05)$ according to the post-hoc test have different letters (a or b or $\mathrm{c}$ or d). ${ }^{*}: p<0.05 ;{ }^{* *}: p<0.01 ; * * *: p<0.001$; NS: non-significant difference.

\subsection{P30 Diet Enhances Epithelial Repair Features}

P30 mice showed a significant thickening of the colonic wall at day 10 (colon weight/length ratio: $0.037 \pm 0.002$ ) when compared to day 7 (P30: $0.025 \pm 0.002, p=0.0059)$ and to P14 diet (P14: $0.030 \pm 0.002, p=0.0142)$. P30-fed mice were indeed the only group which recovered initial colonic length at day 28 (day 0: $6.50 \pm 0.17 \mathrm{~cm}$ vs day $28 \mathrm{P} 30: 5.98 \pm 0.16 \mathrm{~cm}$, NS) contrastingly to other dietary protein groups $(5.70 \pm 0.20 \mathrm{~cm})$. This increase in colon weight and length corresponded to a higher crypt height and a higher number of nuclei per crypt in P30 mice than in the P14 group at day 10 (Figure 3B,C). The percentage of Ki67 positive cells was greater in the colonic tissue of P30 mice (vs P14, $p=0.0467$ ), as illustrated in Figure 3D although 30\% of the mucosa surface on average was devoid of crypts in all groups at day 10, and the number of caspase 3 positive cells was significantly reduced in P30 mice when compared to the other two diets (P30: $0.21 \pm 0.07$ vs P14: $0.70 \pm 0.26$ and P53: $0.98 \pm 0.26$ positive cells per crypt, $p<0.05$ ). These epithelial morphometric changes coincided with increased gene expression of the epithelial repair factor Tgf- $\beta 1$ in P30-fed mice (Table 2). Interestingly, the gene encoding Gpx2, which protects intestinal tissue against oxidative damage, displayed an increased expression in P30 mice at day 10 (P30: $1.21 \pm 0.21$ vs P14: $0.78 \pm 0.06$ and P53: $0.51 \pm 0.12$, $p<0.05$ ). In addition, the genes encoding Tff3, colonic Saa (Serum Amyloid A) and Il-15 were also higher compared to P14 at day 13 (Table 2). 


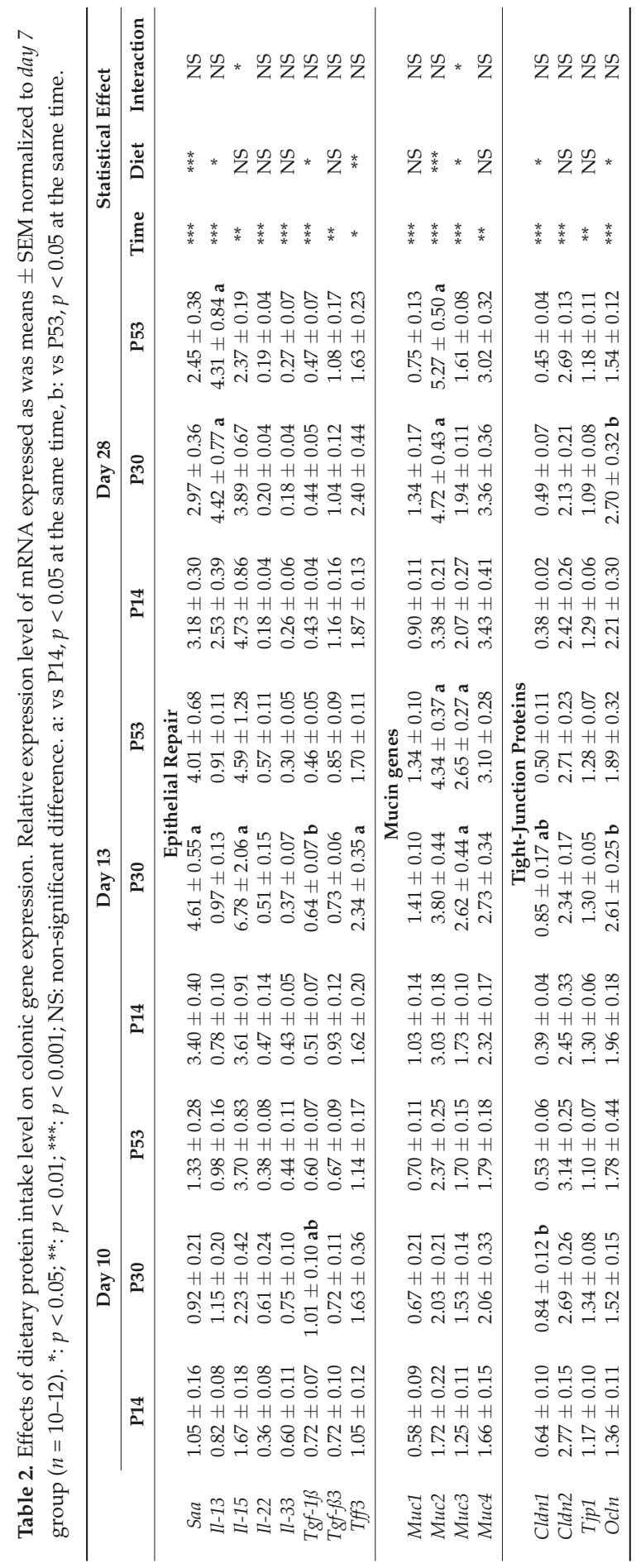




\subsection{P30 Diet Improves Dss-Induced Barrier Function Alterations}

Interestingly, FD4 challenge showed an intestinal permeability reduction for animals receiving a P30 diet at days 9 and 12 compared to the P14 group (Figure 4A) and this was confirmed at the colon level where FD4 flux measured in a Ussing chamber was decreased in P30 mice (7119 $\pm 507 \mathrm{FU})$ when compared to P14 (9945 $\pm 303 \mathrm{FU})$ at day 10 ( $p=0.014)$. This reduction of DSS-induced colon permeability was associated with lower LBP plasmatic concentrations at days 13 and 28 compared to P14 and P53 groups (Figure 4B). In addition, the gene expression of the tight-junction proteins $C l d n 1$ and $O c l n$ was differentially modulated by dietary protein intake. Indeed, $C l d n 1$ gene expression was increased by P30 at days 10 and 13 while Ocln increased at days 13 and 28 (Table 2). This result of Cldn1 expression has been confirmed by western blotting (Figure 4A), where Claudin-1 protein level was higher in P30 colon at day 13. Moreover, ZO-1 was more expressed at day 13 in P30 mice, when compared to the other two groups, although no significant difference at the gene expression level was detected (Tjp1, Table 2) and inter-individual variability was seen (Figure 4C). In addition, the level of dietary protein intake modulated colonic gene expression of several mucins. Indeed, both HP diets increased mRNA expression of the transmembrane mucin $M u c 3$ at day 13 and the main gel-forming mucin Muc2 at day 28 (which was also increased at day 13 by the P53 diet only). In contrast, neither Muc1 nor Muc4 were affected by the diet (Table 2).

A

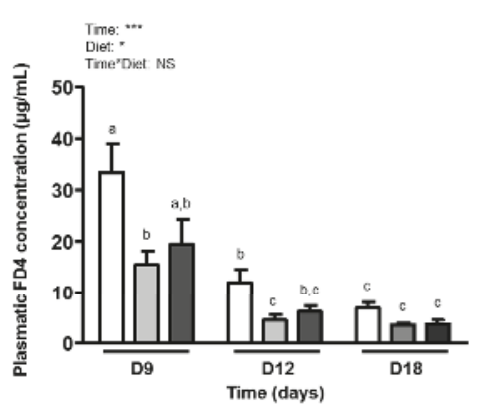

B

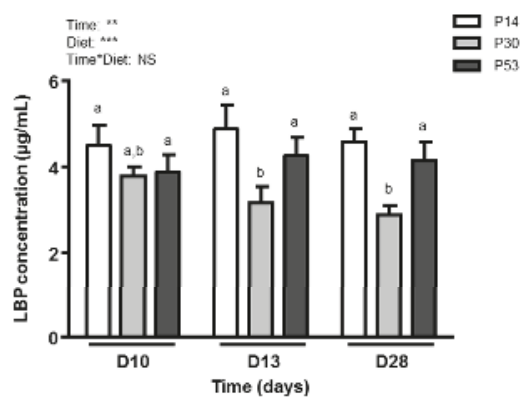

C
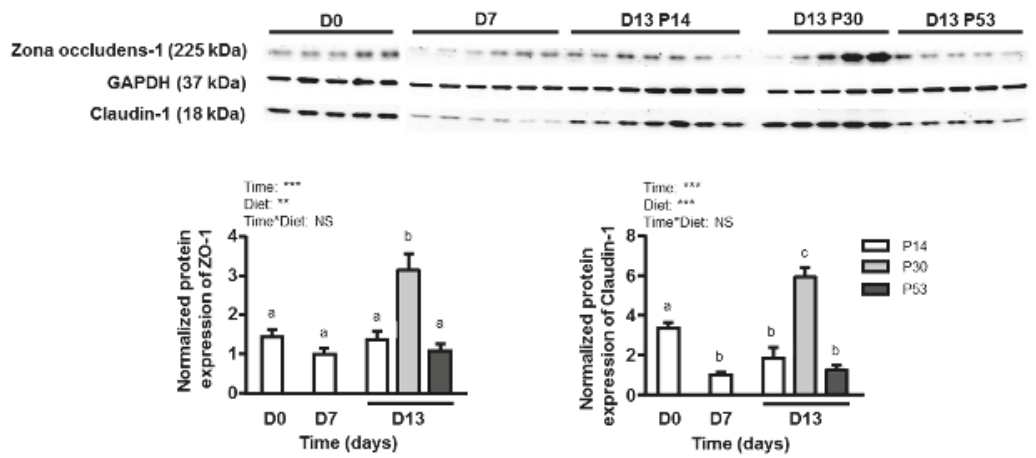

Figure 4. Effects of dietary protein intake level on barrier function after dietary intervention with P14, P30 or P53 diet. (A): Evolution of plasmatic FD4 concentration. (B): Plasmatic concentration of LPS-binding protein (LBP). (C): Western-blot analysis of the tight junction proteins Zona Occludens-1 and Claudin-1, was performed in colonic homogenates of day 13 mice. GAPDH was used as loading control. Images are representative of four Western blots. Means that are significantly different $(p<0.05)$ according to the post-hoc test have different letters (a or b or c). Values are means $\pm \operatorname{SEM}(n=10-12)$. $*: p<0.05 ; * *: p<0.01 ; * * *: p<0.001$; NS: non-significant difference. 
3.4. DSS-Induced Dysbiosis is not Alleviated by Diet, but P30 Diet Increases the Proportions of Butyrate-Producing Bacteria

DSS-induced dysbiosis was not resolved within 28 days, whatever the diet (Table 3). Diversity and richness were still lower than at day $0(6.46 \pm 0.07$ and $794 \pm 55.2$ for Shannon and Chao indexes, respectively) and similar to day 7 ( $4.75 \pm 0.32$ and $633 \pm 43.7$, for Shannon and Chao, respectively). Only the Simpson index was significantly less decreased in P30 group at day 10 when compared to the two other diets. In addition, the number of observed OTUs at day 13 was significantly decreased in P53 mice compared to P30 (Table 3).

The overall microbiota composition at the phylum level for each dietary group is shown in Figure 5A and the data describing microbial composition similarities based on family were compared between the diet groups with PCA with the three diet groups as classifying variables (Figure 5B-D). At day 10, mucosa-adherent microbial samples clustered by diet groups, as evidenced by their significant ecological distance $(\mathrm{R}=0.32, p<0.001$ ) (Figure $5 \mathrm{~B})$. P53 group was mostly explained by Clostridiales (Firmicutes), Enterobacteriaceae (Proteobacteria), Rikenellaceae and Porphyromonaceae (Bacteroidetes) versus Eubacteriaceae (Firmicutes) and Bifidobacteriaceae (Actinobacteria) for P30 group (Figure 5B). Haemophilus (Proteobacteria) and Alloprevotella (Bacteroidetes) proportions were statistically increased in P53 mice at day 10 while Desulfovibrio (Proteobacteria), an $\mathrm{H}_{2} \mathrm{~S}$ producer, showed a higher abundance at day 10 for P30 and P14 diets (vs P53, $p<0.05$ ) (Table 3). However, the quantification of total sulphato-reducing bacteria did not indicate differences at any time for any diet (data not shown). Interestingly, the Bacteroides genus relative percentage was reduced in the P30 group (vs P14 and P53, $p<0.001$ ) while caecal SCFA concentrations were higher (Table 3).

A

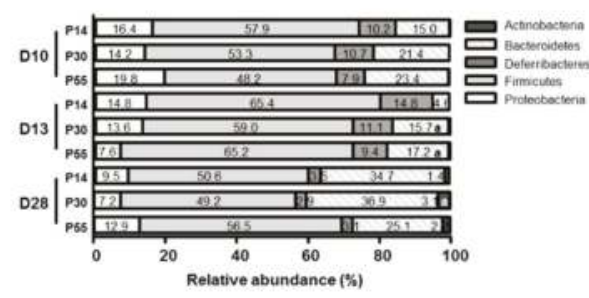

C

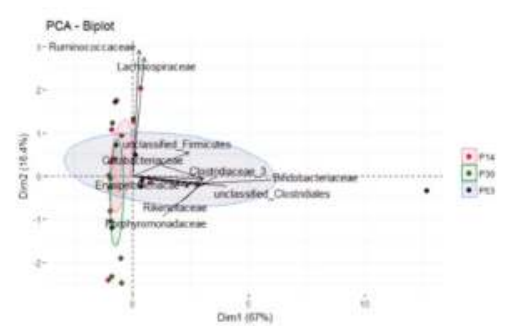

B

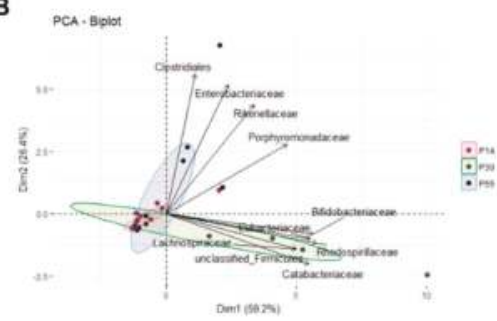

D

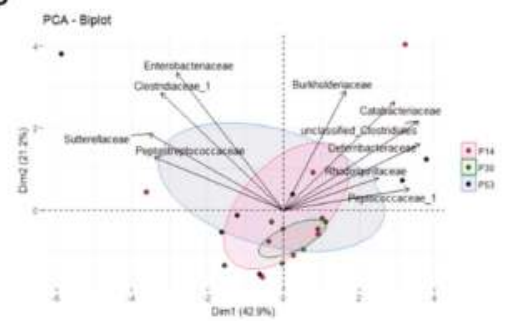

Figure 5. Effects of dietary protein intake on mucosa-associated microbiota composition after 3 (D10), 6 (D13) or 21 days (D28) of dietary intervention with P14, P30 or P53 diet. (A). Relative abundance percentages of bacterial phyla in mucosa-adherent microbiota a: vs P14, $p<0.05$ at the same time. Due to low relative proportions, Tenericutes is not visible in the figure. (B-D): Principal coordinate analysis on family relative abundances of mucosa-adherent microbiota at day 10 (B), day 13 (C) and day 28 (D). 


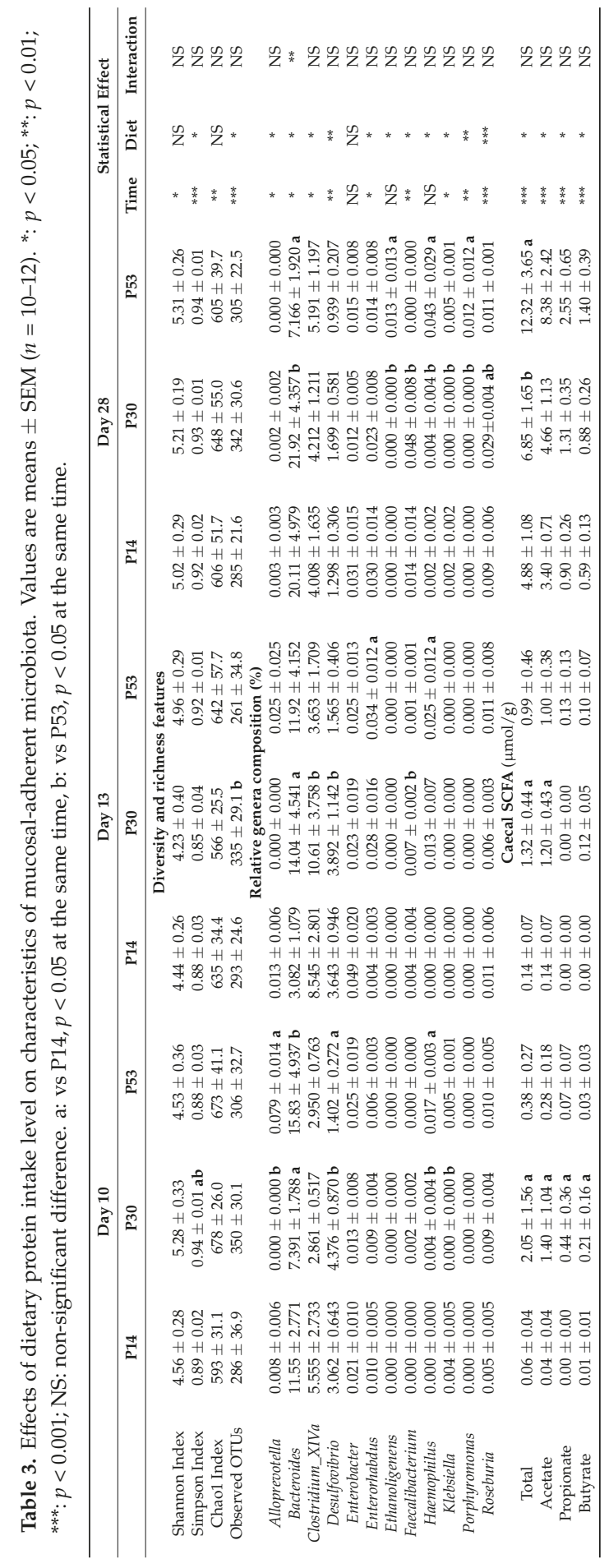


Although Firmicutes phylum proportions remained stable and similar to day 0 (Figure 5) whatever the time and the diet, Clostridium_XIVa and Faecalibacterium proportions were higher in P30 group than in P53 at day 13 ( $p=0.005)$ (Table 3). In contrast to day 10, Bacteroidetes relative abundance was higher with both P30 and P53 diets owing to increased proportions of Bacteroides genus. Moreover, Haemophilus had increased proportions in the P53 group when compared to the P14 group at day 13, as well as Enterorhabdus (Actinobacteria) (Table 3).

At day 28, the impact of dietary protein intake on mucosal-adherent composition was less marked between groups (Figure 5D) especially between P14 and P30 groups (Table 3). However, Actinobacteria, which proportions stayed low before day 28 (day 0-day 13: 0.1\%) (Figure 5A), were two-fold higher in P30 (P30: 3.1\% vs P14: 1.4\%, $p=0.014$ ) and in the P53 group, the proportion of Bifidobacteriaceae being increased in that latter group ( $p=0.021, \mathrm{P} 14: 0.60 \pm 0.28 \%$, P30: $1.70 \pm 0.83 \%$ and P53: $0.93 \pm 0.36 \%$ ). In addition, the protein intake modulated microbiota activities since total caecal SCFA concentrations increased in both HP groups at day 28. Although Deferribacteres proportion was reduced for the three diets when compared to days 10 and 13, reaching day 0 level $(3.7 \pm 1.0 \%$, NS vs day 28 whatever the diet), no other major differences at the phylum level were recorded. Interestingly, Lachnospiraceae family (Firmicutes) relative proportion at day 28 was greater than the one at day 7 (day 7: $13.48 \pm 2.91 \%$ vs day 28: $24.22 \pm 1.82 \%, p=0.0184$ ). Moreover, higher proportions of Faecalibacterium (Clostridiaceae) (vs P53) and Roseburia (vs P53 and P14) in P30 group were observed at day 28 (Table 3). Contrastingly, P53 microbiota composition showed again noticeable taxonomic differences compared to P14 and P30 mice, with increased abundance of the genera Porphyromonas, Ethanoligenens and Haemophilus contrasting with a decreased abundance of Bacteroides (Table 3).

\section{Discussion}

The present study showed that the level of dietary protein intake differentially modulated mucosal healing after an acute colon inflammatory episode. While the P53 diet worsened both the intensity and duration of the inflammation induced by DSS treatment, the P30 diet, when compared to P14 diet, accelerated epithelial repair by favoring the restoration of colon barrier architecture and function.

P30 diet indeed induced crypt hyperproliferation associated with increased gene expression of the repairing factors $T g f-\beta 1$ and $T f f 3$, both factors being known to contribute to the integrity of mucosal surface continuity but in an independent manner [22]. In addition, Saa, which was over-expressed in P30 mice compared to the two other diets, was recently described as a protective factor against colon epithelium acute injury [23]. Altogether, the increased expression of genes encoding these promoting repair factors as well as tight-junction proteins (Claudin-1 and Zona-Occludens 1), likely contributed to the restoration of colon barrier integrity, as evidenced by lower permeability and bacterial translocation-related marker (LBP) in the systemic flow. Additionally, an increased mRNA level of Gpx2, which upregulation is associated with inflammation resolution [24], suggests a lower inflammation-induced oxidative stress in P30 animals. Furthermore, the P30-mediated positive effects on epithelial repair might be related to a modulation of the mucosa-adherent microbiota composition and activities within the first days of colitis resolution. Indeed, the higher relative abundance of bacteria belonging to Lachnospiraceae, Eubacteriaceae and Bifidobacteriaceae families, together with higher concentrations of SCFAs, the major end products of microbiota metabolic activity, may partly explain the beneficial impact of P30 diet on colon mucosa. Interestingly, the P30 diet also longitudinally increased the proportion of Faecalibacterium, a commensal butyrate producer, detected in healthy subjects, whose abundance is reduced in IBD patients and is positively correlated with the maintenance of clinical remission $[25,26]$. Butyrate is well known to be a major fuel for colonic epithelial cells, to exert pluripotent effects on the colon such as the regulation of cell growth and differentiation [27], and to present anti-inflammatory properties [28]. Butyrate deficiency in its concentrations and/or in its transport and metabolism has been indeed observed in the inflamed mucosa [29].

Although minor differences in mucosa-adherent bacterial composition were noticed between the animals fed with P14 or P30 diets in the week following the acute colitis episode (with the notable 
exception regarding the Bacteroides proportion), bacterial populations were severely modulated in P53 mice compared to the two other diets. Indeed, P53 diet consumption in DSS-treated mice decreased the proportions of butyrate-producing bacteria from Clostridium XIVa, Faecalibacterium and Roseburia genera in the mucosa-adherent microbiota. P53 diet-fed mice were also characterized by an increased representation of families and genera previously reported in IBD patients during flares [30,31] such as Alloprevotella, Ethanoligenens, Klebsiella, Porphyromonas and Haemophilus. In parallel, Desulfovibrio, an $\mathrm{H}_{2} \mathrm{~S}$ producer, was reduced in $\mathrm{P} 53$ diet-fed mice as recently reported in the faecal microbiota recovered from UC patients [31], whereas the quantification of sulphato-reducing bacteria was not different between diets. This latter result is somewhat unexpected since an increased protein content in the diet is associated with a higher faecal $\mathrm{H}_{2} \mathrm{~S}$ concentration [32]. However, substrate availability, microbiote composition and metabolic activity are major parameters for fixing the production of bacterial metabolites [13], and other AA-derived bacterial metabolites that are known to exert deleterious effects on intestinal epithelial cells when present in excess, such as ammonium, p-cresol or hydrogen sulphide [9,33-35], that might have been over-produced in P53-fed mice. Our study is in accordance with Llewellyn et al. study that shows that the level of dietary proteins has an impact on colitis severity that is associated with changes in microbiota composition [36]. In this study, the authors have shown that a high-protein diet (41\%) enhanced disease progression when compared to a low-protein diet (6\%). However, comparison between this study and ours should take into account the important experimental designs differences, which were the quantity and quality of proteins in the diets. Indeed, we used a mix of milk proteins containing both whey and caseins (20:80) to mimic cow milk composition, while Llewellyn et al. used casein as the only protein source. Furthermore, diets were provided one week prior to the DSS administration, whereas we started to give our different diets after colitis induction at day 7. Other studies, outside the scope of the present work, are necessary for determining more precisely the changes in the luminal environment in each experimental conditions. Such changes may partly explain that P53-fed mice showed a higher inflammation state but a level of epithelial crypt repair similar to P30-fed mice. Consequently, in addition to the quantity, the source of dietary proteins may modulate the IBD course $[37,38]$ although association between protein intake and IBD risk has not been found in all studies [39,40], highlighting the urgent need for human studies to decipher the role of quantity and source of dietary proteins in IBD.

Furthermore, the reduction of carbohydrate supply in the diet may also influence colon healing. Indeed, P30 diet proportions might be more suitable to cover the requirements of macronutrients (both carbohydrates and proteins) to sustain the metabolic activity of cells [41] and synthesis of macromolecules involved in epithelial repair and restoration of barrier integrity. Additional experiments, such as the measurement of colon mucosal cell bioenergetics and protein synthesis during acute inflammation and following mucosal healing, would help to decipher these points.

\section{Conclusions}

Our results fit with the view that an optimized epithelium repair after an acute inflammatory episode can be obtained through the supply of an appropriate amount of dietary protein. It indeed depends upon a balance between the AA originating from dietary and endogenous proteins, which are available from the blood supply for mucosal healing, and the gut microbial activity towards undigested protein that enters the colon. Our study showed that a moderately high dietary protein intake during a post-inflammatory phase appears beneficial to speed up the restoration of the intestinal mucosa integrity when compared to a normoproteic diet, by affecting several key parameters associated with inflammation and healing during and after the inflammatory flare in the DSS model of colitis. In contrast, a further increase in the dietary protein amount appears counter-productive for such a process. As such, our experimental study is in accordance with the need for an increased protein intake during active IBD [7] but suggests that a threshold protein intake value should be not exceeded to avoid deleterious effects on the patients' inflamed mucosa. 
Author Contributions: S.V.-L., M.A., A.B., M.G., and A.L. performed experiments. S.V.-L., P.L., A.-M.D., R.V. and A.L. analyzed the data. A.L., M.L., C.G. and F.B. conceived and supervised the study. S.V.-L. and A.L. drafted the manuscript. All of the authors read and approved the final manuscript as submitted and agree to be accountable for all aspects of the work.

Funding: This work was supported by Institut National de Recherche Agronomique -AgroParisTech, by the Société Française de Nutrition and the Association François Aupetit. Sandra Vidal is a recipient of a PhD grant from INRA-Université Paris-Saclay (ALIAS program). Funding bodies had no role in the design of the study and collection, analysis, and interpretation of data and in writing the manuscript.

Acknowledgments: The authors greatly acknowledge R. Onifarasoaniaina and M. Favier from the Cochin HistIM Facility, Morgane Dufay who helped to take care of the animals, Maxime Evrard and Julie Bonnereau for their involvement in the experiment. Also, they want to thank Armor Protéines (Saint-Brice-en-Cogles, France) for providing the dietary proteins used to prepare diets.

Conflicts of Interest: The authors declare no conflict of interest.

\section{References}

1. Danese, S.; Fiocchi, C. Ulcerative colitis. N. Engl. J. Med. 2011, 365, 1713-1725. [CrossRef] [PubMed]

2. Neurath, M.F.; Travis, S.P.L. Mucosal healing in inflammatory bowel diseases: A systematic review. Gut 2012, 61, 1619-1635. [CrossRef] [PubMed]

3. Papi, C.; Fascì-Spurio, F.; Rogai, F.; Settesoldi, A.; Margagnoni, G.; Annese, V. Mucosal healing in inflammatory bowel disease: Treatment efficacy and predictive factors. Dig. Liver Dis. 2013, 45, 978-985. [CrossRef] [PubMed]

4. Neurath, M.F. New targets for mucosal healing and therapy in inflammatory bowel diseases. Mucosal Immunol. 2014, 7, 6-19. [CrossRef] [PubMed]

5. Sturm, A.; Dignass, A.U. Epithelial restitution and wound healing in inflammatory bowel disease. World J. Gastroenterol. 2008, 14, 348-353. [CrossRef] [PubMed]

6. Forbes, A.; Goldesgeyme, E.; Paulon, E. Nutrition in inflammatory bowel disease. JPEN. J. Parenter. Enteral Nutr. 2011, 35, 571-580. [CrossRef]

7. Forbes, A.; Escher, J.; Hébuterne, X.; Kłęk, S.; Krznaric, Z.; Schneider, S.; Shamir, R.; Stardelova, K.; Wierdsma, N.; Wiskin, A.E.; et al. ESPEN guideline: Clinical nutrition in inflammatory bowel disease. Clin. Nutr. 2017, 36, 321-347. [CrossRef] [PubMed]

8. Vidal-Lletjós, S.; Beaumont, M.; Tomé, D.; Benamouzig, R.; Blachier, F.; Lan, A. Dietary Protein and Amino Acid Supplementation in Inflammatory Bowel Disease Course: What Impact on the Colonic Mucosa? Nutrients 2017, 9, 310. [CrossRef] [PubMed]

9. Andriamihaja, M.; Davila, A.-M.; Eklou-Lawson, M.; Petit, N.; Delpal, S.; Allek, F.; Blais, A.; Delteil, C.; Tomé, D.; Blachier, F. Colon luminal content and epithelial cell morphology are markedly modified in rats fed with a high-protein diet. Am. J. Physiol. Gastrointest. Liver Physiol. 2010, 299, G1030-G1037. [CrossRef] [PubMed]

10. Blachier, F.; Beaumont, M.; Andriamihaja, M.; Davila, A.-M.; Lan, A.; Grauso, M.; Armand, L.; Benamouzig, R.; Tomé, D. Changes in the luminal environment of the colonic epithelial cells and physiopathological consequences. Am. J. Pathol. 2017, 187, 476-486. [CrossRef] [PubMed]

11. Geypens, B.; Claus, D.; Evenepoel, P.; Hiele, M.; Maes, B.; Peeters, M.; Rutgeerts, P.; Ghoos, Y. Influence of dietary protein supplements on the formation of bacterial metabolites in the colon. Gut 1997, 41, 70-76. [CrossRef] [PubMed]

12. Liu, X.; Blouin, J.-M.; Santacruz, A.; Lan, A.; Andriamihaja, M.; Wilkanowicz, S.; Benetti, P.-H.; Tomé, D.; Sanz, Y.; Blachier, F.; et al. High-protein diet modifies colonic microbiota and luminal environment but not colonocyte metabolism in the rat model: The increased luminal bulk connection. Am. J. Physiol. Gastrointest. Liver Physiol. 2014, 307, G459-G470. [CrossRef] [PubMed]

13. Beaumont, M.; Portune, K.J.; Steuer, N.; Lan, A.; Cerrudo, V.; Audebert, M.; Dumont, F.; Mancano, G.; Khodorova, N.; Andriamihaja, M.; et al. Quantity and source of dietary protein influence metabolite production by gut microbiota and rectal mucosa gene expression: A randomized, parallel, double-blind trial in overweight humans. Am. J. Clin. Nutr. 2017, 106, 1005-1019. [CrossRef] [PubMed] 
14. Blachier, F.; Beaumont, M.; Portune, K.J.; Steuer, N.; Lan, A.; Audebert, M.; Khodorova, N.; Andriamihaja, M.; Airinei, G.; Benamouzig, R.; et al. High-protein diets for weight management: Interactions with the intestinal microbiota and consequences for gut health. A position paper by the my new gut study group. Clin. Nutr. 2018. [CrossRef] [PubMed]

15. Lan, A.; Blais, A.; Coelho, D.; Capron, J.; Maarouf, M.; Benamouzig, R.; Lancha, A.H.; Walker, F.; Tomé, D.; Blachier, F. Dual effects of a high-protein diet on DSS-treated mice during colitis resolution phase. Am. J. Physiol. Gastrointest. Liver Physiol. 2016, 311, G624-G633. [CrossRef] [PubMed]

16. Liu, Y.; Wang, X.; Hu, C.-A. Therapeutic Potential of Amino Acids in Inflammatory Bowel Disease. Nutrients 2017, 9, 920. [CrossRef] [PubMed]

17. Sprong, R.C.; Schonewille, A.J.; van der Meer, R. Dietary cheese whey protein protects rats against mild dextran sulfate sodium-induced colitis: Role of mucin and microbiota. J. Dairy Sci. 2010, 93, 1364-1371. [CrossRef] [PubMed]

18. Faure, M.; Moënnoz, D.; Montigon, F.; Mettraux, C.; Breuillé, D.; Ballèvre, O. Dietary threonine restriction specifically reduces intestinal mucin synthesis in rats. J. Nutr. 2005, 135, 486-491. [CrossRef] [PubMed]

19. Gaifem, J.; Gonçalves, L.G.; Dinis-Oliveira, R.J.; Cunha, C.; Carvalho, A.; Torrado, E.; Rodrigues, F.; Saraiva, M.; Castro, A.G.; Silvestre, R. L-Threonine Supplementation During Colitis Onset Delays Disease Recovery. Front. Physiol. 2018, 9, 1247. [CrossRef] [PubMed]

20. Lan, A.; Blachier, F.; Benamouzig, R.; Beaumont, M.; Barrat, C.; Coelho, D.; Lancha, A.; Kong, X.; Yin, Y.; Marie, J.-C.; et al. Mucosal healing in inflammatory bowel diseases: Is there a place for nutritional supplementation? Inflamm. Bowel Dis. 2015, 21, 198-207. [CrossRef] [PubMed]

21. Chemouny, J.M.; Gleeson, P.J.; Abbad, L.; Lauriero, G.; Boedec, E.; Le Roux, K.; Monot, C.; Bredel, M.; Bex-Coudrat, J.; Sannier, A.; et al. Modulation of the microbiota by oral antibiotics treats immunoglobulin A nephropathy in humanized mice. Nephrol. Dial. Transplant. 2018, 1-10. [CrossRef] [PubMed]

22. Dignass, A.; Lynch-devaney, K.; Kindon, H.; Thim, L.; Podolsky, D.K. Trefoil peptides promote epithelial migration through a transforming growth factor beta-independent pathway. J. Clin. Investig. 1994, 94, 376-383. [CrossRef] [PubMed]

23. Zhang, G.; Liu, J.; Wu, L.; Fan, Y.; Sun, L.; Qian, F.; Chen, D.; Ye, R.D. Elevated Expression of Serum Amyloid a 3 Protects Colon Epithelium against Acute Injury Through TLR2-Dependent Induction of Neutrophil IL-22 Expression in a Mouse Model of Colitis. Front. Immunol. 2018, 9, 1503. [CrossRef] [PubMed]

24. Hiller, F.; Besselt, K.; Deubel, S.; Brigelius-Flohé, R.; Kipp, A.P. GPx2 induction is mediated through STAT transcription factors during acute colitis. Inflamm. Bowel Dis. 2015, 21, 2078-2089. [CrossRef] [PubMed]

25. Sokol, H.; Seksik, P.; Furet, J.P.; Firmesse, O.; Nion-Larmurier, I.; Beaugerie, L.; Cosnes, J.; Corthier, G.; Marteau, P.; Doraé, J. Low counts of faecalibacterium prausnitzii in colitis microbiota. Inflamm. Bowel Dis. 2009, 15, 1183-1189. [CrossRef] [PubMed]

26. Machiels, K.; Joossens, M.; Sabino, J.; De Preter, V.; Arijs, I.; Eeckhaut, V.; Ballet, V.; Claes, K.; Van Immerseel, F.; Verbeke, K.; et al. A decrease of the butyrate-producing species Roseburia hominis and Faecalibacterium prausnitzii defines dysbiosis in patients with ulcerative colitis. Gut 2014, 63, 1275-1283. [CrossRef] [PubMed]

27. Macfarlane, G.T.; Macfarlane, S. Bacteria, colonic fermentation, and gastrointestinal health. J. AOAC Int. 2012, 95, 50-60. [CrossRef] [PubMed]

28. Chang, P.V.; Hao, L.; Offermanns, S.; Medzhitov, R. The microbial metabolite butyrate regulates intestinal macrophage function via histone deacetylase inhibition. Proc. Natl. Acad. Sci. USA 2014, 111, 2247-2252. [CrossRef] [PubMed]

29. Thibault, R.; Blachier, F.; Darcy-Vrillon, B.; de Coppet, P.; Bourreille, A.; Segain, J.-P.P. Butyrate utilization by the colonic mucosa in inflammatory bowel diseases: A transport deficiency. Inflamm. Bowel Dis. 2010, 16, 684-695. [CrossRef] [PubMed]

30. Lucke, K.; Miehlke, S.; Jacobs, E.; Schuppler, M. Prevalence of Bacteroides and Prevotella spp. in ulcerative colitis. J. Med. Microbiol. 2006, 55, 617-624. [CrossRef] [PubMed]

31. Ma, H.-Q.; Yu, T.-T.; Zhao, X.-J.; Zhang, Y.; Zhang, H.-J. Fecal microbial dysbiosis in Chinese patients with inflammatory bowel disease. World J. Gastroenterol. 2018, 24, 1464-1477. [CrossRef] [PubMed]

32. Magee, E.A.; Richardson, C.J.; Hughes, R.; Cummings, J.H. Contribution of dietary protein to sulfide production in the large intestine: An in vitro and a controlled feeding study in humans. Am. J. Clin. Nutr. 2000, 72, 1488-1494. [CrossRef] [PubMed] 
33. Andriamihaja, M.; Lan, A.; Beaumont, M.; Audebert, M.; Wong, X.; Yamada, K.; Yin, Y.; Tomé, D.; Carrasco-Pozo, C.; Gotteland, M.; et al. The deleterious metabolic and genotoxic effects of the bacterial metabolite p-cresol on colonic epithelial cells. Free Radic. Biol. Med. 2015, 85, 219-227. [CrossRef] [PubMed]

34. Beaumont, M.; Andriamihaja, M.; Lan, A.; Khodorova, N.; Audebert, M.; Blouin, J.M.; Grauso, M.; Lancha, L.; Benetti, P.H.; Benamouzig, R.; et al. Detrimental effects for colonocytes of an increased exposure to luminal hydrogen sulfide: The adaptive response. Free Radic. Biol. Med. 2016, 93, 155-164. [CrossRef] [PubMed]

35. Portune, K.J.; Beaumont, M.; Davila, A.-M.; Tomé, D.; Blachier, F. Gut microbiota role in dietary protein metabolism and health-related outcomes: The two sides of the coin. Trends Food Sci. Technol. 2016, 57, 213-232. [CrossRef]

36. Llewellyn, S.R.; Britton, G.J.; Contijoch, E.J.; Vennaro, O.H.; Mortha, A.; Colombel, J.F.; Grinspan, A.; Clemente, J.C.; Merad, M.; Faith, J.J. Interactions Between Diet and the Intestinal Microbiota Alter Intestinal Permeability and Colitis Severity in Mice. Gastroenterology 2018, 154, 1037-1046.e2. [CrossRef] [PubMed]

37. Jantchou, P.; Morois, S.; Clavel-Chapelon, F.; Boutron-Ruault, M.-C.; Carbonnel, F. Animal protein intake and risk of inflammatory bowel disease: The E3N prospective study. Am. J. Gastroenterol. 2010, 105, $2195-2201$. [CrossRef] [PubMed]

38. Jowett, S.L.; Seal, C.J.; Pearce, M.S.; Phillips, E.; Gregory, W.; Barton, J.R.; Welfare, M.R. Influence of dietary factors on the clinical course of ulcerative colitis: A prospective cohort study. Gut 2004, 53, 1479-1484. [CrossRef] [PubMed]

39. Spooren, C.E.G.M.; Pierik, M.J.; Zeegers, M.P.; Feskens, E.J.M.; Masclee, A.A.M.; Jonkers, D.M.A.E. Review article: The association of diet with onset and relapse in patients with inflammatory bowel disease. Aliment. Pharmacol. Ther. 2013, 38, 1172-1187. [CrossRef] [PubMed]

40. Racine, A.; Carbonnel, F.; Chan, S.S.M.; Hart, A.R.; Bas Bueno-De-Mesquita, H.; Oldenburg, B.; Van Schaik, F.D.M.; Tjønneland, A.; Olsen, A.; Dahm, C.C.; et al. Dietary Patterns and Risk of Inflammatory Bowel Disease in Europe: Results from the EPIC Study. Inflamm. Bowel Dis. 2016, 22, 345-354. [CrossRef] [PubMed]

41. Kominsky, D.J.; Campbell, E.L.; Colgan, S.P. Metabolic shifts in immunity and inflammation. J. Immunol. 2010, 184, 4062-4068. [CrossRef] [PubMed] 


\title{
Intestinal Anti-Inflammatory Effect of a Peptide Derived from Gastrointestinal Digestion of Buffalo (Bubalus bubalis) Mozzarella Cheese
}

\author{
Gian Carlo Tenore ${ }^{1,+}{ }^{,}$Ester Pagano ${ }^{1,+}{ }^{+}$, Stefania Lama ${ }^{2}$, Daniela Vanacore ${ }^{2}$, Salvatore Di Maro, \\ Maria Maisto, Raffaele Capasso ${ }^{4}$, Francesco Merlino ${ }^{1}$, Francesca Borrelli ${ }^{1, *}$, Paola Stiuso ${ }^{2, *}$ and \\ Ettore Novellino ${ }^{1}$ \\ 1 Department of Pharmacy, School of Medicine and Surgery, University of Naples Federico II, Via D. \\ Montesano 49, 80131 Naples, Italy; giancarlo.tenore@unina.it (G.C.T.); ester.pagano@unina.it (E.P.); \\ maria.maisto@unina.it (M.M.); francesco.merlino@unina.it (F.M.); ettore.novellino@unina.it (E.N.) \\ 2 Department of Precision Medicine, University of Campania “Luigi Vanvitelli”, Via De Crecchio 7, \\ 80138 Naples, Italy; stefania.lama@unicampania.it (S.L.); daniela.vanacore@unicampania.it (D.V.) \\ 3 DiSTABiF, Università degli Studi della Campania "Luigi Vanvitelli", via Vivaldi 43, 81100 Caserta, Italy; \\ salvatore.dimaro@unicampania.it \\ 4 Department of Agricultural Sciences, University of Naples Federico II, 80055 Portici, Italy; \\ raffaele.capasso@unina.it \\ * Correspondence: franborr@unina.it (F.B.); paola.stiuso@unicampania.it (P.S.); Tel.: +39-081-678665 (F.B.); \\ +39-81-5667635 (P.S.); Fax: +39-081-678403 (F.B.); +39-81-5665869 (P.S.) \\ + These two authors equally contributed to this work.
}

Received: 1 February 2019; Accepted: 6 March 2019; Published: 13 March 2019

\begin{abstract}
Under physiological conditions, the small intestine represents a barrier against harmful antigens and pathogens. Maintaining of the intestinal barrier depends largely on cell-cell interactions (adherent-junctions) and cell-matrix interactions (tight-junctions). Inflammatory bowel disease is characterized by chronic inflammation, which induces a destructuring of the architecture junctional epithelial proteins with consequent rupture of the intestinal barrier. Recently, a peptide identified by Bubalus bubalis milk-derived products (MBCP) has been able to reduce oxidative stress in intestinal epithelial cells and erythrocytes. Our aim was to evaluate the therapeutic potential of MBCP in inflammatory bowel disease (IBD). We studied the effect of MBCP on (i) inflamed human intestinal Caco2 cells and (ii) dinitrobenzene sulfonic acid (DNBS) mice model of colitis. We have shown that $\mathrm{MBCP}$, at non-cytotoxic concentrations, both in vitro and in vivo induced the adherent epithelial junctions organization, modulated the nuclear factor (NF)- $\mathrm{kB}$ pathway and reduced the intestinal permeability. Furthermore, the $\mathrm{MBCP}$ reverted the atropine and tubocurarine injury effects on adherent-junctions. The data obtained showed that MBCP possesses anti-inflammatory effects both in vitro and in vivo. These results could have an important impact on the therapeutic potential of $\mathrm{MBCP}$ in helping to restore the intestinal epithelium integrity damaged by inflammation.
\end{abstract}

Keywords: intestinal inflammation; inflammatory bowel disease; epithelial adherens junctions; bioactive peptides

\section{Introduction}

Inflammatory bowel disease (IBD) is a term used to describe conditions that are characterized by chronic inflammation of the gastrointestinal tract. Crohn's disease and ulcerative colitis are the principal types of inflammatory bowel disease. Worldwide, the prevalence and incidence of IBD is increasing mainly in newly industrialized countries whose societies have become more Westernized [1] Although the etiology of IBD is still unknown, it arises as a result of the interaction of environmental 
or genetic factors and the immune responses [2]. IBD treatment involves the use of anti-inflammatory drugs (such as 5-aminosalicylic acid), corticosteroids, monoclonal antibodies [anti-tumor necrosis factor (TNF)- $\alpha$ antibodies] and vascular adhesion molecules [2,3] (anti-integrin antibodies). However, these drugs are not curative therapies and are mainly used as induction and maintenance therapy. Moreover, for most of these drugs, a high inter-individual variability in a positive response has been reported [3]. Therefore, it is essential to find clinical efficacious alternatives for the treatment of inflammatory bowel disease (IBD). Recently, intestinal permeability has been recognized as a new target for IBD prevention and therapy $[4,5]$. In physiological conditions, the intestinal epithelium selectively absorbs nutrients and represents an efficient barrier to noxious antigens and pathogens. Disruption of the intestinal barrier has been considered a major factor in several inflammatory intestinal diseases [6]. The architecture and function of the intestinal epithelium requires close coordination between enterocyte proliferation and apoptosis, both highly dependent on cell-cell and cell-matrix interactions. Enterocytes are joined to each other by tight junctions and adherens junctions. The main molecular component of the adherens junctions is E-cadherin, a transmembrane protein with five extracellular domains that interdigitate with those of an adjacent cell in a calcium-dependent homophilic manner to form a continuous linear "zipper" structure [7,8]. E-cadherin exerts functional adhesion activity when it is connected to the actin cytoskeleton by the cytoplasmic complex $\alpha$-, $\beta-$, or $\gamma$-catenin in a mutually exclusive manner $[7,8]$. It is well established that, in IBD patients, these junctional complexes undergo a disruption leading to a breaking of the intestinal barrier [9]. Moreover, mucosal biopsies from patients with IBD have increased levels of cytokines, able to regulate intercellular permeability, such as interferon-gamma (IFN- $\gamma$ ) and tumor necrosis factor (TNF- $\alpha$ ), have been observed [10].

Buffalo (Bubalus bubalis) milk is a source of many bioactive peptides that have been demonstrated to play a relevant role in preventing various disorders [11-14]. These bioactive peptides are mainly released during gastrointestinal digestion of milk and milk-products. Recently, novel peptides have been identified from buffalo milk-derived products (MBCP) that are able to reduce $\mathrm{H}_{2} \mathrm{O}_{2}$-induced oxidative stress in intestinal epithelial cells and in erythrocytes $[15,16]$. Among these, MBCP, a peptide isolated from the buffalo milk after in vitro digestion of Mozzarella di Bufala Campana $D O P$, has demonstrated possession of a good stability to brush border exopeptidases and a high bioavailability [16]. In this paper, we evaluated the therapeutic potential of MBCP in IBD. For this purpose, we investigated the effect of $\mathrm{MBCP}$ on (i) adjacent junctions conformation and permeability under inflammatory conditions in an intestinal epithelial cell line, Caco-2 cells and (ii) intestinal inflammation and the associated changes in motility in mice.

\section{Material and Methods}

\subsection{Peptide Synthesis}

MBCP was synthetised using the solid-phase method and purified via HPLC after deprotection, as previously described [16]. MBCP was dissolved in water for in vitro and in vivo experiments.

\subsection{Drugs and Reagents}

2,4,6-dinitrobenzenesulfonic acid (DNBS), croton oil, fluorescein isothiocyanate (FITC)-conjugated dextran (molecular mass 3-5 kDa), betamethasone, atropine, tubocurarine and neutral red (NR) solution were purchased from Sigma (Milan, Italy). TNF- $\alpha$ was obtained from R\&D Systems, Space Import-Export SRL (Milano, Italy). All reagents for cell cultures were obtained from Sigma, Bio-Rad Laboratories (Milan, Italy) and Microtech Srl (Naples, Italy). All chemicals and reagents employed in this study were of analytical grade. 


\subsection{Cell Culture}

Caco-2 cells (American Type Culture Collection, Rockville, MD, USA), a human colorectal adenocarcinoma epithelial cell line, were grown at $37{ }^{\circ} \mathrm{C}$ in a $5 \% \mathrm{CO}_{2}$ atmosphere in h-glucose Minimum essential medium MEM containing 1\% non-essential amino acids and supplemented with $10 \%$ de-complemented fetal bovine serum (FBS), $100 \mathrm{U} \cdot \mathrm{mL}^{-1}$ penicillin, $100 \mathrm{mg} \cdot \mathrm{mL}^{-1}$ streptomycin, $1 \%$ L-glutamine and $1 \%$ sodium pyruvate. The growth of the cells was measured by counting the cells with a Coulter counter (Nexcelom, Lawrence, MA, USA, Cellometer Auto1000). All experiments were performed in triplicate.

\subsection{Animals}

Male adult ICR mice, weighing 20-25 g for upper gastrointestinal transit experiments and 25-30 g for colitis experiments, were purchased from Charles River Laboratories (Calco, Lecco, Italy) and housed in polycarbonate cages under controlled temperature $\left(23 \pm 2{ }^{\circ} \mathrm{C}\right)$, constant humidity $(60 \%)$ and a $12 \mathrm{~h} \mathrm{light/dark}$ cycle. The animals were acclimatized to their environment at least 1 week under the above reported standard conditions with free access to tap water. Mice were fed ad libitum with a standard rodent diet, except for the $24 \mathrm{~h}$ immediately before the intracolonic administration of DNBS or the oral administration of the charcoal meal and for 2-h before the oral gavage of croton oil or MBCP. Mice were randomly allocated to different experimental groups and outcome assessments were performed in single-blind. All experiments were approved by the Institutional Animal Ethics Committee for the use of experimental animals and conformed to guidelines for the safe use and care of experimental animals in accordance with the Italian D.L. no. 116 of 27 January 1992 and associated guidelines in the European Communities Council (86/609/ECC and 2010/63/UE).

\subsection{Cytotoxicity Studies}

We evaluated the effect of $\mathrm{MBCP}$ on Caco-2 cell viability by using a microplate colorimetric assay that measures the ability of viable cells to incorporate and bind the neutral red (NR), a weak cationic dye, in lysosomes. Cells were plated at the appropriate density to obtain a model of undifferentiated and differentiated cells; then, $5 \times 10^{3}$ and $20 \times 10^{3}$ cells per well in 96-well plates for undifferentiated and differentiated cells, respectively. After 24 hours, cells were exposed to various concentrations of MBCP $(9-375 \mu \mathrm{M})$ for 48-h. Then, cells were incubated with NR dye solution $(50 \mu \mathrm{g} / \mathrm{mL})$ for 3-h at $37^{\circ} \mathrm{C}$, and finally washed with phosphate buffered saline (PBS) and lysed with $1 \%$ acetic acid. The absorbance was read at $540 \mathrm{~nm}$ (iMarkTM microplate reader, Bio-Rad, Milano, Italy).

\subsection{Alkaline Phosphatase (ALP) Activity}

ALP activity was used as marker of the degree of differentiation of human Caco-2 cells. Attached and floating cells were washed and lysed with $0.25 \%$ sodium deoxycholate. ALP activity was determined using Sigma Diagnostics ALP reagent (no. 245). Total cellular protein content of the samples was determined in a microassay procedure using the Coomassie protein assay reagent kit (Pierce). ALP activity was calculated as $\mathrm{nmol} / \mathrm{min} / \mathrm{mg}$ of protein.

\subsection{Western Blotting}

For cell extract preparation, the cells were washed twice with ice-cold PBS/bovine serum albumin (BSA), scraped and centrifuged for $30 \mathrm{~min}$ at $4{ }^{\circ} \mathrm{C}$ in $1 \mathrm{~mL}$ of lysis buffer ( $1 \%$ Triton, $0.5 \%$ sodium deoxycholate, $0.1 \mathrm{M} \mathrm{NaCl}$, 1mM Ethylenediamine tetra-acetic acid (EDTA), pH 7.5, $10 \mathrm{mM} \mathrm{Na}_{2} \mathrm{HPO}_{4}$, pH 7.4, 10 mM Phenylmethyl sulfonyl fluoride, $25 \mathrm{mM}$ benzamidin, $1 \mathrm{mM}$ leupeptin, 0.025 units $/ \mathrm{mL}$ aprotinin). Colon tissue were lysed in a specific buffer (1\% NP-40, 0.1\% SDS, $100 \mathrm{mM}$ sodium ortovanadate, $0.5 \%$ sodium deoxycholate in RIPA buffer) in the presence of protease inhibitors ( $4 \mathrm{mg} / \mathrm{mL}$ of leupeptin, aprotinin, pepstatin A, chymostatin, PMSF and $5 \mathrm{mg} / \mathrm{mL}$ of chymotrypsin-like proteases (TPCK)). The homogenates were sonicated twice by three strokes ( $20 \mathrm{~Hz}$ for $20 \mathrm{~s}$ each); after 
centrifugation for $30 \mathrm{~min}$ at $10,000 \times g$, the supernatants were stored at $80^{\circ} \mathrm{C}$. Equal amounts of cell and tissues proteins were separated by SDS-PAGE, electrotransferred to nitrocellulose and reacted with the different antibodies. Blots were then developed using enhanced chemoluminescence detection reagents (SuperSignal West Pico, Pierce) and exposed to X-ray film. All films were scanned by using Quantity One software (BioRad laboratories, Hercules, CA, USA).

\subsection{Immunostaining and Confocal Microscopy}

After $24-\mathrm{h}$ and 48 -h of incubation with TNF- $\alpha(10 \mu \mathrm{M})$, TNF- $\alpha$ plus MBCP $(18 \mu \mathrm{M})$ or TNF- $\alpha$ plus betamethasone $(10 \mu \mathrm{M})$, Caco-2 cells were fixed for 20 min with a $3 \%(w / v)$ paraformaldehyde solution and permeabilized for 10 min with $0.1 \%(w / v)$ Triton X-100 in PBS at room temperature. To prevent nonspecific interactions of antibodies, cells were treated for $2 \mathrm{~h}$ in $5 \%$ BSA in PBS. In another set of experiments, Caco-2 cells were treated with the acetylcholine receptor antagonists atropine $(10 \mu \mathrm{M})$ and tubocurarine $(10 \mu \mathrm{M})$ for 6-h. Immunostaining was carried out by incubation with anti-E-cadherin, anti-actin and anti- $\beta$-catenin antibodies (1:500, Alexa Fluor ${ }^{\circledR}$, BD Pharmingen ${ }^{\mathrm{TM}}$ ). The slides were mounted on microscope slides by Mowiol. The analyses were performed with a Zeiss LSM 510 microscope equipped with a plan-apochromat objective X 63 (NA 1.4) in oil immersion. The nuclei were stained with 4',6-diamidino-2-phenylindole (DAPI).

\subsection{Permeability Assay on Caco-2 Cells}

To evaluate cell permeability, Caco-2 cells were seeded into a transwell filters with a pore diameter of $8 \mu \mathrm{m}$ in 24 -well plates at a density of $2.0 \times 10^{5}$ cells/insert. Further cultivation in the same medium as above allowed the cells to spontaneously differentiate and polarize into the epithelial monolayer within 21 days. The basolateral compartment contained $1.5 \mathrm{~mL}$ of culture medium while apical compartment contained $0.2 \mathrm{~mL}$. After seeding, the cells were treated with $0.07 \mu \mathrm{M}$ of MBCP. The culture medium was replaced 3 times/wk. After 21 days, the filters containing the cell monolayers (with or without MBCP) were treated for $2 \mathrm{~h}$ with TNF- $\alpha(10 \mu \mathrm{M})$ and then with a mannitol-lactulose solution $(0.05 \mathrm{mmol} / \mathrm{L}$, $0.25 \mathrm{~mol} / \mathrm{L}, 0.2 \mathrm{~mL}$ ). After 3 hours, the basolateral solution was collected and both mannitol and lactulose concentration were measured by liquid chromatography-mass spectrometry (LC-MS).

\subsection{Induction of Experimental Colitis}

Colitis was induced in the anesthetized mice by the intracolonic administration of 2,4,6-dinitrobenzene sulfonic acid (DNBS) as previously described [17]. Briefly, DNBS (150 mg/kg), dissolved in $50 \%$ ethanol $(150 \mu \mathrm{L} /$ mouse), was administrated into the distal colon using a polyethylene catheter ( $1 \mathrm{~mm}$ in diameter) via the rectum $(4.5 \mathrm{~cm}$ from the anus). Three days after the DNBS administration, all mice were euthanized by asphyxiation with $\mathrm{CO}_{2}$, the mice abdomen was opened by a midline incision and the colon removed, isolated from surrounding tissues, length measured, opened along the antimesenteric border, rinsed and weighed and then fixed in $10 \%$ formaldehyde for histopathological analysis. MBCP was administrated by oral gavage $(10-100 \mathrm{mg} / \mathrm{kg})$ once a day for 3 consecutive days starting $24 \mathrm{~h}$ after DNBS administration. Animals were euthanized 2 hours after the last administration of MBCP.

\subsection{Haematoxylin-Eosin Staining}

Paraffin-embedded colon tissues were cut into 5-mm sections. The sections were dewaxed in xylene for $10 \mathrm{~min}$ and dehydrated in gradient alcohol. The sections were then stained with haematoxylin for $8 \mathrm{~min}$ and eosin for $5 \mathrm{~min}$. After dehydration, the sections were sealed and examined under a light microscope (Leica DM 2500). Photographs were taken using the Leica DFC320 R2 digital camera [18]. 


\subsection{Immuno-Fluorescence Microscopy}

The fixed slides of colon tissue, were dewaxed, rehydrated and processed. Briefly, antigen retrieval was performed by pressure-cooking slides for $3 \mathrm{~min}$ in $0.01 \mathrm{M}$ citrate buffer ( $\mathrm{pH}$ 6.0). To prevent nonspecific interactions of antibodies, the slides were treated for $2 \mathrm{~h}$ in $5 \%$ BSA in PBS. Immunostaining was carried out by incubation, overnight at $4{ }^{\circ} \mathrm{C}$, with anti-E-cadherin and anti- $\beta$-Catenin antibodies $\left(1: 100\right.$, Alexa Fluor ${ }^{\circledR}$, BD Pharmingen $\left.{ }^{\mathrm{TM}}\right)$. The slides were mounted on microscope slides by Mowiol + DAPI for nuclear staining, and then observed under the optical microscope (Leica DM 5000 B + CTR 5000).

\subsection{Intestinal Permeability Measurement}

The effect of MBCP, at a dose of $100 \mathrm{mg} / \mathrm{kg}$, was also tested on intestinal permeability, using the FITC-Dextran method.Briefly, 2 days after the induction of colitis, mice were gavaged with $600 \mathrm{mg} / \mathrm{kg}$ of FITC-conjugated dextran (molecular mass 3-5 kDa). After $24 \mathrm{~h}$, blood was collected by cardiac puncture, and the FITC-derived fluorescence was immediately analyzed in the serum by a microplate reader (GloMax Explorer System, Promega; excitation wavelengths $485 \pm 14 \mathrm{~nm}$, emission wavelengths $520 \pm 25 \mathrm{~nm}$ ). Serial-diluted FITC dextran was used to generate a standard curve. Intestinal permeability was expressed as the concentrations of FITC $(\mu \mathrm{M})$ detected in the serum.

\subsection{Induction of Intestinal Hypermotility and Upper Gastrointestinal Transit in Mice}

Upper gastrointestinal transit was measured in both physiological and pathological conditions. The hypermotility was induced by the inflammatory agent croton oil (CO) as previously described [19]. Briefly, two doses of $\mathrm{CO}(20 \mu \mathrm{L} /$ mouse $)$ were given for two consecutive days by oral gavage and the upper gastrointestinal transit was measured 4 days after the first administration of CO [20] (i.e., when the maximal inflammatory response associated to the intestinal hypermotility was reported). Mice were deprived of food overnight and then the upper gastrointestinal transit was evaluated by identifying the leading front of an intragastrically administered charcoal meal marker $(10 \%$ charcoal suspension in 5\% gum Arabic, $10 \mathrm{~mL} / \mathrm{kg}$ ) in the small intestine as previously described [19]. Twenty minutes after charcoal administration, mice were euthanized and the small intestine was isolated by cutting at the pyloric and ileocaecal junctions. The distance traveled by the marker was measured and expressed as a percentage of the total length of the small intestine from pylorus to caecum. MBCP was administrated by oral gavage $(5-50 \mathrm{mg} / \mathrm{kg}) 30 \mathrm{~min}$ prior to charcoal administration.

\subsection{Statistical Analysis}

Data are expressed as the mean \pm S.E.M. or S.D. of $n$ experiments. Statistical significance was assessed using the Student's $t$-test for comparing a single treatment mean with a control mean, and a one-way ANOVA followed by a Tukey multiple comparisons test for the analysis of multiple treatment means. Values of $p<0.05$ were considered significant. The $\mathrm{IC}_{50}$ (concentration that produced $50 \%$ inhibition of cell viability) values were calculated using sigmoidal dose response curve-fitting models (Graphpad Prism Software, version 5.03, Inc. Avendia de la Playa La Jolla, CA, USA).

\section{Results}

\subsection{In Vitro Study}

\subsubsection{MBCP Induces Cytotoxic Effects in Caco-2 Cells at Very High Concentrations}

In order to characterize a safety profile of $\mathrm{MBCP}$, we evaluated its effect on the cell viability of pre- (undifferentiated exponentially growing) and post-confluent (differentiated) Caco- 2 cells. MBCP (9-375 $\mu \mathrm{M}$ ), induced in undifferentiated and differentiated Caco-2 cells, a concentration-dependent cell viability inhibition at $48 \mathrm{~h}$ with an $\mathrm{IC}_{50}$ of $65 \mu \mathrm{M}$ (Figure 1 ). Therefore, to characterize the anti-inflammatory effects of $\mathrm{MBCP}$ on Caco-2 cells, subsequent experiments were performed using a 
MBCP concentration that did not induce significant cytotoxic effect in both pre and post-confluent cells (i.e., $18 \mu \mathrm{M}$ ).

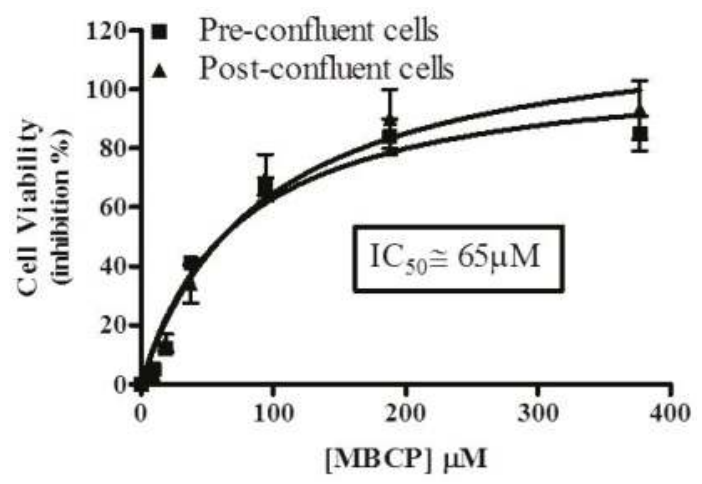

Figure 1. MBCP reduces cell viability in Caco-2 cells. Cell viability was evaluated by the Neutral Red assay in pre and post-confluent Caco-2 cells. Cells were incubated with increasing concentration of MBCP $(9-375 \mu \mathrm{M})$ for $48 \mathrm{~h}$. Each point represents the mean \pm SD of three independent experiments.

\subsubsection{MBCP Stimulates Differentiation in Growing Caco-2 Cells}

The effect of MBCP on cell differentiation was evaluated by measuring the activity of alkaline phosphatase, with the enzyme frequently used as a marker of colon cells differentiation [21] A treatment of undifferentiated Caco-2 cells with $\mathrm{MBCP}(18 \mu \mathrm{M}$ for $48 \mathrm{~h})$ induced a significantly increase $(p<0.05)$ of alkaline phosphatase activity (APA) of about $14 \%$ compared to the untreated Caco-2 cells (control). The APA value was in the Caco-2 cells of $8.6 \pm 0.4$ ( $\mathrm{nmol} / \mathrm{min} / \mathrm{mg}$ protein), while, in MBCP treated Caco-2 cells, it was $10 \pm 0.7$ (mean $\left.\pm \mathrm{SD}, n=3,{ }^{*} p<0.05\right)$

\subsubsection{MBCP Modulates Adherent Junctions Formation in Control and TNF- $\alpha$-Stimulated Caco-2 Cells}

In order to investigate the role of MBCP on adherent junctions (AJs) formation, we studied the cellular organization of E-cadherin and actin in pre-confluent Caco-2 cells by confocal miscroscopy. Caco-2 cells, after $24 \mathrm{~h}$ from seeding, showed a strong cytoplasmic localization of both E-cadherin and actin proteins (Figure 2A-C) that was ameliorated by MBCP treatment (Figure 2D-F). At $48 \mathrm{~h}$, the E-cadherin-actin complex presented a not homogeneous membranous localization in untreated Caco-2 cells (Figure 2G-I). MBCP treatment induced a strong cell-cell contact membranous localization of E-cadherin-Actin complex (Figure 2M-O).

To assess whether MBCP could counteract the AJ-destructuration induced by the pro-inflammatory mediator TNF- $\alpha$, we examined the $\beta$-catenin organization in TNF- $\alpha$-stimulated cells. Caco- 2 cells treated with TNF- $\alpha(10 \mu \mathrm{M}$, for 48 -h) showed a cytoplasmic compartment $\beta$-catenin localization (Figure 3D-F) compared to untreated cells (CTR) (Figure 3A-C). A treatment of Caco-2 cell with MBCP $(18 \mu \mathrm{M})$ induced an enhancement of both E-cadherin and $\beta$-catenin localization at AJs (Figure 2G-I) compared to TNF- $\alpha$ alone. Since corticosteroids are the effective first-line treatment in cytokine-induced inflammation, we treated the Caco- 2 cells (stimulated with TNF- $\alpha$ ) with betamethasone. Betamethasone $(10 \mu \mathrm{M})$ treatment induced a membranous E-cadherin and $\beta$-catenin organization, although a $\beta$-catenin cytoplasmic immunoreactivity persisted (Figure $3 \mathrm{~L}-\mathrm{N}$ ). In our experimental conditions (Caco- 2 cells treated or not with TNF- $\alpha$ ), MBCP accelerated and induced an organization of adjacent junctions in the cells, without changes the E-cadherin, actin and $\beta$-catenin protein expression evaluated by Western blot analysis (data not shown). 


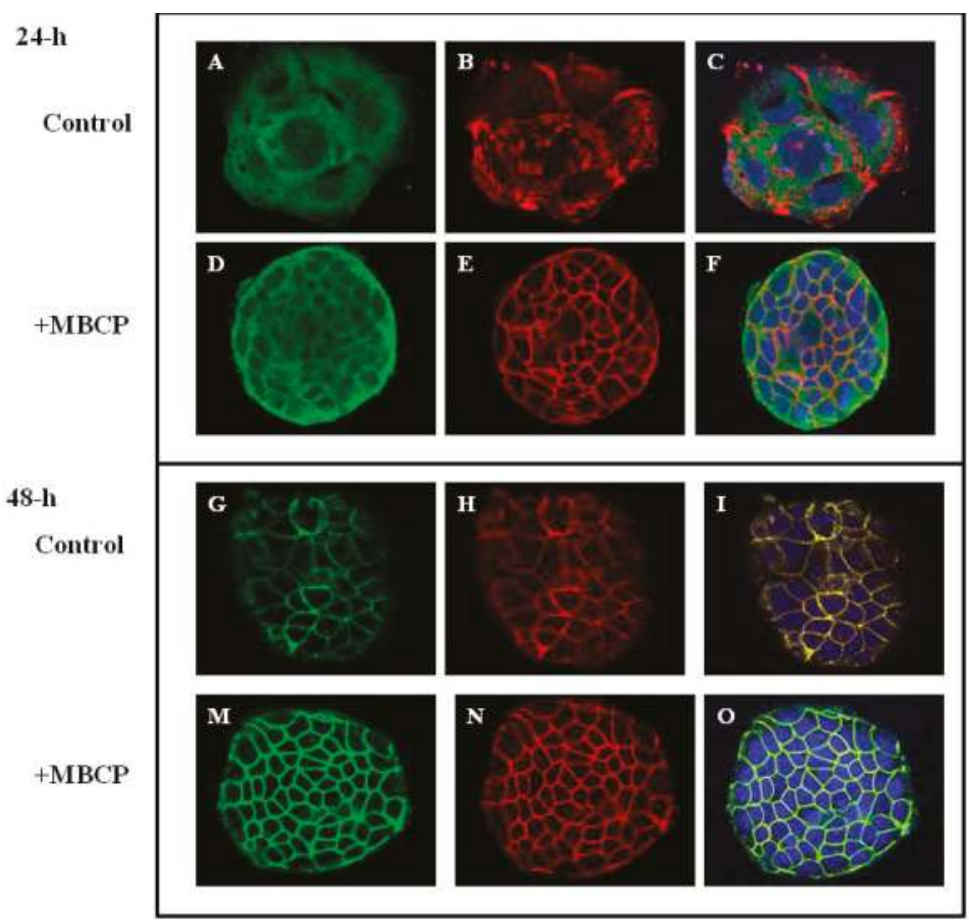

Figure 2. MBCP accelerates the formation of adherens junctions. Confocal microscopy images of Caco-2 cells (A-C,G-I) and MBCP treated Caco-2 cells (panel (D-F,M-O). Cells were treated for 24-h and $48-\mathrm{h}$ with MBCP at $18 \mu \mathrm{M}$ concentration. The merged image is on the right (green, actin; red, E-cadherin; blue, dapi).

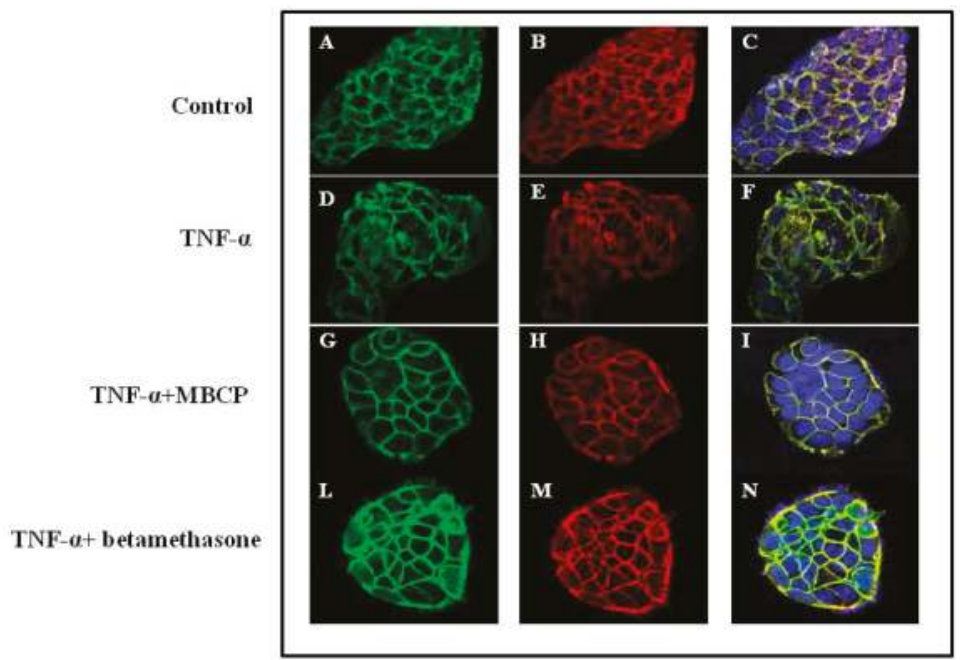

Figure 3. MBCP ameliorates the adherens junctions destructured by TNF- $\alpha$. Confocal microscopy images of untreated Caco-2 cells (panel (A-C), Caco-2 cells treated for $48 \mathrm{~h}$ with TNF- $\alpha(10 \mu \mathrm{M},(\mathrm{D}-\mathrm{F})$ ), TNF $\alpha 10 \mu \mathrm{M}$ plus MBCP $(18 \mu \mathrm{M},(\mathrm{G}-\mathbf{I}))$ or with TNF- $\alpha(10 \mu \mathrm{M})$ plus betamethasone $(\mathbf{L}-\mathbf{N})$. On the right, merged image (green, $\beta$-catenin; red, E-cadherin; blue, dapi). 


\subsubsection{MBCP Counteracts the TNF- $\alpha$ Inflammatory Effect by Modulating the NF-kB Pathway}

It is well known that TNF- $\alpha$ causes the activation of transcription factors, including NF- $\mathrm{KB}$. NF- $\mathrm{kB}$ regulates host inflammatory, immune responses and also stimulates the expression of inducible cyclooxygenase enzymes (COX-2) that contribute to the pathogenesis of the inflammatory process. Therefore, we evaluated, by Western blot analysis, the effects of MBCP on NF- $\mathrm{B}$, pNF-кB, COX-2 and 5 -Lipoxygenases (LOX) expression in TNF- $\alpha$ treated Caco-2 cells. A treatment with TNF- $\alpha$ induced an increase of 5-LOX, p-NF- $\mathrm{KB}$ and COX-2 expression. MBCP $(18 \mu \mathrm{M})$ was able to reduce the expression of p-NF- $\mathrm{BB}, 5$-LOX and COX-2 expression increased by TNF- $\alpha$ (Figure 4). Moreover, MBCP $(18 \mu \mathrm{M})$ reduced the basal expression of NF- $k B$ in untreated Caco-2 cells. Betamethasone $(10 \mu \mathrm{M})$, used as a positive control, significantly reduced 5-LOX expression, but not the NF- $\mathrm{kB}, \mathrm{p}-\mathrm{NF}-\mathrm{kB}$ and COX-2 expression, increased by TNF- $\alpha$.
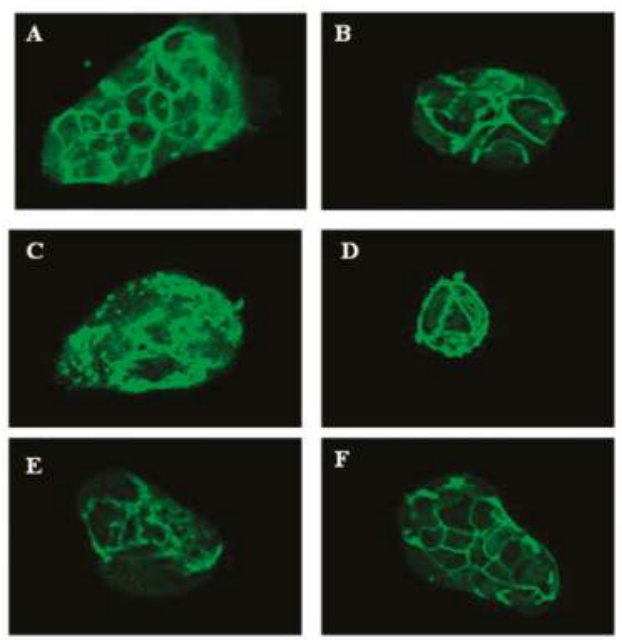

Figure 4. MBCP ameliorates the adherens junctions destructured by atropine and tubocurarine. Confocal microscopy images of untreated Caco-2 cells (A) or Caco-2 cells treated for six hours with $\operatorname{MBCP}(18 \mu \mathrm{M},(\mathbf{B}))$, tubocurarine $(10 \mu \mathrm{M},(\mathbf{C}))$, Tubocurarine plus $\mathrm{MBCP}(\mathbf{D})$, atropine $(10 \mu \mathrm{M},(\mathbf{E}))$ and atropine plus $\mathrm{MBCP}(\mathbf{F})$. The color green represents $\beta$-catenin.

\subsubsection{MBCP Reduces Atropine and Tubocurarine-Induced Adherens Junctions Disorganization}

Several studies reported that the stimulation of the acetylcholine receptors (AChR) enhances epithelial barrier formation $[22,23]$ and ameliorates TNF- $\alpha$ induced barrier dysfunction in intestinal epithelial cells [24]. According to literature, treatment of Caco-2 cells with the AChR antagonists atropine $(10 \mu \mathrm{M})$ or tubocurarine $(10 \mu \mathrm{M})$ induced an disorganization of adherents junctions (Figure 5). MBCP $(18 \mu \mathrm{M})$ attenuated both atropine and tubocurarine AChR antagonist effects on Caco-2 cells adherens junctions, indicating that MBCP treatment could affect the AChR pathway (Figure 5). 

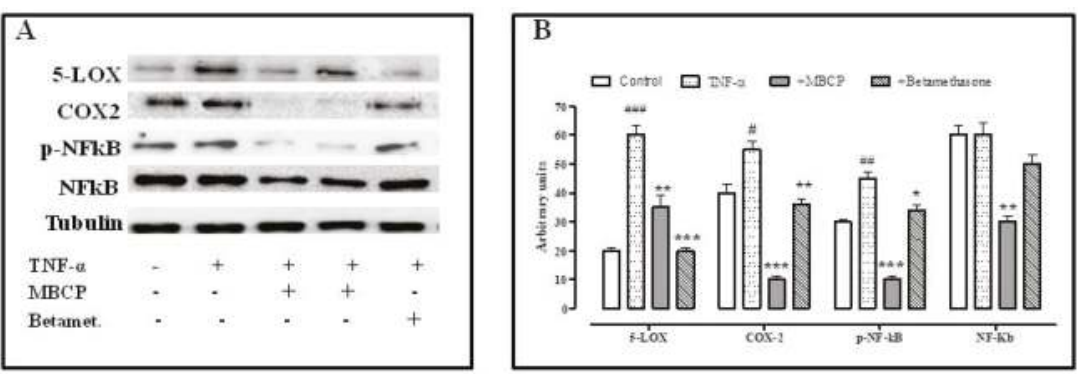

Figure 5. $\mathrm{MBCP}$ reduces the expression of 5-LOX, COX-2, $\mathrm{p}-\mathrm{NF}-\mathrm{kB}$ and NF- $\mathrm{kB}$. (A) Western blot analysis of Caco-2 cells alone or treated with $\mathrm{MBCP}$ or betamethasone. Bands associated with the expression of 5-LOX, COX-2, p-NF- $\mathrm{BB}, \mathrm{NF}-\mathrm{kB}$, and house-keeping $\gamma$-tubulin, after $48 \mathrm{~h}$ of treatment with MBCP $(18 \mu \mathrm{M})$ and betamethasone $(10 \mu \mathrm{M})$. The expression of the house-keeping protein $\gamma$-tubulin was used as loading control. (B) The expression levels of the above proteins are reported as a percentage with respect to the level of the protein in untreated cells, used as control. Bars show the mean $\pm \mathrm{SD}$ of three different determinations. \# $p<0.05$, \#\# $p<0.01$ and \#\#\# $p<0.001$ vs. control; ${ }^{*} p<0.05,{ }^{* *} p<0.01$ and ${ }^{* * *} p<0.001$ vs. TNF- $\alpha$ alone.

\subsubsection{MBCP Inhibits the TNF- $\alpha$-Increased Caco-2 Permeability}

The intestinal epithelial cell line Caco-2 has been used extensively as a model of the human epithelium, as it can be grown in the Transwell system as a differentiated cell monolayer that has selective paracellular permeability to ions and solutes. We used both mannitol and lactulose, intestinal permeability probes, to evaluate the permeability changes in Caco- 2 stimulated with TNF- $\alpha$. Treatment of the Caco-2 cell (see Section 2.9) with TNF- $\alpha(10 \mu \mathrm{M})$ increased the concentration of mannitol in the basolateral solution by about twofold compared to the control cells (cells without TNF- $\alpha$ treatment). [Control of mannitol concentration (mmol/L): $0.01 \pm 0.004$; TNF- $\alpha 0.023 \pm 0.005 *$; mean \pm SD; $* p<0.05]$. The mannitol concentration in the basolateral side of the Caco-2 cells pretreated with MBCP and then incubated with TNF- $\alpha\left(0.009 \pm 0.003 \mathrm{mmol} / \mathrm{L}\right.$, mean $\left.\pm \mathrm{SD} ; \mathrm{n}=3,{ }^{*} p<0.05\right)$ was decreased twofold compared to the TNF- $\alpha$ treated Caco- 2 cells. The MBCP induced any change of the lactulose permeability in the same inflammatory cell condition.

\subsection{In Vivo Study}

\subsubsection{MBCP Reduces the Inflammation in the DNBS Model of Colitis}

The DNBS murine model of colitis was used to assess the in vivo intestinal anti-inflammatory effects of MBCP. DNBS administration $(150 \mathrm{mg} / \mathrm{kg}$ ) caused inflammatory damage, as indicated by the approximately twofold increase in colon weight/colon length ratio $(\mathrm{mg} / \mathrm{cm})$, a simple and reliable marker of inflammation and damage (Figure 6A). MBCP (10-100 mg/kg, by oral gavage), administered for three consecutive days after the inflammatory insult, significantly and in a dose-dependent manner, reduced the effect of DNBS on colon weight/colon length ratio. The effect was significant starting from $30 \mathrm{mg} / \mathrm{kg}$ dose (Figure 6A). The anti-inflammatory effect MBCP was further confirmed by histological analysis. As shown in Figure 6B, DNBS caused a severe inflammatory cellular infiltration and complete destruction of the colon epithelium, compared to control mice (Figure 6B). Our data showed that oral $\mathrm{MBCP}(100 \mathrm{mg} / \mathrm{kg}$ ) reduced the colonic damage induced by DNBS (Figure $6 \mathrm{~B})$.

Considering that the peptide inhibited the phosphorylation of NF-KB in vitro, we investigated if this pathway was involved in the in vivo anti-inflammatory effect of MBCP. As shown in Figure 6C, the phosphorylation of $\mathrm{I} \kappa \mathrm{B} \alpha$ and active NF- $\mathrm{kB}$ expression were increased in the colons of mice treated with DNBS. MBCP (100 mg/kg) was able to significantly reduce these changes induced by DNBS. These results demonstrated that MBCP suppressed NF-KB activation both in the experimental model of colitis and in TNF $\alpha$-stimulated Caco-2 cells. 
A
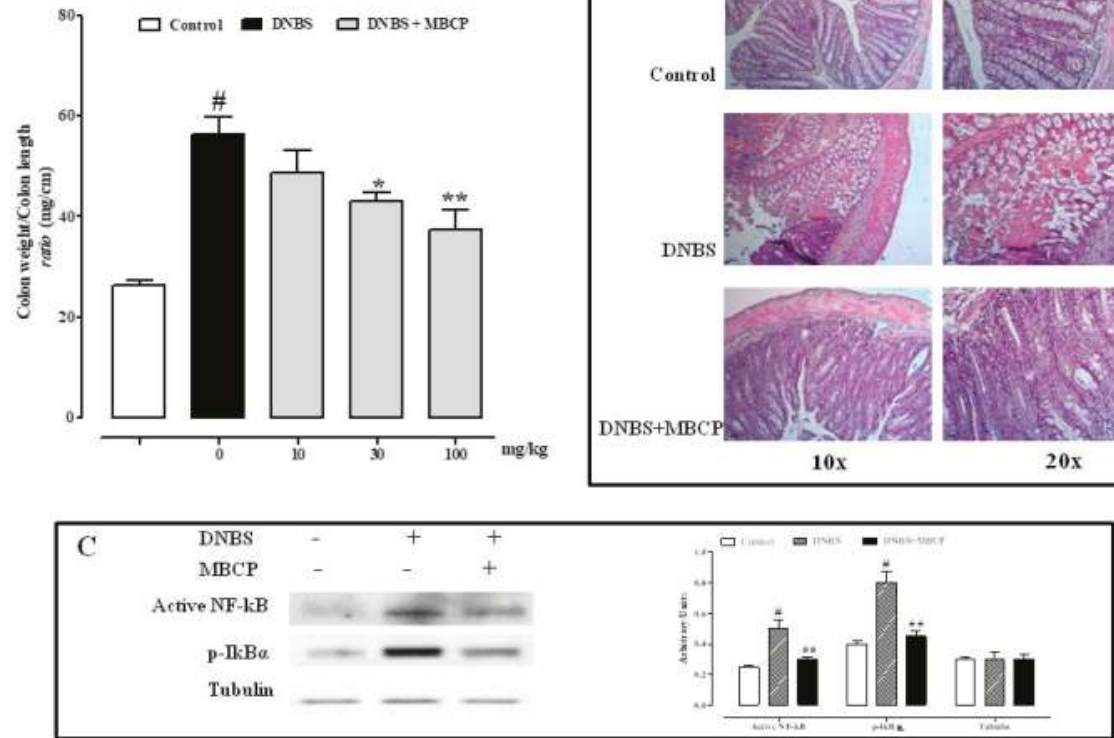

Figure 6. MBCP reduces colon weight/colon length ratio in DNBS-induced colitis in mice. (A) MBCP (10-100 $\mathrm{mg} / \mathrm{kg}$, by oral gavage) was administered once a day starting $24 \mathrm{~h}$ after the induction of colitis by DNBS $(150 \mathrm{mg} / \mathrm{kg})$. Colons were collected three days after DNBS. (B) representative hematoxylin \& eosin stained colon cross-sections of mice treated with vehicle (control), DNBS and DNBS plus MBCP (100 mg/kg by oral gavage). Colons were collected three days after the induction of colitis by DNBS. Original magnification $10 \times$ and $20 \times(\mathrm{A}) .(\mathrm{C})$ Western blot analysis of $\mathrm{p}-\mathrm{NF}-\mathrm{kB}, \mathrm{NF}-\mathrm{\kappa B}$, and house-keeping $\gamma$-tubulin expression in normal mice colon and after the induction of colitis by DNBS.All data are represented as mean \pm SEM of seven mice for each experimental group. Statistical significance was calculated using one-way ANOVA test. $\# p<0.001$ vs. control, ${ }^{*} p<0.05$ and ${ }^{* *} p<0.01$ vs. DNBS alone.

\subsubsection{MBCP Reduces the Intestinal Permeability in Vivo}

The intestinal permeability was increased of the intracolonic administration of DNBS, as revealed by the high concentration of FITC-conjugated dextran in the serum (see Supplementary Files Figure S1). While MBCP $(100 \mathrm{mg} / \mathrm{kg})$, given by oral gavage for three consecutive days, synergistically $(p<0.01)$ partially counteracted the DNBS-induced increase in intestinal permeability (see Supplementary Files Figure S1). Moreover, immunofluorescence analysis showed that DNBS administration caused a destructuration of the colonic AJs associated with an increase of the citoplasmatic expression of E-cadherin and $\beta$-catenin. MBCP $(100 \mathrm{mg} / \mathrm{kg})$ counteracted the effect of DNBS on AJs, (Figure 7), thus confirming the in vitro results on TNF- $\alpha$-stimulated Caco- 2 cells. 


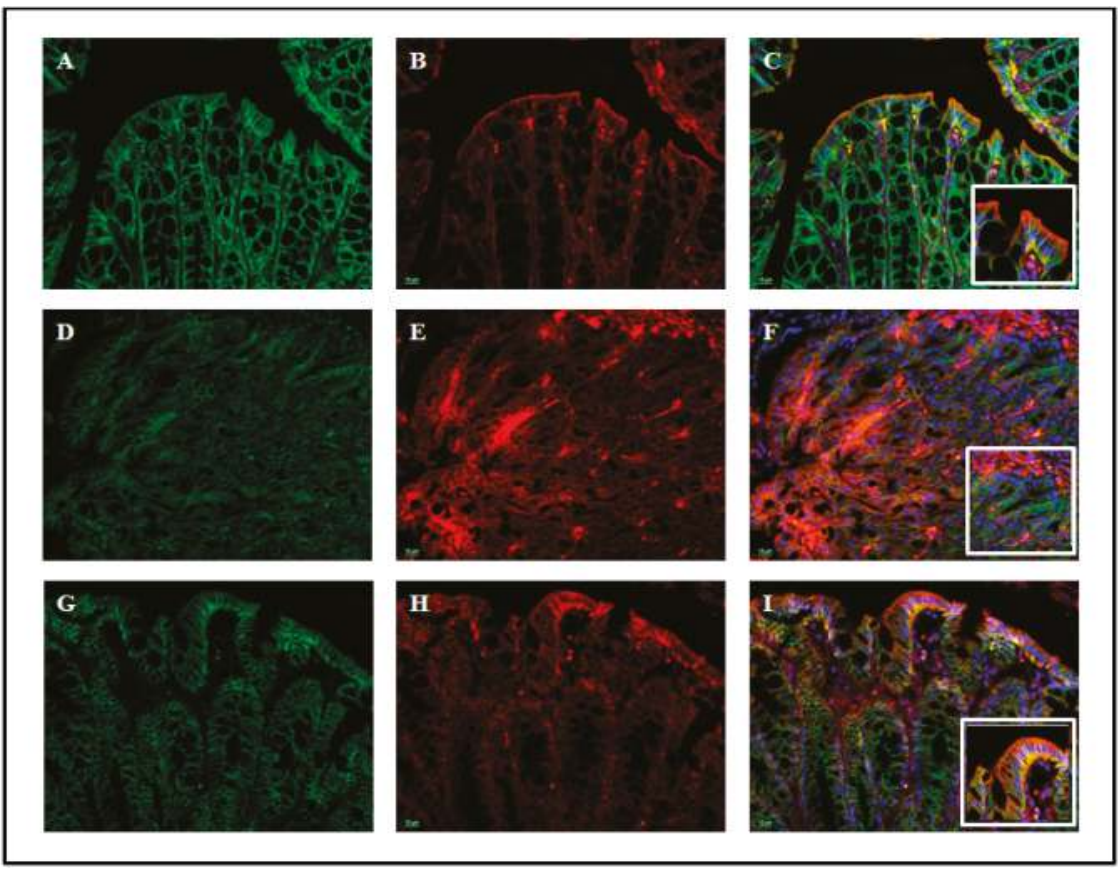

Figure 7. MBCP induces the organization of colonic AJs in DNBS-induced colitis in mice. Immunofluorescence analysis showing the expression of E-cadherin (red) and $\beta$-catenin (green) in control (A-C), DNBS (D-F) and DNBS plus MBCP (G-I) $(100 \mathrm{mg} / \mathrm{kg})$ mice. Original magnification $20 \times$. A magnified portion of the immunofluorescence was shown at higher magnification in the inset of panel $(\mathbf{C}, \mathbf{F}, \mathbf{I})$. The DNBS-induced colitis in mice was treated for three consecutive days after the inflammatory insults with MBCP (100 mg/kg, by oral gavage).

\subsubsection{MBCP Counteracts the Accelerated Upper Gastrointestinal Transit Induced by Croton Oil}

The administration of the flogogen agent croton oil (CO) induced an accelerated upper gastrointestinal transit (GT) 4 days after its first administration. The physiological GT percentage in the mice (control) was of $49.8 \pm 1.4$; whereas GT significantly increased up to values of $65.50 \pm 6.26^{*}$ (mean $\left.\pm \mathrm{SEM} ;{ }^{*} p<0.05\right)$ in CO treated mice. MBCP $(5-50 \mathrm{mg} / \mathrm{kg})$, given by oral gavage $30 \mathrm{~min}$ before the administration of the charcoal, in a dose dependent manner restored the intestinal motility to physiological conditions (\% of GT MBCP 5 mg/kg: $59.43 \pm 2.75 ;$ MBCP $10 \mathrm{mg} / \mathrm{kg}: 56.14 \pm 3.63 ; \mathrm{MBCP}$ $\left.50 \mathrm{mg} / \mathrm{kg}: 39.87 \pm 6.38^{* *} ;{ }^{* *} p<0.01\right)$. MBCP, at the high dose $(50 \mathrm{mg} / \mathrm{kg})$, did not modify the upper gastrointestinal transit in control mice ( $\%$ of GT: control $55 \pm 3.53$; MBCP $48.60 \pm 4.38$ (mean \pm SEM)).

\section{Discussion}

In mammals, the enterocytes are renewed continuously every $4-8$ days through an organized series of events involving proliferation, differentiation and programmed cell death. The proliferation to differentiation transition (PDT) is a critical step in the continual renewal of a normal intestinal epithelium [25]. A useful in vitro model to study the effects of food ingredients on the gut epithelial layer is based on the use of Caco-2 cells for their functional similarity to colonic enterocytes [26]. Indeed, Caco-2 cells express tight junctions, microvilli, enzymes and transporters functionally similar to colonic enterocyte [26]. Moreover, this cell line has the ability to elicit a pro-inflammatory reaction in response to stimulants like TNF- $\alpha$, a known mediator of gastrointestinal mucosal barrier injury [5,27]. In this study, we have shown that a peptide (MBCP) obtained from gastrointestinal digestion of Mozzarella of Bufala Campana DOP is able to modulate the differentiation and permeability in Caco-2 
cells stimulated with TNF- $\alpha$ and to attenuate inflammation and hypermotility in murine models of intestinal inflammation.

\subsection{MBPC Modulates the Differentiation and Permeability in Caco-2 Cells}

Proliferating Caco-2 cells spontaneously initiate the differentiation process when they have reached confluence. The differentiation program starts when specific biochemical events induce the cell-cell contact, through the E-Cadherin/actin/ $\beta$-Catenin complex [28]. Here, we have found that $\mathrm{MBCP}$, at a non-cytotoxic concentration (i.e., $18 \mu \mathrm{M}$ ), induced an increase of intestinal alkaline phosphatase activity in undifferentiated Caco2 cells. Intestinal alkaline phosphatase is a well-known cell differentiation marker and its activity has been inversely correlated with an increased risk of intestinal inflammation development [29]. Moreover, we also found that MBCP treatment, already after $24 \mathrm{~h}$, induced the differentiation program accelerating the E-cadherin-actin membranous organization, thus suggesting a beneficial effect on the AJ. This result is further supported by the experiments with TNF- $\alpha$. According to previous studies [30], we have shown that Caco-2 treatment with TNF- $\alpha$ induced molecular alterations of the E-cadherin organization and consequently a passage of mannitol through the Caco-2 monolayer. MBCP treatment was able to restore the cell-cell junctions, counteracting the breakdown of E-cadherin- $\beta$-catenin complex and reducing the increase of mannitol permeability inducted by TNF- $\alpha$.

According to previous studies, we found that un-stimulated Caco- 2 cells over-expressed COX-2 and NF- $\kappa B$ and TNF- $\alpha$-stimulated Caco-2 cells increased 5-LOX expression. NF- $\kappa B$ transcription factor is a master regulator of the inflammatory response and it is essential for the homeostasis of the immune system. NF- $\mathrm{KB}$ regulates the transcription of genes, such as LOX and COX-2, which control inflammation [31]. Our results demonstrated that MBCP reduced the phosphorylation of NF- $\mathrm{KB}$ as well as COX-2 and 5-LOX expression in TNF- $\alpha$ stimulated cells.

Many mechanisms are responsible for the regulation and stability of adherens junction proteins, one of these possibilities could be the AChR activation. Furthermore, previous studies indicate that cholinergic agonists interfere with NF- $\mathrm{kB}$ pathways preventing IkB $\alpha$ breakdown and p65 nuclear translocation [24,32]. Moreover, cholinergic agonists could prevent gut barrier failure after severe burn injury maintaining intestinal barrier integrity. McGilligan has showed that nicotine decrease Caco-2 permeability by regulating the expression of TJ proteins [33]. Therefore, we measured changes in localization of $\beta$-catenin to determine whether modulation of this protein correlates with the adherens junctions disorganization induced by cholinergic antagonists like atropine and tubocurarine. Confocal microscopy analysis confirmed that both atropine and tubocurarine treatment induced a cytoplasmic accumulation of $\beta$-catenin in Caco- 2 cells. These detrimental effects induced by cholinergic antagonists were counteracted by $\mathrm{MBCP}$ treatment.

\subsection{MBPC Ameliorates Murine Colitis}

The ability of MBCP to restore cell-cell contacts as well as to exert an anti-inflammatory actions was subsequently evaluated in vivo by using the mice model of DNBS-induced colitis. According to previous studies [34,35] intracolonic administration of DNBS induced intestinal inflammation associated to an increase of epithelial permeability. Oral MBCP administration was able to reduce intestinal inflammation as demonstrated by the reduction of colon weight colon length ratio (a simple and reliable marker of inflammation and damage), histological alterations, IkB $\alpha$ phosphorylation and of NF- $\mathrm{kB}$ activation associated with DNBS administration.

Intestinal permeability plays a crucial role in the development of IBD as well as in the IBD ongoing bowel symptoms [5,36]. Moreover, inflammation reduces barrier integrity and affects the normal intestinal permeability [4]. Studies on Caco-2 cells have shown that MBCP restored tight junctions altered by TNF- $\alpha$. Tight junctions are multi-protein complexes that maintain the intestinal barrier while regulating permeability [9]. Therefore, we investigated the effect of MBPC on intestinal permeability in vivo. We adopted an immunofluorescent method through which an orally-administered marker 
(i.e., FITC-conjugated dextran) can be detected in the blood if permeability is impaired. As expected, intestinal permeability increased after DNBS administration and, more importantly, MBCP restored the impaired permeability.

\subsection{MBPC Normalizes Inflammation-Induced Murine Intestinal Hypermotility}

It is clinically well established that inflammation in the gut causes debilitating symptoms due to motility disturbances [37]. To investigate the effect of MBCP on intestinal motility, we administered mice croton oil, which is a flogogen agent able to induce hypermotility as a consequence of intestinal inflammation. By using this experimental model, we have shown that MBPC did not affect motility in healthy mice, but normalized the exaggerated intestinal transit caused by the inflammatory insult. The lack of effect of MBPC in control mice is clinically relevant in the light of the observation that constipation is a very common side effect associated with drugs clinically used to reduce intestinal motility [38,39].

\section{Conclusions}

In conclusion, the data obtained in our study shown that $\mathrm{MBCP}$, a peptide isolated from the buffalo milk after in vitro digestion of Mozzarella di Bufala Campana DOP, exerts anti-inflammatory effects both in vitro and in vivo. This anti-inflammatory effect could be related to its beneficial effects on adherens junctions mainly during an inflammatory process. These results could have an important impact on the therapeutic potential of $\mathrm{MBCP}$ in helping to restore the intestinal epithelium integrity damaged by inflammation, thereby reducing the risk of colorectal cancer.

Supplementary Materials: The following are available online at http:/ /www.mdpi.com/2072-6643/11/3/610/s1, Figure S1: MBCP reduces intestinal permeability in DNBS-induced colitis in mice.

Author Contributions: G.C.T.; P.S.; and E.N.: conceived and designed the experiments; S.L.; E.P.; D.V.; M.M.; R.C.; F.M. and S.D.M.: performed the experiments and analyzed the data; P.S. and F.B.: wrote the paper. G.C.T.; P.S.; E.N.; and F.B.: discussed and confirmed the final manuscript.

Funding: This work was supported by a grant: “Combattere la resistenza tumorale: piattaforma integrata multidisciplinare per un approccio tecnologico innovativo alle oncoterapie-CAMPANIA ONCO TERAPIE" Regione Campania and EiCURE-Regione Campania.

Conflicts of Interest: The authors declare no conflict of interest.

\section{References}

1. Ng, S.C.; Shi, H.Y.; Hamidi, N.; Underwood, F.E.; Tang, W.; Benchimol, E.I.; Panaccione, R.; Ghosh, S.; Wu, J.C.Y.; Chan, F.K.L.; et al. Worldwide incidence and prevalence of inflammatory bowel disease in the 21st century: A systematic review of population-based studies. Lancet 2018, 390, 2769-2778. [CrossRef]

2. Sairenji, T.; Collins, K.L.; Evans, D.V. An Update on Inflammatory Bowel Disease. Prim Care 2017, 44, 673-692. [CrossRef]

3. Lucafò, M.; Franca, R.; Selvestrel, D.; Curci, D.; Pugnetti, L.; Decorti, G.; Stocco, G. Pharmacogenetics of treatments for inflammatory bowel disease. Expert Opin. Drug Metab. Toxicol. 2018, 14, 1209-1223. [CrossRef] [PubMed]

4. Bischoff, S.C.; Barbara, G.; Buurman, W.; Ockhuizen, T.; Schulzke, J.D.; Serino, M.; Tilg, H.; Watson, A.; Wells, J.M. Intestinal permeability-A new target for disease prevention and therapy. BMC Gastroenterol. 2014, 14, 189. [CrossRef] [PubMed]

5. Michielan, A.; D'Incà, R. Intestinal Permeability in Inflammatory Bowel Disease: Pathogenesis, Clinical Evaluation, and Therapy of Leaky Gut. Mediat. Inflamm. 2015, 2015, 628157. [CrossRef]

6. Fasano, A.; Shea-Donohue, T. Mechanisms of disease: The role of intestinal barrier function in the pathogenesis of gastrointestinal autoimmune diseases. Nat. Clin. Pract. Gastroenterol. Hepatol. 2005, 2, 416-422. [CrossRef] [PubMed]

7. Coopman, P.; Djiane, A. Adherens Junction and E-Cadherin complex regulation by epithelial polarity. Cell. Mol. Life Sci. 2016, 73, 3535-3553. [CrossRef] 
8. Brüser, L.; Bogdan, S. Adherens Junctions on the Move-Membrane Trafficking of E-Cadherin. Cold Spring Harb. Perspect. Biol. 2017, 9, a029140. [CrossRef] [PubMed]

9. Edelblum, K.L.; Turner, J.R. The tight junction in inflammatory disease: Communication breakdown. Curr. Opin. Pharmacol. 2009, 9, 715-720. [CrossRef]

10. Utech, M.; Brüwer, M.; Nusrat, A. Tight junctions and cell-cell interactions. Methods Mol. Biol. 2006, 341, 185-195.

11. De Simone, C.; Picariello, G.; Mamone, G.; Stiuso, P.; Dicitore, A.; Vanacore, D.; Chianese, L.; Addeo, F.; Ferranti, P. Characterisation and cytomodulatory properties of peptides from Mozzarella di Bufala Campana cheese whey. J. Pept. Sci. 2009, 15, 251-258. [CrossRef] [PubMed]

12. De Simone, C.; Ferranti, P.; Picariello, G.; Scognamiglio, I.; Dicitore, A.; Addeo, F.; Chianese, L.; Stiuso, P. Peptides from water buffalo cheese whey induced senescence cell death via ceramide secretion in human colon adenocarcinoma cell line. Mol. Nutr. Food Res. 2011, 55, 229-238. [CrossRef] [PubMed]

13. Mohanty, D.P.; Mohapatra, S.; Misra, S.; Sahu, P.S. Milk derived bioactive peptides and their impact on human health-A review. Saudi J. Biol. Sci. 2016, 23, 577-583. [CrossRef] [PubMed]

14. Chanu, K.V.; Thakuria, D.; Kumar, S. Antimicrobial peptides of buffalo and their role in host defenses. Vet. World 2018, 11, 192-200. [CrossRef] [PubMed]

15. Basilicata, M.G.; Pepe, G.; Adesso, S.; Ostacolo, C.; Sala, M.; Sommella, E.; Scala, M.C.; Messore, A.; Autore, G.; Marzocco, S.; et al. Antioxidant Properties of Buffalo-Milk Dairy Products: A $\beta$-Lg Peptide Released after Gastrointestinal Digestion of Buffalo Ricotta Cheese Reduces Oxidative Stress in Intestinal Epithelial Cells. Int. J. Mol. Sci. 2018, 19, 1955. [CrossRef] [PubMed]

16. Tenore, G.C.; Ritieni, A.; Campiglia, P.; Stiuso, P.; Di Maro, S.; Sommella, E.; Pepe, G.; D’Urso, E.; Novellino, E. Antioxidant peptides from "Mozzarella di Bufala Campana DOP" after simulated gastrointestinal digestion: In vitro intestinal protection, bioavailability, and anti-haemolytic capacity. J. Funct. Foods 2015, 15, 365-375.

17. Pagano, E.; Capasso, R.; Piscitelli, F.; Romano, B.; Parisi, O.A.; Finizio, S.; Lauritano, A.; Marzo, V.D.; Izzo, A.A.; Borrelli, F. An Orally Active Cannabis Extract with High Content in Cannabidiol attenuates Chemically-induced Intestinal Inflammation and Hypermotility in the Mouse. Front. Pharmacol. 2016, 7, 341. [CrossRef]

18. Venditti, M.; Fasano, C.; Santillo, A.; Aniello, F.; Minucci, S. First evidence of DAAM1 localization in mouse seminal vesicles and its possible involvement during regulated exocytosis. C. R. Biol. 2018, 341, 228-234. [CrossRef] [PubMed]

19. Capasso, R.; Orlando, P.; Pagano, E.; Aveta, T.; Buono, L.; Borrelli, F.; Di Marzo, V.; Izzo, A.A. Palmitoylethanolamide normalizes intestinal motility in a model of post-inflammatory accelerated transit: Involvement of $\mathrm{CB}_{1}$ receptors and TRPV1 channels. Br. J. Pharmacol. 2014, 171, 4026-4037. [CrossRef] [PubMed]

20. Pol, O.; Puig, M.M. Reversal of tolerance to the antitransit effects of morphine during acute intestinal inflammation in mice. Br. J. Pharmacol. 1997, 122, 1216-1222. [CrossRef] [PubMed]

21. Matsumoto, H.; Erickson, R.H.; Gum, J.R.; Yoshioka, M.; Gum, E.; Kim, Y.S. Biosynthesis of alkaline phosphatase during differentiation of the human colon cancer cell line Caco-2. Gastroenterology 1990, 98, 1199-1207. [CrossRef]

22. Pelissier-Rota, M.; Lainé, M.; Ducarouge, B.; Bonaz, B.; Jacquier-Sarlin, M. Role of cholinergic receptors in colorectal cancer: Potential therapeutic implications of vagus nerve stimulation? J. Cancer Ther. 2013, 4, 1116-1131. [CrossRef]

23. Lesko, S.; Wessler, I.; Gäbel, G.; Petto, C.; Pfannkuche, H. Cholinergic modulation of epithelial integrity in the proximal colon of pigs. Cells Tissues Organs 2013, 197, 411-420. [CrossRef] [PubMed]

24. Khan, M.R.; Uwada, J.; Yazawa, T.; Islam, M.T.; Krug, S.M.; Fromm, M.; Karaki, S.; Suzuki, Y.; Kuwahara, A.; Yoshiki, H.; et al. Activation of muscarinic cholinoceptor ameliorates tumor necrosis factor- $\alpha$-induced barrier dysfunction in intestinal epithelial cells. FEBS Lett. 2015, 589, 3640-3647. [CrossRef] [PubMed]

25. Yang, B.; Cao, L.; Liu, B.; McCaig, C.D.; Pu, J. The transition from proliferation to differentiation in colorectal cancer is regulated by the calcium activated chloride channel A1. PLoS ONE 2013, 8, e60861. [CrossRef]

26. Tanoue, T.; Nishitani, Y.; Kanazawa, K.; Hashimoto, T.; Mizuno, M. In vitro model to estimate gut inflammation using co-cultured Caco-2 and RAW264.7 cells. Biochem. Biophys. Res. Commun. 2008, 374, 565-569. [CrossRef] 
27. Tatiya-Aphiradee, N.; Chatuphonprasert, W.; Jarukamjorn, K. Immune response and inflammatory pathway of ulcerative colitis. J. Basic Clin. Physiol. Pharmacol. 2018, 30, 1-10. [CrossRef]

28. Buhrke, T.; Lengler, I.; Lampen, A. Analysis of proteomic changes induced upon cellular differentiation of the human intestinal cell line Caco-2. Dev. Growth Differ. 2011, 53, 411-426. [CrossRef]

29. Bilski, J.; Mazur-Bialy, A.; Wojcik, D.; Zahradnik-Bilska, J.; Brzozowski, B.; Magierowski, M.; Mach, T.; Magierowska, K.; Brzozowski, T. The Role of Intestinal Alkaline Phosphatase in Inflammatory Disorders of Gastrointestinal Tract. Mediat. Inflamm. 2017, 2017, 9074601. [CrossRef]

30. Yi, J.Y.; Jung, Y.J.; Choi, S.S.; Chung, E. TNF-alpha Downregulates E-cadherin and Sensitizes Response to $\gamma$-irradiation in Caco-2 Cells. Cancer Res. Treat. 2009, 41, 164-170. [CrossRef]

31. Christian, F.; Smith, E.L.; Carmody, R.J. The Regulation of NF-kB Subunits by Phosphorylation. Cells 2016, 5, 12. [CrossRef]

32. Rioux, N.; Castonguay, A. The induction of cyclooxygenase-1 by a tobacco carcinogen in U937 human macrophages is correlated to the activation of NF-kappaB. Carcinogenesis 2000, 21, 1745-1751. [CrossRef] [PubMed]

33. McGilligan, V.E.; Wallace, J.M.; Heavey, P.M.; Ridley, D.L.; Rowland, I.R. The effect of nicotine in vitro on the integrity of tight junctions in Caco-2 cell monolayers. Food. Chem. Toxicol. 2007, 45, 1593-1598. [CrossRef] [PubMed]

34. Borrelli, F.; Fasolino, I.; Romano, B.; Capasso, R.; Maiello, F.; Coppola, D.; Orlando, P.; Battista, G.; Pagano, E.; Di Marzo, V.; et al. Beneficial effect of the non-psychotropic plant cannabinoid cannabigerol on experimental inflammatory bowel disease. Biochem. Pharmacol. 2013, 85, 1306-1316. [CrossRef] [PubMed]

35. Borrelli, F.; Romano, B.; Petrosino, S.; Pagano, E.; Capasso, R.; Coppola, D.; Battista, G.; Orlando, P.; Di Marzo, V.; Izzo, A.A. Palmitoylethanolamide, a naturally occurring lipid, is an orally effective intestinal anti-inflammatory agent. Br. J. Pharmacol. 2015, 172, 142-158. [CrossRef] [PubMed]

36. Chang, J.; Leong, R.W.; Wasinger, V.C.; Ip, M.; Yang, M.; Phan, T.G. Impaired Intestinal Permeability Contributes to Ongoing Bowel Symptoms in Patients with Inflammatory Bowel Disease and Mucosal Healing. Gastroenterology 2017, 153, 723-731. [CrossRef] [PubMed]

37. Brierley, S.M.; Linden, D.R. Neuroplasticity and dysfunction after gastrointestinal inflammation. Nat. Rev. Gastroenterol. Hepatol. 2014, 11, 611-627. [CrossRef]

38. Corsetti, M.; Tack, J. Naloxegol, a new drug for the treatment of opioid-induced constipation. Expert Opin. Pharmacother. 2015, 16, 399-406. [CrossRef]

39. Rao, S.S.; Rattanakovit, K.; Patcharatrakul, T. Diagnosis and management of chronic constipation in adults. Nat. Rev. Gastroenterol. Hepatol. 2016, 13, 295-305. [CrossRef] 


\title{
Synbiotic Supplementation Containing Whole Plant Sugar Cane Fibre and Probiotic Spores Potentiates Protective Synergistic Effects in Mouse Model of IBD
}

\author{
Tanvi Shinde 1,2,*, Agampodi Promoda Perera ${ }^{2}$, Ravichandra Vemuri ${ }^{2}$, Shakuntla V. Gondalia ${ }^{3}$, \\ Avinash V. Karpe ${ }^{4}$, David J. Beale ${ }^{4}$, Sonia Shastri ${ }^{2}$, Benjamin Southam ${ }^{2}$, Rajaraman Eri ${ }^{2}$ and \\ Roger Stanley ${ }^{1, *}$ \\ 1 Centre for Food Safety and Innovation, Tasmanian Institute of Agriculture, University of Tasmania, \\ Launceston, TAS 7250, Australia \\ 2 School of Health Sciences, College of Health and Medicine, University of Tasmania, Launceston, TAS 7250, \\ Australia; agampodi.perera@utas.edu.au (A.P.P.); ravichandra.vemuri@utas.edu.au (R.V.); \\ sonia.shastri@utas.edu.au (S.S.); benjamin.southam@utas.edu.au (B.S.); rajaraman.eri@utas.edu.au (R.E.) \\ 3 Centre for Human Psychopharmacology, Swinburne University of Technology, Hawthorn, VIC 3122, \\ Australia; sgondalia@swin.edu.au \\ 4 Land and Water, Commonwealth Scientific and Industrial Research Organization (CSIRO), Ecosciences \\ Precinct, Dutton Park, QLD 4102, Australia; Avinash.Karpe@csiro.au (A.V.K.); David.Beale@csiro.au (D.J.B.) \\ * Correspondence: tanvi.shinde@utas.edu.au (T.S.); roger.stanley@utas.edu.au (R.S.); \\ Tel.: +61-4-79107345 (T.S.)
}

Received: 29 March 2019; Accepted: 10 April 2019; Published: 11 April 2019

\begin{abstract}
Inflammatory bowel diseases (IBD) are a chronic inflammatory disorders with increasing global incidence. Synbiotic, which is a two-point approach carrying probiotic and prebiotic components in mitigating inflammation in IBD, is thought to be a pragmatic approach owing to the synergistic outcomes. In this study, the impacts of dietary supplementation with probiotic Bacillus coagulans MTCC5856 spores (B. coagulans) and prebiotic whole plant sugar cane fibre (PSCF) was assessed using a murine model of IBD. Eight-week-old C57BL/ 6 mice were fed a normal chow diet supplemented with either B. coagulans, PSCF or its synbiotic combination. After seven days of supplementation, colitis was induced with dextran sulfate sodium (DSS) in drinking water for seven days during the continuation of the supplemented diets. Synbiotic supplementation ameliorated disease activity index and histological score $(-72 \%, 7.38$, respectively), more effectively than either B. coagulans $(-47 \%, 10.1)$ and PSCF $(-53 \%, 13.0)$ alone. Synbiotic supplementation also significantly $(p<0.0001)$ prevented the expression of tight junction proteins and modulated the altered serum IL-1 $\beta$ $(-40 \%)$, IL-10 $(+26 \%)$, and C-reactive protein (CRP) $(-39 \%)$ levels. Synbiotic supplementations also raised the short-chain fatty acids (SCFA) profile more extensively compared to the unsupplemented DSS-control. The synbiotic health outcome effect of the probiotic and prebiotic combinations may be associated with a synergistic direct immune-regulating efficacy of the components, their ability to protect epithelial integrity, stimulation of probiotic spores by the prebiotic fibre, and/or with stimulation of greater levels of fermentation of fibres releasing SCFAs that mediate the reduction in colonic inflammation. Our model findings suggest synbiotic supplementation should be tested in clinical trials.
\end{abstract}

Keywords: synbiotic; prebiotic; probiotic; IBD; Bacillus spores; dietary fibre; sugar cane fibre; ulcerative colitis 


\section{Introduction}

Inflammatory bowel disease (IBD) is a chronic relapsing inflammatory condition of the gastrointestinal tract that comprises two partially overlapping but distinct clinical entities: Crohn's disease (CD) that involves the entire gastrointestinal tract and ulcerative colitis (UC) that is limited to colon and rectum [1]. The incidence of CD and UC has become a global disease with accelerating incidence in countries adopting a Westernised diet, highlighting the urgent need for research into prevention and management of this complex and costly pathology [2]. Although the aetiology and pathogenesis of IBD still remains unclear, emerging evidence supports the involvement of a recurrent tripartite pathophysiological circuit encompassing gut dysbiosis, altered epithelial integrity and defective immune responses [1]. Therefore, preventive and therapeutic approaches that impede or break this inflammatory circuit by resolving one or more of the pathophysiological circuit components are highly sought.

Dietary interventions are increasingly perceived as both preventive and corrective strategies to normalise the dysfunctional microbiome, altered immune and barrier integrity functions to normality in IBD [3-5]. In this regard, probiotic and prebiotic dietary fibres (DF) are thought to be useful in mitigating the inflammatory circuit thereby resolving or preventing the severity of IBD. Both bioactive ingredients can improve inflammatory parameters in the gut by modifying microbiota composition and metabolites, regulating secretion of immunomodulatory molecules and protecting the colonic epithelial barrier [1,6-8]. Synbiotics, being a combination of probiotic and prebiotic ingredients that positively interact, potentially offer prophylactic and therapeutic effects that could function synergistically to confer health benefits to the host.

DFs have shown particular promise in attenuating colonic inflammation in humans [9]. Meanwhile, the underlying mechanisms are likely to be multifactorial: they include dilution of toxins via stool bulking and the production of metabolites, particularly short-chain fatty acids (SCFA), as a result of microbial fermentation is frequently cited as a major potential contributor to the protective effect [10]. DFs consist of edible plant parts that resist digestion and absorption in the small intestine and undergo complete or partial fermentation in the colon. It is an extremely complex group of substances, including non-starch polysaccharides, resistant starch, cellulose and hemicellulose, oligosaccharides, pectins, gums, lignin, and waxes [11]. Much work on DFs, however, has examined various purified ingredients that represent limited chemical complexity, contrasting to those that naturally occur in fruits and vegetables [12]. Nevertheless, the biochemical complexity of DFs is recently being more appreciated to be a vital factor influencing the gut microbial complexity [12-14]. This highlights the prudence of application of prebiotic fibres that are representative of whole plant vegetables and fruits, prepared to retain fibre biochemical complexity. In this context, a process to produce sugar cane fibre by wet diffusion to remove most of the sucrose from cut cane, which is then dried and ground into a flour, has been reported to preserve the cell wall components and retain other intrinsic nutritional biologically active components $[15,16]$. Such fibre, in addition to retaining other micronutrients and polyphenols, also contains both soluble and insoluble benefits as well as rapid- and poor-fermentable fibres and at ratios that more accurately represent natural whole plant foods. In a recent study, such sugar cane fibre has been shown to impart positive effects on human gut microbiota in vitro [17]. The high content of total dietary fibre (87\%) was accounted for with respect to its positive effect in this study. The relative similarity of this sugar cane fibre product to that in other whole plant foods indicates its potential as a convenient supplementary source of dietary fibre that could alter microbial ecology and have a positive influence for IBD attenuation.

Bacillus coagulans is a GRAS (generally recognised as safe) affirmed probiotic that can ferment a variety of plant substrates rich in insoluble cell wall components [18,19] more efficiently than most members of gut microbiota $[20,21]$. It is also known to be capable of modulating the innate immune system by binding and interacting with the gastrointestinal tract epithelium [22,23]. This makes it a suitable probiotic for its synbiotic combination with prebiotic whole plant sugar cane fibre (PSCF) rich in insoluble cell wall fractions. Probiotic Bacillus coagulans MTCC (Microbial Type Culture 
Collection) 5856 spores in addition to exhibiting excellent immunomodulatory effects in vitro, have shown significant survival during simulated gastric transit with substantial adhesion capacity to human colonic epithelial cells [22]. Based on the aforementioned findings, we hypothesised that preconditioning with probiotic B. coagulans MTCC 5856 spores, prebiotic PSCF and its synbiotic combination might repress the onset and/or severity of DSS-induced colitis in mice. This study aimed to evaluate the efficacy of probiotic B. coagulans spores and PSCF, both alone and in combination as synbiotic to ameliorate the onset of experimental colitis in mice and further examine its underlying mechanisms.

\section{Materials and Methods}

\subsection{Probiotic Bacteria and Prebiotic Dietary Fibre}

LactoSpore ${ }^{\circledR}$ (Sabinsa Corporation, East Windsor, NJ, USA) containing the probiotic strain Bacillus coagulans MTCC $5856\left(6 \times 10^{9}\right.$ spores/gm) was produced by Sami Labs Limited (Bangalore, India) and supplied by Sabinsa Corporation (Australia). Kfibre ${ }^{\mathrm{TM}}$, prebiotic whole plant sugar cane fibre (PSCF) was supplied by KFSU Pty Ltd., Queensland, Australia.

\subsection{Animals}

Fifty C57BL/6J (seven week old) mice of both sexes of average weight $19 \mathrm{~g}$ were obtained from the University of Tasmania animal breeding facility and housed in a temperature-controlled environment with a 12-h day /night light cycle. Individual body weights were assessed daily including over an initial acclimation period of seven days. All mice had ad libitum access to radiation-sterilised rodent feed pellets (Barastoc Rat and Mouse, Ridley AgProducts, Melbourne, Victoria, Australia) and autoclaved tap water for drinking during experiments. All animal experiments were approved by the Animal Ethics Committee of the University of Tasmania (ethics approval number: A0015840) and conducted in accordance with the Australian Code of Practice for Care and Use of Animals for Scientific Purposes (8th Edition, 2013). All efforts were made to minimize animals' suffering and to reduce the number of animals used.

\subsection{Study Design and Treatments}

Following one week of acclimation, mice at eight weeks of age were randomly allocated into the following five groups ( $n=10$ per group): (1) Healthy control (HC), (2) DSS-control, (3) probiotic B. coagulans MTCC 5856 spores (B. coagulans), (4) whole plant prebiotic sugar cane fibre (PSCF) supplement, and (5) synbiotic combined supplement. Mice in HC and DSS-control groups received $4 \mathrm{~g}$ chow mash (standard chow pellet blended with water). The B. coagulans group received $4 \mathrm{~g}$ chow mash supplemented with probiotic B. coagulans MTCC 5856 spores $\left(2 \times 10^{9} \mathrm{CFU} /\right.$ day/mouse $)$. The PSCF group received $4 \mathrm{~g}$ chow mash supplemented with Kfibre ${ }^{\mathrm{TM}}$ (200 mg/day/mouse). Synbiotic treatment group mice received $4 \mathrm{~g}$ chow, each supplemented with B. coagulans MTCC 5856 spores $\left(2 \times 10^{9} \mathrm{CFU} /\right.$ day/mouse) and Kfibre ( $200 \mathrm{mg} /$ day/mouse). The chow mash was prepared fresh each day. The mice were single-caged throughout the experiment to measure the defined daily intake of respective treatments from prepared chow mash. The mice were fed these treatments for 14 days. Colitis was induced during the last seven days of the experimental period as previously described [24], by administering $2 \%$ dextran sulfate sodium (DSS; MP Biomedicals, colitis grade average molecular weight: $36,000-50,000$ ) in the drinking water of all groups except for the non-colitic DSS-control mice which received normal drinking water. Mice were sacrificed on day 15 by $\mathrm{CO}_{2}$ asphyxiation.

\subsection{Clinical Scoring and Histological Analysis}

A Disease Activity Index (DAI) was determined daily in all mice by scoring for body weight, hemocult reactivity or presence of gross blood and stool consistency during the week of DSS induction, as detailed in [25]. Stool was collected from individual mice and tested for the presence of blood using 
Hemoccult II slides (Beckman Coulter Inc., Brea, CA, USA). Briefly, the following parameters were used for calculation: (a) body weight loss (score $0=0 \%$, score $1=1-5 \%$, score $2=6-10 \%$, score $3=11-15 \%$ ); (b) stool consistency (score $0=$ normal, score 1 = soft but still formed, score 2 = very soft/loose stool, score 3 = diarrhoea/watery stool); and (c) blood in stool (score $0=$ negative hemoccult, score 1 = positive hemoccult, score 2 = Blood traces in stool visible, score $3=$ rectal bleeding). DAI was determined by combining the scores from these three categories. Body weights were measured for each animal throughout the experiments and expressed as percent weight loss to the weight immediately before DSS treatment. Fecal samples were collected and stored at $-80{ }^{\circ} \mathrm{C}$ on day 14 for metabolite analysis.

After sacrificing the mice, the colons were removed from the caecum to the anus following the method of Perera et al. [26]. The length of the colons from the ileocaecal junction to the rectum were recorded. The colon was subsequently opened along its longitudinal axis and the luminal (mucosal) contents were removed using sterilised $200 \mu \mathrm{L}$ pipette tips prior to weighing the organ. The length and weight of colon and spleen were documented. Spleen weight, colon length, and colon weight/body weight ratio were calculated as macroscopic markers of inflammation. The mucosal and cecal contents were collected for metabolite profiling and stored at $-80^{\circ} \mathrm{C}$. The colon was bisected longitudinally, and one half was prepared using the Swiss roll technique [27] whereas the remaining colonic tissue was dissected out, segregated into proximal colon (PC) and distal colon (DC) and snap-frozen for molecular analyses. Swiss rolls underwent $24 \mathrm{~h}$ fixation in $10 \%(v / v)$ neutral-buffered formalin. Swiss rolls were subsequently transferred to $70 \%$ ethanol prior to progressive dehydration, clearing and infiltration with HistoPrep paraffin wax (Fisher Scientific, Philadelphia, PA, USA). Swiss rolls were then embedded in wax and $5 \mu \mathrm{m}$ sections were cut using a rotary microtome. Sections were stained with haematoxylin and eosin (H and E; HD Scientific, Sydney, Australia). Slides stained with H and E ( $n=8$ per group) graded blindly for the severity of tissue damage at distal and proximal regions as described previously [28,29]. Briefly, frequency of distribution of inflammation graded 0-3, crypt architectural distortion and ulceration graded 0-5, tissue damage graded 0-3, inflammatory infiltrate graded 0-3, goblet cell loss graded 0-3, mucosal thickening (oedema) were graded 0-3. All images were captured on a Leica DM500 microscope using a Leica ICC50 W camera (Leica Microsys-tems, Wetzlar, Germany).

\subsection{Alcian Blue Staining}

DSS-induced alterations in goblet cells and subsequent depletion in synthesis and secretion of mucin glycoprotein (MUC2) was analysed by Alcian blue staining (ab150662 Alcian Blue, pH 2.5 (Mucin Stain), Abcam, Australia) following the manufacturer's instructions. Briefly, paraffin-embedded colon sections ( $n=4$ /group) were stained with Alcian blue, staining the acidic sulphated mucin blue and the counterstained with Safranin O, staining the nuclei red following the method previously described [30]. Computer-assisted image analysis was performed with a Leica DM500 microscope (Leica Microsystems, Wet-zlar, Germany) and Leica ICC50 W camera (Leica Microsys-tems, Wetzlar, Germany). The staining intensity (IOD) was assessed using Image Pro Plus 7.0 (Media Cybernetics, Inc., Rockville, MD, USA) and used for comparison among groups [31].

\subsection{Immunohistochemical Detection of Tight Junction Proteins}

Immunohistochemical detection of epithelial tight junction (TJ) proteins was performed using a Rabbit specific HRP/DAB (ABC) Detection IHC kit (ab64261, Abcam, Australia) following the manufacturer's instruction and as previously described [32]. Following removal of paraffin and rehydration, the tissue sections were exposed to heat-induced epitope retrieval $\left(4 \mathrm{~min}\right.$ at $\left.121{ }^{\circ} \mathrm{C}\right)$ in a sodium citrate buffer, $\mathrm{pH} 6$ in a Decloaking chamber (Biocare Medical, Pachico, CA, USA). After washing the slides in $1 \times$ phosphate buffered saline (PBS) 2 mins/wash, endogenous peroxidase activity was blocked by incubating the slides with hydrogen peroxide block for $10 \mathrm{~min}$. Next, the slides were washed with PBS $(2 \times 2 \mathrm{~min})$ washes and protein block was then applied for $30 \mathrm{~min}$ at room temperature to block non-specific background staining. Following PBS $(1 \times 2 \mathrm{~min})$ wash, colon sections were then incubated with primary antibodies: anti-ZO-1 (NBP1-85046, Novus Biologicals, Australia, 
1:400); anti-occludin (NBP1-87402, Novus, 1:600); anti-claudin-1 (NBP1-77036, Novus, $1 \mu \mathrm{g} / \mathrm{mL}$ ) overnight at $4{ }^{\circ} \mathrm{C}$. Sections were then washed with PBS $(4 \times 2 \mathrm{~min})$ and biotinylated goat anti-rabbit IgG was applied and incubated for $10 \mathrm{~min}$ at room temperature. At the end of incubation, the slides were washed in PBS $(4 \times 2 \mathrm{~min})$ and streptavidin peroxidase was applied to the sections and further incubated for 10 at room temperature. The slides were then thoroughly rinsed with PBS $(4 \times 2 \mathrm{~min})$ before sections were covered with 3,3'-diaminobenzidine (DAB) chromogen and substrate solution for $10 \mathrm{~min}$. Tissue sections were subsequently counterstained with hematoxylin, dehydrated, and mounted with DPX media (Sigma-Aldrich, Sydney, Australia).

Computer-assisted image analysis was performed with a Leica DM500 microscope (Leica Microsystems, Wet-zlar, Germany), Leica ICC50 W camera (Leica Microsys-tems, Wetzlar, Germany), and Image Pro Plus 7.0 (Media Cybernetics, Inc., Rockville, MD, USA) software. The expression of tight junction proteins (TJPs): ZO-1, occludin and claudin-1 was blindly assessed by choosing random five fields on each slide ( $n=4$ /group). Barrier TJ protein expressions and staining intensity in colonic epithelium was expressed as the percentage expression of a respective TJ protein.

\subsection{Myeloperoxidase Activity}

The extent of the inflammatory cell invasion in the colon was examined by the assessment of myeloperoxidase (MPO) activity [24]. Weighed and snap frozen PC and DC specimens $(n=3)$ were analysed for MPO activity using a Myeloperoxidase Activity Assay kit (ab105136, colorimetric, Abcam ${ }^{\circledR}$, Cambridge, UK). Briefly, frozen tissue after washing in cold PBS, was resuspended in MPO assay buffer, before homogenisation with 10-15 passes using an Omni TH tissue homogeniser (Omni International, US) with 10-15 passes. The homogenate was then centrifuged at 13,000 $\mathrm{g}(10$ $\mathrm{min}$ ) and the supernatant assayed for MPO activity as per the manufacturer's instructions. The values are expressed as MPO activity units/g tissue.

\subsection{Tissue Explant Culture and Cytokine Measurements}

PC and DC colon tissues of mice from each group were cut, weighed and washed with cold PBS before transferring to a 12 well plate containing $0.5 \mathrm{~mL} /$ well of RPMI1640 culture medium (In Vitro Technologies Pty Ltd, Melbourne, Australia) supplemented with 10\% v/v fetal calf serum (Gibco, Life Technologies Pty Ltd, Melbourne, Australia), penicillin (100 mU/L), and streptomycin (100 mg/L) (Sigma-Aldrich Pty Ltd, Sydney, Australia) as described previously [26]. After $24 \mathrm{~h}$ of incubation, supernatant was collected from each well, centrifuged and stored at $-80^{\circ} \mathrm{C}$ until further analysis. Serum was collected from blood drawn by cardiac puncture at the end of the study for cytokine analysis.

The cytokine levels in colon tissue $(n=3)$ and serum $(n=3)$ were determined by immunoassay using a Bio-Plex Pro Mouse cytokine 23-plex kit (Bio-Rad \#M60009RDPD, Bio-Rad Laboratories, Gladesville, NSW, Australia) following the manufacturer's instructions and concentrations analysed using a Bio-Plex 200 instrument (Bio-Rad) and Bioplex Manager software, version 6 (Bio-Rad Laboratories) respectively. For tissues, the cytokine levels were normalized by dividing the cytokine results $(\mathrm{pg} / \mathrm{mL})$ by the measured biopsy weight $(\mathrm{g})$. The most significantly altered cytokines are presented as $\mathrm{pg} / \mathrm{g}$ of tissue.

\section{9. iNOS Activity}

The expression of inducible isoform of nitric oxide synthase (iNOS) in colonic epithelial cells in response to pro-inflammatory stimuli [33] was determined in PC and DC specimens using a Nitic Oxide Synthase Activity Assay kit (ab211084, Fluorometric, Abcam ${ }^{\circledR}$, Cambridge, UK). Snap frozen proximal and distal colonic tissues $(n=3)$ were washed in in cold PBS and resuspended in $200 \mu \mathrm{L}$ NOS assay buffer then homogenised by 10-15 passes of an Omni TH tissue homogeniser (Omni International, Tulsa, OK, USA). The homogenate was then centrifuged at $10,000 \times \mathrm{g}\left(10 \mathrm{~min}, 4^{\circ} \mathrm{C}\right)$ and the supernatant then underwent iNOS activity assay activity assay as per the manufacturer's instructions. The amount 
of protein in the lysate was determined using $D C^{\mathrm{TM}}$ Protein Assay (Bio-Rad Laboratories, Australia). The results are expressed as iNOS activity $\mathrm{mU} / \mathrm{mg}$.

\subsection{Serum C-Reactive Protein Analysis}

The levels of C-reactive protein (CRP) in serum from respective groups ( $n=3$ samples/group) were analysed using Mouse C-Reactive Protein/CRP Quantikine Elisa kit (MCRP00, R and D Systems, Australia) following the manufacturer's instructions. The results are expressed as $\mu \mathrm{g} / \mathrm{mL}$.

\subsection{Volatile SCFA Analysis}

Cecal, mucosal and fecal samples ( $n=5$ per group) were prepared and derivatized following the protocol developed by Furuhashi et al. [34] with some modifications. Briefly, cecal, mucosal and fecal samples of $100-150 \mathrm{mg}$ fresh weight (stored at $-80^{\circ} \mathrm{C}$ ) were weighed to $\pm 0.1 \mathrm{mg}$ accuracy. These samples were added to a sterile $1.5 \mathrm{~mL}$ bead-beating tube (NAVY Rino Lysis tubes, Next Advance, Troy, NY, USA). A $1.0 \mathrm{~mL}$ aliquot isobutanol (10\% MilliQ water), (LC-MS grade, Merck, Castle Hill, NSW, Australia) was added to each sample, followed by two $30 \mathrm{~s}, 4000 \mathrm{rpm}$ homogenization pulses sandwiched between a 20-s pause interval (Precellys Evolution Homogenizer, Bertin Instruments, Montigny-le-Bretonneux, France). The samples were subsequently centrifuged at $16,000 \times g$ for 6 min.

The supernatant $(675 \mu \mathrm{L})$ was transferred to a clean round bottomed $2 \mathrm{~mL}$ centrifuge tube (Eppendorf South Pacific Pty. Ltd., Macquarie Park, NSW, Australia) and NaOH (20 mM, 125 L, Merck Pty Ltd., Castle Hill, NSW, Australia) and chloroform (400 $\mu$ L, LC-MS grade, Merck Pty Ltd.,) were added. The samples were briefly vortexed and centrifuged at $16,000 \cdot g$ for $3 \mathrm{~min}$. The aqueous phase (upper layer, $400 \mu \mathrm{L}$ ) was transferred to a new clean round bottomed $2 \mathrm{~mL}$ centrifuge tube (Eppendorf South Pacific Pty. Ltd., Macquarie Park, NSW, Australia) containing a boiling chip (Sigma Aldrich, Castle Hill, NSW,

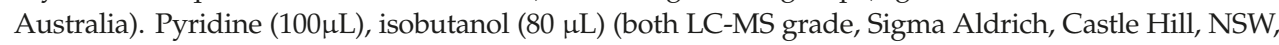
Australia), and MilliQ (Millipore Corporation) water $(70 \mu \mathrm{L})$ were added and the samples were subjected to gentle hand vortexing (swirling action) followed by the addition of $50 \mu \mathrm{L}$ isobutyl chloroformate $(98 \%$ purity, Sigma Aldrich, Castle Hill, NSW, Australia). The tube was kept opened to release any generated gases and was allowed to stand for about one minute. Hexane (150 $\mu \mathrm{L}$, LC-MS grade, Sigma Aldrich, Castle Hill, NSW, Australia) was then added to each tube, which was then capped and vortexed prior to centrifugation at $15,700 \times g$ for $4 \mathrm{~min}$. The upper phase $(100 \mu \mathrm{L})$ was subsequently transferred to clean gas chromatography (GC) autosampler vial fitted with silanized low volume glass inserts; Malathion $(1 \mu \mathrm{L}$, equivalent to $2.5 \mu \mathrm{g}$ dry weight) was added as an internal standard.

The GC-MS analysis was performed on an Agilent 6890B GC oven coupled to a 5977B mass spectrometer (MS) detector (Agilent Technologies, Mulgrave, VIC, Australia) fitted with an MPS autosampler (Gerstel GmbH \& Co.KG, Mülheim an der Ruhr, Germany). The GC oven was fitted with two $15 \mathrm{~m}$ HP-5MS columns (0.25 mm ID and $0.25 \mu \mathrm{m}$ film thickness; 19091S-431 UI (Ultra Inert), Agilent Technologies, VIC, Australia) coupled to each other through a purged ultimate union (PUU) for the use of post-run backflushing. The sample $(1.0 \mu \mathrm{L})$ was introduced via a multimode inlet (MMI) operated in split mode (1:20). The column was maintained at $40{ }^{\circ} \mathrm{C}$ for $5 \mathrm{~min}$, followed by an increase to $250^{\circ} \mathrm{C}$ at a rate of $10^{\circ} \mathrm{C} / \mathrm{min}$. This was followed by a second increment to $310^{\circ} \mathrm{C}$ at a rate of $60^{\circ} \mathrm{C} / \mathrm{min}$. The column was held at $310^{\circ} \mathrm{C}$ for $1 \mathrm{~min}$. The mass spectrometer was kept in extractor ion mode (EI mode) at $70 \mathrm{eV}$. The GC-MS ion source temperature and transfer line were kept at $250{ }^{\circ} \mathrm{C}$ and $280^{\circ} \mathrm{C}$, respectively. Detector voltage was kept at $1054 \mathrm{~V}$. The MS detector was turned off for the first $3 \mathrm{~min}$ and, at 4.0-4.8 $\mathrm{min}$ and 12.5-13.2-min time windows until the excess derivatization reagent (chloroformate/hexane solvents) were eluted from the column. This ensured that the source filament was not saturated and damaged. The scan range was kept in the range of $m / z$ 35-350 (35-350 Daltons). Data acquisition and spectral analysis were performed as described in a previous study [35] and qualitative identification of metabolites was performed according to the Metabolomics Standard Initiative (MSI) chemical analysis workgroup [36] using standard GC-MS reference metabolite libraries (NIST 17, and an in-house CF-based metabolomics library developed 
after Smart et al. [37] with the use of Kovats retention indices based on a reference n-alkane standard (C8-C40 Alkanes Calibration Standard, Sigma-Aldrich, Castle Hill, NSW, Australia).

\subsection{Metabolic Phenotyping Analysis}

Untargeted metabolomic profiling of cecal, mucosal and fecal samples ( $n=5$ per group) were performed using GC-MS analysis as described previously [35].

\subsection{Statistical Analysis}

The samples in the study were randomly chosen for all the analysis to avoid bias. All data are presented as means \pm standard error of the mean (SEM). The statistical analysis was performed with the use of GraphPad Prism Software (Version 7.0, San Diego, CA, USA) The data were evaluated using One-way analysis of variance (ANOVA) followed by Tukey's post-hoc test to determine statistical differences between the groups against the DSS-control samples. For the analysis of DAI and body weight changes during the experimental period, two-way ANOVA followed by Tukey's post-hoc test was used, setting treatment and the time as the variables. A $p$-value of $<0.05$ was considered significant.

\section{Results}

3.1. Effects of B. Coagulans, PSCF and Synbiotic Supplementation on DAI and Macroscopic Inflammatory Markers in DSS-Induced Mice

In comparison with the HC group, the administration of probiotic, prebiotic and synbiotic treatments in the respective groups did not show any sign of toxicity, which was evaluated by body weight increase, food intake and general appearance of the animals. DAI (cumulative score for body weight change, stool consistency and blood in feces) was evaluated to determine the efficacy of the treatments in reducing the severity of disease symptoms in DSS-induced colitis (Figure 1A). Compared with the DSS-control group that showed severe colitis symptoms, pre-conditioning with B. coagulans, PSCF and synbiotic combination significantly reduced the DAI levels as early as day2 until the end of experiment. At the end of the experiment, DAI of DSS-control group was significantly high (5.8 \pm $0.5)(p \leq 0.0001)$ compared with that of $B$. coagulans $(3.1 \pm 0.6,47 \%$ reduction), PSCF $(2.7 \pm 0.5$, $53 \%$ reduction), and synbiotic ( $1.6 \pm 0.2,72 \%$ reduction) groups. DSS induction in DSS-control mice resulted in significant body weight loss until the end of experiment $(-4.13 \pm 1.4 \%)$. In contrast, mice maintained healthy body weight gain with Bacillus $(2.84 \pm 1.7 \%)$, PSCF $(4.25 \pm 1.0 \%)$, and synbiotic $(4.7 \pm 0.7 \%)$ treatments. Interestingly, PSCF was the more effective in reducing DAI starting from day 2 of DSS and in remediating the body weight loss as early as day 5 owing mainly to the impact of improvement in stool consistency on the DAI rating.

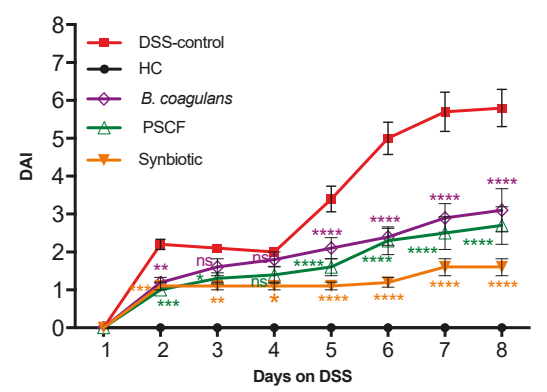

$A$

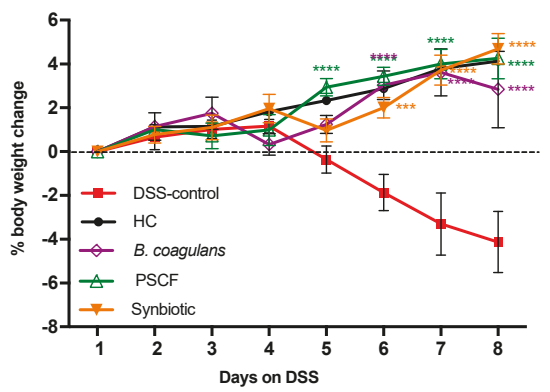

$B$

Figure 1. Cont. 


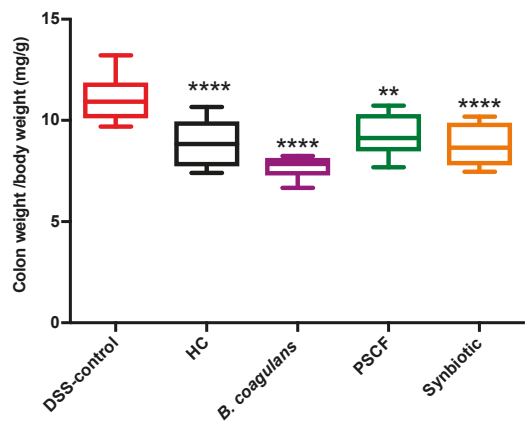

C

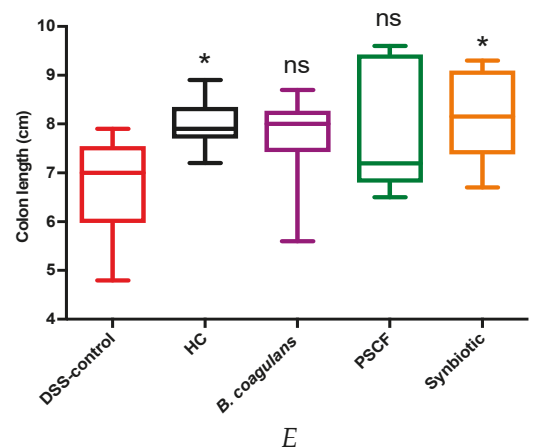

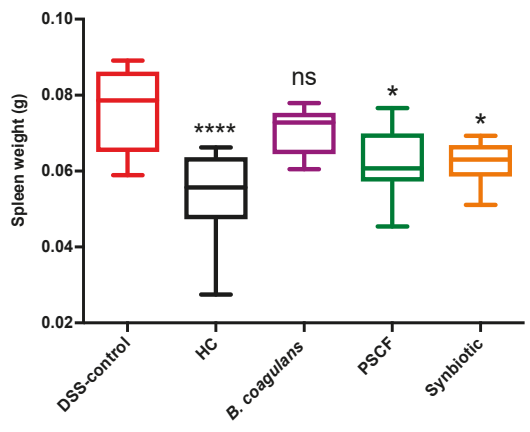

D

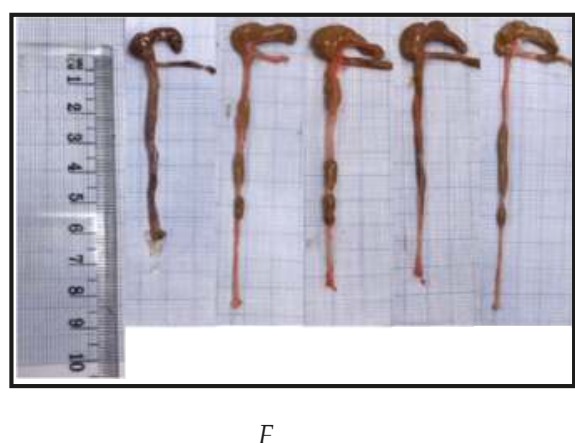

Figure 1. Effect of B. coagulans spores, PSCF and synbiotic in DSS-induced colitis model. (A) Disease Activity Index (DAI), (B) \% body weight change. Statistical significance among groups evaluated by two-way repeated-measures analysis of variance (ANOVA) followed by Tukey's test. ${ }^{*} p<0.05$, ${ }^{* *} p<0.01,{ }^{* * *} p<0.001,{ }^{* * * *} p<0.0001$ vs. DSS-control group and data expressed as mean $\pm \operatorname{SEM}(n=10$ per group). Colon weight/body weight ratio (C), Spleen weight (D), Colon length (E) and Macroscopic appearance of colon (F). Data expressed as mean \pm SEM ( $n=10$ per group), evaluated by one-way ANOVA followed by Tukey's Test. ns-non-significant, HC-Healthy control, PSCF-Prebiotic sugar cane fibre.

The macroscopic evaluation of colonic segments determined the beneficial effects of all three treatments used in our study as evidenced by substantial reduction in colon weight/body weight ratio (B. coagulans, $7.68 \pm 0.2$; PSCF, $9.24 \pm 0.3$ and synbiotic, $8.74 \pm 0.3 \mathrm{mg} / \mathrm{g}$ ) compared with DSS-control group (11.12 $\pm 0.3 \mathrm{mg} / \mathrm{gm}$ ) (Figure 1C). Intestinal inflammation is associated with spleen enlargement [24] and, as expected, relative spleen weight of untreated DSS-control mice was significantly higher $(0.076 \pm 0.004 \mathrm{~g})$ than that of $\mathrm{HC}$ mice $(0.054 \pm 0.003 \mathrm{~g})$. PSCF $(0.062 \pm 0.003 \mathrm{~g})$ and synbiotic $(0.063 \pm 0.002 \mathrm{~g})$ were equally significantly effective in reducing spleen weight while B. coagulans did not affect the relative spleen weight (Figure 1D). In contrast to shortening of colon length (Figure 1E,F) in DSS-control group $(6.75 \pm 0.3 \mathrm{~cm})$, synbiotic treatment proved effective in reducing this outcome by maintaining the colon length $(8.12 \pm 0.2 \mathrm{~cm})$, significantly equal $(p=0.99)$ to that of HC group $(8.01 \pm 0.2 \mathrm{~cm})$. These markers are considered to be directly correlated to the severity of colonic damage in this experimental model of colitis [24]. 
3.2. Effects of B. Coagulans, PSCF and Synbiotic Supplementation on Histological Alterations in DSS-Induced Mice

Histological ( $\mathrm{H}$ and E staining) examination of proximal colon (PC) and distal colon (DC) sections of DSS-induced mice showed altered erosion or destruction of epithelium, crypt distortion, depletion of goblet cells, submucosal oedema, and inflammatory cellular infiltration in the colon, mostly affecting the DC (Figure 2A). HC mice showed no signs of histological colon damage with score 0, while DSS induction in DSS-control mice resulted in a cumulative damage score of $10.5 \pm 0.8$ for the PC and $17.4 \pm 0.5$ for the DC (Figure 2B). Supplementation of DSS-induced mice with B. coagulans, PSCF and synbiotic induced protection and repair of the colonic mucosa. B. coagulans and synbiotic in particular were more effective in retention of colonic structure, protection of crypts and goblet cells and rescued infiltration of inflammatory cells, which resulted in a significant overall reduction of cumulative histological score of DC (10.1 \pm 1.2 and $7.38 \pm 0.7$ respectively). Relatively, PSCF also provided partial significant protection with histological score of $13.0 \pm 1.0$. In contrast, PCSF had no effect in PC $(10.1 \pm 0.5)$ with only B. coagulans and synbiotic treatments being significantly successful in reducing damage to the PC with histological scores of $9.5 \pm 0.7$ and $7.8 \pm 0.3$ respectively. Unlike DSS-control group, there was reduced polymorphic inflammatory infiltrate in the lamina propria and submucosa in probiotic, prebiotic and synbiotic supplemented group. This observation corroborates with the significantly reduced MPO activity in the colon (Figure 2C) of all three treatments compared with the DSS-control group. The attenuation of colonic inflammation in pre-conditioned mice (B. coagulans, PSCF and synbiotic) is probably due to the anti-inflammatory properties of the functional dietary ingredients tested in this study.

\subsection{Effects of B. Coagulans, PSCF and Synbiotic Supplementation on Goblet Cells and Colonic Tight Junction Barrier}

Histological examination of distal colon from DSS mice showed a depletion of goblet cells when compared to HC and pre-conditioned mice. This suggested beneficial effects of probiotic and prebiotic ingredients on the intestinal epithelium potentially through stimulating mucus secretion by goblet cells. Specific staining with Alcian blue was therefore carried out to assess the mucus production following the administration of probiotic, prebiotic and synbiotic treatments. As depicted in Figure 3, in comparison with DSS-control group, there was a higher level of mucus staining with Alcian blue in supplemented mice samples. This implied that there has been an induction of higher levels of mucus secretion in the DSS-challenged mice that received B. coagulans spores, symbiotic, and PSCF supplementaions. Unlike the DSS-controlled samples where, goblet cells were almost entirely destroyed, the mice supplemented with synbiotic and B. coagulans showed protection of the goblet cells. PSCF also partially protected the goblet cells with mucus staining compared with DSS-control.

Immunohistochemical analysis was then performed to evaluate the assembly of the TJs and the integrity of the intestinal barrier. The presence of the TJ proteins-ZO-1, occludin, and claudin- 1 on the tissue sections were analysed (Figure 4). In HC, ZO-1 (Figure 4A) staining was more intense in the apical tight junction complex both at the surface and in the crypts. In addition to showing their presence at the crypt surface, occludin (Figure 4B), and claudin-1 (Figure 4C) proteins stained more strongly at the basolateral membrane of crypts. As previously reported [38,39] such signals were weak or totally absent on the epithelium of DSS-control sections resulting in very low percentage TJ protein expressions. Basolateral and partial apical staining of ZO-1, occludin and claudin-1 was maintained with B. coagulans and synbiotic supplementation in DSS-treated animals. While PSCF was able to partially maintain ZO-1 and claudin-1 staining, such an effect was less evident for occludin. Synbiotic treatment was most effective in preserving the TJ protein expressions in DSS-induced mice further confirming its beneficial effects on the intestinal integrity. 
A

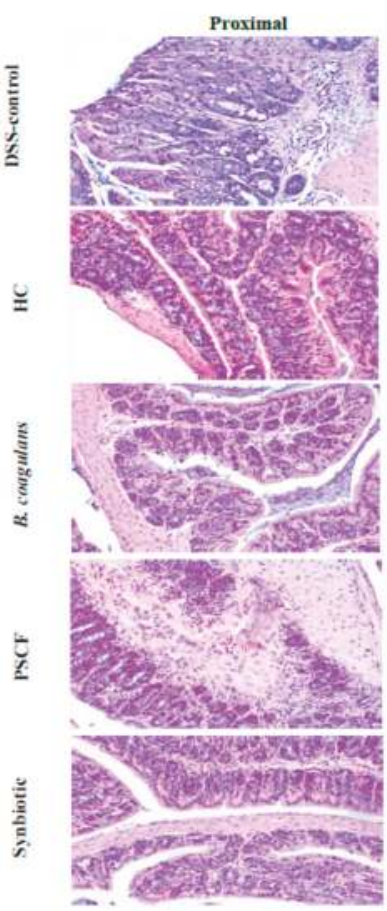

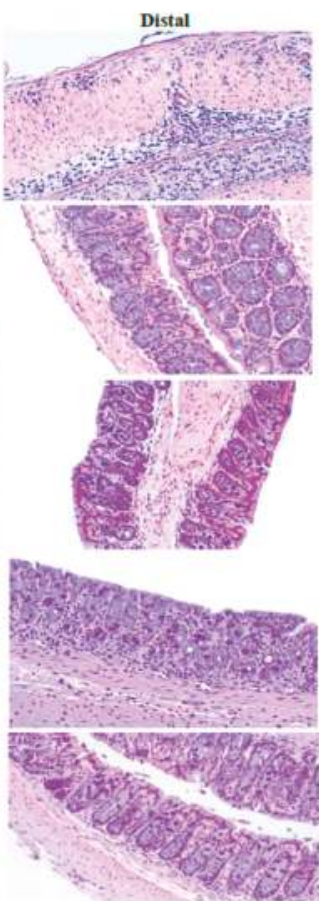

C
B

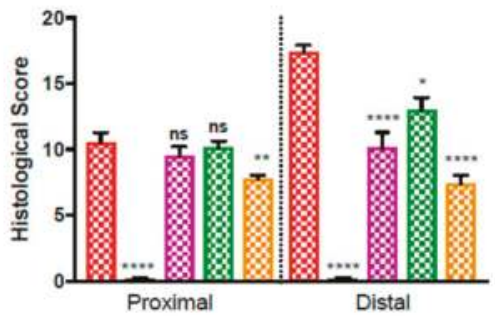

W HC

麻 DSS-control

MB $B$ conguians

BS PSCF

Symbiotc

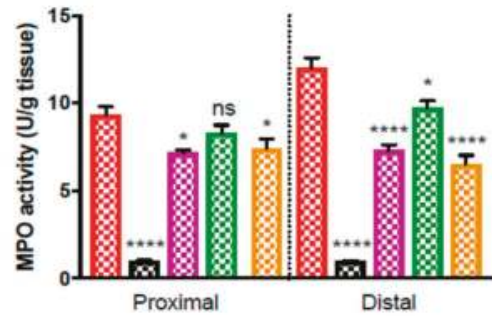

Proximal

Distal

Figure 2. Effect of B. coagulans spores, PSCF and synbiotic treatments on DSS-induced colon injury and inflammation. (A) Histological images of proximal and distal colonic tissues stained with hematoxylin and eosin at $20 \times$ for each experimental group. (B) Histological score calculated after microscopic analyses of proximal and distal sections of the colon. (C) Myeloperoxidase (MPO) activity in colonic tissues was determined by colorimetric assay. Results expressed as mean \pm SEM ( $n=8$ per group), evaluated by one-way ANOVA followed by Tukey's test $\left({ }^{*} p<0.05,{ }^{* *} p<0.01,{ }^{* * * *} p<0.0001\right)$. 

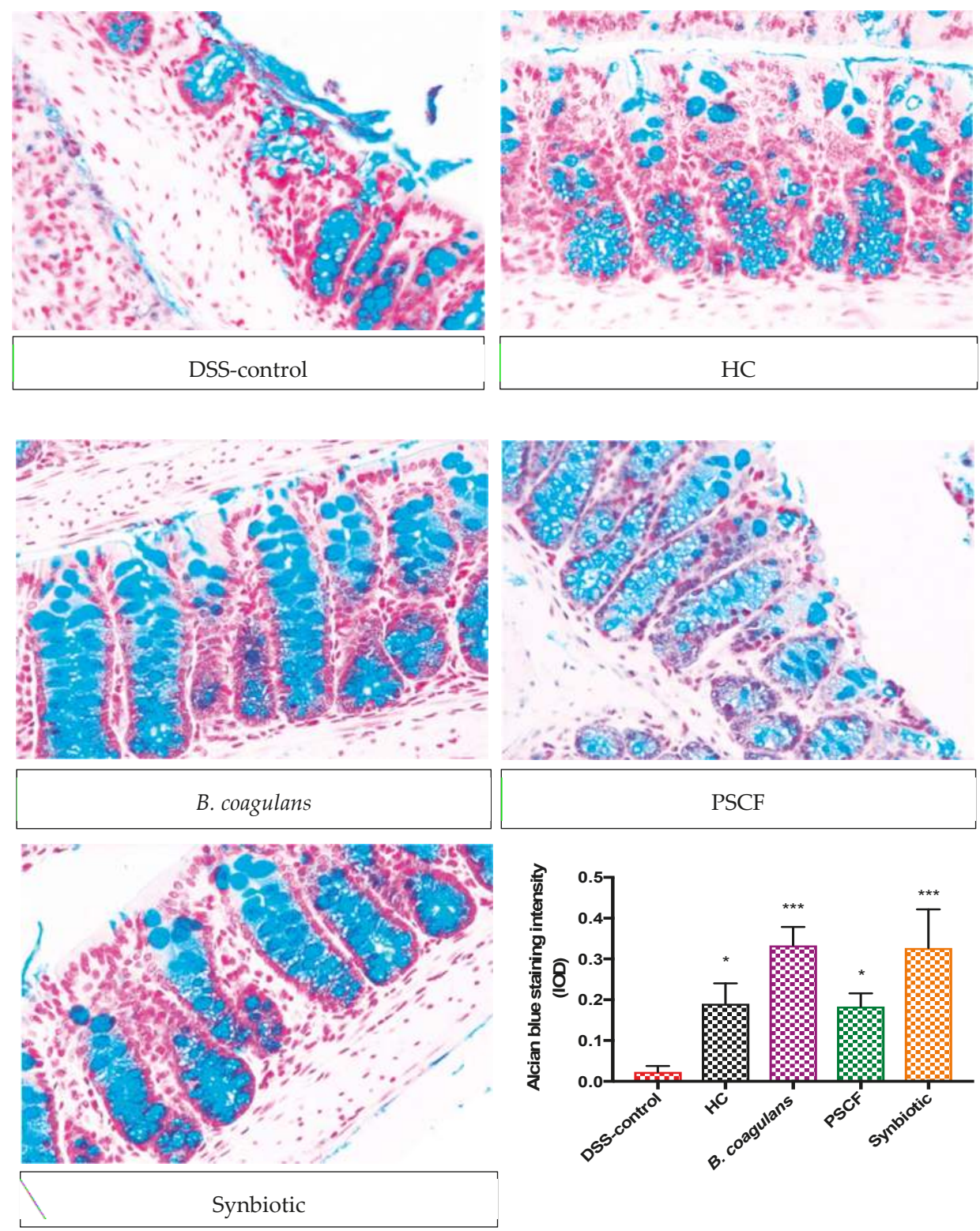

Figure 3. Effect of B. coagulans spores, PSCF and synbiotic on goblet cells. The paraffin embedded sections were stained with Alcian Blue to detect changes in goblet cells and in production of mucus in distal colonic tissue in each experimental group $(40 \times)$ and staining intensity (IOD) of respective group is illustrated in the graph. Results expressed as mean \pm SEM ( $n=4$ per group), evaluated by one-way ANOVA followed by Tukey's test $\left({ }^{*} p<0.05,{ }^{* * *} p<0.001\right)$. 
A $\quad$ ZO-1
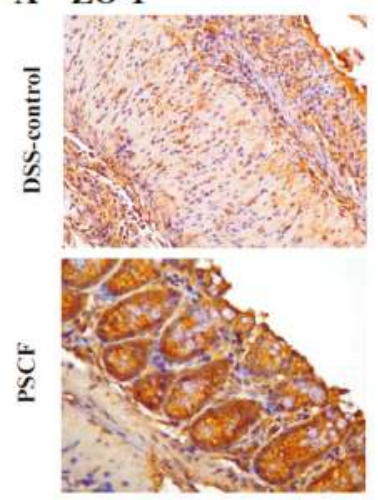

B Occludin

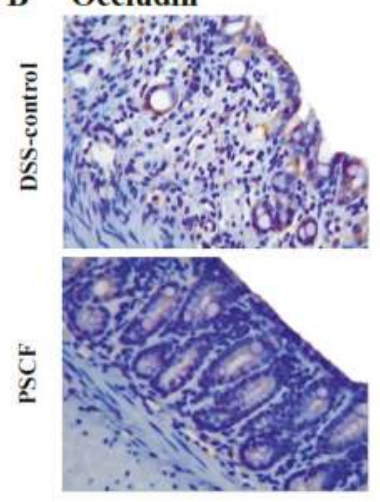

\section{Claudin-1}

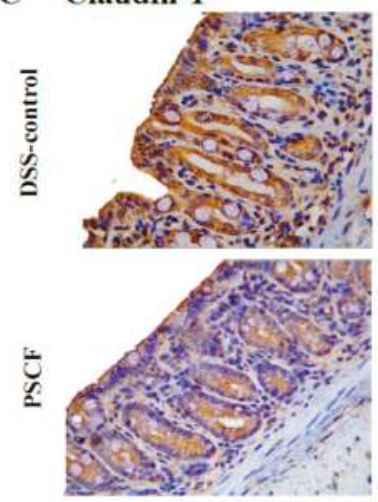

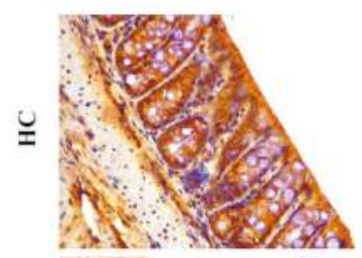
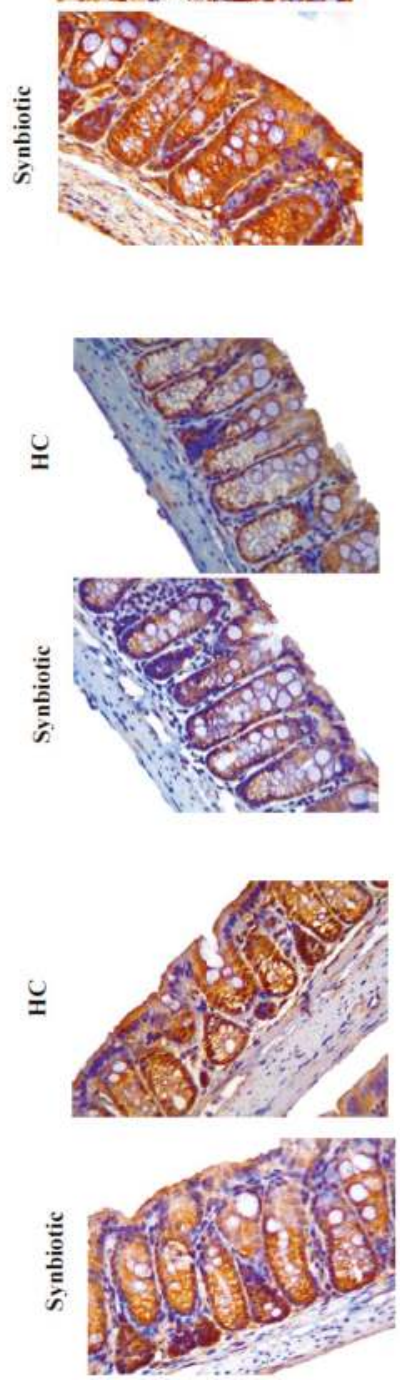
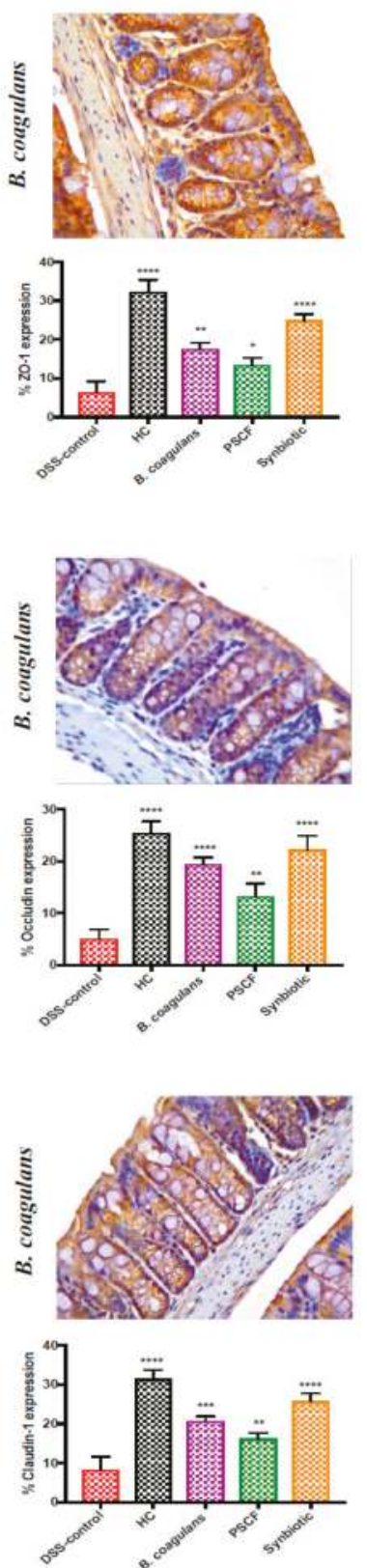

Figure 4. Effects of B. coagulans spores, PSCF and synbiotic on expression of epithelial tight junction proteins. Immunohistochemical detection of (A) ZO-1, (B) Occludin and (C) claudin-1 and its respective percentage of expression in colon at $40 \times$. Data expressed as mean \pm SEM $(n=4$ per group) and statistical significance among groups evaluated by one-way ANOVA followed by Tukey's test * $p<0.05$, ${ }^{* *} p<0.01,{ }^{* * *} p<0.001,{ }^{* * * *} p<0.0001$ vs. DSS-control group. 
3.4. Immunomodulatory Effects of B. Coagulans, PSCF, and Synbiotic Supplementation on Immune Markers in DSS-Induced Mice

The cytokine analysis of the colonic segments and serum measured the intestinal immunomodulatory and anti-inflammatory effects of B. coagulans, PSCF and their synbiotic combination. A beneficial impact of ameliorating the altered immune response induced by DSS intake was noted. Overall, probiotic, prebiotic, and synbiotic treatments remarkably reduced the pro-inflammatory cytokine secretions of IL- $1 \alpha$, IL- $1 \beta$, IL- 6 , IL- 12, TNF- $\alpha$, and IFN- $\gamma$ in proximal and distal colon segments compared with that of the DSS-colitic segments (Figure 5), while no significant effect of supplementations was noted on other cytokine levels (Supplementary Figure S1). B. coagulans and synbiotic treatments were equally effective in maintaining the levels of these altered cytokines relative to that of non-colitic mice while PSCF did not show significant effective reduction of IL-12 cytokine levels (Figure 5D). Synbiotic treatment was statistically more potent in suppressing the elevated levels of IL-1 $\alpha(-90.29 \%)$ and IL-12 $(-67.42 \%)$ compared with B. coagulans $(-85.94 \%,-52.20 \%$, respectively) in the DC. No positive effect was observed in the PC segment for IL-6 and TNF- $\alpha$ levels by PSCF treatment. However, the excellent immunomodulatory effect in the DC for respective cytokines was confirmed (Figure 5C,E), implicating its differential effects in these colonic segments.

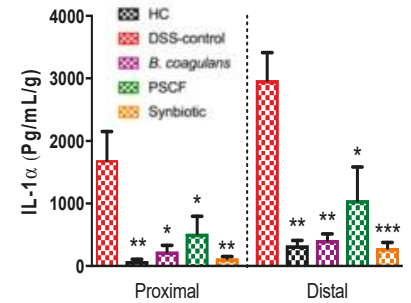

$A$

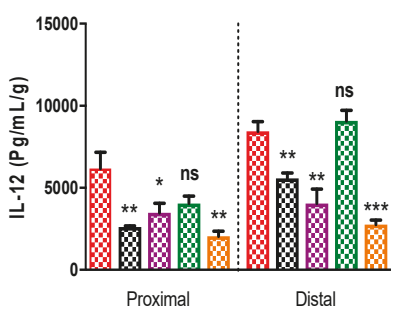

$D$

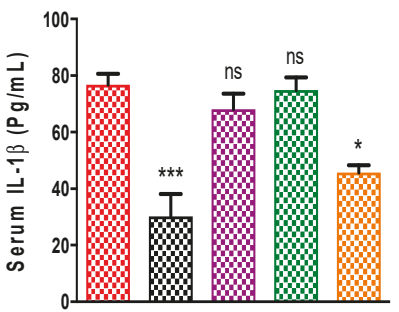

G

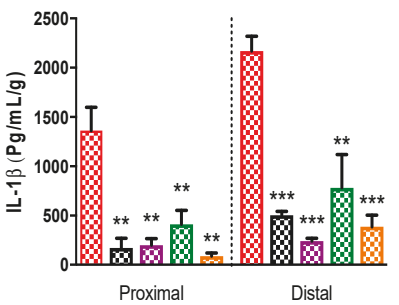

$B$

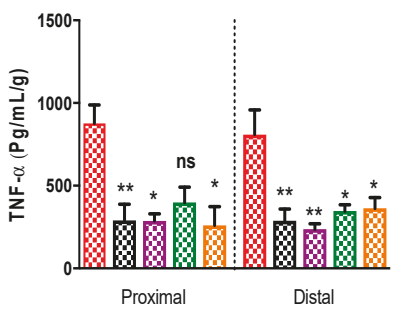

E

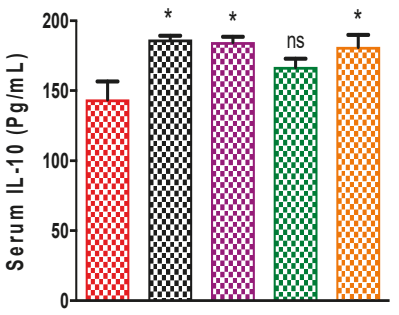

$H$

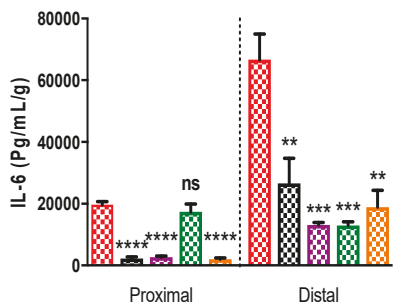

C

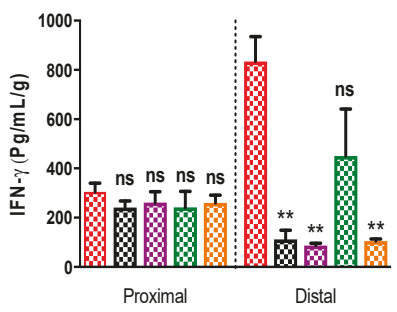

F

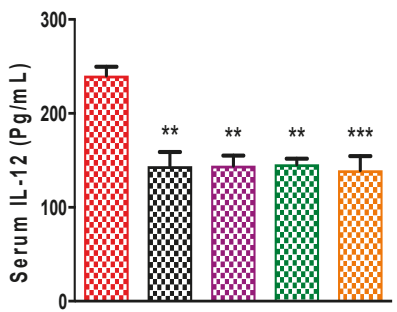

I

Figure 5. Cont. 

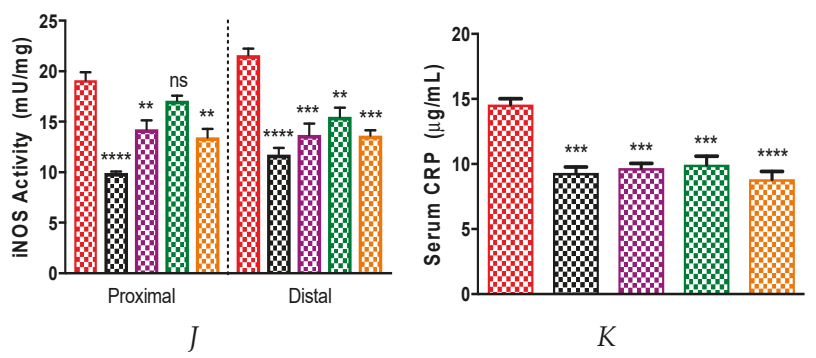

Figure 5. Effect of B. coagulans spores, PSCF and synbiotic on immune markers in colon tissues and blood serum. Protein levels of cytokines including (A) IL-1 $\alpha$, (B) IL-1 $\beta$, (C) IL-6, (D) IL-12, (E) TNF- $\alpha$, (F) IFN- $\gamma$ in proximal and distal colon explants as well as cytokine levels of (G) IL-1 $\beta$, (H) IL-10, and (I) IL-12 in blood serum were analysed by Bio-plex. iNOS activity in colon tissues (J) measured by NOS activity assay and CRP levels in serum $(\mathbf{K})$ by ELISA. Statistical significance among groups evaluated by one-way ANOVA followed by Tukey's test. ${ }^{*} p<0.05,{ }^{* *} p<0.01,{ }^{* * *} p<0.001,{ }^{* * * *} p<0.0001$ vs. DSS-colitic group and data expressed as mean \pm SEM ( $n=3$ per group).

Similar immunomodulatory effects were found on the serum cytokines Il-1 $\beta$, IL-12, and IL-10 (Figure 5G-I), while no significant effect was observed for other serum cytokines (Supplementary Figure S1). All three treatments substantially restored the IL-12 levels to values similar to HC mice, while synbiotic treatment was significantly more efficacious in reducing pro-inflammatory IL-1 $\beta$ levels in serum. Moreover, B. coagulans and synbiotic treatments increased the anti-inflammatory IL-10 levels in serum $(181.2 \pm 8.70,184.7 \pm 3.81 \mathrm{pg} / \mathrm{mL}$ respectively) compared with the DSS-colitic group $(143.8 \pm 12.80 \mathrm{pg} / \mathrm{mL}$ ) (Figure $3 \mathrm{H})$. iNOS activity which is known to be high in colitis in response to pro-inflammatory stimuli [33], was significantly suppressed in both colon segments by all treatments (Figure 5J) compared with DSS-colitic levels. Serum CRP level was significantly higher in the DSS-control group $(14.58 \pm 0.45 \mu \mathrm{g} / \mathrm{mL})$ in comparison with $\mathrm{HC}$ animals $(9.32 \pm 0.45 \mu \mathrm{g} / \mathrm{mL})$. Bacillus, PSCF and synbiotic remarkably normalised the CRP levels $(9.67 \pm 0.34,9.95 \pm 0.65,8.83 \pm 0.59$ $\mu \mathrm{g} / \mathrm{mL}$, respectively) to that of the HC group. These results confirmed the notable anti-inflammatory efficacy of probiotic Bacillus spores, prebiotic PSCF and synbiotic treatments used in our study.

\subsection{Effects of B. coagulans, PSCF and Synbiotic Supplementation on Altered Fecal Metabolic Profile in} DSS-Induced Mice

Fecal metabolites were analysed using GC-MS to gain an overview of changes induced by B. coagulans, PSCF and synbiotic supplementation in DSS-treated mice. A total of 61 metabolites of different functional groups such as sugars, amino acids, volatile fatty acids, and biogenic amines were detected. A supervised partial least squares-discriminant analysis (PLS-DA) was performed to evaluate metabolic phenotyping of each experimental group as shown in Figure 6A. The samples from HC and DSS-control clusters were clearly divergent indicating marked distinction in metabolic patterns between the two groups. While, clusters of supplemented mice samples overlapped with that of HC and DSS-control, clear demarcation of the synbiotic and B. coagulans cluster was noted, with PSCF showing only partial divergence relative to that of DSS-control. This indicates substantial efficacy of B. coagulans to metabolise PSCF that, in turn, induced significant biochemical changes potentially owing to their synergistic effects as evidenced from synbiotic samples. Combination of PLS-DA $\left(R^{2} Y=0.810(p=0.01), Q^{2}=0.710\right)$, VIP scores (Figure $6 \mathrm{~B}$ ) and significance analysis for microarrays (SAM) enabled us to identify potential biomarkers. The results showed 61 metabolites with 40 statistically significant compounds contributing to the clustering, with their SAM scores fold changes and International Chemical Identifiers (InChI) and standard InChI hashes (InChIKey IDs) listed in Table S1. Key metabolic markers making a significant contribution were identified by VIP analysis as displayed in Figure 6B. Among these identified metabolites, noticeable differences between 
DSS-control, and HC samples were noted particularly for succinic acid, stearic acid, and glycerol. Synbiotic supplementation was beneficial in minimising the metabolite alterations induced by DSS (Figure 6B and Table S1).

A

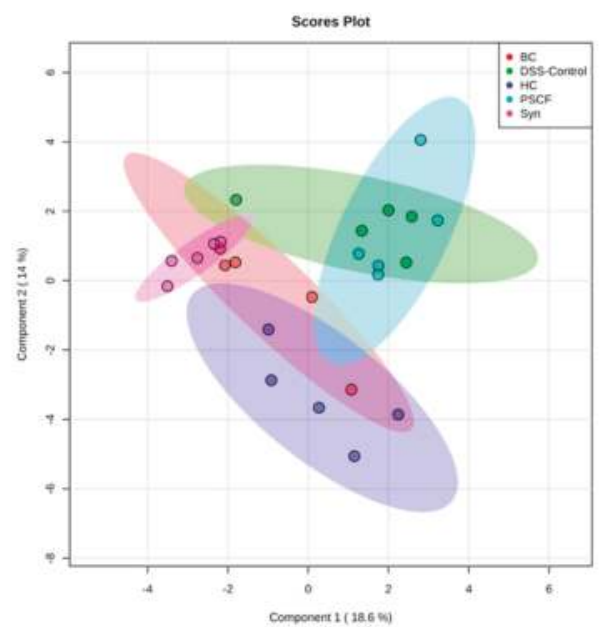

B

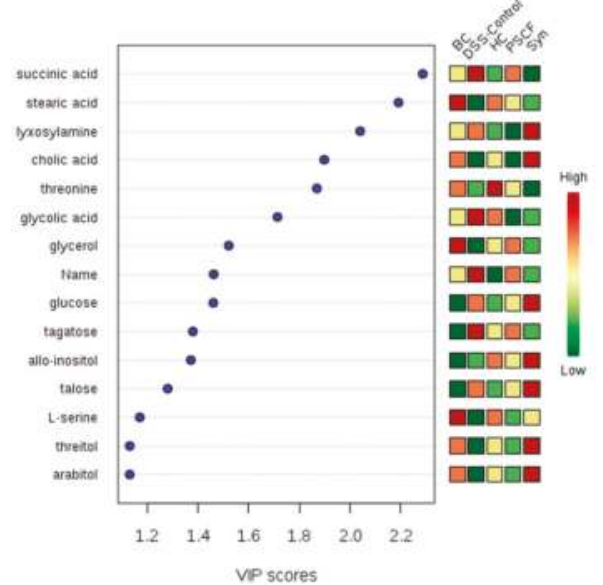

Figure 6. Effect of B. coagulans spores, PSCF and synbiotic on metabolic modulations in DSS-induced colitic mice. (A) 2D-PLS-DA plot showing spatial division among groups that received different supplementations, DSS-control mice that received no supplementation and HC. (B) Key compounds separating the groups based on variable importance projection (VIP) score plot in PLS-DA analysis. (BC-B. coagulans, Syn-synbiotic).

\subsection{Effects of B. Coagulans, PSCF and Synbiotic Supplementation SCFA Levels in DSS-Induced Mice}

As shown in Figure 7, supplementation of DSS-induced mice with B. coagulans, PSCF, and synbiotic treatments induced substantial modulations in the SCFA concentrations and their effects varied across cecal, luminal and fecal contents. Overall, the highest concentration of SCFA were noted in cecal contents compared to mucosal and fecal contents. There were no significant differences between the concentration of acetate and propionate in DSS-control and HC mice. PSCF however, induced a significant increase in the acetate levels in cecal and mucosal samples while, Synbiotic treatment was most significant in elevating acetate concentrations in mucosal-associated samples. All three supplementations were significantly effective in improving the propionate concentrations in cecal and mucosal contents while, only synbiotic supplementation increased propionate levels in fecal samples. 

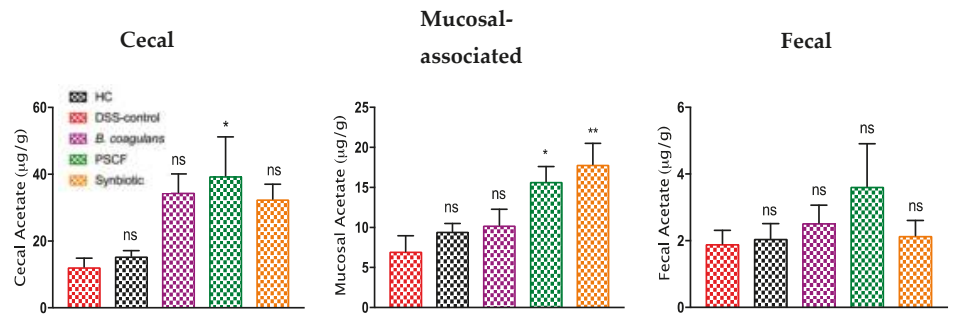

(a)

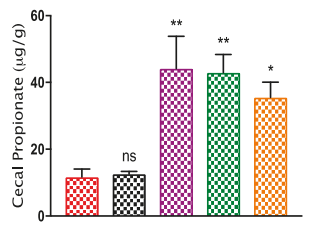

(d)

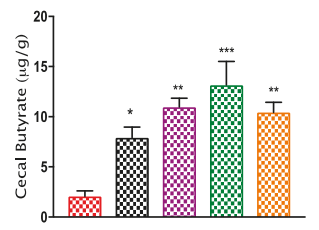

(g)

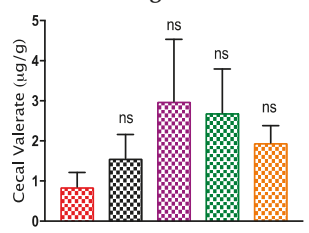

(j)

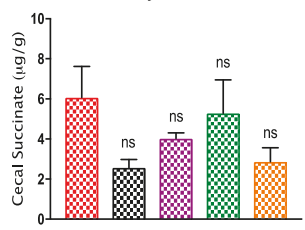

(m) (b)

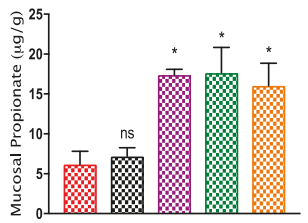

(e)

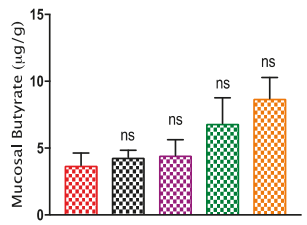

(h)

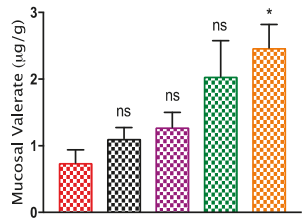

(k)

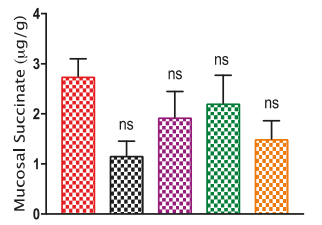

(n) (c)

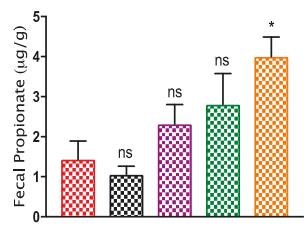

(f)

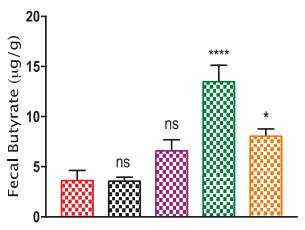

(i)

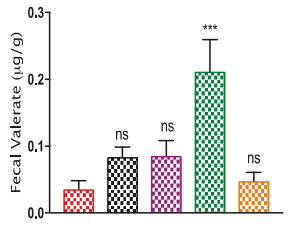

(1)

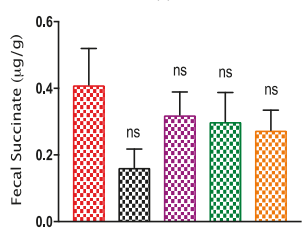

(o)

Figure 7. Effects of B. coagulans spores, PSCF and synbiotic in modulating SCFA concentrations in cecal, mucosal-associated and fecal contents in DSS-induced colitis. Cecal- acetate (a), proionate (d), butyrate $(\mathrm{g})$, valerate $(\mathbf{j})$, succinate $(\mathbf{m})$; mucosal-associated acetate $(\mathbf{b})$, proionate $(\mathbf{e})$, butyrate $(\mathbf{h})$, valerate $(\mathbf{k})$, succinate $(\mathbf{n})$ and fecal- acetate $(\mathbf{c})$, propionate $(\mathbf{f})$, butyrate $(\mathbf{i})$, valerate $(\mathbf{l})$, succinate (o). -Statistical significance among groups evaluated by one-way ANOVA followed by Tukey's test. ${ }^{*} p<0.05,{ }^{* *} p<0.01,{ }^{* * *} p<0.001,{ }^{* * * *} p<0.0001$ vs. DSS-colitic group and data expressed as mean \pm SEM ( $n=5$ per group). ns-non-significant 
Butyrate levels were significantly decreased by DSS administration $(2.05 \pm 0.6 \mu \mathrm{g} / \mathrm{g})$ compared with that of HC mice $(7.91 \pm 1.1 \mu \mathrm{g} / \mathrm{g})$ in the cecum. The decrease in butyrate induced by DSS was maintained at control levels with all three supplements in the cecum with PSCF $(13.2 \pm 2.4 \mu \mathrm{g} / \mathrm{g})$ being significantly more effective than $B$. coagulans $(11 \pm 0.9 \mu \mathrm{g} / \mathrm{g})$ and synbiotic $(10.4 \pm 1.0 \mu \mathrm{g} / \mathrm{g})$. Similarly, in fecal content, PSCF significantly improved butyrate $(13.6 \pm 1.6 \mu \mathrm{g} / \mathrm{g}$ compared with $3.72 \pm 0.1 \mu \mathrm{g} / \mathrm{g}$ for DSS-colitic followed by synbiotic $(8.15 \pm 0.6 \mu \mathrm{g} / \mathrm{g})$ while B. coagulans $(6.69 \pm 0.1$ $\mu \mathrm{g} / \mathrm{g}$ ) had no effect. Synbiotic supplementation resulted in marked increase in mucosal-assocaited valerate while, PSCF caused elevation in its concentration in fecal content. Similarly, synbiotic was more efficient in reducing elevated succinate levels.

\section{Discussion}

Dietary strategies involving probiotic and prebiotic fibre components that function by modulating immune responses, colonic epithelial integrity and microbial composition and related metabolites are being widely investigated for the prevention or reduction of severity of IBD [40,41]. The present study clearly supported the premise that conditioning of the gut with synbiotic supplementation containing compatible probiotic and prebiotic fibre can be greatly beneficial to reducing the symptoms and severity of DSS-induced acute colitis in mice. The observations confirmed substantial anti-inflammatory efficacy by synbiotic supplementation containing B. coagulans and PSCF. This was evidenced by the improvement of clinical symptoms, macroscopic, histological, biochemical, metabolic and immune parameters in the DSS-induced colitic mice model.

The addition of $2 \%(w / v)$ DSS to drinking water for seven days without ameliorating treatments resulted in a progressive rise in DAI (Figure 1A), owing to both body weight loss (Figure 1B) and excretion of diarrheic/bleeding feces. However, the supplementation of DSS-induced mice with B. coagulans, PSCF, and their synbiotic combination significantly attenuated the severity of the DSS damage and improved DAI and macroscopic markers of inflammation (Figure 1C-F). The ability of PSCF to show early effect on DAI and body weight could be related to its high content of insoluble hemicellulose. This fraction has a large water-holding capacity thus, could appropriately contribute to regulating the fecal water content in colitic mice [42,43]. The anti-diarrheal effect of B. coagulans have been previously confirmed [44]. The increased beneficial effects of synbiotic supplementation in reducing the disease severity could be related to the synergistic actions between the probiotic and prebiotic components.

A potentiated synbiotic effect relative to that of B. coagulans and PSCF individually was also evident from the histology of the colon compared with DSS-control mice (Figure 2A,B). Synbiotic supplementation showed substantial protection to the colonic epithelial architecture by mitigating crypt disruption, loss of goblet cells, submucosal edema and epithelial structure damage induced by DSS. Synbiotic supplementation also induced suppression of infiltration of activated neutrophils as evidenced by significant reduction in MPO activity in DSS-induced mice (Figure 2C). The infiltration of activated neutrophils is one of the most prominent histological features observed in IBD and is directly proportional to the MPO activity. Superoxide anions and other reactive species produced by neutrophils leads to tissue necrosis and mucosal dysfunction in IBD [39]. The reduction in MPO activity suggests that synbiotic supplementation imparted an anti-inflammatory effect, in addition to histological protection.

Disruption of intestinal epithelial TJs and impaired epithelial barrier function is a central event in the pathogenesis of IBD and may lead to persistent aberrant immune reactions, thus accelerating gut inflammation and the inflammatory circuit [45]. Synbiotic treatment in our study was the most effective followed by B. coagulans and PSCF in protecting the expression level of TJ proteins (ZO-1, occludin and claudin-1) in DSS-induced mice (Figure 4). TJs create a semi-permeable barrier against paracellular penetration of harmful substances from the gut lumen [45]. Bacillus subtilis intake has been recently confirmed to upregulate the expressions of TJ proteins for improved barrier function in colitic mice [46] and corroborates with our results. Moreover, synbiotic and B. coagulans protected 
the goblet cells and mucin production more effectively than PSCF alone (Figure 3). This indicates the possible ability of these $B$. coagulans MTCC 5856 spores to benefit goblet cell structure and function and needs further investigation to determine the mechanism of this effect. Some Bacillus species have been shown to upregulate mucin glycoproteins and protect colonic mucus layer integrity and goblet cell function [47]. Although we could not confirm if synbiotic supplementation had a stimulating effect on TJ proteins and/or localisation or, instead if it avoided TJ degradation by DSS, we observed that synbiotic supplementation significantly maintained the TJ patterns similar to that of animals in HC. This is indicative that synbiotic supplementation significantly preserved the integrity of the epithelium. The synergy between B. coagulans and PSCF could have imparted excellent protection and/or maintenance of epithelial integrity on DSS-induced mice, thus supporting its application in IBD to reinforce intestinal barrier integrity.

Alterations in the barrier integrity in IBD also leads to aberrant immune responses resulting in an inflammation cascade and tissue damage [48]. Although B. coagulans and PSCF supplementations alone were able to modulate the tested cytokines (IL- $1 \alpha$, IL-1 $\beta$, IL- 6 , IL-12, TNF- $\alpha$, and IFN- $\gamma$ ), a more profound anti-inflammatory effect was observed with synbiotic supplementation in both the colon and serum (Figure 5A-I). A spike in the levels of IL-1 $\beta$, IL-6, and TNF- $\alpha$ have been implicated in human IBD pathogenesis [49]. Such pro-inflammatory cytokines are secreted at high levels by activated lamina propria antigen presenting cells in response to inflammation. Pro-inflammatory cytokines such as IL-6, TNF- $\alpha$, and IL-1 $\beta$ are being targeted by therapeutic approaches to curb the aberrant inflammatory response in IBD due to their roles in the pathogenesis of the disease [50-52]. TNF- $\alpha$ is reported to exert its pro-inflammatory effect through elevated production of IL-6 and IL-1 $\beta$ [53]. This is in line with the present study, where DSS-induction caused elevated secretions of these cytokines in DSS-control group relative to that of HC (Figure 5). Upregulation of pro-inflammatory cytokine levels has also been reported to upregulate iNOS expression and secretion of nitric oxide that causes tissue damage in IBD [54]. The anti-inflammatory effects of synbiotic, B. coagulans and PSCF in suppressing the levels of these pro-inflammatory cytokines as well as reducing iNOS activity in colonic tissues, indicates their immunomodulatory potentials. B. coagulans MTCC 5856 spores in our previous study showed excellent immunomodulatory effect in vitro [22]. Marked reduction in pro-inflammatory cytokines in the colon of DSS-induced mice in the current study further supports its excellent immunomodulatory efficacy in IBD application.

Synbiotic supplementation was the most effective in demonstrating noticeable anti-inflammatory effect by significantly reducing the serum levels of pro-inflammatory IL- $1 \beta$ and IL-12 while concurrently elevating anti-inflammatory IL-10 in the serum. IL-10 plays a prominent role in counterbalancing Th1 and Th17 immune activity in IBD towards a Th2 response by downregulating antigen presentation and subsequent release of proinflammatory cytokines thereby attenuating mucosal inflammation [53]. IL-10 has been reported to play a role in maintaining intestinal barrier integrity possibly owing to effects on zonulin pathway [55]. The ability of $B$. coagulans spores to elicit IL-10 levels in inflammatory condition has been determined in vitro and in human subjects. Thus, the anti-inflammatory efficacy of synbiotic supplementation could be related to major immune-regulating capability of B. coagulans MTCC 5856 spores, thereby supporting its application in synbiotic therapies for IBD. Furthermore, synbiotic treatment also reduced the increased CRP levels in the serum of DSS-induced mice. In the inflammatory state, circulating IL-6 promotes CRP production in the liver and its release into the bloodstream [56]. Elevated levels of CRP has been implicated in human IBD patients [57]. The reduction in overall pro-inflammatory mediators by synbiotic supplementation may be due to either direct immune-regulating effect of the B. coagulans and PSCF on cytokine secretion or it could be owing to their indirect effect on the protection of intestinal barrier integrity. In either case this leads to reduction in luminal antigens and full activation of the innate immune system.

DNA extracted from cecal, fecal and mucosal-associated contents from DSS-induced mice in the present study, failed amplification before 16s rRNA sequencing owing to the presence of DSS 
in the samples that inhibited the PCR amplification as known earlier [58]. The microbiota profiling of DSS samples could therefore not be performed and is the limitation of the study. The levels of microbiota-derived untargeted metabolites and SCFAs were therefore alternatively analysed as signatures of the gut microbiota that contribute to modulating the immune activity of the intestinal mucosa $[59,60]$. The untreated DSS-control mice exhibited distinct fecal metabolic phenotype relative to that of HC. This was reflective of clinical and animal IBD studies that confirmed significant differences in metabolic profiles between healthy and IBD patients [61-63]. The metabolic analysis of supplemented mice in our study resulted in considerable normalization of metabolic profile indicating the positive effects of synergistic combination of B. coagulans and PSCF that induced marked improvement in metabolic pattern. Notably for mice supplemented with PSCF there was not much distinction from the DSS-control group was observed but the synbiotic combination with B. coagulans resulted in an improved metabolic pattern. This marked synergism could be associated with the acceleration of fermentation of insoluble plant cellular materials, such as hemicellulose in PSCF, by the supplemented B. coagulans [18]. The resulting higher levels of fermentation metabolites would thus, in turn, influence other beneficial microbial metabolic activities. This further supports the application of compatible synbiotic components to generate maximum benefits through increased SCFA production.

Increased levels of microbiota-derived SCFAs are inversely associated with dysbiosis in IBD [64]. Of particular interest are higher levels of acetate, propionate and butyrate, which results from fermentation of indigestible carbohydrates from fibre-rich diets. The effects of SCFAs have been studied to in animal models of colitis [42] and clinical UC [65]. Each type of SCFA is likely to contribute to host health [66]. In this study we determined the SCFA profile along the gastro-intestinal tract, analyzing samples across cecal, mucosal-associated, and fecal contents. The concentration of SCFAs varied along the length of the gut, with most abundant levels in the cecum and PC, while it declined towards the DC [66]. In line with a recent study [67], cecum showed the highest levels of all the SCFAs tested in our study irrespective of the supplementation. Cecum is considered the major site for fermentation in the rodent gut and contains the largest pool of microbiota. It therefore generates the most SCFAs. However, some recent studies have also reported changes in the microbiota and associated amounts of metabolites in along different regions of gastro-intestinal (GI) tract [67-69]. Therefore, the overall GI profile is of importance when associating the gut microbiome and metabolites with health outcomes.

All three supplementations caused a substantial increase in concentrations of measured SCFAs compares with that of DSS-control group. However, the potentiated effect of synbiotic supplementation is evidenced by induction of SCFA production along the entire length of the colon compared to B. coagulans supplementation alone where the additional SCFA production capacity was absent past the cecum. This effect highlights the advantage of using a compatible prebiotic fibre as synbiotic companion with a particular probiotic that can metabolize it. The high total dietary fibre content of PSCF (87\%) [17] would contribute to this effect. PSCF supplementation resulted in increased butyrate levels line with an in-vitro study with human gut microbiota utilizing sugar cane fibre [17]. The effect of additional SCFA production in cecum by B. coagulans would not extend to the proximal or distal parts of the colon. In the current study, the elicited extra SCFAs with synbiotic supplementation from cecum to the fecal pellets indicated the ability of B. coagulans to utilize the PSCF to also generate SCFAs after the cecum. The ability of B. coagulans to metabolize a variety of plant fibers for fermentation, including cranberry fibre [70] and fenugreek seeds [71], to produce SCFAs and hemicellulose [18] for lactic acid production has been previously determined.

PSCF supplementation also resulted in increased butyrate levels, correlating with results of an in-vitro study with human gut microbiota utilizing sugar cane fibre [17]. This ability of synbiotic supplementation for eliciting butyrate levels along the entire length of colon could contribute to the beneficial effect observed in the current study. Butyrate is the preferred energy source for colonocytes and has the ability to regulate cytokines thus showing protection against inflammation in UC and 
colorectal cancer [66]. Butyrate has been demonstrated in in vitro [72,73] and in vivo [74] studies to increase epithelial integrity and mucus secretion, consistent with the immunohistological and mucus staining analysis in the present study. The substantial increase in butyrate levels in the cecum by $B$. coagulans supplementation may be due to ability to support the growth and activity of butyrate producers probably via cross-feeding of the lactic acid production. B. coagulans are known to be efficient at producing lactic acid through fermentation of various plant substrates, including hemicellulose $[18,19]$. Lactic acid is reported to be utilized by strictly anaerobic butyrate-producing bacteria of clostridial clusters XIVa for the production of high concentrations of butyric acid [75]. Thus, the synbiotic approach with probiotic bacteria and prebiotic fibre that directly or indirectly influence butyrate production may help to restore intestinal barrier integrity in diseased state. This effect was evidenced by the significant reduction in DSS-induced colonic epithelial damage (Figure 2) by synbiotic supplementation in our study.

Acetate and propionate have also been studied to benefit epithelial integrity via binding with certain metabolite-sensing G-protein-coupled receptors (such as GPR43, GPR109A) and modulating immune response [76-78]. Valerate that has been determined to stimulate intestinal growth and attenuate inflammatory pathogenesis in colitis and cancer to metabolic disorders [79] was increased by synbiotic supplementation. Besides its positive effect in colon, SCFAs have also been exhibited to mediate improved host metabolism and modulate the activity of the enteric nervous system [66], thus providing benefits beyond GI tract. The high levels of immuno-modulatory effects observed in the present study could also possibly be correlated to high SCFA levels induced by synbiotic supplementation owing to the synergistic combination. SCFAs by engaging with engage with GPRs are known to induce immune-modulation leading to a direct local and systemic anti-inflammatory effects $[76,80]$. This further supports the application of synergistic synbiotic combinations to achieve maximum benefits in resolving the inflammatory circuit in IBD.

\section{Conclusions}

This is a detailed study highlighting the site-specific inflammatory and SCFA changes in a mice model of IBD as a result of synbiotic supplementation of the normal diet with prebiotic whole plant fibre and probiotic spores. The synbiotic pre-supplementation resulted in a substantial anti-inflammatory effect, reducing disease severity, colonic damage, and inflammatory mediators while modulating the metabolite and SCFA profiles of DSS-induced gut damage. The research has clearly demonstrated that the supplementation of whole plant PSCF and B. coagulans spores produced a synergistic combination that protected mice against acute damage induced by DSS in mice. The results underscore the significant efficacy of synbiotic applications to increase the beneficial and preventive effects on the host by targeting different mechanistic approaches to resolve inflammation cycle. However, the differences in the evolved biology of humans compared to mice requires caution in translation of the results to impacts on human disease [81]. While mice models do allow the changes in gut microbiota, as a result of pre- and probiotic combinations to be studied in a controlled experiment direct human trials will be needed. The delineation of the synergistic biological actions of probiotic $B$. coagulans spores and prebiotic PSCF in mouse model of IBD provides support for investigating their therapeutic and preventive effects in human IBD. However, the ability to reduce the severity of DSS-induced colitis was demonstrated using pre-supplementation. Human trials should be aimed at proactive prevention or after partial control of inflammatory disease, such as in association with drug treatment.

Patents: The information from this study has been filed as a provisional patent application in Australia titled "Preparation for the Treatment of Inflammatory Bowel Disease using a Whole Plant Fibre Extract from Sugar Cane" with application number 2018902145 and a filing date of 15 June 2018. Information relating to novelty of synergy between probiotic Bacillus coagulans and prebiotic whole plant sugar cane fibre in imparting health benefits is the subject of the patented claim.

Supplementary Materials: The following are available online at http:/ / www.mdpi.com/2072-6643/11/4/818/s1, Figure S1: Effect of B. coagulans spores, PSCF and synbiotic on immune markers in colon tissues and blood serum, 
Table S1: Most significant compounds identified by OPLS-DA and SAM analysis in HC, DSS-control, B. coagulans (BC), PSCF and synbiotic groups.

Author Contributions: Conceptualization: T.S., R.E., and R.S.; data curation: T.S.; formal analysis: T.S., A.P.P., R.V., S.S., and B.S.; funding acquisition: R.E. and R.S.; investigation: T.S.; methodology: T.S., S.V.G., A.V.K., and D.J.B.; resources: S.V.G., A.V.K., D.J.B., R.E., and R.S.; supervision: R.E. and R.S.; validation: R.S.; visualization: T.S.; writing—original draft: T.S.; writing-review and editing: T.S., S.V.G., A.V.K., D.J.B., R.E., and R.S.

Funding: This work was supported by industry grant from KFSU Ltd. (RT111031) allocated to R.S. and R.E.

Acknowledgments: The support from Tasmanian Government for Tasmania Graduate Research Scholarship for the PhD study of T.S. is gratefully acknowledged.

Conflicts of Interest: The authors declare no conflict of interest.

\section{References}

1. Vindigni, S.M.; Zisman, T.L.; Suskind, D.L.; Damman, C.J. The intestinal microbiome, barrier function, and immune system in inflammatory bowel disease: A tripartite pathophysiological circuit with implications for new therapeutic directions. Ther. Adv. Gastroenterol. 2016, 9, 606-625. [CrossRef]

2. Ng, S.C.; Shi, H.Y.; Hamidi, N.; Underwood, F.E.; Tang, W.; Benchimol, E.I.; Panaccione, R.; Ghosh, S.; Wu, J.C.; Chan, F.K. Worldwide incidence and prevalence of inflammatory bowel disease in the 21st century: A systematic review of population-based studies. Lancet 2017, 390, 2769-2778. [CrossRef]

3. Hou, J.K.; Lee, D.; Lewis, J. Diet and inflammatory bowel disease: Review of patient-targeted recommendations. Clin. Gastroenterol. Hepatol. 2014, 12, 1592-1600. [CrossRef]

4. Palumbo, V.; Romeo, M.; Marino Gammazza, A.; Carini, F.; Damiani, P.; Damiano, G.; Buscemi, S.; Lo Monte, A.; Gerges Geagea, A.; Jurjus, A. The long-term effects of probiotics in the therapy of ulcerative colitis: A clinical study. Biomed. Pap. 2016, 160, 372-377. [CrossRef]

5. Abruzzo, A.; Damiano, G.; Altomare, R.; Palumbo, V.; Tomasello, G.; Buscemi, S.; Lo Monte, G.; Maione, C.; Buscemi, G.; Lo Monte, A. The influence of some dietary components on intestinal microbiota. Prog. Nutr. 2016, 18, 205-212.

6. Steed, H.; Macfarlane, G.T.; Blackett, K.L.; Bahrami, B.; Reynolds, N.; Walsh, S.V.; Cummings, J.H.; Macfarlane, S. Clinical trial: The microbiological and immunological effects of synbiotic consumption-A randomized double-blind placebo-controlled study in active crohn's disease. Aliment. Pharmacol. Ther. 2010, 32, 872-883. [CrossRef]

7. Wasilewski, A.; Zielińska, M.; Storr, M.; Fichna, J. Beneficial effects of probiotics, prebiotics, synbiotics, and psychobiotics in inflammatory bowel disease. Inflamm. Bowel Dis. 2015, 21, 1674-1682. [CrossRef]

8. Moroeanu, V.I.; Vamanu, E.; Paun, G.; Neagu, E.; Ungureanu, O.R.; Eremia, S.A.; Radu, G.-L.; Ionescu, R.; Pelinescu, D.R. Probiotic strains influence on infant microbiota in the in vitro colonic fermentation model gis1. Indian J. Microbiol. 2015, 55, 423-429. [CrossRef] [PubMed]

9. Wong, C.; Harris, P.J.; Ferguson, L.R. Potential benefits of dietary fibre intervention in inflammatory bowel disease. Int. J. Mol. Sci. 2016, 17, 919. [CrossRef]

10. Pituch-Zdanowska, A.; Banaszkiewicz, A.; Albrecht, P. The role of dietary fibre in inflammatory bowel disease. Prz. Gastroenterol. 2015, 10, 135. [CrossRef]

11. Galvez, J.; Rodríguez-Cabezas, M.E.; Zarzuelo, A. Effects of dietary fiber on inflammatory bowel disease. Mol. Nutr. Food Res. 2005, 49, 601-608. [CrossRef]

12. Williams, B.A.; Grant, L.J.; Gidley, M.J.; Mikkelsen, D. Gut fermentation of dietary fibres: Physico-chemistry of plant cell walls and implications for health. Int. J. Mol. Sci. 2017, 18, 2203. [CrossRef]

13. Tuohy, K.M.; Conterno, L.; Gasperotti, M.; Viola, R. Up-regulating the human intestinal microbiome using whole plant foods, polyphenols, and/or fiber. J. Agric. Food Chem. 2012, 60, 8776-8782. [CrossRef]

14. Liu, R.H. Health benefits of fruit and vegetables are from additive and synergistic combinations of phytochemicals. Am. J. Clin. Nutr. 2003, 78, 517S-520S. [CrossRef]

15. Edwards, G.; Ball, M. Dietary Supplement for the Treatment of Acid Reflux and Gastro-Oesophageal Reflux Disease (Gord/Gerd). U.S. Patent US20160287657A, 2016.

16. Ball, M.; Edwards, G. Use of Dietary Fibre Supplement in a Food Formulation. U.S. Patent WO2014162303A1, 2016. 
17. Gamage, H.K.; Tetu, S.G.; Chong, R.W.; Bucio-Noble, D.; Rosewarne, C.P.; Kautto, L.; Ball, M.S.; Molloy, M.; Packer, N.H.; Paulsen, I.T. Fibre supplements derived from sugarcane stem, wheat dextrin and psyllium husk have different in vitro effects on the human gut microbiota. Front. Microbiol. 2018, 9, 1618. [CrossRef]

18. Walton, S.L.; Bischoff, K.M.; van Heiningen, A.R.; van Walsum, G.P. Production of lactic acid from hemicellulose extracts by bacillus coagulans mxl-9. J. Ind. Microbiol. Biotechnol. 2010, 37, 823-830. [CrossRef]

19. Konuray, G.; Erginkaya, Z. Potential use of bacillus coagulans in the food industry. Foods 2018, 7, 92. [CrossRef]

20. Slavin, J.L.; Brauer, P.M.; Marlett, J.A. Neutral detergent fiber, hemicellulose and cellulose digestibility in human subjects. J. Nutr. 1981, 111, 287-297. [CrossRef]

21. Leschine, S.B. Cellulose degradation in anaerobic environments. Annu. Rev. Microbiol. 1995, 49, $399-426$. [CrossRef]

22. Shinde, T.; Vemuri, R.; Shastri, M.D.; Perera, A.P.; Tristram, S.; Stanley, R.; Eri, R. Probiotic bacillus coagulans mtcc 5856 spores exhibit excellent in-vitro functional efficacy in simulated gastric survival, mucosal adhesion and immunomodulation. J. Funct. Foods 2019, 52, 100-108. [CrossRef]

23. Baron, M. Original research: A patented strain of bacillus coagulans increased immune response to viral challenge. Postgrad. Med. 2009, 121, 114-118. [CrossRef]

24. Chassaing, B.; Aitken, J.D.; Malleshappa, M.; Vijay-Kumar, M. Dextran sulfate sodium (dss)-induced colitis in mice. Curr. Protoc. Immunol. 2014, 104, 15.25. 1-15.25. 14.

25. Murthy, S.; Cooper, H.S.; Shim, H.; Shah, R.S.; Ibrahim, S.A.; Sedergran, D.J. Treatment of dextran sulfate sodium-induced murine colitis by intracolonic cyclosporin. Dig. Dis. Sci. 1993, 38, 1722-1734. [CrossRef]

26. Perera, A.P.; Fernando, R.; Shinde, T.; Gundamaraju, R.; Southam, B.; Sohal, S.S.; Robertson, A.A.; Schroder, K.; Kunde, D.; Eri, R. Mcc950, a specific small molecule inhibitor of nlrp3 inflammasome attenuates colonic inflammation in spontaneous colitis mice. Sci. Rep. 2018, 8, 8618. [CrossRef]

27. Demon, D.; Kuchmiy, A.; Fossoul, A.; Zhu, Q.; Kanneganti, T.-D.; Lamkanfi, M. Caspase-11 is expressed in the colonic mucosa and protects against dextran sodium sulfate-induced colitis. Mucosal Immunol. 2014, 7, 1480. [CrossRef]

28. Eri, R.; McGuckin, M.A.; Wadley, R. T cell transfer model of colitis: A great tool to assess the contribution of $\mathrm{t}$ cells in chronic intestinal inflammation. In Leucocytes; Springer: Berlin/Heidelberg, Germany, 2012; pp. 261-275.

29. Koelink, P.J.; Wildenberg, M.E.; Stitt, L.W.; Feagan, B.G.; Koldijk, M.; van't Wout, A.B.; Atreya, R.; Vieth, M.; Brandse, J.F.; Duijst, S. Development of reliable, valid and responsive scoring systems for endoscopy and histology in animal models for inflammatory bowel disease. J. Crohn's Colitis 2018, 1, 10. [CrossRef]

30. Sovran, B.; Lu, P.; Loonen, L.M.; Hugenholtz, F.; Belzer, C.; Stolte, E.H.; Boekschoten, M.V.; Van Baarlen, P.; Smidt, H.; Kleerebezem, M. Identification of commensal species positively correlated with early stress responses to a compromised mucus barrier. Inflamm. Bowel Dis. 2016, 22, 826-840. [CrossRef]

31. Li, P.; Zhang, R.; Wang, L.; Gan, Y.; Xu, Y.; Song, L.; Luo, L.; Zhao, C.; Zhang, C.; Ouyang, B. Long-term load duration induces $n$-cadherin down-regulation and loss of cell phenotype of nucleus pulposus cells in a disc bioreactor culture. Biosci. Rep. 2017, 37, BSR20160582. [CrossRef]

32. Lean, Q.Y.; Eri, R.D.; Randall-Demllo, S.; Sohal, S.S.; Stewart, N.; Peterson, G.M.; Gueven, N.; Patel, R.P. Orally administered enoxaparin ameliorates acute colitis by reducing macrophage-associated inflammatory responses. PLoS ONE 2015, 10, e0134259. [CrossRef] [PubMed]

33. Mei, Q.; Xu, J.; Xiang, L.; Hu, Y.; Hu, X.; Xu, Z. Change of nitric oxide in experimental colitis and its inhibition by melatonin in vivo and in vitro. Postgrad. Med. J. 2005, 81, 667-672. [CrossRef] [PubMed]

34. Furuhashi, T.; Sugitate, K.; Nakai, T.; Jikumaru, Y.; Ishihara, G. Rapid profiling method for mammalian feces short chain fatty acids by gc-ms. Anal. Biochem. 2018, 543, 51-54. [CrossRef] [PubMed]

35. Vemuri, R.; Shinde, T.; Gundamaraju, R.; Gondalia, S.; Karpe, A.; Beale, D.; Martoni, C.; Eri, R. Lactobacillus acidophilus dds- 1 modulates the gut microbiota and improves metabolic profiles in aging mice. Nutrients 2018, 10, 1255. [CrossRef]

36. Sansone, S.-A.; Fan, T.; Goodacre, R.; Griffin, J.L.; Hardy, N.W.; Kaddurah-Daouk, R.; Kristal, B.S.; Lindon, J.; Mendes, P.; Morrison, N. The metabolomics standards initiative. Nat. Biotechnol. 2007, 25, 846.

37. Smart, K.F.; Aggio, R.B.; Van Houtte, J.R.; Villas-Bôas, S.G. Analytical platform for metabolome analysis of microbial cells using methyl chloroformate derivatization followed by gas chromatography-mass spectrometry. Nat. Protoc. 2010, 5, 1709. [CrossRef] 
38. Srutkova, D.; Schwarzer, M.; Hudcovic, T.; Zakostelska, Z.; Drab, V.; Spanova, A.; Rittich, B.; Kozakova, H.; Schabussova, I. Bifidobacterium longum ccm 7952 promotes epithelial barrier function and prevents acute dss-induced colitis in strictly strain-specific manner. PLoS ONE 2015, 10, e0134050. [CrossRef]

39. Han, F.; Fan, H.; Yao, M.; Yang, S.; Han, J. Oral administration of yeast $\beta$-glucan ameliorates inflammation and intestinal barrier in dextran sodium sulfate-induced acute colitis. J. Funct. Foods 2017, 35, 115-126. [CrossRef]

40. Al Mijan, M.; Lim, B.O. Diets, functional foods, and nutraceuticals as alternative therapies for inflammatory bowel disease: Present status and future trends. World J. Gastroenterol. 2018, 24, 2673. [CrossRef]

41. Ferguson, L.R.; Shelling, A.N.; Browning, B.L.; Huebner, C.; Petermann, I. Genes, diet and inflammatory bowel disease. Mutat. Res. /Fundam. Mol. Mech. Mutagenesis 2007, 622, 70-83. [CrossRef]

42. Kanauchi, O.; Serizawa, I.; Araki, Y.; Suzuki, A.; Andoh, A.; Fujiyama, Y.; Mitsuyama, K.; Takaki, K.; Toyonaga, A.; Sata, M. Germinated barley foodstuff, a prebiotic product, ameliorates inflammation of colitis through modulation of the enteric environment. J. Gastroenterol. 2003, 38, 134-141. [CrossRef]

43. Mitsuyama, K.; Saiki, T.; Kanauchi, O.; Iwanaga, T.; Tomiyasu, N.; Nishiyama, T.; Tateishi, H.; Shirachi, A.; Ide, M.; Suzuki, A. Treatment of ulcerative colitis with germinated barley foodstuff feeding: A pilot study. Aliment. Pharmacol. Ther. 1998, 12, 1225-1230. [CrossRef]

44. Majeed, M.; Nagabhushanam, K.; Natarajan, S.; Sivakumar, A.; Ali, F.; Pande, A.; Majeed, S.; Karri, S.K. Bacillus coagulans mtcc 5856 supplementation in the management of diarrhea predominant irritable bowel syndrome: A double blind randomized placebo controlled pilot clinical study. Nutr. J. 2016, 15, 21. [CrossRef]

45. Turner, J.R. Intestinal mucosal barrier function in health and disease. Nat. Rev. Immunol. 2009, 9, 799. [CrossRef]

46. Gong, Y.; Li, H.; Li, Y. Effects of bacillus subtilis on epithelial tight junctions of mice with inflammatory bowel disease. J. Interferon Cytokine Res. 2016, 36, 75-85. [CrossRef]

47. Zhang, W.; Zhu, Y.-H.; Zhou, D.; Wu, Q.; Song, D.; Dicksved, J.; Wang, J.-F. Oral administration of a select mixture of bacillus probiotics affects the gut microbiota and goblet cell function following escherichia coli challenge in newly weaned pigs of genotype muc4 that are supposed to be enterotoxigenic e. Coli f4ab/ac receptor negative. Appl. Environ. Microbiol. 2017, 83, e02747-16. [CrossRef]

48. Xavier, R.; Podolsky, D. Unravelling the pathogenesis of inflammatory bowel disease. Nature 2007, $448,427$. [CrossRef]

49. Neurath, M.F. Cytokines in inflammatory bowel disease. Nat. Rev. Immunol. 2014, 14, 329. [CrossRef]

50. Hyams, J.S.; Lerer, T.; Griffiths, A.; Pfefferkorn, M.; Kugathasan, S.; Evans, J.; Otley, A.; Carvalho, R.; Mack, D.; Bousvaros, A. Long-term outcome of maintenance infliximab therapy in children with crohn's disease. Inflamm. Bowel Dis. 2008, 15, 816-822. [CrossRef]

51. Atreya, R.; Mudter, J.; Finotto, S.; Müllberg, J.; Jostock, T.; Wirtz, S.; Schütz, M.; Bartsch, B.; Holtmann, M.; Becker, C. Blockade of interleukin 6 trans signaling suppresses t-cell resistance against apoptosis in chronic intestinal inflammation: Evidence in crohn disease and experimental colitis in vivo. Nat. Med. 2000, 6, 583. [CrossRef]

52. Jobin, C.; Sartor, B.R. Nf-kb signaling proteins as therapeutic targets for inflammatory bowel diseases. Inflamm. Bowel Dis. 2000, 6, 206-213. [CrossRef]

53. Neuman, M.G. Immune dysfunction in inflammatory bowel disease. Transl. Res. 2007, 149, $173-186$. [CrossRef]

54. Soufli, I.; Toumi, R.; Rafa, H.; Touil-Boukoffa, C. Cytokines and nitric oxide in immunopathogenesis of ibd and potential therapeutic approaches. In New Insights into Inflammatory BOWEL disease; InTech: Vienna, Austria, 2016.

55. Groschwitz, K.R.; Hogan, S.P. Intestinal barrier function: Molecular regulation and disease pathogenesis. J. Allergy Clin. Immunol. 2009, 124, 3-20. [CrossRef]

56. Del Giudice, M.; Gangestad, S.W. Rethinking il-6 and crp: Why they are more than inflammatory biomarkers, and why it matters. Brainbehav. Immun. 2018, 70, 61-75. [CrossRef]

57. Solem, C.A.; Loftus, E.V., Jr.; Tremaine, W.J.; Harmsen, W.S.; Zinsmeister, A.R.; Sandborn, W.J. Correlation of c-reactive protein with clinical, endoscopic, histologic, and radiographic activity in inflammatory bowel disease. Inflamm. Bowel Dis. 2005, 11, 707-712. [CrossRef] 
58. Kerr, T.; Ciorba, M.; Matsumoto, H.; Davis, V.; Luo, J.; Kennedy, S.; Xie, Y.; Shaker, A.; Dieckgraefe, B.; Davidson, N. Dextran sodium sulfate inhibition of real-time polymerase chain reaction amplification: A poly-a purification solution. Inflamm. Bowel Dis. 2011, 18, 344-348. [CrossRef]

59. Levy, M.; Thaiss, C.A.; Elinav, E. Metabolites: Messengers between the microbiota and the immune system. Genes Dev. 2016, 30, 1589-1597. [CrossRef]

60. Vernocchi, P.; Del Chierico, F.; Putignani, L. Gut microbiota profiling: Metabolomics based approach to unravel compounds affecting human health. Front. Microbiol. 2016, 7, 1144. [CrossRef]

61. Martin, F.-P.; Su, M.-M.; Xie, G.-X.; Guiraud, S.P.; Kussmann, M.; Godin, J.-P.; Jia, W.; Nydegger, A. Urinary metabolic insights into host-gut microbial interactions in healthy and ibd children. World J. Gastroenterol. 2017, 23, 3643. [CrossRef]

62. Marchesi, J.R.; Holmes, E.; Khan, F.; Kochhar, S.; Scanlan, P.; Shanahan, F.; Wilson, I.D.; Wang, Y. Rapid and noninvasive metabonomic characterization of inflammatory bowel disease. J. Proteome Res. 2007, 6, 546-551. [CrossRef]

63. Robinson, A.M.; Gondalia, S.V.; Karpe, A.V.; Eri, R.; Beale, D.J.; Morrison, P.D.; Palombo, E.A.; Nurgali, K Fecal microbiota and metabolome in a mouse model of spontaneous chronic colitis: Relevance to human inflammatory bowel disease. Inflamm. Bowel Dis. 2016, 22, 2767-2787. [CrossRef]

64. Sun, M.; Wu, W.; Liu, Z.; Cong, Y. Microbiota metabolite short chain fatty acids, gpcr, and inflammatory bowel diseases. J. Gastroenterol. 2017, 52, 1-8. [CrossRef]

65. Breuer, R.I.; Buto, S.K.; Christ, M.L.; Bean, J.; Vernia, P.; Paoluzi, P.; Di Paolo, M.; Caprilli, R. Rectal irrigation with short-chain fatty acids for distal ulcerative colitis. Dig. Dis. Sci. 1991, 36, 185-187. [CrossRef]

66. Koh, A.; De Vadder, F.; Kovatcheva-Datchary, P.; Bäckhed, F. From dietary fiber to host physiology: Short-chain fatty acids as key bacterial metabolites. Cell 2016, 165, 1332-1345. [CrossRef]

67. Kaur, A.; Tuncil, Y.E.; Sikaroodi, M.; Gillevet, P.; Patterson, J.A.; Keshavarzian, A.; Hamaker, B.R. Alterations in the amounts of microbial metabolites in different regions of the mouse large intestine using variably fermentable fibres. Bioact. Carbohydr. Diet. Fibre 2018, 13, 7-13. [CrossRef]

68. Pang, W.; Vogensen, F.K.; Nielsen, D.S.; Hansen, A.K. Faecal and caecal microbiota profiles of mice do not cluster in the same way. Lab. Anim. 2012, 46, 231-236. [CrossRef]

69. Hirano, A.; Umeno, J.; Okamoto, Y.; Shibata, H.; Ogura, Y.; Moriyama, T.; Torisu, T.; Fujioka, S.; Fuyuno, Y.; Kawarabayasi, Y. Comparison of the microbial community structure between inflamed and non-inflamed sites in patients with ulcerative colitis. J. Gastroenterol. Hepatol. 2018, 33, 1590-1597. [CrossRef]

70. Majeed, M.; Nagabhushanam, K.; Arumugam, S.; Natarajan, S.; Majeed, S.; Pande, A.; Beede, K.; Ali, F. Cranberry seed fibre: A promising prebiotic fibre and its fermentation by the probiotic bacillus coagulans mtcc 5856. Int. J. Food Sci. Technol. 2018, 53, 1640-1647. [CrossRef]

71. Majeed, M.; Majeed, S.; Nagabhushanam, K.; Arumugam, S.; Natarajan, S.; Beede, K.; Ali, F. Galactomannan from trigonella foenum-graecum 1. Seed: Prebiotic application and its fermentation by the probiotic bacillus coagulans strain mtcc 5856. Food Sci. Nutr. 2018, 6, 666-673. [CrossRef]

72. Nielsen, D.S.G.; Jensen, B.B.; Theil, P.K.; Nielsen, T.S.; Knudsen, K.E.B.; Purup, S. Effect of butyrate and fermentation products on epithelial integrity in a mucus-secreting human colon cell line. J. Funct. Foods 2018, 40, 9-17. [CrossRef]

73. Zheng, L.; Kelly, C.J.; Battista, K.D.; Schaefer, R.; Lanis, J.M.; Alexeev, E.E.; Wang, R.X.; Onyiah, J.C.; Kominsky, D.J.; Colgan, S.P. Microbial-derived butyrate promotes epithelial barrier function through il-10 receptor-dependent repression of claudin-2. J. Immunol. 2017, 199, 2976-2984. [CrossRef]

74. Simeoli, R.; Mattace Raso, G.; Pirozzi, C.; Lama, A.; Santoro, A.; Russo, R.; Montero-Melendez, T.; Berni Canani, R.; Calignano, A.; Perretti, M. An orally administered butyrate-releasing derivative reduces neutrophil recruitment and inflammation in dextran sulphate sodium-induced murine colitis. Br. J. Pharmacol. 2017, 174, 1484-1496. [CrossRef]

75. Van Immerseel, F.; Ducatelle, R.; De Vos, M.; Boon, N.; Van De Wiele, T.; Verbeke, K.; Rutgeerts, P.; Sas, B.; Louis, P.; Flint, H.J. Butyric acid-producing anaerobic bacteria as a novel probiotic treatment approach for inflammatory bowel disease. J. Med. Microbiol. 2010, 59, 141-143. [CrossRef]

76. Tedelind, S.; Westberg, F.; Kjerrulf, M.; Vidal, A. Anti-inflammatory properties of the short-chain fatty acids acetate and propionate: A study with relevance to inflammatory bowel disease. World J. Gastroenterol. WJG 2007, 13, 2826. [CrossRef] 
77. Maslowski, K.M.; Vieira, A.T.; Ng, A.; Kranich, J.; Sierro, F.; Yu, D.; Schilter, H.C.; Rolph, M.S.; Mackay, F.; Artis, D. Regulation of inflammatory responses by gut microbiota and chemoattractant receptor gpr43. Nature 2009, 461, 1282. [CrossRef]

78. Macia, L.; Tan, J.; Vieira, A.T.; Leach, K.; Stanley, D.; Luong, S.; Maruya, M.; McKenzie, C.I.; Hijikata, A.; Wong, C. Metabolite-sensing receptors gpr43 and gpr109a facilitate dietary fibre-induced gut homeostasis through regulation of the inflammasome. Nat. Commun. 2015, 6, 6734. [CrossRef]

79. Yuille, S.; Reichardt, N.; Panda, S.; Dunbar, H.; Mulder, I.E. Human gut bacteria as potent class i histone deacetylase inhibitors in vitro through production of butyric acid and valeric acid. PLoS ONE 2018, 13, e0201073. [CrossRef]

80. Thorburn, A.N.; Macia, L.; Mackay, C.R. Diet, metabolites, and "western-lifestyle" inflammatory diseases. Immunity 2014, 40, 833-842. [CrossRef]

81. Nguyen, T.L.A.; Vieira-Silva, S.; Liston, A.; Raes, J. How informative is the mouse for human gut microbiota research? Dis. Models Mech. 2015, 8, 1-16. [CrossRef]

(C) 2019 by the authors. Licensee MDPI, Basel, Switzerland. This article is an open access article distributed under the terms and conditions of the Creative Commons Attribution (CC BY) license (http:/ / creativecommons.org/licenses/by/4.0/). 
Review

\title{
Dietary Factors in Sulfur Metabolism and Pathogenesis of Ulcerative Colitis
}

\author{
Levi M. Teigen ${ }^{1}$, Zhuo Geng ${ }^{1}$, Michael J. Sadowsky ${ }^{2}$, Byron P. Vaughn ${ }^{1}$, Matthew J. Hamilton ${ }^{2}$ \\ and Alexander Khoruts ${ }^{1,2, *}$ \\ 1 Division of Gastroenterology, Hepatology and Nutrition, Department of Medicine, University of Minnesota, \\ Minneapolis, MN 55455, USA; teige027@umn.edu (L.M.T.); geng0053@umn.edu (Z.G.); \\ bvaughn@umn.edu (B.P.V.) \\ 2 BioTechnology Insititute, Department of Soil, Water \& Climate, and Department of Plant \& Microbial \\ Biology, University of Minnesota, St. Paul, MN 55108, USA; sadowsky@umn.edu (M.J.S.); \\ hami0192@umn.edu (M.J.H.) \\ * Correspondence: khoru001@umn.edu; Tel.: +1-612-301-3981
}

Received: 24 March 2019; Accepted: 23 April 2019; Published: 25 April 2019

\begin{abstract}
The biogeography of inflammation in ulcerative colitis (UC) suggests a proximal to distal concentration gradient of a toxin. Hydrogen sulfide $\left(\mathrm{H}_{2} \mathrm{~S}\right)$ has long been considered one such toxin candidate, and dietary sulfur along with the abundance of sulfate reducing bacteria (SRB) were considered the primary determinants of $\mathrm{H}_{2} \mathrm{~S}$ production and clinical course of UC. The metabolic milieu in the lumen of the colon, however, is the result of a multitude of factors beyond dietary sulfur intake and SRB abundance. Here we present an updated formulation of the $\mathrm{H}_{2} \mathrm{~S}$ toxin hypothesis for UC pathogenesis, which strives to incorporate the interdependency of diet composition and the metabolic activity of the entire colon microbial community. Specifically, we suggest that the increasing severity of inflammation along the proximal-to-distal axis in UC is due to the dilution of beneficial factors, concentration of toxic factors, and changing detoxification capacity of the host, all of which are intimately linked to the nutrient flow from the diet.
\end{abstract}

Keywords: colon; high-sulfur foods; inflammation; metagenomics; microbiota; sulfur reducing

\section{Introduction}

Inflammatory bowel disease (IBD), which includes both Crohn's disease and ulcerative colitis (UC), is estimated to affect $\sim 3$ million individuals in the U.S. alone and continues to increase in incidence and prevalence worldwide [1]. Epidemiological evidence implicates industrialization, an increasingly western lifestyle, and associated changes in the microbiome with the development of IBD [2-4]. Diet is a major determinant of intestinal microbiota composition and activity $[4,5]$; therefore, it is a focus of intense interest for the mechanistic understanding of IBD pathogenesis.

The risk of IBD development increases in immigrant children from developing countries [6] and substantial changes in the intestinal microbiome, including loss of diversity and displacement of Prevotella by Bacteroides strains, which can be observed following immigration from developing countries to the United States [7]. These findings lend strong support to the notion that changes in the gut microbiota, driven in part by diet, contribute to the pathogenesis of UC. Although attempts to identify consistent, specific 'trigger' foods that predict the development of UC have been unsuccessful, there appears to be an overall trend for an association between an animal-based diet and UC development and activity, while a plant-based diet may be protective against UC [8]. Conceptually, the epidemiological data that demonstrates an increasing prevalence of IBD with westernization lend support to this notion as westernization generally results in a transition from a plant-based to an animal-based diet [9]. 
The unique distribution of gut inflammation seen with UC, typically greatest in the rectum and extending continuously towards the proximal colon, supports the notion that a directionally concentrated toxic substance(s) may be involved in its pathogenesis. While the nature of this compound remains unknown, several lines of evidence, both observational and mechanistic, implicate hydrogen sulfide $\left(\mathrm{H}_{2} \mathrm{~S}\right)$ as a possible candidate. The $\mathrm{H}_{2} \mathrm{~S}$ toxin hypothesis, however, is complicated by the fact that $\mathrm{H}_{2} \mathrm{~S}$ at low levels can be anti-inflammatory [10]. Consequently, the $\mathrm{H}_{2} \mathrm{~S}$ toxin hypothesis may be better viewed in a concentration dependent manner where each individual host likely has a varied tolerance to a specific concentration of $\mathrm{H}_{2} \mathrm{~S}$ above which intestinal damage occurs [11].

To date, attempts to examine the role of diet in the $\mathrm{H}_{2} \mathrm{~S}$ toxin hypothesis have focused primarily on the intake of sulfur-containing foods, without regard for the overall diet composition [12-15]. It is important to consider, however, that outside of a laboratory setting diets are far more complex than can be captured by the measurement of a single or a few nutrients. Therefore, rather than focus on the specific intake of a single dietary component (e.g., sulfur) in the pathogenesis of UC, an emphasis should be placed on overall diet patterns (e.g., animal- vs plant-based diet). This approach has been the focus of a number of recent reviews $[16,17]$, and preliminary data suggest that these types of compositional dietary changes may be beneficial in IBD [18].

The high protein content of animal-based diets provides a greater amount of sulfur that becomes available to distal gut microbiota [19]. Additionally, the higher fat content of a typical animal-based diet may lead to an increase in taurine conjugation to bile acids and a corresponding increase in the quantity of sulfur in the colonic lumen through an endogenous source [20]. Importantly, an animal-based diet pattern will also tend to be low in fiber, which may drive a metabolic shift of microbiota away from carbohydrate and towards protein fermentation and increased mucin degradation (Figure 1) [21,22].

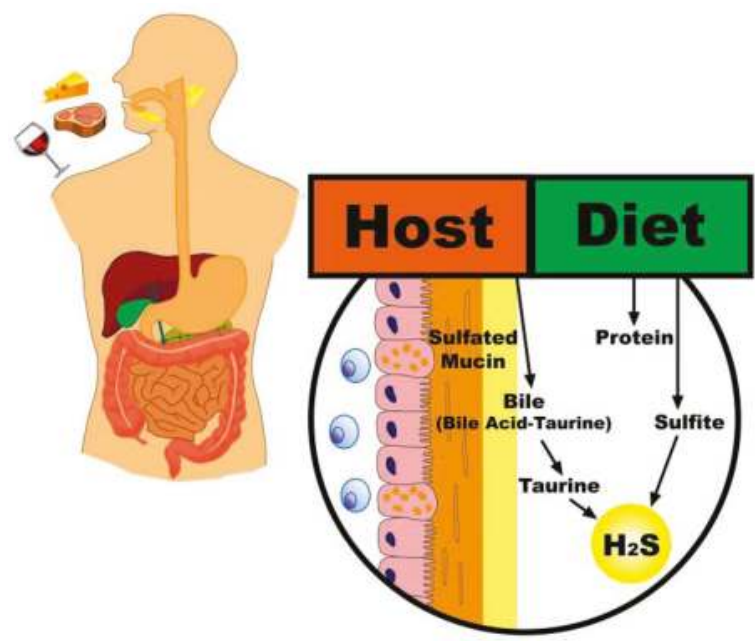

Figure 1. Contributions of an animal-based diet to hydrogen sulfide production. Legend: An animal-based diet results in a greater amount of dietary sulfur available to distal gut microbiota, both directly through sulfur-containing amino acids and indirectly through an increase in taurine conjugated bile acids and mucin degradation.

A complimentary hypothesis to explain the directional biogeography of UC may be the dilution of beneficial microbial metabolites along the luminal flow in the intestine. One such set of products is the short-chain fatty acids (SCFA), which are generated as a result of complex polysaccharide fermentation and play essential roles in colon physiology and mucosal homeostasis. Thus, butyrate (a SCFA), in addition to being a preferred energy source for the colonocytes [23,24], also promotes the presence of regulatory $\mathrm{T}$ cells in the colon [25,26], strengthens the gut barrier function [27], decreases 
epithelial oxygenation [28,29], and inhibits excessive colonic stem cell proliferation [30]. Furthermore, the chemical milieu established by microbial metabolites impact the overall microbial community structure through feedback loops affecting the relative abundances of different microbial trophic groups. Lower $\mathrm{pH}$, driven by higher concentrations of SCFA, favors the growth of methanogens over that of sulfate reducing bacteria (SRB) [31], whereas $\mathrm{H}_{2} \mathrm{~S}$ may increase the oxygenation in colonocytes by inhibiting $\beta$-oxidation [32] and lead to the inhibition of obligate anaerobes that produce SCFA.

Therefore, the updated understanding of the $\mathrm{H}_{2} \mathrm{~S}$ toxin hypothesis for UC pathogenesis presented here strives to account for the activity of the entire microbial community rather than individual microbial subgroups working in isolation (e.g., Desulfovibrio) and appreciates the interdependency of diet composition on both the structure and function of the microbial community. In many respects, this is a synecological approach to study of the relationship between gut microbiota and the development of UC.

\subsection{Toxic Effects of Hydrogen Sulfide}

Although $\mathrm{H}_{2} \mathrm{~S}$ is generated by the host and is increasingly recognized to have a multitude of important beneficial physiologic functions [33], it becomes a potent toxin once its concentration exceeds the detoxifying capacity in the tissue. Specifically, higher amounts of $\mathrm{H}_{2} \mathrm{~S}$ generated in the intestine have the potential to disrupt the gut barrier function, which may be an early and critical initiating event in triggering the onset of UC and the perpetuation of its activity [34]. Traditionally, the $\mathrm{H}_{2} \mathrm{~S}$ toxin hypothesis has focused on the potential injurious effects of sulfide gas on the cellular metabolism of colonocytes, mainly the inhibition of cytochrome $c$ oxidase activity in mitochondria, which induces oxidative stress in a fashion similar to cyanide [35]. Roediger and colleagues demonstrated that $\mathrm{H}_{2} \mathrm{~S}$ inhibits $\beta$-oxidation of butyrate by colonocytes, their preferred energy source [36]. Notably, UC mucosa has lower rates of butyrate uptake and oxidation relative to healthy controls [37]. In summary, oxidative stress and energy starvation caused by excessive $\mathrm{H}_{2} \mathrm{~S}$ concentrations may lead to colonocyte death, penetration of the epithelial barrier by the intestinal microbes and their direct interaction with the mucosal immune system. Resulting inflammation leads to further disruption of the gut barrier, decreased butyrate oxidation [37], and decreased mucosal sulfide detoxification and the subsequent perpetuation of inflammation [38].

In recent years, considerable attention has also been focused on mucus integrity in UC. The mucus layer of the colon consists of an outer (loose) and an inner (dense) layer, the latter being largely impenetrable to the resident intestinal microbiota $[39,40]$. The inner mucus layer in UC patients and in some animal models of IBD is more penetrable to bacteria relative to healthy controls [41]. Mucin glycoproteins form the major building blocks of mucus. The dominant mucin secreted by the goblet cells in the colon is MUC2, which contains cysteine-rich domains that mediate its multimerization through disulfide bonds. $\mathrm{H}_{2} \mathrm{~S}$ can directly break the sulfide bonds in the mucus and disrupt the MUC2 network leading to a loss of mucus viscosity and greater permeability [42].

\subsection{Pre-Clinical Models}

Some of the strongest support for the $\mathrm{H}_{2} \mathrm{~S}$ toxin hypothesis in the literature comes from pre-clinical models. The most commonly used animal model of IBD uses dextran sodium sulfate (DSS) to induce damage to the epithelium [43]. This allows for bacterial translocation from the lumen of the intestine resulting in inflammation [43]. This model is renowned for its simplicity and consistency in producing epithelial damage similar to that seen in IBD and also lends support to a role of sulfur in the pathogenesis of UC in humans.

In IL-10 knockout mice, a common murine model of IBD, a diet high in saturated fat increases the presence of taurine-conjugated bile acids, leading to an expansion of the sulphite-reducing pathobiont Bilophila wadsworthia and a greater severity of inflammation [20]. High fat diets also promote intestinal inflammation in rats as well as adenoma formation in the presence of a mutagen [44]. A high protein diet in a similar model increases SRB abundance and sulfide production and decreases the abundance 
of bacterial taxa associated with SCFA production and the amount of butyrate in stool [45]. Similarly, a high protein diet has been associated with post-weaning diarrhea in piglets [46]. Taken together, a high protein and saturated fat diet (characteristic of a western, animal-based diet) seems to result in an increased capacity for $\mathrm{H}_{2} \mathrm{~S}$ production, decreased capacity for butyrate production, and a potential to cause intestinal inflammation.

\subsection{Clinical Observations}

Mesalamine, a first-line agent in treatment of mild-to-moderate ulcerative colitis, inhibits sulfide production in a dose-dependent manner when added to a fecal slurry in vitro [47]. Furthermore, UC patients taking mesalamine have reduced fecal sulfide relative to patients who do not [47]. It is noteworthy that mesalamine and butyrate are both peroxisome proliferator activated receptor gamma (PPAR- $\gamma$ ) agonists $[48,49]$. Stimulation of PPAR- $\gamma$ promotes $\beta$ oxidation of fatty acids and epithelial hypoxia in the colon, which favors obligate anaerobes and lowers the abundance of Proteobacteria [50]. Interestingly, some antibiotics (e.g., aminoglycosides) which target Proteobacteria (including SRB) have short-term benefits in UC patients [13,51].

Few studies have attempted to link dietary interventions targeting the updated $\mathrm{H}_{2} \mathrm{~S}$ toxin hypothesis (i.e., transition from a western-style diet) with UC. The available handful of reports is limited to small case series or individual case studies (Table 1) [52-56]. These low dietary sulfur interventions generally emphasize transitions to a plant-based, semi-vegetarian diet. While all these experiences describe positive outcomes, conclusions are limited given the small patient numbers. The earliest case series conducted by Roediger et al. included only four patients and relied on symptom and histological criteria [52]. These patients sustained improvement in the activity of UC over 18 months of follow-up, while being maintained on the low sulfur diet. Chiba et al. reported a decreased relapse rate of $\mathrm{UC}$ relative to historical expectations among 60 patients instructed to follow a plant-based diet and followed for up to five years [56].

Table 1. Summary of reports detailing dietary interventions in the treatment of ulcerative colitis (UC) targeting the updated $\mathrm{H}_{2} \mathrm{~S}$ hypothesis.

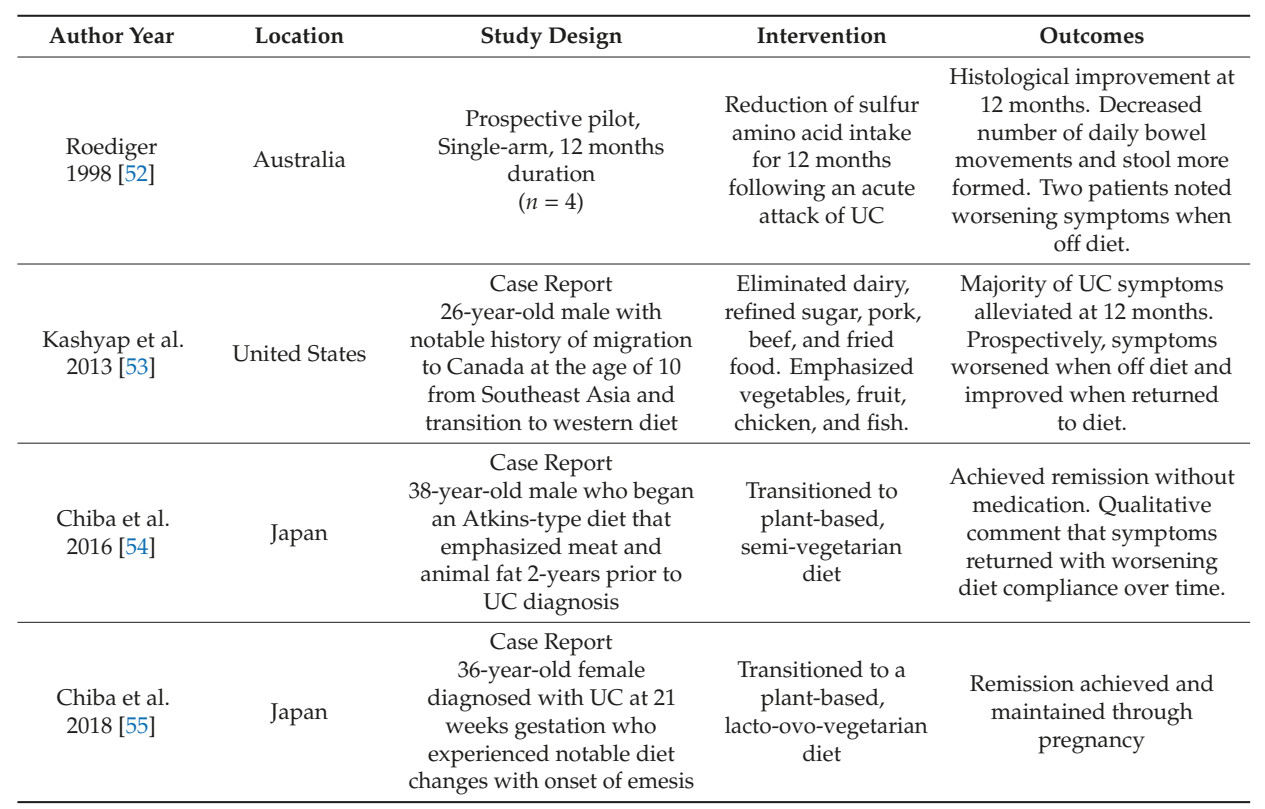


Table 1. Cont.

\begin{tabular}{ccccc}
\hline Author Year & Location & Study Design & Intervention & Outcomes \\
\hline $\begin{array}{c}\text { Chiba et al. } \\
2018[56]\end{array}$ & Japan & $\begin{array}{c}\text { Individuals with } \\
\text { mild UC or UC in } \\
\text { remission were } \\
\text { provided with } \\
\text { plant-based } \\
\text { diet education }\end{array}$ & $\begin{array}{c}\text { Relapse rates extending to } \\
\text { follow-up at } 5 \text { years } \leq 19 \%, \\
\text { which the authors purport is } \\
\text { better than those previously } \\
\text { reported. Diet adherence } \\
\text { decreased over time. }\end{array}$ \\
\hline
\end{tabular}

\section{Sources of Sulfur in the Colon}

\subsection{Dietary Intake}

While sulfur is ubiquitous in the food supply [57], and the fifth most abundant element on earth, its dietary linkage is not well characterized. For example, the USDA Food Composition Database does not include sulfur as a searchable nutrient. The sulfur content of food can be estimated using the sulfur-containing amino acids (methionine and cysteine) as surrogates, but this fails to account for sulfur-containing food modifiers or additives, such as sulfiting agents (e.g., potassium bisulfate, sodium bisulfate), sulfuric acid, or carrageenan [58]. Therefore, the most robust method of estimating dietary sulfur intake, including an estimate for inorganic sulfur, is through a $24-48$ hour urine collection $[59,60]$. Because the primary dietary sources of sulfur are the sulfur-containing amino acids, in practice, quantification of sulfur-containing amino acid content has been used to create low- and high-sulfur diets $[52,61]$.

Dietary input and small intestinal absorption are considered the main determinants of sulfur delivery to the colon [19]. The small intestine has an efficient but saturable dietary sulfate absorptive capacity of $\sim 5-7 \mathrm{mmol} /$ day [62]. Once dietary sulfate intake exceeds $\sim 5-7 \mathrm{mmol} /$ day the amount reaching the colon increases linearly with intake. Therefore, the amount of dietary sulfur that reaches the colon, particularly with increasing levels, can be generally assumed to parallel dietary intake. A number of additional factors, however, such as food preparation (e.g., cooked versus uncooked, cooking temperature and method, ground versus whole), meal consumption habits (e.g., chewing), and transit time have been shown to influence small intestinal absorption of sulfur-containing molecules and the total amount of sulfur reaching the colon [63-67].

\subsection{Endogenous Sources}

The main endogenous sources of sulfur are taurine-conjugated bile acids and mucin. Taurine is a sulfur-containing amino acid that is synthesized in the liver from methionine and cysteine. Diet can influence the rate of taurine conjugation and subsequent presence of taurine conjugated bile acids in the colon [68]. Although the majority of bile acids are re-absorbed in the distal small intestine into the enterohepatic circulation, a small percentage will spill into the colon [69]. Deconjugation of bile acids is an early step in secondary bile metabolism and is mediated by gut microbiota.

Intestinal mucins are glycoproteins that form a mucus barrier along the epithelial surface of the gastrointestinal tract. The primary intestinal mucin MUC2 contains cysteine amino acids as core components of its structure $[39,70]$. Glycosylation of mucin proteins makes up a substantial portion of their size and provides protection of the peptide backbone and the gel-forming capacity [70]. The glycosylation pattern of mucin varies along the intestinal tract with increasing sulfation moving distally through the small intestine and into the colon [71,72]. The colonic mucus barrier is predominately composed of sulfomucins with a trend toward slightly diminished sulfation moving distally ( $100 \%$ sulfation in the right colon and $86 \%$ in the rectum) [73]. The normal sulfation pattern is altered in UC and is associated with the concentration of SRB [73]. 


\section{3. $\mathrm{H}_{2} \mathrm{~S}$ Measurement}

The ideal experimental system to test the role of $\mathrm{H}_{2} \mathrm{~S}$ in UC pathogenesis would be able to measure its intraluminal concentration at different segments in the intestine. Unfortunately, technology to do that does not yet exist; therefore, studies that measure $\mathrm{H}_{2} \mathrm{~S}$ have focused on ex-vivo determination of its production from fecal samples. Fecal concentrations of hydrogen sulfide, however, are notoriously difficult due to the volatility of the gas, low intestinal concentrations, and instrumentation issues. Historically, the majority of work assessing stool sulfide concentrations in UC relied on colorimetric assays $[62,74,75]$. This method requires 'trapping' $\mathrm{H}_{2} \mathrm{~S}$ in fresh fecal samples for measurement and is associated with a number of inherent limitations $[76,77]$. Ion-exchange chromatography has also been used to measure $\mathrm{H}_{2} \mathrm{~S}$ in feces [78].

$\mathrm{H}_{2} \mathrm{~S}$ gas measurements, done using gas chromatography (GC) with sulfur chemiluminescence detection, are considered the reference standard for $\mathrm{H}_{2} \mathrm{~S}$ measurement [79]. These measures, however, cannot be obtained in real time and require in vitro incubation of the fecal sample. Only one study of UC has used GC as the measurement method and found that $\mathrm{H}_{2} \mathrm{~S}$ production was elevated substantially in UC compared to controls over a 24 hour period [80]. Although this method does not capture the $\mathrm{H}_{2} \mathrm{~S}$ content of fresh fecal samples, in vitro incubation may reflect in vivo $\mathrm{H}_{2} \mathrm{~S}$ production capacity of the microbiota, providing a functional measure of the microbiota metabolic capacity. Furthermore, this method allows for interrogation of the capacity of various dietary components to affect $\mathrm{H}_{2} \mathrm{~S}$ production (e.g., more $\mathrm{H}_{2} \mathrm{~S}$ production with cysteine or mucin vs sulfate) [80,81].

\section{Colonic Microbiota and $\mathrm{H}_{2} \mathrm{~S}$ Production}

\subsection{Sulfate-Reducing Bacteria}

Microbial production of $\mathrm{H}_{2} \mathrm{~S}$ is generally thought to be carried out by a limited number of bacteria and archael species. Sulfate-reducing bacteria (SRB) and the dissimilatory sulfur cycle, a form of anaerobic respiration that uses sulfate as the terminal electron acceptor, have been the primary focus of the $\mathrm{H}_{2} \mathrm{~S}$ toxin hypothesis in UC [14]. This pathway generates $\mathrm{H}_{2} \mathrm{~S}$ as an end-product of sulfate reduction. The pathway consists of three enzymatic steps involving ATP sulfurylase, adenosine 5'-phosphosulfate reductase, and sulfite reductase [82]. The final step in the pathway, sulfite reduction, is considered the rate limiting step. The genus Desulfovibrio is regarded as the most abundant SRB in humans [83]. Despite the focus on Desulfovibrio spp. as the primary producer of $\mathrm{H}_{2} \mathrm{~S}$ in the human gut, the number of microbial groups known to be capable of dissimilatory sulfate reduction continues to expand [82,84]. Many of these microbes can also reduce sulfite, dithionite, thiosulfate, elemental sulfur, and several thionates. The three sulfite reductase genes which catalyze the production of hydrogen sulfide are dissimilatory sulfite reductase (dsr), anaerobic sulfite reductase (asr), and cytochrome $c$ sulfite reductase $(m c c A)$ [82].

In humans, the measurement of key enzymes involved in the sulfate reduction pathway is a more specific method for measurement of SRB than measurement of bacterial genera such as Desulfovibrio [85]. This approach identified a unique capacity of SRB genera in UC to generate $\mathrm{H}_{2} \mathrm{~S}$ and induce epithelial apoptosis, compared with healthy controls $[74,86]$. Recent multiomics data in colon cancer lends additional support to the importance of accounting for the functional capacity of microbes (e.g., via measurement of key enzymes) in addition to their community composition [87].

Despite their importance and usefulness, there is a dearth of literature that relies on the quantification of sulfite reductase genes to characterize SRBs in large UC cohorts. Use of this technique in healthy subjects demonstrated that an animal-based diet increases sulfite reductase gene expression [5]. Recent work by Jia et al. used a semi-nested PCR method to detect $d s r B$ DNA, but failed to find a difference in abundance between healthy and UC cohorts ( $n=18$ and $n=14$, respectively) [88]. The agar shake dilution method, which measures SRB growth over a specified incubation period, has often been employed to measure SRB in individuals with UC. Studies done using this methodology found elevated SRB abundance in individuals with UC compared to those without $[47,74,89]$. 


\subsection{Cysteine Degraders}

Although sulfate intake and quantity of SRB has been the primary focus of the $\mathrm{H}_{2} \mathrm{~S}$ toxin hypothesis, there is a growing appreciation for the contribution of protein intake to $\mathrm{H}_{2} \mathrm{~S}$ production. The efficiency of protein degradation is greatest in the distal colon and at neutral to alkaline $\mathrm{pH}$, which suggests a possible relationship with the pathogenesis of UC [90]. A cross-over diet study conducted by Magee and colleagues demonstrated that dietary protein intake positively correlates with fecal sulfide concentrations in healthy individuals [78].

A recent in vitro study that profiled gas production from incubated fecal samples implicated cysteine specifically as a primary driver of $\mathrm{H}_{2} \mathrm{~S}$ production-conversely, the effect of sulfate was small [81]. Cysteine degradation to $\mathrm{H}_{2} \mathrm{~S}$ is catalyzed by enzymes with cysteine desulfhydrase activity. Although the enzymes with cysteine desulfhydrase activity are well characterized in humans [91], identifying enzymes with this activity in microorganisms has proven far more challenging. Escherichia coli possess several enzymes with cysteine desulfhydrase activity, including $O$-acetylserine sulfhydrylase-A, O-acetylserine sulfhydrylase-B, MalY, tryptophanase and cystathionine $\beta$-lyase [92]. Because cysteine likely makes a substantial contribution to $\mathrm{H}_{2} \mathrm{~S}$ production in the colonic lumen, there is a critical need to detail cysteine desulfhydrase activity within the colonic microbiome.

Prevention of excessive cysteine degradation to $\mathrm{H}_{2} \mathrm{~S}$ may underlie the possible protective role of appendectomy for appendicitis in UC [93]. Although the mechanism underlying the purported beneficial effect of appendectomy in UC has yet to be elucidated, one possibility is related to the potential role of the appendix as a reservoir for gut microbes [93]. Specifically, the appendix contains Fusobacterium spp. in its healthy state [94] and appendicitis is associated with an abundance of this bacteria [95]. Fusobacterium spp. contain a number of proteins necessary to metabolize L-cysteine to $\mathrm{H}_{2} \mathrm{~S}[96,97]$. Therefore, removal of the appendix, and subsequently a source of $\mathrm{H}_{2} \mathrm{~S}$ production, may contribute to its possible therapeutic role in the prevention of UC.

\subsection{Interplay Between Functional Microbial Groups}

A number of physicochemical factors shape the composition and functional output of microbial communities, which need to be integrated in order to fully account for the inter-individual differences. One such factor is the availability of hydrogen $\left(\mathrm{H}_{2}\right)$, which is essential as a substrate for anaerobic respiration in the colonic lumen. The groups of bacteria that rely on and compete for $\mathrm{H}_{2}$ for anaerobic respiration are acetogens, methanogens, and SRB (Figure 2) [98]. $\mathrm{H}_{2}$ production results from microbial fermentation of carbohydrates in the lumen of the intestine. Therefore, the balance between $\mathrm{H}_{2}$ production and consumption is often referred to as the 'hydrogen economy' [99]. An imbalance of $\mathrm{H}_{2}$ consumption and production may result in a metabolic environment that consumes NADH for $\mathrm{H}_{2}$ production at the expense of butyrate production [100]. In the context of the $\mathrm{H}_{2} \mathrm{~S}$ toxin hypothesis, it is important to consider possible imbalances in $\mathrm{H}_{2}$ consumption (e.g., altered SRB concentration) or production (e.g., low dietary intake of fermentable carbohydrate) that could create a metabolic environment leading to UC-namely, excessive $\mathrm{H}_{2} \mathrm{~S}$ production [80], mucin degradation [22,41], and diminished butyrate production [5].

Other physical (e.g., intestinal transit time) and chemical factors (e.g., $\mathrm{pH}$ and oxygen tension), also contribute to the microbial community structure in the colon by favoring some groups of microbes over others. Accelerated colon motility is likely to benefit fast-growing microorganisms and disfavor slow growing microorganisms, and constipation is associated with higher carriage of methanogenic archaea and increased methane production [101]. Fermentation of digestible carbohydrate results in the production of SCFA and a drop in luminal pH in the colon relative to the small intestine [102-104]. Increased dietary fiber also results in faster intestinal transit [105]. Indeed, the colon pH is lowest proximally and increases distally, which is likely driven by the decreasing availability of digestible carbohydrate [102]. Mildly acidic $\mathrm{pH}$, characteristic of the proximal colon, is inhibitory to the growth of SRB, but favors the growth of methanogens and some butyrate producers $[30,106,107]$. In contrast, sulfate reduction and $\mathrm{H}_{2} \mathrm{~S}$ production are optimal at an alkaline $\mathrm{pH}$ [108]. 


\section{Acetogens Methanogens}

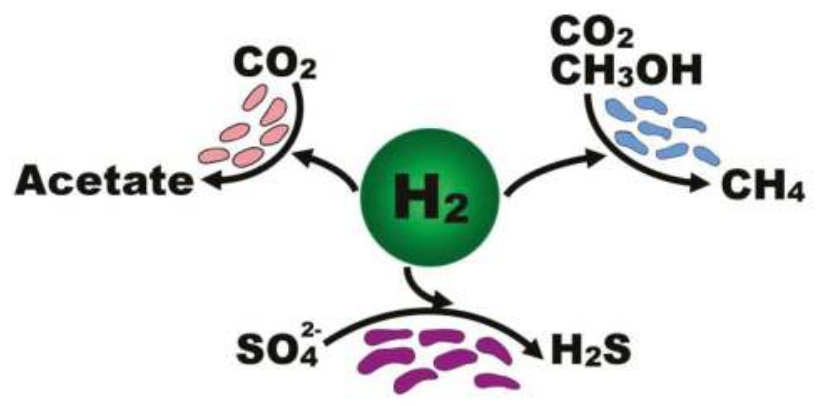

\section{Sulfate Reducing Bacteria}

Figure 2. Bacterial competition for hydrogen for anaerobic respiration in the lumen of the intestine. Legend: Acetogens, methanogens, and sulfate reducing bacteria are the microbial groups that compete for $\mathrm{H}_{2}$ in anaerobic respiration in the lumen of the colon. The availability of hydrogen can shape the composition and functional output of microbial communities.

\section{4. $\mathrm{H}_{2} \mathrm{~S}$ Clearance}

Clearance of hydrogen sulfide in the gut can be viewed as the final component of the $\mathrm{H}_{2} \mathrm{~S}$ toxin hypothesis. Due to its potential deleterious effects, $\mathrm{H}_{2} \mathrm{~S}$ is highly regulated in the cell. $\mathrm{As}_{\mathrm{H}} \mathrm{H}_{2} \mathrm{~S}$ is freely permeable across membranes, luminal concentration in the colon would be expected to correlate with intracellular concentrations [10]. Recent work in animal models demonstrates the relationship between luminal $\mathrm{H}_{2} \mathrm{~S}$ and intracellular regulation [109]. Although a small amount of $\mathrm{H}_{2} \mathrm{~S}$ present in the lumen of the colon is able to be cleared through flatus [110], the primary pathway for $\mathrm{H}_{2} \mathrm{~S}$ clearance is via intracellular sulfide oxidation. Interestingly, this pathway may be facilitated by cyanide, which has been proposed by Levitt and colleagues to explain the well-documented beneficial role of smoking in UC [111].

Colonic mucosa possesses a very efficient system for $\mathrm{H}_{2} \mathrm{~S}$ detoxification, and defects in these pathways are hypothesized to contribute to pathogenesis of colitis [111,112]. In earlier work, thiol methyltransferase and rhodanese were thought to have a major role in the detoxification of intestinal $\mathrm{H}_{2} \mathrm{~S}$ [113]. However, the investigators found conflicting results when using these enzymes to estimate the $\mathrm{H}_{2} \mathrm{~S}$ detoxification capacity in $\mathrm{UC}[38,114]$. Around the time this work was being conducted, however, a more detailed and comprehensive understanding of $\mathrm{H}_{2} \mathrm{~S}$ detoxification was being developed that implicates sulfide quinone oxidoreductase (SQR) as the rate-limiting enzyme in sulfur oxidation $[115,116]$.

The SQR enzyme is located within the inner mitochondrial membrane [117]. The reaction of $\mathrm{H}_{2} \mathrm{~S}$ with SQR results in oxidation of $\mathrm{H}_{2} \mathrm{~S}$ to a small-molecule persulfide and reduction of coenzyme $\mathrm{Q}$ (CoQ), which links $\mathrm{H}_{2} \mathrm{~S}$ oxidation with complex III of the electron transport chain [117]. The sulfur transfer from $\mathrm{H}_{2} \mathrm{~S}$ to a small molecule acceptor occurs via a cysteine intermediate within the SQR enzyme. Therefore, the availability of a small molecule acceptor is believed to be the rate-limiting step in the SQR reaction [118]. The antioxidant glutathione (GSH) is also believed to be the primary acceptor in a physiological setting, but sulfite has also been proposed as an alternative acceptor [118]. If GSH is utilized as the acceptor, the result is formation of glutathione persulfide (GSSH), which is then utilized by persulfide dioxygenase (ETHE1) or rhodanese to form sulfite or thiosulfate, respectively [117,119]. If sulfite is used as the acceptor, thiosulfate is produced, and rhodanese instead converts thiosulfate to GSSH, which is then converted to sulfite by ETHE1 $[117,119]$. In a steady state, GSH has been estimated to be favored $\sim 5: 1$ over sulfite [118], but elevated intracellular sulfite concentrations or depletion of 
GSH have been suggested to inhibit SQR $[116,118,119]$. Furthermore, although $\mathrm{H}_{2} \mathrm{~S}$ oxidation appears to be a robust pathway for intracellular control of $\mathrm{H}_{2} \mathrm{~S}$ levels, an excess of $\mathrm{H}_{2} \mathrm{~S}$ may paradoxically result in feedback inhibition of SQR from the electron transport chain as a result of its deleterious effect on cytochrome $c$ oxidase activity [119].

The $\mathrm{H}_{2} \mathrm{~S}$ detoxification capacity and expression of enzymes involved in the $\mathrm{H}_{2} \mathrm{~S}$ detoxification pathway (SQR, ETHE1, and thiosulfate sulfurtransferase) have been shown to follow a general trend of highest in the proximal colon to lowest in the rectum [120]. This expression pattern lends further support to the $\mathrm{H}_{2} \mathrm{~S}$ toxin hypothesis as the progression of $\mathrm{UC}$ progresses from the lowest to the highest $\mathrm{H}_{2} \mathrm{~S}$ detoxification capacity and enzyme expression (Figure 3). In addition, GSH synthesis has been demonstrated to be impaired in IBD [121].
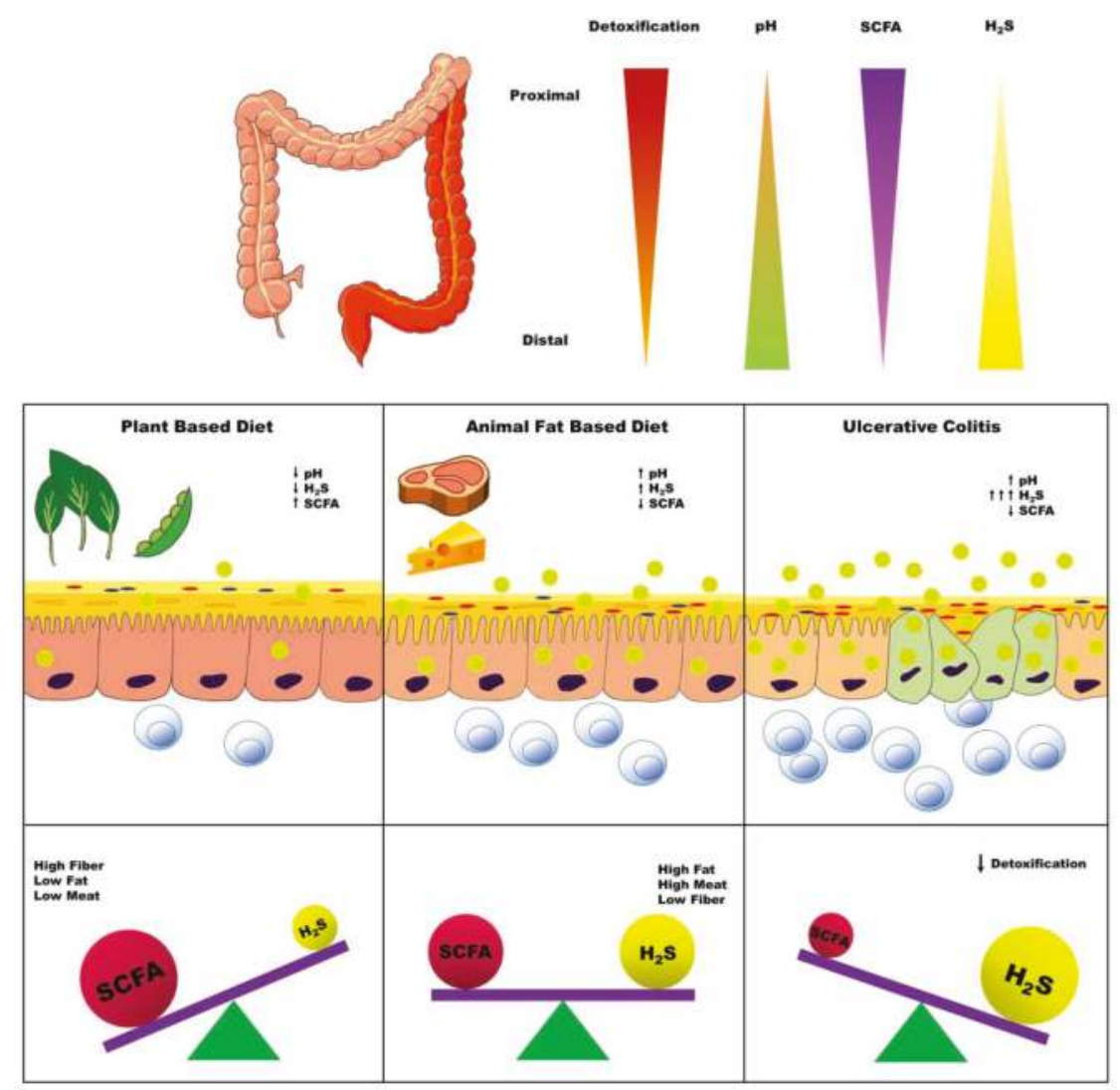

Figure 3. Overview of metabolic milieu and influence of diet in the pathogenesis of ulcerative colitis. Legend: The distribution of inflammation seen with ulcerative colitis is typically greatest in the rectum and extends continuously towards the proximal colon with varying severity. This pattern of inflammation parallels decreasing expression of the host $\mathrm{H}_{2} \mathrm{~S}$ detoxifying enzymes, rising $\mathrm{pH}$, and decreasing concentrations of short-chain fatty acids (SCFA). A plant-based diet promotes greater production of SCFA, but not $\mathrm{H}_{2} \mathrm{~S}$. SCFA production decreases in an animal-based diet, while $\mathrm{H}_{2} \mathrm{~S}$ production increases; however, there is a sufficient capacity for $\mathrm{H}_{2} \mathrm{~S}$ detoxification to prevent epithelial cell damage. Nonetheless, a decreased capacity for $\mathrm{H}_{2} \mathrm{~S}$ detoxification results in inflammation, which in turn further exacerbates the gradients of SCFA and $\mathrm{H}_{2} \mathrm{~S}$ concentrations along the proximal-to-distal colon axis. 


\section{Dietary Factors Influencing Colonic $\mathrm{H}_{2} \mathrm{~S}$ Production}

\subsection{Protein}

Dietary protein has been shown to be a robust driver of in vitro and in vivo $\mathrm{H}_{2} \mathrm{~S}$ production $[78,81]$ Furthermore, the efficiency of protein degradation is inverse to that of carbohydrate fermentation (and SCFA production) and parallels that of UC pathogenesis (highest in the distal colon and decreasing proximally) [90]. Dietary protein is efficiently absorbed in the small intestine, but the amount reaching the colon is thought to be positively correlated with the amount consumed [90].

In both human and animal studies, a high protein diet results in fecal microbiota changes that increase $\mathrm{H}_{2} \mathrm{~S}$ production and decrease SCFA production [5,45]. Increases in SRB and Bacteroidetes sp. observed in UC also occur in a high protein diet $[5,45,122]$ and observed decreases in specific butyrate producing species within a high protein diet (F. Prausnitzii, Akkermansia, and Ruminococcus) mirror those seen in UC [45,122-124].

Sulfur-containing amino acids are the primary source of dietary sulfur. Because of limited information on the sulfur content of food, outside of a metabolic unit, a 'high' or 'low' sulfur diet is defined according to cysteine and methionine concentration [52]. Therefore, a 'low sulfur' diet is actually a 'sulfur-containing amino acid-controlled diet', rather than a true 'sulfur-controlled diet' [52] In general, a sulfur-containing amino acid-controlled diet results in a shift from a more traditional western diet (high in animal protein and fat, and low in fiber) to more of a plant-based diet (high in fiber, low in animal protein and fat). Recently published diet composition data from a small sulfur-containing amino acid diet intervention study $(n=4)$ conducted in the U.S. suggests that a typical U.S. diet is already high in sulfur-containing amino acids; a transition from a baseline diet to a 'high sulfur' diet results in minor diet composition changes, while a transition from a baseline to a 'low sulfur' diet results in a substantial transition from an animal-based to a plant-based diet (resulting in increased fiber, and decreased protein and fat intake) [61].

As described above, $\mathrm{H}_{2} \mathrm{~S}$ production capacity is often characterized by quantifying SRB, but cysteine desulfhydrase activity provides another route of $\mathrm{H}_{2} \mathrm{~S}$ production for the colonic microbiota that is often left unassessed. In fact, in vitro modeling using healthy human feces suggests that the contribution of cysteine to $\mathrm{H}_{2} \mathrm{~S}$ production is substantially more than that from sulfate [81].

\subsection{Carbohydrate and Fiber}

High carbohydrate availability in the colon, as noted above, promotes microbial groups able to utilize carbohydrate substrates, but also affects other aspects of microbial metabolism and especially impacts protein degradation. The addition of fermentable fiber to healthy human feces in an in vitro setting drastically reduces $\mathrm{H}_{2} \mathrm{~S}$ production from any source (e.g., cysteine, sulfate) [81]. Lower $\mathrm{pH}$ associated with microbial carbohydrate fermentation also leads to inhibition of dissimilatory aromatic amino acid metabolism [125]. Interestingly, stool $\mathrm{pH}$ is lower in vegan and vegetarian individuals relative to omnivores, consistent with greater abundance of carbohydrates in the proximal colon, faster colon transit, and higher delivery of SCFA to the distal colon [126]. This finding underscores the importance of overall diet composition when considering the relative proportions of different end-products of microbial metabolism.

\subsection{Dietary Fat}

Although there is little data supporting a direct role of dietary fat in the $\mathrm{H}_{2} \mathrm{~S}$ toxin hypothesis, animal fat was shown in a murine model to result in increased production of taurine-conjugated bile acids and a bloom of the sulfite-reducer Bilophila wadsworthia in the colonic microenvironment [127]. A high fat diet in mice is associated with lesser production of SCFA and greater production of $\mathrm{H}_{2} \mathrm{~S}$, even when the protein content of high fat chow is lower relative to regular chow [128]. It is obvious that a relative contribution of macronutrients to $\mathrm{H}_{2} \mathrm{~S}$ production is difficult to isolate in a single comparison, 
given that an increase in one will correspondingly necessitate reduction in at least one other to maintain the same caloric intake.

\subsection{Endogenous Sulfur}

The most significant source of endogenous sulfur (both as sulfate and cysteine) is intestinal mucin. The mucous layer of the colon is composed of two layers [40], the microbiota is abundant in the outer loose mucous layer, while the inner dense mucous layer-largely devoid of bacteria-maintains a barrier between the colonic microbiota and colonocytes $[39,40]$. The composition of the colon microbiota has been shown to influence mucin secretion, breakdown, structure, and the glycosylation pattern [22,42,129-131]. Conversely, mucin glycosylation patterns have been shown to influence the colonic microbiota [132]. Therefore, the $\mathrm{H}_{2} \mathrm{~S}$ toxin hypothesis must consider these complex relationships between the mucin, microbiota, and diet. A characteristically high sulfur diet (high in animal protein and fat, and low in fiber) leads to nutrient deprivation for the microbiota and results in increased mucin desulfating sulfatase activity and mucin degradation $[5,22,131,133]$. This pattern is remarkably similar to that seen in UC [134-136]. The phenotypic changes resulting in increased mucin breakdown (e.g., low fiber) and $\mathrm{H}_{2} \mathrm{~S}$ production (e.g., high protein/sulfur intake) $[5,22,45,78]$ may produce $\mathrm{H}_{2} \mathrm{~S}$ concentrations that overwhelm the detoxification capacity of colonocytes and contribute to $\mathrm{H}_{2} \mathrm{~S}$ toxicity.

\subsection{Miscellaneous Dietary Factors}

Finally, it is also important to consider factors that may correlate with sulfur content in the diet but are not related to sulfur metabolism. An increased intake of heme, present in its highest concentrations in red meat, can have cytotoxic effects on colonocytes [42]. Consumption of processed foods expose individuals to additives such as phosphates, nitrates, and emulsifiers, which have been shown to influence the composition of microbiota, mucin layer thickness, and intestinal inflammation [137-140]. Even specific cooking methods may alter the potential of diet to impact the intestinal microbiome and microbiota-host interactions. Both enzymatic and non-enzymatic mechanisms of protein modification may also influence intestinal $\mathrm{H}_{2} \mathrm{~S}$ production. Thus, glycation of dietary protein can to shift the colonic microbiota towards greater abundance of SRB and lesser production of butyrate [64]. Conversely, there are likely undiscovered or underappreciated components of a plant-based diet that may be protective in UC [141].

\subsection{Inflammatory Factors}

Onset of inflammation triggers a number of positive feedback loops that amplify the detrimental effects of $\mathrm{H}_{2} \mathrm{~S}$ on the homeostasis of colonic mucosa. Increased synthesis of inducible nitric oxide synthetase (iNOS) results in increased release of nitrate, which contributes to dysbiosis by encouraging the expansion of facultative anaerobic Proteobacteria [28]. Nitric oxide also impairs $\mathrm{H}_{2} \mathrm{~S}$ detoxification [109], thus lowering the threshold concentration where $\mathrm{H}_{2} \mathrm{~S}$ becomes toxic to epithelial cells. Epithelial injury results in crypt hyperplasia and loss of epithelial hypoxia, which are detrimental to butyrate-producing obligate anaerobes. In animal models and human studies, SCFA (and specifically butyrate) production is inhibited in the presence of inflammation [122-124,142]. Inflammation is also correlated with a lower BcoAT gene content of fecal microbiota, consistent with lower butyrate production capacity [143]. Collectively, these changes hasten the collapse of the gut barrier to intestinal microbes and inhibit regulatory immune circuits, such as regulatory $\mathrm{T}$ cells, which further augment the inflammatory response.

\subsection{Interventions Targeting the Colon Microbiota}

The underlying mechanisms that make up the $\mathrm{H}_{2} \mathrm{~S}$ toxin hypothesis are supported by changes produced with fermentable fiber supplementation in individuals with UC (increased butyrate production) [144] and findings from a recent fecal microbial transplantation (FMT) trial that demonstrated a correlation between sustained remission and increased butyrate production, 
while increased abundances of Bacteroidetes and Proteobacteria correlated with no response or relapse [122]. Notably, members of the Bacteroidetes phylum contain mucin desulfating sulfatase enzymes $[122,145,146]$. The FMT in UC findings have also been recently expanded upon by Wei et al. who demonstrated a role for fiber in maintaining the gut microbiota composition in individuals with UC following FMT [147].

The role of carbohydrate, and more specifically fermentable carbohydrate, intake within the $\mathrm{H}_{2} \mathrm{~S}$ toxin hypothesis may be its overall impact on protein degradation by colonic bacteria. These findings are supported by a diet study in healthy volunteers [148], where administration of metronidazole effectively reduced SRB counts, but fecal concentration of $\mathrm{H}_{2} \mathrm{~S}$ remained unchanged. In contrast, consumption of fermentable fiber (oligofructose) resulted in a decrease in fecal $\mathrm{H}_{2} \mathrm{~S}$, concomitant increase in SCFA concentrations, but failure to influence SRB counts. The impact of fiber on microbial $\mathrm{H}_{2} \mathrm{~S}$ production is further supported by recent data demonstrating a decrease in SRB with a high brassica diet (sulfur-containing vegetables) [149]. Although brassica vegetables are technically considered to be high sulfate foods, they also have a high fiber content. These findings underscore the beneficial role for the latter within the $\mathrm{H}_{2} \mathrm{~S}$ toxin hypothesis and support a clinical approach focusing on plant versus animal-based diets rather than high- and low-sulfur diets.

It is important to consider that while short-term changes in diet can affect activities of different microbial groups in the intestine, they do not change the overall individual-specific taxonomic composition of microbiota $[61,150]$. Short-term low carbohydrate, high protein diets are among the most popular approaches used to achieve weight loss. Such diets do result in decreases in relative abundance of some butyrate producing bacteria, as well as altered fecal metabolite profiles, including lower content of SCFA and fiber-derived antioxidant phenolic acids [151]. It is possible that such dietary changes can even trigger the onset of UC, which may be reversed with increasing plant-derived dietary components $[54,56]$. However, long-term diets low in microbiota-accessible carbohydrates can lead to complete extinctions of microbial taxa [152]. Therefore, it is possible that most consistent benefits of dietary therapies will also require transplantation of relevant microbiota, optimized in capacity for the individual patients.

\section{Conclusions}

There is an intriguing body of literature supporting a relationship between dietary sulfur intake and the $\mathrm{H}_{2} \mathrm{~S}$ toxin hypothesis in the development of UC. To date, the focus of the $\mathrm{H}_{2} \mathrm{~S}$ toxin hypothesis in UC has primarily been focused on the abundance and activity of SRB (e.g., Desulfovibrio) and dietary sulfur intake. However, this provides only a partial window on the total activity of the microbiota that is relevant to UC pathogenesis. An updated approach to the $\mathrm{H}_{2} \mathrm{~S}$ hypothesis has to incorporate interactions between different microbial groups as well as multiple components of the diet. A compelling case does exist that a typical western diet, which tends to be high in protein and low in fermentable fiber, may promote the accumulation of harmful products such as $\mathrm{H}_{2} \mathrm{~S}$ and even increase their toxicity, and is also associated with a lower production of beneficial products such as butyrate. Patients are intensely interested in adjunctive dietary therapies for UC. Multiple technological developments that allow compositional and functional measurements of entire microbial communities should facilitate the development and testing of novel microbiota-targeting interventions that include the dietary management of IBD.

Author Contributions: Conceptulization, L.M.T, A.K.; writing-original draft preparation, L.M.T.; writing—review and editing, L.M.T, M.J.S., B.P.V., M.J.H., Z.G., A.K.; supervision, A.K.

Funding: This research received no external funding.

Acknowledgments: This work was partially supported by grants from the Healthy Foods Healthy Lives Institute, the Allen Foundation, and Achieving Cures Together.

Conflicts of Interest: B.P.V. has received compensation from speaking and/or ad boards from Abbvie and Janssen and has received grant support from Roche, Takeda, Celgene and Diasorin. 


\section{References}

1. Kaplan, G.G. The global burden of IBD: From 2015 to 2025. Nat. Rev. Gastroenterol. Hepatol. 2015, 12, 720-727. [CrossRef]

2. Ng, S.C.; Shi, H.Y.; Hamidi, N.; Underwood, F.E.; Tang, W.; Benchimol, E.I.; Panaccione, R.; Ghosh, S.; $\mathrm{Wu}$, J.C.Y.; Chan, F.K.L.; et al. Worldwide incidence and prevalence of inflammatory bowel disease in the 21st century: A systematic review of population-based studies. Lancet 2017, 390, 2769-2778. [CrossRef]

3. Ng, S.C.; Kaplan, G.G.; Tang, W.; Banerjee, R.; Adigopula, B.; Underwood, F.E.; Tanyingoh, D.; Wei, S.C.; Lin, W.C.; Lin, H.H.; et al. Population Density and Risk of Inflammatory Bowel Disease: A Prospective Population-Based Study in 13 Countries or Regions in Asia-Pacific. Am. J. Gastroenterol. 2019, 114, $107-115$. [CrossRef]

4. Rothschild, D.; Weissbrod, O.; Barkan, E.; Kurilshikov, A.; Korem, T.; Zeevi, D.; Costea, P.I.; Godneva, A.; Kalka, I.N.; Bar, N.; et al. Environment dominates over host genetics in shaping human gut microbiota. Nature 2018, 555, 210-215. [CrossRef] [PubMed]

5. David, L.A.; Maurice, C.F.; Carmody, R.N.; Gootenberg, D.B.; Button, J.E.; Wolfe, B.E.; Ling, A.V.; Devlin, A.S.; Varma, Y.; Fischbach, M.A.; et al. Diet rapidly and reproducibly alters the human gut microbiome. Nature 2014, 505, 559-563. [CrossRef] [PubMed]

6. Benchimol, E.I.; Mack, D.R.; Guttmann, A.; Nguyen, G.C.; To, T.; Mojaverian, N.; Quach, P.; Manuel, D.G. Inflammatory bowel disease in immigrants to Canada and their children: A population-based cohort study. Am. J. Gastroenterol. 2015, 110, 553-563. [CrossRef] [PubMed]

7. Vangay, P.; Johnson, A.J.; Ward, T.L.; Al-Ghalith, G.A.; Shields-Cutler, R.R.; Hillmann, B.M.; Lucas, S.K.; Beura, L.K.; Thompson, E.A.; Till, L.M.; et al. US Immigration Westernizes the Human Gut Microbiome. Cell 2018, 175, 962-972.e10. [CrossRef]

8. Hou, J.K.; Abraham, B.; El-Serag, H. Dietary intake and risk of developing inflammatory bowel disease: A systematic review of the literature. Am. J. Gastroenterol. 2011, 106, 563-573. [CrossRef] [PubMed]

9. Kaplan, G.G.; Ng, S.C. Understanding and Preventing the Global Increase of Inflammatory Bowel Disease. Gastroenterology 2017, 152, 313-321.e2. [CrossRef] [PubMed]

10. Yang, G. Hydrogen sulfide in cell survival: A double-edged sword. Expert Rev. Clin. Pharmacol. 2011, 4, 33-47. [CrossRef]

11. Barton, L.L.; Ritz, N.L.; Fauque, G.D.; Lin, H.C. Sulfur Cycling and the Intestinal Microbiome. Dig. Dis. Sci. 2017, 62, 2241-2257. [CrossRef]

12. Pitcher, M.C.L.; Cummings, J.H. Hydrogen sulphide: A bacterial toxin in ulcerative colitis? Gut 1996, 39, 1-4. [CrossRef] [PubMed]

13. Roediger, W.E.W.; Moore, J.; Babidge, W. Colonic sulfide in pathogenesis and treatment of ulcerative colitis. Dig. Dis. Sci. 1997, 42, 1571-1579. [CrossRef] [PubMed]

14. Rowan, F.E.; Docherty, N.G.; Coffey, J.C.; O'Connell, P.R. Sulphate-reducing bacteria and hydrogen sulphide in the aetiology of ulcerative colitis. Br. J. Surg. 2009, 96, 151-158. [CrossRef] [PubMed]

15. Blachier, F.; Davila, A.M.; Mimoun, S.; Benetti, P.H.; Atanasiu, C.; Andriamihaja, M.; Benamouzig, R.; Bouillaud, F.; Tome, D. Luminal sulfide and large intestine mucosa: Friend or foe? Amino Acids 2010, 39, 335-347. [CrossRef]

16. Blachier, F.; Beaumont, M.; Andriamihaja, M.; Davila, A.M.; Lan, A.; Grauso, M.; Armand, L.; Benamouzig, R.; Tome, D. Changes in the Luminal Environment of the Colonic Epithelial Cells and Physiopathological Consequences. Am. J. Pathol. 2017, 187, 476-486. [CrossRef]

17. Levine, A.; Sigall Boneh, R.; Wine, E. Evolving role of diet in the pathogenesis and treatment of inflammatory bowel diseases. Gut 2018, 67, 1726-1738. [CrossRef]

18. Chiba, M.; Abe, T.; Tsuda, H.; Sugawara, T.; Tsuda, S.; Tozawa, H.; Fujiwara, K.; Imai, H. Lifestyle-related disease in Crohn's disease: Relapse prevention by a semi-vegetarian diet. World J. Gastroenterol. 2010, 16, 2484-2495. [CrossRef]

19. Florin, T.; Neale, G.; Gibson, G.R.; Christl, S.U.; Cummings, J.H. Metabolism of dietary sulphate: Absorption and excretion in humans. Gut 1991, 32, 766-773. [CrossRef]

20. Devkota, S.; Wang, Y.; Musch, M.W.; Leone, V.; Fehlner-Peach, H.; Nadimpalli, A.; Antonopoulos, D.A.; Jabri, B.; Chang, E.B. Dietary-fat-induced taurocholic acid promotes pathobiont expansion and colitis in Il10-/- mice. Nature 2012, 487, 104-108. [CrossRef] 
21. Holmes, A.J.; Chew, Y.V.; Colakoglu, F.; Cliff, J.B.; Klaassens, E.; Read, M.N.; Solon-Biet, S.M.; McMahon, A.C.; Cogger, V.C.; Ruohonen, K.; et al. Diet-Microbiome Interactions in Health Are Controlled by Intestinal Nitrogen Source Constraints. Cell Metab. 2017, 25, 140-151. [CrossRef] [PubMed]

22. Desai, M.S.; Seekatz, A.M.; Koropatkin, N.M.; Kamada, N.; Hickey, C.A.; Wolter, M.; Pudlo, N.A.; Kitamoto, S.; Terrapon, N.; Muller, A.; et al. A Dietary Fiber-Deprived Gut Microbiota Degrades the Colonic Mucus Barrier and Enhances Pathogen Susceptibility. Cell 2016, 167, 1339-1353.e1321. [CrossRef]

23. Wong, J.M.; de Souza, R.; Kendall, C.W.; Emam, A.; Jenkins, D.J. Colonic health: Fermentation and short chain fatty acids. J. Clin. Gastroenterol. 2006, 40, 235-243. [CrossRef] [PubMed]

24. Roediger, W.E. Role of anaerobic bacteria in the metabolic welfare of the colonic mucosa in man. Gut 1980, 21, 793-798. [CrossRef] [PubMed]

25. Furusawa, Y.; Obata, Y.; Fukuda, S.; Endo, T.A.; Nakato, G.; Takahashi, D.; Nakanishi, Y.; Uetake, C.; Kato, K.; Kato, T.; et al. Commensal microbe-derived butyrate induces the differentiation of colonic regulatory $\mathrm{T}$ cells. Nature 2013, 504, 446-450. [CrossRef] [PubMed]

26. Arpaia, N.; Campbell, C.; Fan, X.; Dikiy, S.; van der Veeken, J.; deRoos, P.; Liu, H.; Cross, J.R.; Pfeffer, K.; Coffer, P.J.; et al. Metabolites produced by commensal bacteria promote peripheral regulatory T-cell generation. Nature 2013, 504, 451-455. [CrossRef] [PubMed]

27. Finnie, I.A.; Dwarakanath, A.D.; Taylor, B.A.; Rhodes, J.M. Colonic mucin synthesis is increased by sodium butyrate. Gut 1995, 36, 93-99. [CrossRef]

28. Byndloss, M.X.; Olsan, E.E.; Rivera-Chavez, F.; Tiffany, C.R.; Cevallos, S.A.; Lokken, K.L.; Torres, T.P.; Byndloss, A.J.; Faber, F.; Gao, Y.; et al. Microbiota-activated PPAR-gamma signaling inhibits dysbiotic Enterobacteriaceae expansion. Science 2017, 357, 570-575. [CrossRef]

29. Litvak, Y.; Byndloss, M.X.; Tsolis, R.M.; Baumler, A.J. Dysbiotic Proteobacteria expansion: A microbial signature of epithelial dysfunction. Curr. Opin. Microbiol. 2017, 39, 1-6. [CrossRef] [PubMed]

30. Christl, S.U.; Eisner, H.D.; Dusel, G.; Kasper, H.; Scheppach, W. Antagonistic effects of sulfide and butyrate on proliferation of colonic mucosa: A potential role for these agents in the pathogenesis of ulcerative colitis. Dig. Dis. Sci. 1996, 41, 2477-2481. [CrossRef]

31. O'Flaherty, V.; Mahony, T.; O'Kennedy, R.; Colleran, E. Effect of pH on growth kinetics and sulphide toxicity thresholds of a range of methanogeic, syntrophic and sulphate-reducing bacteria. Process Biochem. 1998, 33, 555-569. [CrossRef]

32. Babidge, W.; Millard, S.; Roediger, W. Sulfides impair short chain fatty acid beta-oxidation at acyl-CoA dehydrogenase level in colonocytes: Implications for ulcerative colitis. Mol. Cell. Biochem. 1998, 181, 117-124. [CrossRef]

33. Wallace, J.L.; Blackler, R.W.; Chan, M.V.; Da Silva, G.J.; Elsheikh, W.; Flannigan, K.L.; Gamaniek, I.; Manko, A.; Wang, L.; Motta, J.P.; et al. Anti-inflammatory and cytoprotective actions of hydrogen sulfide: Translation to therapeutics. Antioxid. Redox Signal. 2015, 22, 398-410. [CrossRef] [PubMed]

34. Odenwald, M.A.; Turner, J.R. The intestinal epithelial barrier: A therapeutic target? Nat. Rev. Gastroenterol. Hepatol. 2017, 14, 9-21. [CrossRef]

35. Jiang, J.; Chan, A.; Ali, S.; Saha, A.; Haushalter, K.J.; Lam, W.L.; Glasheen, M.; Parker, J.; Brenner, M.; Mahon, S.B.; et al. Hydrogen Sulfide-Mechanisms of Toxicity and Development of an Antidote. Sci. Rep. 2016, 6, 20831. [CrossRef]

36. Roediger, W.E.; Duncan, A.; Kapaniris, O.; Millard, S. Reducing sulfur compounds of the colon impair colonocyte nutrition: Implications for ulcerative colitis. Gastroenterology 1993, 104, 802-809. [CrossRef]

37. De Preter, V.; Arijs, I.; Windey, K.; Vanhove, W.; Vermeire, S.; Schuit, F.; Rutgeerts, P.; Verbeke, K. Impaired butyrate oxidation in ulcerative colitis is due to decreased butyrate uptake and a defect in the oxidation pathway. Inflamm. Bowel Dis. 2012, 18, 1127-1136. [CrossRef] [PubMed]

38. De Preter, V.; Arijs, I.; Windey, K.; Vanhove, W.; Vermeire, S.; Schuit, F.; Rutgeerts, P.; Verbeke, K. Decreased mucosal sulfide detoxification is related to an impaired butyrate oxidation in ulcerative colitis. Inflamm. Bowel Dis. 2012, 18, 2371-2380. [CrossRef] [PubMed]

39. Johansson, M.E.; Hansson, G.C. Immunological aspects of intestinal mucus and mucins. Nat. Rev. Immunol. 2016, 16, 639-649. [CrossRef] [PubMed]

40. Johansson, M.E.; Phillipson, M.; Petersson, J.; Velcich, A.; Holm, L.; Hansson, G.C. The inner of the two Muc2 mucin-dependent mucus layers in colon is devoid of bacteria. Proc. Natl. Acad. Sci. USA 2008, 105, 15064-15069. [CrossRef] [PubMed] 
41. Johansson, M.E.; Gustafsson, J.K.; Holmen-Larsson, J.; Jabbar, K.S.; Xia, L.; Xu, H.; Ghishan, F.K.; Carvalho, F.A.; Gewirtz, A.T.; Sjovall, H.; et al. Bacteria penetrate the normally impenetrable inner colon mucus layer in both murine colitis models and patients with ulcerative colitis. Gut 2014, 63, 281-291. [CrossRef]

42. Ijssennagger, N.; Belzer, C.; Hooiveld, G.J.; Dekker, J.; van Mil, S.W.; Muller, M.; Kleerebezem, M.; van der Meer, R. Gut microbiota facilitates dietary heme-induced epithelial hyperproliferation by opening the mucus barrier in colon. Proc. Natl. Acad. Sci. USA 2015, 112, 10038-10043. [CrossRef]

43. Eichele, D.D.; Kharbanda, K.K. Dextran sodium sulfate colitis murine model: An indispensable tool for advancing our understanding of inflammatory bowel diseases pathogenesis. World J. Gastroenterol. 2017, 23, 6016-6029. [CrossRef]

44. Zhu, Q.C.; Gao, R.Y.; Wu, W.; Guo, B.M.; Peng, J.Y.; Qin, H.L. Effect of a high-fat diet in development of colonic adenoma in an animal model. World J. Gastroenterol. 2014, 20, 8119-8129. [CrossRef]

45. Mu, C.; Yang, Y.; Luo, Z.; Guan, L.; Zhu, W. The Colonic Microbiome and Epithelial Transcriptome Are Altered in Rats Fed a High-Protein Diet Compared with a Normal-Protein Diet. J. Nutr. 2016, 146, $474-483$. [CrossRef]

46. Heo, J.M.; Kim, J.C.; Hansen, C.F.; Mullan, B.P.; Hampson, D.J.; Pluske, J.R. Effects of feeding low protein diets to piglets on plasma urea nitrogen, faecal ammonia nitrogen, the incidence of diarrhoea and performance after weaning. Arch. Anim. Nutr. 2008, 62, 343-358. [CrossRef]

47. Pitcher, M.C.; Beatty, E.R.; Cummings, J.H. The contribution of sulphate reducing bacteria and 5-aminosalicylic acid to faecal sulphide in patients with ulcerative colitis. Gut 2000, 46, 64-72. [CrossRef] [PubMed]

48. Dubuquoy, L.; Rousseaux, C.; Thuru, X.; Peyrin-Biroulet, L.; Romano, O.; Chavatte, P.; Chamaillard, M.; Desreumaux, P. PPARgamma as a new therapeutic target in inflammatory bowel diseases. Gut 2006, 55, 1341-1349. [CrossRef]

49. Alex, S.; Lange, K.; Amolo, T.; Grinstead, J.S.; Haakonsson, A.K.; Szalowska, E.; Koppen, A.; Mudde, K.; Haenen, D.; Al-Lahham, S.; et al. Short-chain fatty acids stimulate angiopoietin-like 4 synthesis in human colon adenocarcinoma cells by activating peroxisome proliferator-activated receptor gamma. Mol. Cell. Biol. 2013, 33, 1303-1316. [CrossRef]

50. Xu, J.; Chen, N.; Wu, Z.; Song, Y.; Zhang, Y.; Wu, N.; Zhang, F.; Ren, X.; Liu, Y. 5-Aminosalicylic Acid Alters the Gut Bacterial Microbiota in Patients with Ulcerative Colitis. Front. Microbiol. 2018, 9, 1274. [CrossRef]

51. Burke, D.A.; Axon, A.T.; Clayden, S.A.; Dixon, M.F.; Johnston, D.; Lacey, R.W. The efficacy of tobramycin in the treatment of ulcerative colitis. Aliment. Pharmacol. Ther. 1990, 4, 123-129. [CrossRef]

52. Roediger, W.E.W. Decreased sulphur aminoacid intake in ulcerative colitis. Lancet 1998, 351. [CrossRef]

53. Kashyap, P.C.; Reigstad, C.S.; Loftus, E.V., Jr. Role of diet and gut microbiota in management of inflammatory bowel disease in an Asian migrant. J. Allergy Clin. Immunol. 2013, 132, 250-250.e255. [CrossRef] [PubMed]

54. Chiba, M.; Tsuda, S.; Komatsu, M.; Tozawa, H.; Takayama, Y. Onset of Ulcerative Colitis during a Low-Carbohydrate Weight-Loss Diet and Treatment with a Plant-Based Diet: A Case Report. Perm. J. 2016, 20, 80-84. [CrossRef] [PubMed]

55. Chiba, M.; Sugawara, T.; Komatsu, M.; Tozawa, H. Onset of Ulcerative Colitis in the Second Trimester after Emesis Gravidarum: Treatment with Plant-based Diet. Inflamm. Bowel Dis. 2018, 24, e8-e9. [CrossRef]

56. Chiba, M.; Nakane, K.; Tsuji, T.; Tsuda, S.; Ishii, H.; Ohno, H.; Watanabe, K.; Ito, M.; Komatsu, M.; Yamada, K.; et al. Relapse Prevention in Ulcerative Colitis by Plant-Based Diet Through Educational Hospitalization: A Single-Group Trial. Perm. J. 2018, 22. [CrossRef] [PubMed]

57. Florin, T.H.J.; Neale, G.; Goretski, S.; Cummings, J.H. The sulfate content of foods and beverages. J. Food Compos. Anal. 1993, 6, 140-151. [CrossRef]

58. U.S. Food and Drug Administration. Food Additive Status List. Available online: https://www.fda.gov/food/ ingredientspackaginglabeling/foodadditivesingredients/ucm091048.htm (accessed on 15 August 2018).

59. Curno, R.; Magee, E.A.; Edmond, L.M.; Cummings, J.H. Studies of a urinary biomarker of dietary inorganic sulphur in subjects on diets containing 1-38 mmol sulphur/day and of the half-life of ingested 34SO4(2-). Eur. J. Clin. Nutr. 2008, 62, 1106-1115. [CrossRef]

60. Magee, E.A.; Curno, R.; Edmond, L.M.; Cummings, J.H. Contribution of dietary protein and inorganic sulfur to urinary sulfate: Toward a biomarker of inorganic sulfur intake. Am. J. Clin. Nutr. 2004, 80, 137-142. [CrossRef] 
61. Dostal Webster, A.; Staley, C.; Hamilton, M.J.; Huang, M.; Fryxell, K.; Erickson, R.; Kabage, A.J.; Sadowsky, M.J.; Khoruts, A. Influence of short-term changes in dietary sulfur on the relative abundances of intestinal sulfate-reducing bacteria. Gut Microbes 2019, 1-11. [CrossRef]

62. Florin, T.H. Hydrogen sulphide and total acid-volatile sulphide in faeces, determined with a direct spectrophotometric method. Clin. Chim. Acta 1991, 196, 127-134. [CrossRef]

63. Buffiere, C.; Gaudichon, C.; Hafnaoui, N.; Migne, C.; Scislowsky, V.; Khodorova, N.; Mosoni, L.; Blot, A.; Boirie, Y.; Dardevet, D.; et al. In the elderly, meat protein assimilation from rare meat is lower than that from meat that is well done. Am. J. Clin. Nutr. 2017, 106, 1257-1266. [CrossRef]

64. Mills, D.J.; Tuohy, K.M.; Booth, J.; Buck, M.; Crabbe, M.J.; Gibson, G.R.; Ames, J.M. Dietary glycated protein modulates the colonic microbiota towards a more detrimental composition in ulcerative colitis patients and non-ulcerative colitis subjects. J. Appl. Microbiol. 2008, 105, 706-714. [CrossRef] [PubMed]

65. Oberli, M.; Marsset-Baglieri, A.; Airinei, G.; Sante-Lhoutellier, V.; Khodorova, N.; Remond, D.; Foucault-Simonin, A.; Piedcoq, J.; Tome, D.; Fromentin, G.; et al. High True Ileal Digestibility but Not Postprandial Utilization of Nitrogen from Bovine Meat Protein in Humans Is Moderately Decreased by High-Temperature, Long-Duration Cooking. J. Nutr. 2015, 145, 2221-2228. [CrossRef]

66. Pennings, B.; Groen, B.B.; van Dijk, J.W.; de Lange, A.; Kiskini, A.; Kuklinski, M.; Senden, J.M.; van Loon, L.J. Minced beef is more rapidly digested and absorbed than beef steak, resulting in greater postprandial protein retention in older men. Am. J. Clin. Nutr. 2013, 98, 121-128. [CrossRef] [PubMed]

67. Remond, D.; Machebeuf, M.; Yven, C.; Buffiere, C.; Mioche, L.; Mosoni, L.; Mirand, P.P. Postprandial whole-body protein metabolism after a meat meal is influenced by chewing efficiency in elderly subjects. Am. J. Clin. Nutr. 2007, 85, 1286-1292. [CrossRef] [PubMed]

68. Ridlon, J.M.; Wolf, P.G.; Gaskins, H.R. Taurocholic acid metabolism by gut microbes and colon cancer. Gut Microbes 2016, 7, 201-215. [CrossRef] [PubMed]

69. Di Ciaula, A.; Garruti, G.; Lunardi Baccetto, R.; Molina-Molina, E.; Bonfrate, L.; Wang, D.Q.; Portincasa, P. Bile Acid Physiology. Ann. Hepatol. 2017, 16, s4-s14. [CrossRef]

70. Johansson, M.E.; Larsson, J.M.; Hansson, G.C. The two mucus layers of colon are organized by the MUC2 mucin, whereas the outer layer is a legislator of host-microbial interactions. Proc. Natl. Acad. Sci. USA 2011, 108 (Suppl. 1), 4659-4665. [CrossRef]

71. Holmen Larsson, J.M.; Thomsson, K.A.; Rodriguez-Pineiro, A.M.; Karlsson, H.; Hansson, G.C. Studies of mucus in mouse stomach, small intestine, and colon. III. Gastrointestinal Muc5ac and Muc2 mucin O-glycan patterns reveal a regiospecific distribution. Am. J. Physiol. Gastrointest. Liver Physiol. 2013, 305, G357-G363. [CrossRef] [PubMed]

72. Robbe, C.; Capon, C.; Coddeville, B.; Michalski, J.-C. Structural diversity and specific distribution of O-glycans in normal human mucins along the intestinal tract. Biochem. J. 2004, 384, 307-316. [CrossRef] [PubMed]

73. Lennon, G.; Balfe, A.; Bambury, N.; Lavelle, A.; Maguire, A.; Docherty, N.G.; Coffey, J.C.; Winter, D.C.; Sheahan, K.; O'Connell, P.R. Correlations between colonic crypt mucin chemotype, inflammatory grade and Desulfovibrio species in ulcerative colitis. Colorectal Dis. 2014, 16, O161-O169. [CrossRef] [PubMed]

74. Gibson, G.R.; Cummings, J.H.; Macfarlane, G.T. Growth and activities of sulphate-reducing bacteria in gut contents of healthy subjects and patients with ulcerative colitis. FEMS Microbiol. Ecol. 1991, 86, 103-112. [CrossRef]

75. Moore, J.; Babidge, W.; Millard, S.; Roediger, W. Colonic luminal hydrogen sulfide is not elevated in ulcerative colitis. Dig. Dis. Sci. 1998, 43, 162-165. [CrossRef]

76. Hughes, M.N.; Centelles, M.N.; Moore, K.P. Making and working with hydrogen sulfide: The chemistry and generation of hydrogen sulfide in vitro and its measurement in vivo: A review. Free Radic. Biol. Med. 2009, 47, 1346-1353. [CrossRef]

77. Kolluru, G.K.; Shen, X.; Bir, S.C.; Kevil, C.G. Hydrogen sulfide chemical biology: Pathophysiological roles and detection. Nitric Oxide 2013, 35, 5-20. [CrossRef] [PubMed]

78. Magee, E.A.; Richardson, C.J.; Hughes, R.; Cummings, J.H. Contribution of dietary protein to sulfide production in the large intestine: An in vitro and a controlled feeding study in humans. Am. J. Clin. Nutr. 2000, 72, 1488-1494. [CrossRef] [PubMed]

79. Vitvitsky, V.; Banerjee, R. $\mathrm{H}_{2} \mathrm{~S}$ analysis in biological samples using gas chromatography with sulfur chemiluminescence detection. Methods Enzymol. 2015, 554, 111-123. [CrossRef] [PubMed] 
80. Levine, J.; Ellis, C.J.; Furne, J.K.; Springfield, J.; Levitt, M.D. Fecal hydrogen sulfide production in ulcerative colitis. Am. J. Gastroenterol. 1998, 93, 83-87. [CrossRef]

81. Yao, C.K.; Rotbart, A.; Ou, J.Z.; Kalantar-Zadeh, K.; Muir, J.G.; Gibson, P.R. Modulation of colonic hydrogen sulfide production by diet and mesalazine utilizing a novel gas-profiling technology. Gut Microbes 2018, 1-13. [CrossRef]

82. Anantharaman, K.; Hausmann, B.; Jungbluth, S.P.; Kantor, R.S.; Lavy, A.; Warren, L.A.; Rappe, M.S.; Pester, M.; Loy, A.; Thomas, B.C.; et al. Expanded diversity of microbial groups that shape the dissimilatory sulfur cycle. ISME J. 2018, 12, 1715-1728. [CrossRef]

83. Scanlan, P.D.; Shanahan, F.; Marchesi, J.R. Culture-independent analysis of desulfovibrios in the human distal colon of healthy, colorectal cancer and polypectomized individuals. FEMS Microbiol. Ecol. 2009, 69, 213-221. [CrossRef]

84. Muller, A.L.; Kjeldsen, K.U.; Rattei, T.; Pester, M.; Loy, A. Phylogenetic and environmental diversity of DsrAB-type dissimilatory (bi)sulfite reductases. ISME J. 2015, 9, 1152-1165. [CrossRef] [PubMed]

85. Christophersen, C.T.; Morrison, M.; Conlon, M.A. Overestimation of the abundance of sulfate-reducing bacteria in human feces by quantitative PCR targeting the Desulfovibrio 16S rRNA gene. Appl. Environ. Microbiol. 2011, 77, 3544-3546. [CrossRef] [PubMed]

86. Coutinho, C.; Coutinho-Silva, R.; Zinkevich, V.; Pearce, C.B.; Ojcius, D.M.; Beech, I. Sulphate-reducing bacteria from ulcerative colitis patients induce apoptosis of gastrointestinal epithelial cells. Microb. Pathog. 2017, 112, 126-134. [CrossRef] [PubMed]

87. Hale, V.L.; Jeraldo, P.; Mundy, M.; Yao, J.; Keeney, G.; Scott, N.; Cheek, E.H.; Davidson, J.; Green, M.; Martinez, C.; et al. Synthesis of multi-omic data and community metabolic models reveals insights into the role of hydrogen sulfide in colon cancer. Methods 2018. [CrossRef] [PubMed]

88. Jia, W.; Whitehead, R.N.; Griffiths, L.; Dawson, C.; Bai, H.; Waring, R.H.; Ramsden, D.B.; Hunter, J.O.; Cauchi, M.; Bessant, C.; et al. Diversity and distribution of sulphate-reducing bacteria in human faeces from healthy subjects and patients with inflammatory bowel disease. FEMS Immunol. Med. Microbiol. 2012, 65, 55-68. [CrossRef] [PubMed]

89. Duffy, M.; O’Mahony, L.; Coffey, J.C.; Collins, J.K.; Shanahan, F.; Redmond, H.P.; Kirwan, W.O. Sulfate-Reducing Bacteria Colonize Pouches Formed for Ulcerative Colitis but Not for Familial Adenomatous Polyposis. Dis. Colon Rectum 2002, 45, 384-388. [CrossRef]

90. Windey, K.; De Preter, V.; Verbeke, K. Relevance of protein fermentation to gut health. Mol. Nutr. Food Res. 2012, 56, 184-196. [CrossRef] [PubMed]

91. Kimura, H. Metabolic turnover of hydrogen sulfide. Front. Physiol. 2012, 3, 101. [CrossRef]

92. Awano, N.; Wada, M.; Mori, H.; Nakamori, S.; Takagi, H. Identification and functional analysis of Escherichia coli cysteine desulfhydrases. Appl. Environ. Microbiol. 2005, 71, 4149-4152. [CrossRef]

93. Sahami, S.; Kooij, I.A.; Meijer, S.L.; Van den Brink, G.R.; Buskens, C.J.; Te Velde, A.A. The Link between the Appendix and Ulcerative Colitis: Clinical Relevance and Potential Immunological Mechanisms. Am. J. Gastroenterol. 2016, 111, 163-169. [CrossRef] [PubMed]

94. Rogers, M.B.; Brower-Sinning, R.; Firek, B.; Zhong, D.; Morowitz, M.J. Acute Appendicitis in Children Is Associated with a Local Expansion of Fusobacteria. Clin. Infect. Dis. 2016, 63, 71-78. [CrossRef] [PubMed]

95. Zhong, D.; Brower-Sinning, R.; Firek, B.; Morowitz, M.J. Acute appendicitis in children is associated with an abundance of bacteria from the phylum Fusobacteria. J. Pediatr. Surg. 2014, 49, 441-446. [CrossRef]

96. Basic, A.; Blomqvist, M.; Dahlen, G.; Svensater, G. The proteins of Fusobacterium spp. involved in hydrogen sulfide production from L-cysteine. BMC Microbiol. 2017, 17, 61. [CrossRef] [PubMed]

97. Basic, A.; Blomqvist, S.; Carlen, A.; Dahlen, G. Estimation of bacterial hydrogen sulfide production in vitro. J. Oral Microbiol. 2015, 7, 28166. [CrossRef] [PubMed]

98. Wolf, P.G.; Biswas, A.; Morales, S.E.; Greening, C.; Gaskins, H.R. $\mathrm{H}_{2}$ metabolism is widespread and diverse among human colonic microbes. Gut Microbes 2016, 7, 235-245. [CrossRef]

99. Carbonero, F.; Benefiel, A.C.; Gaskins, H.R. Contributions of the microbial hydrogen economy to colonic homeostasis. Nat. Rev. Gastroenterol. Hepatol. 2012, 9, 504-518. [CrossRef]

100. Macfarlane, S.; Macfarlane, G.T. Regulation of short-chain fatty acid production. Proc. Nutr. Soc. 2003, 62, 67-72. [CrossRef] 
101. Kunkel, D.; Basseri, R.J.; Makhani, M.D.; Chong, K.; Chang, C.; Pimentel, M. Methane on breath testing is associated with constipation: A systematic review and meta-analysis. Dig. Dis. Sci. 2011, 56, 1612-1618. [CrossRef]

102. Macfarlane, G.T.; Gibson, G.R.; Cummings, J.H. Comparison of fermentation reactions in different regions of the human colon. J. Appl. Bacteriol. 1992, 72, 57-64.

103. Rao, S.S.; Kuo, B.; McCallum, R.W.; Chey, W.D.; DiBaise, J.K.; Hasler, W.L.; Koch, K.L.; Lackner, J.M.; Miller, C.; Saad, R.; et al. Investigation of colonic and whole-gut transit with wireless motility capsule and radiopaque markers in constipation. Clin. Gastroenterol. Hepatol. 2009, 7, 537-544. [CrossRef]

104. Videla, S.; Vilaseca, J.; Antolin, M.; Garcia-Lafuente, A.; Guarner, F.; Crespo, E.; Casalots, J.; Salas, A.; Malagelada, J.R. Dietary inulin improves distal colitis induced by dextran sodium sulfate in the rat. Am. J. Gastroenterol. 2001, 96, 1486-1493. [CrossRef] [PubMed]

105. de Vries, J.; Miller, P.E.; Verbeke, K. Effects of cereal fiber on bowel function: A systematic review of intervention trials. World J. Gastroenterol. 2015, 21, 8952-8963. [CrossRef]

106. Walker, A.W.; Duncan, S.H.; McWilliam Leitch, E.C.; Child, M.W.; Flint, H.J. pH and peptide supply can radically alter bacterial populations and short-chain fatty acid ratios within microbial communities from the human colon. Appl. Environ. Microbiol. 2005, 71, 3692-3700. [CrossRef] [PubMed]

107. Duncan, S.H.; Louis, P.; Thomson, J.M.; Flint, H.J. The role of $\mathrm{pH}$ in determining the species composition of the human colonic microbiota. Environ. Microbiol. 2009, 11, 2112-2122. [CrossRef]

108. Gibson, G.R.; Cummings, J.H.; Macfarlane, G.T.; Allison, C.; Segal, I.; Vorster, H.H.; Walker, A.R.P. Alternative pathways for hydrogen disposal during fermentation in the human colon. Gut 1990, 31, 679-683. [CrossRef]

109. Beaumont, M.; Andriamihaja, M.; Lan, A.; Khodorova, N.; Audebert, M.; Blouin, J.M.; Grauso, M.; Lancha, L.; Benetti, P.H.; Benamouzig, R.; et al. Detrimental effects for colonocytes of an increased exposure to luminal hydrogen sulfide: The adaptive response. Free Radic. Biol. Med. 2016, 93, 155-164. [CrossRef] [PubMed]

110. Suarez, F.; Furne, J.; Springfield, J.; Levitt, M. Insights into human colonic physiology obtained from the study of flatus composition. Am. Physiol. Soc. 1997, 272, G1028-G1033. [CrossRef] [PubMed]

111. Levitt, M.D.; Furne, J.; Springfield, J.; Suarez, F.; DeMaster, E. Detoxification of hydrogen sulfide and methanethiol in the cecal mucosa. J. Clin. Investig. 1999, 104, 1107-1114. [CrossRef] [PubMed]

112. Furne, J.; Springfield, J.; Koenig, T.; DeMaster, E.; Levitt, M.D. Oxidation of hydrogen sulfide and methanethiol to thiosulfate by rat tissues: A specialized function of the colonic mucosa. Biochem. Pharmacol. 2001, 62, 255-259. [CrossRef]

113. Picton, R.; Eggo, M.C.; Merrill, G.A.; Langman, M.J.S.; Singh, S. Mucosal protection against sulphide: Importance of the enzyme rhodanese. Gut 2002, 50, 201-205. [CrossRef] [PubMed]

114. Picton, R.; Eggo, M.C.; Langman, M.J.; Singh, S. Impaired detoxication of hydrogen sulfide in ulcerative colitis? Dig. Dis. Sci. 2007, 52, 373-378. [CrossRef]

115. Wilson, K.; Mudra, M.; Furne, J.; Levitt, M. Differentiation of the roles of sulfide oxidase and rhodanese in the detoxification of sulfide by the colonic mucosa. Dig. Dis. Sci. 2008, 53, 277-283. [CrossRef]

116. Landry, A.P.; Ballou, D.P.; Banerjee, R. Modulation of Catalytic Promiscuity during Hydrogen Sulfide Oxidation. ACS Chem. Biol. 2018, 13, 1651-1658. [CrossRef]

117. Filipovic, M.R.; Zivanovic, J.; Alvarez, B.; Banerjee, R. Chemical Biology of $\mathrm{H}_{2} \mathrm{~S}$ Signaling through Persulfidation. Chem. Rev. 2018, 118, 1253-1337. [CrossRef]

118. Landry, A.P.; Ballou, D.P.; Banerjee, R. $\mathrm{H}_{2} \mathrm{~S}$ oxidation by nanodisc-embedded human sulfide quinone oxidoreductase. J. Biol. Chem. 2017, 292, 11641-11649. [CrossRef] [PubMed]

119. Libiad, M.; Yadav, P.K.; Vitvitsky, V.; Martinov, M.; Banerjee, R. Organization of the human mitochondrial hydrogen sulfide oxidation pathway. J. Biol. Chem. 2014, 289, 30901-30910. [CrossRef] [PubMed]

120. Mimoun, S.; Andriamihaja, M.; Chaumontet, C.; Atanasiu, C.; Benamouzig, R.; Blouin, J.M.; Tome, D.; Bouillaud, F.; Blachier, F. Detoxification of $\mathrm{H}(2) \mathrm{S}$ by differentiated colonic epithelial cells: Implication of the sulfide oxidizing unit and of the cell respiratory capacity. Antioxid. Redox Signal. 2012, 17, 1-10. [CrossRef] [PubMed]

121. Sido, B.; Hack, V.; Hochlehnert, A.; Lipps, H.; Herfarth, C.; Droge, W. Impairment of intestinal glutathione synthesis in patients with inflammatory bowel disease. Gut 1998, 42, 485-492. [CrossRef]

122. Fuentes, S.; Rossen, N.G.; van der Spek, M.J.; Hartman, J.H.; Huuskonen, L.; Korpela, K.; Salojarvi, J.; Aalvink, S.; de Vos, W.M.; D'Haens, G.R.; et al. Microbial shifts and signatures of long-term remission in ulcerative colitis after faecal microbiota transplantation. ISME J. 2017, 11, 1877-1889. [CrossRef] 
123. Bajer, L.; Kverka, M.; Kostovcik, M.; Macinga, P.; Dvorak, J.; Stehlikova, Z.; Brezina, J.; Wohl, P.; Spicak, J.; Drastich, P. Distinct gut microbiota profiles in patients with primary sclerosing cholangitis and ulcerative colitis. World J. Gastroenterol. 2017, 23, 4548-4558. [CrossRef]

124. Vermeire, S.; Joossens, M.; Verbeke, K.; Wang, J.; Machiels, K.; Sabino, J.; Ferrante, M.; Van Assche, G.; Rutgeerts, P.; Raes, J. Donor Species Richness Determines Faecal Microbiota Transplantation Success in Inflammatory Bowel Disease. J. Crohn's Colitis 2016, 10, 387-394. [CrossRef]

125. Smith, E.A.; Macfarlane, G.T. Enumeration of human colonic bacteria producing phenolic and indolic compounds: Effects of $\mathrm{pH}$, carbohydrate availability and retention time on dissimilatory aromatic amino acid metabolism. J. Appl. Bacteriol. 1996, 81, 288-302. [CrossRef]

126. Zimmer, J.; Lange, B.; Frick, J.S.; Sauer, H.; Zimmermann, K.; Schwiertz, A.; Rusch, K.; Klosterhalfen, S.; Enck, P. A vegan or vegetarian diet substantially alters the human colonic faecal microbiota. Eur. J. Clin. Nutr. 2012, 66, 53-60. [CrossRef]

127. Devkota, S.; Chang, E.B. Interactions between Diet, Bile Acid Metabolism, Gut Microbiota, and Inflammatory Bowel Diseases. Dig. Dis. 2015, 33, 351-356. [CrossRef]

128. Leone, V.; Gibbons, S.M.; Martinez, K.; Hutchison, A.L.; Huang, E.Y.; Cham, C.M.; Pierre, J.F.; Heneghan, A.F.; Nadimpalli, A.; Hubert, N.; et al. Effects of diurnal variation of gut microbes and high-fat feeding on host circadian clock function and metabolism. Cell Host Microbe 2015, 17, 681-689. [CrossRef]

129. Barcelo, A.; Claustre, J.; Moro, F.; Chayvialle, J.-A.; Cuber, J.-C. Plaisancia. Mucin secretion is modulated by luminal factors in the isolated vascularly perfused rat colon. Gut 2000, 46, 218-224. [CrossRef] [PubMed]

130. Deplancke, B.; Finster, K.; Vallen Graham, W.; Collier, C.T.; Thurmond, J.E.; Gaskins, H.R. Gastrointestinal and Microbial Responses to Sulfate-Supplemented Drinking Water in Mice. Exp. Biol. Med. 2003, 228, 424-433. [CrossRef]

131. Schroeder, B.O.; Birchenough, G.M.H.; Stahlman, M.; Arike, L.; Johansson, M.E.V.; Hansson, G.C.; Backhed, F. Bifidobacteria or Fiber Protects against Diet-Induced Microbiota-Mediated Colonic Mucus Deterioration. Cell Host Microbe 2018, 23, 27-40.e27. [CrossRef] [PubMed]

132. Croix, J.A.; Carbonero, F.; Nava, G.M.; Russell, M.; Greenberg, E.; Gaskins, H.R. On the relationship between sialomucin and sulfomucin expression and hydrogenotrophic microbes in the human colonic mucosa. PLoS ONE 2011, 6, e24447. [CrossRef] [PubMed]

133. Rey, F.E.; Gonzalez, M.D.; Cheng, J.; Wu, M.; Ahern, P.P.; Gordon, J.I. Metabolic niche of a prominent sulfate-reducing human gut bacterium. Proc. Natl. Acad. Sci. USA 2013, 110, 13582-13587. [CrossRef]

134. Dwarakanath, A.D.; Campbell, B.J.; Tsai, H.H.; Sunderland, D.; Hart, C.A.; Rhodes, J.M. Faecal mucinase activity assessed in inflammatory bowel disease using $14 \mathrm{C}$ threonine labelled mucin substrate. Gut 1995, 37, 58-62. [CrossRef] [PubMed]

135. Press, A.G.; Hauptmann, I.A.; Hauptmann, L.; Fuchs, B.; Fuchs, M.; Ewe, K.; Ramadori, G. Gastrointestinal $\mathrm{pH}$ profiles in patients with inflammatory bowel disease. Aliment Pharmacol. Ther. 1998, 12, $673-678$. [CrossRef] [PubMed]

136. Tsai, H.H.; Dwarakanath, A.D.; Hart, C.A.; Milton, J.D.; Rhodes, J.M. Increased faecal mucin sulphatase activity in ulcerative colitis: A potential target for treatment. Gut 1995, 36, 570-576. [CrossRef] [PubMed]

137. Chassaing, B.; Koren, O.; Goodrich, J.K.; Poole, A.C.; Srinivasan, S.; Ley, R.E.; Gewirtz, A.T. Dietary emulsifiers impact the mouse gut microbiota promoting colitis and metabolic syndrome. Nature 2015, 519, 92-96. [CrossRef]

138. Roberts, C.L.; Keita, A.V.; Duncan, S.H.; O’Kennedy, N.; Soderholm, J.D.; Rhodes, J.M.; Campbell, B.J. Translocation of Crohn's disease Escherichia coli across M-cells: Contrasting effects of soluble plant fibres and emulsifiers. Gut 2010, 59, 1331-1339. [CrossRef] [PubMed]

139. Sugihara, K.; Masuda, M.; Nakao, M.; Abuduli, M.; Imi, Y.; Oda, N.; Okahisa, T.; Yamamoto, H.; Takeda, E.; Taketani, Y. Dietary phosphate exacerbates intestinal inflammation in experimental colitis. J. Clin. Biochem. Nutr. 2017, 61, 91-99. [CrossRef]

140. Swidsinski, A.; Ung, V.; Sydora, B.C.; Loening-Baucke, V.; Doerffel, Y.; Verstraelen, H.; Fedorak, R.N. Bacterial overgrowth and inflammation of small intestine after carboxymethylcellulose ingestion in genetically susceptible mice. Inflamm. Bowel Dis. 2009, 15, 359-364. [CrossRef]

141. Sadar, S.S.; Vyawahare, N.S.; Bodhankar, S.L. Ferulic acid ameliorates TNBS-induced ulcerative colitis through modulation of cytokines, oxidative stress, iNOs, COX-2, and apoptosis in laboratory rats. EXCLI J. 2016, 15, 482-499. [CrossRef] 
142. Zheng, H.; Chen, M.; Li, Y.; Wang, Y.; Wei, L.; Liao, Z.; Wang, M.; Ma, F.; Liao, Q.; Xie, Z. Modulation of Gut Microbiome Composition and Function in Experimental Colitis Treated with Sulfasalazine. Front. Microbiol. 2017, 8, 1703. [CrossRef] [PubMed]

143. Laserna-Mendieta, E.J.; Clooney, A.G.; Carretero-Gomez, J.F.; Moran, C.; Sheehan, D.; Nolan, J.A.; Hill, C.; Gahan, C.G.M.; Joyce, S.A.; Shanahan, F.; et al. Determinants of Reduced Genetic Capacity for Butyrate Synthesis by the Gut Microbiome in Crohn's Disease and Ulcerative Colitis. J. Crohn's Colitis 2018, 12, $204-216$. [CrossRef] [PubMed]

144. Valcheva, R.; Koleva, P.; Martinez, I.; Walter, J.; Ganzle, M.G.; Dieleman, L.A. Inulin-type fructans improve active ulcerative colitis associated with microbiota changes and increased short-chain fatty acids levels. Gut Microbes 2018, 1-24. [CrossRef]

145. Earley, H.; Lennon, G.; Balfe, A.; Kilcoyne, M.; Clyne, M.; Joshi, L.; Carrington, S.; Martin, S.T.; Coffey, J.C.; Winter, D.C.; et al. A Preliminary Study Examining the Binding Capacity of Akkermansia muciniphila and Desulfovibrio spp., to Colonic Mucin in Health and Ulcerative Colitis. PLoS ONE 2015, 10, e0135280. [CrossRef]

146. Tsai, H.H.; Sunderland, D.; Gibson, G.R.; Hart, C.A.; Rhodes, J.M. A novel mucin sulphatase from human faeces: Its identification, purification and characterization. Clin. Sci. 1992, 82, 447-454. [CrossRef]

147. Wei, Y.; Gong, J.; Zhu, W.; Tian, H.; Ding, C.; Gu, L.; Li, N.; Li, J. Pectin enhances the effect of fecal microbiota transplantation in ulcerative colitis by delaying the loss of diversity of gut flora. BMC Microbiol. 2016, 16, 255. [CrossRef]

148. Lewis, S.; Brazier, J.; Beard, D.; Nazem, N.; Proctor, D. Effects of metronidazole and oligofructose on faecal concentrations of sulphate-reducing bacteria and their activity in human volunteers. Scand. J. Gastroenterol. 2009, 40, 1296-1303. [CrossRef]

149. Kellingray, L.; Tapp, H.S.; Saha, S.; Doleman, J.F.; Narbad, A.; Mithen, R.F. Consumption of a diet rich in Brassica vegetables is associated with a reduced abundance of sulphate-reducing bacteria: A randomised crossover study. Mol. Nutr. Food Res. 2017, 61. [CrossRef]

150. Wu, G.D.; Chen, J.; Hoffmann, C.; Bittinger, K.; Chen, Y.Y.; Keilbaugh, S.A.; Bewtra, M.; Knights, D.; Walters, W.A.; Knight, R.; et al. Linking long-term dietary patterns with gut microbial enterotypes. Science 2011, 334, 105-108. [CrossRef]

151. Russell, W.R.; Gratz, S.W.; Duncan, S.H.; Holtrop, G.; Ince, J.; Scobbie, L.; Duncan, G.; Johnstone, A.M.; Lobley, G.E.; Wallace, R.J.; et al. High-protein, reduced-carbohydrate weight-loss diets promote metabolite profiles likely to be detrimental to colonic health. Am. J. Clin. Nutr. 2011, 93, 1062-1072. [CrossRef]

152. Sonnenburg, E.D.; Smits, S.A.; Tikhonov, M.; Higginbottom, S.K.; Wingreen, N.S.; Sonnenburg, J.L. Diet-induced extinctions in the gut microbiota compound over generations. Nature 2016, 529, 212-215. [CrossRef] [PubMed]

(C) 2019 by the authors. Licensee MDPI, Basel, Switzerland. This article is an open access article distributed under the terms and conditions of the Creative Commons Attribution (CC BY) license (http://creativecommons.org/licenses/by/4.0/). 


\title{
A Review of Dietary Therapy for IBD and a Vision for the Future
}

\author{
Nicole Green, Talya Miller, David Suskind and Dale Lee * \\ Department of Pediatric Gastroenterology and Hepatology, Seattle Children's Hospital, Seattle, WA 98105, USA; \\ nicole.green@seattlechildrens.org (N.G.); talya.miller@seattlechildrens.org (T.M.); \\ david.suskind@seattlechildrens.org (D.S.) \\ * Correspondence: dale.lee@seattlechildrens.org; Tel.: +1-206-987-7339
}

Received: 31 March 2019; Accepted: 24 April 2019; Published: 26 April 2019

\begin{abstract}
Inflammatory bowel disease (IBD) is a chronic inflammatory condition affecting the gastrointestinal tract. The rising incidence of IBD has been associated with urbanization and shifts toward a Westernized diet. The intestinal microbiome has been a focus of disease pathogenesis and also therapeutic intervention. Dietary therapy for IBD has been well-studied with exclusive enteral nutrition, a formula-based diet with the exclusion of foods. In addition, interest in food-based exclusion diets has been increasing, with patients and families leading the charge. Challenges with dietary therapy for IBD include the lack of understanding of a detailed mechanistic pathway to explain the impact of diet on IBD pathogenesis and the difficult nature of designing and implementing dietary clinical trials. Epidemiological studies have demonstrated associations and intervention studies have demonstrated efficacy, but specific dietary targets remain as hypotheses at present. Current IBD therapy focuses on suppression of the immune system, yet the incomplete efficacy of present drugs suggests that other therapies must be developed and employed. Dietary interventions, with known ability to modulate the intestinal microbiome, are a unique opportunity to improve outcomes in IBD. Dietary intervention trials are challenging, and capturing both broad dietary patterns as well as exposure to individual food compounds is important. With increasing patient interest and preliminary research in dietary therapy indicating efficacy, it is imperative to further advance the science of utilizing diet in IBD, as well as to support patients by proactively addressing diet within their care plan.
\end{abstract}

Keywords: inflammatory bowel disease; Crohn; ulcerative colitis; diet; nutrition; exclusive enteral nutrition

\section{Background}

Nutritional interventions play a central role in the treatment and management of inflammatory bowel disease (IBD). While malnutrition is a common presentation in IBD, nutritional interventions have not only focused on correcting nutrient deficiencies but also on improving disease activity and symptoms. In addition to other societies, the North American Society for Pediatric Gastroenterology Hepatology and Nutrition has issued clinical guidelines emphasizing the importance of nutritional interventions in pediatric IBD. These guidelines focus on the use of exclusive enteral nutrition (EEN), a formula-based therapy, in pediatric Crohn's disease [1-3]. Since the publication of these guidelines, further research has expanded the concept of food-based nutritional intervention in patients with IBD. Recent research has shown the impact of diet on all three components of the IBD paradigm: the intestinal microbiome, mucosal integrity, and the immune system. With known influence on disease pathogenesis and perpetuation, diet in IBD is developing into a powerful, state-of-the-art treatment for IBD. 
The history of nutritional therapy for IBD began with initial observations in hospitalized adults with severe Crohn's disease who improved on EEN [4,5]. Since the initial report of EEN in IBD in the late 1970s, there have been over 200 publications on EEN with multiple meta-analyses showing that the use of EEN in children with Crohn's disease is as effective as corticosteroids at inducing remission of active inflammation [6,7]. In adults with Crohn's disease, EEN has shown conflicting results. A 2018 Cochrane Database Systematic Review comparing EEN versus steroids showed greater efficacy of steroids [8]. It is unclear whether the perceived difference between efficacy in pediatric and adult EEN studies is related to compliance with therapy, greater duration of disease, or prior exposures to immunosuppressive drugs. Two studies in adults with newly diagnosed Crohn's disease showed similar efficacy with EEN involving a nasogastric tube option $[9,10]$.

Immunosuppressive therapies are not effective at treating IBD for all patients and carry risk. The foods we eat have been hypothesized to play a role in pathogenesis as well as exacerbation of inflammation for many years. With basic science research showing the effect of dietary exposures on the intestinal microbiome as well as mucosal integrity, renewed interest has emerged on the ability of diet to treat the inflammatory burden characterizing patients with IBD. Despite the complexities and difficulty in doing clinical nutrition research, epidemiologic research has identified dietary components that are protective and risk factors for the development of IBD [11,12]. In addition, many diets have been reported to be efficacious in small case series, including the specific carbohydrate diet (SCD), IBD anti-inflammatory diet (IBD-AID), Crohn's disease exclusion diet (CDED), and semi-vegetarian diet [13]. The SCD developed by Dr. Sydney Haas, a pediatrician, in the 1930s to treat patients with celiac disease has been one of the better studies of exclusion diets used in IBD [14]. It was popularized in the late 20th century by Elaine Gottschall, whose daughter's ulcerative colitis was successfully treated with the SCD by Dr. Haas [15]. The SCD and other exclusion diets have risen in popularity among patients, and research efforts are underway attempting to keep pace with patient demand.

\section{IBD Pathogenesis}

The pathogenesis of IBD, while not completely elucidated, is thought to be multifactorial with interplay between genetics, the environment, and the immune system. The major environmental factors associated with IBD include shifts in diet, antibiotics, other medications, air pollution, infections, major life stressors, and smoking [12,16]. Diet is an environmental exposure that has changed over time, is modifiable, and has increasingly become a component of the treatment paradigm for IBD. A look at the epidemiologic patterns of IBD, with highest prevalence in Northern Europe and North America and rapidly increasing incidence in more-industrialized countries, has led to the theory that a Westernized lifestyle, and in particular diet, is linked to the rising incidence of IBD [17].

Understanding the role of dietary therapy in IBD requires an appreciation of the intestinal microbiome and intestinal mucosal integrity or barrier function. The intestinal microbiota consists of thousands of bacterial species with a gene pool 150 times larger than the human genome, with tremendous functional diversity [18]. The intestinal mucosal barrier consists of epithelial cells connected by tight junctions, antimicrobial factors, and overlying inner and outer mucus layers that limit the interaction between the microbiome and immune system [19]. In IBD, the homeostasis between the microbiome, intestinal barrier, and immune system is disrupted resulting in dysbiosis, increased barrier permeability, and inflammation [20].

The intestinal microbiome is highly adaptable and responsive to environmental factors. From the time of birth, environmental factors such as mode of delivery (vaginal versus caesarean section) and feeding method (breast feeding versus formula feeding) influence the microbiome [21,22]. Dietary patterns have a profound effect on the gut microbiome, and Wu et al., demonstrated that a high fat and animal protein diet compared to a high carbohydrate diet was associated with significant differences in the intestinal microbiome [23]. Numerous studies have highlighted differences in the microbiome between individuals with IBD and healthy individuals, with an overall trend towards loss of diversity of the microbiota in IBD [24-27]. A decrease in certain Clostridium clusters is a consistent finding 
among studies $[20,25]$. These species stimulate regulator $\mathrm{T}$ cells, resulting in immune tolerance and reduced gastrointestinal inflammation [20,28]. Conversely, members of the family Enterobacteriaceae are consistently elevated in IBD [20]. These include the well-known intestinal pathogens Campylobacter spp., Salmonella spp., and Escherichia coli. Whether these patterns are a cause or consequence of intestinal inflammation remains debatable. In the healthy state, the microbiota comes into contact with epithelial cells and the immune system in a highly controlled manner [29]. In IBD, the mucosal barrier is compromised, resulting in translocation of the intestinal microbiota and resultant immune system activation and inflammatory response [30].

\section{Scientific Basis of Dietary Therapy for IBD}

Many studies have evaluated the ability of diet to modulate intestinal microbiota and influence epithelial barrier function. Low fiber diets have been linked to IBD with a posited mechanism of reduction in short-chain fatty acid production by commensal bacteria whose preferred energy source is fiber [31]. Butyrate, a short-chain fatty acid, is essential for colonic health and the major energy source for colonocytes [32]. In addition to supporting intestinal barrier function, short-chain fatty acids also promote immune tolerance by promoting development of T-regulatory cells $[33,34]$. Food additives are commonly consumed by patients with IBD, and specific dietary emulsifiers (carboxymethylcellulose and polysorbate 80 ) have been shown to induce low grade inflammation and metabolic syndrome in wild type mice and promote colitis in genetically predisposed IL-10 knockout mice [35,36]. The emulsifiers perturbed the host microbiota, resulting in increased inflammatory potential with a rise in the number of mucolytic bacteria, and erosion of the protective mucous layer. At present, efforts are underway to better evaluate the frequency of exposure to food additives and the effect of these compounds on the intestinal system.

A summary of clinical trials and data reporting on the outcomes of dietary therapies in IBD are well described in greater detail elsewhere [3,37-39]. Importantly, many of these trials are smaller in size, deemed to produce a lower grade of evidence, and limited by the lack of long-term outcome data. In a recently published Cochrane review, Limketkai et al. analyzed 18 randomized controlled trials, comprising 1878 participants, published between 1965 and 2018 [37]. The intervention diets involved complete exclusion or significant limitation of one or more food groups. Diets included low refined carbohydrates; low microparticles; low calcium; low red and processed meat; low disaccharides, grain, saturated fats, red, and processed meat; symptoms-guided diets; highly-restricted organic diet; milk-free; Alberta-based anti-inflammatory diet; and Carrageenan-free diet. The different studies looked at various outcomes, including induction of remission, clinical relapse, surrogate biomarkers of inflammation, endoscopic improvement, health-related quality of life, and the need for surgery. This review concluded that no firm conclusions could be reached about the effect of these dietary interventions on IBD.

Though these included studies may be superior in that they are randomized controlled studies, additional studies on EEN and broader restrictions diets have shown clearer impact on IBD [40-42]. A key distinction from the randomized trials is the more profound nature of dietary intervention with EEN and many restrictions diets. Though not all controlled studies, the findings in these numerous, heterogeneous studies suggest the impact of dietary interventions on inflammation and clinical outcomes. Together with strong patient desire for dietary guidance and the opportunity for greater efficacy with conventional immunosuppression, larger more definitive studies are needed.

The most rigorously studied dietary interventions in IBD is exclusive enteral nutrition (EEN), a formula-based therapy for Crohn's disease. Numerous studies in children and adolescents have demonstrated the ability of EEN to induce remission of active Crohn's disease in $80-85 \%$ of patients $[43,44]$. EEN is equivalent to corticosteroid therapy in inducing clinical remission and superior at achieving endoscopic mucosal healing [43,45]. EEN is a first-line therapy for pediatric Crohn's disease in many parts of the world, and the treatment protocol typically involves administration of formula to provide $100 \%$ of caloric needs and exclusion of food for $6-8$ weeks [1]. 
The exact mechanism by which EEN exerts its effect is unknown. Hypothesized mechanisms (Table 1) include limiting antigen exposure, antigenic monotony, enhancing nutritional status and nutrient delivery, altering the microbiome and immune response, and avoidance of deleterious compounds $[3,5,20,46]$. As EEN and exclusion diets are extremely different interventions, it is likely that the mechanism by which they impact disease is likewise different. Despite disease activity improvement, studies looking at fecal metagenomics in children with Crohn's disease have found that EEN seems to decrease gut microbiota diversity and promote a more "dysbiotic" state when compared to healthy controls [47]. Microbial functional capacity also decreased with EEN as did genes encoding proteins involved in B-complex vitamin biosynthesis [47]. In studies evaluating changes to the intestinal microbiome in patients with IBD treated with conventional medical therapies, dysbiosis improved with therapy [48]. Clearly therefore, the relationship between the beneficial effects of EEN and alterations in the gut microbiota needs further characterization and may result from alterations in beneficial or harmful metabolites produced by the bacteria.

Table 1. Hypothesized Mechanisms of Action for Diet Therapy in Inflammatory Bowel Disease (IBD).

\begin{tabular}{|c|c|c|c|}
\hline Category of Mechanism & Hypothesized Mechanism & EEN & Exclusion Diets \\
\hline \multirow{3}{*}{ Mechanical/physical } & Liquid nutrition & $\mathrm{x}$ & \\
\hline & Alteration of gut motility & $x$ & $x$ \\
\hline & Gut rest & $\mathrm{x}$ & \\
\hline \multirow{2}{*}{ Nutritional status } & Improved nutrient delivery & $x$ & \\
\hline & Improved caloric delivery & $\mathrm{x}$ & \\
\hline \multirow{3}{*}{ Epithelial barrier } & Decreased permeability & $\mathrm{x}$ & $\mathrm{x}$ \\
\hline & Restitution of intestinal barrier & $\mathrm{x}$ & $\mathrm{x}$ \\
\hline & Improved delivery of fiber (SCFA * production) & & $x$ \\
\hline \multirow{4}{*}{ Immune system } & Limited antigen exposure & $x$ & \\
\hline & Antigenic monotony & $x$ & \\
\hline & Direct anti-inflammatory effect & $\mathrm{x}$ & $\mathrm{x}$ \\
\hline & Alteration in bile acids & $\mathrm{x}$ & $\mathrm{x}$ \\
\hline \multirow{2}{*}{ Microbiome } & Shift of gut microbiome & $\mathrm{x}$ & $\mathrm{x}$ \\
\hline & Stabilize gut microbiome & $\mathrm{x}$ & $\mathrm{x}$ \\
\hline \multirow{2}{*}{ Specific avoidances } & Avoidance of food additives & & $\mathrm{x}$ \\
\hline & Avoidance of deleterious food substances & $\mathrm{x}$ & $\mathrm{x}$ \\
\hline \multirow{2}{*}{ Anti-inflammatory effect } & Provide beneficial substance & $\mathrm{x}$ & $\mathrm{x}$ \\
\hline & Increased antioxidant consumption & & $\mathrm{x}$ \\
\hline
\end{tabular}

EEN established the ability of diet to drive Crohn's disease into remission, but EEN is difficult to sustain as long-term maintenance therapy and is not effective for ulcerative colitis. Exclusion diets, though, are practical as long-term therapy and have been found to be useful in both Crohn's disease and ulcerative colitis. One of the most studied exclusion diets is the specific carbohydrate diet (SCD). This diet removes all grains, sweeteners (except for honey), processed food, and all milk products except for hard cheeses and yogurt fermented longer than $24 \mathrm{~h}$. Both clinical and laboratory improvement have been reported in pediatric and adult patients with IBD [49-52]. One study over 12 weeks in children and adolescents used capsule endoscopy and demonstrated mucosal healing [53]. Therapy with the SCD has been shown to result in significant changes in microbial composition [49]. The SCD has been the best-studied food-based diet for IBD, and one survey with 417 respondents found patients reported significant improvement in symptoms, but that only $17 \%$ of patients partnered with a healthcare professional in the implementation of the SCD [50].

The Crohn's disease exclusion diet (CDED) is based on the hypothesis that components of the Western diet promote a pro-inflammatory microbiome and may disrupt the mucosal barrier. The diet focuses on excluding gluten, dairy, gluten-free baked goods, animal fat, emulsifiers, and all canned 
or processed foods. A prospective cohort of pediatric and adult participants with mild to moderate Crohn's disease treated with partial enteral nutrition (formula providing approximately $50 \%$ of daily calories) and the CDED showed success in achieving induction of clinical remission [41]. The IBD anti-inflammatory diet (IBD-AID) is derived from the SCD. It is a whole foods-based diet that restricts the intake of complex carbohydrates such as refined sugar, gluten-based grains, and certain starches from the diet, but also incorporates ingestion of prebiotics and probiotics. The diet also incorporates phases of food textures. In a small retrospective case series of patients with IBD on the IBD-AID for at least 4 weeks, all demonstrated improvement in clinical symptoms [54].

In a study of a semi-vegetarian diet in patients with remission of Crohn's disease induced by either medical therapy or surgery, patients maintained a greater rate of clinical remission over 2 years [55]. The CD-TREAT diet hypothesizes that a whole-food diet based on the composition of Modulen ${ }^{\mathrm{TM}}$, a commonly utilized formula for EEN in Europe, can emulate the efficacy of EEN [56]. In the component of the study evaluating healthy adults, similar microbiome and metabolome changes were seen with both the CD-TREAT diet and EEN. In the part of the study evaluating five children with active Crohn's disease, the CD-TREAT diet resulted in a significant decline in fecal calprotectin, though this surrogate marker of intestinal inflammation remained elevated at both 4 and 8 weeks.

The worsening of dysbiosis and decrease in butyrate production demonstrated with EEN therapy is seemingly counterintuitive, as is the lack of any fiber content in the formulas commonly utilized for EEN $[57,58]$. It may be the case that EEN acts via a unique mechanism of action to impact inflammation in IBD in comparison to the action of restrictions diets. As the gut microbiome can both drive inflammation and respond to underlying inflammation, further elucidation of the complex interaction between diet, microbiome, and host will help guide future therapy. With the paradoxical findings seen in EEN versus whole food restriction diets, changes occurring while transitioning from one diet to another may provide unique insights into mechanisms.

Epidemiologic studies have demonstrated greater risk of developing IBD with increased intake of total fat, polyunsaturated fatty acids, omega- 6 fatty acids, and meat, while fruits, vegetables, and fiber intake have been shown to have protective effects [11]. Greater meat intake has been associated with both increased risk of ulcerative colitis relapse in adults and decreased rate of achieving remission of Crohn's disease in children on partial enteral nutrition therapy [59,60]. Conversely the only existing randomized study on red meat showed no association between red meat or processed meat consumption and risk of Crohn's disease relapse, as measured by self-reported symptoms [61]. A limitation of these studies evaluating individual dietary components is the potential for confounding factors due to numerous associations between individual foods consumed [62].

While some dietary interventions have demonstrated impacts on inflammation, other interventions, such as the low FODMAP diet, have been demonstrated to impact functional gastrointestinal symptoms in patients with IBD [63-65]. A variety of exclusion diets have shown efficacy at treating inflammation in small case series reports, but additional studies are needed to better substantiate these findings. While these studies suggest that specific food components may be deleterious, it may be the complex interactions of food components within the food matrix with the intestinal microbiome that trigger and perpetuate the cycle of inflammation in IBD. At present, the mechanism by which dietary interventions impact inflammation in IBD remains unknown. Studies involving the microbiome, metabolome, and proteome are beginning to shed insight, and will help guide the movement to more targeted dietary therapeutic approaches in the future. The mechanism of action for dietary therapies requires further study in both clinical and preclinical models.

\section{Contrasting Pharmacological and Dietary Therapy}

The goals of IBD therapy include resolution of symptoms, mucosal healing, improved quality of life, nutritional restitution, and minimizing adverse effects associated with therapy. For both nutritional therapy and conventional medical therapy, disease remission is often a singular focus, but the broader goals of the patient are important in the discussions about therapy. Primary outcomes in dietary 
and drug trials for IBD, though, should be the same, with a focus on evaluation of objective markers of inflammation, including labs, imaging, endoscopic findings, and surrogate markers of intestinal inflammation. An ongoing challenge for dietary trials is the ability to fund trials that are not sponsored by corporations with a financial incentive to drive development of a new therapeutic.

EEN in pediatric Crohn's disease has been demonstrated to lead to high rates of remission $(85 \%)$ and superior mucosal healing when compared to treatment with corticosteroids $[40,43,66]$. Immunosuppressive therapy for IBD with monoclonal antibody anti-TNF-alpha drugs (e.g., infliximab or adalimumab), with or without concomitant immunomodulatory drugs are widely viewed as one of the most efficacious approaches to inducing clinical remission. A randomized-controlled trial in adults with newly diagnosed Crohn's disease demonstrated steroid-free remission at week 26 for $56.8 \%$ of patients on infliximab plus azathioprine, and $44.4 \%$ for those on infliximab alone [67]. A trial of infliximab in children demonstrated clinical remission at week 10 in $58.9 \%$ of participants, and $55.8 \%$ at week 54 [68]. In the ACT1 and ACT2 trials of infliximab in adult ulcerative colitis, clinical remission at week 8,30 , and 54 was never above $40 \%$ [69]. Similarly, a pediatric trial of infliximab in ulcerative colitis showed week 54 remission in less than $40 \%$ of study participants [70]. These data suggest that medications, though effective, do not induce remission in all patients with IBD, and room exists to further optimize therapy with newer medical therapies, and dietary interventions.

Like drugs, dietary therapy is not effective for all patients, and future personalization of dietary therapy is an opportunity. Dietary therapy can be utilized as stand-alone monotherapy, or diet can also be utilized concomitant with immunosuppressive drugs. A study in Japan evaluating the utility of partial enteral nutrition therapy (greater than $900 \mathrm{kcal} /$ day from enteral nutrition with formula) with infliximab demonstrated a significant decrease in disease recurrence over the course of nearly 2 years [71]. Patients commonly employ dietary modifications to address symptoms but also attempt to control inflammation with diet [72]. Implementation of dietary changes is often guided by individual research and anecdotal experience, and the majority of patients are not guided by their medical team to involve diet as part of their IBD therapy plan [13]. At present the push to study and employ dietary measures is strongly driven by patients, while many medical practitioners remain skeptical and poorly informed about dietary interventions. As an example, despite overwhelming evidence for the use of EEN in pediatric CD, U.S. physicians still use EEN to a much lesser extent than their European and Canadian colleagues [73].

That patients and families will employ dietary therapy despite the rigor this involves suggests that tangible benefits exist. Given that therapies may be intensive for weeks to months (e.g., EEN), or require changes to dietary patterns long-term, the presence of family and community support can be key in the success of the patient's ability to adhere to the treatment. There has been limited data published on the patient experience while on EEN; however, a study of 29 families of patients with CD who chose EEN as induction therapy showed $59 \%$ of patients would repeat EEN if needed. However, $72 \%$ of parents would be more interested in a solid food based exclusion diet option [74].

There is generally a lesser degree of concern regarding the safety of nutritional therapy when compared to immunosuppressive drug therapies (Table 2). However, potential side effects can include nutritional inadequacy, psychosocial impact, and a potential for loss to medical follow-up. Psychosocial impact in the setting of nutritional therapy was evaluated by the measure of quality of life (QOL) in children and adolescents in a trial over 8 weeks with anti-TNF therapy, partial enteral nutrition, and exclusive enteral nutrition therapy [60]. This study demonstrated that children with Crohn's disease who achieved clinical remission on any of the three intervention arms had a significant increase in the quality of life measured by the IMPACT-III questionnaire, a validated disease-specific measure of QOL in pediatric IBD. This study evaluated sub-domains of QOL and found that body image-associated QOL was superior in the EEN group compared to the anti-TNF therapy group. Exclusion diets can be isolating and difficult to maintain, and certain patients may not be a good fit for dietary therapy. For those who demonstrate interest in dietary therapy, close monitoring, support of their family and friends, and partnership with the broader medical team (including physician, dietitian, 
and psychologist) helps with maintaining compliance and ensuring appropriateness of an ongoing dietary approach to IBD.

Table 2. Comparison of Dietary Therapy and Drug Therapy.

\begin{tabular}{ccc}
\hline & Dietary Therapy & Drug Therapy \\
\hline Driven by patient/family interest & ++ & + \\
Ability to personalize & ++ & \\
Directly impacts quality of life & ++ & + \\
Importance of family support & ++ & + \\
Challenges with compliance & ++ & \\
Challenges with study design & ++ & ++ \\
Risk of nutrient deficiency & ++ & ++ \\
Works in combination with other therapies & ++ & ++ \\
Physician-driven & & ++ \\
Targets known pathway & & ++ \\
Greater risk of infection & & ++ \\
Greater risk of cancers & & ++ \\
Insurance coverage & & \\
\hline
\end{tabular}

* Independent of disease activity. ${ }^{* *}$ Formula companies have supported the design and implementation of some studies on EEN, but the majority of dietary trials do not have corporate backing.

Since dietary therapies can be employed without physician prescription, there is potential for loss of medical follow-up. Without partnership with the medical team, dietary therapy may result in inadequate nutritional intake and a lack of appropriate objective evaluation of clinical, laboratory, and mucosal remission. When considering potential side effects of nutritional therapy, medical professionals must acknowledge that many patients are on dietary therapy as treatment for their IBD without formal medical guidance [50]. At a minimum, the medical team should engage patients in discussion about their dietary patterns, understand the basics of commonly utilized IBD exclusion diets, and partner with patients to closely monitor nutritional status and disease activity. Medical therapies require formal prescription from a healthcare professional and insurance provides coverage, or at least provides coverage of alternative medications. At present, dietary therapy for IBD is not broadly reimbursed by insurance. Further data on efficacy of exclusion diets is needed, but even for established therapy like EEN for Crohn's disease, insurance companies do not provide reimbursement in the United States.

Despite the many challenges around utilizing dietary therapy for IBD, dietary interventions are eagerly sought and employed by patients. The desire to avoid or lessen the degree of immunosuppression or supplement existing medical therapies are common drivers. Given the burden associated with dietary modification when considering cost, time for food preparation, and dietary restriction, it is important to recognize that nutritional therapy may not be an appealing option for many patients. For these reasons, not all patients and families will have equitable access to or interest in nutritional therapies. No studies have yet shown the variation in average costs between the different dietary interventions.

\section{Vision for the Future of Diet and IBD}

Dietary trials present challenges, but the ability to use nutrition to treat disease is a unique opportunity. It has long been accepted that dietary exposures are associated with risk of developing IBD, but diet as therapy has not been fully integrated into the broader paradigm of IBD therapy (Table 3). Diet represents a fundamental aspect of daily living and has infinite variability; diet is a potential target for profound intervention. Dietary intervention with EEN or even a shift in fat and fiber consumption results in a tangible shift in the intestinal microbiome [23,47,75]. Efforts have been placed into the development of therapeutic approaches to selectively modulate the intestinal 
microbiome, including prebiotics, probiotics, antibiotics, and fecal transplantation, but persistent shaping of the microbiome requires ongoing maintenance of these therapies [76]. Dietary therapies that reshape patterns of food consumption may alter exposures to deleterious substances such as select food additives, directly influence the intestinal microbiome, and also have a direct impact on the functioning of the host immune system [3].

Table 3. Dietary Therapy for IBD: Now and the Future.

\begin{tabular}{c} 
Current Accepted Place of Dietary Therapy \\
EEN in pediatric Crohn's disease \\
Dietary therapies for symptom control \\
Nutritional therapy to support malnutrition \\
\hline Major Areas Requiring Research \\
\hline Impact of dietary therapies with/without concomitant immunosuppression \\
Impact of dietary therapies long-term \\
Mechanism of action for dietary therapies \\
Exclusion diets-dietary components of importance \\
Cost-benefit analysis of dietary interventions \\
Targeted dietary approaches to selectively modulate microbiome
\end{tabular}

Many individuals institute dietary changes based on varied sources of information and also as a reaction to the onset of IBD symptoms $[13,77]$. Recommendations are of variable validity, and better data on the role of diet in the setting of active IBD is needed. The data on EEN therapy has led to the utilization of this as standard therapy for induction of remission in pediatric Crohn's disease. The limitation of EEN is the difficulty in sustaining the intervention. Food-based IBD trials can involve exclusion, supplementation, or dramatic changes in dietary pattern [62]. Thus far, it appears unlikely that focused exclusion or supplementation have a role in IBD therapy, but more profound changes in dietary pattern have shown promise. Though individual dietary components may be deleterious (such as refined sugars, high fat, and specific food additives), it may be the case that the effect of these components are balanced or attenuated by other components (e.g., fiber, antioxidants, bioactive molecules). As such, we suggest that future dietary trials in IBD should consider the global dietary pattern, while also considering the role of individual dietary components. At present, published trials for exclusion diets in IBD often include a description of the general principles of the diet, but do not further characterize the variability within the proposed intervention and the impact this has on clinical outcomes. Challenges to consider in food-based dietary trials in IBD include issues with participant compliance, the measurement of compliance, and variability within a particular dietary intervention.

Though randomized controlled trials are the gold standard for the generation of evidence to drive clinical practice, innovative study design such as n-of- 1 studies, and data from larger cohort studies, together with preclinical data, will also help to better substantiate the existing literature supporting dietary therapies in IBD. The paradigm of relying only on data from controlled trials is challenging in the setting of dietary interventions requiring profound changes to habitual diet, the need to maintain compliance, and the cost of implementing such trials.

As diet can be a primary or adjunctive therapy, the magnitude of effect considered as significant may be different for dietary trials as compared to drug trials. For example, if a dietary intervention results in lack of endoscopic mucosal healing but significantly decreases inflammatory burden as measured by biochemical markers, this may suggest that the intervention has a promising role as an adjunct to conventional immunosuppressive therapy. As the mechanism by which dietary therapy impacts IBD involves gut luminal exposures, the microbiome, and triggering of the mucosal immune system, diet has the potential to be utilized alongside medications to improve outcomes and durability, and minimize risks associated with immunosuppression. Drugs are not commonly brought to market with known partial efficacy, and they are not tested as frequently as adjuncts to existing therapies, but this is different for dietary therapy. Dietary trials demonstrating complete remission or even some 
response to therapy may have utility, as the cost and side-effect profile of dietary therapy is generally favorable for dietary therapy.

For novel pharmacologic therapeutics, often the initial identification and targeting of a specific molecular pathway guides drug development. The mechanism by which diet impacts IBD has yet to be fully elucidated [38,78]. Though the microbiome and specific deleterious substances have been identified, as of yet no definitive molecular pathway target exists for dietary therapy. At present, basic scientific research is ongoing to better elucidate mechanisms of action for both EEN and exclusion diets on treatment of active IBD, but empiric clinical trials are being pushed forward as well. Unlike drugs, with guidance, diet can be modulated within the bounds of safety, balance, and nutritionally-adequacy. Empiric dietary changes guided by experience may have value, but systematic evaluation of dietary interventions with trials is needed to produce the data to demonstrate efficacy beyond anecdote. Publication bias in favor of positive studies and poorly designed clinical studies are a limitation to the existing data for dietary therapy in IBD and need to be better addressed moving forward. A variety of exclusion diets have been proposed as therapy for IBD, and with systematic evaluation these trials will inform pre-clinical research, which in turn will help guide future trials.

Close partnership between the patient, gastroenterologist, and dietitian is necessary when utilizing dietary therapy to treat IBD. The discussion about the concept of integrating dietary therapy, goals of therapy, concerns, and a close monitoring plan should begin early. In some situations, diet will be used as monotherapy and it is of particular importance that close follow-up and monitoring of objective markers of inflammation occur, so as to prevent disease progression, select nutrient deficiencies, or global malnutrition. For physicians, the most common IBD treatment paradigms have dismissed the role of diet. A partnership with acknowledgement of the need for further clinical trial data, inherent uncertainty of efficacy for all IBD therapies, and the potential for benefit with dietary interventions will help guide progress towards better understanding of the utility of dietary therapy for individuals with IBD.

Author Contributions: N.G. contributed in design, authorship, and editing of the manuscript. She has approved of the final draft submitted. T.M. contributed in design, authorship, and editing of the manuscript. She has approved of the final draft submitted. D.S. contributed in design, authorship, and editing of the manuscript. He has approved of the final draft submitted. D.L. contributed in design, authorship, and editing of the manuscript. He has approved of the final draft submitted.

Funding: This research received no external funding.

Conflicts of Interest: The authors declare no conflicts of interest.

\section{References}

1. Critch, J.; Day, A.S.; Otley, A.; King-Moore, C.; Teitelbaum, J.E.; Shashidhar, H.; NASPGHAN IBD Committee. Use of enteral nutrition for the control of intestinal inflammation in pediatric Crohn disease. J. Pediatr. Gastroenterol. Nutr. 2012, 54, 298-305. [CrossRef] [PubMed]

2. Kleinman, R.E.; Baldassano, R.N.; Caplan, A.; Griffiths, A.M.; Heyman, M.B.; Issenman, R.M.; Lake, A.M. Nutrition support for pediatric patients with inflammatory bowel disease: A clinical report of the North American society for pediatric gastroenterology, hepatology and nutrition. J. Pediatr. Gastroenterol. Nutr. 2004, 39, 15-27. [CrossRef]

3. Lee, D.; Albenberg, L.; Compher, C.; Baldassano, R.; Piccoli, D.; Lewis, J.D.; Wu, G.D. Diet in the pathogenesis and treatment of inflammatory bowel diseases. Gastroenterology 2015, 148, 1087-1106. [CrossRef]

4. Voitk, A.J.; Brown, R.A.; McArdle, A.H.; Hinchey, E.J.; Gurd, F.N. Clinical uses of an elemental diet: Preliminary studies. Can. Med. Assoc. J. 1972, 107, 123-129. [PubMed]

5. Voitk, A.J.; Echave, V.; Feller, J.H.; Brown, R.A.; Gurd, F.N. Experience with elemental diet in the treatment of inflammatory bowel disease. Is this primary therapy? Arch. Surg. 1973, 107, 329-333. [CrossRef] [PubMed]

6. Ricour, C.; Duhamel, J.F.; Nihoul-Fekete, C. Use of parenteral and elementary enteral nutrition in the treatment of Crohn's disease and ulcerative colitis in children. Arch. Fr. Pediatr. 1977, 34, 505-513. [PubMed]

7. Navarro, J.; Vargas, J.; Cezard, J.P.; Charritat, J.L.; Polonovski, C. Prolonged constant rate elemental enteral nutrition in Crohn's disease. J. Pediatr. Gastroenterol. Nutr. 1982, 1, 541-546. [CrossRef] [PubMed] 
8. Narula, N.; Dhillon, A.; Zhang, D.; Sherlock, M.E.; Tondeur, M.; Zachos, M. Enteral nutritional therapy for induction of remission in Crohn's disease. Cochrane Database Syst. Rev. 2018, 4, CD000542. [CrossRef] [PubMed]

9. Okada, M.; Yao, T.; Yamamoto, T.; Takenaka, K.; Imamura, K.; Maeda, K.; Fujita, K. Controlled trial comparing an elemental diet with prednisolone in the treatment of active Crohn's disease. Hepatogastroenterology 1990, $37,72-80$.

10. O'Morain, C.; Segal, A.W.; Levi, A.J. Elemental diet as primary treatment of acute Crohn's disease: A controlled trial. Br. Med. J. (Clin. Res. Ed.) 1984, 288, 1859-1862. [CrossRef]

11. Hou, J.K.; Abraham, B.; El-Serag, H. Dietary intake and risk of developing inflammatory bowel disease: A systematic review of the literature. Am. J. Gastroenterol. 2011, 106, 563-573. [CrossRef]

12. Ananthakrishnan, A.N. Epidemiology and risk factors for IBD. Nat. Rev. Gastroenterol. Hepatol. 2015, 12, 205-217. [PubMed]

13. Hou, J.K.; Lee, D.; Lewis, J. Diet and inflammatory bowel disease: Review of patient-targeted recommendations. Clin. Gastroenterol. Hepatol. 2014, 12, 1592-1600. [CrossRef] [PubMed]

14. Haas, S.V.; Haas, M.P. The treatment of celiac disease with the specific carbohydrate diet; report on 191 additional cases. Am. J. Gastroenterol. 1955, 23, 344-360. [PubMed]

15. Gottschall, E. Breaking the Viscious Cycle, 2nd ed.; Kirkton Press Limited: Baltimore, ON, Canada, 1994.

16. Ananthakrishnan, A.N.; Bernstein, C.N.; Iliopoulos, D.; Macpherson, A.; Neurath, M.F.; Ali, R.A.R.; Vavricka, S.R.; Fiocchi, C. Environmental triggers in IBD: A review of progress and evidence. Nat. Rev. Gastroenterol. Hepatol. 2018, 15, 39-49. [CrossRef] [PubMed]

17. Ng, S.C.; Shi, H.Y.; Hamidi, N.; Underwood, F.E.; Tang, W.; Benchimol, E.I.; Panaccione, R.; Ghosh, S.; $\mathrm{Wu}$, J.C.Y.; Chan, F.K.L.; et al. Worldwide incidence and prevalence of inflammatory bowel disease in the 21st century: A systematic review of population-based studies. Lancet 2018, 390, 2769-2778. [CrossRef]

18. Qin, J.; Li, R.; Raes, J.; Arumugam, M.; Burgdorf, K.S.; Manichanh, C.; Nielsen, T.; Pons, N.; Levenez, F.; Yamada, T.; et al. A human gut microbial gene catalogue established by metagenomic sequencing. Nature 2010, 464, 59-65. [CrossRef] [PubMed]

19. Turner, J.R. Intestinal mucosal barrier function in health and disease. Nat. Rev. Immunol. 2009, 9, 799-809. [PubMed]

20. Vindigni, S.M.; Zisman, T.L.; Suskind, D.L.; Damman, C.J. The intestinal microbiome, barrier function, and immune system in inflammatory bowel disease: A tripartite pathophysiological circuit with implications for new therapeutic directions. Ther. Adv. Gastroenterol. 2016, 9, 606-625. [CrossRef]

21. Fallani, M.; Young, D.; Scott, J.; Norin, E.; Amarri, S.; Adam, R.; Aguilera, M.; Khanna, S.; Gil, A.; Edwards, C.A.; et al. Intestinal microbiota of 6-week-old infants across Europe: Geographic influence beyond delivery mode, breast-feeding, and antibiotics. J. Pediatr. Gastroenterol. Nutr. 2010, 51, 77-84. [CrossRef]

22. Penders, J.; Thijs, C.; Vink, C.; Stelma, F.F.; Snijders, B.; Kummeling, I.; Van den Brandt, P.A.; Stobberingh, E.E. Factors influencing the composition of the intestinal microbiota in early infancy. Pediatrics 2006, 118, 511-521. [CrossRef] [PubMed]

23. Wu, G.D.; Chen, J.; Hoffmann, C.; Bittinger, K.; Chen, Y.Y.; Keilbaugh, S.A.; Meenakshi, B.; Dan, K.; William, A.W.; Rob, K.; et al. Linking long-term dietary patterns with gut microbial enterotypes. Science 2011, 334, 105-108. [CrossRef]

24. Michail, S.; Durbin, M.; Turner, D.; Griffiths, A.M.; Mack, D.R.; Hyams, J.; Leleiko, N.; Kenche, H.; Stolfi, A.; Wine, E. Alterations in the gut microbiome of children with severe ulcerative colitis. Inflamm. Bowel Dis. 2012, 18, 1799-1808. [CrossRef] [PubMed]

25. Manichanh, C.; Rigottier-Gois, L.; Bonnaud, E.; Gloux, K.; Pelletier, E.; Frangeul, L.; Nalin, R.; Jarrin, C.; Marteau, P.; Roca, J. Reduced diversity of faecal microbiota in Crohn's disease revealed by a metagenomic approach. Gut 2006, 55, 205-211. [CrossRef]

26. Ott, S.J.; Musfeldt, M.; Wenderoth, D.F.; Hampe, J.; Brant, O.; Fölsch, U.R.; Timmis, K.N.; Schreiber, S. Reduction in diversity of the colonic mucosa associated bacterial microflora in patients with active inflammatory bowel disease. Gut 2004, 53, 685-693. [CrossRef] [PubMed]

27. Sheehan, D.; Moran, C.; Shanahan, F. The microbiota in inflammatory bowel disease. J. Gastroenterol. 2015, 50, 495-507. [CrossRef] [PubMed] 
28. Atarashi, K.; Tanoue, T.; Shima, T.; Imaoka, A.; Kuwahara, T.; Momose, Y.; Cheng, G.; Yamasaki, S.; Saito, T.; Ohba, Y. Induction of colonic regulatory T cells by indigenous Clostridium species. Science 2011, 331, 337-341. [CrossRef]

29. de Souza, H.S.; Fiocchi, C. Immunopathogenesis of IBD: Current state of the art. Nat. Rev. Gastroenterol. Hepatol. 2016, 13, 13-27. [CrossRef]

30. Merga, Y.; Campbell, B.J.; Rhodes, J.M. Mucosal barrier, bacteria and inflammatory bowel disease: Possibilities for therapy. Dig. Dis. 2014, 32, 475-483. [CrossRef]

31. Canani, R.B.; Costanzo, M.D.; Leone, L.; Pedata, M.; Meli, R.; Calignano, A. Potential beneficial effects of butyrate in intestinal and extraintestinal diseases. World J. Gastroenterol. 2011, 17, 1519-1528. [CrossRef]

32. Van Immerseel, F.; Ducatelle, R.; De Vos, M.; Boon, N.; Van De Wiele, T.; Verbeke, K.; Rutgeerts, P.; Sas, B.; Louis, P.; Flint, H.J. Butyric acid-producing anaerobic bacteria as a novel probiotic treatment approach for inflammatory bowel disease. J. Med. Microbiol. 2010, 59, 141-143. [CrossRef]

33. Smith, P.M.; Howitt, M.R.; Panikov, N.; Michaud, M.; Gallini, C.A.; Bohlooly-y, M.; Glickman, J.N.; Garrett, W.S The microbial metabolites, short-chain fatty acids, regulate colonic Treg cell homeostasis. Science 2013, 341, 569-573. [CrossRef] [PubMed]

34. Thorburn, A.N.; Macia, L.; Mackay, C.R. Diet, metabolites, and "western-lifestyle" inflammatory diseases. Immunity 2014, 40, 833-842. [CrossRef] [PubMed]

35. Chassaing, B.; Koren, O.; Goodrich, J.K.; Poole, A.C.; Srinivasan, S.; Ley, R.E.; Gewirtz, A.T. Dietary emulsifiers impact the mouse gut microbiota promoting colitis and metabolic syndrome. Nature 2015, 519, 92-96. [CrossRef] [PubMed]

36. Lee, D.; Swan, C.K.; Suskind, D.; Wahbeh, G.; Vanamala, J.; Baldassano, R.N.; Leonard, M.B.; Lampe, J.W. Children with Crohn's disease frequently consume select food additives. Dig. Dis. Sci. 2018, 63, 2722-2728. [CrossRef] [PubMed]

37. Limketkai, B.N.; Iheozor-Ejiofor, Z.; Gjuladin-Hellon, T.; Parian, A.; Matarese, L.E.; Bracewell, K.; MacDonald, J.K.; Gordon, M.; Mullin, G.E. Dietary interventions for induction and maintenance of remission in inflammatory bowel disease. Cochrane Database Syst. Rev. 2019, 2, CD012839. [CrossRef]

38. Knight-Sepulveda, K.; Kais, S.; Santaolalla, R.; Abreu, M.T. Diet and inflammatory bowel disease. Gastroenterol. Hepatol. 2015, 11, 511-520.

39. Halmos, E.P.; Gibson, P.R. Dietary management of IBD-insights and advice. Nat. Rev. Gastroenterol. Hepatol. 2015, 12, 133-146. [CrossRef]

40. Day, A.S.; Lopez, R.N. Exclusive enteral nutrition in children with Crohn's disease. World J. Gastroenterol. 2015, 2, 6809-6816. [CrossRef]

41. Sigall-Boneh, R.; Pfeffer-Gik, T.; Segal, I.; Zangen, T.; Boaz, M.; Levine, A. Partial enteral nutrition with a Crohn's disease exclusion diet is effective for induction of remission in children and young adults with Crohn's disease. Inflamm. Bowel Dis. 2014, 20, 1353-1360. [CrossRef]

42. Suskind, D.L.; Cohen, S.A.; Brittnacher, M.J.; Wahbeh, G.; Lee, D.; Shaffer, M.L.; Braly, K.; Hayden, H.S.; Klein, J.; Gold, B.; et al. Clinical and fecal microbial changes with diet therapy in active inflammatory bowel disease. J. Clin. Gastroenterol. 2018, 52, 155. [CrossRef] [PubMed]

43. Borrelli, O.; Cordischi, L.; Cirulli, M.; Paganelli, M.; Labalestra, V.; Uccini, S.; Russo, P.M.; Cucchiara, S. Polymeric diet alone versus corticosteroids in the treatment of active pediatric Crohn's disease: A randomized controlled open-label trial. Clin. Gastroenterol. Hepatol. 2006, 4, 744-753. [CrossRef] [PubMed]

44. Day, A.S.; Whitten, K.E.; Sidler, M.; Lemberg, D.A. Systematic review: Nutritional therapy in paediatric Crohn's disease. Aliment. Pharmacol. Ther. 2008, 27, 293-307. [CrossRef]

45. Gorard, D.A.; Hunt, J.B.; Payne-James, J.J.; Palmer, K.R.; Rees, R.G.; Clark, M.L.; Farthing, M.J.; Misiewicz, J.J.; Silk, D.B. Initial response and subsequent course of Crohn's disease treated with elemental diet or prednisolone. Gut 1993, 34, 1198-1202. [CrossRef]

46. Rajendran, N.; Kumar, D. Role of diet in the management of inflammatory bowel disease. World J. Gastroenterol. 2010, 16, 1442-1448. [CrossRef]

47. Quince, C.; Ijaz, U.Z.; Loman, N.; Eren, A.M.; Saulnier, D.; Russell, J.; Haig, S.; Calus, S.; Quick, J.; Barclay, A.; et al. Extensive modulation of the fecal metagenome in children with crohn's disease during exclusive enteral nutrition. Am. J. Gastroenterol. 2015, 110, 1718-1729. [CrossRef]

48. Ni, J.; Wu, G.D.; Albenberg, L.; Tomov, V.T. Gut microbiota and IBD: Causation or correlation? Nat. Rev. Gastroenterol. Hepatol. 2017, 14, 573-584. [CrossRef] 
49. Lane, E.R.; Lee, D.; Suskind, D.L. Dietary therapies in pediatric inflammatory bowel disease: An evolving inflammatory bowel disease paradigm. Gastroenterol. Clin. 2017, 46, 731-744. [CrossRef]

50. Suskind, D.L.; Wahbeh, G.; Cohen, S.A.; Damman, C.J.; Klein, J.; Braly, K.; Shaffer, M.; Lee, D. Patients perceive clinical benefit with the specific carbohydrate diet for inflammatory bowel disease. Dig. Dis. Sci. 2016, 61, 3255-3260. [CrossRef]

51. Suskind, D.L.; Wahbeh, G.; Gregory, N.; Vendettuoli, H.; Christie, D. Nutritional therapy in pediatric Crohn disease: The specific carbohydrate diet. J. Pediatr. Gastroenterol. Nutr. 2014, 58, 87-91. [CrossRef]

52. Kakodkar, S.; Farooqui, A.J.; Mikolaitis, S.L.; Mutlu, E.A. The specific carbohydrate diet for inflammatory bowel disease: A case series. J. Acad. Nutr. Diet. 2015, 115, 1226-1232. [CrossRef]

53. Cohen, S.A.; Gold, B.D.; Oliva, S.; Lewis, J.; Stallworth, A.; Koch, B.; Laura, E.; Mason, D. Clinical and mucosal improvement with specific carbohydrate diet in pediatric Crohn disease. J. Pediatr. Gastroenterol. Nutr. 2014, 59, 516-521. [CrossRef]

54. Olendzki, B.C.; Silverstein, T.D.; Persuitte, G.M.; Ma, Y.; Baldwin, K.R.; Cave, D. An anti-inflammatory diet as treatment for inflammatory bowel disease: A case series report. Nutr. J. 2014, 13, 5. [CrossRef]

55. Chiba, M.; Abe, T.; Tsuda, H.; Sugawara, T.; Tsuda, S.; Tozawa, H.; Fujiwara, K.; Imai, H. Lifestyle-related disease in Crohn's disease: Relapse prevention by a semi-vegetarian diet. World J. Gastroenterol. 2010, 16, 2484-2495. [CrossRef]

56. Svolos, V.; Hansen, R.; Nichols, B.; Quince, C.; Ijaz, U.Z.; Papadopoulou, R.T.; Ewards, C.A.; Watson, D.; Alghamdi, A.; Brejnrod, A.; et al. Treatment of active crohn's disease with an ordinary food-based diet that replicates exclusive enteral nutrition. Gastroenterology 2019, 56, 1354-1367. [CrossRef]

57. MacLellan, A.; Moore-Connors, J.; Grant, S.; Cahill, L.; Langille, M.G.I.; Van Limbergen, J. The impact of Exclusive Enteral Nutrition (EEN) on the gut microbiome in crohn's disease: A review. Nutrients 2017, 9, 447. [CrossRef]

58. Gerasimidis, K.; Bertz, M.; Hanske, L.; Junick, J.; Biskou, O.; Aguilera, M.; Garrick, V.; Russell, R.K.; Blaut, M.; McGrogan, P.; Edwards, C.A. Decline in presumptively protective gut bacterial species and metabolites are paradoxically associated with disease improvement in pediatric Crohn's disease during enteral nutrition. Inflamm. Bowel Dis. 2014, 20, 861-871. [CrossRef]

59. Jowett, S.L.; Seal, C.J.; Pearce, M.S.; Phillips, E.; Gregory, W.; Barton, J.R.; Welfare, M.R. Influence of dietary factors on the clinical course of ulcerative colitis: A prospective cohort study. Gut 2004, 53, 1479-1484. [CrossRef]

60. Lee, D.; Baldassano, R.N.; Otley, A.R.; Albenberg, L.; Griffiths, A.M.; Compher, C.; Chen, E.Z.; Li, H.; Gilroy, E.; Nessel, L.; et al. Comparative effectiveness of nutritional and biological therapy in North American children with active crohn's disease. Inflamm. Bowel Dis. 2015, 21, 1786-1793. [CrossRef]

61. Albenberg, L.; Brensinger, C.M.; Wu, Q.; Gilroy, E.; Kappelman, M.D.; Sandler, R.S.; Lewis, J.D. A diet low in red and processed meat does not reduce rate of crohn's disease flares. Gastroenterology 2019. [CrossRef]

62. Lewis, J.D.; Albenberg, L.; Lee, D.; Kratz, M.; Gottlieb, K.; Reinisch, W. The Importance and challenges of dietary intervention trials for inflammatory bowel disease. Inflamm. Bowel Dis. 2017, 23, 181-191. [CrossRef]

63. Pedersen, N.; Ankersen, D.V.; Felding, M.; Wachmann, H.; Végh, Z.; Molzen, L.; Burisch, J.; Andersen, J.R.; Munkholm, P. Low-FODMAP diet reduces irritable bowel symptoms in patients with inflammatory bowel disease. World J. Gastroenterol. 2017, 23, 3356-3366. [CrossRef] [PubMed]

64. Cox, S.R.; Prince, A.C.; Myers, C.E.; Irving, P.M.; Lindsay, J.O.; Lomer, M.C.; Whelan, K. Fermentable carbohydrates [FODMAPs] exacerbate functional gastrointestinal symptoms in patients with inflammatory bowel disease: A randomised, double-blind, placebo-controlled, cross-over, re-challenge trial. J. Crohn's Colitis 2017, 11, 1420-1429. [CrossRef] [PubMed]

65. Prince, A.C.; Myers, C.E.; Joyce, T.; Irving, P.; Lomer, M.; Whelan, K. Fermentable carbohydrate restriction (Low FODMAP Diet) in clinical practice improves functional gastrointestinal symptoms in patients with inflammatory bowel disease. Inflamm. Bowel. Dis. 2016, 22, 1129-1136. [CrossRef] [PubMed]

66. Heuschkel, R.B. Enteral nutrition in children with Crohn's disease. J. Pediatr. Gastroenterol. Nutr. 2000, 31 , 575. [CrossRef]

67. Colombel, J.F.; Sandborn, W.J.; Reinisch, W.; Mantzaris, G.J.; Kornbluth, A.; Rachmilewitz, D.; Lichtiger, S.; D'Haens, G.; Diamond, R.H.; Broussard, D.L.; et al. Infliximab, azathioprine, or combination therapy for Crohn's disease. N. Engl. J. Med. 2010, 362, 1383-1395. [CrossRef] 
68. Hyams, J.; Crandall, W.; Kugathasan, S.; Griffiths, A.; Olson, A.; Johanns, J.; Liu, G.; Travers, S.; Heuschkel, R.; Markowitz, J.; et al. Induction and maintenance infliximab therapy for the treatment of moderate-to-severe Crohn's disease in children. Gastroenterology 2007, 132, 863-873. [CrossRef]

69. Rutgeerts, P.; Sandborn, W.J.; Feagan, B.G.; Reinisch, W.; Olson, A.; Johanns, J.; Travers, S.; Rachmilewitz, D.; Hanauer, S.B.; Lichtenstein, G.R.; et al. Infliximab for induction and maintenance therapy for ulcerative colitis. N. Engl. J. Med. 2005, 353, 2462-2476. [CrossRef]

70. Hyams, J.; Damaraju, L.; Blank, M.; Johanns, J.; Guzzo, C.; Winter, H.S.; Kugathasan, S.; Cohen, S.; Markowitz, J.; Escher, J.C.; et al. Induction and maintenance therapy with infliximab for children with moderate to severe ulcerative colitis. Clin. Gastroenterol. Hepatol. 2012, 10, 391-399. [CrossRef]

71. Hirai, F.; Ishihara, H.; Yada, S.; Esaki, M.; Ohwan, T.; Nozaki, R.; Ashizuka, S.; Inatsu, H.; Ohi, H.; Aoyagi, K.; et al. Effectiveness of concomitant enteral nutrition therapy and infliximab for maintenance treatment of Crohn's disease in adults. Dig. Dis. Sci. 2013, 58, 1329-1334. [CrossRef]

72. Zallot, C.; Quilliot, D.; Chevaux, J.B.; Peyrin-Biroulet, C.; Guéant-Rodriguez, R.M.; Freling, E.; Collet-Fenetrier, B.; Williet, N.; Ziegler, O.; et al. Dietary beliefs and behavior among inflammatory bowel disease patients. Inflamm. Bowel Dis. 2013, 19, 66-72. [CrossRef]

73. Stewart, M.; Day, A.S.; Otley, A. Physician attitudes and practices of enteral nutrition as primary treatment of paediatric Crohn disease in North America. J. Pediatr. Gastroenterol. Nutr. 2011, 52, 38-42. [CrossRef] [PubMed]

74. Svolos, V.; Gerasimidis, K.; Buchanan, E.; Curtis, L.; Garrick, V.; Hay, J.; Laird, S.; Munro, J.; Gaya, D.R.; Russell, R.K.; Hansen, R. Dietary treatment of Crohn's disease: Perceptions of families with children treated by exclusive enteral nutrition, a questionnaire survey. BMC Gastroenterol. 2017, 17, 14. [CrossRef] [PubMed]

75. Lewis, J.D.; Chen, E.Z.; Baldassano, R.N.; Otley, A.R.; Griffiths, A.M.; Lee, D.; Bittinger, K.; Bailey, A.; Friedman, E.S.; Hoffmann, C.; et al. Inflammation, antibiotics, and diet as environmental stressors of the gut microbiome in pediatric crohn's disease. Cell Host Microbe 2017, 22, 247. [CrossRef] [PubMed]

76. Hansen, J.J.; Sartor, R.B. Therapeutic manipulation of the microbiome in IBD: Current results and future approaches. Curr. Treat. Options Gastroenterol. 2015, 13, 105-120. [CrossRef] [PubMed]

77. Cohen, A.B.; Lee, D.; Long, M.D.; Kappelman, M.D.; Martin, C.F.; Sandler, R.S.; Lewis, J.D. Dietary patterns and self-reported associations of diet with symptoms of inflammatory bowel disease. Dig. Dis. Sci. 2013, 58, 1322-1328. [CrossRef]

78. Sigall-Boneh, R.; Levine, A.; Lomer, M.; Wierdsma, N.; Allan, P.; Fiorino, G.; Gatti, S.; Jonkers, D.; Kierkus, J.; Melgar, S. Research gaps in diet and nutrition in inflammatory bowel disease. A topical review by D-ECCO working group [Dietitians of ECCO]. J. Crohns Colitis 2017, 11, 1407-1419. [PubMed] 
Article

\title{
Combination of Lactobacillus acidophilus and Bifidobacterium animalis subsp. lactis Shows a Stronger Anti-Inflammatory Effect than Individual Strains in HT-29 Cells
}

\author{
Sing-Chung Li ${ }^{1}$, Wei-Fang Hsu ${ }^{1}$, Jung-Su Chang ${ }^{1,2}$ and Chun-Kuang Shih ${ }^{1,3,4, *}$ \\ 1 School of Nutrition and Health Sciences, College of Nutrition, Taipei Medical University, \\ Taipei 11031, Taiwan; sinchung@tmu.edu.tw (S.-C.L.); greg_hsu@hotmail.com (W.-F.H.); \\ susanchang@tmu.edu.tw (J.-S.C.) \\ 2 Graduate Institute of Metabolism and Obesity Sciences, College of Nutrition, Taipei Medical University, \\ Taipei 11031, Taiwan \\ 3 School of Food Safety, College of Nutrition, Taipei Medical University, Taipei 11031, Taiwan \\ 4 Master Program in Food Safety, College of Nutrition, Taipei Medical University, Taipei 11031, Taiwan \\ * Correspondence: ckshih@tmu.edu.tw; Tel.: +886-(2)-2736-1661 (ext. 6569)
}

Received: 28 March 2019; Accepted: 22 April 2019; Published: 27 April 2019

\begin{abstract}
Inflammatory bowel disease (IBD) is an emerging health problem associated with the dysregulation of the intestinal immune system and microbiome. Probiotics are able to reduce inflammatory responses in intestinal epithelial cells (IECs). However, entire signaling pathways and the interaction between different probiotics have not been well-documented. This study was designed to investigate the anti-inflammatory effects and mechanisms of single and combined probiotics. HT-29 cells were induced by lipopolysaccharide (LPS) and tumor necrosis factor (TNF)- $\alpha$, treated with Lactobacillus acidophilus, Bifidobacterium animalis subsp. lactis or their combination and analyzed for inflammation-related molecules. Both L. acidophilus and B. animalis subsp. lactis reduced interleukin (IL)-8 secretion and the expressions of phosphorylated p65 nuclear factor-kappa B ( $p$-p65 NF- $\mathrm{kB}$ ), phosphorylated p38 mitogen-activated protein kinase ( $p$-p38 MAPK), vascular cell adhesion molecule-1 (VCAM-1) and cyclooxygenase-2 (COX-2), while they increased toll-like receptor 2 (TLR2) expression. L. acidophilus did not decrease intercellular adhesion molecule-1 (ICAM-1) but enhanced the inhibitory efficacy of $B$. animalis subsp. lactis. Combined probiotics showed the best anti-inflammatory activity. These results suggest that L. acidophilus and B. animalis subsp. lactis may exert a potent anti-inflammatory effect through modulating TLR2-mediated NF-KB and MAPK signaling pathways in inflammatory IECs. Both strains, especially their combination, may be novel adjuvants for IBD therapy.
\end{abstract}

Keywords: intestinal epithelial cells; inflammation; probiotics; Lactobacillus acidophilus; Bifidobacterium animalis subsp. lactis

\section{Introduction}

Inflammatory bowel disease (IBD), including ulcerative colitis (UC) and Crohn's disease (CD), is traditionally a common chronic gastrointestinal disease in several Western countries. The highest reported prevalence rates of IBD are in Europe (505/100,000 for UC in Norway and 322/100,000 for CD in Germany) and North America (286/100,000 for UC in the USA and 319/100,000 for CD in Canada) [1]. The overall prevalence of IBD has exceeded $0.3 \%$ in North America and many European countries [1]. However, IBD has become a global disease with accelerating incidence in newly industrialized countries in Asia, Africa and South America [1]. According to a recent study of Yen et al. [2], both the 
incidence and prevalence of IBD increased continuously in Taiwan from 2001 to 2015. Improved hygiene, civilization and Westernization may be possible causes [2]. Several studies suggest that IBD is an emerging health problem in the world [1-3]. The reason for the global increase in this initially Western-prevalent disease is still unknown, but it may be closely associated with environmental and social changes, such as the adoption of Western lifestyle and diet [1,2]. The rise of IBD in adults and children has been observed in Western developed countries, while gut microbiota and dietary fiber-rich diet of rural African individuals may protect them from the threat of IBD [4].

The etiology of IBD is thought to relate to a complex interaction of genetic and environmental factors, including the dysregulation of the enteric immune system and alterations in the intestinal microbiome [5]. Patients with IBD have typical dysbiosis characterized by an increase in pro-inflammatory bacteria, a decrease in anti-inflammatory bacteria and a reduction in bacterial diversity compared to the healthy population [5]. A reduced diversity of the gut microbiota in IBD patients is largely due to a low abundance of probiotics belonging to the Lactobacillus and Bifidobacterium genera [6]. IBD is characterized by mucosal and systemic inflammatory responses to the gut microbiome, which is the outcome of a lack of mucosal immune tolerance [6]. It is well-known that chronic inflammation is related to carcinogenesis and thus long-standing IBD is considered as a risk factor of colorectal cancer (CRC), especially colitis-associated colorectal cancer (CAC). In recent years, high incidence rates of CRC have been observed in Taiwan and other countries where the rates were historically low [7]. This increasing trend of CRC is similar to that of IBD in many countries. Both CRC and IBD are now global health issues that cannot be ignored.

The mucosal tissue of the intestine contains the largest part of the immune system in humans. It is estimated that $70 \%$ of immune cells are present in the gut [8]. Intestinal epithelial cells (IECs) provide a physical and chemical barrier between the intestinal lumen and the lamina propria [8]. They represent the first contact point for bacteria within the gut and thus prevent microbial penetration and induce communication for immune recognition of intestinal bacteria [9]. The activation of the pro-inflammatory genes in IECs in response to challenges by bacterial products such as lipopolysaccharide (LPS) or pro-inflammatory cytokines such as tumor necrosis factor (TNF)- $\alpha$ is associated with acute and chronic intestinal inflammation [10]. However, bacterial ligands may not directly alter the inflammation of IECs, although bacterial cell wall components, such as LPS and lipoteichoic acid (LTA), contribute to toll-like receptor (TLR)-mediated inflammation [11]. Ligands from pathogenic bacteria efficiently activate monocytes and macrophages through the secretion of pro-inflammatory cytokines such as TNF- $\alpha$, interleukin (IL)-1 $\beta$, IL-6 and IL-8 [11]. In contrast, ligands from probiotic bacteria only minimally induce TNF- $\alpha$ production [11]. These studies suggest that it is reasonable to simulate the inflammatory environment of the intestine using a combination of bacterial ligands and pro-inflammatory cytokines.

Probiotics have beneficial effects on the host through their ability to modulate the mucosal immune system. They have been shown to both augment/modulate homeostatic immune defenses and to ameliorate infection, inflammation and allergy by modulating gut function [12]. Treatment with probiotics has been considered to be potentially effective and safe in patients with IBD. It has been reported that individual probiotic species have variable potential to stimulate the mucosal immune system [13]. Probiotics differentially modulate IECs' responses via the activation or suppression of distinct signaling pathways, such as TLR, nuclear factor-kappa B (NF-KB) and mitogen-activated protein kinase (MAPK) signaling pathways, in a strain-dependent manner, including strains of the same species $[12,14]$.

The majority of probiotic microorganisms belong to the genera Lactobacillus and Bifidobacterium, and most Lactobacillus and Bifidobacterium species belong to probiotics [15]. Lactobacillus and Bifidobacterium species have been shown to reduce inflammatory responses, including NF- $\mathrm{kB}$ activation and IL-8 production, in inflammatory IECs, rodent colitis models and patients with IBD $[9,16]$. However, not all probiotics exert constant anti-inflammatory effects in experimental models of intestinal inflammation. For example, L. acidophilus, B. lactis and L. casei have an anti-inflammatory activity in the 2,4,6-trinitrobenzene sulfonic acid (TNBS) model of rat colitis but each probiotic shows 
its own anti-inflammatory profile [17]. It would be interesting to compare different probiotics in the same experimental model, in order to establish the best profile in a given setting and to further apply this new concept for IBD therapy [17]. A recent meta-analysis study demonstrated that results from clinical trials of probiotics on IBD were inconsistent [5]. Among all trials included in this meta-analysis, some showed an improvement in the maintenance or induction of remission by probiotics, while others failed to show any benefit [5]. This could be due to the species or strains of probiotics used, or the methodological differences among studies. Some studies found that probiotics may be as effective as 5-aminosalicylates (5-ASAs), a common drug for the treatment of IBD, in preventing relapse of quiescent UC but other studies showed that there was no benefit of probiotics over placebo in inducing remission of active UC [5]. However, when only trials of VSL\#3-a mixed preparation of probiotics—were considered, there appeared to be a benefit. VSL\#3 may be effective in inducing remission in patients with active UC [5]. These findings suggest that combination of probiotics seems to be more effective in anti-inflammation than single strains. However, the detailed anti-inflammatory mechanism of each probiotic and their interactions are still unclear.

Although some strains of probiotics belonging to the Lactobacillus and Bifidobacterium genera have been shown to possess anti-inflammatory and immunomodulatory activities, their entire signaling pathways have not been well-documented. There are only limited studies that have examined the inflammation-associated signaling pathways of L. acidophilus and B. animalis subsp. lactis, two common strains of probiotics used in fermented dairy products, especially the comparison and interaction between these probiotics. The aim of the present study was to investigate the anti-inflammatory effects and associated mechanisms of L. acidophilus, B. animalis subsp. lactis and their combination in LPS- and TNF- $\alpha$-induced inflammatory IECs.

\section{Materials and Methods}

\subsection{Cell Culture}

Human colon cancer cell line HT-29 cells were purchased from the Food Industry Research and Development Institute (Hsinchu, Taiwan) and incubated in 9-cm culture dishes as a monolayer in Roswell Park Memorial Institute (RPMI) 1640 medium supplemented with 10\% fetal bovine serum (FBS), $1 \%$ L-glutamine $(v / v)$ and $1 \%$ penicillin/streptomycin and maintained at $37{ }^{\circ} \mathrm{C}$ in a humidified atmosphere of $5 \% \mathrm{CO}_{2}$. Cells were sub-cultured following enzymatic digestion using $1 \%$ trypsin solution.

\subsection{Bacterial Strains}

L. acidophilus and B. animalis subsp. lactis (Chr. Hansen, Denmark) were grown in the Man-Rogosa-Sharpe (MRS) broth overnight at $37^{\circ} \mathrm{C}$ in anaerobic conditions. Viable bacteria were counted by plating serial dilutions on MRS agar. Both strains of probiotics were heat-inactivated by water-bath at $60^{\circ} \mathrm{C}$ for $30 \mathrm{~min}$, then collected by centrifugation at $500 \times \mathrm{g}$ for $10 \mathrm{~min}$ at $4{ }^{\circ} \mathrm{C}$ and washed in phosphate-buffered saline (PBS), as described in previous studies $[18,19]$.

\subsection{Treatments of Cells}

HT-29 cells were seeded in a 24 -well microtiter plate at a density of $3 \times 10^{5}$ cells/well in volume of $400 \mu \mathrm{L}$ medium for one day. Cells as an inflammatory group were treated with non-FBS medium containing LPS $(1 \mu \mathrm{g} / \mathrm{mL})$ and TNF- $\alpha(20 \mathrm{ng} / \mathrm{mL})$ from Escherichia coli O58:B5 (L6529 Sigma-Aldrich, St. Louis, MO, USA) for $24 \mathrm{~h}$ [20]. Cells as intervention groups were added to probiotics at the designated multiplicity of infection (MOI) of $0.1,1,10$ [21], and incubated in $5 \% \mathrm{CO}_{2}$ at $37^{\circ} \mathrm{C}$ with non-FBS medium containing LPS and TNF- $\alpha$. The culture medium was harvested and analyzed for IL- 8 secretion by enzyme-linked immunosorbent assay (ELISA). We chose the doses of probiotics with an inhibitory effect on IL-8 secretion for comparing the anti-inflammatory effects of single and combined 
probiotics. Cells were incubated with or without probiotics (L. acidophilus, B. animalis subsp. lactis or both), then medium and cells were collected and analyzed by ELISA and Western blot, respectively.

\subsection{Cell Viability Assay}

HT-29 cells were seeded in a 96-well microtiter plate at a density of $1 \times 10^{4}$ cells/well in volume of $100 \mu \mathrm{L}$ medium. Cells were treated with L. acidophilus, B. animalis subsp. lactis or both and with LPS and TNF- $\alpha$ for $24 \mathrm{~h}$. After the above treatments, cells were incubated with 3-(4,5-dimethylthiazol-2-yl)-2,5-diphenyltetrazolium bromide (MTT) dye $(0.5 \mu \mathrm{g} / \mu \mathrm{L})$ at $37^{\circ} \mathrm{C}$ for $4 \mathrm{~h}$. Absorbance of the product was read at $570 \mathrm{~nm}$ by ELISA reader.

\subsection{Analysis of IL-8 Secretion by ELISA}

IL-8 levels in HT-29 conditioned media were measured by a commercially available ELISA kit (BioLegend, San Diego, CA, USA).

\subsection{Analysis of Protein Expression by Western Blot}

The analytical procedures were modified from previous studies [22,23]. Cells were sonicated for $20 \mathrm{~min}$ and then lysed cells were centrifuged at $10,000 \times \mathrm{g}$ for $10 \mathrm{~min}$ at $4{ }^{\circ} \mathrm{C}$. Supernatants were collected and the protein concentration was determined using the Bio-Rad Bradford assay (Bio-Rad, Hercules, CA, USA). Samples (each containing $30 \mu \mathrm{g}$ of supernatant protein) were mixed with NuPAGE LDS sample buffer and $\beta$-mercaptoethanol (Invitrogen, Carlsbad, CA, USA), heated at $95{ }^{\circ} \mathrm{C}$ for $10 \mathrm{~min}$, cooled to room temperature and electrophoretically resolved on $10 \%$ NuPAGE Bis-Tris gels (Invitrogen, Carlsbad, CA, USA). Proteins were then transferred to polyvinylidene difluoride (PVDF) membrane, which was then blocked with blocking buffer (TBST containing 5\% albumin) at room temperature for $1 \mathrm{~h}$. The blot was probed with TLR2 (1:1000 dilution, BioVision, Milpitas, CA, USA), phosphorylated p65 NF-кB ( $p$-p65 NF-кB, 1:1000 dilution, Bioworld, Dublin, OH, USA), phosphorylated p38 MAPK ( $p$-p38 MAPK, 1:1000 dilution, BioVision, Milpitas, CA, USA), cyclooxygenase-2 (COX-2, 1:1000 dilution, Santa Cruz Biotechnology, Santa Cruz, CA, USA), intercellular adhesion molecule-1 (ICAM-1, 1:1000 dilution, BioVision, Milpitas, CA, USA) or vascular cell adhesion molecule-1 (VCAM-1, 1:1000 dilution, Abbiotec, San Diego, CA, USA) polyclonal antibody at $4{ }^{\circ} \mathrm{C}$ for $16 \mathrm{~h}$. After being washed extensively to eliminate nonspecific binding, the membrane was incubated with the secondary alkaline phosphatase-labeled antibody at room temperature for $1 \mathrm{~h}$ and incubated with 5-bromo-4-chloro-3-indolyl-phosphate/nitroblue tetrazolium (BCIP/NBT) substrate solution (Sigma-Aldrich Inc., St. Louis, MO, USA) for $20 \mathrm{~min}$ in the dark. The reactive solution was removed, and the reaction was stopped using 50\% methanol. Blot intensities were quantified using Bio-Rad Quantity One software (Bio-Rad Laboratories, Hercules, CA, USA). All experimental data are triplicates.

\subsection{Statistical Analysis}

Data are expressed as means and standard deviations. The difference among groups was analyzed by one-way analysis of variance (ANOVA) and Duncan's multiple range test using SAS software (SAS Institute, Cary, NC, USA). The significant difference was set at $p<0.05$. 


\section{Results}

\subsection{Effects of Different Doses of Single Probiotics on IL-8 Secretion in Inflammatory HT-29 Cells}

HT-29 cells stimulated with LPS and TNF- $\alpha$ secreted significantly higher IL-8 levels than did unstimulated cells (Figure 1A,B). L. acidophilus at MOI of 1 and 10 significantly decreased IL-8 secretion in inflammatory HT-29 cells (Figure 1A). B. animalis subsp. lactis at MOI of 10 showed an inhibitory effect on IL-8 secretion (Figure 1B). As a result, we chose L. acidophilus at MOI of 1 as group A, B. animalis subsp. lactis at MOI of 10 as group $\mathrm{B}$, and combination of L. acidophilus at MOI of 1 and $B$. animalis subsp. lactis at $\mathrm{MOI}$ of 10 as group $\mathrm{AB}$ for further studies.
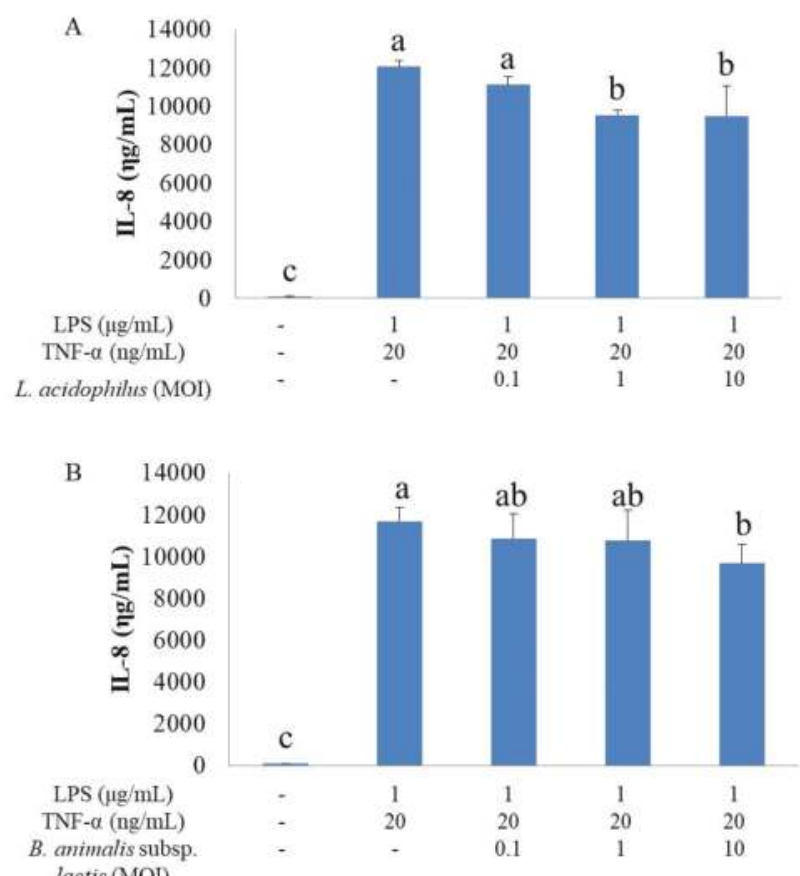

Figure 1. Effects of different doses of single probiotics on IL-8 secretion in inflammatory HT-29 cells. All values except for control are mean \pm SD $(n=3)$. Bars with the same letter are not significantly different from one another determined by Duncan's multiple range test, $p<0.05$. (A) The inhibitory effect of L. acidophilus on IL-8 secretion; (B) the inhibitory effect of B. animalis subsp. lactis on IL-8 secretion.

\subsection{Effects of Pro-Inflammatory Factors and Probiotics on Cell Viability}

The viabilities of HT-29 cells were not different among groups after $24 \mathrm{~h}$ of incubation (Figure 2), showing that pro-inflammatory factors (LPS and TNF- $\alpha$ ) and probiotics (alone or in combination) did not affect the growth of HT-29 cells. 


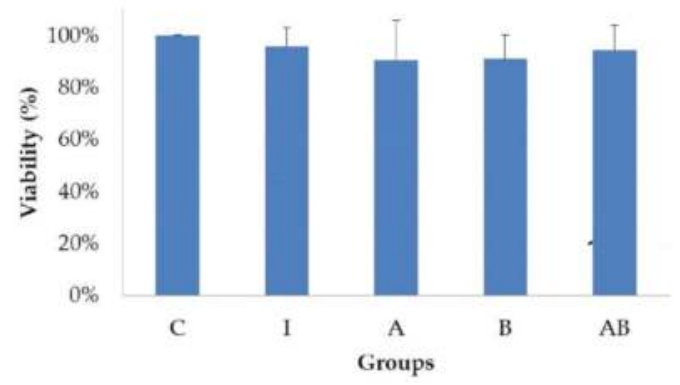

Figure 2. Effects of pro-inflammatory factors and probiotics on viability of HT-29 cells. All values except for control are mean $\pm \mathrm{SD}(\mathrm{n}=3)$. There is no significant difference among groups determined by Duncan's multiple range test, $p<0.05$. C, control; I, TNF- $\alpha(20 \mathrm{ng} / \mathrm{mL})$ with LPS $(1 \mu \mathrm{g} / \mathrm{mL})$; A, L. acidophilus at MOI of 1 with TNF- $\alpha(20 \mathrm{ng} / \mathrm{mL})$ and LPS $(1 \mu \mathrm{g} / \mathrm{mL}) ; \mathrm{B}$, B. animalis subsp. lactis at MOI of 10 with TNF- $\alpha(20 \mathrm{ng} / \mathrm{mL})$ and LPS $(1 \mu \mathrm{g} / \mathrm{mL}) ; \mathrm{AB}$, L. acidophilus at MOI of 1 and B. animalis subsp. lactis at MOI of 10 with TNF- $\alpha(20 \mathrm{ng} / \mathrm{mL})$ and LPS $(1 \mu \mathrm{g} / \mathrm{mL})$. Control group is used as the reference.

\subsection{Effects of Probiotics on IL-8 Secretion in Inflammatory HT-29 Cells}

A shown in Figure 3, all probiotics-treated inflammatory HT-29 cells (groups A, B and AB) had significantly lower IL-8 levels than did cells stimulated with LPS and TNF- $\alpha$ (group I). In addition, the IL-8 level was significantly lower in group AB than in either group A or B.

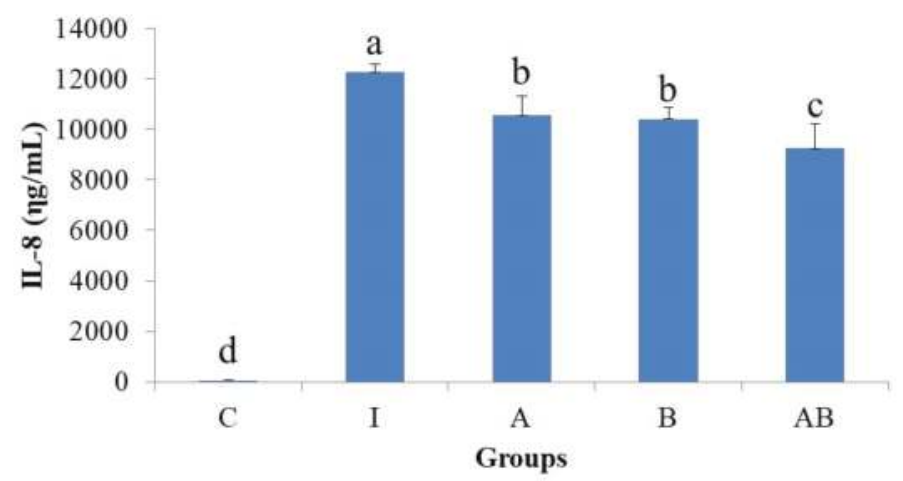

Figure 3. Effects of probiotics on IL-8 secretion in inflammatory HT-29 cells. All values except for control are mean $\pm \mathrm{SD}(\mathrm{n}=3)$. Bars with the same letter are not significantly different from one another determined by Duncan's multiple range test, $p<0.05$. C, control; I, TNF- $\alpha(20 \mathrm{ng} / \mathrm{mL})$ with lipopolysaccharide (LPS) $(1 \mu \mathrm{g} / \mathrm{mL}) ; \mathrm{A}$, L. acidophilus at MOI of 1 with TNF- $\alpha(20 \mathrm{ng} / \mathrm{mL})$ and LPS $(1 \mu \mathrm{g} / \mathrm{mL}) ; \mathrm{B}$, B. animalis subsp. lactis at MOI of 10 with TNF- $\alpha(20 \mathrm{ng} / \mathrm{mL})$ and LPS $(1 \mu \mathrm{g} / \mathrm{mL})$; $\mathrm{AB}$, L. acidophilus at MOI of 1 and B. animalis subsp. lactis at MOI of 10 with TNF- $\alpha(20 \mathrm{ng} / \mathrm{mL})$ and LPS $(1 \mu \mathrm{g} / \mathrm{mL})$.

\subsection{Effects of Probiotics on the Expression of Inflammation-Related Proteins in Inflammatory HT-29 Cells}

Groups $A, B$ and $A B$ had significantly higher expression of TLR2 (Figure $4 A$ ) and lower expressions of $p$-p65 NF- $\mathrm{kB}$ (Figure 4B), p-p38 MAPK (Figure 4C) and COX-2 (Figure 4D) than did group I. Group $\mathrm{AB}$ showed the best inhibitory effect on the expressions of inflammation-related proteins, including p-p65 NF-кB (Figure 4B), p-p38 MAPK (Figure 4C) and COX-2 (Figure 4D). B. animalis subsp. lactis treatment (groups $\mathrm{B}$ and $\mathrm{AB}$ ) significantly decreased ICAM-1 expression compared with group I, and this inhibitory effect was more potent in group $\mathrm{AB}$ than in group $\mathrm{B}$ (Figure $4 \mathrm{E}$ ). L. acidophilus alone (group A) had no inhibitory effect on ICAM-1 expression but it enhanced the efficacy of B. animalis 
subsp. lactis when they were used in combination (group AB, Figure 4E). All probiotics-treated groups (groups A, B and AB) had significantly lower VCAM-1 expressions than did group I, while L. acidophilus treatment (groups $\mathrm{A}$ and $\mathrm{AB}$ ) showed a better inhibitory effect on VCAM-1 than did B. animalis subsp. lactis alone (group B), as shown in Figure $4 \mathrm{~F}$.
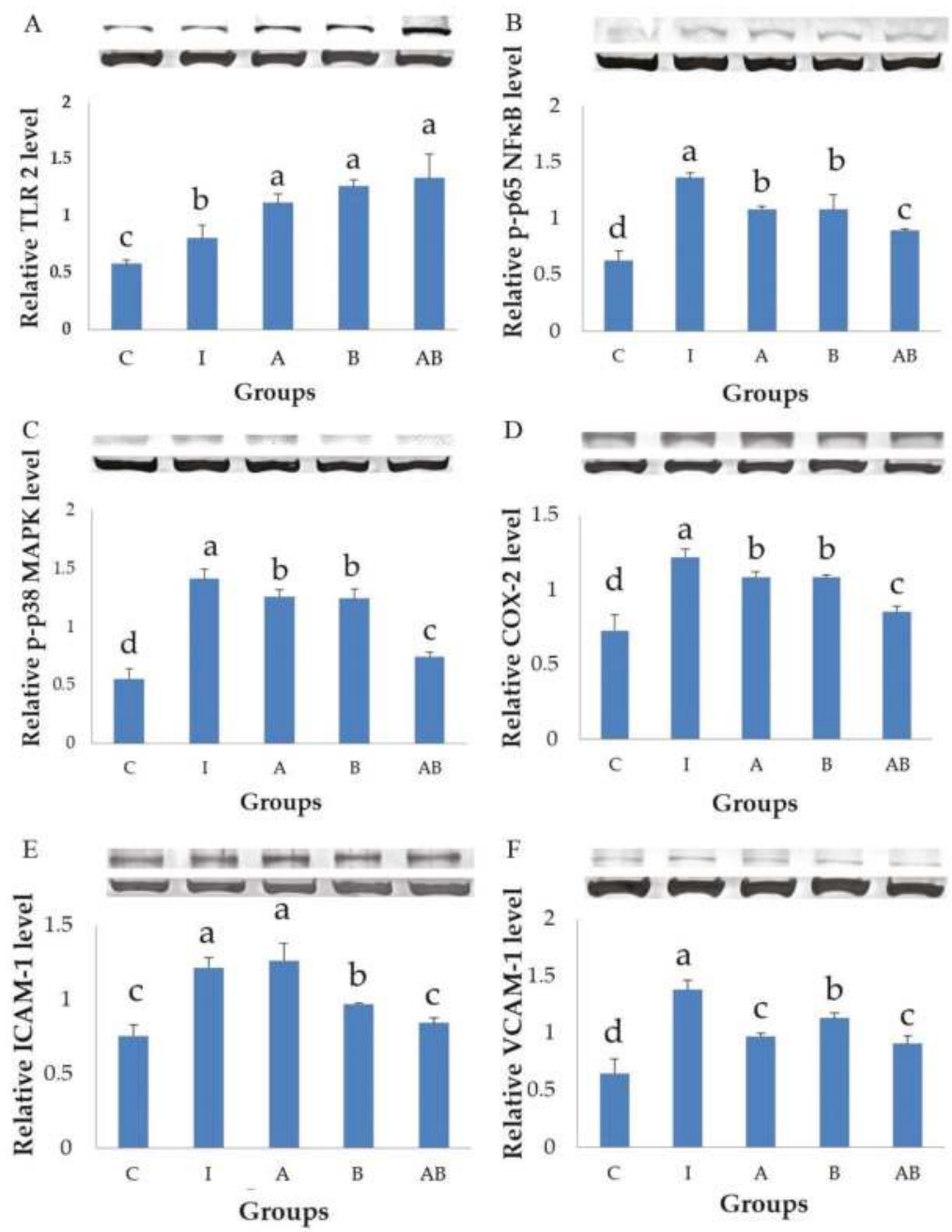

Figure 4. Effects of probiotics on the expression of inflammation-related proteins in inflammatory HT-29 cells. All values are mean $\pm S D(n=3)$. Bars with the same letter are not significantly different from one another determined by Duncan's multiple range test, $p<0.05$. C, control; I, TNF- $\alpha(20 \mathrm{ng} / \mathrm{mL})$ with LPS $(1 \mu \mathrm{g} / \mathrm{mL}) ; A$, L. acidophilus at MOI of 1 with TNF- $\alpha(20 \mathrm{ng} / \mathrm{mL})$ and LPS $(1 \mu \mathrm{g} / \mathrm{mL}) ; \mathrm{B}$, B. animalis subsp. lactis at MOI of 10 with TNF- $\alpha(20 \mathrm{ng} / \mathrm{mL})$ and LPS $(1 \mu \mathrm{g} / \mathrm{mL}) ; \mathrm{AB}$, L. acidophilus at MOI of 1 and B. animalis subsp. lactis at MOI of 10 with TNF- $\alpha(20 \mathrm{ng} / \mathrm{mL})$ and LPS $(1 \mu \mathrm{g} / \mathrm{mL})$. (A), expression of toll-like receptor 2 (TLR2); (B), expression of phosphorylated p65 nuclear factor kappa B ( $p$-p65 $\mathrm{NFKB}$ ); (C), expression of phosphorylated p38 mitogen-activated protein kinase ( $p$-p38 MAPK); (D), expression of cyclooxygenase-2 (COX-2); (E), expression of intercellular adhesion molecule-1 (ICAM-1); (F), expression of vascular cell adhesion molecule-1 (VCAM-1). 


\section{Discussion}

The present study demonstrated that both L. acidophilus and B. animalis subsp. lactis significantly reduced the secretion of IL- 8 and the expressions of $p$-p65 NF- $\mathrm{kB}, p$-p38 MAPK, VCAM-1 and COX-2, as well as increased the expression of TLR2 in LPS- and TNF- $\alpha$-induced inflammatory IECs. B. animalis subsp. lactis significantly reduced the expression of ICAM-1 and this inhibitory effect was enhanced by L. acidophilus, which did not affect ICAM-1 expression directly. The combination of two strains showed the best anti-inflammatory activity. These results suggest that both L. acidophilus and B. animalis subsp. lactis possess a potent anti-inflammatory activity in inflammatory IECs and there is an interaction between these two strains of probiotics.

Lactobacillus is an important genus of probiotics, which plays an essential role of immunomodulation in the intestinal mucosa. For example, L. acidophilus DDS-1 demonstrated a superior survival rate, good adhesion capacity and strong immunomodulatory effect under experimental conditions with or without LPS stimulation [24]. Most L. acidophilus species can be well adapted and are able to survive during harsh conditions of digestion [24]. Due to this stability, L. acidophilus has been considered an ideal vehicle for mucosal-targeted delivery of drugs [24]. The amount of L. acidophilus remained almost unchanged in the gut for a long time [25].

The modulatory effects of L. acidophilus on intestinal inflammation and the immune system are dependent on the stimulation in IECs. L. acidophilus significantly increased COX-2 expression and prostaglandin $\mathrm{E}_{2}\left(\mathrm{PGE}_{2}\right)$ secretion in unstimulated Colo320 cells, but not in TNF- $\alpha$-induced cells [26]. L. acidophilus NCFM could rapidly but transiently induce the production of pro-inflammatory cytokines and chemokines in unstimulated Caco-2 cells [21]. In addition, L. acidophilus NCFM activated the pathogen-associated molecular pattern (PAMP) receptor TLR2 and enhanced the phosphorylation of p65 NF- $\mathrm{kB}$ and $\mathrm{p} 38$ MAPK in unstimulated Caco-2 cells [21]. The activation of TLR2-mediated NF- $\mathrm{kB}$ and MAPK signaling pathways played a key role in the production of cytokines and chemokines in IECs [21].

In contrast, L. acidophilus showed an anti-inflammatory effect in IECs stimulated by bacterial ligands or pro-inflammatory cytokines. L. acidophilus Bar13 were effective in inhibiting IL-8 production in HT-29 cells stimulated by TNF- $\alpha$, IL-1 $\beta$ or LPS [27]. L. acidophilus DDS-1 significantly upregulated anti-inflammatory IL-10 and downregulated pro-inflammatory TNF- $\alpha$ production and IL-8 levels in LPS-treated HT-29 cells [24]. L. acidophilus LAB20 increased the transepithelial electrical resistance (TER) of enterocyte monolayers and thus strengthened intestinal barrier function [16]. L. acidophilus LAB20 also showed anti-inflammatory capacity by attenuating the LPS-induced IL-8 production in HT-29 cells [16]. The results suggest that this strain may balance the IL-8 expression of IECs in response to stimulation by LPS from Gram-negative bacteria in the intestine. Our study used LPS and TNF- $\alpha$ to induce inflammatory responses of HT-29 cells and the results were in agreement with the above studies, showing an anti-inflammatory effect of L. acidophilus. Some animal studies also demonstrated an anti-inflammatory activity of L. acidophilus in the intestinal tract. L. acidophilus administration reduced colonic leukotriene $\mathrm{B}_{4}\left(\mathrm{LTB}_{4}\right)$ production, inducible nitrogen oxide synthase (iNOS) expression and myeloperoxidase (MPO) activity in the TNBS model of rat colitis [17]. L. acidophilus inhibited colitis by inducing goblet cell differentiation, interfering with endoplasmic reticulum stress and suppressing NF- $\mathrm{kB}$ activation in dextran sulfate sodium (DSS)-treated mice [28]. These findings suggest that L. acidophilus can be used as a potent immunomodulator for IBD treatment.

Probiotics modulate immune- and inflammation-associated signaling pathways by gastrointestinal digested products of probiotics and bacterial metabolites. The active compounds of L. acidophilus for the anti-inflammatory effect may include either cell components or secretory factors. Cell wall components play a critical role in modulating intestinal inflammation and immune function. Some components of L. acidophilus, such as cell wall-associated polysaccharides, proteins and LTAs, could directly inhibit NF-kB activation in HT-29 cells [28]. The surface-layer protein (SLP) from L. acidophilus is closely related to a human health-promoting effect via modulation of the immune system. For example, SLP extracted from L. acidophilus NCFM was found to inhibit the production of reactive oxygen species (ROS), 
nitrogen oxide (NO), PGE 2 , TNF- $\alpha$ and IL-1 $\beta$ through attenuating the activation of MAPK and NF- $\mathrm{kB}$ in LPS-induced RAW264.7 cells [29]. This SLP inhibited NO and $\mathrm{PGE}_{2}$ production by downregulating the protein expression levels of iNOS and COX-2 [29]. On the other hand, the cell-free supernatant of L. acidophilus was able to downregulate IL-8 expression in HT-29 cells stimulated by LPS [30]. Otte et al. (2009) indicated that distinct probiotics could specifically and significantly decrease the induced COX-2 expression in IECs, most likely mediated by released factors such as protein, butyrate and propionate and in part by bacterial DNA [26]. A preliminary study of Kainulainen et al. (2015) suggested that exopolysaccharide (EPS) of L. acidophilus LAB20 may have a role in its immunomodulatory activity [16].

Bifidobacteria make up 3-5\% of adult microflora and thus constitute one of the predominant species of the human colonic microflora [31]. They are more abundant in human gut microbiota than Lactobacilli [32] and showed a moderate to low adhesion capacity of IECs [24]. B. animalis subsp. lactis has well-established probiotic characteristics and proven beneficial health effects for gastrointestinal health and immune function [33]. This strain also has potent anti-inflammatory and anti-proliferative effects [10]. The capacity of Bifidobacteria to inhibit LPS-induced NF- $\kappa B$ activation is strain-dependent in IECs. For example, B. bifidum was reported as a promising candidate for probiotic intervention in inflammatory diseases of the gastrointestinal tract [31]. A recent study screened new probiotic strains for IBD management by combining in vitro and in vivo methods and found that the best anti-inflammatory capacity in peripheral blood mononuclear cells (PBMCs) from healthy donors were observed for Bifidobacteria, while the anti-inflammatory profile of Lactobacilli was strain-dependent [6]. B. bifidum PI22, the strain that exhibited the most protective capacity against acute colitis, was only slightly efficacious against chronic colitis in TNBS-treated mice. This strain inhibited the expression of genes encoding chemokine C-X-C motif ligand 2 (CXCL2), IL-1 $\beta$, TNF- $\alpha$, IL-6 and IL-17 [6]. In contrast, $B$. lactis LA804 which was less efficacious in the acute model was the most protective against chronic colitis by downregulating the expression of genes for CXCL2, IL-1 $\beta$ and TNF- $\alpha$ in TNBS-treated mice [6].

Similar to L. acidophilus, the modulatory effects of B. animalis subsp. lactis on intestinal inflammation and the immune system are also dependent on the stimulation used in IECs and animal models. B. lactis BB12 transiently triggered the phosphorylation of p 65 NF- $\mathrm{kB}$ and p38 MAPK in unstimulated native epithelium after the initial monoassociation of rats and in unstimulated Mode-K IECs [34]. TLR2 played an important role in mediating the initial interaction of B. lactis BB12 with the intestinal epithelium [34]. This study suggested that probiotics could transiently trigger innate signal transduction and pro-inflammatory gene expression in the intestinal epithelium in the early stages of bacterial colonization [34].

In contrast, $B$. animalis subsp. lactis showed an anti-inflammatory effect in IECs stimulated by bacterial ligands or pro-inflammatory cytokines and in animals treated with pro-inflammatory compounds. B. lactis inhibited NF- $\mathrm{kB}$ and its regulated genes for COX-2, matrix metalloproteinase-9 (MMP-9) and vascular endothelial growth factor (VEGF) in HT-29 cells stimulated with IL-1 $\beta$, TNF- $\alpha$ or LPS [10]. Another study showed that B. animalis ssp. lactis UABla-12 was able to downregulate IL-8 levels in LPS-treated HT-29 cells [24]. Our study used LPS and TNF- $\alpha$ to induce inflammatory responses of HT-29 cells and showed an anti-inflammatory effect of B. animalis subsp. lactis, in agreement with previous studies. Animal studies also confirmed the anti-inflammatory activity of this strain of probiotics. For example, B. lactis treatment reduced colonic TNF- $\alpha$ production and the expressions of iNOS and COX-2 in TNBS model of rat colitis [17]. B. lactis prevented DSS-induced acute colitis and $C A C$ in mice and could be a therapeutic agent for IBD as well as a potential preventive agent for CAC [10].

Previous studies found that Bifidobacterium strains' DNA was effective in limiting epithelial pro-inflammatory responses in IL-10-deficient mice and in HT-29 cells challenged with TNF- $\alpha$; however, the pro-inflammatory response to LPS in HT-29 cells was not inhibited by probiotic DNA [31]. These authors indicated that the anti-inflammatory compound is neither actively secreted by Bifidobacteria nor released from lysed cells. The mechanisms by which whole cells of Bifidobacteria 
inhibit inflammatory responses are distinct from their DNA [31]. Recent studies have focused on the surface-associated structural components of Bifidobacteria, such as EPS, peptidoglycan, LTA and lipoglycans [35]. The biosynthesis of EPS has been described in several species of Bifdobacterium and it seems that these molecules play a relevant role in their immunomodulatory and anti-inflammatory capabilities [36].

The synergistic effect of different probiotic strains may have a higher impact on host cells compared to single probiotic strains. Some studies on the anti-inflammatory effects of probiotics were performed using mixtures of different probiotic strains, mainly probiotics belonging to Lactobacillus and Bifidobacterium genera [31]. Clinical reports have found that administration of mixed probiotics exhibits a combinatorial effect in the host [35]. The enhanced efficacy of multi-strain/combinatorial therapy may arise due to a combination of several probiotic mechanisms, with each strain making their own contributions [35]. Some combined probiotics have been shown to possess a good anti-inflammatory activity in vivo and in vitro. A combination of Streptococcus thermophilus ATCC19258 and L. acidophilus ATCC4356 had a pronounced effect on the phosphorylation of p38 MAPK and extracellular signal regulated kinase (ERK)1/2 in HT-29 cells with or without the stimulation of interferon- $\gamma$ (IFN- $\gamma$ ) or TNF- $\alpha$ [30]. In addition, this probiotic combination reduced the phosphorylation of the nuclear factor of kappa light polypeptide gene enhancer in B-cells inhibitor $\alpha$ (IкB- $\alpha$ ) [37]. These results suggest that the probiotic combination may activate the MAPK signaling pathway but inhibit the NF- $\mathrm{KB}$ signaling pathway in IECs challenged by pro-inflammatory cytokines. VSL\#3 probiotics significantly ameliorated the disease activity index (DAI), prevented the decrease of tight junction proteins including occludin and zonula occludens (ZO)-1 and increased the expression of $p$-p38 MAPK and phosphorylated ERK ( $p$-ERK) in DSS-treated rats [38]. Furthermore, tight junction proteins (occludin and ZO-1), p-p38 MAPK and $p$-ERK in the VSL\#3 group were significantly higher than those in either the control or TNF- $\alpha$ group in TNF- $\alpha$-induced HT-29 cells [38]. This study suggests that VSL\#3 probiotics may enhance the tight junction and protect the epithelial barrier in vivo and in vitro by activating the p38 MAPK and ERK signaling pathways. Ultrabletique, a probiotic cocktail, exerted an anti-inflammatory effect and contributed to the rapid recovery of DSS-induced acute colitis in mice [39]. The results were associated with reduced expressions of TLR4, iNOS and NF-KB in colonic tissues [39].

There are limited studies investigating the interaction between different probiotics. L. acidophilus Bar13 and B. longum Bar33 were effective in inhibiting IL-8 secretion in HT-29 cells stimulated by TNF- $\alpha$, IL-1 $\beta$ or LPS, and B. longum Bar33 presented the highest effect [27]. Both strains showed the potential to protect enterocytes against an acute inflammatory response [27]. However, a combination of these two strains did not show an additive effect. Another study demonstrated that each of L.acidophilus, B. lactis or their combination had an individual modulatory impact on the immune system, including regulatory $\mathrm{T}$ (Treg) cells and effector T helper (Th) cells, of UC patients [13]. However, there is no synergistic effect between these two strains. B. lactis had a more stimulatory effect on the Treg cells of UC patients, although it also stimulated the effector Th cells [13]. Based on the IL-10 and transforming growth factor- $\beta$ (TGF- $\beta$ ) levels of PBMCs stimulated by probiotics, the authors supposed that $B$. lactis could be a better candidate for Treg cells stimulation compared with L. acidophilus and mixed probiotics in UC patients. Our study found that both L. acidophilus and B. animalis subsp. lactis significantly suppressed inflammation in LPS- and TNF- $\alpha$-stimulated HT-29 cells and that the combination of these two strains showed the best anti-inflammatory effect. Our results also indicated that TLR, NF- $\mathrm{KB}$ and MAPK signaling pathways and their associated factors, such as adhesion molecules and inflammatory mediators, played important roles in the observed anti-inflammatory activity. The two strains may have an interaction through these pathways.

TLRs are pattern recognition receptors (PRR) expressed by various cells in the gastrointestinal tract, including IECs [8]. TLRs represent the first point of contact between environment and organism and are powerful molecular regulators by which the immune system may sense the environment and protect the host from pathogens or endogenous threats [40]. TLRs are membrane bound and provide pathogen surveillance, which upon ligand binding, activate NF-kB signaling and lead to the production 
of pro-inflammatory cytokines, chemokines and antimicrobial peptides [12]. TLR signaling in IECs plays several crucial roles in maintaining healthy gut epithelial barrier that includes proliferation of epithelial cells, production of antimicrobials, maintenance of tight junction and modulation of immune system [36]. TLR signaling in the gut is involved in either maintaining intestinal homeostasis or inducing an inflammatory response [8]. Under normal conditions, IECs show low expression of TLRs and are therefore unresponsive to TLR stimuli; under inflammatory conditions, epithelial TLR expression is increased, which contributes to both inflammation and immune tolerance [8]. Epithelial TLR2 activation has been described to protect against barrier disruption in IECs [8].

TLR2 recognizes a wide range of ligands, among which the best characterized are lipoproteins highly expressed on the outer membrane of Gram-positive bacteria [40]. The cell wall of probiotic bacteria contains peptidoglycan, lipoproteins and lipopeptides, described as TLR2 ligands, and thus TLR2 plays an important role in the recognition of anti-inflammatory probiotic bacteria [36]. The immunomodulatory role of LTA in probiotic Lactobacillus through TLR2 recognition is well established. Although these biopolymers have long been identified in Bifidobacterium, their roles in PRR-mediated interaction in host have not yet been well studied [35]. In contrast, the activation of TLR4 by LPS from Gram-negative bacteria leads to the secretion of pro-inflammatory mediators in the intestinal mucosa, mainly through the activation of the transcription factor NF- $\mathrm{KB}$ [39]. TLR2 ligation may induce a suppressive effect on TLR4-mediated inflammatory responses via the expression or activation of TLR negative regulators [12]. The present study showed that L. acidophilus and B. animalis subsp. lactis, either alone or in combination, significantly enhanced TLR2 expression in HT-29 cells stimulated with LPS and TNF- $\alpha$, suggesting the potential of these probiotics in protecting IECs against bacteria- and cytokine-mediated inflammation.

NF- $\mathrm{kB}$ transcriptional system in IEC plays an essential role in the regulation of inflammation in patients with various intestinal disorders [10]. It regulates the transcription of a series of genes involved in acute responses to injury and in chronic intestinal inflammation including the genes for IL-1 $\beta$, TNF- $\alpha$, IL-6, IL-8, COX-2, ICAM-1, and so on [31]. NF- - B is activated through a wide range of stimuli such as TNF- $\alpha$, IL-1 $\beta$ and LPS [31] and acts as a central regulator of the IEC innate immune response and as an essential transcription factor for integrating the pro-inflammatory response to infection with enteroinvasive bacteria [41]. In addition, NF-kB, as an important downstream pathway in LPS-mediated signal responses, is also closely related to tumor growth, inflammation and apoptosis [29]. Probiotics can alter the NF-kB pathway at many different levels including degradation, ubiquitination and inhibition of proteasome function [39]. Induction of the nuclear export of complexes formed by nuclear factor NF- $\mathrm{kB}$ and peroxisome proliferator-activated receptor- $\gamma$ (PPAR- $\gamma$ ) is another anti-inflammatory mechanism induced by probiotics [39].

MAPK proteins, including ERK, c-Jun N-terminal kinase (JNK), and p38 protein kinases, are activated to act on their respective substrates to regulate the inflammatory response in LPS-stimulated cells [29]. Activation of the MAPK signaling pathway by LPS can indirectly activate the downstream NF- $\mathrm{kB}$ pathway, initiate protein expression and stimulate complex physiological responses [29]. Probiotics can affect the MAPK signaling pathway independent of NF- $\mathrm{KB}$ signaling. Suppression of $\mathrm{p} 38$ phosphorylation has been associated with the inhibition of IL-8 secretion without impacting on IL-8 mRNA levels or the activation of NF-KB [14]. Probiotic-induced changes in phosphorylation levels of p38, JNK and ERK1/2 MAPKs can affect cytokine secretion and apoptosis [14].

The present study showed that LPS and TNF- $\alpha$ stimulation significantly increased the phosphorylated forms of p65 NF-KB and p38 MAPK in HT-29 cells, suggesting the activation of NF- $\mathrm{KB}$ and MAPK signaling pathways. Inflammatory cells treated with either L. acidophilus or B. animalis subsp. lactis had significantly lower expression of phosphorylated p65 NF- $\mathrm{kB}$ and p38 MAPK proteins compared with untreated cells. Furthermore, the combination of L. acidophilus and B. animalis subsp. lactis showed the best inhibitory effect on the activation of NF- $\mathrm{KB}$ and MAPK. These results suggest that the two strains of probiotics may exert an anti-inflammatory effect by modulating NF- $\kappa \mathrm{B}$ and MAPK signaling pathways in IECs. 
IL-8 is a chemokine produced by many types of cells, including IECs [11]. IL-8 is responsible for the initiation of inflammatory cascades and the recruitment of neutrophils into the mucosa of lesions [16]. It also plays important roles in angiogenesis and the metastasis of tumors [11]. In response to enteropathogens infection the IECs release IL-8 and other pro-inflammatory molecules that initiate an acute inflammatory response [27]. A prolonged infection can result in a massive and long-lasting IL-8 release by IECs [27]. LPS, IL- $1 \alpha$, IL-1 $\beta$, IL-10, IFN- $\gamma$ and TNF- $\alpha$ regulate IL-8 production in various cell types [11]. HT-29 cells produce IL- 8 after stimulation by TNF- $\alpha$ and IL-1 $\beta$, but not by IFN- $\gamma$, IL-10 or IL-13 [11]. TNF- $\alpha$ plays a guiding role in the production of IL-8, and NF- $\mathrm{kB}$ establishes a feedback mechanism via autocrine/paracrine factors to regulate the expression and activation of IL-8 [42].

COX-2 plays an important role in inflammation. A variety of extracellular and intracellular stimuli, including LPS and TNF- $\alpha$, rapidly induce the expression of COX-2 [43]. There are strain- and stimulation-specific effects of probiotics on COX-2 expression in IECs. Both probiotic E. coli Nissle and mixed probiotics VSL\#3 had no effect on basal COX-2 expression but decreased stimulated COX-2 activity, protein expression and $\mathrm{PGE}_{2}$ secretion, while L. acidophilus increased COX-2 expression and $\mathrm{PGE}_{2}$ secretion in unstimulated Colo320 and SW480 IECs and in gastrin-induced Colo320 cells but not in TNF- $\alpha$-induced Colo320 cells [26]. Although the precise mechanisms regulating COX-2 expression in IECs have not been fully clarified, activator protein-1 (AP-1) and p38 MAPK are the only regulatory elements identified in IECs so far [26].

The present study found that stimulation by LPS and TNF- $\alpha$ significantly increased the secretory level of IL-8 and the expression of COX-2 in HT-29 cells, showing a typical inflammatory response. In contrast, the inflammatory cells treated with either L. acidophilus or B. animalis subsp. lactis had significantly lower IL-8 level and COX-2 expression compared to those without probiotics treatment. Additionally, combination of L. acidophilus and B. animalis subsp. lactis showed the best inhibitory effect on IL-8 secretion and COX-2 expression. These effects are in agreement with those on NF- $\mathrm{KB}$ and MAPK and suggest that these two strains of probiotics are able to suppress inflammation by modulating IL-8 secretion and COX-2 expression in IECs.

Cell adhesion molecules (CAMs) are categorized as cadherins, selectins, integrins and immunoglobulin-like adhesion molecules (IgCAMs) such as mucosal addressin cell adhesion molecule 1 (MAdCAM-1), ICAM-1 and VCAM-1. They are required for the binding of leukocytes to tissue components and the directional movement of leukocytes toward inflamed sites [44]. Human IECs are normally lack of ICAM- 1 and VCAM- 1 expressions but induction of their expressions has been reported in response to pro-inflammatory cytokines such as IL-1 $\beta$, IL-6 and IFN- $\gamma$ [45]. Our study also induced the expressions of ICAM- 1 and VCAM- 1 by LPS and TNF- $\alpha$. ICAM- 1 and VCAM- 1 are closely related to NF- $\mathrm{KB}$ and MAPK signaling pathways. The binding sites for NF- $\mathrm{KB}$ have been identified in the promoter regions of the genes for ICAM-1 and VCAM-1 [46]. Another study has indicated that there is a possible relationship between inhibition of MAPKs and decreased expression of ICAM- 1 and VCAM-1 [45].

ICAM-1 is constitutively expressed by several types of cells, including leukocytes, endothelial cells and IECs $[47,48]$. The major agonists for ICAM- 1 expression are bacterial products (such as LPS) and pro-inflammatory cytokines (such as TNF- $\alpha$, IL-1 and IFN- $\gamma$ ) released at inflammatory sites [47]. Expression of ICAM-1 in human IECs is upregulated following infection with invasive bacteria and stimulation with TNF- $\alpha$ and IFN- $\gamma$ [47]. Transcription of ICAM- 1 is under regulation by cytokines such as IL- $1 \beta$, TNF- $\alpha$ and IFN- $\gamma$ as well as by growth factors and stress oxidants through various signaling pathways, including NF- $\mathrm{KB}$ and AP-1 pathways [44]. ICAM-1 plays an important role in both innate and adaptive immune responses. It is involved in the transendothelial migration of leukocytes to inflammatory sites, as well as interactions between antigen presenting cells (APC) and T cells [48]. ICAM-1 on IECs is involved in the immune defense, including attraction of neutrophils to the surface defect, adhesion of transmigrated neutrophils to face the luminal content and binding of microbial surface molecules [44]. Both active UC and CD are associated with marked infiltration of inflammatory 
cells, while ICAM-1 is involved in leukocyte infiltration [49]. ICAM-1 plays a specific role in the pathogenesis of IBD, so loss of ICAM-1 function may be effective in controlling IBD [44].

VCAM-1 is inducible and predominantly expressed in endothelial cells and also on the surface of other cells, including macrophages and cancer cells [50]. The expression of VCAM-1 is activated by pro-inflammatory cytokines, including TNF- $\alpha$, and also by ROS, oxidized low-density lipoprotein (oLDL), high glucose concentration, TLR agonists (such as LPS) and shear stress [50]. VCAM-1 is an important modulator of lymphocyte and monocyte trafficking [46]. VCAM-1 was originally identified as a cell adhesion molecule that helps regulate inflammation-associated vascular adhesion and the transendothelial migration of leukocytes such as macrophages and T cells [50]. Recent evidence suggests that VCAM-1 is closely associated with the progression of various immunological disorders and cancers, so it is a potential therapeutic target in these diseases [50]. Experimental studies have shown that blockade of VCAM-1 results in a higher inhibition of leukocyte adhesion in colonic venules and a potent effect on preventing or reversing established inflammation in various animal models [51-53].

In human intestinal microvascular endothelial cells, ICAM-1 is constitutively expressed and VCAM-1 is not detectable in basal conditions but a marked increase in both adhesion molecules is observed after challenges of these cells with IL-1 $\beta$, TNF- $\alpha$ or LPS [46]. In the murine intestine, the constitutive level of VCAM-1 expression is substantially lower than that of ICAM-1; however, a significant increase in the endothelial cell surface density of VCAM-1 is noted by cytokine stimulation [46]. Remarkable increases in VCAM-1 expression have also been documented in rodent models of chemically induced colitis, as well as in colitis of IL-10 knockout mice [46,51,52]. In these animal models, the expression of ICAM-1 was not increased or was only slightly elevated, whereas increased expressions of MAdCAM- 1 and VCAM- 1 were observed. VCAM-1, in contrast with ICAM-1, is not involved in physiological leukocyte recirculation and selective blockade of its function might attenuate the inflammatory response without altering physiological immune mechanisms [46]. Specific blockade of the adhesion molecules such as ICAM-1 involved in leukocyte recruitment to the inflamed intestine is considered to be one of the most promising therapeutic targets for IBD [53,54].

Limited studies have investigated the effect of probiotics on adhesion molecules. Riedel et al. (2006) reported that B. bifidum S17 decreased mRNA level of ICAM-1 in LPS-induced HT-29 cells [31]. Angulo et al. (2006) demonstrated that $L$. casei attenuated leukocyte recruitment in experimental colitis induced by TNBS in rats [55]. This effect was possibly related to the suppression of ICAM-1 upregulation, although VCAM-1 expression was unaffected. Chu et al. (2010) indicated that L. plantarum treatment improved the histological damage score and interfered with the upregulation of adhesion molecules such as ICAM-1 in IL-10 knockout mice [49]. In addition to whole probiotics, the cell wall components of probiotics also play an important role in regulation of adhesion molecules and other inflammatory-related factors. Kim et al. (2012) showed that LTA from L. plantarum inhibited TNF- $\alpha$-induced inflammatory responses, including increased IL-8, iNOS, NO and ICAM-1, in HT-29 cells through inhibition of the NF- $\mathrm{KB}$ and MAPK signaling pathways [11].

Our study successfully induced strong expressions of ICAM-1 and VCAM-1 in HT-29 cells stimulated with LPS and TNF- $\alpha$. Interestingly, L. acidophilus and B. animalis subsp. lactis showed a different impact on ICAM-1 and VCAM-1. L. acidophilus and B. animalis subsp. lactis, either alone or in combination, significantly decreased VCAM-1 expression in inflammatory HT-29 cells compared with those untreated with probiotics. L. acidophilus showed higher efficacy on VCAM-1 inhibition than did B. animalis subsp. lactis. However, L. acidophilus alone did not inhibit ICAM-1 expression but enhanced the efficacy of $B$. animalis subsp. lactis which significantly decreased ICAM-1 expression in inflammatory HT-29 cells. It shows a synergistic effect between two strains of probiotics. ICAM-1, associated with the recruitment and adhesion of neutrophils, is known to play a determining role in acute inflammatory conditions, while VCAM- 1 is more relevant as a mediator of chronic inflammatory diseases in which the recruitment of lymphocytes is the main cellular event $[44,55]$. Blocking ICAM-1 and VCAM-1 may have therapeutic benefits for the acute inflammatory component of IBD in SAMP-1/Yi adoptive 
transfer model mice [56]. These findings suggest that B. animalis subsp. lactis may have the potential to alleviate acute inflammation in the gut, while L. acidophilus may be a candidate for remission of chronic intestinal inflammation. Combination of two strains seems to have the best anti-inflammatory activity in the intestinal tract and may be a candidate adjuvant for IBD treatment.

\section{Conclusions}

In conclusion, the present study showed that L. acidophilus and B. animalis subsp. lactis exerted a potent anti-inflammatory effect through modulating TLR2-mediated NF- $\mathrm{KB}$ and MAPK signaling pathways in inflammatory IECs. Both strains, especially their combination, may have the potential to be developed as a novel adjuvant for IBD therapy.

Author Contributions: Conceptualization, S.-C.L., W.-F.H., J.-S.C. and C.-K.S.; Methodology, S.-C.L., W.-F.H., J.-S.C. and C.-K.S.; Data Analysis, S.-C.L. and W.-F.H.; Original Draft Preparation, S.-C.L., W.-F.H., J.-S.C. and C.-K.S.; Review, Editing \& Final approval of the manuscript, C.-K.S.

Funding: This research was funded by Ministry of Science and Technology, Taiwan (NSC102-2313-B-038-006, MOST103-2313-B-038-004 \& MOST104-2320-B-038-024).

Acknowledgments: This study was supported by the grants from the Ministry of Science and Technology, Taiwan (NSC102-2313-B-038-006, MOST103-2313-B-038-004 \& MOST104-2320-B-038-024).

Conflicts of Interest: The authors declare no conflict of interest.

\section{References}

1. Ng, S.C.; Shi, H.Y.; Hamidi, N.; Underwood, F.E.; Tang, W.; Benchimol, E.I.; Panaccione, R.; Ghosh, S.; $\mathrm{Wu}$, J.C.Y.; Chan, F.K.L.; et al. Worldwide incidence and prevalence of inflammatory bowel disease in the 21st century: A systematic review of population-based studies. Lancet 2018, 390, 2769-2778. [CrossRef]

2. Yen, H.H.; Weng, M.T.; Tung, C.C.; Wang, Y.T.; Chang, Y.T.; Chang, C.H.; Shieh, M.J.; Wong, J.M.; Wei, S.C. Epidemiological trend in inflammatory bowel disease in Taiwan from 2001 to 2015: A nationwide populationbased study. Intest. Res. 2019, 17, 54-62. [CrossRef] [PubMed]

3. Molodecky, N.A.; Soon, I.S.; Rabi, D.M.; Ghali, W.A.; Ferris, M.; Chernoff, G.; Benchimol, E.I.; Panaccione, R.; Ghosh, S.; Barkema, H.W.; et al. Increasing incidence and prevalence of the inflammatory bowel diseases with time, based on systematic review. Gastroenterology 2012, 142, 46-54. [CrossRef]

4. De Filippo, C.; Cavalieri, D.; Di Paola, M.; Ramazzotti, M.; Poullet, J.B.; Massart, S.; Collini, S.; Pieraccini, G.; Lionetti, P. Impact of diet in shaping gut microbiota revealed by a comparative study in children from Europe and rural Africa. Proc. Natl. Acad. Sci. USA 2010, 107, 14691-14696. [CrossRef] [PubMed]

5. Derwa, Y.; Gracie, D.J.; Hamlin, P.J.; Ford, A.C. Systematic review with meta-analysis: The efficacy of probiotics in inflammatory bowel disease. Aliment. Pharm. 2017, 46, 389-400. [CrossRef]

6. Alard, J.; Peucelle, V.; Boutillier, D.; Breton, J.; Kuylle, S.; Pot, B.; Holowacz, S.; Grangette, C. New probiotic strains for inflammatory bowel disease management identified by combining in vitro and in vivo approaches. Benef. Microbes 2018, 9, 317-331. [CrossRef] [PubMed]

7. Torre, L.A.; Siegel, R.L.; Ward, E.M.; Jemal, A. Global cancer incidence and mortality rates and trends-An update. Cancer Epidemiol. Biomark. Prev. 2016, 25, 16-27. [CrossRef]

8. De Kivit, S.; Tobin, M.C.; Forsyth, C.B.; Keshavarzian, A.; Landay, A.L. Regulation of intestinal immune responses through tlr activation: Implications for pro-and prebiotics. Front Immunol. 2014, 5, 60. [CrossRef] [PubMed]

9. Kaci, G.; Lakhdari, O.; Dore, J.; Ehrlich, S.D.; Renault, P.; Blottiere, H.M.; Delorme, C. Inhibition of the NF-kappaB pathway in human intestinal epithelial cells by commensal Streptococcus salivarius. Appl. Environ. Microbiol. 2011, 77, 4681-4684. [CrossRef] [PubMed]

10. Kim, S.W.; Kim, H.M.; Yang, K.M.; Kim, S.A.; Kim, S.K.; An, M.J.; Park, J.J.; Lee, S.K.; Kim, T.I.; Kim, W.H.; et al. Bifidobacterium lactis inhibits NF-kappaB in intestinal epithelial cells and prevents acute colitis and colitis-associated colon cancer in mice. Inflamm. Bowel Dis. 2010, 16, 1514-1525. [CrossRef]

11. Kim, H.; Jung, B.J.; Jung, J.H.; Kim, J.Y.; Chung, S.K.; Chung, D.K. Lactobacillus plantarum lipoteichoic acid alleviates TNF-alpha-induced inflammation in the HT-29 intestinal epithelial cell line. Mol. Cells 2012, 33, 479-486. [CrossRef] [PubMed] 
12. Llewellyn, A.; Foey, A. Probiotic modulation of innate cell pathogen sensing and signaling events. Nutrients 2017, 9, 1156. [CrossRef] [PubMed]

13. Sheikhi, A.; Shakerian, M.; Giti, H.; Baghaeifar, M.; Jafarzadeh, A.; Ghaed, V.; Heibor, M.R.; Baharifar, N.; Dadafarin, Z.; Bashirpour, G. Probiotic yogurt culture Bifidobacterium animalis subsp. lactis BB-12 and Lactobacillus acidophilus LA-5 modulate the cytokine secretion by peripheral blood mononuclear cells from patients with ulcerative colitis. Drug Res. 2016, 66, 300-305. [CrossRef]

14. Thomas, C.M.; Versalovic, J. Probiotics-host communication: Modulation of signaling pathways in the intestine. Gut Microbes 2010, 1, 148-163. [CrossRef]

15. De Vrese, M.; Schrezenmeir, J. Probiotics, prebiotics, and synbiotics. Adv. Biochem. Eng. Biotechnol. 2008, 111, 1-66. [PubMed]

16. Kainulainen, V.; Tang, Y.; Spillmann, T.; Kilpinen, S.; Reunanen, J.; Saris, P.E.; Satokari, R. The canine isolate Lactobacillus acidophilus LAB20 adheres to intestinal epithelium and attenuates LPS-induced IL-8 secretion of enterocytes in vitro. BMC Microbiol. 2015, 15, 4. [CrossRef]

17. Peran, L.; Camuesco, D.; Comalada, M.; Bailon, E.; Henriksson, A.; Xaus, J.; Zarzuelo, A.; Galvez, J. A comparative study of the preventative effects exerted by three probiotics, Bifidobacterium lactis, Lactobacillus casei and Lactobacillus acidophilus, in the TNBS model of rat colitis. J. Appl. Microbiol. 2007, 103, 836-844. [CrossRef] [PubMed]

18. Ostad, S.; Salarian, A.; Ghahramani, M.; Fazeli, M.; Samadi, N.; Jamalifar, H. Live and heat-inactivated lactobacilli from feces inhibit Salmonella typhi and Escherichia coli adherence to Caco-2 cells. Folia Microbiol. 2009, 54, 157-160. [CrossRef]

19. Bevilacqua, A.; Cagnazzo, M.T.; Caldarola, C.; Ciuffreda, E.; Dragano, A.R.; Franchino, S.; Lauriola, R.; Pacifico, A.; Corbo, M.R.; Sinigaglia, M. Bifidobacteria as potential functional starter cultures: A case study by msc students in food science and technology (University of Foggia, Southern Italy). Food Nutr. Sci. 2012, 3, 55. [CrossRef]

20. Lee, S.K.; Kim, T.I.; Kim, Y.K.; Choi, C.H.; Yang, K.M.; Chae, B.; Kim, W.H. Cellular differentiation-induced attenuation of LPS response in HT-29 cells is related to the down-regulation of TLR4 expression. Biochem. Biophys. Res. Commun. 2005, 337, 457-463. [CrossRef]

21. Jiang, Y.; Lü, X.; Man, C.; Han, L.; Shan, Y.; Qu, X.; Liu, Y.; Yang, S.; Xue, Y.; Zhang, Y. Lactobacillus acidophilus induces cytokine and chemokine production via NF-kB and p38 mitogen-activated protein kinase signaling pathways in intestinal epithelial cells. Clin. Vaccine Immunol. 2012, 19, 603-608. [CrossRef]

22. Bradford, M.M. A rapid and sensitive method for the quantitation of microgram quantities of protein utilizing the principle of protein-dye binding. Anal. Biochem. 1976, 72, 248-254. [CrossRef]

23. Jeong, C.H.; Seok, J.S.; Petriello, M.C.; Han, S.G. Arsenic downregulates tight junction claudin proteins through p38 and NF-кB in intestinal epithelial cell line, HT-29. Toxicology 2017, 379, 31-39. [CrossRef]

24. Vemuri, R.; Shinde, T.; Shastri, M.D.; Perera, A.P.; Tristram, S.; Martoni, C.J.; Gundamaraju, R.; Ahuja, K.D.K.; Ball, M.; Eri, R. A human origin strain Lactobacillus acidophilus DDS-1 exhibits superior in vitro probiotic efficacy in comparison to plant or dairy origin probiotics. Int. J. Med. Sci. 2018, 15, 840-848. [CrossRef] [PubMed]

25. Agah, S.; Alizadeh, A.M.; Mosavi, M.; Ranji, P.; Khavari-Daneshvar, H.; Ghasemian, F.; Bahmani, S.; Tavassoli, A. More protection of Lactobacillus acidophilus than Bifidobacterium bifidum probiotics on azoxymethane-induced mouse colon cancer. Probiotics Antimicrob. Proteins 2018. [CrossRef]

26. Otte, J.M.; Mahjurian-Namari, R.; Brand, S.; Werner, I.; Schmidt, W.E.; Schmitz, F. Probiotics regulate the expression of COX-2 in intestinal epithelial cells. Nutr. Cancer 2009, 61, 103-113. [CrossRef]

27. Candela, M.; Perna, F.; Carnevali, P.; Vitali, B.; Ciati, R.; Gionchetti, P.; Rizzello, F.; Campieri, M.; Brigidi, P. Interaction of probiotic lactobacillus and bifidobacterium strains with human intestinal epithelial cells: Adhesion properties, competition against enteropathogens and modulation of il-8 production. Int. J. Food Microbiol. 2008, 125, 286-292. [CrossRef]

28. Kim, D.H.; Kim, S.; Lee, J.H.; Kim, J.H.; Che, X.; Ma, H.W.; Seo, D.H.; Kim, T.I.; Kim, W.H.; Kim, S.W.; et al. Lactobacillus acidophilus suppresses intestinal inflammation by inhibiting endoplasmic reticulum stress. J. Gastroenterol. Hepatol. 2019, 34, 178-185. [CrossRef]

29. Wang, H.; Zhang, L.; Xu, S.; Pan, J.; Zhang, Q.; Lu, R. Surface-layer protein from Lactobacillus acidophilus NCFM inhibits lipopolysaccharide-induced inflammation through MAPK and NF-kB signaling pathways in RAW264. 7 cells. J. Agric. Food Chem. 2018, 66, 7655-7662. [CrossRef] 
30. De Marco, S.; Sichetti, M.; Muradyan, D.; Piccioni, M.; Traina, G.; Pagiotti, R.; Pietrella, D. Probiotic cell-free supernatants exhibited anti-inflammatory and antioxidant activity on human gut epithelial cells and macrophages stimulated with LPS. Evid. Based Complement. Altern. Med. 2018, 2018, 1756308. [CrossRef] [PubMed]

31. Riedel, C.U.; Foata, F.; Philippe, D.; Adolfsson, O.; Eikmanns, B.J.; Blum, S. Anti-inflammatory effects of bifidobacteria by inhibition of LPS-induced NF-kappaB activation. World J. Gastroenterol. 2006, 12, 3729-3735. [CrossRef]

32. Pham, T.; Teoh, K.T.; Savary, B.J.; Chen, M.H.; McClung, A.; Lee, S.O. In vitro fermentation patterns of rice bran components by human gut microbiota. Nutrients 2017, 9, 1237. [CrossRef] [PubMed]

33. Jungersen, M.; Wind, A.; Johansen, E.; Christensen, J.E.; Stuer-Lauridsen, B.; Eskesen, D. The science behind the probiotic strain Bifidobacterium animalis subsp. lactis BB-12. Microorganisms 2014, 2, 92-110. [CrossRef] [PubMed]

34. Ruiz, P.A.; Hoffmann, M.; Szcesny, S.; Blaut, M.; Haller, D. Innate mechanisms for bifidobacterium lactis to activate transient pro-inflammatory host responses in intestinal epithelial cells after the colonization of germ-free rats. Immunology 2005, 115, 441-450. [CrossRef] [PubMed]

35. Sarkar, A.; Mandal, S. Bifidobacteria-Insight into clinical outcomes and mechanisms of its probiotic action. Microbiol. Res. 2016, 192, 159-171. [CrossRef]

36. Wachi, S.; Kanmani, P.; Tomosada, Y.; Kobayashi, H.; Yuri, T.; Egusa, S.; Shimazu, T.; Suda, Y.; Aso, H.; Sugawara, M. Lactobacillus delbrueckii TUA $4408 \mathrm{~L}$ and its extracellular polysaccharides attenuate enterotoxigenic Escherichia coli-induced inflammatory response in porcine intestinal epitheliocytes via toll-like receptor-2 and 4. Mol. Nutr. Food Res. 2014, 58, 2080-2093. [CrossRef]

37. Resta-Lenert, S.; Barrett, K.E. Probiotics and commensals reverse TNF-alpha- and IFN-gamma-induced dysfunction in human intestinal epithelial cells. Gastroenterology 2006, 130, 731-746. [CrossRef] [PubMed]

38. Dai, C.; Zhao, D.-H.; Jiang, M. VSL\#3 probiotics regulate the intestinal epithelial barrier in vivo and in vitro via the p38 and erk signaling pathways. Int. J. Mol. Med. 2012, 29, 202-208.

39. Toumi, R.; Soufli, I.; Rafa, H.; Belkhelfa, M.; Biad, A.; Touil-Boukoffa, C. Probiotic bacteria lactobacillus and bifidobacterium attenuate inflammation in dextran sulfate sodium-induced experimental colitis in mice. Int. J. Immunopathol. Pharm. 2014, 27, 615-627. [CrossRef]

40. Hug, H.; Mohajeri, M.H.; La Fata, G. Toll-like receptors: Regulators of the immune response in the human gut. Nutrients 2018, 10, 203. [CrossRef]

41. Elewaut, D.; DiDonato, J.A.; Kim, J.M.; Truong, F.; Eckmann, L.; Kagnoff, M.F. NF-kB is a central regulator of the intestinal epithelial cell innate immune response induced by infection with enteroinvasive bacteria. J. Immunol. 1999, 163, 1457-1466.

42. Zhang, J.; Yi, R.; Qian, Y.; Sun, P.; Zhao, X.; Yang, Z. Lactobacillus plantarum CQPC06 activity prevents dextran sulfate sodium-induced colitis by regulating the IL-8 pathway. J. Food Sci. 2018, 83, 2653-2661. [CrossRef] [PubMed]

43. Williams, C.S.; Mann, M.; DuBois, R.N. The role of cyclooxygenases in inflammation, cancer, and development. Oncogene 1999, 18, 7908-7916. [CrossRef]

44. Vainer, B. Intercellular adhesion molecule-1 (ICAM-1) in ulcerative colitis: Presence, visualization, and significance. Apmis 2010, 118, 1-43. [CrossRef]

45. Gallicchio, M.; Rosa, A.C.; Dianzani, C.; Brucato, L.; Benetti, E.; Collino, M.; Fantozzi, R. Celecoxib decreases expression of the adhesion molecules ICAM-1 and VCAM-1 in a colon cancer cell line (HT29). Br. J. Pharm. 2008, 153, 870-878. [CrossRef] [PubMed]

46. Panés, J.; Peñalva, M.; Piqué, J. New therapeutic targets in inflammatory bowel disease (IBD): Cell adhesion molecules. Inmunología 2003, 22, 1-12.

47. Huang, G.T.; Eckmann, L.; Savidge, T.C.; Kagnoff, M.F. Infection of human intestinal epithelial cells with invasive bacteria upregulates apical intercellular adhesion molecule-1 (ICAM-1) expression and neutrophil adhesion. J. Clin. Investig. 1996, 98, 572-583. [CrossRef]

48. Lawson, C.; Wolf, S. ICAM-1 signaling in endothelial cells. Pharm. Rep. 2009, 61, 22-32. [CrossRef]

49. Chu, Z.X.; Chen, H.Q.; Ma, Y.L.; Zhou, Y.K.; Zhang, M.; Zhang, P.; Qin, H.L. Lactobacillus plantarum prevents the upregulation of adhesion molecule expression in an experimental colitis model. Dig. Dis. Sci. 2010, 55, 2505-2513. [CrossRef] 
50. Kong, D.H.; Kim, Y.K.; Kim, M.R.; Jang, J.H.; Lee, S. Emerging roles of vascular cell adhesion molecule-1 (VCAM-1) in immunological disorders and cancer. Int. J. Mol. Sci. 2018, 19, 1057. [CrossRef]

51. Sans, M.; Panes, J.; Ardite, E.; Elizalde, J.I.; Arce, Y.; Elena, M.; Palacin, A.; Fernandez-Checa, J.C.; Anderson, D.C.; Lobb, R.; et al. VCAM-1 and ICAM-1 mediate leukocyte-endothelial cell adhesion in rat experimental colitis. Gastroenterology 1999, 116, 874-883. [CrossRef]

52. Soriano, A.; Salas, A.; Salas, A.; Sans, M.; Gironella, M.; Elena, M.; Anderson, D.C.; Piqué, J.M.; Panés, J. VCAM-1, but not ICAM-1 or MadCAM-1, immunoblockade ameliorates DSS-induced colitis in mice. Lab. Investig. 2000, 80, 1541. [CrossRef] [PubMed]

53. Danese, S.; Panés, J. Development of drugs to target interactions between leukocytes and endothelial cells and treatment algorithms for inflammatory bowel diseases. Gastroenterology 2014, 147, 981-989. [CrossRef] [PubMed]

54. Reinisch, W.; Hung, K.; Hassan-Zahraee, M.; Cataldi, F. Targeting endothelial ligands: ICAM-1/alicaforsen, MadCAM-1. J. Crohns Colitis 2018, 12, S669-S677. [CrossRef]

55. Angulo, S.; Llopis, M.; Antolin, M.; Gironella, M.; Sans, M.; Malagelada, J.R.; Pique, J.M.; Guarner, F.; Panes, J. Lactobacillus casei prevents the upregulation of ICAM-1 expression and leukocyte recruitment in experimental colitis. Am. J. Physiol. Gastrointest. Liver Physiol. 2006, 291, G1155-G1162. [CrossRef]

56. Burns, R.C.; Rivera-Nieves, J.; Moskaluk, C.A.; Matsumoto, S.; Cominelli, F.; Ley, K. Antibody blockade of ICAM-1 and VCAM-1 ameliorates inflammation in the SMAP-1/Yit adoptive transfer model of Crohn's disease in mice. Gastroenterology 2001, 121, 1428-1436. [CrossRef]

(C) 2019 by the authors. Licensee MDPI, Basel, Switzerland. This article is an open access article distributed under the terms and conditions of the Creative Commons Attribution (CC BY) license (http://creativecommons.org/licenses/by/4.0/). 


\title{
The Role of Vitamin D in Inflammatory Bowel Disease: Mechanism to Management
}

\author{
Jane Fletcher ${ }^{1, *}$, Sheldon C. Cooper ${ }^{2}$, Subrata Ghosh ${ }^{3,4}$ and Martin Hewison 5 \\ 1 Nutrition Nurses, University Hospitals Birmingham NHS Trust, Queen Elizabeth Hospital Birmingham, \\ Mindelsohn Way, Edgbaston, Birmingham B15 2TH 1, UK \\ 2 Gastroenterology Department, University Hospitals Birmingham NHS Trust, Queen Elizabeth Hospital \\ Birmingham, Mindelsohn Way, Edgbaston, Birmingham B15 2WB 2, UK; sheldon.cooper@uhb.nhs.uk \\ 3 NIHR Biomedical Research Centre, University Hospitals Birmingham NHS Foundation Trust, \\ Queen Elizabeth Hospital Birmingham, Mindelsohn Way, Edgbaston, Birmingham B15 2TH, UK; \\ s.ghosh@bham.ac.uk \\ 4 Institute of Translational Medicine, University of Birmingham, Birmingham B15 2TH, UK \\ 5 Institute of Metabolism and Systems Research, The University of Birmingham, \\ Birmingham B15 2TT, UK; m.hewison@bham.ac.uk \\ * Correspondence: jane.fletcher@uhb.nhs.uk; Tel.: +44-121-371-4561
}

Received: 12 April 2019; Accepted: 29 April 2019; Published: 7 May 2019

\begin{abstract}
Vitamin D has been linked to human health benefits that extend far beyond its established actions on calcium homeostasis and bone metabolism. One of the most well studied facets of extra-skeletal vitamin $\mathrm{D}$ is its activity as an immuno-modulator, in particular its potent anti-inflammatory effects. As a consequence, vitamin D deficiency has been associated with inflammatory diseases including inflammatory bowel disease (IBD). Low serum levels of the major circulating form of vitamin D, 25-hydroxyvitamin D (25-OH-D) are significantly more prevalent in patients with IBD, particularly in the winter and spring months when UV-induced synthesis of vitamin D is lower. Dietary malabsorption of vitamin D may also contribute to low serum $25(\mathrm{OH}) \mathrm{D}$ in IBD. The benefits of supplementation with vitamin D for IBD patients are still unclear, and improved vitamin D status may help to prevent the onset of IBD as well as ameliorating disease severity. Beneficial effects of vitamin D in IBD are supported by pre-clinical studies, notably with mouse models, where the active form of vitamin $\mathrm{D}, 1$,25-dihydroxyvitamin $\mathrm{D}(1,25-(\mathrm{OH}) 2 \mathrm{D})$ has been shown to regulate gastrointestinal microbiota function, and promote anti-inflammatory, tolerogenic immune responses. The current narrative review aims to summarise the different strands of data linking vitamin D and IBD, whilst also outlining the possible beneficial effects of vitamin D supplementation in managing IBD in humans.
\end{abstract}

Keywords: vitamin D; IBD; Crohn's disease; ulcerative colitis; supplementation; deficiency

\section{Introduction}

Inflammatory bowel diseases (IBD) are chronic, disabling diseases precipitating inflammation and ulceration throughout the gastro-intestinal tract (GIT). IBD is characterised by diarrhea, nocturnal defecation, abdominal pain, weight loss, and fatigue. The two principal forms of IBD are Crohn's disease (CD) and ulcerative colitis (UC). Where UC usually only affects the colon, CD may affect the entire GIT from mouth to anus. IBD is common in developed, westernised countries with highest prevalence estimates of; UC 505 per 100,000 and CD 322 per 100,000 population in Europe and UC 249 per 100,000 and CD 319 per 100,000 population in North America [1]. The incidence of IBD is rapidly increasing in newly industrialised countries strongly implicating environmental factors [2] The pathogenesis of IBD is not fully understood, but key influences are thought to include genetics, 
environmental factors, immune response, and gut microbiota [3]. The gut microbiota has emerged as an important feature in innate and adaptive immunity [4], with microbial colonisation of the gut being essential for the development of a mature immune system [5]. One explanation for the ongoing increase in IBD is the adverse effect of modern lifestyles on the composition and function of gut microbiota, including high saturated fat/high sugar diets and the use of antibiotics [6].

It is recognised that the availability of vitamin D is important in regulating gut mucosal immunity [3]; with studies suggesting that vitamin D may affect gut epithelial integrity, innate immune barrier function, and the development and function of T cells [7-9]. Although vitamin D deficiency is common in people with IBD it is not established if this is a cause or a consequence of the disease. However, there are suggestions that in genetically predisposed individuals, vitamin D deficiency may be a contributing factor in the development of IBD [7]. There is growing evidence that vitamin D status may affect disease activity. As such consideration should be given to screening and management of vitamin D deficiency in this patient group [10-12].

This narrative review explores the prevalence of vitamin D deficiency in people with IBD and the possible benefits to patients in treating this deficiency. Consideration is given to the management of vitamin D deficiency via exposure to sunlight, dietary sources and supplementation.

\section{Vitamin D Deficiency in IBD}

\subsection{Defining Vitamin D Deficiency}

Vitamin D-deficiency is a common health issue across the globe, but it is especially prevalent in Northern European countries. Despite this, defining vitamin D deficiency in human populations remains a contentious issue. In 2010 the Institute of Medicine (IOM) defined vitamin deficiency as serum concentrations of 25-OH-D less than $20 \mathrm{ng} / \mathrm{mL}$ ( $50 \mathrm{nmol} / \mathrm{L}$ ) [13]. Subsequently the Endocrine Society issued slightly different guidelines, defining vitamin D insufficiency as being serum 25-OH-D levels below $30 \mathrm{ng} / \mathrm{mL}$ (75 nmol/L) [14]. In the UK, the Scientific Advisory Committee on Nutrition (SACN) have taken a different approach [15], recommending that a serum 25-hydroxyvitamin D (25-OH-D) level of $<25 \mathrm{nmol} / \mathrm{L}$ is the threshold at which vitamin D deficiency, and associated complications such as poor musculoskeletal health, is likely to develop. However, other guidelines and studies suggest that levels of $25-\mathrm{OH}-\mathrm{D}$ below $25-50 \mathrm{nmol} / \mathrm{L}$ constitute deficiency with levels $>75 \mathrm{nmol} / \mathrm{L}$ indicating sufficiency [14,16-19]. The threshold between 51-74 nmol/L 25-OH-D may then be termed insufficiency. Crucially, these varying recommendations for vitamin D sufficiency and insufficiency continue to be based on the classical skeletal actions of vitamin $\mathrm{D}$, and there are no current recommendations for more recently reported extra-skeletal actions of vitamin $\mathrm{D}$ and additional effects are associated with pharmacologic doses of vitamin D.

Both the Endocrine Society [14] and the National Osteoporosis Society [20] recommend that screening for vitamin D deficiency should be carried out in those at risk of vitamin D deficiency; they include people with IBD/malabsorptive disorders in the at risk group. Treatment for deficiency is recommended at a cut off of $<50 \mathrm{nmol} / \mathrm{L}[14,20]$. Nonetheless, current UK National Institute for Health and Care Excellence (NICE) and American College of Gastroenterology (ACG) guidance on the management of patients with CD and UC [21-24] do not address vitamin D deficiency in these patients.

\subsection{Prevalence of Deficiency in IBD}

Vitamin D deficiency is a public health concern with the NICE recommending oral supplementation in specific population groups including infants and children under 4 years, pregnant or breastfeeding women, people over 65 years of age and those who have low or no exposure to the sun. For example, those who cover their skin for cultural reasons, are housebound or confined indoors [25]. The population prevalence of vitamin D deficiency (serum $25-\mathrm{OH}-\mathrm{D}<40 \mathrm{nmol} / \mathrm{L}$ ) in some westernised countries is reported to be between $30 \%$ [26,27] and $47 \%$ [28] with the highest levels reported for those people with darkly pigmented skin. However, people with IBD are likely to be at increased risk of developing 
vitamin D deficiency for a number of reasons including: impaired absorption of nutrients and bile salt malabsorption, restricted dietary intake, and medical advice to avoid/protect against sunlight exposure while taking immuno-suppressive treatments such as thiopurines [29].

A number of studies have evaluated the prevalence of vitamin D deficiency and insufficiency in people with IBD [30] (Table 1). Prevalence of vitamin D deficiency is highest during winter and spring and lowest during summer and autumn [31]. Where available reported prevalence in Table 1 corresponds with winter/spring levels. These studies suggest that vitamin D deficiency is generally higher in patients with CD than UC and usually higher than that of the general population. However, previous work by the authors suggest it is not standard clinical practice to measure vitamin $\mathrm{D}$ in these groups [30]. It is also important to recognise that whilst serum 25-OH-D deficiency has been widely reported for $\mathrm{CD}$ and $\mathrm{UC}$, this may not necessarily reflect the status of other vitamin D metabolites. Notably analysis of the active form of vitamin D, 1,25-dihydroxyvitamin D (1,25-(OH)2D) has shown higher serum levels of this metabolite in patients with CD relative to UC patients [32]. The suggestion from this and other studies is that the increased inflammation in $C D$ is associated with enhanced extra-renal conversion of 25-OH-D to 1,25- $(\mathrm{OH}) 2 \mathrm{D}$ to support gastrointestinal anti-inflammatory immune responses. Measurement of serum levels of 1,25-(OH)2D is generally rare, but it is interesting to note that serum levels of this metabolite correlated inversely with bone mineral density in CD patients [32]. The functional impact of vitamin D on skeletal homeostasis in IBD is still unclear, and it is possible that link between elevated serum 1,25-(OH)2D and bone loss simply reflects the greater severity of inflammatory disease. Inflammation itself can drive osteopenia through pro-inflammatory cytokines [33]. Impaired vitamin D status has been reported to be a risk factor for low bone mineral density (BMD) in IBD patients in some studies [34], but not in others [35], and single nucleotide polymorphism variants of the gene for the vitamin $\mathrm{D}$ receptor $(V D R)$ have been shown to protect against low BMD in patients with IBD [36]. Supplementation with vitamin D and calcium has been shown to improve BMD in pediatric [37] and adult [38] IBD patients.

Table 1. Prevalence of vitamin D deficiency and insufficiency in inflammatory bowel disease (IBD) [30].

\begin{tabular}{|c|c|c|c|c|c|c|}
\hline Study & $n$ & $\begin{array}{l}\text { Age Years } \\
\text { Mean (SD) }\end{array}$ & Condition & $\begin{array}{c}25(\mathrm{OH}) \mathrm{D} \\
<75 \mathrm{nmol} / \mathrm{L} \\
(\%)\end{array}$ & $\begin{array}{c}25(\mathrm{OH}) \mathrm{D} \\
<50 \mathrm{nmol} / \mathrm{L} \\
(\%)\end{array}$ & $\begin{array}{c}25(\mathrm{OH}) \mathrm{D} \\
<30 \mathrm{nmol} / \mathrm{L} \\
(\%)\end{array}$ \\
\hline \multirow{3}{*}{ Bours et al [39] } & 185 & $50(15)$ & UC & & 34 & \\
\hline & 131 & 47 (15) & $\mathrm{CD}$ & & 44 & \\
\hline & 57 & $41(13)$ & UC & & 44 & \\
\hline \multirow[t]{2}{*}{ Caviezel et al [40] } & 99 & $41(14)$ & $\mathrm{CD}$ & & 58 & \\
\hline & 25 & $48(15)$ & IBS & & 28 & \\
\hline \multirow{2}{*}{ Frigstad et al [41] } & 178 & 39 & UC & & 44 & 7 \\
\hline & 230 & 40 & $\mathrm{CD}$ & & 53 & 8 \\
\hline Gilman et al [42] & 50 & $38(10)$ & $\mathrm{CD}$ & & 44 & 6 \\
\hline \multirow{2}{*}{ Kabbani et al [43] } & 368 & $44(10)$ & UC & 29.9 & & \\
\hline & 597 & & CD & 30 & & \\
\hline \multirow{2}{*}{ Kuwabara et al [44] } & 41 & $39(15)$ & UC & & 60 & \\
\hline & 29 & $32(7)$ & CD & & 100 & \\
\hline \multirow{2}{*}{ McCarthy et al [45] } & 32 & $37(11)$ & $\mathrm{CD}$ & & 50 & 41 \\
\hline & 32 & 37 (11) & $\mathrm{HC}$ & & 25 & 1 \\
\hline \multirow{2}{*}{ Pappa et al [46] } & 36 & $15(3)$ & UC & & & 25 \\
\hline & 94 & $15(4)$ & CD & & & 38 \\
\hline & 112 & $16(4)$ & CD & & & 16 \\
\hline Siffledeen et al [48] & 242 & $40(10)$ & $\mathrm{CD}$ & & 22 & 8 \\
\hline \multirow{2}{*}{ Suibhne et al [49] } & 81 & $36(11)$ & CD & 90 & 63 & \\
\hline & 70 & $36(9)$ & $\mathrm{HC}$ & & 51 & \\
\hline \multirow{2}{*}{ Ulitsky et al [50] } & 101 & 42 & UC & 67 & 46 & \\
\hline & 18 & $16(2)$ & UC & 83 & 50 & 28 \\
\hline \multirow{2}{*}{ Veit et al [51] } & 40 & $17(2)$ & $\mathrm{CD}$ & 73 & 40 & 15 \\
\hline & 116 & $15(2)$ & $\mathrm{HC}$ & 75 & 27 & 10 \\
\hline
\end{tabular}

$\mathrm{UC}=$ Ulcerative colitis; $\mathrm{CD}=$ Crohn's disease; IBS = irritable bowel syndrome; HC = healthy control. 


\subsection{Vitamin D Deficiency in IBD—Cause or Consequence}

Though vitamin D deficiency in IBD is well documented it is unclear if this is a cause or a consequence of the disease. It is notable that the highest prevalence of the disease appears in more temperate climates with lower levels of sunlight [8]. Some studies have suggested a correlation between vitamin D deficiency and increased disease activity [41,43,52-54]. Low vitamin D levels associated with IBD may be due to dietary restriction or low UV light exposure, but it is also important to recognise that genetic factors also contribute to variations in circulating vitamin D [55]. Single nucleotide polymorphisms (SNP) for components of the vitamin D system have been shown to contribute to the variation of serum 25-OH-D levels in IBD patients [56]. However, the contribution of SNPs to serum levels of 25-OH-D in IBD patients is very small (3\%) [56], and subsequent Mendelian randomisation analyses have not shown any link between genetic determinants of vitamin D status and risk of IBD [57]. It is still unclear whether these observations support a causative relationship between vitamin $\mathrm{D}$ status and the development of IBD. A prospective cohort study of 72,719 women (age, 40-73 years) enrolled in the Nurses' Health Study found that a higher predicted vitamin D status was associated with a reduced risk of developing CD [58]. However, it would seem that grounds for a causative relationship are most likely to be found in laboratory evidence of the functional effects of vitamin D, suggesting an effect of the active form of vitamin D on immune responses related to IBD [59].

\section{Functional Effects of Vitamin D in IBD-Analysis of In Vitro and In Vivo Models}

The mechanistic basis for a role for vitamin D in IBD stems firstly from studies of immuno-modulatory properties of $1,25-(\mathrm{OH}) 2 \mathrm{D}$. These include antibacterial [60-62] and anti-inflammatory $[63,64]$ actions on cell from the innate and adaptive immune system that modulate the pathology of gastrointestinal dysregulation and inflammation. Vitamin D also appears to play a pivotal role in the maintenance of gastrointestinal barrier integrity by regulating proteins associated with epithelial cell gap junctions [65-67]. The barrier function of vitamin D is also linked to its impact on the gastrointestinal microbiota, with serum 25-OH-D status in humans being correlated with changes in gastrointestinal bacterial genera associated with inflammatory immune responses $[68,69]$. In this way vitamin D has the potential to both prevent the onset of IBD via effects on barrier function and microbiota homeostasis, and also ameliorate disease progression through anti-inflammatory immune responses.

The beneficial effects of vitamin D on gastrointestinal barrier function and immune surveillance are strongly supported by studies of mouse models that have incorporated dietary vitamin $\mathrm{D}$ restriction and genetic manipulation of the vitamin D metabolic and signaling system. Mice lacking the gene for the vitamin D receptor (VDR) that binds 1,25-(OH)2D show increased severity of experimentally-induced colitis that mimics IBD [70]. Similar observations have also been made for mice lacking the gene for $1 \alpha$-hydroxylase, the enzyme that converts $25-\mathrm{OH}-\mathrm{D}$ to $1,25-(\mathrm{OH}) 2 \mathrm{D}$ [71]. Thus, in mice, inability to synthesise or recognise 1,25-(OH)2D was associated with increased IBD severity. This is due, in part, to impaired anti-inflammatory adaptive immune function [70], but has also been linked to disruption of the normal gastrointestinal epithelial barrier [66]. Interestingly, in mice with dietary vitamin D restriction increased colitis severity was associated with elevated levels of normal commensal bacteria within the gastrointestinal sub-mucosal epithelium, further underlining the importance of vitamin $\mathrm{D}$ in maintaining barrier integrity and surveillance of the gut microbiota [72]. The bioavailability of vitamin D for barrier and immunomodulatory activity within the gastrointestinal tract may also be influenced by the serum vitamin D binding protein (DBP) that transports vitamin D metabolites in the circulation. Although 25-OH-D binds with high affinity to DBP, it has been reported that the unbound or 'free' fraction of serum 25-OH-D is the most biologically active for immune responses to vitamin D [73]. Thus, the interaction between DBP and 25-OH-D may play a key role in gastrointestinal responses to vitamin D. For example, mice raised on diets containing exclusively vitamin D2 showed much lower serum levels of 25-OH-D compared to mice raised on vitamin D3 only. However, because 25-OH-D2 binds with lower affinity to DBP than 25-OH-D3, the levels of free 25-OH-D were similar 
in the two groups of mice and both showed similar patterns of experimentally induced colitis [74]. These observations suggest that free 25-OH-D may be a more sensitive marker of the protective effects of vitamin D on IBD. In addition to observations from mouse models, evidence from studies of humans also supports a link between vitamin D and IBD. This includes reports that $1,25-(\mathrm{OH}) 2 \mathrm{D}$ is a potent stimulator of nucleotide-binding oligomerisation domain containing 2 (NOD2), an intracellular pathogen-recognition receptor [75]. Mutations in the gene for NOD2 (CARD15) are associated with the development of $\mathrm{CD}[76,77]$, and vitamin D status may therefore act as a key environmental trigger for those individuals who are genetically predisposed to $C D$. Figure 1 is a schematic representation of the expression of VDR and the CYP27B1 gene (responsible for the production of $1 \alpha$-hydroxylase) in human colonic epithelial cells and antigen presenting cells.

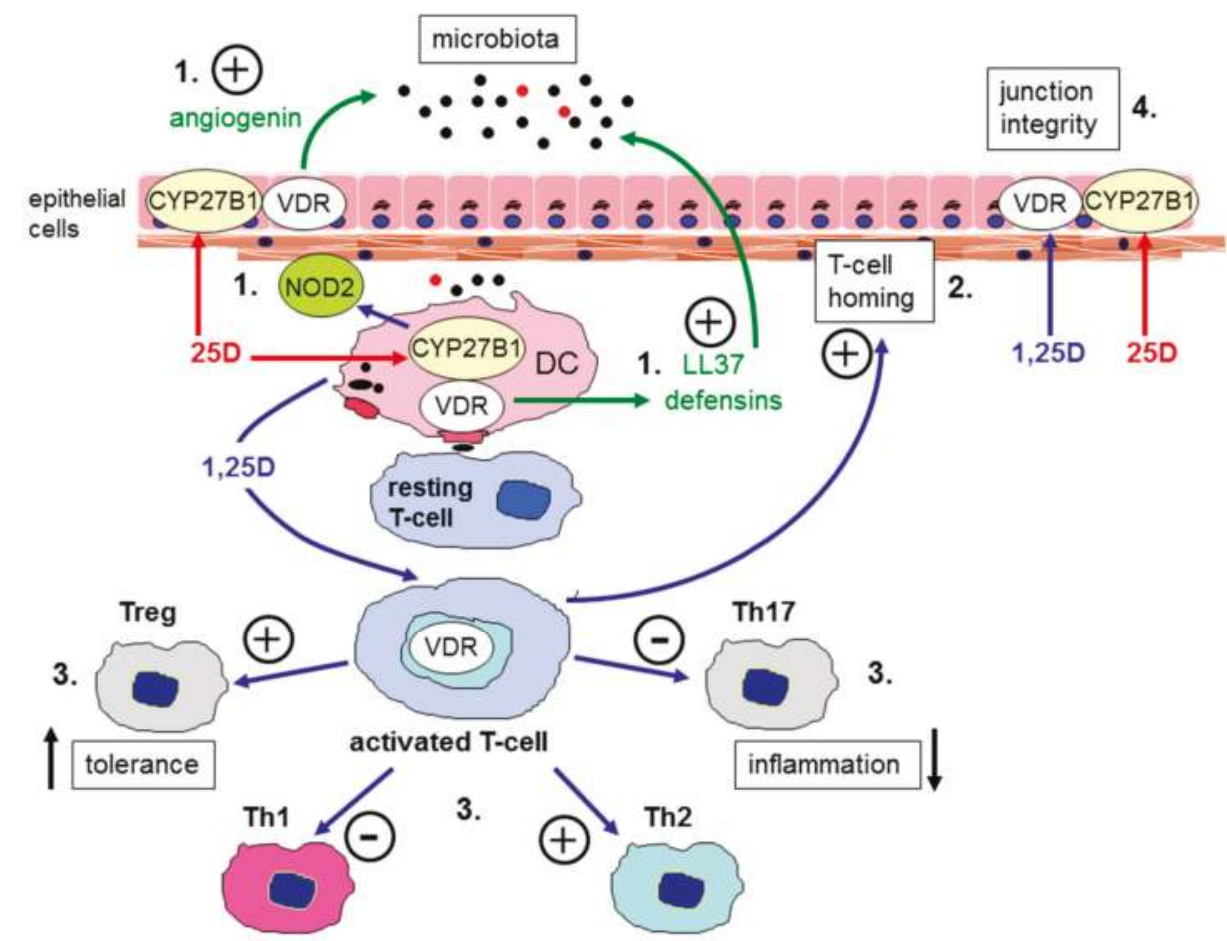

Figure 1. Vitamin $\mathrm{D}$ and barrier function in the gastrointestinal tract. Schematic representation of the expression of the vitamin D receptor (VDR) and vitamin D-activating enzyme (CYP27B1) in human colonic epithelial cells, antigen presenting cells such as dendritic cells (DC), and T cells. Immune responses to vitamin D occur either via systemic 1,25-dihydroxyvitamin D (1,25-(OH)2D) or local conversion of 25-hydroxyvitamin D (25-OH-D) to 1,25-(OH)2D. Possible target mechanisms include: 1) interface with microbiota (induction of antibacterials such as angiogenin, cathelcidin (LL37), defensins or intracellular pathogen recognition proteins such as nucleotide-binding oligomerisation domain containing 2 (NOD2)); 2) T cell homing to sites of inflammation; 3) suppression of inflammatory Th17 and Th1 cells and induction of tolerogenic Treg and Th2 cells; 4) enhanced expression of epithelial membrane junction proteins

\section{Managing Vitamin D Deficiency in IBD—Sources of Vitamin D}

\subsection{Sunlight Exposure}

The main source of vitamin D in humans is via synthesis in the body stimulated by sunlight exposure [78]. The vitamin D precursor 7-dehydrocholesterol is produced by the liver and is stored in 
the skin. On exposure of the skin to ultraviolet B-light, this is converted to pre-vitamin D3 and enters the circulation. In the liver pre-vitamin D3 is converted to the inactive form 25(OH)D3. Renal conversion of 25(OH)D3 produces active form 1,25- $(\mathrm{OH}) 2 \mathrm{D} 3$ [78].

Lack of adequate exposure to sunlight is likely to lead to vitamin D deficiency with levels $<25 \mathrm{nmol} / \mathrm{L}$ [15]. However, it is difficult to determine what adequate exposure is. The British Dermatology Association in their 2010 consensus statement on the use of sun-exposure to prevent vitamin D deficiency noted the results of Rhodes et al [79]; the time to produce vitamin D is generally short and certainly before skin begins to burn or redden. The study found that sunlight exposure for an equivalent of 13 minutes of UK summer midday sun (approximate latitude $53.4808^{\circ} \mathrm{N}, 2.2426^{\circ} \mathrm{W}$ ), three times per week over a six-week period was sufficient to increase serum vitamin D levels above $50 \mathrm{nmol} / \mathrm{L}$. The study was carried out in Caucasian participants with one third of their skin exposed to simulated sunlight and wearing no sunscreen [79]. Darker skin required a longer exposure to sunlight to produce vitamin $\mathrm{D}$, so, it would not be possible to have a single recommendation for all skin types. In addition, Rhodes et al note that excessive cutaneous exposure to sunlight may in fact lead to degradation of vitamin D but they do not quantify what excessive exposure is [79]. A more recent global consensus statement on the treatment and prevention of rickets suggests that the use of sunlight exposure to prevent or treat vitamin D deficiency is not feasible [80]. The authors note that there is no safe threshold of ultra violet exposure that allows for sufficient vitamin D synthesis without increasing the risk of skin cancer [80]. This is of particular relevance to patients with IBD, where there is an increased risk of non-melanoma skin cancer in those who have received thiopurines to treat IBD [81].

\subsection{Dietary Sources}

The majority of naturally occurring vitamin $\mathrm{D}$ in food is found in animal products [82] and, as in humans, the quantity of vitamin D found in animal products will vary according to the vitamin D status of the animal. This will be influenced by the animal's diet, if they receive vitamin D supplementation in their feed and their exposure to sunlight [83]. In the USA the addition of vitamin D to livestock feed has been shown to improve the quality of the meat [84]. Despite the emphasis on animal sources of vitamin D, Black et al [85], stress the importance of further research in to edible plant-based foods in providing vitamin $\mathrm{D}$ in the diet.

SACN [15] confirmed that the recommended daily intake for vitamin D from all sources (food and oral supplementation) is $400 \mathrm{IU}$ per day for those aged 4 years and above. SACN estimate that this is a sufficient amount to maintain vitamin D levels $>25 \mathrm{nmol} / \mathrm{L}$. However, this remains below the levels recommended by the Endocrine Society and National Osteoporosis Society $[14,20]$. Nonetheless, maintaining this moderate intake from food sources alone is difficult where there are few foods containing significant quantities of vitamin D.

NHS Choices [86] recommend the following sources of vitamin D:

- $\quad$ oily fish—such as herring, mackerel, salmon and sardines

- liver

- red meat

- $\quad$ egg yolks

- fortified foods—such as fat spreads and breakfast cereals

There are notable differences in the amount of vitamin $\mathrm{D}$ in each of these foods. Oily fish is a good source of vitamin D even though there are inter-source variations, such as differences between wild salmon and farmed salmon. However, oily fish may not be commonly consumed in some national diets. An analysis of UK Biobank dietary intake data showed that, of 164,573 sets of participant data, only $20 \%$ reported consuming two portions of oily fish per week [87]. The main dietary sources of vitamin D intake in UK adults aged 19-64 were; oily fish (11\% of vitamin D intake), cereals (13\%), eggs and egg dishes (13\%), fortified fats (19\%), and meat (providing $30 \%$ of total vitamin D intake) [15]. 
The UK National Diet and Nutrition Survey [88] reports that in adults aged 19-64 years, mean daily intake of vitamin D from dietary sources alone was $112 \mathrm{IU}$. When oral supplementation was included intakes increased to $356 \mathrm{IU}$ in men and $136 \mathrm{IU}$ in women, still below the modest recommended $400 \mathrm{IU} /$ day.

\section{Dietary Intake of Vitamin D in People with IBD}

Studies have suggested that people with IBD may restrict their diet to help manage the symptoms of their disease. This has been shown particularly in people with CD and CD-related strictures [89] and even in remission diet may be sub-optimal. A cross sectional study was carried out with $67 \mathrm{CD}$ patients in remission in Canada. Results showed that intake of vitamins C, D, niacin, thiamin, magnesium, phosphorus, potassium, and zinc were all significantly lower in CD patients compared to a control sample $(p<0.05)$ [90]. A recent UK cross sectional study of 67 patients with IBD (40 CD, 23 UC) found that $97 \%$ of patients reported food avoidance [91]. Commonly avoided foods were vegetables and wheat products. Mean vitamin D intake was $283 \mathrm{IU} /$ day in the reported cohort. A further small cross-sectional [92] study of dietary intake in 31 patients with IBD in Iceland reported that patients avoided dairy $(60 \%)$ and processed meat products $(55 \%)$ to manage symptoms of their IBD. Some patients reported that fish had a positive effect on their symptoms (22\%). A cross-sectional study carried out in the Netherlands [93] of 165 patients with IBD compared to healthy controls, demonstrated that patients with IBD consumed more meat and poultry with an average difference of 15.0g/day (95\% CI 8.50-21.4); less dairy products at $-36.3 \mathrm{~g} /$ day (95\% CI -65.8--6.84); and slightly less fish at $-1.42 \mathrm{~g} /$ day $(95 \% \mathrm{CI}-0.94--3.79)$. The findings of these studies would suggest that patients with IBD are unlikely to consume adequate amounts of vitamin D rich foods to achieve the moderate recommended intake.

\subsection{Vitamin D Supplementation}

Oral supplements containing vitamin D are widely available both over the counter and in the form of prescription only medicines. Some may be single or multi-nutrient preparations. Supplements usually contain either ergocalciferol (vitamin D2) or cholecalciferol (vitamin D3); both forms of vitamin $\mathrm{D}$ are metabolised by the liver to 25(OH)D. However, studies suggest that vitamin D2 appears to yield less 25-OH-D than an equal amount of vitamin D3, with vitamin D3 being more effective at raising blood levels of 25-OH-D in humans [94,95]. For this reason, vitamin D3 supplementation is often recommended [14,20].

The dose of vitamin D supplementation required to prevent deficiency is an issue for debate. The European Food Safety Authority [96] has set an upper tolerable limit for vitamin D supplementation of 4,000 IU daily in adults. In line with the SACN report [15], current NICE [25] recommendations are 400 IU vitamin D daily. The IOM [13] suggest an intake of 600 IU daily to maintain a 25-OH-D level of $>50 \mathrm{nmol} / \mathrm{L}$, with the Endocrine Society [14] recommending a daily intake for adults of 1500 IU-2000IU. However, these guidelines do not address the risks for people with inflammatory, malabsorptive diseases such as IBD. A recent review has suggested that in IBD doses of 1800 IU-10,000 IU daily may be required [97]. With such a wide dosing range it is difficult to determine the amount that is likely to be most effective.

\section{Benefits of Treating Vitamin D Deficiency in IBD}

It is widely accepted that vitamin D supplementation should be provided during corticosteroid treatment in IBD for prevention of deterioration of bone health, forming standard guidance. There is increasing interest in research investigating prescribing vitamin D supplementation for modulating inflammatory biochemical processed in IBD [97]. Oral supplementation of vitamin D has been shown to be safe with only minor side-effects which on the whole are generally tolerated among children, with no difference with dosage regimes such as $2000 \mathrm{IU} /$ day versus 50,000 IU/week [98,99]. Adult studies show similar safety profiles [97], with meta-analysis confirming this with the most 
common side-effects including thirst, nausea, dry mouth, headaches, minor gastrointestinal upset, drowsiness, and fatigue $[100,101]$.

Supplementation of 40,000IU of cholecalciferol weekly successfully significantly increased vitamin D levels in patients with active UC and among subjects with inactive UC and non-UC sufferers. Moreover, there was an associated significant reduction in inflammatory markers of colitis: both C-reactive protein (CRP) and fecal calprotectin [69]. This is not a finding in every study, however. A study from Korea whilst establishing a negative correlation between vitamin D levels and CRP in those with $C D$, supplementation had no impact upon CRP and disease indices; no association seen with vitamin D levels, CRP and disease indices in those with UC [102]. Whilst not meeting statistical significance $(p=0.06)$ relapse of CD was less common among subjects supplemented with 1200IU of vitamin D3, a group also showing an increase in serum vitamin D3 with supplementation [101].

With immuno-modulatory and biologic therapy increasing the incidence of infections, it is of interest that upper respiratory tract infections were less commonly observed in those supplemented, and indeed the greatest protective effect was observed among those with vitamin D deficiency provided with a modest 500 IU per day (versus placebo) [103].

Vitamin D also impacts upon hospitalisation and need for surgery. In an observational study of patients with both UC and CD, those with an insufficient serum vitamin D (25-OH-D) had an increased risk of requiring both hospitalisation and surgical intervention than those who were never deficient. This was independent of treatment strategies with immuno-modulators and anti-TNF alpha medication. This study measured vitamin D levels and different time points and identified those in whom the levels rose to normal, the majority of whom were taking supplements. This group significantly reduced their risk of requiring surgery with a drop in CRP also noted [12]. Whilst the study by Govani et al [104] did not examine vitamin D levels, they used the US National inpatient sample database, identifying patients with $\mathrm{CD}$ requiring surgery and established a UV exposure index based at 3-digit zip-code level. It was identified that those exposed to greater UV had a reduced risk of surgical intervention [104].

Vitamin D deficiency has been shown to be a risk for the development of colo-rectal cancer in people with IBD [105]. Whilst the study by Ananthakrishnan et al does not examine supplementation of vitamin D, their study of 2809 patients with vitamin D assay results (at least one) and development of cancer revealed that deficiency of vitamin D was associated with an increased risk of cancer: odds ratio $1.82(95 \%$ CI 1.25-2.65), with an $8 \%$ reduction in risk for every $1 \mathrm{ng} / \mathrm{mL}$ increase in vitamin D level [105]. A recent randomised controlled trial on recurrence of adenoma in people without an inflammatory bowel disease showed less-promising results [106]. In the trial participants were randomised to receive either vitamin D, calcium, both agents, or placebo. No significant difference was found in any of the treatment regimens on the recurrence of adenoma. However, it is important to note that only participants with 25-OH-D levels $>30 \mathrm{nmol} / \mathrm{L}<225 \mathrm{nmol} / \mathrm{L}$ were included [106]. Therefore, it can be argued that these results do not relate to a population who are vitamin $\mathrm{D}$ deficient such as those with IBD. Further research is warranted into the effects of treatment of vitamin D deficiency in IBD and the impact on the development of colo-rectal cancers in this group.

IBD and anemia are linked, with the action of hepcidin implicated. Observational data has confirmed vitamin D deficiency is associated with increased hepcidin concentration and anemia [107]. This observational data has been strengthened with an interventional study revealing that even a short period of two-weeks of vitamin D supplementation was sufficient to not only increase serum vitamin $\mathrm{D}$, but reduce serum CRP and hepcidin levels [108].

Vitamin D effects have been postulated to have an immuno-modulatory effect; a vitamin D analogue was shown to act as a TNF-alpha inhibitor of peripheral blood mononuclear cells, especially when used in conjunction with Infliximab, a TNF-alpha inhibitor [109]. Observation among 173 subjects receiving anti-TNF alpha medication showed those with a normal vitamin D level had an increased odds ratio 2.64 (95\% CI 1.31-5.32, $p=0.0067)$ of having achieved remission at 3-months compared with those with low levels [110]. This finding is echoed in another retrospective observational study revealing that patients with IBD with an insufficient vitamin D level at initiation of anti-TNF alpha 
treatment were more likely to stop treatment due to loss of response (HR 3.49; 95\%CI 1.34-9.09), again suggesting a beneficial effect of vitamin D repletion [111].

Meta-analysis of randomised control trials have been clear in identifying that supplementation, whilst on the whole was well tolerated and did increase serum vitamin D levels, were too heterogeneous in nature with regards to dosing and follow up-periods to draw any significant findings with improvements in serum inflammatory markers of CRP and ESR. There was however recognition that there were significantly fewer relapses in those supplemented with vitamin D (OR 0.34; $95 \% \mathrm{CI}$ $0.20-0.58$ ) but analysis by dosing did not yield further significant findings [100].

\section{Conclusions}

Vitamin D plays a significant role in the maintenance of gastrointestinal barrier integrity, surveillance of the gut microbiota and inflammatory immune responses. These mechanisms are important in both preventing the development of IBD and ameliorating symptoms of the disease. Nonetheless, vitamin D deficiency is common in people with IBD with prevalence being higher than the general population and somewhat higher in CD than UC [30]. The reasons for this are multi-factorial but include lack of sun exposure due to immuno-suppressive treatments, dietary restrictions [90], and impaired absorption of nutrients.

Whilst vitamin D deficiency is more common among those with IBD, data has been unable to establish if the relationship is causative or as a result of inflammation. Many serum markers of nutrition are affected by inflammation, making it challenging to understand cause or effect. However, through understanding of the mechanisms of action there is emerging evidence that vitamin D deficiency may be implicated in disease severity, if not in part the etiology of IBD.

There is a wealth of evidence revealing that intervention with oral vitamin D supplementation is safe and well tolerated. It has been more challenging to identify the advantages of restoring vitamin D levels in clinical disease progression, but more studies are beginning to reveal benefits. Aside from the well recognised skeletal effects, there is growing evidence for IBD disease outcome benefits from the normalisation of 25-OH-D serum levels in people with IBD. Benefits include: reduced risk of surgery in those with CD [12], reduction in inflammatory markers [69], reduction in the development of anemia [112], improved response to anti-TNF alpha treatment [111], and a reduction in the risk of colo-rectal cancer [105]. One study revealed a reduction in upper respiratory tract infections [91]. These benefits warrant attention to the vitamin D status of people with IBD. Several key questions remain to be answered. Firstly, it is still unclear what is the optimal circulating level(s) of 25-OH-D for prevention and/or management of IBD. Current guidelines such as the Institute of Medicine in North America [13], and Science Advisory Council on Nutrition in the UK [15] have been developed based on the regulation of calcium homeostasis and bone metabolism. As yet there are no similar guidelines for extra-skeletal effects of vitamin D. This is further complicated by the recent report of a human subject with homozygous deletion of the vitamin D binding protein (DBP) gene [113]. The woman in question had almost undetectable circulating levels of 25-OH-D but was nevertheless normocalcemic and had only had mild changes in bone metabolism, but had severe ankylosing spondylitis. This case report suggests that total serum measurement of 25-OH-D may not be the most accurate determinant of vitamin D 'status', but rather unbound or free 25-OH-D (which was relatively normal in the woman with the DBP gene deletion) is the driving force behind many actions of vitamin D [114]. This is likely to be an important consideration in future studies of vitamin D supplementation and clinical impact.

Maintaining or improving vitamin D intake by diet or increased sun exposure is problematic for people with IBD. Improvement in vitamin D status can be achieved by oral supplementation. There is however a lack of consistent outcome data for significant improvement in IBD outcomes, with variation in duration and dosing of supplementation, and many studies being retrospective in nature. The data surrounding enhanced response to biologic therapies with normalised vitamin D levels suggest that, with more interventional, double-blind, placebo controlled randomised trials, that individualisation of 
IBD management with supplementation of vitamin D in those who are deficient offers an important strategy in improving clinical outcomes.

Author Contributions: Conceptualisation, J.F., S.C.C. and M.H.; Writing-Original Draft Preparation, J.F., S.C.C., S.G. and M.H.; Writing-Review and Editing, J.F., S.C.C., S.G. and M.H.; Visualisation, J.F.; Supervision, S.C.C. and M.H.

Funding: No Funding is declared for this review.

Conflicts of Interest: S.C.C. has received honoraria from Baxter and Novartis, and educational sponsorship from Fresenius-Kabi/Calea. M.H., S.G. and J.F. declare no conflicts of interest.

\section{References}

1. Molodecky, N.A.; Soon, I.S.; Rabi, D.M.; Ghali, W.A.; Ferris, M.; Chernoff, G.; Benchimol, E.I.; Panaccione, R.; Ghosh, S.; Barkema, H.W.; et al. Increasing Incidence and Prevalence of the Inflammatory Bowel Diseases With Time, Based on Systematic Review. Gastroenterology 2012, 142, 46-54. [CrossRef]

2. Ng, S.C.; Shi, H.Y.; Hamidi, N.; Underwood, F.E.; Tang, W.; Benchimol, E.I.; Panaccione., R.; Ghosh, S.; $\mathrm{Wu}$, J.C.Y.; et al. Worldwide incidence and prevalence of inflammatory bowel disease in the 21st century: A systematic review of population-based studies. Lancet 2018, 390, 2769-2778. [CrossRef]

3. De Souza, H.S.P.; Fiocchi, C. Immunopathogenesis of IBD: current state of the art. Nat. Rev. Gastroenterol. Hepatol. 2015, 13, 13-27. [CrossRef]

4. Alexander, K.L.; Targan, S.R.; Elson, C.O., III. Microbiota activation and regulation of innate and adaptive immunity. Immunol. Rev. 2014, 260, 206-220. [CrossRef] [PubMed]

5. Maynard, C.L.; Elson, C.O.; Hatton, R.D.; Weaver, C.T. Reciprocal Interactions of the Intestinal Microbiota and Immune System. Nat. Cell Boil. 2012, 489, 231-241. [CrossRef] [PubMed]

6. Bernstein, C.N.; Shanahan, F. Disorders of a modern lifestyle: reconciling the epidemiology of inflammatory bowel diseases. Gut 2008, 57, 1185-1191. [CrossRef]

7. Cantorna, M.T. Vitamin D and its role in immunology: Multiple sclerosis, and inflammatory bowel disease. Prog. Biophys. Mol. Boil. 2006, 92, 60-64. [CrossRef]

8. Raman, M.; Milestone, A.N.; Walters, J.R.; Hart, A.L.; Ghosh, S. Vitamin D and gastrointestinal diseases: Inflammatory bowel disease and colorectal cancer. Therap. Adv. Gastroenterol. 2011, 4, 49-62. [CrossRef]

9. Raftery, T.; Martineau, A.R.; Greiller, C.L.; Ghosh, S.; McNamara, D.; Bennett, K.; Meddings, J.; O'Sullivan, M. Effects of vitamin D supplementation on intestinal permeability, cathelicidin and disease markers in Crohn's disease: Results from a randomised double-blind placebo-controlled study. United Eur. Gastroenterol. J. 2015, 3, 294-302. [CrossRef] [PubMed]

10. Yang, L.; Weaver, V.; Smith, J.P.; Bingaman, S.; Hartman, T.J.; Cantorna, M.T. Therapeutic Effect of Vitamin D Supplementation in a Pilot Study of Crohn's Patients. Clin. Transl. Gastroenterol. 2013, 4, e33. [CrossRef]

11. Bancil, A.S.; Poullis, A.; Samman, S.; Darnton-Hill, I. The Role of Vitamin D in Inflammatory Bowel Disease. Healthcare 2015, 3, 338-350. [CrossRef] [PubMed]

12. Ananthakrishnan, A.N.; Cagan, A.; Gainer, V.S.; Cai, T.; Cheng, S.-C.; Savova, G.; Chen, P.; Szolovits, P.; Xia, Z.; De Jager, P.L.; et al. Normalization of Plasma 25-hydroxy Vitamin D is Associated with Reduced Risk of Surgery in Crohn's Disease. Inflamm. Bowel Dis. 2013, 19, 1921-1927. [CrossRef] [PubMed]

13. Ross, A.C.; Manson, J.E.; Abrams, S.A.; Aloia, J.F.; Brannon, P.M.; Clinton, S.K.; Durazo-Arvizu, R.A.; Gallagher, J.C.; Gallo, R.L.; Jones, G.; et al. The 2011 Report on Dietary Reference Intakes for Calcium and Vitamin D From the Institute of Medicine: What Clinicians Need to Know. Obstet. Gynecol. 2011, 66, 356-357.

14. Holick, M.F.; Binkley, N.C.; Bischoff-Ferrari, H.A.; Gordon, C.M.; Hanley, D.A.; Heaney, R.P.; Murad, M.H.; Weaver, C.M. Evaluation, Treatment, and Prevention of Vitamin D Deficiency: an Endocrine Society Clinical Practice Guideline. J. Clin. Endocrinol. Metab. 2011, 96, 1911-1930. [CrossRef]

15. Scientific Advisory Committee on Nutrition. SACN Vitamin D and Health Report; Scientific Advisory Committee on Nutrition: London, UK, 2016.

16. Institute of Medicine (US) Committee to Review Dietary Reference Intakes for Vitamin D and Calcium. Dietary Reference Intakes for Calcium and Vitamin D; Ross, A.C., Taylor, C.L., Yaktine, A.L., Del Valle, H.B., Eds.; National Academies Press (US): Washington, DC, USA, 2011. 
17. Dawson-Hughes, B.; Mithal, A.; Bonjour, J.-P.; Boonen, S.; Burckhardt, P.; Fuleihan, G.E.-H.; Josse, R.G.; Lips, P.; Morales-Torres, J.; Yoshimura, N. IOF position statement: vitamin D recommendations for older adults. Osteoporos. Int. 2010, 21, 1151-1154. [CrossRef]

18. Hanley, D.A.; Cranney, A.; Jones, G.; Whiting, S.J.; Leslie, W.D.; Cole, D.E.; Atkinson, S.A.; Josse, R.G.; Feldman, S.; Kline, G.A.; et al. Vitamin D in adult health and disease: a review and guideline statement from Osteoporosis Canada. Can. Med Assoc. J. 2010, 182, E610-E618. [CrossRef]

19. Okazaki, R.; Ozono, K.; Fukumoto, S.; Inoue, D.; Yamauchi, M.; Minagawa, M.; Michigami, T.; Takeuchi, Y.; Matsumoto, T.; Sugimoto, T. Assessment criteria for vitamin D deficiency/insufficiency in Japan: Proposal by an expert panel supported by the Research Program of Intractable Diseases, Ministry of Health, Labour and Welfare, Japan, the Japanese Society for Bone and Mineral Research and the Japan Endocrine Society [Opinion]. J. Bone Miner. Metab. 2017, 35, 1-5. [PubMed]

20. Francis, R.; Aspray, T.; Bowring, C.; Fraser, W.; Gittoes, N.; Javaid, M.; Macdonald, H.; Patel, S.; Selby, P.; Tanna, N. National Osteoporosis Society practical clinical guideline on vitamin D and bone health. Maturitas 2015, 80, 119-121. [CrossRef]

21. National Institute for Health and Care Excellence. CG 152 Crohn's disease: Management in adults, children and young people; NICE: London, UK, 2012.

22. National Institute for Health and Care Excellence. CG 166 Ulcerative Colitis: Management in Adults, Children and Young People; NICE: London, UK, 2013.

23. Lichtenstein, G.R.; Loftus, E.V.; Isaacs, K.L.; Regueiro, M.D.; Gerson, L.B.; E Sands, B. ACG Clinical Guideline: Management of Crohn's Disease in Adults. Am. J. Gastroenterol. 2018, 113, 481-517. [CrossRef]

24. Rubin, D.T.; Ananthakrishnan, A.N.; Siegel, C.A.; Sauer, B.G.; Long, M.D. ACG Clinical Guideline: Ulcerative Colitis in Adults. Am. J. Gastroenterol. 2019, 114, 384-413. [CrossRef]

25. National Institute for Health and Care Excellence. Vitamin D: Supplement Use in Specific Population Groups PH56; NICE: London, UK, 2017.

26. Larose, T.L.; Chen, Y.; Camargo, C.A., Jr.; Langhammer, A.; Romundstad, P.; Mai, X.M. Factors associated with vitamin D deficiency in a Norwegian population: the HUNT Study. J. Epidemiol. Community Health 2014, 68, 165-170. [CrossRef]

27. Schwalfenberg, G.; Genuis, S.; Hiltz, M.; Schwalfenberg, G. Addressing vitamin D deficiency in Canada: A public health innovation whose time has come. Public Health 2010, 124, 350-359. [CrossRef]

28. Hypponen, E.; Power, C. Hypovitaminosis D in British adults at age $45 \mathrm{y}$ : nationwide cohort study of dietary and lifestyle predictors. Am. J. Clin. Nutr. 2007, 85, 860-868. [CrossRef]

29. Fletcher, J. Vitamin D deficiency in patients with inflammatory bowel disease. Br. J. Nurs. 2016, 25, 846-851. [CrossRef] [PubMed]

30. Fletcher, J.; Swift, A. Vitamin D screening in patients with inflammatory bowel disease. Gastrointest. Nurs. 2017, 15, 16-23. [CrossRef]

31. Hossein-Nezhad, A.; Holick, M.F. Vitamin D for Health: A Global Perspective. Mayo Proc. 2013, 88, 720-755. [CrossRef] [PubMed]

32. Abreu, M.T.; Kantorovich, V.; A Vasiliauskas, E.; Gruntmanis, U.; Matuk, R.; Daigle, K.; Chen, S.; Zehnder, D.; Lin, Y.-C.; Yang, H.; et al. Measurement of vitamin D levels in inflammatory bowel disease patients reveals a subset of Crohn's disease patients with elevated 1,25-dihydroxyvitamin D and low bone mineral density. Gut 2004, 53, 1129-1136. [CrossRef]

33. Ghosh, S.; Cowen, S.; Hannan, W.; Ferguson, A. Low bone mineral density in Crohn's disease, but not in ulcerative colitis, at diagnosis. Gastroenterology 1994, 107, 1031-1039. [CrossRef]

34. Abraham, B.P.; Prasad, P.; Malaty, H.M. Vitamin D deficiency and corticosteroid use are risk factors for low bone mineral density in inflammatory bowel disease patients. Am. J. Dig. Dis. 2014, 59, 1878-1884. [CrossRef]

35. Maratova, K.; Hradsky, O.; Matyskova, J.; Copova, I.; Soucek, O.; Sumnik, Z.; Bronsky, J. Musculoskeletal system in children and adolescents with inflammatory bowel disease: normal muscle force, decreased trabecular bone mineral density and low prevalence of vertebral fractures. Eur. J. Pediatr. 2017, 176, 1355-1363. [CrossRef] [PubMed] 
36. Szymczak-Tomczak, A.; Krela-Kaźmierczak, I.; Kaczmarek-Ryś, M.; Hryhorowicz, S.T.; Stawczyk-Eder, K.; Szalata, M.; Skrzypczak-Zielińska, M.; Łykowska-Szuber, L.; Eder, P.; Michalak, M.; et al. Vitamin D receptor (VDR) TaqI polymorphism, vitamin D and bone mineral density in patients with inflammatory bowel diseases. Adv. Clin. Exp. Med. 2019, 28. [CrossRef]

37. Maratova, K.; Matyskova, J.; Copova, I.; Zarubova, K.; Sumnik, Z.; Hradsky, O.; Soucek, O.; Bronsky, J. Supplementation with $2000 \mathrm{IU}$ of Cholecalciferol Is Associated with Improvement of Trabecular Bone Mineral Density and Muscle Power in Pediatric Patients with IBD. Inflamm. Bowel Dis. 2017, 23, 514-523.

38. Bakker, S.F.; Dik, V.K.; Witte, B.I.; Lips, P.; Roos, J.C.; Van Bodegraven, A.A. Increase in bone mineral density in strictly treated Crohn's disease patients with concomitant calcium and vitamin D supplementation. J. Crohn's Coliti 2013, 7, 377-384. [CrossRef]

39. Bours, P.H.; Wielders, J.P.; Vermeijden, J.R.; van de Wiel, A. Seasonal variation of serum 25-hydroxyvitamin $\mathrm{D}$ levels in adult patients with inflammatory bowel disease. Osteoporos Int. 2011, 22, 2857-2867. [CrossRef] [PubMed]

40. Caviezel, D.; Maissen, S.; Niess, J.H.; Kiss, C.; Hruz, P. High Prevalence of Vitamin D Deficiency among Patients with Inflammatory Bowel Disease. Inflamm. Intest. Dis. 2017, 2, 200-210. [CrossRef] [PubMed]

41. Frigstad, S.O.; Høivik, M.; Jahnsen, J.; Dahl, S.R.; Cvancarova, M.; Grimstad, T.; Berset, I.; Huppertz-Hauss, G.; Hovde, Ø.; Torp, R. Vitamin D deficiency in inflammatory bowel disease: prevalence and predictors in a Norwegian outpatient population. Scand. J. Gastroenterol. 2017, 52, 100-106. [CrossRef]

42. Gilman, J.; Shanahan, F.; Cashman, K.D. Determinants of vitamin D status in adult Crohn's disease patients, with particular emphasis on supplemental vitamin D use. Eur. J. Clin. Nutr. 2006, 60, 889-896. [CrossRef]

43. A Kabbani, T.; E Koutroubakis, I.; E Schoen, R.; Ramos-Rivers, C.; Shah, N.; Swoger, J.; Regueiro, M.; Barrie, A.; Schwartz, M.; Hashash, J.G.; et al. Association of Vitamin D Level With Clinical Status in Inflammatory Bowel Disease: A 5-Year Longitudinal Study. Am. J. Gastroenterol. 2016, 111, 712-719. [CrossRef]

44. Kuwabara, A.; Tanaka, K.; Tsugawa, N.; Nakase, H.; Tsuji, H.; Shide, K.; Kamao, M.; Chiba, T.; Inagaki, N.; Okano, T;; et al. High prevalence of vitamin K and D deficiency and decreased BMD in inflammatory bowel disease. Osteoporos Int. 2009, 20, 935-942. [CrossRef]

45. McCarthy, D.; Duggan, P.; O’Brien, M.; Kiely, M.; McCarthy, J.; Shanahan, F.; Cashman, K.D. Seasonality of vitamin D status and bone turnover in patients with Crohn's disease. Aliment. Pharmacol. Ther. 2005, 21, 1073-1083. [CrossRef]

46. Pappa, H.M.; Gordon, C.M.; Saslowsky, T.M.; Zholudev, A.; Horr, B.; Shih, M.-C.; Grand, R.J. Vitamin D Status in Children and Young Adults With Inflammatory Bowel Disease. PEDIATRICS 2006, 118, 1950-1961. [CrossRef] [PubMed]

47. Sentongo, A.T.; Semaeo, E.J.; Stettler, N.; A Piccoli, D.; A Stallings, V.; Zemel, B.S. Vitamin D status in children, adolescents, and young adults with Crohn disease. Am. J. Clin. Nutr. 2002, 76, 1077-1081. [CrossRef]

48. Siffledeen, J.S.; Siminoski, K.; Steinhart, H.; Greenberg, G.; Fedorak, R.N. The Frequency of Vitamin D Deficiency in Adults with Crohn's Disease. Can. J. Gastroenterol. 2003, 17, 473-478. [CrossRef]

49. Suibhne, T.N.; Cox, G.; Healy, M.; O'Morain, C.; O'Sullivan, M. Vitamin D deficiency in Crohn's disease: Prevalence, risk factors and supplement use in an outpatient setting. J. Crohns Colitis 2012, 6, 182-188. [CrossRef]

50. Ulitsky, A.; Ananthakrishnan, A.N.; Naik, A.; Skaros, S.; Zadvornova, Y.; Binion, D.G.; Issa, M. Vitamin D deficiency in patients with inflammatory bowel disease: association with disease activity and quality of life. JPEN 2011, 35, 308-316. [CrossRef]

51. Veit, L.E.; Maranda, L.; Fong, J.; Nwosu, B.U. The Vitamin D Status in Inflammatory Bowel Disease. PLoS ONE 2014, 9, e101583. [CrossRef]

52. Hassan, V.; Hassan, S.; Seyed-Javad, P.; Ahmad, K.; Asieh, H.; Maryam, S.; Farid, F.; Siavash, A. Association between Serum $25(\mathrm{OH})$ Vitamin D Concentrations and Inflammatory Bowel Diseases (IBDs) Activity. Med J. 2013, 68, 34-38.

53. Garg, M.; Rosella, O.; Lubel, J.S.; Gibson, P.R. Association of Circulating Vitamin D Concentrations with Intestinal but Not Systemic Inflammation in Inflammatory Bowel Disease. Inflamm. Bowel Dis. 2013, 19, 2634-2643. [CrossRef] [PubMed]

54. Jørgensen, S.P.; Hvas, C.L.; Agnholt, J.; Christensen, L.A.; Heickendorff, L.; Dahlerup, J.F. Active Crohn's disease is associated with low vitamin D levels. J. Crohn's Colitis 2013, 7, e407-e413. 
55. Wang, T.J.; Zhang, F.; Richards, J.B.; Kestenbaum, B.; Van Meurs, J.B.; Berry, D.; Kiel, D.P.; Streeten, E.A.; Ohlsson, C.; Koller, D.L.; et al. Common genetic determinants of vitamin D insufficiency: a genome-wide association study. Lancet 2010, 376, 180-188. [CrossRef]

56. Ananthakrishnan, A.N.; Cagan, A.; Cai, T.; Gainer, V.S.; Shaw, S.Y.; Churchill, S.; Karlson, E.W.; Murphy, S.N.; Kohane, I.; Liao, K.P.; et al. Common genetic variants influence circulating vitamin D levels in inflammatory bowel diseases. Inflamm. Bowel Dis. 2015, 21, 2507-2514. [CrossRef]

57. Lund-Nielsen, J.; Vedel-Krogh, S.; Kobylecki, C.J.; Brynskov, J.; Afzal, S.; Nordestgaard, B.G. Vitamin D and Inflammatory Bowel Disease: Mendelian Randomization Analyses in the Copenhagen Studies and UK Biobank. J. Clin. Endocrinol. Metab. 2018, 103, 3267-3277. [CrossRef]

58. Ananthakrishnan, A.N.; Khalili, H.; Higuchi, L.M.; Bao, Y.; Korzenik, J.R.; Giovannucci, E.L.; Richter, J.M.; Fuchs, C.S.; Chan, A.T. Higher predicted vitamin D status is associated with reduced risk of Crohn's disease. Gastroenterology 2012, 142, 482-489. [CrossRef]

59. Ananthakrishnan, A.N. Editorial: Vitamin D and IBD: Can We Get Over the "Causation" Hump? Am. J. Gastroenterol. 2016, 111, 720-722. [CrossRef]

60. Liu, P.T.; Stenger, S.; Li, H.; Wenzel, L.; Tan, B.H.; Krutzik, S.R.; Ochoa, M.T.; Schauber, J.; Wu, K.; Meinken, C.; et al. Toll-Like Receptor Triggering of a Vitamin D-Mediated Human Antimicrobial Response. Science 2006, 311, 1770-1773. [CrossRef] [PubMed]

61. Bacchetta, J.; Zaritsky, J.J.; Sea, J.L.; Chun, R.F.; Lisse, T.S.; Zavala, K.; Nayak, A.; Wesseling-Perry, K.; Westerman, M.; Hollis, B.W.; et al. Suppression of iron-regulatory hepcidin by vitamin D. J. Am. Soc. Nephrol. 2014, 25, 564-572. [CrossRef]

62. Hewison, M. Antibacterial effects of vitamin D. Nat. Rev. Endocrinol. 2011, 7, 337-345. [CrossRef] [PubMed]

63. Cantorna, M.T. Vitamin D, multiple sclerosis and inflammatory bowel disease. Arch. Biochem. Biophys. 2012, 523, 103-106. [CrossRef] [PubMed]

64. Adams, J.S.; Hewison, M. Unexpected actions of vitamin D: new perspectives on the regulation of innate and adaptive immunity. Nat. Clin. Pr. Endocrinol. Metab. 2008, 4, 80-90. [CrossRef]

65. Zhang, Y.-G.; Wu, S.; Lu, R.; Zhou, D.; Zhou, J.; Carmeliet, G.; Petrof, E.; Claud, E.C.; Sun, J. Tight junction CLDN2 gene is a direct target of the vitamin D receptor. Sci. Rep. 2015, 5, 10642. [CrossRef]

66. Liu, W.; Chen, Y.; Golan, M.A.; Annunziata, M.L.; Du, J.; Dougherty, U.; Kong, J.; Musch, M.; Huang, Y.; Pekow, J.; et al. Intestinal epithelial vitamin D receptor signaling inhibits experimental colitis. J. Clin. Investig. 2013, 123, 3983-3996. [CrossRef]

67. Kong, J.; Zhang, Z.; Musch, M.W.; Ning, G.; Sun, J.; Hart, J.; Bissonnette, M.; Li, Y.C. Novel role of the vitamin D receptor in maintaining the integrity of the intestinal mucosal barrier. Am. J. Physiol. Liver Physiol. 2008, 294, 208-216. [CrossRef]

68. Luthold, R.V.; Fernandes, G.R.; Franco-De-Moraes, A.C.; Folchetti, L.G.; Ferreira, S.R.G. Gut microbiota interactions with the immunomodulatory role of vitamin D in normal individuals. Metab. Clin. Exp. 2017, 69, 76-86. [CrossRef]

69. Garg, M.; Hendy, P.; Ding, J.N.; Shaw, S.; Hold, G.; Hart, A. The Effect of Vitamin D on Intestinal Inflammation and Faecal Microbiota in Patients with Ulcerative Colitis. J. Crohn?s Coliti 2018, 12, 963-972. [CrossRef]

70. Froicu, M.; Weaver, V.; Wynn, T.A.; McDowell, M.A.; Welsh, J.E.; Cantorna, M.T. A Crucial Role for the Vitamin D Receptor in Experimental Inflammatory Bowel Diseases. Mol. Endocrinol. 2003, 17, $2386-2392$. [CrossRef]

71. Liu, N.; Nguyen, L.; Chun, R.F.; Lagishetty, V.; Ren, S.; Wu, S.; Hollis, B.; DeLuca, H.F.; Adams, J.S.; Hewison, M. Altered Endocrine and Autocrine Metabolism of Vitamin D in a Mouse Model of Gastrointestinal Inflammation. Endocrinology 2008, 149, 4799-4808. [CrossRef] [PubMed]

72. Lagishetty, V.; Misharin, A.V.; Liu, N.Q.; Lisse, T.S.; Chun, R.F.; Ouyang, Y.; McLachlan, S.M.; Adams, J.S.; Hewison, M. Vitamin D deficiency in mice impairs colonic antibacterial activity and predisposes to colitis. Endocrinology 2010, 151, 2423-2432. [CrossRef]

73. Chun, R.F.; Lauridsen, A.L.; Suon, L.; Zella, L.A.; Pike, J.W.; Modlin, R.L.; Martineau, A.R.; Wilkinson, R.J.; Adams, J.; Hewison, M. Vitamin D-Binding Protein Directs Monocyte Responses to 25-Hydroxy- and 1,25-Dihydroxyvitamin D. J. Clin. Endocrinol. Metab. 2010, 95, 3368-3376. [CrossRef]

74. Larner, D.P.; Jenkinson, C.; Chun, R.F.; Westgate, C.S.J.; Adams, J.S.; Hewison, M. Free versus total serum 25-hydroxyvitamin D in a murine model of colitis. J. Steroid Biochem. Mol. Biol. 2019, 189, 204-209. [CrossRef] [PubMed] 
75. Wang, T.T.; Dabbas, B.; Laperriere, D.; Bitton, A.J.; Soualhine, H.; Tavera-Mendoza, L.E.; Dionne, S.; Servant, M.J.; Bitton, A.; Seidman, E.G.; et al. Direct and indirect induction by 1,25-dihydroxyvitamin D3 of the NOD2/CARD15-defensin beta2 innate immune pathway defective in Crohn disease. J. Biol. Chem. 2010, 285, 2227-2231. [CrossRef] [PubMed]

76. Ogura, Y.; Bonen, D.K.; Inohara, N.; Nicolae, D.L.; Chen, F.F.; Ramos, R.; Britton, H.; Moran, T.; Karaliuskas, R.; Kirschner, B.S.; et al. A frameshift mutation in NOD2 associated with susceptibility to Crohn's disease. Nature 2001, 411, 603-606. [CrossRef] [PubMed]

77. Hugot, J.P.; Chamaillard, M.; Zouali, H.; Lesage, S.; Cézard, J.P.; Belaiche, J.; Almer, S.; Tysk, C.; O’Morain, C.A.; Gassull, M.; et al. Association of NOD2 leucine-rich repeat variants with susceptibility to Crohn's disease. Nature 2001, 411, 599-603. [CrossRef]

78. Reich, K.M.; Fedorak, R.N.; Madsen, K.; I Kroeker, K. Vitamin D improves inflammatory bowel disease outcomes: Basic science and clinical review. World J. Gastroenterol. 2014, 20, 4934-4947. [CrossRef] [PubMed]

79. Rhodes, L.E.; Webb, A.R.; Fraser, H.I.; Kift, R.; Durkin, M.T.; Allan, D.; O’Brien, S.J.; Vail, A.; Berry, J.L. Recommended Summer Sunlight Exposure Levels Can Produce Sufficient but Not the Proposed Optimal 25(OH)D Levels at UK Latitudes. J. Invest. Dermatol. 2010, 130, 1411-1418. [CrossRef] [PubMed]

80. Munns, C.F.; Shaw, N.; Kiely, M.; Specker, B.L.; Thacher, T.D.; Ozono, K.; Michigami, T.; Tiosano, D.; Mughal, M.Z.; Mäkitie, O.; et al. Global Consensus Recommendations on Prevention and Management of Nutritional Rickets. J. Clin. Endocrinol. Metab. 2016, 101, 394-415. [CrossRef] [PubMed]

81. Magro, F.; Peyrin-Biroulet, L.; Sokol, H.; Aldeger, X.; Costa, A.; Higgins, P.D.; Joyce, J.C.; Katsanos, K.H.; Lopez, A.; De Xaxars, T.M.; et al. Extra-intestinal malignancies in inflammatory bowel disease: Results of the 3rd ECCO Pathogenesis Scientific Workshop (III). J. Crohn's Colitis 2014, 8, 31-44. [CrossRef] [PubMed]

82. Schmid, A.; Walther, B. Natural Vitamin D Content in Animal Products1. Adv. Nutr. Int. J. 2013, 4, 453-462. [CrossRef]

83. Roseland, J.; Phillips, K.M.; Patterson, K.Y.; Pehrsson, P.R.; Taylor, C.L. Chapter 60: Vitamin D in foods: An evolution of knowledge. In Vitamin D; Feldman, D., Ed.; Elsevier: New York, NY, USA, 2018; pp. 41-77.

84. Carnagey, K.M.; Huff-Lonergan, E.; Trenkle, A.; Wertz-Lutz, A.E.; Horst, R.L.; Beitz, D.C. Use of 25-hydroxyvitamin D3 and vitamin E to improve tenderness of beef from the longissimus dorsi of heifers1,2. J. Sci. 2008, 86, 1649-1657. [CrossRef]

85. Black, L.J.; Lucas, R.M.; Sherriff, J.L.; Björn, L.O.; Bornman, J.F. In Pursuit of Vitamin D in Plants. Nutrients 2017, 9, 136. [CrossRef]

86. NHS. Vitamins and Minerals: Vitamin D. Available online: https://www.nhs.uk/conditions/vitamins-andminerals/vitamin-d/ (accessed on 15 April 2019).

87. Shafique, M.; Russell, S.; Murdoch, S.; Bell, J.D.; Guess, N. Dietary intake in people consuming a low-carbohydrate diet in the UK Biobank. J. Hum. Nutr. Diet. 2018, 31, 228-238. [CrossRef]

88. Public Health England. National Diet and Nutrition Survey: Results from Years 1, 2, 3 and 4; Crown Copyright: London, UK, 2017.

89. Bergeron, F.; Bouin, M.; LeMoyne, M.; Presse, N.; D'Aoust, L.; D'Aoust, L. Food avoidance in patients with inflammatory bowel disease: What, when and who? Clin. Nutr. 2018, 37, 884-889. [CrossRef] [PubMed]

90. Taylor, L.; Almutairdi, A.; Shommu, N.; Fedorak, R.; Ghosh, S.; Reimer, R.A.; Panaccione, R.; Raman, M. Cross-Sectional Analysis of Overall Dietary Intake and Mediterranean Dietary Pattern in Patients with Crohn's Disease. Nutrients 2018, 10, 1761. [CrossRef] [PubMed]

91. Krishnamoorthy, R.; Jeanes, Y. PWE-115 Dietary patterns in inflammatory bowel disease-intolerances, quality of life and calcium/vitamin D intake. Gut 2018, 67, A175-A176.

92. Vidarsdottir, J.B.; Johannsdottir, S.E.; Thorsdottir, I.; Björnsson, E.; Ramel, A. A cross-sectional study on nutrient intake and -status in inflammatory bowel disease patients. Nutr. J. 2016, 15, 219. [CrossRef] [PubMed]

93. Opstelten, J.L.; De Vries, J.H.; Wools, A.; Siersema, P.D.; Oldenburg, B.; Witteman, B.J. Dietary intake of patients with inflammatory bowel disease: A comparison with individuals from a general population and associations with relapse. Clin. Nutr. 2018, (in press). [CrossRef]

94. Tripkovic, L.; Lambert, H.; Hart, K.; Smith, C.P.; Bucca, G.; Penson, S.; Chope, G.; Hyppönen, E.; Berry, J.; Vieth, R.; et al. Comparison of vitamin D2 and vitamin D3 supplementation in raising serum 25-hydroxyvitamin D status: a systematic review and meta-analysis123. Am. J. Clin. Nutr. 2012, 95, 1357-1364. [CrossRef] 
95. Glendenning, P.; Chew, G.T.; Inderjeeth, C.A.; Taranto, M.; Fraser, W.D. Calculated free and bioavailable vitamin D metabolite concentrations in vitamin D-deficient hip fracture patients after supplementation with cholecalciferol and ergocalciferol. Bone 2013, 56, 271-275. [CrossRef]

96. EFSA Panel on Dietetic Products, Nutrition and Allergies (NDA). Scientific Opinion on the Tolerable Upper Intake Level of vitamin D. EFSA J. 2012, 10, 2813.

97. Hlavaty, T.; Krajcovicova, A.; Payer, J. Vitamin D therapy in inflammatory bowel diseases: Who, in what form, and how much? J Crohns Colitis 2015, 9, 198-209. [CrossRef] [PubMed]

98. Pappa, H.M.; Mitchell, P.D.; Jiang, H.; Kassiff, S.; Filip-Dhima, R.; DiFabio, D.; Quinn, N.; Lawton, R.C.; Bronzwaer, M.E.S.; Koenen, M.; et al. Maintenance of Optimal Vitamin D Status in Children and Adolescents With Inflammatory Bowel Disease: A Randomized Clinical Trial Comparing Two Regimens. J. Clin. Endocrinol. Metab. 2014, 99, 3408-3417. [CrossRef] [PubMed]

99. Pappa, H.M.; Mitchell, P.D.; Jiang, H.; Kassiff, S.; Filip-Dhima, R.; DiFabio, D.; Quinn, N.; Lawton, R.C.; Varvaris, M.; Van Straaten, S.; et al. Treatment of Vitamin D Insufficiency in Children and Adolescents with Inflammatory Bowel Disease: A Randomized Clinical Trial Comparing Three Regimens. J. Clin. Endocrinol. Metab. 2012, 97, 2134-2142. [CrossRef]

100. Li, J.; Chen, N.; Wang, D.; Zhang, J.; Gong, X. Efficacy of vitamin D in treatment of inflammatory bowel disease: A meta-analysis. Medicine 2018, 97, e12662. [CrossRef]

101. Jørgensen, S.P.; Agnholt, J.; Glerup, H.; Lyhne, S.; Villadsen, G.E.; Hvas, C.L.; Bartels, L.E.; Kelsen, J.; Christensen, L.A.; Dahlerup, J.F. Clinical trial: vitamin D3 treatment in Crohn's disease - a randomized double-blind placebo-controlled study. Aliment. Pharmacol. Ther. 2010, 32, 377-383. [CrossRef]

102. Jun, J.C.; Yoon, H.; Choi, Y.J.; Shin, C.M.; Park, Y.S.; Kim, N.Y.; Lee, D.H.; Kim, J.S. Tu1715 - The Effect of Vitamin D Administration on Inflammatory Marker in Patients with Inflammatory Bowel Disease. Gastroenterology 2018, 154. [CrossRef]

103. Arihiro, S.; Nakashima, A.; Matsuoka, M.; Suto, S.; Uchiyama, K.; Kato, T.; Mitobe, J.; Komoike, N.; Itagaki, M.; Miyakawa, Y.; et al. Randomized Trial of Vitamin D Supplementation to Prevent Seasonal Influenza and Upper Respiratory Infection in Patients With Inflammatory Bowel Disease. Inflamm. Bowel Dis. 2019. [CrossRef]

104. Govani, S.M.; Higgins, P.D.; Stidham, R.W.; Montain, S.J.; Waljee, A.K. Increased ultraviolet light exposure is associated with reduced risk of inpatient surgery among patients with Crohn's disease. J. Crohns Colitis 2015, 9, 77-81. [CrossRef]

105. Ananthakrishnan, A.N.; Cheng, S.C.; Cai, T.; Cagan, A.; Gainer, V.S.; Szolovits, P.; Shaw, S.Y.; Churchill, S.; Karlson, E.W.; Murphy, S.N.; et al. Association between reduced plasma 25-hydroxy vitamin D and increased risk of cancer in patients with inflammatory bowel diseases. Clin. Gastroenterol. Hepatol. 2014, 12, 821-827. [CrossRef]

106. Baron, J.A.; Barry, E.L.; Mott, L.A.; Rees, J.R.; Sandler, R.S.; Snover, D.C.; Bostick, R.M.; Ivanova, A.; Cole, B.F.; Ahnen, D.J.; et al. A Trial of Calcium and Vitamin D for the Prevention of Colorectal Adenomas. New Engl. J. Med. 2015, 373, 1519-1530. [CrossRef]

107. Syed, S.; Michalski, E.S.; Tangpricha, V.; Chesdachai, S.; Kumar, A.; Prince, J.; Ziegler, T.R.; Suchdev, P.S.; Kugathasan, S.; Ms, S.S.M. Vitamin D status is Associated with Hepcidin and Hemoglobin concentrations in Children with Inflammatory Bowel Disease. Inflamm. Bowel Dis. 2017, 23, 1650-1658. [CrossRef]

108. Moran-Lev, H.; Weisman, Y.; Cohen, S.; Deutsch, V.; Cipok, M.; Bondar, E.; Lubetzky, R.; Mandel, D. The interrelationship between hepcidin, vitamin $\mathrm{D}$, and anemia in children with acute infectious disease. Pediatr. Res. 2018, 84, 62-65. [CrossRef]

109. Stio, M.; Treves, C.; Martinesi, M.; D’Albasio, G.; Bagnoli, S.; Bonanomi, A.G. Effect of Anti-TNF Therapy and Vitamin D Derivatives on the Proliferation of Peripheral Blood Mononuclear Cells in Crohn's Disease. Am. J. Dig. Dis. 2004, 49, 328-335. [CrossRef]

110. Winter, R.W.; Collins, E.; Cao, B.; Carrellas, M.; Crowell, A.M.; Korzenik, J.R. Higher 25-hydroxyvitamin $\mathrm{D}$ levels are associated with greater odds of remission with anti-tumour necrosis factor-alpha medications among patients with inflammatory bowel diseases. Aliment. Pharmacol. Ther. 2017, 45, 653-659. [CrossRef]

111. Zator, Z.A.; Cantu, S.M.; Konijeti, G.G.; Nguyen, D.D.; Sauk, J.; Yajnik, V.; Ananthakrishnan, A.N. Pretreatment 25-hydroxyvitamin D levels and durability of anti-tumor necrosis factor-alpha therapy in inflammatory bowel diseases. JPEN 2014, 38, 385-391. [CrossRef] 
112. Fialho, A.; Fialho, A.; Kochhar, G.; Shen, B. Association between vitamin D deficiency and anemia in inflammatory bowel disease patients with ileostomy. J. Coloproctology 2015, 35, 139-145. [CrossRef]

113. Henderson, C.M.; Fink, S.L.; Bassyouni, H.; Argiropoulos, B.; Brown, L.; Laha, T.J.; Jackson, K.J.; Lewkonia, R.; Ferreira, P.; Hoofnagle, A.N.; et al. Vitamin D-Binding Protein Deficiency and Homozygous Deletion of the GC Gene. New Engl. J. Med. 2019, 380, 1150-1157. [CrossRef]

114. Chun, R.F.; Peercy, B.E.; Orwoll, E.S.; Nielson, C.M.; Adams, J.S.; Hewison, M. Vitamin D and DBP: The free hormone hypothesis revisited. J. Steroid Biochem. Mol. Boil. 2014, 144, 132-137. [CrossRef] 


\title{
Implications of the Westernized Diet in the Onset and Progression of IBD
}

\author{
Fernando Rizzello ${ }^{1,+}$, Enzo Spisni ${ }^{2, *}{ }^{\dagger}$, Elisabetta Giovanardi ${ }^{1}$, Veronica Imbesi ${ }^{1}$, \\ Marco Salice ${ }^{1}$, Patrizia Alvisi ${ }^{3}$, Maria Chiara Valerii ${ }^{1,2,+}$ and Paolo Gionchetti ${ }^{1,+}$ \\ 1 Department of Medical and Surgical Sciences, University of Bologna, Via Massarenti 9, 40138 Bologna, Italy; \\ fernando.rizzello@unibo.it (F.R.); elisabett.giovanardi@studio.unibo.it (E.G.); \\ veronica.imbesi@studio.unibo.it (V.I.); marco.salice@hotmail.com (M.S.); chiaravalerii@hotmail.it (M.C.V.); \\ paolo.gionchetti@unibo.it (P.G.) \\ 2 Department of Biological, Geological and Environmental Sciences, University of Bologna, Via Selmi 3, \\ 40126 Bologna, Italy \\ 3 Pediatric Unit, Maggiore Hospital, Largo Bartolo Nigrisoli, 2, 40133 Bologna, Italy; \\ Patrizia.alvisi@ausl.bologna.it \\ * Correspondence: enzo.spisni@unibo.it; Tel.: +39-51-209-4147; Fax: +39-51-209-4286 \\ + F.R., E.S., M.C.V. and P.G. equally contributed.
}

Received: 7 April 2019; Accepted: 4 May 2019; Published: 8 May 2019

\begin{abstract}
Inflammatory bowel diseases (IBD) are currently considered multifactorial pathologies in which various combined environmental factors act on a genetic background, giving rise to a chronic inflammation of the gastrointestinal tract. Among the various environmental factors, it now seems clear that the diet plays the major role in IBD onset and progression. Several clinical studies have attempted to understand the impact of diet in the development and progression of these diseases in order to establish useful guidelines for their management. However, the modest and sometimes contradictory results did not lead to the definition of shared dietary suggestions. On the other hand, food fads and recommendations based on anecdotal episodes are often followed by IBD patients to improve their diet. This review provides a critical overview of existing data on the role of diet as a risk factor for IBD. The methodology used was that of analyzing the results of clinical studies conducted on diet and IBD over the last 12 years through PubMed, as well as analyzing the most relevant studies on nutrients and their possible roles in IBD through the knowledge of the mechanisms by which they can modulate the microbiota or the intestinal physiology.
\end{abstract}

Keywords: Inflammatory Bowel Disease (IBD); Mediterranean Diet; Western-style Diet (WSD); Nutritional Approach

\section{IBD and Environmental Risk Factors}

The etiology of inflammatory bowel disease (IBD) remains unclear. The most accredited theory is the interplay between genetic susceptibility and exposure to environmental risk factors, such as pollution, stress, diet, pathogen infections, antibiotic treatment and cigarette smoking, also known as the exposome paradigm [1]. Many studies evidence a rise in both the incidence and prevalence of IBD in traditionally low-incidence areas, such as southern Europe, Asia and most developing countries where Western lifestyle and nutritional habits are progressively being adopted [2]. The prevalence of IBD has also increased significantly in the pediatric population [3].

Many studies have concluded that people emigrating from low-prevalence regions, such as Asia, to high-prevalence countries like northern Europe are at greater risk of developing IBD. This is especially true for first-generation immigrant children who show similar IBD incidence and prevalence patterns to the indigenous population [4]. 
These findings would seem to reduce the importance of a genetic predisposition to IBD and incriminate major lifestyle changes for the rising IBD rates observed in developing countries, in immigrant populations moving to high-prevalence countries, and children in developed countries. These considerations suggest that environmental factors significantly modify the expression of Crohn's Disease (CD) and Ulcerative Colitis (UC). IBD would therefore appear to be a worldwide disease linked to environmental factors such as the diet, lifestyle and pollutants prevalent in industrialized urban societies [4,5].

Diet is a key environmental factor. It plays a major role in the homeostasis of the gut microenvironment, influencing the gut's microbial composition and functioning, the gut barrier, host immunity and the gut physiology by regulating gut hormone release [6,7]. The diet in the developed "Western" world is very different from the traditional diet of previous generations when the prevalence of IBD was considerably lower. The most radical change has been the switch from a plant-based to an animal-sourced diet. This radically modified the gut microbiota and microbiome decreasing the amount of bacteria involved in fiber degradation, such as Prevotella and Treponema. [8,9]. Another important change is the increased presence of xenobiotics in food and especially in meat. Xenobiotics are chemical substance that are not naturally produced or expected to be present within the organism and that mainly derive from environmental pollution or from pesticide, widely used in conventional agriculture. Xenobiotics can undergo the bio-magnification process in intensively farmed animal meat. In fact, changes in the gut microbiome and xenobiotic metabolism have been found in children with Crohn's disease [10].

Recent data suggest that another important change brought about by the Western diet is an overall higher calorie intake, especially from sugar, refined carbohydrates, animal proteins and ultra-processed foods. The role of these latter in gut homeostasis is still under investigation [11], and there are not in-depth studies linking ultra-processed foods and IBD, however, it is becoming increasingly evident that some food additives have negative effects on the gut microbiota.

In conclusion, diet may well play a key role in both the pathogenesis and clinical course of IBD. As a result, diet should become an effective tool to optimize the efficacy of the conventional treatment of these diseases.

\section{The Microbiota and IBD}

The human gut is colonized by a specific and dynamic subset of trillions of microorganisms. Fundamental for our body functions, these microorganisms weigh about $1 \mathrm{~kg}$ - significantly more than all the cells of the human body-and contain more than 100 times as many genes as the human genome. They constitute the so-called 'gut microbiota' of the host's gastrointestinal tract [12].

The human microbiota comprises different types of microorganisms, including bacteria (prokaryotes), archaea, fungi, protists, and viruses. Its composition differs significantly in the different regions of the gut. Indeed, the displacement of microbial communities within the intestinal tract can sometimes give rise to pathologies such as the small intestinal bacterial overgrowth (SIBO) syndrome [13].

The gut microbiota and its host exist in a symbiotic relationship. Together they form a sort of "super-organism" with a wide range of functions. These include digesting substrates, producing nutrients, developing and regulating the immune system, preventing the growth of harmful microorganisms by forming a barrier against colonization or invasion, and detoxifying certain xenobiotics [14]. An adequate biodiversity of the microbiota plays an important role not only for the correct functions of the intestine but also in other organs and systems, such as the enteric and central nervous system [15]. Brain and microbiota interactions have led to the recognition of a new term called "microbiota-gut-brain axis". This term refers to a two-way communication system that allows intestinal microbes to change the physiology of the brain and the brain to modulate the intestinal functions. The evidence of this two-way communication comes from multiple studies that have shown that stressful events in an individual's life can alter the composition of the intestinal microbiota, 
as well as reciprocally, the infection of intestinal pathogenic bacteria can induce anxiogenic behavior in mice [16,17]. Moreover, impaired responses of the central nervous system to stressors have also been clearly demonstrated in patients with microbiota alterations due to active Crohn's disease [18].

Another characteristic of the gut microbiota is its inter-individual variability due to genetic predisposition and factors such as environmental microbes, geography, climate, sanitation, pollution, diet and lifestyle (Figure 1). It follows that the composition of an individual's microbiota changes continuously in a mutualistic response to extrinsic and intrinsic variables. Excessive alterations of the gut microbiota lead to an intestinal imbalance between protective and harmful bacteria, called dysbiosis. This condition seems to be responsible for the inadequate response of the immune system to both external and intestinal epitopes, and may contribute to the loss of oral tolerance. This imbalance can have serious effects during infancy and early childhood when the microbiota is still maturing [19]. Dysbiosis may also be a leading contributory cause of several gut microbiota-related diseases, including IBD $[20,21]$.

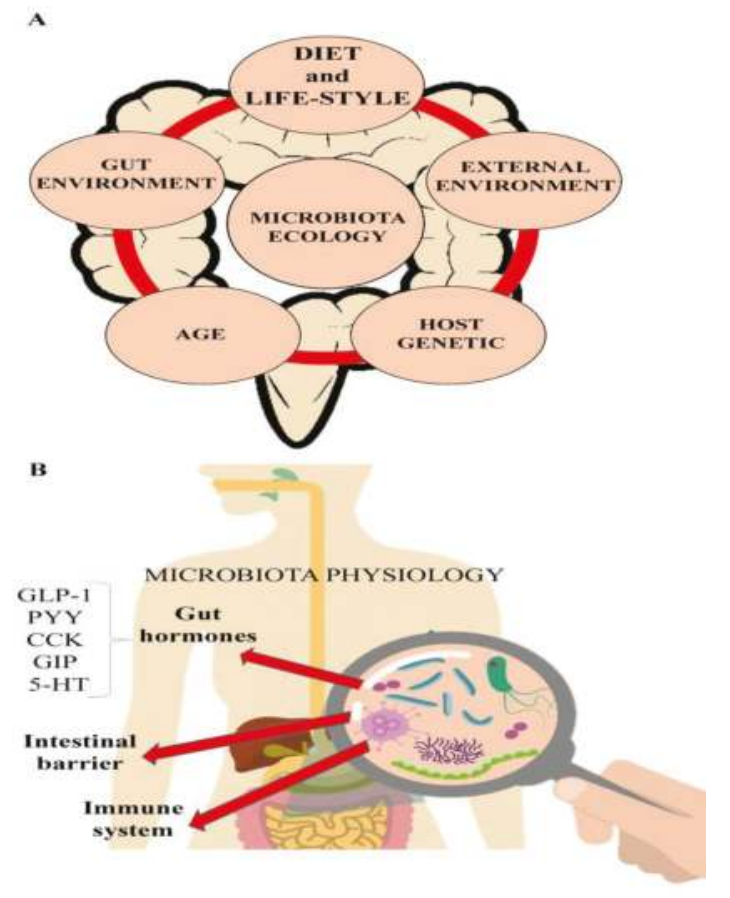

Figure 1. Factors implicated in the modulation of the microbiota ecology (A) and complex microbiota interactions with the gastrointestinal physiology (B). PYY, Peptide YY; GLP-1, Glucagon-like peptide-1; CCK, Cholecystokinin; GIP, Gastric inhibitory polypeptide; 5-HT, 5-hydroxytryptamine or Serotonin.

While many studies have clearly demonstrated the presence of severe intestinal permeability alterations during the active phases of IBD, permeability seems to recover during the remission phase. It should be noted, however, that modest alterations in intestinal permeability are difficult to be measured in vivo [22].

IBD are associated with alterations in the composition of the intestinal microbiota, namely a significant reduction of bacterial diversity, an increase in several pro-inflammatory components called "pathobionts", such as Proteobacteria and Actinobacteria, and the depletion of "health-promoting" anti-inflammatory bacterial genus, such as Faecalibacterium and Roseburia [23].

During the acute phases of IBD, altered intestinal permeability causes commensal bacteria and microbial antigens to translocate from the lumen to the submucosa, giving rise to local and 
systemic inflammation. These translocations activate the immune cells, which in turn release numerous pro-inflammatory cytokines, activating an inflammatory loop and inducing chronic intestinal inflammation [24].

The microbiota's plasticity allows the gut microbiome to respond rapidly to changes in dietary patterns. The fermentable carbohydrates, protein, fat, and sugars ingested modulate the levels of beneficial and harmful microbes, toxic microbial metabolites and protective metabolites, such as short-chain fatty acids (SCFAs) [25].

Dietary fibers, polysaccharides, sugars (i.e. lactose) and peptides that are not digested by host enzymes in the upper gut are partly degraded by the gut microbiota in the cecum and colon through a process called fermentation. The major products of fiber fermentation are SCFAs, in particular, acetate, propionate, and butyrate [26]. SCFAs play important multifactorial roles in energy homeostasis, host immune function $[25,27]$ and general gut physiology $[25,28]$ (Table 1$)$. As a result, the amount of SCFAs synthesized, especially in the colon, has been adopted as an indirect measure of microbiota health [28]. High salt consumption also affects the gut microbiome of both mice and humans by lowering the intestinal survival of Lactobacillaceae [29].

Table 1. The multifactorial role of the SCFAs in energy homeostasis in host immune function and host physiology $[25,27,28]$. GABA, gamma-Aminobutyric acid; CNS, Central Nervous System; ENS, Enteric Nervous system; PYY, Peptide YY; GLP-1, Glucagon-like peptide-1.

\begin{tabular}{lll}
\hline CNS Activity & Metabolic Regulations & Immune Homeostasis \\
\hline & Energy source & Enhancement of IL-10 (DC) \\
GABA production in CNS & Intestinal gluconeogenesis & IL-18 biosynthesis (IEC) \\
Modulation of ENS and CNS function & Antilipolytic activity (WAT) & Treg polarization \\
Serotonin biosynthesis & Lepitin production (WAT) & Reduction of TNF, IL-6, IFN (DC) \\
& Release of major appetite and glucose & IEC barrier fortification \\
& regulatory peptides (PYY, GPL1) (L-cell) & IgA promotion \\
\hline
\end{tabular}

The modulation of intestinal microbiota can be also implemented through probiotics. Probiotics have been defined by the World Health Organization (WHO) as "live microorganisms which, when administered in adequate quantities, confer health benefits to the host". They can play beneficial effects on the digestive system, through multiple mechanisms including interference with potential pathogenic microorganisms, improvement of the barrier function, immunomodulation and the modulation of neurotransmitter synthesis [30,31]. The impact of probiotics on IBD has been the subject of numerous studies. Data derived from studies on animal IBD models showed a high potential of different bacterial strains in modulating the clinical course of experimental IBD. Instead, on humans the experimental results showed little evidence of efficacy on Crohn's disease (CD), modest data on Ulcerative Colitis (UC) but a good support for patients affected by pouchitis, the inflammation of the ileal pouch which is created in the management of IBD patients [32].

It has recently been demonstrated that several dietary components can alter the intestinal microbiota. These include xenobiotics belonging to the group of environmental contaminants, but also additives normally used for the preparation of ultra-processed foods. The xenobiotics capable to have negative effects on the microbiota include organochlorine pesticides [33], glyphosate [34] and perfluorooctanesulfonic acid [35]. All these components have been found in human breast milk, and may therefore negatively modulate the microbial ecology of babies whose unstable microbiota could rapidly develop into a permanently altered gut environment [35]. The effect of food additives added to ultra-processed foods will be discussed in detail below.

Studies of IBD-associated microbiome have evidenced the important role played by the gut environment in the manifestation of IBD. It has also been observed that among the environmental factors associated with microbiota development and alterations, diet plays a key role in modulating the composition and functions of the microbiome and epithelial barrier, and may even influence epigenetic changes. It follows that the food may deeply modulate the microbiome-gut axis and therefore it may 
represent an important therapeutic tool to counteract dysbiosis and modify the natural history of IBD, bearing out the motto "We are what we eat" [7].

\section{Evolution of the Human Diet}

The first dramatic change in human nutrition occurred about 10,000 years ago during the Neolithic age with the transition of the hunter/gatherer society to one based on agriculture and livestock farming. This change profoundly modified man's diet. Instead of a wide range of different foods that changed with the seasons and with the migratory patterns of animals, our diet became restricted to a few domesticated plants and farmed animal species. For the human metabolism this meant a dramatic change in glycemic load, fatty acid composition, macro- and micro-nutrient concentration and total fiber content. Indeed, in evolutionary terms, this dietary change is so recent that the human species has not had enough time to adapt its gastro-intestinal physiology [36]. Many foods common in the new diet were completely absent in the hunter/gatherer society. Milk, for example, was consumed only during breastfeeding, and dairy products were completely absent [37]. The first evidence of dairy milk consumption has been dated to about 6,000 years ago [2,38]. Cereals are another interesting example. At the beginning of the Holocene age (12,000-10,000 years ago), cereals—always unrefined-were still rarely eaten. The first evidence of white flour production has been traced back to around 3500 Before Christ (BC) in ancient Egypt [39], but the widespread consumption of highly refined flours began only 150-200 years ago [40].

The second truly revolutionary change in the human diet was the introduction of industrialized food following the Second World War. Driven by the so-called "green revolution", which made widespread use of agricultural chemicals, industrialized food production led to a huge rise in the availability of refined cereals, sugar, cheese, butter and coffee (Figure 2). Before this, sugar had been a rare component of the human diet. The first evidence of crystallized sugar can be traced back to $500 \mathrm{BC}$ in India [41]. Before that, the only source of sugar had been honey, available in very limited amounts and only at certain times of the year. Even subsequently, sugar remained a rare commodity. Before the industrial revolution, the annual average sugar consumption in the United States was $6.8 \mathrm{~kg}$ per person while in 1970 it exceeded $55 \mathrm{~kg}$, and in 2000 stood at $69 \mathrm{~kg}$ [42].

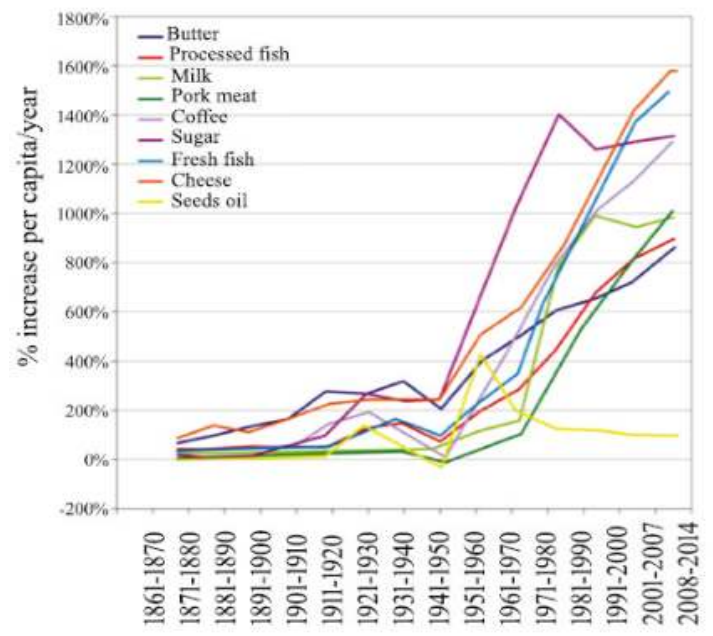

Figure 2. Variation in average food consumption of the population in Italy, one of the countries where the Mediterranean diet was initially discovered, from the end of the 1800s to the early 2000s. The basis of comparison (100\%) is the period 1861-1871. Data source: Italian National Research Institute for Food and Nutrition (INRAN), 2013. 
Industrialization and the advent of intensive farming also involved a change in farmed animal feed. As a result, the quality of farmed meat, and especially its fat content, is today completely different from the wild animal meat consumed by hunter/gatherers. This is because mammal fat stores are localized in subcutaneous and abdominal adipose tissue in the form of saturated fatty acids (SFAs), while polyunsaturated and monounsaturated fatty acids (PUFAs and MUFAs) prevail in the muscles. It has been estimated that prior to domestication, wild animals consumed most of their subcutaneous and abdominal fat reserves, with the result that our hunter ancestors ate meat with high levels of muscle-stored PUFAs and MUFAs and little or no SFAs [43-46]. Industrial farming, on the other hand, has led to hyper-fed animals with high percentages of accumulated SFAs $[45,46]$.

First extracted around 5000 years ago, vegetable oils were not initially used as a food. It was only with industrialization and the introduction of new extraction techniques that seed oils became products for human consumption (Figure 2). With the advent of hydrogenation in 1980, hydrogenated vegetable fats suddenly became part of our daily diet. Overall, the increase in dietary fat has certainly had an impact on the microbiota and GI diseases [47].

Salt consumption seems to have started at the beginning of the Neolithic age, although its massive consumption only began with the industrial revolution. This has led to an inversion of our ingested sodium/potassium ratio compared to the Neolithic diet. Today, $90 \%$ of the salt in the Western-style diet (WSD) comes from processed foods [36]. Food industrialization has also caused a sharp drop in the consumption of fruits and vegetables, and therefore fibers, vitamins and potassium [44,48]. In conclusion, industrialization and the resultant change in diet caused a sudden rise in man's net acid load after digestion, also known as the Potential Renal Acid Load, PRAL. This is because staple WSD foods like cheese, refined cereals and meat are net acid producers while the vegetable-rich diet of our pre-agricultural ancestors was a net base producer [49].

The dramatic change in man's diet is still ongoing. Ultra-processed foods, recently classified as the NOVA-4 group [50], are now readily available and today account for over $50 \%$ of the total energy intake of the United States population [11,51].

\section{The Western-Style Diet and Its Pro-Inflammatory Foods}

Compared to traditional regional diets, such as Mediterranean, Indian, Japanese and South East Asian, the WSD contains significantly higher amounts of simple refined carbohydrates, saturated fats, red meat, dairy products and industrialized foods, and a concomitantly lower quantity of vegetables, fruits, legumes, whole cereals, raw foods and fibers in general (Figure 3). In fact, the WSD consists mainly of calorie-dense foods high in saturated fats, glycemic carbohydrates and animal proteins. Many studies have looked at the association between WSD and obesity, hypertension [52], chronic kidney disease [53] and other non-communicable diseases [54]. Their findings clearly show that the WSD may promote intestinal inflammation through various mechanisms, including alterations of the microbiota [55-58]. Although the relationship between the WSD and IBD has been only partially investigated [59,60], it is nonetheless true that the WSD contains nutrients able to elicit a direct or indirect pro-inflammatory effect on the intestine through one of the three components involved in gut physiology: the immune system, the microbiota, and the intestinal barrier. Moreover, the WSD is low in nutrients and micronutrients with anti-inflammatory and anti-oxidant proprieties. It is therefore not unreasonable to consider the transition to the WSD, also defined as diet westernization, as one of the major environmental factors responsible for the rising incidence and prevalence of IBD. A very interesting in-depth study on the effects of this dietary switch on the intestinal microbiota of children has been recently published [61]. The authors clearly demonstrate that the gradual increase of animal proteins (meat and dairy products), saturated fats, and processed and refined foods to the rural vegetarian diet of urban African children drastically changed their microbial profiles and gut functioning. The sharp drop in fiber intake as a result of the dietary change was found to lead to a concomitant fall in microbial populations able to ferment dietary fibers and a sharp rise in other bacterial groups able to metabolize animal proteins, animal fats and sugars. The westernization of 
the diet has involved and overwhelmed even the traditional oriental diets, such as the Japanese or that of South East Asia, which are diets geographically associated with a low prevalence of IBD [62]. The potential implications of WSD components on the gut immune system, the microbiota and the intestinal permeability will be analyzed in detail in the following sections of this review.

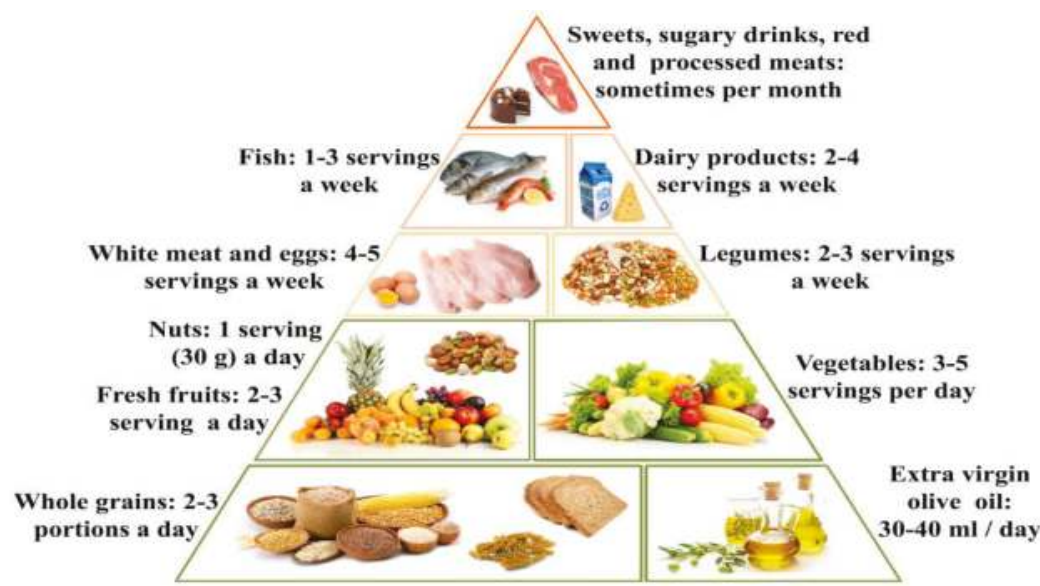

Mediterranean food pyramid

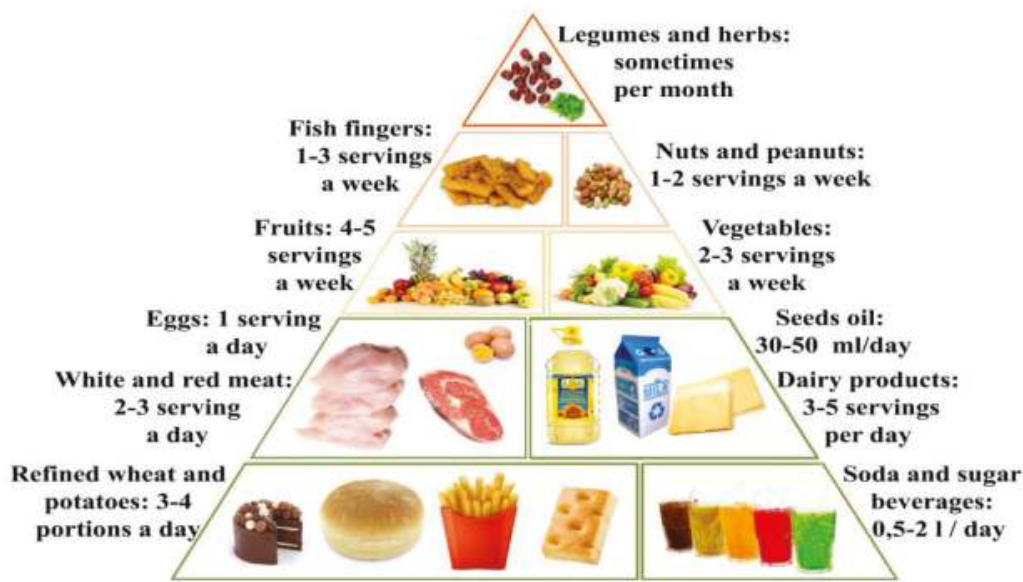

Western-style food pyramid

Figure 3. Comparison between the Mediterranean diet (upper) and the Western-style diet (lower) pyramids. The Mediterranean diet pyramid is inspired by the eating habits of Greece, Southern Italy, and Spain before 1950s. The principal aspects of this diet include high consumption of fruits, vegetables, unrefined cereals, legumes and olive oil, moderate to high consumption of fish, moderate consumption of dairy products and low consumption of non-fish meat.

\section{Carbohydrates}

Carbohydrates are classified on the basis of their chemical structure into mono- and di-saccharides (simple sugars, such as glucose, fructose, sucrose), oligosaccharides (fructo- and galactooligosaccharides), and polysaccharides (starch, cellulose, inulin). A fraction of the carbohydrates 
ingested remains undigested in the small intestine and passes to the large intestine to be fermented and/or excreted. For this reason, carbohydrates are also classified according to their absorbability in the small intestine. For example, simple sugars and starches are hydrolyzed and absorbed in the small intestine and are therefore known as available carbohydrates. Inulin, fructo-oligosaccharides and galacto-oligosaccharides, known as resistant starches, are not hydrolyzed in the small intestine but fermented by the large bowel microbiota, and are therefore called unavailable carbohydrates. Finally, insoluble fibers including plant cell-wall polysaccharides, such as cellulose and pectin, and some storage polysaccharides, such as inulin and oligosaccharides, are also called non-digestible components because they pass through the digestive tract largely intact, exerting a laxative effect by changing the osmotic properties of the luminal content and by increasing the intestinal fecal mass. The quality of the carbohydrate ingested, for example, whether mono-, di- or oligo-saccharides, also seems to impact the clinical course of IBD. In fact, a correlation has been observed between fructose and lactose malabsorption and IBD severity $[59,63]$. In light of this evidence, a fructose- and lactose-free diet has been proposed for IBD patients [59].

Lactose malabsorption is frequently detected in IBD, especially in CD patients. However, prevalence is ethnicity dependent, being higher among Asian populations. Moreover, although lactose avoidance may improve symptoms in patients with lactose intolerance, evidence of objective disease improvement is currently lacking. Studies of a lactose-free diet in IBD patients are currently underway [59].

High consumption of fermentable carbohydrates such as glucose, fructose, sucrose, lactose or polyols (sorbitol, mannitol, xylitol and maltitol) has been shown to exceed the intestine's absorptive ability, increasing dysbiosis and gut permeability, thus promoting inflammation. These fermentable carbohydrates, which include oligo-, di-, and monosaccharides and polyol, are known as FODMAPs (Table 2). Being poorly absorbed, they are rapidly fermented by bacteria in the gut, giving rise to a high osmotic load. This is the rationale behind the FODMAP-exclusion diet. Firstly proposed for Irritable Bowel Syndrome (IBS) patients, the FODMAP-exclusion diet was applied for a limited period to a few IBD patients [64] in an attempt to alleviate their IBS-like symptoms, such as bloating, flatulence, crampy pain, and diarrhea, but proved very limited benefit. Moreover, since undernutrition is a common feature of IBD, the use of this very restrictive diet should be carefully considered [64].

Table 2. Carbohydrates included or excluded in the low-FODMAPs diet.

\begin{tabular}{|c|c|c|}
\hline Food Group & Include & Exclude \\
\hline Fruit & $\begin{array}{l}\text { Bananas, strawberry, raspberry, blueberry, orange, } \\
\text { mandarin, clementine, cantaloupe, grapes, melons, lemon, } \\
\text { lime, kiwi, passion fruit }\end{array}$ & $\begin{array}{l}\text { Apple, applesauce, apricots, blackberries, cherries, } \\
\text { nectarines, pears, peach, plum, prune, watermelon, } \\
\text { grapefruit, dried fruit }\end{array}$ \\
\hline Vegetables & $\begin{array}{l}\text { Carrots, celery, corn, alfalfa, bean sprouts, bell pepper, } \\
\text { broccoli (<1/2 cup), bok choy, cucumber, eggplant, green } \\
\text { bean, kale, lettuce, potato, spinach, spring onion (green } \\
\text { top), squash, tomato, turnip, zucchini }\end{array}$ & $\begin{array}{l}\text { Brussels sprouts, asparagus, avocado, beetroot, } \\
\text { cauliflower, cabbage, garlic, leek, mushroom, onion, } \\
\text { pea shallot, snow pea, sweet corn, sweet potato }\end{array}$ \\
\hline Grains & Rice, oats & Wheat, rye \\
\hline Legumes & Certain legumes (soya) & Many legumes (chickpeas, lentils, beans) \\
\hline Dairy & $\begin{array}{l}\text { Lactose-free yoghurt and milk; almond, coconut, rice or } \\
\text { soy "milk", hard cheese, low-lactose cheese }\end{array}$ & $\begin{array}{l}\text { Cow, goat, sheep milk, buttermilk, soymilk, soft } \\
\text { cheese cream and ice cream }\end{array}$ \\
\hline Beverage & Fruit juice and vegetable juices from permitted foods, wine & $\begin{array}{l}\text { Soft drinks, sports drinks, fruit juice and vegetable } \\
\text { juices from unpermitted foods, alcohol }\end{array}$ \\
\hline Other & Maple syrup & Honey and sweeteners \\
\hline
\end{tabular}

Turning to complex carbohydrates, several epidemiological studies have observed associations between the consumption of refined carbohydrates and the risk of developing IBD. However, these data are not sufficiently statistically significant to allow confirmation of this hypothesis [7]. Nonetheless, the WSD contains many different refined carbohydrates, such as sucrose and starch found especially in soft drinks, white bread, cakes, and desserts, all high-density energy-rich but nutrient-poor products. In the long term, they may cause alterations of the gut microbiota and immune system, increasing the risk for many diseases including IBD [65]. Other dietary approaches have been proposed in an attempt 
to improve IBD symptoms. These include the "specific carbohydrate diet" (SCD), first described by Haas and co-workers in 1924 to treat celiac disease, and subsequently applied to IBD patients [66,67]. The SCD excludes gluten and complex carbohydrates since they are poorly absorbed and lead to bacterial fermentation, which in turn induces dysbiosis in the gut lumen due to pathobiont expansion (Table 3). Only monosaccharides such as glucose, fructose and galactose are permitted. Clinical trials, albeit involving very few patients, reported improvements in bowel symptoms, with some patients able to discontinue at least one of their IBD medications at the end of the SCD trial period [67].

Table 3. Carbohydrates included or excluded in the Specific Carbohydrate Diet (SCD).

\begin{tabular}{|c|c|c|}
\hline Food Group & Include & Exclude \\
\hline Fruit & All fresh fruit & Canned fruit (because of possible added sugars and starches) \\
\hline Vegetables & Fresh vegetables & $\begin{array}{l}\text { Canned or frozen vegetables (because of possible added } \\
\text { sugars and starches), potatoes and yams }\end{array}$ \\
\hline Grains & None & All cereal grain \\
\hline Legumes & Certain legumes (lentils, split pea) & Certain legumes (chickpeas, soybeans) and soybean "milk" \\
\hline Dairy & $\begin{array}{l}\text { Lactose-free dairy (milk, cheese and } \\
\text { yogurt) }\end{array}$ & Milk and all dairy products \\
\hline Beverage & Wine & Instant tea, coffee and beer \\
\hline Other & Honey, & Corn syrup, chocolate \\
\hline
\end{tabular}

In conclusion, if poorly absorbed, all carbohydrates may be fermented, promoting dysbiosis and inflammation. An excess of any kind of carbohydrate in IBD patients with intestinal malabsorption may easily exacerbate existing intestinal dyshomeostasis, which in turn may contribute to dysmetabolism of other nutrients, such as fats.

It is therefore reasonable to conclude that IBD diets should carefully monitor the intake of simple and complex carbohydrates and strictly limit lactose, especially in patients intolerant to this sugar. In fact, exclusion of specific carbohydrates (known as carbohydrate monotony) is a common feature of the different diet-based IBD therapies, in light of the suggestion that carbohydrate variation could be linked to IBD pathogenesis [68]. It does, however, seem unlikely that a single sub-group of foods could be the cause of IBD.

\section{Proteins}

Several mechanisms of action have been postulated to explain the possible negative impact of high protein (HP) consumption in IBD patients. One hypothesis is that the intestine's limited capacity to assimilate proteins, especially in IBD patients, leads to non-absorbed proteins to reach the colonic lumen where they alter microbiota composition by reducing the abundance of bacteria like Roseburia/E.Rectale, considered health-promoting microorganisms for their ability to produce butyrate [69]. In fact, HP diets appear to induce IBD-like dysbiosis [70]. Rats fed a HP diet showed increased prokaryotic protease activity, a phenomenon apparently linked to the pathogenesis of IBD [71]. HP diets may also lead to an imbalance of metabolites of protein catabolism by colonic bacteria (ammonium, hydrogen sulfide, p-cresol and phenol), which could damage colonocytes and the intestinal barrier [72,73]. Several animal studies on colitis have also demonstrated that proteins derived from red meat, milk and dairy products can exacerbate colitis. However, human epidemiological data only partially support these findings. This is probably due to the complexity of the human diet and diverging nutritional data collection methods [70]. In fact, two epidemiological studies have shown a correlation between IBD and high animal protein intake derived from meat and fish but not from eggs or dairy products, while no correlation was found with the consumption of vegetable proteins [74,75]. Two meta-analyzes conducted in 2011 and 2015 also indicate that the consumption of red, white and processed meat may be considered risk factors for the development of IBD [6,76]. Conversely, two more recent studies have produced conflicting results as to a correlation between meat consumption and IBD activity. The first, by Opstelten et al. [77] involving 165 patients with IBD in remission and 1469 healthy controls, indicated that although IBD patients eat more meat than controls, 
there was no correlation with disease exacerbation. The second study on 53 patients with active disease and 50 in remission concluded that meat is a risk factor for active disease [77,78]. In addition to the protein metabolism mechanism already discussed, it has also been hypothesized that the effect of meat consumption on the onset and course of these diseases could be linked to the cooking process, which notoriously leads to the formation of carcinogenic or mutagenic molecules such as heterocyclic amines, acrylamide and polycyclic aromatic hydrocarbon, associated with endothelial damage and altered immune responses [79]. However, the most convincing studies on meat and IBD have focused on the risk of high saturated and high n-6 polyunsaturated fatty acid (PUFA) intake as a consequence of high meat (especially red meat) consumption.

Gastro-resistant proteins are another point of attention when structuring an IBD diet since the abundance of gastro-resistant proteins in some foods can alter intestinal permeability. Gluten is a gastro-resistant protein complex. Its digestion gives rise to toxic and antigenic peptides (especially alpha-gliadin peptides), which may not only interfere with the tight junction between enterocytes but also with enterocyte survival, affecting the intestinal barrier. This, together with strong evidence of gluten sensitivity in IBD patients [80], has led to the gluten-free diet being recommended for IBD sufferers. Indeed, a gluten-free diet is often self-imposed by patients in an attempt to alleviate symptoms. Ancient wheat varieties, although seemingly giving rise to similar amounts of toxic and antigenic gluten peptides [81], have been shown to ameliorate gut and systemic inflammation in a range of patients, including IBS sufferers [82]. For this reason, ancient varieties of low immunogenic impact grains, such as Senatore Cappelli (Triticum turgidum durum) and Enkir (Triticum monococcum), or a novel grain variety with low gluten immunogenicity like Tritordeum [83] should be considered for inclusion in the IBD diet. Being gluten-free, rice has also been suggested as a complex carbohydrate suitable for the IBD diet. This includes whole grain rice, provided it is cooked at length [84].

Milk and dairy products contain large quantities of caseins, gastro-resistant proteins known to increase dysbiosis, inflammation and intestinal permeability in the mouse gut [85]. For this reason, milk and dairy foods are excluded in most IBD diets and indeed, commonly restricted by IBD patients themselves [86]. On the other hand, fermented milk products have shown positive effects on IBD patients. Kefir was administered in a randomized controlled trial to 25 IBD patients with positive effects on clinical parameters, such as erythrocyte sedimentation rate, C-reactive protein, bloating, general-feeling scores, and the Lactobacillus bacterial load evaluated in patient feces [87]. Yogurt was administered to IBD patients in a double-blind placebo-controlled clinical trial with beneficial effects on the mean numbers of Lactobacillus, Bifidobacterium, and Bacteroides in the feces of the treated group [88].

In conclusion, although performed with only a few patients, the studies conducted so far would seem to suggest that the IBD diet should reduce the overall amount of meat, eliminate red and processed meat, and eliminate or greatly reduce gluten and dairy products (caseins), with the sole exception of yogurt and kefir.

\section{Fats}

Recent epidemiological data attribute a protective effect to n-3 PUFAs in UC prevention and therapy $[89,90]$. Conversely, consumption of higher ratios of n-6/n-3 PUFAs has been associated with increased UC incidence. The role of n-3 PUFAs in CD prevention and therapy, however, appears complex and controversial [89]. The physiological basis for PUFAs' role in IBD is that the eicosanoids produced by $n-3$ and n- 6 PUFA are key immune response regulators, being precursors of several pro-inflammatory (n-6 PUFA) and anti-inflammatory (n-3 PUFA) molecules. It has been demonstrated that n-6 PUFAs are more available than n-3 PUFA for eicosanoid metabolism in the UC colon mucosa. For this reason, dietary supplementation with n-3 PUFA, and especially eicosapentaenoic acid (EPA), can reduce the production of prostaglandin E2, thromboxane B2 and hydroxyeicosatetraenoic acid inflammatory mediators [91-93]. The anti-inflammatory effects of n-3 PUFA have also been linked to the synthesis of molecules like resolvins, protectins and maresins, which counteract IBD-related dysbiosis [94], and the down-regulation of pro-inflammatory genes [95]. However, two IBD studies 
have indicated that PUFA intake may have a different impact in CD compared to UC patients. In their study on 137 UC patients and 38 healthy controls, Wiese and collaborators [96] showed that the concentration of inflammatory tissue cytokines in UC patients was directly related to the amount of serum SFAs and inversely related to total PUFAs, EPA and docosahexaenoic acid (DHA), confirming the protective role of n-3 PUFAs in UC. But a recent study by Scoville and collaborators [97] on 116 CD patients and 27 healthy controls showed that n-3 PUFA serum concentrations were directly related to disease severity. In particular, the authors found EPA concentration to be strongly related to pro-inflammatory serum cytokine concentrations. This datum not only suggests that fatty acid dysmetabolism occurs in patients with CD but also that n-3 PUFA and EPA serum levels may also have a negative effect in CD patients. Conversely, no correlation with SFA concentration was observed in UC patients [97]. Interestingly, both studies, which administered the same amounts of meat, total fat, and SFA to IBD patients and healthy controls, found that n-6 PUFA/n-3 PUFA ratios were similar in $\mathrm{CD}$ patients and healthy subjects, despite higher respect the WHO recommendations.

In summary, we may conclude that high fat intake should be avoided in IBD sufferers since high fat diets may lead to an accumulation of secondary bile acids, such as deoxycholic acid, which can inhibit Bacteroidetes and Firmicutes phyla growth, leading to IBD-like dysbiosis [7]. However, a prospective study carried on 170,805 women showed no correlation between fat consumption and IBD risk, but rather a positive correlation between high trans-unsaturated fat intake and UC risk [89]. In addition, the intake of n-3 PUFA and EPA in particular, within foods or supplements, may have positive effects on health, but only for UC patients.

\section{Dietary Fibers}

Dietary fibers are non-digestible complex carbohydrates present in plants. They are grouped according to their solubility or insolubility in water. Since humans lack fiber-specific enzymes, fibers remain mainly undigested and are therefore not absorbed by the gastrointestinal tract. However, small amounts of the ingested fibers are fermented by the bacteria in the gut, especially in the colon. Present in fruits, vegetables, cereals and legumes, dietary fibers can exert a protective role against IBD through four independent mechanisms [90]:

Reducing intestinal transit time;

Increasing the synthesis of SCFAs such as acetate, butyrate and propionate, which may positively modulate intestinal inflammation;

Improving gut microbiota composition, thus counteracting IBD-related dysbiosis;

Contributing to the maintenance of intestinal barrier functions.

No conclusive studies exist evidencing the efficacy of dietary fibers in slowing IBD progression [98]. A study conducted by Andersen and collaborators [90] showed no association between average fiber intake (total fiber, fiber from fruit, vegetables and cereals) and the subsequent development of CD or UC. Moreover, it has been shown that fibers may exacerbate gastrointestinal (GI) symptoms, such as abdominal distension, flatulence, constipation, and diarrhea in IBD patients during remission periods [99]. It is therefore reasonable to postulate that a high fiber diet is not beneficial for individuals with insufficient "fiber-degrading bacteria" in their gut microbiota [100]. However, while this type of diet may be contraindicated for patients during disease flares, it is nonetheless highly recommended, with appropriate adjustments, after remission in an attempt to approximate the $30 \mathrm{~g} / \mathrm{die}$ WHO recommended intake.

As suggested by Eswaran and collaborators [99], patients in remission should receive a diet with low levels of insoluble fibers, such as those in skinned vegetables like zucchini, carrots, eggplants, green beans and chards. The vegetables should be very well cooked and consumed as a cream, at least initially. Peeled fresh fruit is another option, providing small quantities of insoluble fiber. Putting ingredients through a juice extractor will further reduce the amount of insoluble fiber present in fruit, vegetables and legumes while maintaining most of the soluble fiber fraction, vitamin and mineral content. There is also some unsubstantiated evidence that fruit and vegetable consumption may have 
a protective immune-modulating effect on IBD patients since they contain protective compounds that may help to prevent disease flares [101]. Another source of insoluble fibers is whole grain cereals. Here too, it seems appropriate to include cereals in the diet only after remission, doing it gradually to avoid worsening of the disease-related GI symptoms [84,99]. Although many IBD patients avoid foods containing fibers, this may lead to micronutrient deficiency and aggravate the malnutrition often presented by sufferers of this disease.

\section{Micronutrients}

IBD patients tend to suffer from nutrient malabsorption and/or have erroneous nutritional patterns [102], with the result that many are likely to present micronutrient, vitamin and mineral deficiencies. Since micronutrient deficiency may affect immune system physiology and thus facilitate disease relapse, diet or supplementation adjustment strategies could be a key to improove patients' quality of life [103]. The main vitamin deficiencies found in IBD-patients are of fat-soluble vitamins A (retinol and retinoids) and D, and water-soluble vitamins B12 and B9 (folic acid). Experimental models have provided some evidence that vitamin A (provided as retinol) supplementation attenuates intestinal inflammation and improves the gut environment, with significant benefits to health [65]. As antioxidants, vitamins A have a probable protective role against free radicals and oxidative damage, both of which are increased in these patients [104].

As well as regulating bone, calcium, and phosphorus metabolism, Vitamin D has a key role in intestinal defense mechanisms, regulating the adaptive and innate immune systems and suppressing microbial invasion of the epithelium $[105,106]$. Vitamin D deficiency has been detected in $16 \%$ to $95 \%$ of IBD sufferers, mainly in CD patients [107]. This may be due to several factors, such as intestinal malabsorption, low exposure to sunlight, lack of physical activity, or insufficient dietary intake. Therapy targeting the vitamin D3 signaling pathway has been suggested in IBD since it may regulate both innate and adaptive immune functions. However, the overall effect of vitamins on IBD is still not well understood, and the clinical studies performed have shown contrasting results. A Danish study conducted between 2005 and 2008 randomized 94 IBD patients to receive oral vitamin D3 or placebo. Although patients receiving vitamin D3 showed a reduced risk of clinical relapse (from $29 \%$ to $13 \%$ ), this was not statistically significant [108]. Similarly, a more recent Iranian study on 108 IBD patients found that oral vitamin D3 supplementation gave rise to lower serum TNF levels, which, however, were also not statistically significant [109].

Folic acid, or folate, is available in several vegetables and is absorbed in the duodenum and proximal jejunum. Both micronutrients are essential for cell metabolism, and folate deficiency is often correlated with vitamin B12 deficiency, this latter mainly available in animal products. Despite their ample availability in food, folate and vitamin B12 absorption occurs in areas often damaged by phlogosis, which may be the cause of impaired uptake in IBD-patients [110].

Trace elements are another important avenue of ongoing research into the prevention and control of IBD. Iron deficiency is very frequent in IBD and the leading cause of anemia among IBD patients. In a recent study, $27 \%$ of all CD and $21 \%$ of all UC patients were found to have anemia [111]. An iron-, heme- and nonheme-rich diet or oral/parenteral supplementation is therefore mandatory. However, iron-and especially ferrous sulfate-is a known pro-oxidative agent, raising concern that oral iron may increase oxidative stress in patients with colitis [112].

Zinc is an essential micronutrient, an enzyme co-factor involved in cellular immunity, cell growth and wound healing. Zinc deficiency has been associated with excessive loss of GI secretions due to chronic diarrhea or fistula drainage in IBD patients. Prevalent in $15 \%$ to $40 \%$ of IBD patients, it has also been associated with hospitalization, surgery and other disease complications [113].

Selenium is a non-metal trace element existing in both the inorganic and organic form. It is translationally incorporated as selenocysteine into selenoproteins, which can modulate various cellular pathways involved in inflammation. Adequate selenium intake is thus essential for normal immune functions [114]. In fact, selenium deficiency has been correlated with gastrointestinal inflammation and 
altered gut microbiota composition [115]. Epidemiological studies suggest that selenium serum levels are inversely correlated with IBD incidence. Moreover, selenium blood concentrations were found to be significantly lower in Caucasians and Asiatic IBD patients, especially in CD [116,117]. Although further studies are necessary to assess the mechanism underlying the effect of selenium deficiency in IBD, it would seem important to ensure adequate selenium intake in all IBD patients.

\section{Food Additives and Ultra-Processed Food}

Ultra-processed foods (NOVA 4 classification) are the result of a series of industrial processes, many requiring sophisticated equipment and technology. Ultra-processed food manufacture entails fractioning whole foods into different substances, their chemical modification and subsequent assembly with other modified and unmodified food products. The industrial techniques used include extrusion, molding, pre-frying, and the addition of "cosmetic" palatability additives [50]. The presence of high levels of ultra-processed food in the diet has already been linked to a number of non-communicable diseases including cancer, obesity and type 1 and 2 diabetes [118-120]. Despite this, however, the role of these foods on the intestinal microbiota, and on IBD in particular, has never been investigated. However, there is well-documented scientific evidence of the negative effects on the microbiota of food additives typically present in a considerable number of ultra-processed foods. Various studies have clearly shown the negative effect of non-caloric artificial sweeteners often present in carbonated soft drinks on microbiome composition and functioning [121]. The sweetener sucralose has been shown to reduce the relative amount of Clostridium cluster XIVa in mice feces [122]. Dietary emulsifiers have also been shown to directly alter human microbiota composition and gene expression in an ex vivo model [123], while maltodextrins, commonly used for the production of soft drinks, candy and sport energy products, have been found to cause microbiota alterations [124] and affect gut epithelial cells, reducing mucus production and exacerbating gut inflammation [125].

The commonly used food additive carrageenan has been shown to induce intestinal inflammation in animal models. Although there is little evidence of the role carrageenan may play in the development of IBD since direct carrageenan assessment trials in man are unethical, animal studies have clearly demonstrated that this additive induces IBD-like histopathological features, alters the microbiome, and disrupts the intestinal epithelial barrier [126]. Red meat, eggs naturally contain high levels of sulfites due to their high cysteine content, which can become a substrate for the production of hydrogen sulfide $\left(\mathrm{H}_{2} \mathrm{~S}\right)$ by sulfate-reducing bacteria. However, sulfites are also abundantly used as food additives to limit bacterial contamination. Generally considered safe for human consumption, they are present in food and alcoholic beverages in quantities up to 5000 parts per million (ppm). However, even at lower concentrations, sulfites have been shown to damage beneficial bacteria, such as Lactobacillus and S. thermophilus in the human gut [127]. Sulfites can be produced by intestinal microbiota even starting from taurine (from the bacterium B. wadsworthia) which is a typical ingredient of energy drinks or from chondroitin sulfate, a widely used food supplement in the treatment of osteoarthritis [128]. Some studies have found that patients with UC show a higher production of $\mathrm{H}_{2} \mathrm{~S}$ in the gut, but it is not clear whether this increased synthesis is simply linked to the increased intestinal inflammation [128].

\section{Alcohol}

Small quantities of alcohol are first metabolized in the stomach. Larger amounts are absorbed in the small intestine and may reach the large bowel from the bloodstream where it is metabolized by luminal bacterial alcohol dehydrogenase, producing the toxic compound acetaldehyde $[129,130]$. Severe dysbiosis has been associated with chronic alcohol abuse. In addition, the high sugar content of some alcoholic drinks may be associated with osmotic diarrhea [131]. A study of 52 Crohn's and 38 UC patients with an alcohol consumption pattern similar to the general population found that although $75 \%$ of the IBD patients reported worsening of their symptoms, this could not be related to alcohol intake [132]. In 2018, a prospective cohort study of 262,451 subjects enrolled in six countries showed no association between alcohol use and the onset of IBD [133]. Interestingly, two studies have found 
a correlation between UC disease activity and sulfite, an additive commonly used also in processed alcoholic drinks $[134,135]$. In conclusion, although there are insufficient data to define alcohol as a risk factor for IBD, its toxic effect on colon mucosa strongly suggests limiting alcohol intake in IBD sufferers.

\section{Nutritional approach in IBD}

Recently, numerous new dietary philosophies have become popular. These include the paleo and the vegan diets, often recommended in the management of gastrointestinal diseases such as IBD. However, there is no clinical evidence to support their use in IBD [113]. The only nutritional approach with strong evidence of efficacy is Exclusive Enteral Nutrition (EEN), a diet based on foods classified according to their nitrogen content as: elemental (amino acid based), semi-elemental (oligopeptide based) and polymeric (whole protein based). EEN has proved as efficacious as steroid treatment in inducing remission of CD in children and adults but not of UC [136]. Although the above-mentioned low FODMAPs diet has been mainly used to alleviate symptoms in IBS patients, there are currently no consistent data supporting its use in IBD. A pilot study conducted on 52 CD and 20 UC patients showed that $50 \%$ of patients had an improvement in abdominal pain, bloating, and diarrhea [137]. A double-blind controlled placebo cross-over trial was conducted with 32 patients with quiescent disease and IBS-like symptoms that had proved responsive to a Low-FODMAP diet. Patients were randomly assigned to receive fructans, galacto-oligosaccharides, sorbitol, glucose or placebo. The study found that only fructans exacerbated symptoms in patients, which casts into question the real usefulness of the low FODMAPs diet in IBD [138]. Another uncontrolled clinical trial included a semi-vegetarian diet as an "add-on therapy" to the biological drugs used to induce remission. 26 adults and 11 children with recently diagnosed $C D$ and 9 adults with relapsing $C D$ received a lacto-ovo semi-vegetarian diet that included fish once a week and meat once every 2 weeks. Results showed an increase of the expected remission rate in all patients [139]. Yet another study of CD patients on a semi-vegetarian diet for two years reported the absence of CD relapse in 15 of the 16 patients treated [140]. The IgG exclusion diet, on the other hand, is a personalized regime based on preliminary blood titration of food IgG. Two studies conducted by the same research group have demonstrated the efficacy of this diet. The first, an open-label pilot study, was conducted on 40 symptomatic CD patients [141] while the second was a randomized study enrolling 76 subjects with active CD. In this second study, the foods most frequently excluded were milk, beef, pork and eggs. The personalized diet significantly improved patients' quality of life and disease activity, measured using the Crohn's Disease Activity Index (CDAI) and Harvey-Bradshaw Index (HBI) [142].

Although SCD diet is one of the most popular among IBD patients, it has many restrictions making compliance difficult. Obih and collaborators [143] conducted a retrospective study on 26 IBD children who had followed the SCD for 3-48 months, 18 of whom were receiving concomitant medication therapy. 12 of the 26 patients showed improvements in their clinical and inflammatory markers. It should, however, be noted that in the medium and long term the SCD diet may cause adverse effects, such as weight loss and nutritional deficiencies. Patients following this diet must therefore be carefully monitored.

Another study on the SCD, involving 50 adult IBD (mainly colonic CD) patients, obtained a better symptom score and a higher quality of life. Some patients were even able to maintain clinical remission without medication [144]. The fecal microbiota of patients on the SCD was observed to have a higher biodiversity index compared to matched patients eating a WSD [144]. These data suggest that the SCD may be a good starting point from which to plan a dietary regime for IBD patients, especially in the maintenance phase.

\section{Conclusions}

Although the study data are still often unsubstantiated, it is important not to underestimate the role diet can play in supporting conventional IBD therapies, not only helping to induce and maintain clinical remission, but above all, improving patient quality of life. 
While controversy still surrounds the exact role played by diet in the development of IBD, there are indications that diet may significantly modulate disease onset and activity. No formal dietary guidelines exist, however, for patients with IBD. The wide variability of study results is due in part to the design limitations of what are largely underpowered, retrospective studies, and in part to the heterogeneous disease subtypes (UC, CD) recruited to the studies.

The complexity of the human diet makes it very difficult to identify individual foods as risk factors. It is in fact more likely that complex nutritional patterns very different from either the traditional Mediterranean diet or other traditional diets matching our evolutionary status may impact pathogenesis and the clinical course of IBD. In evolutionary terms, in fact, people in industrialized countries can be described as nutritionally mismatched and more susceptible - to degrees depending on their genetic background - the many non-communicable diet-related diseases, including IBD.

We now know the mechanisms of action whereby certain food groups may have a negative impact on the microbiota, triggering intestinal inflammation or intestinal permeability. We also know that the Western population tends to eat excessive amounts of "dangerous" foodstuffs. As mentioned above, the reason why studies have failed to provide strong evidence of dietary efficacy could be due to individual variability as well the small patient series studied. However, as one of the factors involved in the onset and progression of IBD, diet requires further in-depth study, especially since IBD patients tend to have disordered and unbalanced eating patterns. Although guidelines on IBD prevention and treatment are starting to appear (Figure 4) [60], it must be remembered, however, that they are based more on logical assumptions and animal models than on evidence provided by broad controlled human clinical studies. 


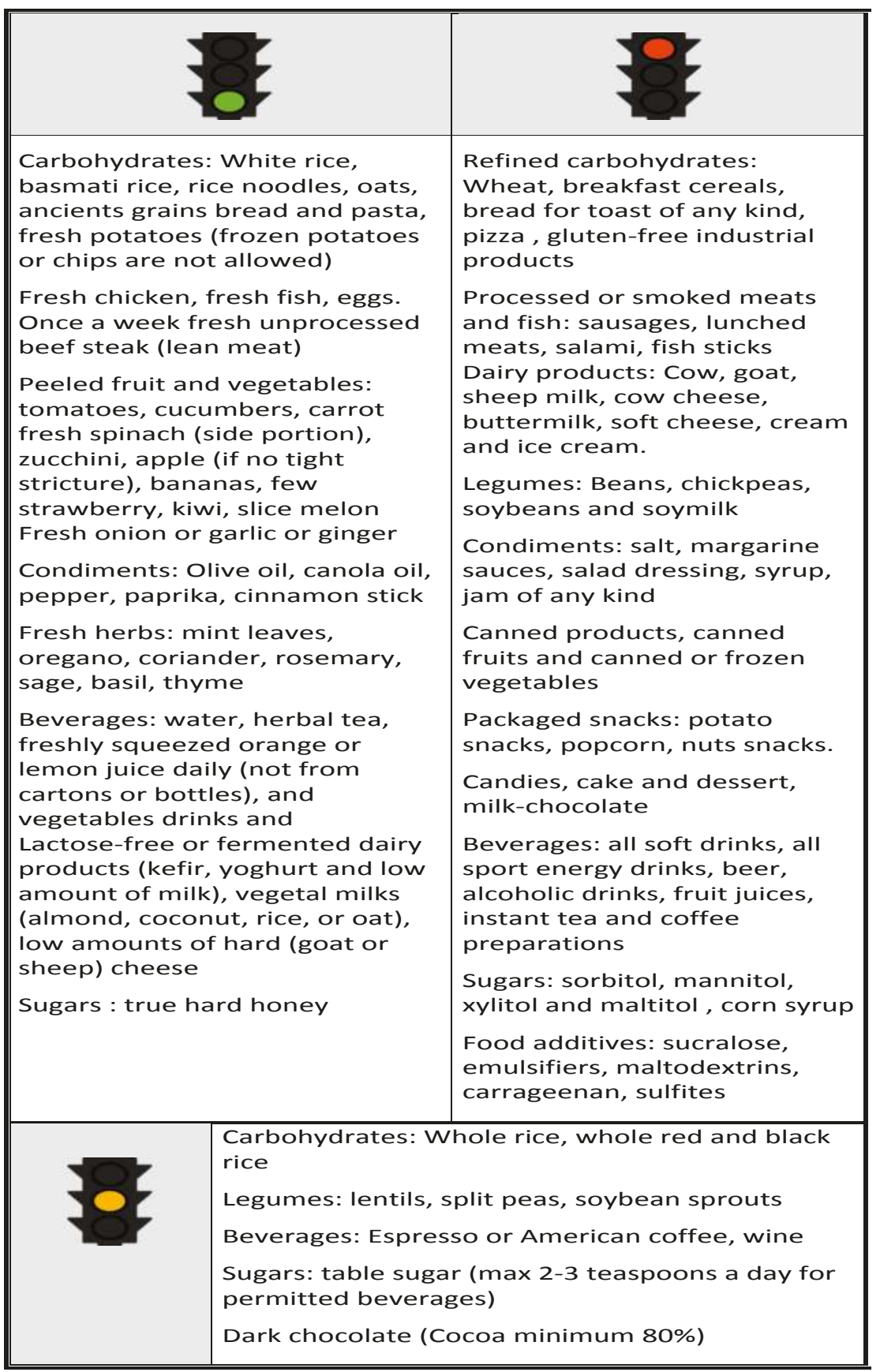

Figure 4. A possible diet for IBD: dietary foods and factors potentially affecting positively or negatively host intestinal barrier, immunity and microbioma in IBD patients. 
Author Contributions: Writing-original draft preparation, F.R., E.S., M.C.V. and P.G.; writing-review and editing, E.S., M.C.V., V.I., E.G., M.S. and P.A.

Funding: This research received no external funding.

Conflicts of Interest: The authors declare no conflict of interest.

\section{References}

1. Leso, V.; Ricciardi, W.; Iavicoli, I. Occupational risk factors in inflammatory bowel disease. Eur. Rev. Med. Pharmacol. Sci. 2015, 19, 2838-2851.

2. Loftus, E.V., Jr. Clinical epidemiology of inflammatory bowel disease: Incidence, prevalence, and environmental influences. Gastroenterology 2004, 126, 1504-1517. [CrossRef] [PubMed]

3. Benchimol, E.I.; Bernstein, C.N.; Bitton, A.; Carroll, M.W.; Singh, H.; Otley, A.R.; Vutcovici, M.; El-Matary, W.; Nguyen, G.C.; Griffiths, A.M.; et al. Trends in epidemiology of pediatric inflammatory bowel disease in Canada: Distributed network analysis of multiple population-based provincial health administrative databases. Am. J. Gastroenterol. 2017, 112, 1120-1134. [CrossRef] [PubMed]

4. Molodecky, N.A.; Panaccione, R.; Ghosh, S.; Barkema, H.W.; Kaplan, G.G. Alberta Inflammatory Bowel Disease Consortium. Challenges associated with identifying the environmental determinants of the inflammatory bowel diseases. Inflamm. Bowel Dis. 2011, 17, 1792-1799. [CrossRef]

5. Ng, S.C.; Bernstein, C.N.; Vatn, M.H.; Lakatos, P.L.; Loftus, E.V., Jr.; Tysk, C.; O’Morain, C.; Moum, B.; Colombel, J.F. Epidemiology and Natural History Task Force of the International Organization of Inflammatory Bowel Disease (IOIBD). Geographical variability and environmental risk factors in inflammatory bowel disease. Gut 2013, 62, 630-649. [CrossRef] [PubMed]

6. Hou, J.K.; Abraham, B.; El-Serag, H. Dietary intake and risk of developing inflammatory bowel disease: A systematic review of the literature. Am. J. Gastroenterol. 2011, 106, 563-573. [CrossRef]

7. Levine, A.; Sigall Boneh, R.; Wine, E. Evolving role of diet in the pathogenesis and treatment of inflammatory bowel diseases. Gut 2018, 67, 1726-1738. [CrossRef] [PubMed]

8. Zuo, T.; Kamm, M.A.; Colombel, J.F.; Ng, S.C. Urbanization and the gut microbiota in health and inflammatory bowel disease. Nat. Rev. Gastroenterol. Hepatol. 2018, 15, 440-452. [CrossRef]

9. David, L.A.; Maurice, C.F.; Carmody, R.N.; Gootenberg, D.B.; Button, J.E.; Wolfe, B.E.; Ling, A.V.; Devlin, A.S.; Varma, Y.; Fischbach, M.A.; et al. Diet rapidly and reproducibly alters the human gut microbiome. Nature 2014, 505, 559-563. [CrossRef]

10. Dunn, K.A.; Moore-Connors, J.; MacIntyre, B.; Stadnyk, A.; Thomas, N.A.; Noble, A.; Mahdi, G.; Rashid, M.; Otley, A.R.; Bielawski, J.P.; et al. The Gut microbiome of pediatric Crohn's disease patients differs from healthy controls in genes that can influence the balance between a healthy and dysregulated immune response. Inflamm. Bowel Dis. 2016, 22, 2607-2618. [CrossRef] [PubMed]

11. Martínez Steele, E.; Baraldi, L.G.; Louzada, M.L.; Moubarac, J.C.; Mozaffarian, D.; Monteiro, C.A. Ultra-processed foods and added sugars in the US diet: Evidence from a nationally representative cross-sectional study. BMJ. 2016, 6, e009892. [CrossRef] [PubMed]

12. Rajilić-Stojanović, M.; De Vos, W.M. The first 1000 cultured species of the human gastrointestinal microbiota. FEMS Microbiol. Rev. 2014, 38, 996-1047. [CrossRef]

13. Human Microbiome Project Consortium. Structure, function and diversity of the healthy human microbiome. Nature 2012, 486, 207-214. [CrossRef] [PubMed]

14. Aleksandrova, K.; Romero-Mosquera, B.; Hernandez, V. Diet, Gut microbiome and epigenetics: Emerging links with inflammatory bowel diseases and prospects for management and prevention. Nutrients 2017, 9 , E962. [CrossRef] [PubMed]

15. Dinan, T.G.; Cryan, J.F. The microbiome-gut-brain axis in health and disease. Gastroenterol. Clin. North Am. 2017, 46, 77-89. [CrossRef]

16. Mukhtar, K.; Nawaz, H.; Abid, S. Functional gastrointestinal disorders and gut-brain axis: What does the future hold? Wolrd J. Gastroenterol. 2019, 25, 552-566. [CrossRef] [PubMed]

17. Goehler, L.E.; Park, S.M.; Opitz, N.; Lyte, M.; Gaykema, R.P. Campylobacter jejuni infection increases anxiety-like behavior in the holeboard: Possible anatomical substrates for viscerosensory modulation of exploratory behavior. Brain Behav. Immun. 2008, 22, 354-366. [CrossRef] 
18. Agostini, A.; Ballotta, D.; Righi, S.; Moretti, M.; Bertani, A.; Scarcelli, A.; Sartini, A.; Ercolani, M.; Nichelli, P.; Campieri, M.; et al. Stress and brain functional changes in patients with Crohn's disease: A functional magnetic resonance imaging study. Neurogastroenterol. Motil. 2017, 29, 1-10. [CrossRef]

19. Frolkis, A.; Dieleman, L.A.; Barkema, H.W.; Panaccione, R.; Ghosh, S.; Fedorak, R.N.; Madsen, K.; Kaplan, G.G.; Alberta IBD Consortium. Environment and the inflammatory bowel diseases. Can. J. Gastroenterol. 2013, 27, e18-e24. [CrossRef]

20. Viggiano, D.; Ianiro, G.; Vanella, G.; Bibbò, S.; Bruno, G.; Simeone, G.; Mele, G. Gut barrier in health and disease: Focus on childhood. Eur. Rev. Med. Pharmacol. Sci. 2015, 19, 1077-1085.

21. Lopetuso, L.R.; Scaldaferri, F.; Bruno, G.; Petito, V.; Franceschi, F.; Gasbarrini, A. The therapeutic management of gut barrier leaking: The emerging role for mucosal barrier protectors. Eur. Rev. Med. Pharmacol. Sci. 2015, 19, 1068-1076.

22. Carrière, J.; Darfeuille-Michaud, A.; Nguyen, H.T. Infectious etiopathogenesis of Crohn's disease. World J. Gastroenterol 2014, 20, 12102-12117. [CrossRef]

23. Kostic, A.D.; Xavier, R.J.; Gevers, D. The microbiome in inflammatory bowel diseases: Current status and the future ahead. Gastroenterology 2014, 146, 1489-1499. [CrossRef] [PubMed]

24. De Souza, H.S.; Fiocchi, C. Immunopathogenesis of IBD: Current state of the art. Nat. Rev. Gastroenterol Hepatol. 2016, 13, 13-27. [CrossRef]

25. Koh, A.; De Vadder, F.; Kovatcheva-Datchary, P.; Bäckhed, F. From Dietary Fiber to Host Physiology: Short-Chain Fatty Acids as Key Bacterial Metabolites. Cell 2016, 165, 1332-1345. [CrossRef]

26. Macfarlane, G.T.; Macfarlane, S. Bacteria, colonic fermentation, and gastrointestinal health. J. AOAC Int. 2012, 95, 50-60. [CrossRef] [PubMed]

27. Tilg, H.; Moschen, A. Microbiota and diabetes: An evolving relationship. Gut 2014, 63, 1513-1521. [CrossRef]

28. Nastasi, C.; Candela, M.; Bonefeld, C.M.; Geisler, C.; Hansen, M.; Krejsgaard, T.; Biagi, E.; Andersen, M.H.; Brigidi, P.; Ødum, N.; et al. The effect of short-chain fatty acids on human monocyte-derived dendritic cells. Sci. Rep. 2015, 5, 16148. [CrossRef]

29. Wilck, N.; Matus, M.G.; Kearney, S.M.; Olesen, S.W.; Forslund, K.; Bartolomaeus, H.; Haase, S.; Mähler, A.; Balogh, A.; Markó, L.; et al. Salt-responsive gut commensal modulates TH17 axis and disease. Nat. Cell Boil. 2017, 551, 585-589. [CrossRef]

30. Ritchie, M.L.; Romanuk, T.N. A meta-analysis of probiotic efficacy for gastrointestinal diseases. PLOS ONE 2012, 7, e34938. [CrossRef] [PubMed]

31. Sánchez, B.; Delgado, S.; Blanco-Míguez, A.; Lourenço, A.; Gueimonde, M.; Margolles, A. Probiotics, gut microbiota, and their influence on host health and disease. Mol. Nutr. Food Res. 2017, 61. [CrossRef]

32. Abraham, B.P.; Quigley, E.M.M. Probiotics in inflammatory bowel disease. Gastroenterol. Clin. North Am. 2017, 46, 769-782. [CrossRef] [PubMed]

33. Liu, Q.; Shao, W.; Zhang, C.; Xu, C.; Wang, Q.; Liu, H.; Sun, H.; Jiang, Z.; Gu, A. Organochloride pesticides modulated gut microbiota and influenced bile acid metabolism in mice. Environ. Pollut. 2017, 226, 268-276. [CrossRef]

34. Mao, Q.; Manservisi, F.; Panzacchi, S.; Mandrioli, D.; Menghetti, I.; Vornoli, A.; Bua, L.; Falcioni, L.; Lesseur, C.; Chen, J.; et al. The Ramazzini Institute 13-week pilot study on glyphosate and Roundup administered at human-equivalent dose to Sprague Dawley rats: Effects on the microbiome. Environ. Health. 2018, 17, 50. [CrossRef] [PubMed]

35. Iszatt, N.; Janssen, S.; Lenters, V.; Dahl, C.; Stigum, H.; Knight, R.; Mandal, S.; Peddada, S.; González, A.; Midtvedt, T.; et al. Environmental toxicants in breast milk of Norwegian mothers and gut bacteria composition and metabolites in their infants at 1 month. Microbiome 2019, 7, 34. [CrossRef] [PubMed]

36. Cordain, L.; Eaton, S.B.; Sebastian, A.; Mann, N.; Lindeberg, S.; Watkins, B.A.; O'Keefe, J.H.; Brand-Miller, J. Origins and evolution of the Western diet: Health implications for the 21st century. Am. J. Clin. Nutr. 2005, 81, 341-354. [CrossRef]

37. Zeder, M.A.; Hesse, B. The initial domestication of goats (Capra hircus) in the Zagros mountains 10,000 years ago. Science 2000, 287, 2254-2257. [CrossRef] [PubMed]

38. Copley, M.S.; Berstan, R.; Dudd, S.N.; Docherty, G.; Mukherjee, A.J.; Straker, V.; Payne, S.; Evershed, R.P. Direct chemical evidence for widespread dairying in prehistoric Britain. Proc. Natl. Acad. Sci. USA 2003, 100, 1524-1529. [CrossRef]

39. Cleave, T.L. The Saccharine Disease; Stonebridge Press: Bristol, UK, 1974; pp. 6-27. 
40. Storck, J.; Teague, W.D. Flour for Man's Bread, a History of Milling; University of Minnesota Press: Minneapolis, MS, USA, 1952.

41. Galloway, P.J.; Thomson, G.A.; Fisher, B.M.; Semple, C.G. Insulin-induced hypoglycemia induces a rise in C-reactive protein. Diabetes Care. 2000, 23, 861-862. [CrossRef]

42. Food Availability (Per Capita) Data System (Table: Sugar and sweeteners). Available online: https: //www.ers.usda.gov/data-products/food-availability-per-capita-data-system/ (accessed on 6 May 2019).

43. Cordain, L.; Watkins, B.A.; Florant, G.L.; Kelher, M.; Rogers, L.L.; Li, Y. Fatty acid analysis of wild ruminant tissues: Evolutionary implications for reducing diet-related chronic disease. Eur. J. Clin. Nutr. 2002, 56, 181-191. [CrossRef]

44. Cordain, L. The nutritional characteristics of a contemporary diet based upon Paleolithic food groups. JANA 2002, 5, 15-24.

45. Stahl, W.R. Organ weights in primates and other mammals. Science 1965, 150, 1039-1042. [CrossRef] [PubMed]

46. Rule, D.C.; Broughton, K.S.; Shellito, S.M.; Maiorano, G. Comparison of muscle fatty acid profiles and cholesterol concentrations of bison, beef cattle, elk, and chicken. J. Anim. Sci. 2002, 80, 1202-1211. [CrossRef]

47. Zhang, M.; Yang, X.J. Effects of a high fat diet on intestinal microbiota and gastrointestinal diseases. World J. Gastroenterol 2016, 28, 8905-8909. [CrossRef]

48. Frassetto, L.; Morris, R.C., Jr.; Sellmeyer, D.E.; Todd, K.; Sebastian, A. Diet, evolution and aging-The pathophysiologic effects of the post-agricultural inversion of the potassium-to-sodium and base-to-chloride ratios in the human diet. Eur. J. Nutr. 2001, 40, 200-213. [CrossRef] [PubMed]

49. Cordain, L.; Gotshall, R.; Eaton, S.B.; Eaton, S.B., 3rd. Physical activity, energy expenditure and fitness: An evolutionary perspective. Int. J. Sports Med. 1998, 19, 328-335. [CrossRef]

50. Monteiro, C.A.; Cannon, G.; Levy, R.B.; Moubarac, J.C.; Louzada, M.L.; Rauber, F.; Khandpur, N.; Cediel, G.; Neri, D.; Martinez-Steele, E.; et al. Ultra-processed foods: What they are and how to identify them. Public. Health. Nutr. 2019, 22, 936-941. [CrossRef]

51. Kim, H.; Hu, E.A.; Rebholz, C.M. Ultra-processed food intake and mortality in the USA: Results from the Third National Health and Nutrition Examination Survey (NHANES III, 1988-1994). Public Health Nutr. 2019, 21, 1-9. [CrossRef]

52. Rai, S.K.; Fung, T.T.; Lu, N.; Keller, S.F.; Curhan, G.C.; Choi, H.K. The dietary approaches to stop hypertension (DASH) diet, Western diet, and risk of gout in men: Prospective cohort study. BMJ 2017, 357, j1794. [CrossRef]

53. Hariharan, D.; Vellanki, K.; Kramer, H. The Western diet and chronic kidney disease. Curr. Hypertens. Rep. 2015, 17, 16. [CrossRef]

54. Melaku, Y.A.; Renzaho, A.; Gill, T.K.; Taylor, A.W.; Dal Grande, E.; De Courten, B.; Baye, E.; Gonzalez-Chica, D.; Hyppönen, E.; Shi, Z.; et al. Burden and trend of diet-related non-communicable diseases in Australia and comparison with 34 OECD countries, 1990-2015: Findings from the Global Burden of Disease Study 2015. Eur. J. Nutr. 2018, 58, 1299-1313. [CrossRef]

55. Smith, P.M.; Howitt, M.R.; Panikov, N.; Michaud, M.; Gallini, C.A.; Bohlooly-Y, M.; Glickman, J.N.; Garrett, W.S. The microbial metabolites, short chain fatty acids, regulate colonic Treg cell homeostasis. Science 2013, 341, 569-573. [CrossRef]

56. Devkota, S.; Wang, Y.; Musch, M.W.; Leone, V.; Fehlner-Peach, H.; Nadimpalli, A.; Antonopoulos, D.A.; Jabri, B.; Chang, E.B. Dietary-fat-induced taurocholic acid promotes pathobiont expansion and colitis in Il10-/- mice. Nature 2012, 487, 104-108. [CrossRef]

57. Chassaing, B.; Koren, O.; Goodrich, J.K.; Poole, A.C.; Srinivasan, S.; Ley, R.E.; Gewirtz, A.T. Dietary emulsifiers impact the mouse gut microbiota promoting colitis and metabolic syndrome. Nature. Cell Biol. 2015, 519, 92-96. [CrossRef] [PubMed]

58. Zinöcker, M.K.; Lindseth, I.A. The Western diet-microbiome-host interaction and its role in metabolic disease. Nutrients 2018, 10, E365. [CrossRef] [PubMed]

59. Limdi, J.K. Dietary practices and inflammatory bowel disease. Indian. J. Gastroenterol. 2018, 37, $284-292$. [CrossRef]

60. Marion-Letellier, R.; Savoye, G.; Ghosh, S. IBD: In food we trust. J. Crohns Colitis 2016, 10, 1351-1361. [CrossRef] 
61. De Filippo, C.; Di Paola, M.; Ramazzotti, M.; Albanese, D.; Pieraccini, G.; Banci, E.; Miglietta, F.; Cavalieri, D.; Lionetti, P. Diet, Environments, and gut microbiota: A preliminary investigation in children living in rural and urban burkina faso and italy. Front. Microbiol. 2017, 8, 1979. [CrossRef]

62. Chiba, M.; Nakane, K.; Komatsu, M. Westernized diet is the most ubiquitous environmental factor in inflammatory bowel disease. Perm. J. 2019, 23, 18-107. [CrossRef] [PubMed]

63. Dixon, L.J.; Kabi, A.; Nickerson, K.P.; McDonald, C. Combinatorial effects of diet and genetics on inflammatory bowel disease pathogenesis. Inflamm. Bowel Dis. 2015, 21, 912-922. [CrossRef] [PubMed]

64. Gibson, P.R. Use of the low-FODMAP diet in inflammatory bowel disease. J. Gastroenterol. Hepatol. 2017, 32 , 40-42. [CrossRef]

65. Statovci, D.; Aguilera, M.; MacSharry, J.; Melgar, S. The Impact of Western diet and nutrients on the microbiota and immune response at mucosal interfaces. Front. Immunol. 2017, 8, 838. [CrossRef]

66. Hou, J.K.; Lee, D.; Lewis, J. Diet and inflammatory bowel disease: Review of patient-targeted recommendations. Clin. Gastroenterol Hepatol. 2014, 12, 1592-1600. [CrossRef]

67. Olendzki, B.C.; Silverstein, T.D.; Persuitte, G.M.; Ma, Y.; Baldwin, K.R.; Cave, D. An anti-inflammatory diet as treatment for inflammatory bowel disease: A case series report. Nutr. J. 2014, 13, 5. [CrossRef] [PubMed]

68. Britto, S.; Kellermayer, R. Carbohydrate monotony as protection and treatment for inflammatory bowel disease. J. Crohns Colitis 2019. [CrossRef]

69. Russell, W.R.; Gratz, S.W.; Duncan, S.H.; Holtrop, G.; Ince, J.; Scobbie, L.; Duncan, G.; Johnstone, A.M.; Lobley, G.E.; Wallace, R.J.; et al. High-protein, reduced-carbohydrate weight-loss diets promote metabolite profiles likely to be detrimental to colonic health. Am. J. Clin. Nutr. 2011, 93, 1062-1072. [CrossRef] [PubMed]

70. Vidal-Lletjós, S.; Beaumont, M.; Tomé, D.; Benamouzig, R.; Blachier, F.; Lan, A. Dietary protein and amino acid supplementation in inflammatory bowel disease course: What impact on the colonic mucosa? Nutrients 2017, 9, 310. [CrossRef]

71. Steck, N.; Hoffmann, M.; Sava, I.G.; Kim, S.C.; Hahne, H.; Tonkonogy, S.L.; Mair, K.; Krueger, D.; Pruteanu, M.; Shanahan, F.; et al. Enterococcus faecalis metalloprotease compromises epithelial barrier and contributes to intestinal inflammation. Gastroenterology 2011, 141, 959-971. [CrossRef]

72. Blachier, F.; Beaumont, M.; Andriamihaja, M.; Davila, A.-M.; Lan, A.; Grauso, M.; Armand, L.; Benamouzig, R.; Tomé, D. Changes in the luminal environment of the colonic epithelial cells and physiopathological consequences. Am. J. Pathol. 2017, 187, 476-486. [CrossRef]

73. Blachier, F.; Beaumont, M.; Kim, E. Cysteine-derived hydrogen sulfide and gut health: A matter of endogenous or bacterial origin. Curr. Opin. Clin. Nutr. Metab. Care 2019, 22, 68-75. [CrossRef]

74. Shoda, R.; Matsueda, K.; Yamato, S.; Umeda, N. Epidemiologic analysis of Crohn disease in Japan: Increased dietary intake of n-6 polyunsaturated fatty acids and animal protein relates to the increased incidence of Crohn disease in Japan. Am. J. Clin. Nutr. 1996, 63, 741-745. [CrossRef] [PubMed]

75. Jantchou, P.; Morois, S.; Clavel-Chapelon, F.; Boutron-Ruault, M.C.; Carbonnel, F. Animal protein intake and risk of inflammatory bowel disease: The E3N prospective study. Am. J. Gastroenterol. 2010, 105, 2195-2201. [CrossRef] [PubMed]

76. Ge, J.; Wang, D.; Fan, L. Effect of personalized nutrition guidance on the birth rate of fetal macrosomia in Chinese population: A meta-analysis of nine randomized controlled trials. Cell Biochem. Biophys. 2015, 72, 669-674. [CrossRef] [PubMed]

77. Opstelten, J.L.; De Vries, J.H.M.; Wools, A.; Siersema, P.D.; Oldenburg, B.; Witteman, B.J.M. Dietary intake of patients with inflammatory bowel disease: A comparison with individuals from a general population and associations with relapse. Clin. Nutr. 2018, S0261-5614, 31202-31203. [CrossRef] [PubMed]

78. Tasson, L.; Canova, C.; Vettorato, M.G.; Savarino, E.; Zanotti, R. Influence of diet on the course of inflammatory bowel disease. Dig. Dis. Sci. 2017, 62, 2087-2094. [CrossRef]

79. Wang, Z.W.; Ji, F.; Teng, W.J.; Yuan, X.G.; Ye, X.M. Risk factors and gene polymorphisms of inflammatory bowel disease in population of Zhejiang, China. World J. Gastroenterol. 2011, 17, 118-122. [CrossRef] [PubMed]

80. Limketkai, B.N.; Sepulveda, R.; Hing, T.; Shah, N.D.; Choe, M.; Limsui, D.; Shah, S. Prevalence and factors associated with gluten sensitivity in inflammatory bowel disease. Scand. J. Gastroenterol. 2018, 53, 147-151. [CrossRef] [PubMed] 
81. Bianca, D.; Ficco, M.; Prandi, B.; Amaretti, A.; Anfelli, I.; Leonardi, A.; Raimondi, S.; Pecchioni, N.; De Vita, P.; Faccini, A.; et al. Comparison of gluten peptides and potential prebiotic carbohydrates in old and modern Triticum turgidum ssp. genotypes. Food Res. Int. 2018, 120, 568-576.

82. Dinu, M.; Whittaker, A.; Pagliai, G.; Benedettelli, S.; Sofi, F. Ancient wheat species and human health: Biochemical and clinical implications. J. Nutr. Biochem. 2018, 52, 1-9. [CrossRef]

83. Vaquero, L.; Comino, I.; Vivas, S.; Rodríguez-Martín, L.; Giménez, M.J.; Pastor, J.; Sousa, C.; Barro, F. Tritordeum: A novel cereal for food processing with good acceptability and significant reduction in gluten immunogenic peptides in comparison with wheat. J. Sci. Food Agric. 2018, 98, 2201-2209. [CrossRef]

84. Reddavide, R.; Rotolo, O.; Caruso, M.G.; Stasi, E.; Notarnicola, M.; Miraglia, C.; Nouvenne, A.; Meschi, T.; De' Angelis, G.L.; Di Mario, F.; et al. The role of diet in the prevention and treatment of inflammatory bowel diseases. Acta Biomed. 2018, 89, 60-75. [PubMed]

85. Llewellyn, S.R.; Britton, G.J.; Contijoch, E.J.; Vennaro, O.H.; Mortha, A.; Colombel, J.F.; Grinspan, A.; Clemente, J.C.; Merad, M.; Faith, J.J. Interactions between diet and the intestinal microbiota alter intestinal permeability and colitis severity in mice. Gastroenterology 2018, 154, 1037-1046. [CrossRef]

86. Lim, H.-S.; Kim, S.-K.; Hong, S.-J. Food elimination diet and nutritional deficiency in patients with inflammatory bowel disease. Clin. Nutr. Res. 2018, 7, 48-55. [CrossRef]

87. Yılmaz, İ.; Dolar, M.E.; Özpınar, H. Effect of administering kefir on the changes in fecal microbiota and symptoms of inflammatory bowel disease: A randomized controlled trial. Turk. J. Gastroenterol. 2019, 30, 242-253. [CrossRef] [PubMed]

88. Shadnoush, M.; Hosseini, R.S.; Khalilnezhad, A.; Navai, L.; Goudarzi, H.; Vaezjalali, M. Effects of probiotics on gut microbiota in patients with inflammatory bowel disease: A double-blind, placebo-controlled clinical trial. Korean J. Gastroenterol. 2015, 65, 215. [CrossRef] [PubMed]

89. Ananthakrishnan, A.N.; Khalili, H.; Konijeti, G.G.; Higuchi, L.M.; de Silva, P.; Fuchs, C.S.; Willett, W.C.; Richter, J.M.; Chan, A.T. Long-term intake of dietary fat and risk of ulcerative colitis and Crohn's disease. Gut 2014, 63, 776-784. [CrossRef]

90. Andersen, V.; Chan, S.; Luben, R.; Khaw, K.T.; Olsen, A.; Tjonneland, A.; Kaaks, R.; Grip, O.; Bergmann, M.M.; Boeing, H.; et al. Fiber intake and the development of inflammatory bowel disease: A European prospective multi-centre cohort study (EPIC-IBD). J. Crohns Colitis 2018, 12, 129-136. [CrossRef] [PubMed]

91. Scaioli, E.; Sartini, A.; Bellanova, M.; Campieri, M.; Festi, D.; Bazzoli, F.; Belluzzi, A. Eicosapentaenoic acid reduces fecal levels of calprotectin and prevents relapse in patients with ulcerative colitis. Clin. Gastroenterol. Hepatol. 2018, 16, 1268-1275.e2. [CrossRef] [PubMed]

92. Calder, P.C. Marine omega-3 fatty acids and inflammatory processes: Effects, mechanisms and clinical relevance. Biochim. Biophys. Acta 2015, 1851, 469-484. [CrossRef]

93. Lee, T.H.; Hoover, R.L.; Williams, J.D.; Sperling, R.I.; Ravalese, J., 3rd; Spur, B.W.; Robinson, D.R.; Corey, E.J.; Lewis, R.A.; Austen, K.F. Effect of dietary enrichment with eicosapentaenoic and docosahexaenoic acids on in vitro neutrophil and monocyte leukotriene generation and neutrophil function. New Engl. J. Med. 1985, 312, 1217-1224. [CrossRef] [PubMed]

94. Serhan, C.N. Pro-resolving lipid mediators are leads for resolution physiology. Nature 2014, 510, 92-101. [CrossRef]

95. Scaioli, E.; Liverani, E.; Belluzzi, A. The imbalance between n-6/n-3 polyunsaturated fatty acids and inflammatory bowel disease: A comprehensive review and future therapeutic perspectives. Int. J. Mol. Sci. 2017, 18, 2619. [CrossRef] [PubMed]

96. Wiese, D.M.; Horst, S.N.; Brown, C.T.; Allaman, M.M.; Hodges, M.E.; Slaughter, J.C.; Druce, J.P.; Beaulieu, D.B.; Schwartz, D.A.; Wilson, K.T.; et al. Serum fatty acids are correlated with inflammatory cytokines in ulcerative colitis. PLOS ONE 2016, 11, e0156387. [CrossRef]

97. Scoville, E.A.; Allaman, M.M.; Adams, D.W.; Motley, A.K.; Peyton, S.C.; Ferguson, S.L.; Horst, S.N.; Williams, C.S.; Beaulieu, D.B.; Schwartz, D.A.; et al. Serum polyunsaturated fatty acids correlate with serum cytokines and clinical disease activity in Crohn's disease. Sci. Rep. 2019, 9, 2882. [CrossRef] [PubMed]

98. Benjamin, J.L.; Hedin, C.R.H.; Koutsoumpas, A.; Ng, S.C.; McCarthy, N.E.; Hart, A.L.; Kamm, M.A.; Sanderson, J.D.; Knight, S.C.; Forbes, A.; et al. Randomised, double-blind, placebo-controlled trial of fructo-oligosaccharides in active Crohn's disease. Gut 2011, 60, 923-929. [CrossRef]

99. Eswaran, S.; Muir, J.; Chey, W.D. Fiber and functional gastrointestinal disorders. Am. J. Gastroenterol. 2013, 108, 718-727. [CrossRef] 
100. Sugihara, K.; Morhardt, T.L.; Kamada, N. The role of dietary nutrients in inflammatory bowel disease. Front. Immunol. 2019, 9, 3183. [CrossRef] [PubMed]

101. Boeing, H.; Bechthold, A.; Bub, A.; Ellinger, S.; Haller, D.; Kroke, A.; Leschik-Bonnet, E.; Müller, M.J.; Oberritter, H.; Schulze, M.B.; et al. Critical review: Vegetables and fruit in the prevention of chronic diseases. Eur. J. Nutr. 2012, 51, 637-663. [CrossRef]

102. Taylor, L.; Almutairdi, A.; Shommu, N.; Fedorak, R.; Ghosh, S.; Reimer, R.A.; Panaccione, R.; Raman, M. Cross-sectional analysis of overall dietary intake and Mediterranean dietary pattern in patients with Crohn's disease. Nutrients 2018, 10, 1761. [CrossRef] [PubMed]

103. Malavia, D.; Crawford, A.; Wilson, D. Nutritional immunity and fungal pathogenesis: The struggle for micronutrients at the host-pathogen interface. Adv. Microb. Physiol. 2017, 70, 85-103.

104. Soares-Mota, M.; Silva, T.A.; Gomes, L.M.; Pinto, M.A.; Mc Mendonça, L.; Farias, M.L.F.; Nunes, T.; Ramalho, A.; Zaltman, C. High prevalence of vitamin A deficiency in Crohn's disease patients according to serum retinol levels and the relative dose-response test. World J. Gastroenterol. 2015, 21, 1614-1620. [CrossRef] [PubMed]

105. Ardesia, M.; Ferlazzo, G.; Fries, W. Vitamin D and inflammatory bowel disease. BioMed Res. Int. 2015, 2015, 1-16. [CrossRef]

106. Ooi, J.H.; Li, Y.; Rogers, C.J.; Cantorna, M.T. Vitamin D regulates the gut microbiome and protects mice from dextran sodium sulfate-induced colitis. J. Nutr. 2013, 143, 1679-1686. [CrossRef] [PubMed]

107. Ko, K.H.; Kim, Y.S.; Lee, B.K.; Choi, J.H.; Woo, Y.M.; Kim, J.Y.; Moon, J.S. Vitamin D deficiency is associated with disease activity in patients with Crohn's disease. Intest. Res. 2018, 17, 70-77. [CrossRef] [PubMed]

108. Jørgensen, S.P.; Agnholt, J.; Glerup, H.; Lyhne, S.; Villadsen, G.E.; Hvas, C.L.; Bartels, L.E.; Kelsen, J.; Christensen, L.A.; Dahlerup, J.F. Clinical trial: Vitamin D3 treatment in Crohn's disease - a randomized double-blind placebo-controlled study. Aliment. Pharmacol. Ther. 2010, 32, 377-383. [CrossRef] [PubMed]

109. Dadaei, T.; Safapoor, M.H.; Asadzadeh Aghdaei, H.A.; Balaii, H.; Pourhoseingholi, M.A.; Naderi, N.; Zojaji, H.; Azimzadeh, P.; Mohammadi, P.; Zali, M.R. Effect of vitamin D3 supplementation on TNF- $\alpha$ serum level and disease activity index in Iranian IBD patients. Gastroenterol. Hepatol. Bed Bench 2015, 8, 49-55. [PubMed]

110. Pan, Y.; Liu, Y.; Guo, H.; Jabir, M.S.; Liu, X.; Cui, W.; Li, D. Associations between folate and vitamin B12 levels and inflammatory bowel disease: A meta-analysis. Nutrients 2017, 9, E382. [CrossRef] [PubMed]

111. Madanchi, M.; Fagagnini, S.; Fournier, N.; Biedermann, L.; Zeitz, J.; Battegay, E.; Zimmerli, L.; Vavricka, S.R.; Rogler, G.; Scharl, M.; et al. The relevance of vitamin and iron deficiency in patients with inflammatory bowel diseases in patients of the swiss IBD cohort. Inflamm. Bowel Dis. 2018, 24, 1768-1779. [CrossRef]

112. Goldsmith, J.R.; Sartor, R.B. The role of diet on intestinal microbiota metabolism: Downstream impacts on host immune function and health, and therapeutic implications. J. Gastroenterol. 2014, 49, 785-798. [CrossRef]

113. Kakodkar, S.; Mutlu, E.A. Diet as a therapeutic option for adult inflammatory bowel disease. Gastroenterol. Clin. North Am. 2017, 46, 745-767. [CrossRef]

114. Stoffaneller, R.; Morse, N.L. A review of dietary selenium intake and selenium status in Europe and the Middle East. Nutrients 2015, 7, 1494-1537. [CrossRef]

115. Kudva, A.K.; Shay, A.E.; Prabhu, K.S. Selenium and inflammatory bowel disease. Am. J. Physiol. Gastrointest. Liver Physiol. 2015, 309, G71-G77. [CrossRef]

116. Reimund, J.-M.; Hirth, C.; Koehl, C.; Baumann, R.; Duclos, B. Antioxidant and immune status in active Crohn's disease. A possible relationship. Clin. Nutr. 2000, 19, 43-48. [CrossRef]

117. Han, Y.M.; Yoon, H.; Lim, S.; Sung, M.-K.; Shin, C.M.; Park, Y.S.; Kim, N.; Lee, D.H.; Kim, J.S. Risk factors for vitamin D, zinc, and selenium deficiencies in Korean patients with inflammatory bowel disease. Gut Liver 2017, 11, 363-369. [CrossRef]

118. Costa, C.S.; Rauber, F.; Leffa, P.S.; Sangalli, C.N.; Campagnolo, P.D.B.; Vitolo, M.R. Ultra-processed food consumption and its effects on anthropometric and glucose profile: A longitudinal study during childhood. Nutr. Metab. Cardiovasc. Dis. 2019, 29, 177-184. [CrossRef]

119. Fiolet, T.; Srour, B.; Sellem, L.; Kesse-Guyot, E.; Allès, B.; Méjean, C.; Deschasaux, M.; Fassier, P.; Latino-Martel, P.; Beslay, M.; et al. Consumption of ultra-processed foods and cancer risk: Results from NutriNet-Santé prospective cohort. BMJ 2018, 360, k322. [CrossRef] [PubMed] 
120. Aguayo-Patrón, S.V.; Calderón de la Barca, A.M. Old Fashioned vs. Ultra-Processed-Based current diets: Possible Implication in the increased susceptibility to type 1 diabetes and celiac disease in childhood. Foods 2017, 6, E100. [CrossRef]

121. Rother, K.I.; Conway, E.M.; Sylvetsky, A.C. How non-nutritive sweeteners influence hormones and health Trends. Endocrinol. Metab. 2018, 29, 455-467. [CrossRef]

122. Uebanso, T.; Ohnishi, A.; Kitayama, R.; Yoshimoto, A.; Nakahashi, M.; Shimohata, T.; Mawatari, K.; Takahashi, A. Effects of low-dose non-caloric sweetener consumption on gut microbiota in mice. Nutrients 2017, 9, 560. [CrossRef]

123. Chassaing, B.; Van De Wiele, T.; De Bodt, J.; Marzorati, M.; Gewirtz, A.T. Dietary emulsifiers directly alter human microbiota composition and gene expression ex vivo potentiating intestinal inflammation. Gut 2017, 66, 1414-1427. [CrossRef]

124. Baer, D.J.; Stote, K.S.; Henderson, T.; Paul, D.R.; Okuma, K.; Tagami, H.; Kanahori, S.; Gordon, D.T.; Rumpler, W.V.; Ukhanova, M.; et al. The metabolizable energy of dietary resistant maltodextrin is variable and alters fecal microbiota composition in adult men. J. Nutr. 2014, 144, 1023-1029. [CrossRef]

125. Laudisi, F.; Di Fusco, D.; DiNallo, V.; Stolfi, C.; Di Grazia, A.; Marafini, I.; Colantoni, A.; Ortenzi, A.; Alteri, C.; Guerrieri, F.; et al. The food additive maltodextrin promotes endoplasmic reticulum stress-driven mucus depletion and exacerbates intestinal inflammation. Cell. Mol. Gastroenterol. Hepatol. 2019, 7, 457-473. [CrossRef] [PubMed]

126. Martino, J.V.; Van Limbergen, J.; Cahill, L.E. The role of carrageenan and carboxymethylcellulose in the development of intestinal inflammation. Front. Pediatr. 2017, 5, 96. [CrossRef]

127. Irwin, S.V.; Fisher, P.; Graham, E.; Malek, A.; Robidoux, A. Sulfites inhibit the growth of four species of beneficial gut bacteria at concentrations regarded as safe for food. PLOS ONE 2017, 12, e0186629. [CrossRef]

128. Lewis, J.D.; Abreu, M.T. Diet as a trigger or therapy for inflammatory bowel diseases. Gastroenterology 2017, 152, 398-414.e6. [CrossRef]

129. Bode, C.; Bode, J.C. Effect of alcohol consumption on the gut. Best Pr. Clin. Gastroenterol. 2003, 17, 575-592. [CrossRef]

130. Salaspuro, M. Bacteriocolonic pathway for ethanol oxidation: Characteristics and implications. Ann. Med. 1996, 28, 195-200. [CrossRef] [PubMed]

131. Mantzouranis, G.; Fafliora, E.; Saridi, M.; Tatsioni, A.; Glanztounis, G.; Albani, E.; Katsanos, K.H.; Christodoulou, D.K. Alcohol and narcotics use in inflammatory bowel disease. Ann. Gastroenterol. 2018, 31, 649-658. [CrossRef]

132. Swanson, G.R.; Sedghi, S.; Farhadi, A.; Keshavarzian, A. Pattern of alcohol consumption and its effect on gastrointestinal symptoms in inflammatory bowel disease. Alcohol 2010, 44, 223-228. [CrossRef]

133. Bergmann, M.M.; Hernandez, V.; Bernigau, W.; Boeing, H.; Chan, S.S.; Luben, R.; Khaw, K.T.; Van Schaik, F.; Oldenburg, B.; Bueno-De-Mesquita, B.; et al. No association of alcohol use and the risk of ulcerative colitis or Crohn's disease: Data from a European Prospective cohort study (EPIC). Eur. J. Clin. Nutr. 2017, 71, 512-518. [CrossRef]

134. Magee, E.A.; Edmond, L.M.; Tasker, S.M.; Kong, S.C.; Curno, R.; Cummings, J.H. Associations between diet and disease activity in ulcerative colitis patients using a novel method of data analysis. Nutr. J. 2005, 4, 7. [CrossRef] [PubMed]

135. Jowett, S.L.; Seal, C.J.; Pearce, M.S.; Phillips, E.; Gregory, W.; Barton, J.R.; Welfare, M.R. Influence of dietary factors on the clinical course of ulcerative colitis: A prospective cohort study. Gut 2004, 53, 1479-1484. [CrossRef] [PubMed]

136. Zachos, M.; Tondeur, M.; Griffiths, A.M. Enteral nutritional therapy for induction of remission in Crohn's disease. Cochrane Database Syst. Rev. 2007, CD000542. [CrossRef]

137. Gearry, R.B.; Irving, P.M.; Barrett, J.S.; Nathan, D.M.; Shepherd, S.J.; Gibson, P.R. Reduction of dietary poorly absorbed short-chain carbohydrates (FODMAPs) improves abdominal symptoms in patients with inflammatory bowel disease-a pilot study. J. Crohns Colitis 2009, 3, 8-14. [CrossRef]

138. Cox, S.R.; Prince, A.C.; Myers, C.E.; Irving, P.M.; Lindsay, J.O.; Lomer, M.C.; Whelan, K. Fermentable carbohydrates [FODMAPs] exacerbate functional gastrointestinal symptoms in patients with inflammatory bowel disease: A randomised, double-blind, placebo-controlled, cross-over, re-challenge trial. J. Crohns Colitis 2017, 11, 1420-1429. [CrossRef] 
139. Chiba, M.; Abe, T.; Tsuda, H.; Sugawara, T.; Tsuda, S.; Tozawa, H.; Fujiwara, K.; Imai, H. Lifestyle-related disease in Crohn's disease: Relapse prevention by a semi-vegetarian diet. World J. Gastroenterol. 2010, 16, 2484-2495. [CrossRef] [PubMed]

140. Chiba, M.; Nakane, K.; Tsuji, T.; Tsuda, S.; Ishii, H.; Ohno, H.; Watanabe, K.; Ito, M.; Komatsu, M.; Yamada, K.; et al. Relapse prevention in ulcerative colitis by plant-based diet through educational hospitalization: A single-group trial. Perm. J. 2018, 22, 17-167. [CrossRef]

141. Rajendran, N.; Kumar, D. Food-specific IgG4-guided exclusion diets improve symptoms in Crohn's disease: A pilot study. Colorectal Dis. 2011, 13, 1009-1013. [CrossRef]

142. Gunasekeera, V.; Mendall, M.A.; Chan, D.; Kumar, D. treatment of Crohn's disease with an IgG4-guided exclusion diet: A randomized controlled trial. Dig. Dis. Sci. 2016, 61, 1148-1157. [CrossRef]

143. Obih, C.; Wahbeh, G.; Lee, D.; Braly, K.; Giefer, M.; Shaffer, M.L.; Nielson, H.; Suskind, D.L. Specific carbohydrate diet for pediatric inflammatory bowel disease in clinical practice within an academic IBD center. Nutrition 2016, 32, 418-425. [CrossRef] [PubMed]

144. Kakodkar, S.; Farooqui, A.J.; Mikolaitis, S.L.; Mutlu, E.A. The specific carbohydrate diet for inflammatory bowel disease: A case series. J. Acad. Nutr. Diet. 2015, 115, 1226-1232. [CrossRef] [PubMed]

(C) 2019 by the authors. Licensee MDPI, Basel, Switzerland. This article is an open access article distributed under the terms and conditions of the Creative Commons Attribution (CC BY) license (http://creativecommons.org/licenses/by/4.0/). 


\title{
Influence of Vitamin D Deficiency on Inflammatory Markers and Clinical Disease Activity in IBD Patients
}

\author{
Pedro López-Muñoz ${ }^{1}$ (D) Belén Beltrán ${ }^{1,2,3, *}$, Esteban Sáez-González ${ }^{1,2}$, Amparo Alba ${ }^{4}$, \\ Pilar Nos ${ }^{1,2,3}$ and Marisa Iborra 1,2,3 \\ 1 IBD Unit, Department of Gastroenterology, Hospital Universitari i Politècnic la Fe, 46026 Valencia, Spain; \\ pedro.lopez8928@gmail.com (P.L.-M.); esteban.digestivo@gmail.com (E.S.-G.); nos_pil@gva.es (P.N.); \\ marisaiborra@hotmail.com (M.I.) \\ 2 IBD Research Group, Medical Research Institute Hospital la Fe (IIS La Fe), 46026 Valencia, Spain \\ 3 Networked Biomedical Research Center for Hepatic and Digestive Diseases (CIBEREHD), Institute of Health \\ Carlos III, 28029 Madrid, Spain \\ 4 Department of Clinical Analysis, Hospital Universitari i Politècnic la Fe, 46026 Valencia, Spain; \\ alba_amp@gva.es \\ * Correspondence: belenbeltranniclos@gmail.com; Tel./Fax: +34-961246257
}

Received: 31 March 2019; Accepted: 8 May 2019; Published: 11 May 2019

\begin{abstract}
Vitamin D has recently been discovered to be a potential immune modulator. Low serum vitamin D levels have been associated with risk of relapse and exacerbation of clinical outcomes in Crohn's disease (CD) and ulcerative colitis (UC). A retrospective, longitudinal study was conducted to determine the association between vitamin $\mathrm{D}$ levels and inflammatory markers and clinical disease activity in inflammatory bowel disease (IBD). In addition, circulating $25(\mathrm{OH}) \mathrm{D}_{3}$ progression was evaluated according to vitamin D supplementation. Participants were separated into three groups according to their vitamin D level: severe deficiency (SD), moderate deficiency (MD) and sufficiency (S). Serum $25(\mathrm{OH}) \mathrm{D}_{3}$ was inversely correlated with faecal calprotectin (FC) for $\mathrm{CD}$ and UC but was only correlated with C-reactive protein (CRP) for UC patients. In the multivariate analysis of FC, CRP and fibrinogen (FBG), we predicted the presence of a patient in the SD group with $80 \%$ accuracy. A deficiency of $25(\mathrm{OH}) \mathrm{D}_{3}$ was associated with increased hospitalisations, flare-ups, the use of steroids and escalating treatment. Supplemental doses of vitamin D were likely to be insufficient to reach adequate serum levels of $25(\mathrm{OH}) \mathrm{D}_{3}$. Vitamin $\mathrm{D}$ intervention studies are warranted to determine whether giving higher doses of vitamin D in IBD might reduce intestinal inflammation or disease activity.
\end{abstract}

Keywords: vitamin D; Crohn's disease; ulcerative colitis; faecal calprotectin; C-reactive protein

\section{Introduction}

Vitamin D is a lipophilic hormone synthesised in the skin under the influence of ultraviolet (UV) sunlight. Although most foods contain little vitamin D, it can be obtained from the diet to a lesser extent. The most important sources of this vitamin are fatty fish and egg yolk [1,2].

UV type B light exposure causes 7-dehydrocholesterol transformation into cholecalciferol or vitamin $\mathrm{D}_{3}$. However, this UV-mediated conversion varies with time of the year, latitude and altitude. During approximately six months of the year, UVB radiation intensity is insufficient for vitamin D synthesis at $45^{\circ}$ latitude near sea level (e.g., France or Italy). This period of time increases with the distance from the equator and is also known as vitamin D winter [3]. Along these lines, in countries where there is limited skin exposure to sunlight a dietary supply of vitamin D is important. Cholecalciferol is hydroxylated in the liver into 25-hydroxyvitamin D3 (25(OH)D3) and subsequently in the kidney into 1,25-dihydroxyvitamin D3 (1,25(OH)2D3), or calcitriol, the active metabolite [4] 
(Figure 1). These two steps are catalysed by the enzymes 25-hydroxylase (CYP2R1) and $1 \alpha$-hydroxylase (CYP27B1) that belong to the family of cytochrome P450 mixed function oxidases (CYPs). CYP2R1 has only been identified in the microsomal fraction of liver. However, although the kidney is the main source of $1,25(\mathrm{OH})_{2} \mathrm{D}_{3}$, several other tissues also express CYP27B1. The regulation of the renal CYP27B1 differs from that of the extrarenal version [5]. In this way, renal CYP27B1 is activated by the parathyroid hormone (PTH) and inhibited by fibroblast growth factor 23 (FGF23) as well as by $1,25(\mathrm{OH})_{2} \mathrm{D}_{3}$ itself. Elevated calcium suppresses CYP27B1 by means of the suppression of PTH, and elevated phosphate suppresses CYP27B1 by means of FGF23 stimulation, although these ions can have direct effects on renal CYP27B1 on their own [5]. Thus, it is known that CYP27B1 is expressed in numerous tissues where vitamin $\mathrm{D}_{3}$ can act as an intracrine or paracrine signal. In this way, several immune system cell types express both CYP27B1 and the vitamin D receptor, with CYP27B1 production controlled by a number of immune-specific inputs [3].

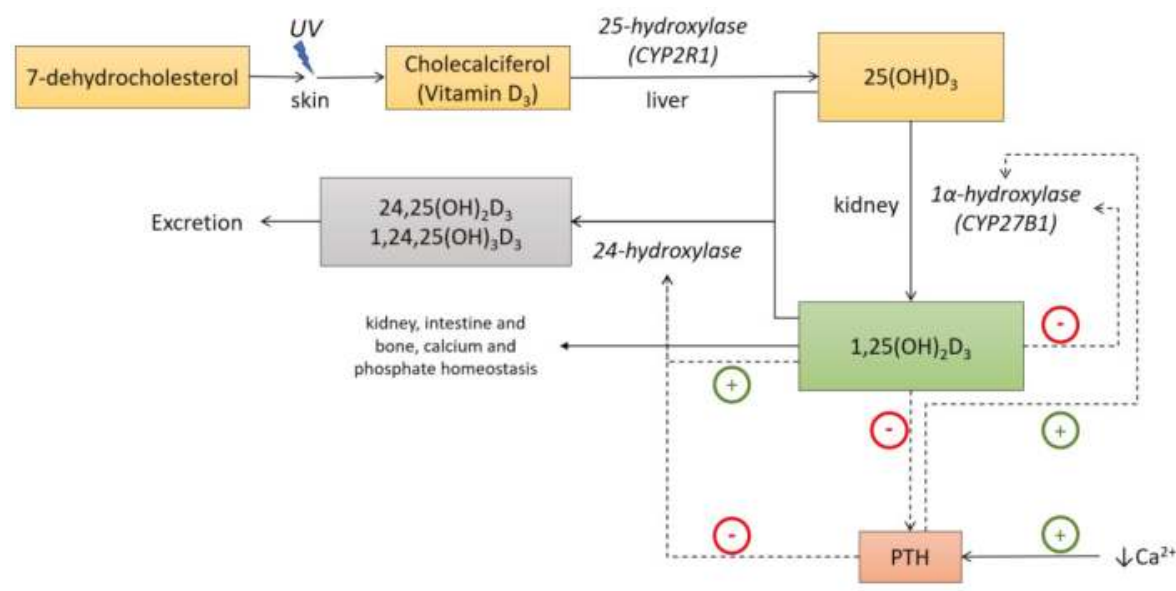

Figure 1. Vitamin D metabolism and functions. Under ultraviolet B light (UVB) exposure, 7-dehydroxycholesterol is converted to vitamin $\mathrm{D}_{3}$ in the skin. First, hydroxylation occurs in the liver where it is converted to 25-hydroxyvitamin $\mathrm{D}_{3}$. 25-hydroxyvitamin $\mathrm{D}_{3}$ is further converted in the kidney to its active metabolite, $1 \alpha, 25$-dihydroxyvitamin $\mathrm{D}_{3}$. In the kidney, $1 \alpha$-hydroxylase is stimulated by the parathyroid hormone (PTH) and feedback inhibited by $1 \alpha, 25$-dihydroxyvitamin $\mathrm{D}_{3}$. $1 \alpha, 25$-dihydroxyvitamin $\mathrm{D}_{3}$ targets the intestine, kidney and bone to regulate calcium and phosphate homeostasis. Adapted from Lim et al [6].

The half-life of $1,25(\mathrm{OH})_{2} \mathrm{D}_{3}$ is very short, ranging from 20 to 40 hours [7]; therefore, its serum determination is not clinically relevant. However, the $25(\mathrm{OH}) \mathrm{D}_{3}$ half-life ranges from 12 to 19 days [8], so it appears to be the most reliable source of systemic vitamin D. $1,25(\mathrm{OH})_{2} \mathrm{D}_{3}$ has its effect on the classic target organs such as bone, intestine and kidney and stimulates calcium transport from these organs to the peripheral blood stream [4].

Calcitriol can influence the expression of targets genes by binding to the vitamin D receptor (VDR), which is present in many cell populations and tissues, such as immune cells. Vitamin D promotes the intestinal absorption of calcium and phosphate and is critical for bone growth and remodelling. Now, however, it is well established that the physiological importance of vitamin D levels extends beyond its classical role in calcium and bone metabolism. Serum vitamin D levels have been associated with inflammatory diseases, such as inflammatory bowel disease (IBD), rheumatoid arthritis, systemic lupus erythematosus, multiple sclerosis, atherosclerosis, and asthma. Regarding inflammation, it has become clear that vitamin D inhibits production of proinflammatory cytokines like IL-6 or TNF $\alpha$ in monocytes via the inhibition of p38 MAP kinase [9], and it can promote anti-inflammatory T-cell pathways to stimulate the antimicrobial effects of macrophages [10]. 
In terms of gut homeostasis, vitamin D plays a role in protecting the intestinal epithelial barrier, immunity and microbiota, all of which are directly involved in IBD [11].

In-vitro 1,25(OH)2D3 treatment can protect the intestinal epithelial barrier in ulcerative colitis (UC) patients by regulating various pathways involved in tight junction proteins [12]

Similar studies have proposed that lack of VDR leads to hyperfunction of Claudin-2. Claudin-2 is an epithelial protein that forms a water channel to permit the paracellular passage of water through its pores in the epithelium, and its expression is restricted to colonic proliferative cells. An overexpression of Claudin-2 permits intestinal leakage, and is associated with active disease in IBD and reduction of intestinal VDR [13].

Other genetic changes in the VDR gene can contribute to a greater risk for CD. Patients with Crohn's disease (CD) who are homozygous for a certain single nucleotide polymorphism (SNP) in the VDR gene have lower levels of VDR protein expressed in serum monocytes. These individuals have an overactivation of lymphocytic adhesion molecules and a higher risk of developing a penetrating phenotype of CD [14].

Vitamin D has effects on both innate and adaptive immune pathways. 1,25(OH)2D3 modulates the expression of cationic antimicrobial peptides like defensins and cathelicidins. VDR signals through distal enhancers in the NOD2 gene, leading to the expression of defensin beta 2 in macrophages. However, this response was absent in macrophages from patients with CD homozygous for nonfunctional NOD2 variants [15]. It should be noted that NOD2 is also known as IBD1. This gene is located on chromosome 16 , in the susceptibility locus for CD, which is responsible for familial aggregation of this disease [16] It has also been reported that DNA copy number of the beta-defensin gene cluster is highly polymorphic within the healthy population, whereas $C D$ patients appear to present a lower copy number. This results in a diminished beta-defensin expression, predisposing patients to major susceptibility to colonic CD [17]. An induction of human cathelicidin antimicrobial peptide gene was observed under the influence of 1,25(OH)2D3 in colon cancer cells and other tissues [18,19]. Vitamin D is also involved in immune regulation by modulating the role of dendritic cells and macrophages through two methods: firstly, 1,25(OH)2D3 inhibits IL-12, a pivotal interleukin in Th1 development [20]; secondly, it promotes IL-10 to stop maturation from dendritic cells to serum monocytes and leads to apoptosis in mature dendritic cells [21]. Regarding adaptive immunity, 1,25(OH)2D3 affects T-cell polarisation by inhibiting T helper 1(Th) (IFN-gamma production) and promoting Th2 cell development (IL-4, IL-5, and IL-10 production) from naïve T-cells [22]. It has been demonstrated in controlled studies that higher vitamin D levels in UC patients were associated with protective anti-inflammatory serum cytokine profiles [23].

Gut microbiota have become the main research topic in intestinal homeostasis. One recent study demonstrated that individuals with significant intake of vitamin $D$ had different microbiota strains from those without a significant vitamin D intake. These patients had plenty of Bacteroidetes, Prevotella and Megasphera strains, traditionally associated with a noninflammatory status [24]. In another study, administration of vitamin D to CD patients resulted in significant change in the gut microbiota with a high abundance of some strains such as Alistipes and Roseburia that were not observed in healthy controls [25]. In murine induced colitis, Alistipes finegoldii played a protective role [26]. Roseburia genii are part of commensal bacteria that produce short-chain fatty acids, especially butyrate, affecting colonic motility, immunity maintenance and anti-inflammatory properties [27]. Therefore, administration of vitamin $\mathrm{D}$ might have a positive effect on $\mathrm{CD}$ by modulating the intestinal bacterial composition and also by increasing the abundance of potential beneficial bacterial strains [25].

Regarding the epidemiology of vitamin D deficiency in IBD, there is a systematic review and meta-analysis that defined this deficiency as serum 25(OH)D3 below $25 \mathrm{ng} / \mathrm{mL}$. In this study, the prevalence was $38.1 \%$ in CD and $31.6 \%$ in UC [28].

The latest evidence suggests that low vitamin D levels are involved in changing disease activity and inflammatory markers in IBD. However, these studies sometimes fail to confirm which comes first: are vitamin D levels an independent predictor of clinical activity or it is just a mere bystander of increased inflammation? 
Due to chronic malabsorption, IBD patients and particularly those with CD are at greater risk of certain nutritional deficiencies. Micronutrients, including hydro and lipophilic vitamins, iron, calcium and zinc, are the most common problems [29]. Malabsorption, maldigestion and upper protein requirements are directly related to clinical disease activity.

It is unknown why quiescent IBD has a greater prevalence of vitamin D deficiency than other risk groups [30]. Even in patients with IBD in clinical remission, vitamin D malabsorption has been confirmed. This fact led to controversy among researchers about oral intake as standard supplementation. According to several prospective studies, IBD patients with vitamin D deficiency are at greater risk of relapse, hospitalisations and flare-ups [2,31-35]. Schäffler et al. described a correlation between the use of a TNF-alpha inhibitor and higher vitamin D levels in CD patients. This correlation could be explained by a better disease control of these patients [34]. However, they detected a correlation between a higher disease activity and lower vitamin D levels in UC but not in $\mathrm{CD}$. Moreover, the same authors found that in CD located in the small intestine, CD patients after small intestine resections showed significant lower vitamin $D$ levels. Other resections did not lead to changes in the vitamin D levels; therefore, the small intestine plays an important role in vitamin D absorption, particularly in IBD [34]. Despite the immune-modulation role of vitamin D confirmed in translational studies, knowledge about its molecular action in IBD is still scarce. Exhaustive study of vitamin D immune pathways is imperative to identifying new supplementation strategies that correct this deficiency. It is also necessary to establish true sufficiency levels of vitamin D to have a goal for supplementation therapy.

As a result of these findings, our group posed a study to assess outcomes in IBD activity depending on vitamin D status. Thus, the association between vitamin D deficiency and inflammatory markers and clinical disease activity could be evaluated. We also hypothesised that oral supplementation according to practice guidelines was not enough to acquire vitamin D sufficiency.

\section{Materials and Methods}

\subsection{Study Design and Patient Enrolment}

We conducted a retrospective, longitudinal and observational study of patients with UC and CD, defined by European Crohn's and Colitis Organization (ECCO) guidelines criteria, which had serial determinations of vitamin D serum samples from the IBD Unit of Hospital Universitari i Politècnic la $\mathrm{Fe}$, a tertiary centre, between 2015 and 2018. Formal consent was collected from all the patients and an ethical committee, according to the declaration of Helsinki, approved the study.

A total of 84 patients divided into three groups according to the $25(\mathrm{OH}) \mathrm{D} 3(\mathrm{ng} / \mathrm{mL})$ level were enrolled in our study. The first vitamin D serum sample collected was used as a baseline to separate patients into groups and according to core event. A cut-off of $<15 \mathrm{ng} / \mathrm{mL}$ was considered severe deficiency (SD), $>15$ and $<30 \mathrm{ng} / \mathrm{mL}$ moderate deficiency (MD) and $>30 \mathrm{ng} / \mathrm{mL}$ sufficiency (S). Progression of vitamin D and supplementation from baseline was evaluated. Clinical disease activity (flare-ups, hospitalisations, times to visit IBD clinic, escalating treatment, use of steroids) in the six months before and the six months after the core event was monitored. In addition, inflammatory markers (CRP, FC and fibrinogen (FBG)) were collected to explore the inflammatory status around the vitamin D core event. Two determinations before and two determinations after to the core event were needed for patient inclusion (Figure 2). Two or more consecutive 25(OH)D3 determinations were also required. Other relevant descriptive variables: age, sex, IBD (EC or UC), extraintestinal manifestations, and current therapy (anti-TNF, immunomodulators, etc.) were considered. We sought active supplementation, establishing at least 25,000 IU (international units) of oral, subcutaneous or intramuscular cholecalciferol monthly as adequate supplementation. 


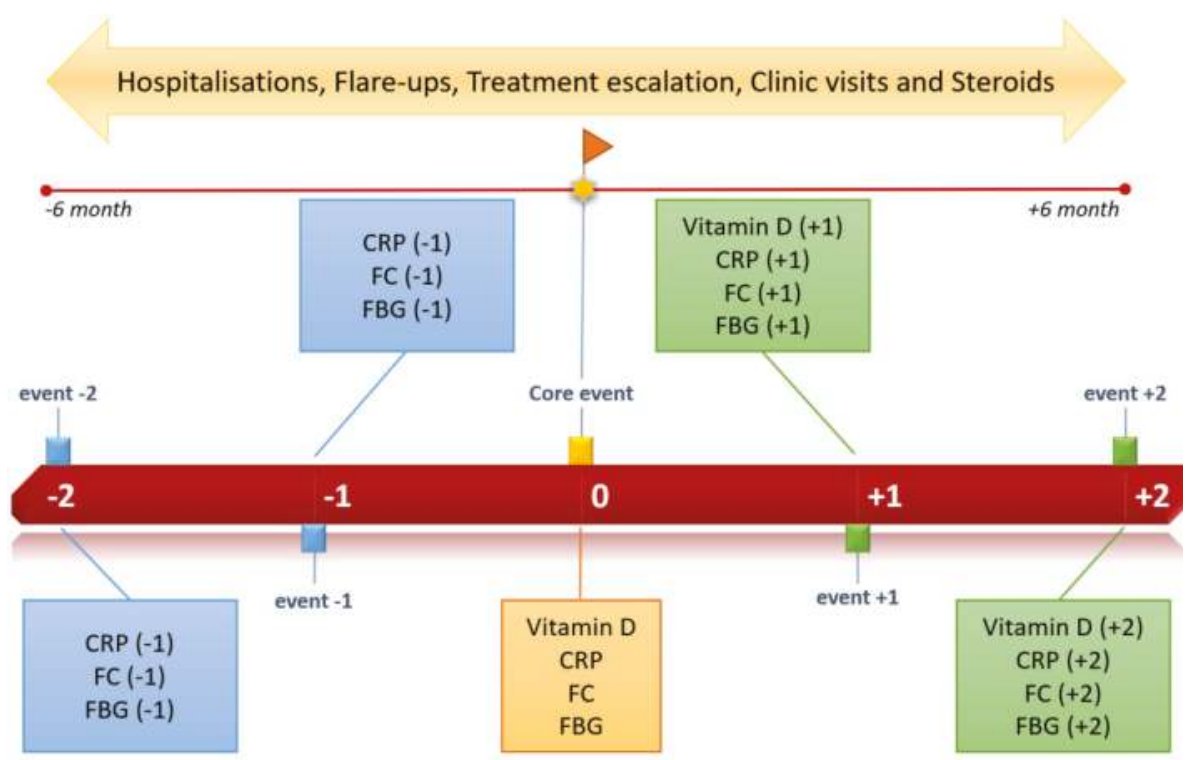

Figure 2. Study timeline.

Patients under 18 years, pregnant patients, those with a previous colectomy in UC, those who had CD surgery in the last three months, those with colorectal cancer or another tumour, those with a severe infection during the time of study and those with malabsorption disease were excluded from enrolment.

Disease activity and laboratory markers were collected from our software OrionClinic (Version 11). Suitable patients to enroll in this study were found by crossing vitamin D measurements from iGestlab software (Cointec iGestlab @ 2013. v1.9.9.815, Orihuela, Alicante, Spain) and IBD Unit clinical histories. Patients with several vitamin D samples, inflammatory markers and IBD diagnosis were filtered to assess enrolment.

Serum $25(\mathrm{OH}) \mathrm{D}_{3}$ levels $(\mathrm{ng} / \mathrm{mL})$ were measured by automated chemiluminescence analyser LIAISON ${ }^{\circledR}$ XL (DiaSorin, Sialuggia, Italy).

A total of 252 patients from IBD Unit and vitamin D measurements were evaluated to enroll. Ultimately, 41 patients with SD, 28 with MD and 16 with sufficient vitamin D levels met the inclusion criteria.

\subsection{Statistical Analysis}

An ANOVA test on ranks (Kruskal-Wallis test) was performed to compare inflammatory markers with the three different Vitamin D groups (severe deficiency, moderate deficiency and sufficiency). Moreover, a multiple comparison procedure was conducted to quantify the extent of the differences between the groups. The Pearson correlation test was applied to study calprotectin-CRP-fibrinogen correlations with vitamin D. The evolution of vitamin D, FC, CRP and FBG was evaluated by mixed linear regression models. A random effect factor was included to amend the nonindependence of the data (longitudinal study).

Given some patients were supplemented with vitamin D, a comparative study was done with this group and the nonsupplemented ones to analyse the evolution of this parameter. Additionally, the association between vitamin D levels and markers of clinical activity (hospitalisations, flare-ups, escalating treatment, number of times they visited the clinic and the use of steroids) was studied through logistic regression models. 
All models included the covariates sex, age and type of disease (CD or UC) as possible confounding factors. R statistic software (3.5.1 version, https://www.r-project.org/), Sigmaplot (SYSTAT, San José, CA, USA) and Minitab (State College, PA, USA) were used in this work. A significance level of $5 \%$ was selected. Multivariate discriminant analysis was performed with SIMCA-P (Umetrics, Umeå, Sweden).

\section{Results}

This study evaluated three concerns: Firstly, the correlation between vitamin D levels, expressed as $25(\mathrm{OH}) \mathrm{D} 3$, with both systemic and intestinal inflammation in IBD patients. Secondly, the association between vitamin D deficiency and clinical disease activity markers, collected in a period of time between six months before and after the randomly selected vitamin D core event. During this period, data on admissions, flare-ups, times the patient needed to visit the clinic, the use of steroids and the need for escalating treatment were collected. Thirdly, which patients benefitted from active supplementation was explored.

\subsection{Descriptive Statistics}

First, a normality study for the continuous quantitative variables (vitamin D, FC, CRP and FBG) was carried out. None of the inflammatory markers (FC, CRP, FBG) followed a normal distribution but their logarithmic transformation leads to data that were normally distributed (Figure 3). However, the vitamin D central values did follow a normal distribution.
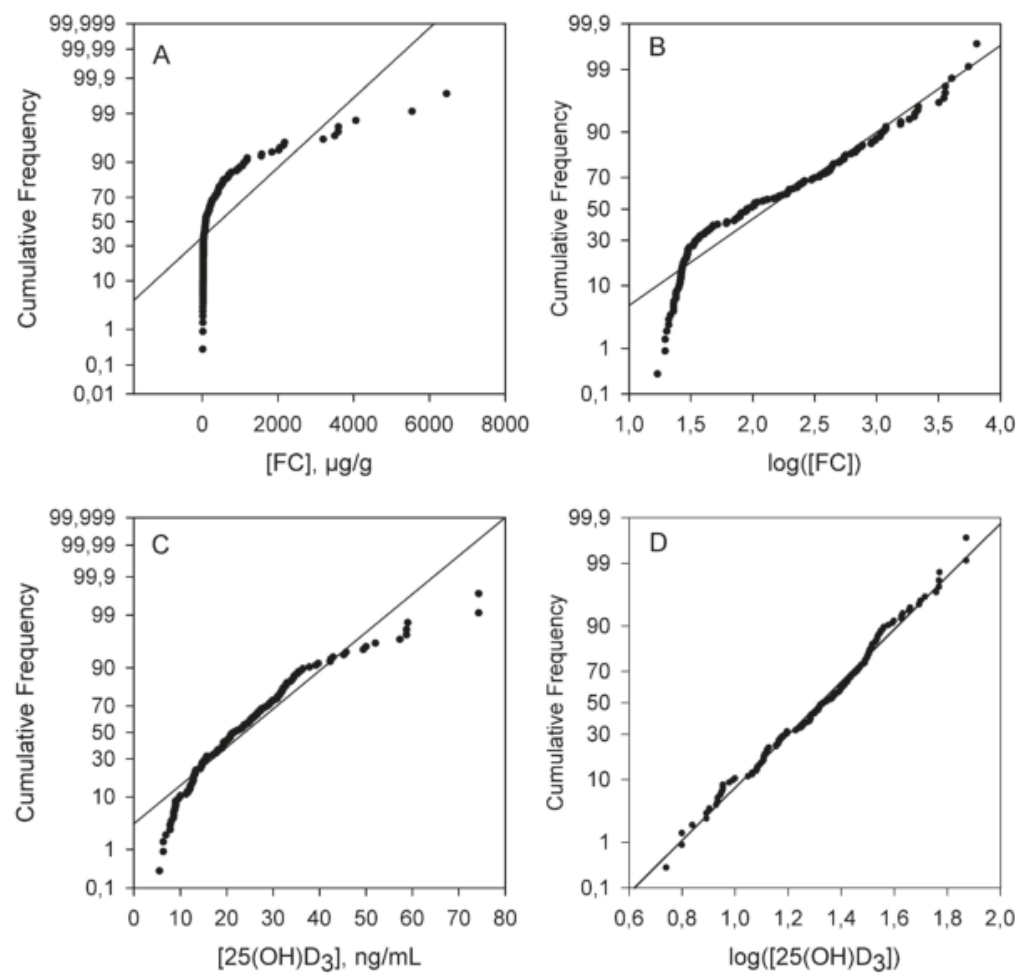

Figure 3. Normality study of FC (A,B) and vitamin D (C,D). Note the nonparametric distribution of FC.

Tables 1 and 2 show some of the statistical parameters obtained from the descriptive parametric and nonparametric study. The data have been grouped according to their vitamin D content. The central 
and dispersion values derived from the parametric and nonparametric treatment of the data are shown Table 1 shows the parametric descriptive statistics where the deviation of the data with respect to normal behavior is clearly shown. The high values of MSSD (mean of the squared successive differences) clearly indicate a non-random distribution of the results. To obtain a more accurate description of central values and dispersion of inflammatory markers according to their vitamin D content, a robust descriptive statistical study is shown in Table 2. The median of the of inflammatory markers tends to increase when lower vitamin D levels were found. The significant differences found between the groups are noteworthy. The median of all determinations of FC in the group SD was $345.7 \mu \mathrm{g} / \mathrm{g}$, while in the MD and S groups it was 63.5 and $29.7 \mu \mathrm{g} / \mathrm{g}$, respectively. The medians of the CRP determinations in the SD, MD and S groups were 5.6, 1.5 and $1.4 \mathrm{mg} / \mathrm{dL}$, respectively.

Table 1. Statistical parameters obtained from vitamin D and inflammatory markers grouped according to vitamin D content.

\begin{tabular}{|c|c|c|c|c|c|c|c|c|}
\hline \multirow[b]{2}{*}{ Variable } & \multirow[b]{2}{*}{ Group } & \multirow[b]{2}{*}{ Mean } & \multirow[b]{2}{*}{ StDev } & \multicolumn{2}{|c|}{$\mathrm{CI}^{\mathrm{a}}(95 \%)$} & \multirow[b]{2}{*}{ Skewness } & \multirow[b]{2}{*}{ Kurtosis } & \multirow[b]{2}{*}{ MSSD } \\
\hline & & & & CI- & $\mathrm{CI}+$ & & & \\
\hline \multirow[t]{3}{*}{ Vitamin D } & $S$ & 40.43 & 12.7 & 37.0 & 43.9 & 1.34 & 1.42 & 78 \\
\hline & MD & 24.36 & 4.7 & 23.3 & 25.5 & 0.32 & -0.16 & 17 \\
\hline & SD & 17.83 & 8.1 & 16.3 & 19.3 & 0.85 & 0.39 & 65 \\
\hline \multirow[t]{3}{*}{ CRP } & S & 2.28 & 2.37 & 1.8 & 2.8 & 1.82 & 3.5 & 1.5 \\
\hline & MD & 2.80 & 3.07 & 2.3 & 3.3 & 1.71 & 3.01 & 4.3 \\
\hline & SD & 23.6 & 51.0 & 16 & 31 & 4.48 & 24.2 & 1102 \\
\hline \multirow[t]{3}{*}{$\mathrm{FC}$} & S & 54 & 64 & 39 & 69 & 4.09 & 19.72 & 2567 \\
\hline & MD & 141 & 164 & 111 & 172 & 1.81 & 3.00 & 15,020 \\
\hline & SD & 868 & 1278 & 665 & 1070 & 2.31 & 5.24 & 508,909 \\
\hline \multirow[t]{3}{*}{ FBG } & S & 423 & 93 & 401 & 445 & 0.35 & -0.51 & 4140 \\
\hline & MD & 412 & 107 & 392 & 432 & 0.57 & -0.13 & 6703 \\
\hline & SD & 497 & 133 & 475 & 517 & 0.08 & -0.77 & 5576 \\
\hline
\end{tabular}

a confidence interval; MSSD: mean of the squared successive differences.

Table 2. Robust statistical parameters obtained from vitamin D and inflammatory markers grouped according to vitamin $\mathrm{D}$ content.

\begin{tabular}{|c|c|c|c|c|c|c|c|c|}
\hline \multirow[b]{2}{*}{ Variable } & \multirow[b]{2}{*}{ Group } & \multirow[b]{2}{*}{ Min } & \multirow[b]{2}{*}{ Q1 } & \multirow[b]{2}{*}{ Median } & \multirow[b]{2}{*}{ Q3 } & \multirow[b]{2}{*}{ Max } & \multicolumn{2}{|c|}{$\mathrm{CI}^{\mathrm{a}}(95 \%)$} \\
\hline & & & & & & & CI- & $\mathrm{CI}+$ \\
\hline \multirow[t]{3}{*}{ Vitamin D } & $S$ & 20.5 & 32.2 & 36.2 & 43.0 & 74.30 & 34.1 & 43.9 \\
\hline & MD & 15.3 & 20.6 & 24.7 & 27.4 & 37.5 & 22.4 & 25.5 \\
\hline & $\mathrm{SD}$ & 4.5 & 12.2 & 15.1 & 22.7 & 45.7 & 14.3 & 19.3 \\
\hline \multirow[t]{3}{*}{ CRP } & S & 0.20 & 0.60 & 1.45 & 2.97 & 11.8 & 0.9 & 2.0 \\
\hline & MD & 0.20 & 0.60 & 1.50 & 3.82 & 16.5 & 1.1 & 2.2 \\
\hline & SD & 0.30 & 1.90 & 5.60 & 15.6 & 364 & 4.5 & 7 \\
\hline \multirow[t]{3}{*}{ FC } & S & 19.6 & 26.7 & 29.7 & 50.7 & 434 & 28 & 37 \\
\hline & MD & 17 & 27.6 & 63 & 229 & 738 & 43 & 98 \\
\hline & SD & 19 & 97 & 346 & 1065 & 6450 & 216 & 444 \\
\hline \multirow[t]{3}{*}{ FBG } & S & 262 & 356 & 411 & 491 & 664 & 387 & 446 \\
\hline & MD & 201 & 325 & 406 & 475 & 700 & 365 & 432 \\
\hline & SD & 151 & 390 & 478 & 602 & 813 & 456 & 502 \\
\hline
\end{tabular}




\subsection{Qualitative Variables Study}

Other qualitative variables were also considered and appear in Table 3. The mean age of the patients was 43.7 years, and 39 of the patients were women (46\%). Some 60 patients had CD $(71 \%)$ and 25 presented as UC (29\%). Forty-four patients $(52 \%)$ were supplemented with vitamin D, almost all of them in the SD group. Extraintestinal manifestations were clustered into articular (eight patients) and dermatological manifestations (two patients). Four cases of $C D$ with perianal disease were observed. We collected data on the treatments given during the period of study: biologics (anti-TNF $\alpha$, vedolizumab, ustekinumab), immunosuppressive agents (azathioprine, methotrexate, 6-mercaptopurine and tacrolimus) and the need for steroids therapy.

Six patients from the SD group required a combination of vedolizumab and steroids during the time of study. None of the individuals in the MD or S groups were on vedolizumab. Some 30 patients from the SD group were on anti-TNF $\alpha$ therapy, 12 of them in combination with azathioprine and eight of them with steroids requirement. However, only 17 patients were on anti-TNF $\alpha$ therapy in MD and $\mathrm{S}$ groups altogether. Only three of them required steroids and seven patients were on combination therapy with azathioprine.

Table 3. Baseline clinical characteristics of patients meeting inclusion criteria.

\begin{tabular}{|c|c|c|}
\hline Clinical Characteristic & & $\begin{array}{l}\text { Number of Participants (Percentage) or } \\
\text { Mean } \pm \text { Standard Deviation }\end{array}$ \\
\hline Age, years & & $43.7 \pm 15.9$ \\
\hline Female sex & & $39(46)$ \\
\hline Crohn's disease & & $60(71)$ \\
\hline Ulcerative colitis & & $25(29)$ \\
\hline Perianal disease & & $4(5)$ \\
\hline Extraintestinal manifestations & & $10(12)$ \\
\hline Vitamin D supplementation & & $44(52)$ \\
\hline \multirow{6}{*}{ Current therapy } & Anti-TNF $\alpha$ & $47(55)$ \\
\hline & Vedolizumab & $8(9)$ \\
\hline & Ustekinumab & $1(1)$ \\
\hline & Steroids & $21(25)$ \\
\hline & Azathioprine & $31(36)$ \\
\hline & Methotrexate & $2(2)$ \\
\hline
\end{tabular}

Clinical disease activity markers collected included flare-ups, hospitalisations, number of times patients visited the clinic, changing or escalating treatment and need for steroid therapy. We found remarkable results between the groups according to vitamin D levels. Among the patients with SD, 16 patients $(41 \%)$ were hospitalised at least once and 23 patients $(58.9 \%)$ had at least one flare-up during the time of the study (Figure 4). In the MD group along with the S group, only four flare-ups and one hospitalisation were reported. In the SD group, patients visited the clinic an average of 5.33 times, while in the MD and S groups they visited 3.61 times.

Changes or escalations in current therapy were made for $69.23 \%$ of the patients with SD, while only $8.7 \%$ in the MD and S groups required such changes. Eighteen patients in the SD group $(46.15 \%)$ required steroids during the time of study, while only three patients in the SD and S groups did $(6.53 \%)$. 


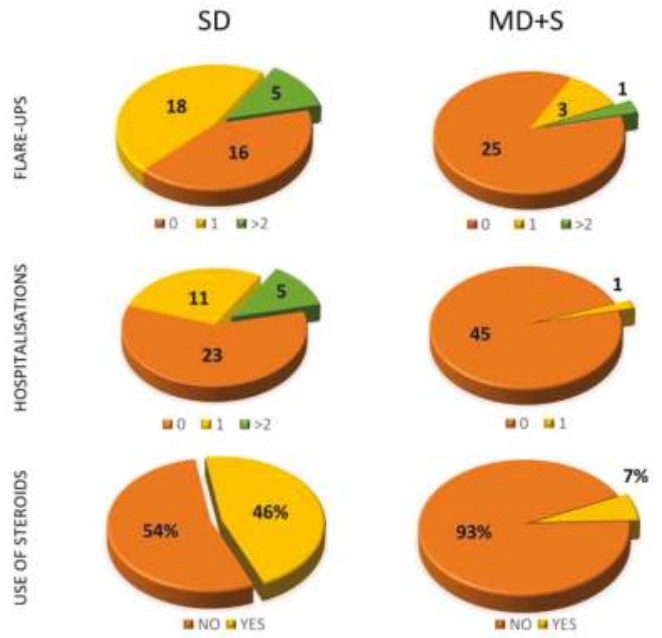

Figure 4. Results of the study of certain qualitative variables depending on vitamin D levels (SD or $\mathrm{MD}+\mathrm{S})$.

\subsection{Linear Correlation Study of Quantitative Variables}

Table 4 shows the median and interquartile range values of inflammatory markers. In nonsupplemented patients, the median and interquartile range of FC increased in cases of severe deficiency. This also occurred when vitamin D was supplemented, but to a lesser extent. A similar behaviour was observed for CRP in terms of the median but not for the interquartile range.

Table 4. Median and interquartile range of FC and CRP grouped by vitamin D levels for patients with and without vitamin D supplementation.

\begin{tabular}{llcccc}
\hline & & \multicolumn{2}{c}{ FC, $\mu \mathrm{g} / \mathrm{g}$} & \multicolumn{2}{c}{ CRP, $\mathbf{m g} / \mathbf{d L}$} \\
\cline { 3 - 6 } & Group & Median & Q3-Q1 & Median & Q3-Q1 \\
\hline \multirow{3}{*}{ Not supplemented } & S & 33 & 25 & 1.3 & 1.8 \\
& MD & 85 & 211 & 1.9 & 4.2 \\
& SD & 524 & 2230 & 8.9 & 3.1 \\
Supplemented & S & 29 & 38 & 2.3 & 4.6 \\
& MD & 55 & 328 & 2.1 & 4.9 \\
& SD & 362 & 943 & 6.7 & 3.1 \\
\hline
\end{tabular}

A nonparametric linear correlation study of vitamin D with FC, CRP and FBG was performed. A Pearson correlation coefficient $(\mathrm{R})$ of $-0.533(p<0.001)$ was obtained for vitamin D and FC in all patients. When two groups of patients were considered, both CD and UC showed a good correlation (Figure 5). As can be seen, this correlation was almost independent of the disease, given there was no significant differences between both slopes for UC and CD $(p=0.686)$.

For CRP, in patients with UC and CD, a Pearson correlation coefficient of -0.512 and $-0.16(p=0.01$ and 0.256$)$, respectively, was obtained (Figure 5). These results demonstrate a strong correlation between CRP and vitamin D exclusively in patients with $U C$, not in $C D$. Therefore, there are significant differences between the correlation slopes $(p=0.02)$. No linear correlation was found between FBG values and vitamin $\mathrm{D}$ levels ( $\mathrm{R}$ near 0 ).

The statistical normality study of FC, CRP and FBG showed a non-normal distribution and, as a consequence, a Kruskal-Wallis test for groups comparison was performed. Significant differences for FC were found between the medians of the SD $(345 \mu \mathrm{g} / \mathrm{g}), \mathrm{MD}(46 \mu \mathrm{g} / \mathrm{g})$ and S (30 $\mu \mathrm{g} / \mathrm{g})$ groups 
with a $p$-value $<0.001$ (Figure 6). A similar study for CRP also showed significant differences $(p=0.01)$ while no differences were obtained for FBG. Figure 7 shows box and whisker plots of inflammatory markers depending on vitamin D levels. Data were normalised for representation purposes. As can be seen, there were significant differences at $99 \%\left(^{* *}\right)$ in the contents of CRP and FC depending on the vitamin D levels.

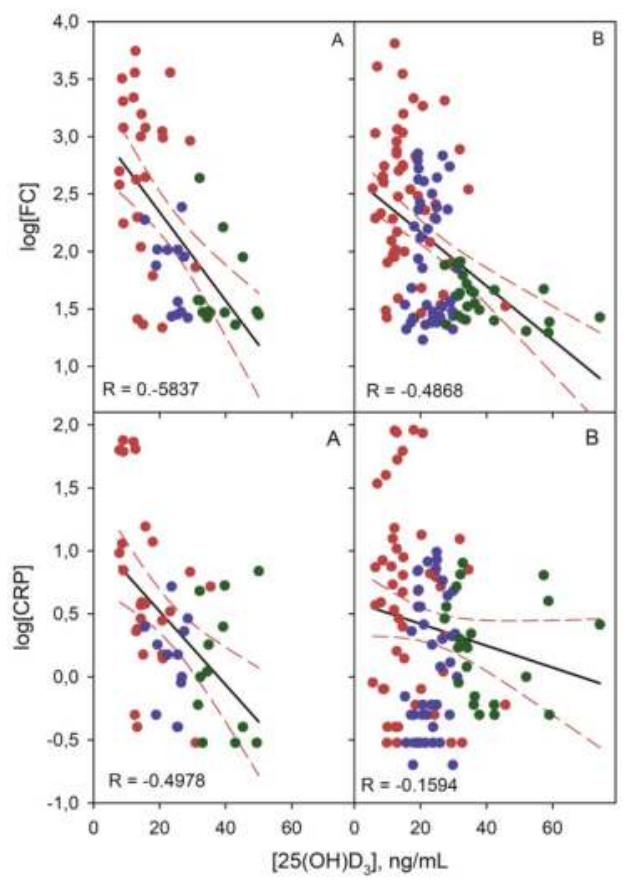

Figure 5. Linear correlation graphs of FC and CRP (log transform) with vitamin D for UC (A) and CD (B) patients. Red, blue and green points correspond to SD, MD and S vitamin D level, respectively.

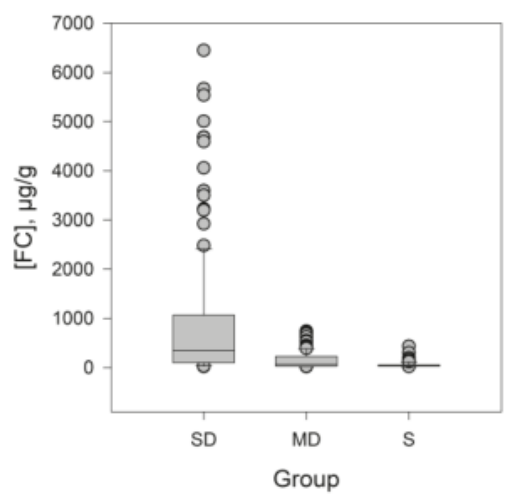

Figure 6. Box and whisker plot to represent the nonparametric variable FC, the values inside the box conform the 25 th and 75 th percentile, while the horizontal line that crosses the box marks the median. The values outside the whiskers are considered atypical. 


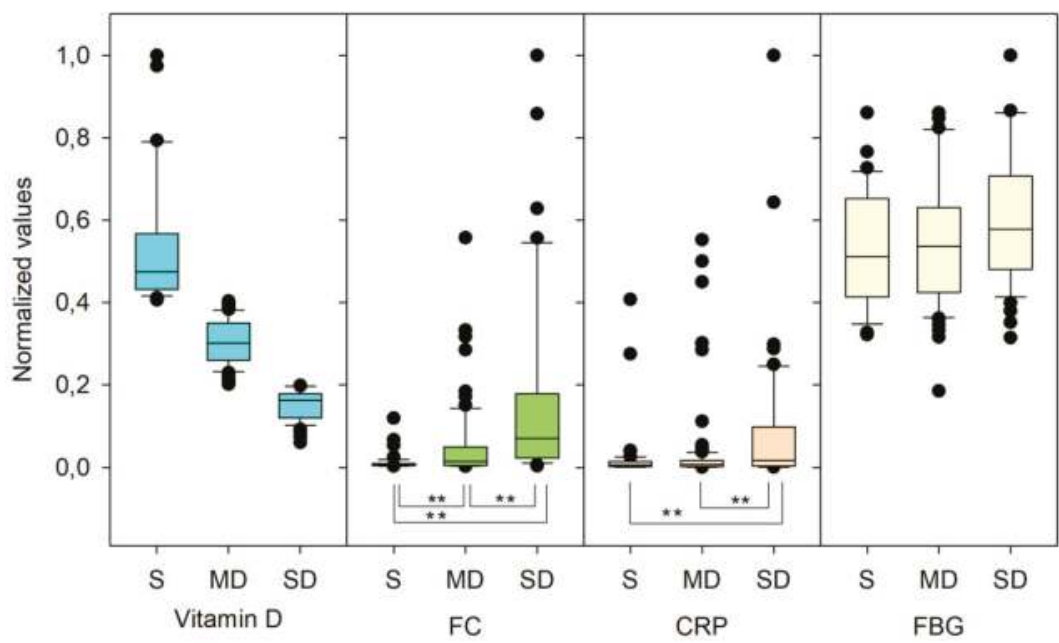

Figure 7. Box and whisker plot using normalised values of inflammatory markers depending on vitamin D levels. ${ }^{* *}$ ) correspond to significant differences at $99 \%$ (Dun's test for multiple comparisons on the Kruskal-Wallis test).

A multivariate discriminant analysis of FC, CRP and FBG shows a discriminant level of $80 \%$. In this way, $74 \%$ of SD patients and $85 \%$ of S patients were correctly classified by the model.

\subsection{Vitamin D Progression}

The progression of vitamin D levels over time was evaluated. Patients were divided according to active supplementation. The mean vitamin $\mathrm{D}$ determination in the third event did not reach more than $25 \mathrm{ng} / \mathrm{mL}$ of vitamin D despite supposedly adequate supplementation. The Bonferroni test indicated significant differences between situations 0 with 1 and 0 with 2, while showing no differences between situations 1 and 2. This behaviour was the same in the group of patients who were supplemented and not supplemented (Figure 8).

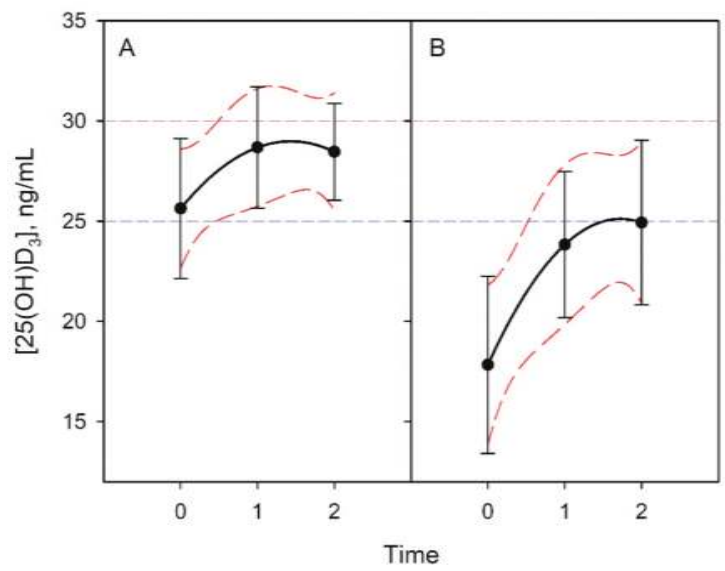

Figure 8. Evolution of vitamin D content in nonsupplemented individuals (A). The graph presents the lines of regression of the contents and their slopes. Active supplementation (B) did not guarantee levels of sufficiency in these patients. 


\subsection{Categorical Variables Study}

For the categorical variables, a logistic regression analysis was used. Negative binomial regression showed an association between the SD group and a higher probability of having flare-ups, hospitalisations and clinic visit ( $p$-value $=0.002$ ). Graphically, it can be seen that for vitamin $\mathrm{D}$ levels below $20 \mathrm{ng} / \mathrm{mL}$, the flare-up and hospitalisation rates increased exponentially regardless of the group (Figure 9).

There was a correlation between those patients in the SD group and the probability of receiving treatment with steroids. Another correlation was also observed with the probability of introducing changes or the need for escalating treatment. All these results were statistically significant $(p<0.001)$.

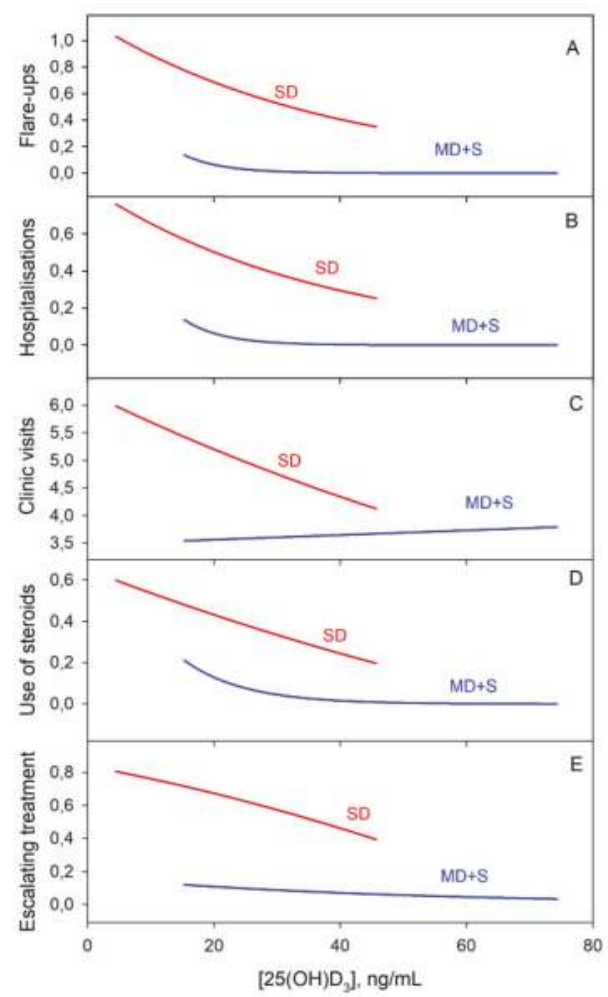

Figure 9. Logistic regression of clinical disease activity markers with vitamin D content. From A to E: Flare-ups, hospitalisations, clinic visits, use of steroids and escalating treatment, respectively.

\section{Discussion}

In this retrospective longitudinal study, we succeeded in describing an inverse correlation between serum vitamin D levels and both systemic inflammatory markers (CRP) and intestinal inflammatory markers (FC). Our findings confirm that vitamin D levels are also correlated with clinical disease activity, as represented in hospitalisations, flare-ups, number of clinic visits, use of steroids and changes in therapy. Additionally, we evaluated the vitamin D progression under oral supplementation, finding an insufficient absorptive capacity.

Other studies support the association between vitamin $\mathrm{D}$ and inflammation. In a cross-sectional study, Meckel et al. [32] demonstrated that serum $25(\mathrm{OH}) \mathrm{D}_{3}$ concentrations are inversely correlated with endoscopic and histologic inflammation and disease activity. In addition, they found that $25(\mathrm{OH}) \mathrm{D}_{3}$ concentrations correlated with the mucosal expression of VDR as well as epithelial junction 
proteins. Moreover, in a retrospective cohort of 60 patients, it was reported that vitamin D was inversely correlated with erythrocyte sedimentation rate (ESR), FC and clinical disease activity measured by Harvey-Bradshaw Index > 5 [36].

Supporting the results of our study, Kabbani et al. [37], in one of the largest prospective cohort studies, demonstrated that patients with low vitamin D levels required more hospitalisations, biologic therapy, steroids, surgeries and healthcare utilisation. We have demonstrated a strong correlation between vitamin D and FC as other authors [38] have done in similar studies. We describe an inverse correlation between vitamin D status and systemic inflammation (CRP levels) in UC. In another prospective study, Gubatan et al. [39] demonstrated that those patients with vitamin levels below $35 \mathrm{ng} / \mathrm{mL}$ were at greater risk of future clinical relapse.

However, there are some uncertainties not resolved yet concerning vitamin D influence on IBD. Several translational studies are emerging to attempt to understand its hormonal pathophysiology, and there are some interventional studies trying to evaluate its active supplementation. Despite the importance of sunlight exposure in vitamin D levels, only a few studies have considered the season where the sample was collected. Baseline vitamin D levels are not yet established to evaluate deficiency and sufficiency. Thus, it is difficult to assess which patients are at real risk of developing clinical consequences. In our study, we set that limit at $<15 \mathrm{ng} / \mathrm{mL}$, but other authors set it at $<20 \mathrm{or}<35 \mathrm{ng} / \mathrm{mL}$. The extent of the supplementation that should be given to our patients and what leads to sufficiency during IBD clinical activity is also unclear.

In our study, several limitations must be considered. Despite describing a strong association between vitamin D deficiency and higher inflammatory markers and disease activity, we cannot confirm a causal relationship due to the retrospective collection of data. A notable cohort study of patients was included in our study, but the inclusion enrolment was in only one single centre by the main researcher. Thus, methodological flaws are possible, including selection bias. Finally, our study is observational and limited by the inability to account for potential unmeasured confounders. Although we evaluated active vitamin D supplementation, we did not consider baseline dietary vitamin D intake, summer or winter season measurement, body mass index, physical activity and adherence to medical therapy and supplementation. There is a relationship between vitamin $\mathrm{D}$ receptor genes (VDR), autophagy and gut microbial assemblage that is essential for maintaining intestinal homeostasis and contributes to the pathophysiology of IBD. However, genetic data regarding VDR polymorphisms has not been considered in this study.

Garg et al. [40] designed an interventional study on vitamin D supplementation where the enrolled IBD patients were given up to 10,000 IU (international units) of oral cholecalciferol. Despite achieving improved symptom-based activity scores and a mean of $41.6 \mathrm{ng} / \mathrm{mL}$ of $25(\mathrm{OH}) \mathrm{D}_{3}$, no significant reduction in FC levels was observed. It is remarkable that there was no control group in this study. Sharifi et al. [41], on the other hand, in a randomised trial, demonstrated a significant decrease in CRP and ESR levels with a single dose of 30,000 IU intramuscular vitamin D supplement. These results support the findings in our study in terms of insufficient supplementation. Doses recommended by guidelines, although suitable for bone health, appear to be clearly insufficient for IBD patients. Considering chronic oral malabsorption, future studies are warranted to explore significant differences in clinical scores or intestinal inflammation depending on the route of vitamin D administration. A higher target $25(\mathrm{OH}) \mathrm{D}_{3}$ concentrations during active supplementation should also be evaluated to clarify beneficial outcomes in IBD patients.

\section{Conclusions}

Vitamin D deficiency is now recognised as another predictor of clinical disease activity according to recent research. This study demonstrates that low serum circulating $25(\mathrm{OH}) \mathrm{D}_{3}$ is associated with intestinal inflammation (FC) in IBD and systemic inflammation (CRP) in UC. Deficiency of $25(\mathrm{OH}) \mathrm{D}_{3}$ is associated with more hospitalisations, flare-ups, use of steroids and escalating treatment. Standard vitamin D oral dose of supplementation is probably insufficient to reach normal serum $25(\mathrm{OH}) \mathrm{D}_{3}$. 
Vitamin D intervention studies are warranted to determine whether raising serum $25(\mathrm{OH}) \mathrm{D}_{3}$ level with higher doses of oral vitamin D in IBD may reduce intestinal inflammation or disease activity.

Author Contributions: P.L.-M.: planning and/or conducting the study, collecting and/or interpreting data, and/or drafting the manuscript. He approved the final draft submitted. B.B.: planning and/or conducting the study, collecting and/or interpreting data, and/or drafting the manuscript. She approved the final draft submitted. E.S.-G.: collecting and/or interpreting data. He approved the final draft submitted. She approved the final draft submitted. A.A.: providing laboratory support in vitamin D measurement, collecting and/or interpreting data. She approved the final draft submitted. P.N.: collecting and/or interpreting data. She approved the final draft submitted. M.I.: planning and/or conducting the study, collecting and/or interpreting data, and/or drafting the manuscript. She approved the final draft submitted.

Funding: This research received no external funding.

Acknowledgments: We are very thankful to Victoria Fornes from the Department of Biostatistics of Hospital la Fe.

Conflicts of Interest: The authors declare no conflict of interest.

\section{References}

1. Stamp, T.C.; Haddad, J.G.; Twigg, C.A. Comparison of oral 25-hydroxycholecalciferol, vitamin D, and ultraviolet light as determinants of circulating 25-hydroxyvitamin D. Lancet 1977, 1, 1341-1343. [CrossRef]

2. Alrefai, D.; Jones, J.; El-Matary, W.; Whiting, S.J.; Aljebreen, A.; Mirhosseini, N.; Vatanparast, H. The Association of Vitamin D Status with Disease Activity in a Cohort of Crohn's Disease Patients in Canada. Nutrients 2017, 9. [CrossRef] [PubMed]

3. White, J.H. Vitamin D metabolism and signaling in the immune system. Rev. Endocr. Metab. Disord. 2012, 13, 21-29. [CrossRef] [PubMed]

4. Lips, P. Vitamin D physiology. Prog. Biophys. Mol. Biol. 2006, 92, 4-8. [CrossRef]

5. Bikle, D.D. Vitamin D metabolism, mechanism of action, and clinical applications. Chem. Biol. 2014, 21, 319-329. [CrossRef]

6. Lim, W.-C.; Hanauer, S.B.; Li, Y.C. Mechanisms of Disease: Vitamin D and inflammatory bowel disease. Nat. Clin. Pract. Gastroenterol. Hepatol. 2005, 2, 308. [CrossRef] [PubMed]

7. Levine, B.S.; Song, M. Pharmacokinetics and efficacy of pulse oral versus intravenous calcitriol in hemodialysis patients. J. Am. Soc. Nephrol. 1996, 7, 488-496.

8. Jones, K.S.; Assar, S.; Harnpanich, D.; Bouillon, R.; Lambrechts, D.; Prentice, A.; Schoenmakers, I. 25(OH)D-2 Half-Life Is Shorter Than 25(OH)D-3 Half-Life and Is Influenced by DBP Concentration and Genotype. J. Clin. Endocrinol. Metab. 2014, 99, 3373-3381. [CrossRef] [PubMed]

9. Wobke, T.K.; Sorg, B.L.; Steinhilber, D. Vitamin D in inflammatory diseases. Front. Physiol. 2014, 5, 244. [CrossRef] [PubMed]

10. Cantorna, M.T.; Zhu, Y.; Froicu, M.; Wittke, A. Vitamin D status, 1,25-dihydroxyvitamin D3, and the immune system. Am. J. Clin. Nutr. 2004, 80,1717s-1720s. [CrossRef]

11. Gubatan, J.; Moss, A.C. Vitamin D in inflammatory bowel disease: More than just a supplement. Curr. Opin. Gastroenterol. 2018, 34, 217-225. [CrossRef]

12. Stio, M.; Retico, L.; Annese, V.; Bonanomi, A.G. Vitamin D regulates the tight-junction protein expression in active ulcerative colitis. Scand. J. Gastroenterol. 2016, 51, 1193-1199. [CrossRef] [PubMed]

13. Zhang, Y.G.; Lu, R.; Xia, Y.; Zhou, D.; Petrof, E.; Claud, E.C.; Sun, J. Lack of Vitamin D Receptor Leads to Hyperfunction of Claudin-2 in Intestinal Inflammatory Responses. Inflamm. Bowel Dis. 2019, 25, 97-110. [CrossRef]

14. Gisbert-Ferrandiz, L.; Salvador, P.; Ortiz-Masia, D.; Macias-Ceja, D.C.; Orden, S.; Esplugues, J.V.; Calatayud, S.; Hinojosa, J.; Barrachina, M.D.; Hernandez, C. A Single Nucleotide Polymorphism in the Vitamin D Receptor Gene Is Associated with Decreased Levels of the Protein and a Penetrating Pattern in Crohn's Disease. Inflamm. Bowel Dis. 2018, 24, 1462-1470. [CrossRef] [PubMed]

15. Wang, T.T.; Dabbas, B.; Laperriere, D.; Bitton, A.J.; Soualhine, H.; Tavera-Mendoza, L.E.; Dionne, S.; Servant, M.J.; Bitton, A.; Seidman, E.G.; et al. Direct and indirect induction by 1,25-dihydroxyvitamin D3 of the NOD2/CARD15-defensin beta2 innate immune pathway defective in Crohn disease. J. Biol. Chem. 2010, 285, 2227-2231. [CrossRef] 
16. Hugot, J.P.; Chamaillard, M.; Zouali, H.; Lesage, S.; Cezard, J.P.; Belaiche, J.; Almer, S.; Tysk, C.; O'Morain, C.A.; Gassull, M.; et al. Association of NOD2 leucine-rich repeat variants with susceptibility to Crohn's disease. Nature 2001, 411, 599-603. [CrossRef] [PubMed]

17. Fellermann, K.; Stange, D.E.; Schaeffeler, E.; Schmalzl, H.; Wehkamp, J.; Bevins, C.L.; Reinisch, W.; Teml, A.; Schwab, M.; Lichter, P.; et al. A chromosome 8 gene-cluster polymorphism with low human beta-defensin 2 gene copy number predisposes to Crohn disease of the colon. Am. J. Hum. Genet. 2006, 79, 439-448. [CrossRef]

18. Gombart, A.F.; Borregaard, N.; Koeffler, H.P. Human cathelicidin antimicrobial peptide (CAMP) gene is a direct target of the vitamin $D$ receptor and is strongly up-regulated in myeloid cells by 1,25-dihydroxyvitamin D3. FASEB J. 2005, 19, 1067-1077. [CrossRef]

19. Wang, T.T.; Nestel, F.P.; Bourdeau, V.; Nagai, Y.; Wang, Q.; Liao, J.; Tavera-Mendoza, L.; Lin, R.; Hanrahan, J.W.; Mader, S.; et al. Cutting edge: 1,25-dihydroxyvitamin D3 is a direct inducer of antimicrobial peptide gene expression. J. Immunol. 2004, 173, 2909-2912. [CrossRef]

20. D'Ambrosio, D.; Cippitelli, M.; Cocciolo, M.G.; Mazzeo, D.; Di Lucia, P.; Lang, R.; Sinigaglia, F.; Panina-Bordignon, P. Inhibition of IL-12 production by 1,25-dihydroxyvitamin D3. Involvement of NF-kappaB downregulation in transcriptional repression of the p40 gene. J. Clin. Investig. 1998, 101, 252-262. [CrossRef]

21. Penna, G.; Adorini, L. 1 Alpha,25-dihydroxyvitamin D3 inhibits differentiation, maturation, activation, and survival of dendritic cells leading to impaired alloreactive T cell activation. J. Immunol. 2000, 164, 2405-2411. [CrossRef] [PubMed]

22. Boonstra, A.; Barrat, F.J.; Crain, C.; Heath, V.L.; Savelkoul, H.F.; O'Garra, A. 1alpha,25-Dihydroxyvitamin d3 has a direct effect on naive CD4(+) T cells to enhance the development of Th2 cells. J. Immunol. 2001, 167, 4974-4980. [CrossRef] [PubMed]

23. Gubatan, J.; Mitsuhashi, S.; Longhi, M.S.; Zenlea, T.; Rosenberg, L.; Robson, S.; Moss, A.C. Higher serum vitamin D levels are associated with protective serum cytokine profiles in patients with ulcerative colitis. Cytokine 2018, 103, 38-45. [CrossRef]

24. Luthold, R.V.; Fernandes, G.R.; Franco-de-Moraes, A.C.; Folchetti, L.G.; Ferreira, S.R. Gut microbiota interactions with the immunomodulatory role of vitamin D in normal individuals. Metabolism 2017, 69, 76-86. [CrossRef] [PubMed]

25. Schaffler, H.; Herlemann, D.P.R.; Klinitzke, P.; Berlin, P.; Kreikemeyer, B.; Jaster, R.; Lamprecht, G. Vitamin D administration leads to a shift of the intestinal bacterial composition in Crohn's disease patients, but not in healthy controls. J. Dig. Dis. 2018, 19, 225-234. [CrossRef]

26. Dziarski, R.; Park, S.Y.; Kashyap, D.R.; Dowd, S.E.; Gupta, D. Pglyrp-Regulated Gut Microflora Prevotella falsenii, Parabacteroides distasonis and Bacteroides eggerthii Enhance and Alistipes finegoldii Attenuates Colitis in Mice. PLoS ONE 2016, 11. [CrossRef] [PubMed]

27. Tamanai-Shacoori, Z.; Smida, I.; Bousarghin, L.; Loreal, O.; Meuric, V.; Fong, S.B.; Bonnaure-Mallet, M.; Jolivet-Gougeon, A. Roseburia spp.: A marker of health? Future Microbiol. 2017, 12, 157-170. [CrossRef] [PubMed]

28. Del Pinto, R.; Pietropaoli, D.; Chandar, A.K.; Ferri, C.; Cominelli, F. Association Between Inflammatory Bowel Disease and Vitamin D Deficiency: A Systematic Review and Meta-analysis. Inflamm. Bowel Dis. 2015, 21, 2708-2717. [CrossRef]

29. Filippi, J.; Al-Jaouni, R.; Wiroth, J.B.; Hebuterne, X.; Schneider, S.M. Nutritional deficiencies in patients with Crohn's disease in remission. Inflamm. Bowel Dis. 2006, 12, 185-191. [CrossRef] [PubMed]

30. Raftery, T.; Merrick, M.; Healy, M.; Mahmud, N.; O’Morain, C.; Smith, S.; McNamara, D.; O'Sullivan, M. Vitamin D Status Is Associated with Intestinal Inflammation as Measured by Fecal Calprotectin in Crohn's Disease in Clinical Remission. Dig. Dis. Sci. 2015, 60, 2427-2435. [CrossRef]

31. Frigstad, S.O.; Hoivik, M.; Jahnsen, J.; Dahl, S.R.; Cvancarova, M.; Grimstad, T.; Berset, I.P.; Huppertz-Hauss, G.; Hovde, O.; Torp, R.; et al. Vitamin D deficiency in inflammatory bowel disease: Prevalence and predictors in a Norwegian outpatient population. Scand. J. Gastroenterol. 2017, 52, 100-106. [CrossRef] [PubMed]

32. Meckel, K.; Li, Y.C.; Lim, J.; Kocherginsky, M.; Weber, C.; Almoghrabi, A.; Chen, X.; Kaboff, A.; Sadiq, F.; Hanauer, S.B.; et al. Serum 25-hydroxyvitamin D concentration is inversely associated with mucosal inflammation in patients with ulcerative colitis. Am. J. Clin. Nutr. 2016, 104, 113-120. [CrossRef] 
33. Santos-Antunes, J.; Nunes, A.C.; Lopes, S.; Macedo, G. The Relevance of Vitamin D and Antinuclear Antibodies in Patients with Inflammatory Bowel Disease Under Anti-TNF Treatment: A Prospective Study. Inflamm. Bowel Dis. 2016, 22, 1101-1106. [CrossRef] [PubMed]

34. Schaffler, H.; Schmidt, M.; Huth, A.; Reiner, J.; Glass, A.; Lamprecht, G. Clinical factors are associated with vitamin D levels in IBD patients: A retrospective analysis. J. Dig. Dis. 2018, 19, 24-32. [CrossRef] [PubMed]

35. Venkata, K.V.R.; Arora, S.S.; Xie, F.L.; Malik, T.A. Impact of vitamin D on the hospitalization rate of Crohn's disease patients seen at a tertiary care center. World J. Gastroenterol. 2017, 23, 2539-2544. [CrossRef]

36. Scolaro, B.L.; Barretta, C.; Matos, C.H.; Malluta, E.F.; Almeida, I.B.T.d.; Braggio, L.D.; Bobato, S.; Specht, C.M Deficiency of vitamin $\mathrm{D}$ and its relation with clinical and laboratory activity of inflammatory bowel diseases. JCOL 2018, 38, 99-104. [CrossRef]

37. Kabbani, T.A.; Koutroubakis, I.E.; Schoen, R.E.; Ramos-Rivers, C.; Shah, N.; Swoger, J.; Regueiro, M.; Barrie, A.; Schwartz, M.; Hashash, J.G.; et al. Association of Vitamin D Level with Clinical Status in Inflammatory Bowel Disease: A 5-Year Longitudinal Study. Am. J. Gastroenterol. 2016, 111, 712-719. [CrossRef]

38. Garg, M.; Rosella, O.; Lubel, J.S.; Gibson, P.R. Association of circulating vitamin D concentrations with intestinal but not systemic inflammation in inflammatory bowel disease. Inflamm. Bowel Dis. 2013, 19, 2634-2643. [CrossRef]

39. Gubatan, J.; Mitsuhashi, S.; Zenlea, T.; Rosenberg, L.; Robson, S.; Moss, A.C. Low Serum Vitamin D During Remission Increases Risk of Clinical Relapse in Patients with Ulcerative Colitis. Clin. Gastroenterol. Hepatol. 2017, 15, 240-246. [CrossRef]

40. Garg, M.; Rosella, O.; Rosella, G.; Wu, Y.; Lubel, J.S.; Gibson, P.R. Evaluation of a 12-week targeted vitamin D supplementation regimen in patients with active inflammatory bowel disease. Clin. Nutr. 2018, 37, 1375-1382. [CrossRef]

41. Sharifi, A.; Hosseinzadeh-Attar, M.J.; Vahedi, H.; Nedjat, S. A randomized controlled trial on the effect of vitamin D3 on inflammation and cathelicidin gene expression in ulcerative colitis patients. Saudi J. Gastroenterol. 2016, 22, 316-323. [CrossRef] [PubMed]

(C) 2019 by the authors. Licensee MDPI, Basel, Switzerland. This article is an open access article distributed under the terms and conditions of the Creative Commons Attribution (CC BY) license (http://creativecommons.org/licenses/by/4.0/). 


\title{
Bases for the Adequate Development of Nutritional Recommendations for Patients with Inflammatory Bowel Disease
}

\author{
Esteban Sáez-González ${ }^{1,2, \dagger}$, Beatriz Mateos ${ }^{1,2,+}{ }^{\text {, Pedro López-Muñoz }}{ }^{1}$, Marisa Iborra ${ }^{1,2,3}$, \\ Inés Moret ${ }^{1,2,3}$, Pilar Nos ${ }^{1,2,3}$ and Belén Beltrán 1,2,3,* \\ 1 Inflammatory Bowel Disease Unit, Gastroenterology Department, La Fe University and Polytechnic \\ Hospital, 46026 Valencia, Spain; esteban.digestivo@gmail.com (E.S.-G.); bmateosa@gmail.com (B.M.); \\ pedro.lopez8928@gmail.com (P.L.-M.); marisaiborra@hotmail.com (M.I.); Ines.Moret@uv.es (I.M.); \\ pilarnos@gmail.com (P.N.) \\ 2 Inflammatory Bowel Disease Research Group, Medical Research Institute Hospital La Fe (IIS La Fe), \\ 46026 Valencia, Spain \\ 3 Biomedical Research Network Center for Liver and Digestive Diseases (CIBEREHD), 28029 Madrid, Spain \\ * Correspondence: belenbeltranniclos@gmail.com; Tel./Fax: +34-961-245-859/+34-961-246-257 \\ $\dagger$ These authors contribute equally to this manuscript.
}

Received: 2 April 2019; Accepted: 10 May 2019; Published: 12 May 2019

\begin{abstract}
Inflammatory bowel disease (IBD) is a chronic and relapsing inflammatory condition of the gastrointestinal tract; it is a heterogeneous and multifactorial disorder resulting from a complex interplay between genetic variation, intestinal microbiota, the host immune system and environmental factors such as diet, drugs, breastfeeding and smoking. The interactions between dietary nutrients and intestinal immunity are complex. There is a compelling argument for environmental factors such as diet playing a role in the cause and course of IBD, given that three important factors in the pathogenesis of IBD can be modulated and controlled by diet: intestinal microbiota, the immune system and epithelial barrier function. The aim of this review is to summarize the epidemiological findings regarding diet and to focus on the effects that nutrients exert on the intestinal mucosa-microbiota-permeability interaction. The nature of these interactions in IBD is influenced by alterations in the nutritional metabolism of the gut microbiota and host cells that can influence the outcome of nutritional intervention. A better understanding of diet-host-microbiota interactions is essential for unravelling the complex molecular basis of epigenetic, genetic and environmental interactions underlying IBD pathogenesis as well as for offering new therapeutic approaches for the treatment of IBD.
\end{abstract}

Keywords: diet; inflammatory bowel disease; microbiota; intestinal barrier; nutrients; immunity

\section{Introduction}

Inflammatory bowel disease (IBD) is a chronic and relapsing inflammatory condition of the gastrointestinal tract. Crohn's disease (CD) and ulcerative colitis (UC) are the two principal types of IBD. Its prevalence has been increasing worldwide, with the highest incidence found in Western countries [1]. The precise etiology of IBD remains unclear; however, interactions between genetic, microbiotic and environmental factors are associated with its pathogenesis [2]. There is a compelling argument for environmental factors such as diet playing a role in the cause and course of IBD [3], given that three important factors in the pathogenesis of IBD can be modulated and controlled by diet: intestinal microbiota, the immune system and epithelial barrier function.

Patients with IBD tend to present with malnutrition, especially when they are having a flare or have chronic, only partially controlled, intestinal inflammation. Currently, to deal appropriately with malnutrition is considered a clinical quality criterion, especially for preventing the complications 
that can arise under a malnutrition situation (e.g., infections, postsurgical complications, immunity alterations). Thus, there is a tendency to at least consider the patient's nutritional status before surgery [4], and a guide has recently been published with dietary recommendations [5]. However, the gastroenterologist typically gives little advice to patients with IBD regarding daily diet or nutrient considerations. Patients are often told, "eat what you can tolerate," and discussion on diet is typically very limited. Despite the scarce attention gastroenterologists give to nutrients, approximately $40 \%$ of patients with CD believe that diet can control symptoms, and approximately $80 \%$ believe diet is important in the overall management of the disease [6]. Some $40 \%$ of patients with IBD have attempted various diet therapies, often without the assistance of a physician or dietician [7].

Perturbations related to dietary intake are thought to relate to the consumption of a dietary pattern that negatively alters gut microbiota composition and intestinal permeability [8]. Recent experimental evidence has suggested a crucial factor of barrier dysfunction in the onset of IBD [9]. The aim of this review is to summarize the epidemiological findings regarding diet and to focus on the effects that nutrients exert on intestinal mucosa-microbiota-permeability interaction.

\section{Diet Influences Inflammatory Bowel Disease: Epidemiological Clues}

Epidemiological studies have shown associations between the intake of specific dietary components and the risk of developing IBD [10-17]; however, association does not necessarily imply causality. These studies must be considered with caution, given that methodological inconsistencies and a recall bias could be affecting the results observed. Furthermore, in a complex disease with dietary triggers, it can be difficult to unravel the role of individual dietary risk factors because dietary patterns often involve exposure to clusters of nutrients. These clusters are favored by the industrial processing of food.

Classically, no environmental factor has been claimed to impact directly on the pathogenesis of IBD, apart from tobacco [18]. However, a recent systematic review and meta-analysis have shown that breastfeeding provides a protective factor against the development of $\mathrm{CD}$ and $\mathrm{UC}$, both in pediatric and adult-onset disease. This inverse association is consistent in studies worldwide and supports the recommendation of breastfeeding in infancy to reduce the risk of IBD development [19]. Further research on the effects of breast milk on the microbiome and modulation of the innate immune system are needed.

A systematic review of the literature, initially including 2085 publications, was published in 2011 [20]. Nineteen studies fulfilled the inclusion criteria, containing a total of 2609 patients with IBD (1340 UC and 1269 CD). The authors concluded that a high intake of total fats, polyunsaturated fatty acids (PUFAs), omega-6 fatty acids and meat were consistently associated with increased risk of developing $\mathrm{UC}$ as well as $\mathrm{CD}$. High vegetable intake was consistently associated with decreased risk of UC, whereas fiber and fruit intake were consistently associated with reduced risk of CD.

Dietary fats are associated with an increased or decreased risk for IBD, depending on the type of fat. Among 170,805 women followed over 26 years, a high intake of dietary long-chain omega-3 PUFAs was associated with a reduced risk of UC. In contrast, high intake of trans-unsaturated fats was associated with an increased risk of UC [15]. Various data have been extracted from an analysis of 366,351 individuals with IBD from the European Prospective Investigation into Cancer and Nutrition study cohort. Globally, a dietary imbalance with a high consumption of sugar and soft drinks and a low consumption of vegetables has been associated with UC risk [12]. Regarding fats, a sub-study analyzing 126 incident cases of UC showed that the patients ranking in the superior quartile of linoleic acid intake had a higher risk of developing UC [21]. Similarly, another analysis considering 70 incident cases of $C D$ has shown that higher intake of the omega-3 PUFA docosahexaenoic acid prevents CD development [22]. A recent population-based study of environmental risk factors shows an increased risk of $\mathrm{CD}$ with frequent fast-food consumption (Western diet) before the diagnosis [23]. A Western diet typically contains high levels of omega-6 PUFAs and low levels of omega-3 PUFAs. The ratio between the intake of omega- 6 and omega-3 PUFAs is considered a risk factor for IBD. 
Data from the Nurses' Health Study including 170,776 participants showed that higher long-term fruit intake was associated with a lower risk of CD but not UC [15]. Multiple studies have reported a negative association between dietary fiber intake from fruits or fruits and vegetables and subsequent risk of $\mathrm{CD}$. Fiber is the dietary component with the greatest agreement in epidemiological studies. In a recent prospective study [24], fiber from fruit has also been implicated in preventing the establishment of pouchitis in patients with UC. It is important to remember that the fiber implicated in these effects is soluble fiber, which is fermentable by bacteria. Soluble fiber is usually less present in food; however, the proportion of insoluble to soluble fiber could be the factor limiting tolerance to various foods.

Animal proteins have also been claimed to increase the risk of IBD [10]. A prospective study (E3N) that included 67,581 French women showed that high animal protein intake was associated with a significantly increased the risk of IBD, particularly with UC. However, other smaller studies have not confirmed this risk [20]. In a separate study evaluating dietary intake and relapse of UC, red meat was found to have the strongest association (odds ratio 5.19; 95\% confidence interval 2.09-12.9) with relapse [14].

Food additives have been progressively increasing in processed food. Both sweeteners and emulsifiers, used to enhance the texture and stability of foods, have been shown to disrupt host-microbiota interaction and to facilitate intestinal inflammation [25]. However, it is difficult to ascertain whether the additives are risk factors because most of the questionnaires used to assess dietary intake do not evaluate food additives.

Currently, it seems clear that dietary patterns are more important than individual foods for considering the risk of IBD. The consumption of vegetables, fruits, olive oil, fish, grains and nuts (prudent diet) was associated with a decreased risk of developing CD in both boys and girls in a Canadian pediatric study. A Mediterranean dietary pattern is high in extra-virgin olive oil, vegetables, fruit, legumes, nuts and seeds, with a moderate consumption of fish, poultry and milk products, and is low in processed foods, baked goods and red and processed meat. This dietary pattern is high in monounsaturated fats, omega-3 PUFAs, fermentable fiber and polyphenols, and adherence to a Mediterranean dietary pattern is associated with lower levels of inflammation biomarkers [26].

\section{Impact of Nutrients on Intestinal Permeability, Microbiome and Immunity}

Dietary fiber is not digested or absorbed by host cells, given that mammalian cells largely lack the necessary enzymes to degrade them. Instead, dietary fiber is subjected to bacterial fermentation in the gastrointestinal tract. Although a wide range of bacteria ferment dietary fiber, each bacterium has a substrate preference based on its enzymatic activity. Thus, dietary intervention can remodel the gut microbial composition by customizing the content of dietary fiber [27]. Short-chain fatty acids (SCFAs), such as acetate, propionate and butyrate, are the major end products of microbial fermentation of dietary fiber. They are key energy substrates used by colonocytes [28]. Butyrate enhances intestinal epithelial barrier function via hypoxia-inducible factor-1, which regulates the integrity of epithelial tight junctions [28-30]. It also increases the synthesis of the MUC2 protein, the main component of intestinal mucus [31,32]. Thus, a lack of dietary fiber might compromise epithelial integrity and mucus production due to insufficient SCFA generation, resulting in impaired intestinal barrier function [33]. Recently, it has been observed that consumption of a low-fiber diet leads to the disruption of intestinal barrier function through a mechanism that is independent of SCFAs. In the absence of dietary fiber, some commensal bacteria use host mucus glycans to meet their energy needs [34]. As a result, these mucolytic bacteria become the predominant species within the gut microbiota. Importantly, a bloom of mucolytic bacteria results in the degradation of the colonic mucus layer that renders the host susceptible to enteric pathogens [34] (Figure 1). These changes facilitate bacterial adherence and translocation into the epithelium, which introduce immunomodulatory effects. In summary, a diet poor in soluble fiber can condition both dysbiosis and a subsequent decrease in the mucus layer, which increases the permeability already affected by the fiber-poor diet. 


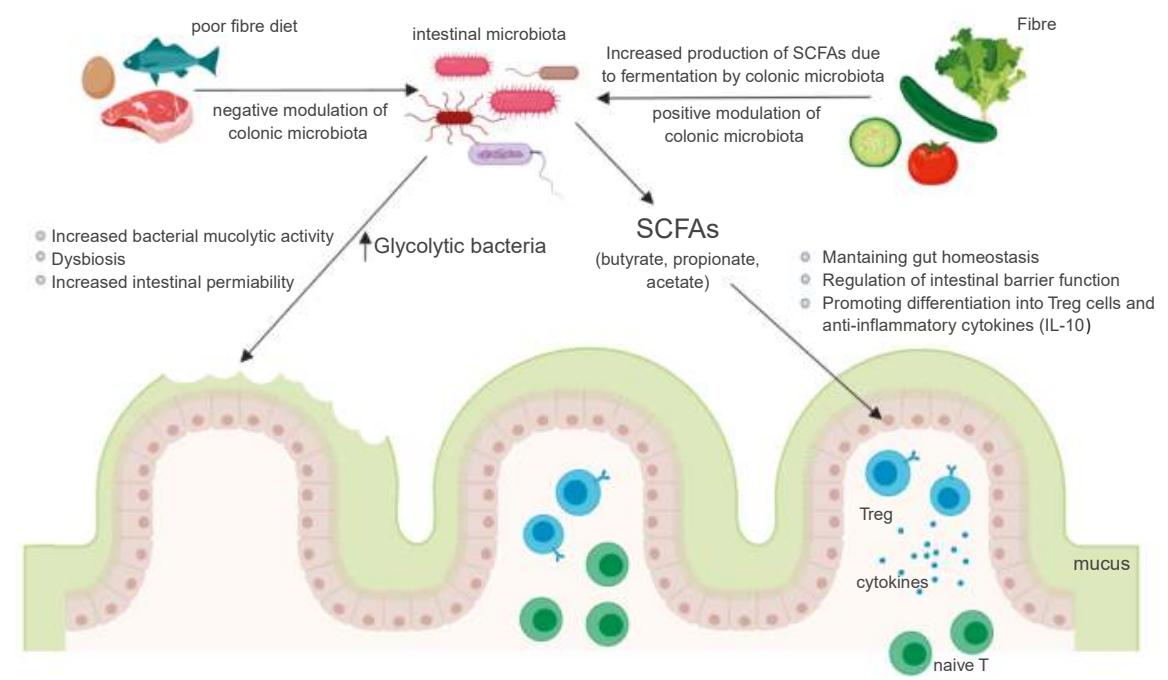

Figure 1. Interaction of dietary fiber with the gut microbiota.

The gut microbiota is thought to play a crucial role in human health and prevention of disease through a variety of mechanisms, including the production of short-chain fatty acids (SCFAs), which are important for maintaining gut homeostasis and optimal immune function. Ingested fiber can influence fecal microbiota profiles, cause changes in the complex gastrointestinal environment and promote the growth of bacteria in general and potentially beneficial bacteria in particular.

Fat- and refined sugar-rich diets can condition a low production of butyrate, which has been described in patients with $C D$, together with a diminution in the butyrate receptor [35] Butyrate plays an important role in downregulating inflammation by suppressing transcription of cytokines and increasing differentiation and the population of lamina propria Tregs $[33,35,36]$. Butyrate can also enhance innate immunity by upregulating defensins and cathelicidins in animal models [36]. Defensins have been reported to be permanently decreased in patients with IBD, and whether a dietary intervention could reverse this condition deserves further investigation. High-fat diets (without high sugars) have been shown to increase tumor necrosis factor (TNF)- $\alpha$ and interferon- $\gamma$ expression, and to decrease levels of colonic Tregs [37].

Dietary amino acids play important roles in gut homeostasis. Amino acid metabolic profiles in the blood, urine, feces and intestinal tissues are also altered in patients with IBD and correlate with the severity of the disease. Additionally, metagenomic studies have revealed that amino acid biosynthesis genes are downregulated and amino acid transporter genes are upregulated in the gut microbiome of patients with IBD, indicating that the gut microbiota lessens the production of amino acids and increases the rate of their use [38,39]. Certain amino acids are critical for immune T-cell function as well as for the proliferation of macrophages [40,41]. Thus, the demand for certain amino acids by host cells and the gut microbiota can increase as a consequence of inflammation.

Tryptophan is an essential amino acid that can be found in fish, meat and cheese. Its metabolites, such as kynurenine, indole-3-aldehyde and indole-3-acetic acid, can act as ligands for the aryl hydrocarbon receptor, a critical regulator of immunity and inflammation involved in adaptive immunity and intestinal barrier function [42-44]. Indoleamine 2,3 dioxygenase-1 (IDO1) is ubiquitously expressed in epithelial cells, dendritic cells and macrophages. IDO1 is the first step in the kynurenine pathway, a major route for tryptophan catabolism. IDO1 regulates the differentiation and maturation of adaptive immune cells [45]. Kynurenine is an initial metabolite of IDO1-mediated tryptophan catabolism and the kynurenine/tryptophan ratio is a surrogate marker of IDO1 activity. A recent clinical study has shown that serum tryptophan levels are lower and the kynurenine/tryptophan ratio is elevated in 
patients with IBD compared with healthy controls [15]. Additionally, indoleamine 2,3-dioxygenase 1 (IDO1) mRNA expression in colonic tissues is significantly higher in IBD and correlates with disease severity, suggesting the kynurenine pathway is upregulated in IBD.

Arginine and glutamine are two semi-essential amino acids that can impact gut homeostasis and innate immunity. Both are diminished in prospective cohorts of patients with IBD, and both are implicated in wound repair mechanisms [46]. Furthermore, arginine regulates macrophage differentiation into the M1 or M2 phenotype following environmental cytokine guidance, whereas glutamine reduces oxidative stress and cytokine production via downregulation of the NF-kB and signal transducer and activator of transcription proteins (STAT) signaling pathways.

The upregulation of amino acid metabolic pathways is one of the major inflammation-related metabolic shifts in the host and the microbiota. In other words, host cells and resident microbes have an increased demand for certain amino acids in the context of IBD. Thus, dietary supplementation of these amino acids is a key strategy for the treatment of IBD. However, it is noteworthy to mention that other amino acids can fuel pro-inflammatory responses in IBD, and restriction of certain amino acids can attenuate intestinal inflammation. A more thorough understanding of the specific roles various amino acids play in IBD and supplying the optimal amount of anti-inflammatory amino acids while limiting the consumption of proinflammatory amino acids can lead to the development of effective amino acid-based dietary interventions.

\section{Mesenteric Fat and Epigenetic Considerations}

Today, patients with IBD sometimes have malnutrition with obesity, associated with metabolic syndrome. Obesity has also been associated with the development of more active IBD, with a higher demand for therapeutic resources. Patients with IBD, especially CD, present fat-wrapping or "creeping fat," which corresponds to ectopic adipose tissue extending from the mesenteric attachment and covering the majority of the small and large intestinal surface. Mesenteric adipose tissue in patients with IBD presents several morphological and functional alterations; e.g., it is more infiltrated with immune cells such as macrophages and T-cells. This fat tissue is not an "innocent bystander," but actively contributes to intestinal and systemic inflammatory responses [47]. It overexpresses peroxisome proliferator-activated receptor gamma (PPAR- $\gamma$ ), which is a "master" regulator of the adipogenesis program and the major orchestrator of visceral adipose tissue Treg accumulation, phenotypes and function. Mesenteric adipose tissue is colonized by luminal bacteria, and PPAR- $\gamma$ expression is modulated by several bacterial stimuli. Thus, the presence of metabolically active adipose tissue should be taken into consideration when attempting to change the alterations facilitating bacterial adherence and/or clearance and permeability regulation of innate/adaptive immunity.

Similarly, epigenetic modulation of gene expression should be contemplated when considering how other environmental factors can affect the mucosa-microbiome-immunity interplay. A Westernized high-fat diet, full of refined carbohydrates, is strongly associated with the development of IBD, contrary to a diet high in fruit, vegetables and omega-3 PUFAs, which is protective against it [26]. Epigenetic mechanisms could explain the connection between genes and environmental factors in triggering the development of IBD. The definition of epigenetics refers to heritable alterations of gene expression events that are caused independently of genetic information carried by the primary DNA sequence. The main epigenetic mechanisms controlling gene expression include DNA methylation, histone modifications and small and long noncoding RNAs [48]. The main objective of epigenetics is to modulate gene expression without modifying the basic DNA sequence. All the mechanisms involved in epigenetics are involved in correct cell development, differentiation, function and homeostasis. Moreover, these mechanisms are influenced by exposure to environmental factors, persist through mitosis and meiosis and, more importantly, can be reversed. Recent advances have indicated that epigenetic variation has an important influence on interactions between nutrients and the genome, which modifies disease risk [49,50]. Diet is known to influence epigenetic changes associated with disease and to modify gene expression patterns in a state of disturbed immunity. A number of nutrients 
have been shown to modulate immune responses and can potentially counteract inflammatory processes [51]. Some studies have suggested that secondary plant metabolites, such as polyphenols, might modulate gene expression, chromatin remodeling and DNA methylation [52]. Epigenetic effects have also been shown for other dietary components, such as curcumin [53]. Moreover, specific components of the Mediterranean diet, particularly nuts and extra-virgin olive oil, have recently been found to be able to induce methylation changes in several peripheral white blood cells, showing a role for specific fatty acids in epigenetic modulation [54]. Thus, considering the status of epigenetic mechanisms in particular patients, it is important to understand whether a dietary intervention could help or not.

\section{Diet as Possible Therapy}

Despite the fact that a growing body of knowledge related to diet is emerging in terms of IBD pathogenesis, there is no strong evidence for dietary interventions helping to induce remission or to maintain response. Dietary therapies, such as enteral exclusive nutrition (EEN), have traditionally and primarily been used to induce remission in early or new-onset CD. EEN has been shown to be effective for inducing clinical remission, improving nutritional status, improving body composition, inducing mucosal healing and decreasing proinflammatory cytokines in pediatric and adult patients with CD. EEN has been extensively used for induction of remission in pediatric $C D$, in which avoidance of steroids is critical for childhood growth. However, EEN has been less often used for adult patients. Although some studies have assessed EEN in adults, most were less conclusive and had multiple confounding factors; thus, this practice is uncommon in adults with CD [55]. EEN involves the exclusive use of liquid nutrition in the form of medical formulas, without exposure to other foods, usually for 6-8 weeks. This effect does not depend on the protein source (a type of formula), but it does depend on the exclusion of ordinary table food [56]. Several recent pediatric studies have demonstrated and confirmed that EEN can induce remission in $60 \%-86 \%$ of children, accompanied by a significant decrease in inflammatory markers, such as the erythrocyte sedimentation rate, C-reactive protein and fecal calprotectin [57-59]. This recent body of literature has evaluated the long-term benefits associated with the use of EEN in mild-to-moderate pediatric CD. In comparison with the use of steroids, and corrected for disease severity, EEN has been associated with higher remission rates, better growth and longer steroid-free periods, but it did not differ in regard to other outcomes such as relapse. The benefit of EEN was lost when partial enteral nutrition (PEN) was used instead, with access to a free diet granted to patients [60]; thus, reaffirming the principle of exclusivity, suggesting that the mechanism depends on the exclusion of a free diet. However, a recent study has shown that PEN can be effective in combination with a "prudent pattern diet" [61]. In this study, PEN, with an exclusion diet based on components hypothesized to affect the microbiome or intestinal permeability, was effective for induction of remission in children and young adults with $\mathrm{CD}$. Several randomized controlled trials are being developed in order to confirm this observation (NCT01728870, NCT 02843100, NCT02231814).

The idea of maintaining remission with diet is compelling and challenging at the same time. On the one hand, once remission is established, it is certainly possible that diet will be sufficient to maintain homeostasis and to prevent the cascade of proinflammatory events leading to flare-ups; however, long-term studies are challenging to perform, and adherence becomes a serious concern. Long-term studies on the maintenance of remission have largely focused on the use of semi-elemental EEN [62,63]. These data show promise for the use of semi-elemental EEN in the maintenance of CD. On the other hand, an interesting idea has been revealed by a recent study showing that, in patients with moderate to severe CD undergoing infliximab treatment (anti-TNF $\alpha$ therapy), combined EEN therapy of $\geq 600 \mathrm{kcal} /$ day led to an increase in the remission maintenance rate, preventing loss of response to infliximab therapy [64]. These studies illustrate the potential benefit of elemental nutrition in the maintenance of remission in patients with $\mathrm{CD}$; however, there are as yet no data to support this hypothesis. 
Regarding fatty acids, PUFAs comprise two main groups of fats: omega- 3 and omega- 6 fatty acids. Omega- 6 is considered an inflammatory fat, whereas omega- 3 is anti-inflammatory. In human IBD, a systematic review has not supported the notion that supplementation of omega-3 fatty acids can induce and maintain remission of IBD [65]. The latest Cochrane review has reached a similar conclusion [66]. Interestingly, several studies have demonstrated that different genotypes can be associated with the variable response to nutritional intervention with omega-3 PUFAs. For example, genetic polymorphisms of TNF $\alpha$ and PPAR $\gamma$ have been associated with an altered response to nutritional intervention with omega-3 PUFAs [67]. In IBD, genetic polymorphisms, such as CYP4F3 and caspase $9+93 \mathrm{C} / \mathrm{T}$, modify the association between dietary fatty acid intake and risk of IBD [68]. Thus, specific mutations should also be considered before considering dietary therapeutic interventions.

\section{Conclusions}

Apart from EEN in specific situations, no strong evidence has been found to provide solid dietary intervention recommendations for patients with IBD. However, evidence and knowledge are accumulating and have suggested that pertinent studies should be prospectively developed in order to characterize potential dietary therapeutic possibilities. This body of knowledge leads us to recommend that a "prudent" diet or a "Mediterranean diet" should be advised to patients. Given that diet has been shown to be important for intestinal homeostasis and to avoid disruption of intestinal permeability, the consumption of vegetables, fruits, olive oil, fish, grains and nuts should be recommended, together with avoidance of the use of excessive additives and industrialized food processing.

Dietary recommendations for patients with IBD should emerge in a personalized manner, taking into consideration nutrient knowledge, the individual mucosa-microbiome-immunity interplay, the status of epigenetic changes and creeping fat metabolism. Dietary recommendations cannot be generalized for all patients, however. Given that the above elements differ per patient, no single dietary intervention can offer the same improvement for all patients. Thus, future studies should take this factor into account and be designed accordingly.

Author Contributions: Conceptualization, B.B., P.N. and I.M.; Writing-Original Draft Preparation, E.S.-G. and B.M.; Writing-Review \& Editing, E.S.-G., I.M. and P.L.-M.; Supervision, P.N. and M.I.

Funding: This research received no external funding.

Conflicts of Interest: The authors declare no conflict of interest.

\section{References}

1. Cosnes, J.; Gowerroisseau-Rousseau, C.; Seksik, P.; Cortot, A. Epidemiology and natural history of inflammatory bowel diseases. Gastroenterology 2011, 140, 1785-1794. [CrossRef] [PubMed]

2. Ananthakrishnan, A.N. Epidemiology and risk factors for IBD. Nat. Rev. Gastroenterol. Hepatol. 2015, 12, 205-217. [CrossRef]

3. Dutta, A.K.; Chacko, A. Influence of environmental factors on the onset and course of inflammatory bowel disease. World J. Gastroenterol. 2016, 22, 1088-1100. [CrossRef] [PubMed]

4. Zangenberg, M.S.; Horesh, N.; Kopylov, U.; El-Hussuna, A. Preoperative optimization of patients with inflammatory bowel disease undergoing gastrointestinal surgery: A systematic review. Int. J. Colorectal. Dis. 2017, 32, 1663-1676. [CrossRef] [PubMed]

5. Forbes, A.; Escher, J.; Hébuterne, X.; Kłęk, S.; Krznaric, Z.; Schneider, S.; Shamir, R.; Stardelova, K.; Wierdsma, N.; Wiskin, A.E.; et al. ESPEN guideline: Clinical nutrition in inflammatory bowel disease. Clin. Nutr. 2017, 36, 321-347. [CrossRef] [PubMed]

6. McDonald, P.J.; Fazio, V.W. What can Crohn's patients eat? Eur. J. Clin. Nutr. 1988, 42, 703-708. [PubMed]

7. Moser, G.; Tillinger, W.; Sachs, G.; Maier-Dobersberger, T.; Wyatt, J.; Vogelsang, H.; Lochs, H.; Gangl, A. Relationship between the use of unconventional therapies and disease-related concerns: A study of patients with inflammatory bowel disease. J. Psychosom. Res. 1996, 40, 503-509. [CrossRef]

8. Maynard, C.L.; Elson, C.O.; Hatton, R.D.; Weaver, C.T. Reciprocal interactions of the intestinal microbiota and immune system. Nature 2012, 489, 231-241. [CrossRef] [PubMed] 
9. Martini, E.; Krug, S.M.; Siegmund, B.; Neurath, M.F.; Becker, C. Mend Your Fences: The epithelial Barrier and its Relationship With Mucosal Immunity in Inflammatory Bowel Disease. Cell Mol. Gastroenterol. Hepatol. 2017, 23, 33-46. [CrossRef]

10. Jantchou, P.; Morois, S.; Clavel-Chapelon, F.; Boutron-Ruault, M.C.; Carbonnel, F. Animal protein intake and risk of inflammatory bowel disease: The E3N prospective study. Am. J. Gastroenterol. 2010, 105, 2195-2201. [CrossRef]

11. D'Souza, S.; Levy, E.; Mack, D.; Israel, D.; Lambrette, P.; Ghadirian, P.; Deslandres, C.; Morgan, K.; Seidman, E.G.; Amre, D.K. Dietary patterns and risk for Crohn's disease in children. Inflamm. Bowel Dis. 2008, 14, 367-373. [CrossRef]

12. Racine, A.; Carbonnel, F.; Chan, S.S.; Hart, A.R.; Bueno-de-Mesquita, H.B.; Oldenburg, B.; van Schalk, F.D.; Tjønneland, A.; Dahm, C.C.; Key, T.; et al. Dietary patterns and risk of inflammatory bowel disease in Europe: Results from the EPIC study. Inflamm. Bowel Dis. 2016, 22, 345-354. [CrossRef]

13. Shoda, R.; Matsueda, K.; Yamato, S.; Umeda, N. Epidemiologic analysis of Crohn disease in Japan: Increased dietary intake of n-6 polyunsaturated fatty acids and animal protein relates to the increased incidence of Crohn disease in Japan. Am. J. Clin. Nutr. 1996, 63, 741-745. [CrossRef] [PubMed]

14. Jowett, S.L.; Seal, C.J.; Pearce, M.S.; Phillips, E.; Gregory, W.; Barton, J.R.; Welfare, M.R. Influence of dietary factors on the clinical course of ulcerative colitis: A prospective cohort study. Gut 2004, 53, 1479-1484. [CrossRef]

15. Ananthakrishnan, A.N.; Khalili, H.; Konijeti, G.G.; Higuchi, L.M.; de Silva, P.; Korzenik, J.R.; Fuchs, C.S.; Willett, W.C.; Richter, J.M.; Chan, A.T. A prospective study of long-term intake of dietary fiber and risk of crohn's disease and ulcerative colitis. Gastroenterology 2013, 145, 970-977. [CrossRef]

16. Costea, I.; Mack, D.R.; Lemaitre, R.N.; Israel, D.; Marcil, V.; Ahmad, A.; Amre, D.K. Interactions between the dietary polyunsaturated fatty acid ratio and genetic factors determine susceptibility to pediatric Crohn's disease. Gastroenterology 2014, 146, 929-931. [CrossRef]

17. Chan, S.S.; Luben, R.; van Schaik, F.; Oldenburg, B.; Bueno-de-Mesquita, H.B.; Hallmans, G.; Karling, P.; Lindgren, S.; Grip, O.; Key, T.; et al. Carbohydrate intake in the etiology of Crohn's disease and ulcerative colitis. Inflamm. Bowel Dis. 2014, 20, 2013-2021. [CrossRef] [PubMed]

18. Torres, J.; Caprioli, F.; Katsanos, K.H.; Lobatón, T.; Micic, D.; Zerôncio, M.; Van Assche, G.; Lee, J.C.; Lindsay, J.O.; Rubin, D.T.; et al. Predicting outcomes to optimize disease management in inflammatory bowel diseases. J. Crohns. Colitis. 2016, 10, 1385-1394. [CrossRef] [PubMed]

19. Xu, L.; Lochhead, P.; Ko, Y.; Claggett, B.; Leong, R.W.; Ananthakrishnan, A.N. Systematic review with meta-analysis: Breastfeeding and the risk of Crohn's disease and ulcerative colitis. Aliment. Pharmacol. Ther. 2017, 46, 780-789. [CrossRef] [PubMed]

20. Hou, J.K.; Abraham, B.; El-Serag, H. Dietary intake and risk of developing inflammatory bowel disease: A systematic review of the literature. Am. J. Gastroenterol. 2011, 106, 563-573. [CrossRef] [PubMed]

21. Tjonneland, A.; Overvad, K.; Bergmann, M.M.; Nagel, G.; Linseisen, J.; Hallmans, G.; Palmqvist, R.; Sjodin, H.; Hagglund, G.; Berglund, G.; et al. Linoleic acid, a dietary n-6 polyunsaturated fatty acid, and the aetiology of ulcerative colitis: A nested case-control study within a European prospective cohort study. Gut 2009, 58, 1606-1611, Epub 23 Jul 2009. [CrossRef]

22. Chan, S.S.; Luben, R.; Olsen, A.; Tjonneland, A.; Kaaks, R.; Lindgren, S.; Grip, O.; Bergmann, M.M.; Boeing, H.; Hallmans, G.; et al. Association between high dietary intake of the n-3 polyunsaturated fatty acid docosahexaenoic acid and reduced risk of Crohn's disease. Aliment. Pharmacol. Ther. 2014, 39, 834-842. [CrossRef]

23. Niewiadomski, O.; Studd, C.; Wilson, J.; Williams, J.; Hair, C.; Knight, R.; Prewett, E.; Dabkowski, P.; Alexander, S.; Allen, B.; et al. Influence of food and lifestyle on the risk of developing inflammatory bowel disease. Intern. Med. J. 2016, 46, 669-676. [CrossRef] [PubMed]

24. Godny, L.; Maharshak, N.; Reshef, L.; Goren, I.; Yahav, L.; Fliss-Isakov, N.; Gophna, U.; Tulchinsky, H.; Dotan, I. Fruit consumption is associated with alterations in microbial composition and lower rates of pouchitis. J. Crohns. Colitis. 2019, 4. [CrossRef] [PubMed]

25. Chassaing, B.; Koren, O.; Goodrich, J.K.; Poole, A.C.; Srinivasan, S.; Ley, R.E.; Gewirtz, A.T. Dietary emulsifiers impact the mouse gut microbiota promoting colitis and metabolic syndrome. Nature 2015, 519, 92-96. [CrossRef] [PubMed] 
26. Taylor, L.; Almutairdi, A.; Shommu, N.; Fedorak, R.; Ghosh, S.; Reimer, R.A.; Panaccione, R.; Raman, M. Cross-sectional analysis of overall dietary intake and mediterranean dietary pattern in patients with crohn's disease. Nutrients 2018, 10, 1761. [CrossRef] [PubMed]

27. Salyers, A.A.; West, S.E.; Vercellotti, J.R.; Wilkins, T.D. Fermentation of mucins and plant polysaccharides by anaerobic bacteria from the human colon. Appl. Environ. Microbiol. 1977, 34, 529-533. [PubMed]

28. Marsman, K.E.; McBurney, M.I. Dietary fiber increases oxidative metabolism in colonocytes but not in distal small intestinal enterocytes isolated from rats. J. Nutr. 1995, 125, 273-282. [CrossRef]

29. Kelly, C.J.; Zheng, L.; Campbell, E.L.; Saeedi, B.; Scholz, C.C.; Bayless, A.J.; Wilson, K.E.; Glover, L.E.; Kominsky, D.J.; Magnuson, A.; et al. Crosstalk between Microbiota-Derived Short-Chain Fatty Acids and Intestinal Epithelial HIF Augments Tissue Barrier Function. Cell Host. Microbe. 2015, 17, 662-671. [CrossRef] [PubMed]

30. Saeedi, B.J.; Kao, D.J.; Kitzenberg, D.A.; Dobrinskikh, E.; Schwisow, K.D.; Masterson, J.C.; Kendrick, A.A.; Kelly, C.J.; Bayless, A.J.; Kominsky, D.J.; et al. HIF-dependent regulation of claudin-1 is central to intestinal epithelial tight junction integrity. Mol. Biol. Cell. 2015, 26, 2252-2262. [CrossRef] [PubMed]

31. Peng, L.; Li, Z.R.; Green, R.S.; Holzman, I.R.; Lin, J. Butyrate enhances the intestinal barrier by facilitating tight junction assembly via activation of AMP-activated protein kinase in Caco-2 cell monolayers. J. Nutr. 2009, 139, 1619-1625. [CrossRef] [PubMed]

32. Burger-van Paassen, N.; Vincent, A.; Puiman, P.J.; van der Sluis, M.; Bouma, J.; Boehm, G.; van Goudoever, J.B.; van Seuningen, I.; Renes, I.B. The regulation of intestinal mucin MUC2 expression by short-chain fatty acids: Implications for epithelial protection. Biochem. J. 2009, 420, 211219. [CrossRef] [PubMed]

33. Furusawa, Y.; Obata, Y.; Fukuda, S.; Endo, T.A.; Nakato, G.; Takahashi, D.; Nakanishi, Y.; Uetake, C.; Kato, K.; Kato, T.; et al. Commensal microbe-derived butyrate induces the differentiation of colonic regulatory $\mathrm{T}$ cells. Nature 2013, 504, 446-450. [CrossRef] [PubMed]

34. Desai, M.S.; Seekatz, A.M.; Koropatkin, N.M.; Kamada, N.; Hickey, C.A.; Wolter, M.; Pudlo, N.A.; Kitamoto, S.; Terrapon, N.; Muller, A.; et al. A dietary fiber-deprived gut microbiota degrades the colonic mucus barrier and enhances pathogen susceptibility. Cell 2016, 167, 1339-1353.e21. [CrossRef]

35. Bach Knudsen, K.E.; Lærke, H.N.; Hedemann, M.S.; Nielsen, T.S.; Ingerslev, A.K.; Gundelund Nielsen, D.S.; Theil, P.K.; Purup, S.; Hald, S.; Schioldan, A.G.; et al. Impact of Diet-Modulated Butyrate Production on Intestinal Barrier Function and Inflammation. Nutrients 2018, 10, 1499. [CrossRef]

36. Wang, H.; Shi, P.; Zuo, L.; Dong, J.; Zhao, J.; Liu, Q.; Zhu, W. Dietary Non-digestible polysaccharides ameliorate intestinal epithelial barrier dysfunction in il-10 knockout mice. J. Crohns. Colitis. 2016, 10, 1076-1086. [CrossRef] [PubMed]

37. Ma, X.; Torbenson, M.; Hamad, A.R.; Soloski, M.J.; Li, Z. High-fat diet modulates non-CD1d-restricted natural killer $\mathrm{T}$ cells and regulatory $\mathrm{T}$ cells in mouse colon and exacerbates experimental colitis. Clin. Exp. Immunol. 2008, 151, 130-138. [CrossRef] [PubMed]

38. Morgan, X.; Tickle, T.; Sokol, H.; Gevers, D.; Devaney, K.; Ward, D.; Reyes, J.A.; Shah, S.A.; LeLeiko, N.; Snapper, S.B.; et al. Dysfunction of the intestinal microbiome in inflammatory bowel disease and treatment. Genome. Biol. 2012, 13, R79. [CrossRef]

39. Davenport, M.; Poles, J.; Leung, J.M.; Wolff, M.J.; Abidi, W.M.; Ullman, T.; Mayer, L.; Cho, I.; Loke, P. Metabolic alterations to the mucosal microbiota inflammatory bowel disease. Inflamm. Bowel. Dis. 2014, 20, 723-731. [CrossRef]

40. Ren, W.; Liu, G.; Yin, J.; Tan, B.; Wu, G.; Bazer, F.W.; Peng, Y.; Yin, Y. Amino-acid transporters in T-cell activation and differentiation. Cell Death Dis. 2017, 8, 1-9. [CrossRef]

41. Langston, P.K.; Shibata, M.; Horng, T. Metabolism Supports Macrophage Activation. Front. Immunol. 2017, 8 , 61. [CrossRef] [PubMed]

42. Palego, L.; Betti, L.; Rossi, A.; Giannaccini, G. Tryptophan biochemistry: Structural, nutritional, metabolic, and medical aspects in humans. J. Amino Acids 2016, 2016, 1-13. [CrossRef] 
43. Lee, J.S.; Cella, M.; McDonald, K.G.; Garlanda, C.; Kennedy, G.D.; Nukaya, M.; Mantovani, A.; Kopan, R.; Bradfield, C.A.; Newberry, R.D.; et al. AHR drives the development of gut ILC22 cells and postnatal lymphoid tissues via pathways dependent on and independent of Notch. Nat. Immunol. 2012, 13, 144-152. [CrossRef] [PubMed]

44. Mezrich, J.D.; Fechner, J.H.; Zhang, X.; Johnson, B.P.; Burlingham, W.J.; Bradfield, C.A. An interaction between kynurenine and the aryl hydrocarbon receptor can generate regulatory T cells. J. Immunol. 2010, 185, 3190-3198. [CrossRef] [PubMed]

45. El-Zaatari, M.; Bass, A.J.; Bowlby, R.; Zhang, M.; Syu, L.J.; Yang, Y.; Grasberger, H.; Shreiner, A.; Tan, B.; Bishu, S.; et al. Indoleamine 2,3-Dioxygenase 1, increased in human gastric pre-neoplasia, promotes inflammation and metaplasia in mice and is associated with type II hypersensitivity/autoimmunity. Gastroenterology 2018, 154, 140-153.e17. [CrossRef] [PubMed]

46. Wagner, S.J.; Schmidt, A.; Effenberger, M.J.P.; Gruber, L.; Danier, J.; Haller, D. Semisynthetic diet ameliorates Crohn's disease-like ileitis in TNF $\triangle \mathrm{ARE} / \mathrm{WT}$ mice through antigen-independent mechanisms of gluten. Inflamm. Bowel Dis. 2013, 19, 1285-1294. [CrossRef]

47. Gonçalves, P.; Magro, F.; Martel, F. Metabolic inflammation in inflammatory bowel disease: Crosstalk between adipose tissue and bowel. Inflamm. Bowel Dis. 2015, 21, 453-467. [CrossRef]

48. Ventham, N.T.; Kennedy, N.A.; Nimmo, E.R.; Satsangi, J. Beyond gene discovery in inflammatory bowel disease: The emerging role of epigenetics. Gastroenterology 2013, 145, 293-308. [CrossRef]

49. Legaki, E.; Gazouli, M. Influence of environmental factors in the development of inflammatory bowel diseases. World J. Gastrointest. Pharmacol. Ther. 2016, 7, 112-125. [CrossRef]

50. Aleksandrova, K.; Romero-Mosquera, B.; Hernandez, V. Diet, gut microbiome and epigenetics: Emerging links with inflammatory bowel diseases and prospects for management and prevention. Nutrients 2017, 30, 962. [CrossRef]

51. Burdge, G.C.; Hoile, S.P.; Lillycrop, K.A. Epigenetics: Are there implications for personalised nutrition? Curr. Opin. Clin. Nutr. Metab. Care 2012, 15, 442-447. [CrossRef]

52. Remely, M.; Lovrecic, L.; de la Garza, A.L.; Migliore, L.; Peterlin, B.; Milagro, F.I.; Martinez, A.J.; Haslberger, A.G. Therapeutic perspectives of epigenetically active nutrients. Br. J. Pharm. 2015, 172, 2756-2768. [CrossRef]

53. Reuter, S.; Gupta, S.C.; Park, B.; Goel, A.; Aggarwal, B.B. Epigenetic changes induced by curcumin and other natural compounds. Genes Nutr. 2011, 6, 93-108. [CrossRef] [PubMed]

54. Arpón, A.; Milagro, F.I.; Razquin, C.; Corella, D.; Estruch, R.; Fitó, M.; Marti, A.; Martínez-González, M.A.; Ros, E.; Salas-Salvadó, J.; et al. Impact of consuming extra-virgin olive oil or nuts within a mediterranean diet on dna methylation in peripheral white blood cells within the predimed-navarra randomized controlled trial: A role for dietary lipids. Nutrients 2017, 10, 15. [CrossRef]

55. Ruemmele, F.M.; Veres, G.; Kolho, K.L.; Griffiths, A.; Levine, A.; Escher, J.C.; Amil-Dias, J.; Barabino, A.; Braegger, C.P.; Bronsky, J.; et al. Consensus guidelines of ECCO/ESPGHAN on the medical management of pediatric Crohn's disease. J. Crohns Colitis. 2014, 8, 1179-1207. [CrossRef] [PubMed]

56. Lee, D.; Baldassano, R.N.; Otley, A.R.; Albenberg, L.; Griffiths, A.M.; Compher, C.; Chen, E.Z.; Li, H.; Gilroy, E.; Nessel, L.; et al. Comparative effectiveness of nutritional and biological therapy in north american children with active crohn's disease. Inflamm. Bowel Dis. 2015, 21, 1786-1793. [CrossRef] [PubMed]

57. Levine, A.; Turner, D.; Pfeffer-Gik, T.; Amil-Dias, J.; Veres, G.; Shaoul, R.; Staiano, A.; Escher, J.; Kolho, K.L.; Paerregaard, A.; et al. Comparison of outcomes parameters for induction of remission in new onset pediatric Crohn's disease: Evaluation of the porto IBD group "growth relapse and outcomes with therapy" (GROWTH CD) study. Inflamm. Bowel Dis. 2014, 20, 278-285. [CrossRef] [PubMed]

58. Connors, J.; Basseri, S.; Grant, A.; Giffin, N.; Mahdi, G.; Noble, A.; Rashid, M.; Otley, A.; Van-Limbergen, J. Exclusive enteral nutrition therapy in paediatric crohn's disease results in long-term avoidance of corticosteroids: Results of a propensity-score matched cohort analysis. J. Crohns Colitis. 2017, 11, 1063-1070. [CrossRef]

59. Cohen-Dolev, N.; Sladek, M.; Hussey, S.; Turner, D.; Veres, G.; Koletzko, S.; Martin de Carpi, J.; Staiano, A.; Shaoul, R.; Lionetti, P.; et al. Differences in outcomes over time with exclusive enteral nutrition compared with steroids in children with mild to moderate crohn's disease: Results from the growth cd study. J. Crohns Colitis. 2018, 12, 306-312. [CrossRef] 
60. Levine, A.; Wine, E. Effects of enteral nutrition on Crohn's disease: Clues to the impact of diet on disease pathogenesis. Inflamm. Bowel Dis. 2013, 19, 1322-1329. [CrossRef]

61. Sigall-Boneth, R.; Pfeffer-Gik, T.; Segal, I.; Zangen, T.; Boaz, M.; Levine, A. Partial enteral nutrion with a Crohn's disease exclusion diet is effective for induction of remission in children and young adults with Crohn's disease. Inflamm. Bowel Dis. 2014, 20, 1353-1360. [CrossRef] [PubMed]

62. Takagi, S.; Utsunomiya, K.; Kuriyama, S.; Yokoyama, H.; Takahashi, S.; Iwabuchi, M.; Takahashi, S.; Kinouchi, Y.; Hiwatashi, N.; Funayama, Y.; et al. Effectiveness of an "half elemental diet" as maintenance therapy for Crohn's disease: A randomized controlled trial. Aliment. Pharmacol. Ther. 2006, 24, 1333-1340. [CrossRef]

63. Yamamoto, T.; Nakahigashi, M.; Umegae, S.; Kitagawa, T.; Matsumoto, K. Impact of long-term enteral nutrition on clinical and endoscopic recurrence after resection for Crohn's disease: A prospective, non-randomized, parallel, controlled study. Aliment. Pharmacol. Ther. 2007, 25, 67-72. [CrossRef]

64. Hisamatsu, T.; Kunisaki, R.; Nakamura, S.; Tsujikawa, T.; Hirai, F.; Nakase, H.; Watanabe, K.; Yokoyama, K.; Nagahori, M.; Kanai, T.; et al. Effect of elemental diet combined with infliximab dose escalation in patients with Crohn's disease with loss of response to infliximab: CERISIER trial. Intest. Res. 2018, 16, 494-498. [CrossRef]

65. Turner, D.; Shah, P.S.; Steinhart, A.H.; Zlotkin, S.; Griffiths, A.M. Maintenance of remission in inflammatory bowel disease using omega-3 fatty acids (fish oil): A systematic review and meta-analyses. Inflamm. Bowel. Dis. 2011, 17, 336-345. [CrossRef] [PubMed]

66. Turner, D.; Zlotkin, S.H.; Shah, P.S.; Griffiths, A.M. Omega 3 fatty acids (fish oil) for maintenance of remission in Crohn's disease. Cochrane Database Syst. Rev. 2009, 21, CD006320. [CrossRef]

67. Paradis, A.M.; Fontaine-Bisson, B.; Bossé, Y.; Robitaille, J.; Lemieux, S.; Jacques, H.; Lamarche, B.; Tchernof, A.; Couture, P.; Vohl, M.C. The peroxisome proliferator-activated receptor alpha Leu162Val polymorphism influences the metabolic response to a dietary intervention altering fatty acid proportions in healthy men. Am. J. Clin. Nutr. 2005, 81, 523-530. [CrossRef]

68. Ananthakrishnan, A.N.; Khalili, H.; Song, M.; Higuchi, L.M.; Lochhead, P.; Richter, J.M.; Chan, A.T. Genetic polymorphisms in fatty acid metabolism modify the association between dietary n3: n6 intake and risk of ulcerative colitis: A prospective cohort study. Inflamm. Bowel Dis. 2017, 23, 1898-1904. [CrossRef]

(C) 2019 by the authors. Licensee MDPI, Basel, Switzerland. This article is an open access article distributed under the terms and conditions of the Creative Commons Attribution (CC BY) license (http://creativecommons.org/licenses/by/4.0/). 


\title{
Inflammatory Bowel Diseases and Food Additives: To Add Fuel on the Flames!
}

\author{
Rachel Marion-Letellier 1,2,*, Asma Amamou 1,2, Guillaume Savoye 1,2,3 and Subrata Ghosh ${ }^{4}$ \\ 1 INSERM unit 1073, Normandie University, UNIROUEN, 22 boulevard Gambetta, F-76183 Rouen, France; \\ asma.amamou@etu.univ-rouen.fr (A.A.); guillaume.savoye@chu-rouen.fr (G.S.) \\ 2 Institute for Research and Innovation in Biomedicine (IRIB), Normandie University, UNIROUEN, \\ F-76183 Rouen, France \\ 3 Department of Gastroenterology, Rouen University Hospital, 1 rue de Germont, F-76031 Rouen, France \\ 4 Institute of Translational Medicine, University of Birmingham, Birmingham B15 2TT, UK; \\ sughosh@ymail.com \\ * Correspondence: rachel.letellier@univ-rouen.fr
}

Received: 28 April 2019; Accepted: 16 May 2019; Published: 18 May 2019

\begin{abstract}
Inflammatory bowel diseases (IBDs) develop in genetically predisposed individuals in response to environmental factors. IBDs are concomitant conditions of industrialized societies, and diet is a potential culprit. Consumption of ultra-processed food has increased over the last decade in industrialized countries, and epidemiological studies have found associations between ultra-processed food consumption and chronic diseases. Further studies are now required to identify the potential culprit in ultra-processed food, such as a poor nutritional composition or the presence of food additives. In our review, we will focus on food additives, i.e., substances from packaging in contact with food, and compounds formed during production, processing, and storage. A literature search using PubMed from inception to January 2019 was performed to identify relevant studies on diet and/or food additive and their role in IBDs. Manuscripts published in English from basic science, epidemiological studies, or clinical trials were selected and reviewed. We found numerous experimental studies highlighting the key role of food additives in IBD exacerbation but epidemiological studies on food additives on IBD risk are still limited. As diet is a modifiable environmental risk factor, this may offer a scientific rationale for providing dietary advice for IBD patients.
\end{abstract}

Keywords: colitis; food additive; diet; emulsifiers; high salt diet; inflammatory bowel diseases

\section{Introduction}

The most common types of inflammatory bowel diseases (IBDs) are Crohn's disease (CD) and ulcerative colitis (UC). IBD etiology is unknown, but IBDs develop in genetically predisposed individuals in response to environmental factors and the result of an exacerbated mucosal immune response to intestinal microbiota. IBDs are concomitant conditions of industrialized societies [1] Indeed, IBD prevalence has continued to increase in Western countries, and newly industrialized countries in Asia, the Middle East, Africa, and South America have exhibited a rapid increase of IBD prevalence [1].

Environmental factors are supposed to play a decisive role in the pathogenesis of IBDs. Diet is considered to be a potential culprit and we previously reviewed the potential effect of specific nutrients in IBDs [2]. With this study, we aimed to focus on another potential dietary culprit: food additives.

Consumption of ultra-processed food (UPF) has increased over the last decade, in particular in industrialized societies [3-5], and studies from the French web-based NutriNet-Santé cohort have found an association between UPF consumption and chronic diseases, such as a higher cancer risk [6]. UPF now represent an important part of the diet of French individuals: UPF accounted for $16 \%$ of 
food consumed by weight, corresponding to $33 \%$ of total energy intake [7]. In addition, the authors also reported an association between these dietary patterns and a higher irritable bowel syndrome risk (OR Q4 vs. Q1 [95\% CI]: 1.25 [1.12-1.39], p-trend < 0.0001) [7]. Similar associations between UPF consumption and chronic diseases are globally observed, such as metabolic syndrome in the US [4] or depression in Spain [5]. Further studies are now required to identify the potential culprit in UPF, such as a poor nutritional composition or the presence of food additives. In our review, we will focus on food additives, i.e., substances from packaging in contact with food, and compounds formed during production, processing, and storage.

\section{IBD Patients and Dietary Beliefs}

Diet is a crucial point for IBD patients [8-10] and exclusion diets are commonly reported. In a French study, the dietary beliefs of 244 IBD patients were studied using a questionnaire of 14 items [8]. The majority of IBD patients (58\%) believed that food can play a role in causing a relapse and this strongly affects quality of life because IBD patients may refuse outdoor dining for fear of causing IBD symptoms [8]. In a study conducted in the UK, 400 IBD patients received a dietary questionnaire and data from this study were in accordance with the French study. In this study, deteriorating symptoms were associated with certain foods from $60 \%$ of IBD patients [10]. Similar results were found in a Dutch study conducted in 294 IBD patients through an online questionnaire [9]. Interestingly, $81 \%$ of IBD patients from the Dutch study reported that their main nutritional knowledge came from their own experience, while $71 \%$ reported that they received professional dietary advice mainly from dieticians [9]. In a Spanish study, the majority of IBD patients $(86 \%)$ refrained from eating some foods to avoid a worsening of flare-ups [11]. In pediatric IBD populations, food avoidance is also commonly reported for $53 \%$ of patients [12].

\section{A Pinch of Salt}

Processed food is a high provider of dietary sodium chloride $(\mathrm{NaCl})$. Few recent studies have evaluated the potential impact of dietary salt in colitis models [13-15] (Figure 1). Tubbs et al. calculated the $\mathrm{NaCl}$ content of a series of available foods in grocery and fast food restaurants using the SELF Nutrition database, and they reported that these foods contained approximately $4 \% \mathrm{w} / \mathrm{w} \mathrm{NaCl}$ [13]. Increased concentration of $\mathrm{NaCl}$ from 10 to $80 \mathrm{mM}$ induced inflammatory cytokine production, such as the IL (interleukin)-23/IL-17 pathway in normal intestinal lamina propria mononuclear cells [14]. High-salt diet (HSD) exacerbated chemically induced colitis, while pharmacological inhibition of p38/MAPK abrogated its effect in both models [14]. Tubbs et al. demonstrated the deleterious effect of HSD in numerous colitis models, such IL-10/- or infectious colitis [13]. Interestingly, Aguiar et al. reported that dietary salt exacerbated colitis but by itself can trigger gut inflammation by increasing intestinal permeability and inflammatory histological score [15]. More recently, HSD also has been shown to have a deleterious impact on intestinal microbiota by decreasing Lactobaccilus levels and short-chain fatty acid production [16].

These studies raised the potential role of dietary salt as an environmental trigger for IBD development by creating a deleterious environment that is more vulnerable to inflammatory insults.

Dietary phosphate has been less studied than dietary salt. Nevertheless, dietary inorganic phosphate is abundant in processed food as a food additive, in particular in fast foods and processed meats. Phosphate intake is two or three times higher [17]: $1655 \mathrm{mg} /$ day for men and $1190 \mathrm{mg} /$ day for women in the US compared to dietary reference intake at $700 \mathrm{mg} /$ day in industrialized countries. Its deleterious effect on intestinal inflammation has also been demonstrated. Sugihara et al. fed Sprague-Dawley rats with a diet containing $0.5 \%$ to $1.5 \%$ phosphate for 7 days before colitis induction [18]. As standard animal diet contains approximatively $5000 \mathrm{mg} / \mathrm{kg}$ of phosphate, i.e., $0.5 \%$, the range of phosphate from $0.5 \%$ to $1.5 \%$ used in the study mimics the human exposure range from 1- to 3-fold of the recommended dietary intake. Dietary phosphate exacerbates colitis by accentuating body weight loss by increasing disease activity index and by activating NF (Nuclear factor)-кB [18]. The authors of the study also 
found an in vitro increased inflammatory response by $2 \mathrm{mM}$ phosphate in liposaccharide (LPS)-treated RAW264 cells [18].

To our knowledge, only one study has reported clinical data concerning salt consumption and IBDs [19]. In a US cohort from 194,711 women, the authors of this study reported that dietary intake of potassium $\left(P_{\text {trend }}=0.005\right)$ but not sodium $\left(P_{\text {trend }}=0.440\right)$ was inversely associated with risk of CD [19]. The authors did not observe any significant association between both dietary potassium and sodium and UC risk [19].

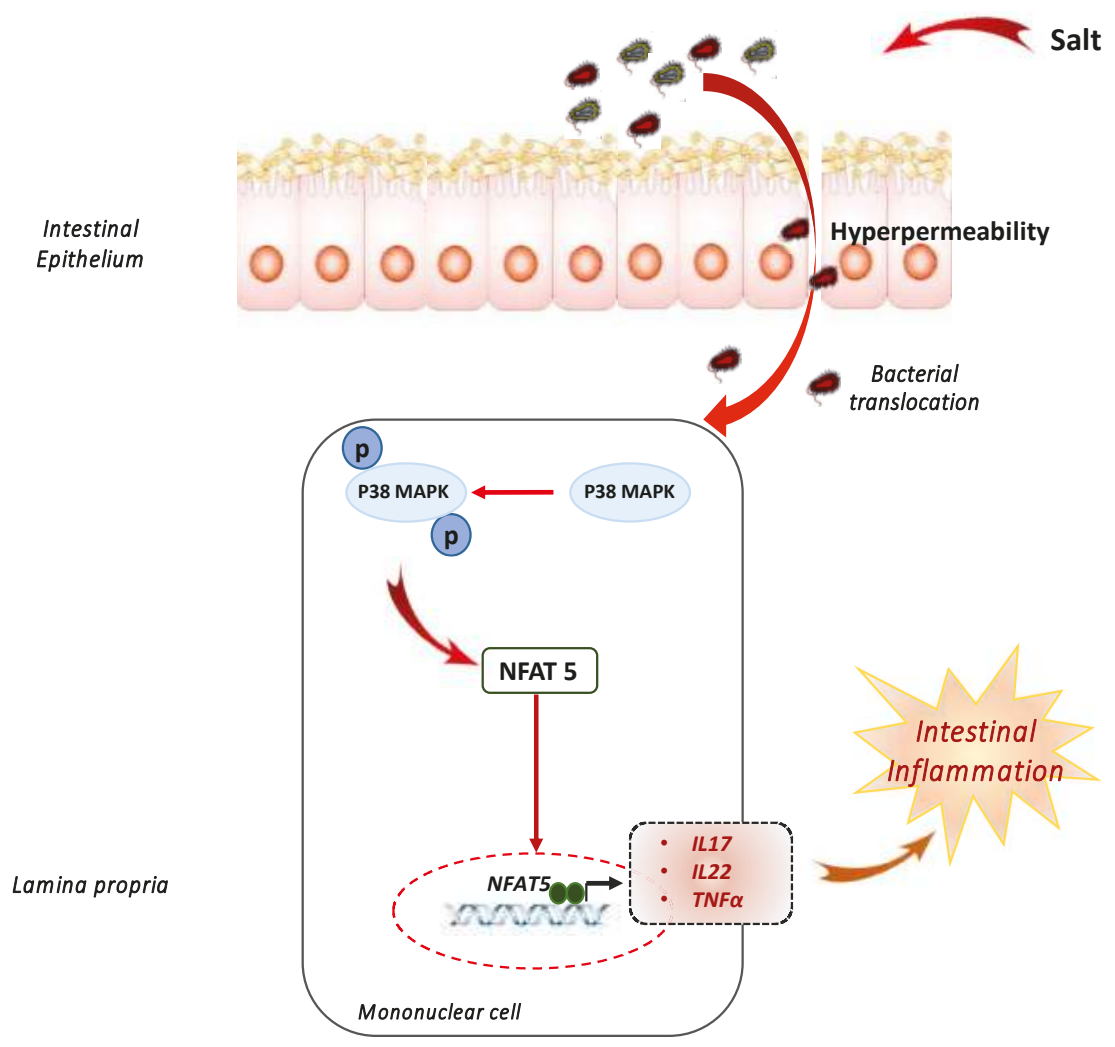

Figure 1. High-salt diet promotes intestinal inflammation. High-salt diet exposure led to higher intestinal permeability and dysbiosis. High-salt diet induced p38 MAPK phosphorylation and NFAT5 in lamina propria mononuclear cells activation with a subsequent expression of inflammatory cytokines expression such as IL17, IL22, and TNF $\alpha$.

\section{Take the Bitter with the Sweet}

Carbohydrates are obviously part of a normal diet but their excessive consumption is a feature of the Westernized diet. In addition, industrialized food contains hidden sugars, such as lactose as a texturing agent in sausages. There is currently no evidence of an association between carbohydrate intake and IBD risk in epidemiological studies [20]. Results from the prospective EPIC (European Prospective Investigation into Cancer and Nutrition) study, when excluding cases occurring within the first 2 years after dietary assessment, found a positive association between a "high sugar and soft drinks" pattern and UC risk (1.68 [1.00-2.82]; $\left.P_{\text {trend }}<0.05\right)$. When the authors considered the foods most associated with the pattern, high consumers of sugar and soft drinks were at higher UC risk only if they had low intakes of vegetables [21], but the amount of consumed sugar was not mentioned. 
Those with pediatric IBDs had a lower intake of carbohydrates compared to the general population, in particular a decreased intake of food types high in sugar [12].

Polysaccharides are commonly added in UPF as emulsifiers, coating agents, stabilizers, or bulking agents. These ingredients enable a better palatability and a longer shelf like of these products. Some of these ingredients have been studied in experimental models of IBDs [22-25].

Nickerson et al. focused their studies on the effect of the dietary polysaccharide maltodextrin to bacterial-induced intestinal inflammation [22-24]. Maltodextrin is commonly found in processed food and medications. Nickerson et al. demonstrated that maltodextrin can promote Salmonella survival in multiple cell types [24]. They also studied the effect of maltodextrin on CD-associated adherent-invasive Escherichia coli (AIEC) and found that the presence of maltodextrin enhanced AIEC adhesion by a better biofilm formation [22]. Maltodextrin consumption may promote intestinal dysbiosis and contribute to disease susceptibility. In a recent study, maltodextrin in drinking water exacerbated intestinal inflammation in an indomethacin-induced enteropathy model or in DSS (Dextran sodium sulfate)-colitis. The mechanisms behind maltodextrin's deleterious effects involved endoplasmic reticulum stress and led to mucin-2 depletion [25].

The consumption of sweeteners is becoming more and more frequent in the US general population. A recent study on 16,942 participants reported that $25 \%$ of children and $41 \%$ of adults in the US consumed foods and beverages containing low-calorie sweeteners [26]. Two large Swedish prospective cohort studies consisting of 83,042 participants did not find any association between sweetened beverages consumption and risk of later-onset CD or UC [27]. In South Korea, food additive consumption (such as sucralose) cannot explain the increased incidence of IBDs [28]. Increased IBD incidence started before the 1980s in South Korea, while sucralose was only approved in 2000 [28]. A recent study demonstrated the deleterious impact of an artificial sweetener called Splenda in experimental IBDs [29]. Splenda contains $1 \%$ sucralose and $99 \%$ maltodextrin. The authors of this study investigated the impact of 6 weeks of supplementation with Splenda in SAMP1/YitFc mice that spontaneously developed ileitis. Splenda supplementation induced ileal myeloperoxidase activity and promoted dysbiosis, in particular an expansion of proteobacteria [29].

Carrageenan is a polysaccharide commonly used as a food additive. In experimental models, it is also commonly used to induce an inflammatory response in vitro or in vivo [30,31]. The daily consumption of carrageenan in the US diet is approximatively $250 \mathrm{mg}$ per adult. Recently, a small clinical trial was conducted to evaluate the potential benefit of a carrageenan-free diet in UC patients [32]. Patients were instructed to follow a carrageenan-free diet by avoiding carrageenan-containing-food and received either 100 to $200 \mathrm{mg}$ of carrageenan capsules or placebo capsules. Carrageenan-supplemented patients had some higher inflammatory parameters, such as a higher clinical disease index and higher IL-6 and fecal calprotectin [32]. Carrageenan also has an impact on intestinal microbiota [33]. Colitis induction by carrageenan at $20 \mathrm{mg} / \mathrm{L}$ in drinking water decreased the level of an anti-inflammatory bacteria Akkermansia muciniphila [30].

Chassaing et al. did a remarkable study on two emulsifiers, carboxymethylcellulose (CMC) and polysorbate-80 (P80), on gut barrier [34-36]. They added emulsifiers in the mouse drinking water at a relative low level for 12 weeks in wild-type or IL-10-deficient mice [35]. Dietary emulsifiers altered gut microbiota composition and induced hyperpermeability [35]. Emulsifiers promote colitis in IL-10 ${ }^{-/}$mice by increasing inflammatory markers such as fecal lipocalin-2 or myeloperoxidase activity [35]. They demonstrated microbiota involvement in mediating the emulsifier effect by transferring intestinal microbiota from emulsifier-treated mice into unexposed germ-free mice; upon doing so, they observed that colonized germ-free mice had the same response [35]. They confirmed in a second study the direct impact of the emulsifiers on the microbiota using a mucosal simulator of the human intestinal microbial ecosystem; they observed that emulsifiers-promoted flagellin levels [36]. In another study, Chassaing et al. investigated whether emulsifiers can impact colon carcinogenesis [34]. They exposed mice with colitis-associated cancer to the same emulsifiers for 13 weeks and they observed that emulsifiers promote carcinogenesis through intestinal microbiota 
alteration, the establishment of a pro-inflammatory environment, and increased proliferation marker Ki67 [34]. Interestingly, they demonstrated in a recent study that emulsifiers also had a significant impact on anxiety [37], and psychological co-morbidities are commonly observed in IBD patients and IBD experimental models [38].

One-third of IBD patient underwent irritable bowel syndrome-like symptoms [39] and a recent meta-analysis confirmed that dietary restriction of fermentable oligosaccharide, disaccharide, monosaccharide, and polyol (FODMAP) reduced functional gastrointestinal symptoms such as bloating [40]. In heathy volunteers, mechanisms behind low-FODMAP diet involved decreased Bifidobacterium and reduced breath hydrogen [41].

\section{Put the Pedal to the Metal}

Aluminum is an abundant element commonly used in our environment. It is used in processed foods and cooking materials such as food packaging or foils. Cosmetic products and drugs are also a source of aluminum. The European Food Safety Authority set the tolerable weekly intake of $1 \mathrm{mg}$ aluminum $/ \mathrm{kg}$ body weight. Pineton de Chambrun et al. investigated the dietary effect of aluminum in colitis models. The strength of this study from Lille University is that the aluminum dose of $1.5 \mathrm{mg} / \mathrm{kg} /$ day that they used was relevant to human exposure [42]. Four weeks of oral administration of aluminum has been shown to worsen chemically-induced colitis models. Indeed, macroscopic and histologic scores and myeloperoxidase activity were higher in colitis mice exposed to aluminum. They also found a similar effect in IL-10/- mice. Exposure of epithelial cells lines with aluminum phosphate increased constitutive or LPS-induced cytokines mRNA level. Interestingly, aluminum exposure also delays mucosal repair capacity by inhibiting proliferating processes [43]. IBD patients often suffer from visceral pain even in remission periods [39], and a recent study from the same team has shown that dietary exposure of aluminum also induced visceral hypersensitivity in rodents [44]. This effect persisted over time after treatment cessation and involved mast cell activation [44].

\section{To Add or Not to Add}

Titanium dioxide $\left(\mathrm{TiO}_{2}\right)$ is a food pigment which increases opacity and confers a white color [45]. Dietary sources of $\mathrm{TiO}_{2}$ are numerous, from sweets to toothpaste, and it is often used to provide a natural whiteness and opacity to foods, such as icing on cakes. $\mathrm{TiO}_{2}$ has an optimal particle diameter of 200-300 nm, but a nano-sized fraction can be found because of manufacturing processes. These $\mathrm{TiO}_{2}$ nanoparticles, in Europe listed as E171, are commonly used as a food additive. We previously investigated the effects of microparticles on macrophages from CD patients, and we observed that microparticles alone did not induce an immune response [46]. By contrast, microparticles act as adjuvants to induce potent cytokine responses in the presence of bacterial antigens such as LPS [46]. In addition, macrophage function has been shown to be impaired in the presence of high concentrations of microparticles [46]. Houdeau et al. investigated the specific effect of E171 on gut barrier [47] at a relevant range for human exposure, because modeling of human exposure to $\mathrm{TiO}_{2}$ estimates an exposure of 2-3 mg/kg/day for children under the age of ten [48]. After one week of dietary exposure to $\mathrm{TiO}_{2}$ at $10 \mathrm{mg} / \mathrm{kg}$, titanium can be detected in Peyer's patches in rats [47] and it is associated with an unfavorable immune cell balance: an increased $\mathrm{CD} 11 \mathrm{~b} / \mathrm{c}^{+} \mathrm{CD} 103^{+} \mathrm{MHC}-\mathrm{II}^{+}$dendritic cell proportion and a decreased proportion of regulatory $\mathrm{T}$ cells [47]. This $\mathrm{TiO}_{2}$ effect is not transient because it lasts after 100 days of $\mathrm{TiO}_{2}$ treatment [47]. In addition, long-term exposition for 100 days is associated with low-grade colon inflammation and induced aberrant crypt foci in a chemically induced carcinogenesis model [47]. The effect of dietary exposure to titanium dioxide at 50 to $500 \mathrm{mg} / \mathrm{kg}$ on colitis development has been investigated [49]. Dietary titanium dioxide exacerbates acute DSS colitis by inducing a shortening of the colon and increasing infiltration and histological inflammatory scores [49]. The same experiment was performed in inflammasome Nlrp3-/- mice, and the results showed that dietary $\mathrm{TiO}_{2}$ at $500 \mathrm{mg} \cdot \mathrm{kg}^{-1}$ did not reproduce colitis exacerbation in Nlrp3/- compared to the response of the wild type mice. The mechanisms behind the dietary effect of $\mathrm{TiO}_{2}$ on colitis 
involved NLRP3. In vitro works demonstrated the accumulation of $\mathrm{TiO}_{2}$ in human intestinal epithelial and macrophage cell lines in a dose-dependent manner [49]. In addition, $\mathrm{TiO}_{2}$ at 20 to $100 \mu \mathrm{g} / \mathrm{mL}$ stimulates oxidative stress and increased intestinal permeability in human intestinal epithelial cell lines. Rogler et al. found that patients with active UC exhibited increased blood levels of titanium [49] In extra-intestinal organs, dietary exposure at 2.5 to $10 \mathrm{mg} / \mathrm{kg}$ to $\mathrm{TiO}_{2}$ for 6 months upregulated renal TGF $\beta$ and SMAD, signaling pathways involved in fibrosis [50]. We speculate that dietary $\mathrm{TiO}_{2}$ may also promote IBD-induced intestinal fibrosis, but this effect has not been yet documented. Two recent studies have proposed a dietary intervention to counteract $\mathrm{TiO}_{2}$-induced inflammation in mice. The administration of both flavonoids naringenin or quercetin inhibits $\mathrm{TiO}_{2}$-induced arthritis by decreasing cytokine production and oxidative stress [51,52].

Oral bisphenol A is a chemical used in food packages. Oral bisphenol A at a dose lower to the tolerable daily intake was able to induce intestinal hyperpermeability in rats, and this effect occurs in a dose-dependent manner [53]. In trinitrobenzene sulfonic acid (TNBS)-induced colitis, perinatal exposure of dietary bisphenol A increased myeloperoxidase activity in female rats but not in male rats [53].

Dietary bisphenol A induced visceral hypersensitivity in response to colorectal distension [53]. A recent study highlighted the effect of bisphenol A in chemically induced colitis in ovariectomized female mice, and the authors demonstrated that bisphenol A exposure led to a worsened colitis through intestinal microbiota metabolites, such as a decreased level of tryptophan [54].

Food heat treatment leads to neoformed compounds such as Maillard reaction products. Exposure to these Maillard reaction products alleviated inflammatory response [55] and dysbiosis in colitis models [56].

\section{Exclusion Diets}

Exclusion diets are commonly followed by IBD patients [57]. These exclusion diets have poor or no scientific rationale but expose IBD patients to nutritional deficiencies. The last European Society for Clinical Nutrition and Metabolism (ESPEN) guidelines about clinical nutrition in IBD patients declared that there is no IBD diet in active disease (strong consensus—-96\% agreement) [58]. We will limit our section to a few exclusion diets that may explain some benefits to limit food additive exposure.

Exclusive enteral nutrition (EEN) is recommended as first intention treatment to induce remission in children or teenagers with CD [58], although EEN is not recommended in adult patients. The mechanisms behind EEN are not fully understood, but it may involve dysbiosis correction or gut barrier maintenance [59]. EEN mechanisms may also involve the absence of specific deleterious food components, such as food additive exposure.

There is also concern about the presence of pesticides in the food chain, although there is still no evidence connecting pesticides to IBDs [60]. A recent study from the NutriNet French cohort found that more frequent organic food consumption decreased cancer risk [61].

We previously mentioned the effect of nanoparticles in gut barrier dysfunction in preclinical models, and two clinical trials have investigated the impact of low or microparticle-free diet in IBD patients. The first one was a very small trial in $20 \mathrm{CD}$ patients that showed a beneficial effect of low or microparticle-free diet in ileal CD patients, such as decreased corticosteroids intake [62].

The second study included 84 active CD patients for 16 weeks [63] and the authors did not find any benefit of the microparticle exclusion on inflammatory disease activity index, fecal calprotectin, or intestinal permeability [63]. We previously reported the effects of carrageenan on gut barrier in preclinical models. A small pilot study investigated the effect of carrageenan-free diet in 12 UC patients [32]. Three out of ten UC patients who followed the carrageenan-free diet for 12 months relapsed compared to three out of five patients in the control group (RR (Relative risk) $0.50,95 \% \mathrm{CI}$ 0.15 to 1.64$)$, but it may be difficult to conclude that there are potentially beneficial effects because of the very small size of the study. 
Lactose-free diets are common in IBD patients. In a small recent study on 78 IBD patients from Iceland, $60 \%$ (47 patients) reported limiting their dairy consumption or even excluding it, but only eight of them used calcium supplements [64]. Acquired lactase deficiency is commonly reported in CD patients, in particular in proximal CD. In a clinical trial in 77 UC patients published in 1965, milk-free diets had only a benefit for one out of five patients [65] and did not justify dairy-restricted diets in all IBD patients. In 29 pediatric UC patients, exclusion of cow milk proteins had no influence on induction and/or maintenance of remission [66].

Paleolithic diet is another exclusion diet without scientific rationale. Its principles are that our gastrointestinal system did not evolve alongside our modernized diet and may explain numerous inflammatory diseases. This diet excludes a lot of foodstuffs, such as ultra-processed foods or cereals, and is limited to wild meats and a non-cereal, plant-based diet. To our knowledge, the only published data about paleo diet and IBDs is a Hungarian case report about a teen CD patient's refractory to treatments. This CD patient had a clinical remission after 2 weeks on a paleo diet and was still in remission 15 months later [67]. Extrapolation from a unique case control study is not permitted, and exclusion of so many foodstuffs may be associated with numerous nutritional deficiencies.

One-third of IBD patients underwent IBS-like symptoms, and partial exclusion of some fermentable components may be associated with benefits to functional symptoms. For example, in a small study with 16 CD patients in remission, a strict exclusion diet of wheat and dairy products for 2 weeks significantly decreased function symptoms such as abdominal pain [68]. In a prospective study with 89 IBD patients in remission, a low-FODMAP diet for 6 weeks reduced IBS-like symptoms while it increased quality of life in patients [69].

Exclusion diets are not recommended and may expose patients to numerous nutritional deficiencies. In addition, cofounding factors are often observed, leading to unjustified dietary exclusion. Recently, a double-blind clinical trial identified that fructan composition, instead of gluten, induced functional symptoms in patients with self-reported non-celiac gluten sensitivity [69]. Similar results were previously reported concerning lactose intolerance [70]. Avoiding some foods has been identified as a risk factor for malnutrition in IBD patients in a Spanish cohort [71]. Of note, malnutrition increased further complications, such as higher surgery and hospitalization rates, and malnutrition is associated with altered quality of life in IBD patients [11,72]. In addition, exclusion diets may limit socializing and dining because many IBD patients reportedly refused dining out because doing so would not conform with their exclusion diet [11]. Exclusion of dairy products such as in a lactose-free diet or paleo diet may increase osteoporosis risk in IBD patients, while gluten-free diets are associated with decreased microbiota diversity.

\section{Conclusions}

Diet is a modifiable environmental risk factor and food additives may act as potentiators of disease. Diet is a crucial point for IBD patients and exclusion diets appear to offer an alternative allowing for relative control of disease development. Nevertheless, the last ESPEN guidelines did not recommend any exclusion diet for induction or maintenance of remission in IBD patients but advised IBD patients to undergo counselling by a dietitian to avoid malnutrition. In addition, it has been recently reported that IBD patients in remission have an unbalanced dietary profile for essential nutrients [73]. Rather than exclusion diets, home-made food may be prioritized to decrease food additive amounts and enable patients to control their exposure to many hidden ingredients, such as added salt or sugars. Home-made food is already recommended in the last nutritional guidelines in France and, contrary to exclusion diets, cooking can represent a form of a convivial time, allowing a better quality of life with increased socialization. In addition, obesity is more and more frequent in IBD patients [74] and food preparation from scratch may be associated with a decreased risk of obesity [75] or decreased amount of dietary energy from ultra-processed food [76].

Funding: This research received no external funding. 
Conflicts of Interest: The authors declare no conflict of interest.

\section{Abbreviations}

$\begin{array}{ll}\text { AIEC } & \text { Adherent-invasive Escherichia coli } \\ \text { CD } & \text { Crohn's disease } \\ \text { CMC } & \text { carboxymethylcellulose } \\ \text { EEN } & \text { exclusive enteral nutrition } \\ \text { ESPEN } & \text { European Society for Clinical Nutrition and Metabolism } \\ \text { FODMAP } & \text { fermentable oligosaccharide, disaccharide monosaccharide, and polyol } \\ \text { HSD } & \text { High-salt diet } \\ \text { IBD } & \text { inflammatory bowel diseases } \\ \text { LPS } & \text { liposaccharide } \\ \text { NFAT5 } & \text { nuclear factor of activated T cells } 5 \\ \text { NLRP } & \text { NOD-like receptor pyrin } \\ \text { P80 } & \text { polysorbate-80 } \\ \text { SCFA } & \text { short-chain fatty acid } \\ \text { SMAD } & \text { small mothers against decapentaplegic } \\ \text { TNBS } & \text { trinitrobenzene sulfonic acid } \\ \text { TiO2 } & \text { titanium dioxide } \\ \text { UC } & \text { ulcerative colitis } \\ \text { UPF } & \text { ultra-processed food }\end{array}$

\section{References}

1. Kaplan, G.G.; $\mathrm{Ng}$, S.C. Understanding and preventing the global increase of inflammatory bowel disease. Gastroenterology 2017, 152, 313-321. [CrossRef]

2. Marion-Letellier, R.; Savoye, G.; Ghosh, S. IBD: In food we trust. J. Crohn's Colitis 2016, 10, $1351-1361$. [CrossRef]

3. Marron-Ponce, J.A.; Tolentino-Mayo, L.; Hernandez, F.M.; Batis, C. Trends in ultra-processed food purchases from 1984 to 2016 in Mexican households. Nutrients 2018, 11, 45. [CrossRef]

4. Steele, E.M.; Juul, F.; Neri, D.; Rauber, F.; Monteiro, C.A. Dietary share of ultra-processed foods and metabolic syndrome in the US adult population. Prev. Med. 2019. [CrossRef]

5. Gomez-Donoso, C.; Sanchez-Villegas, A.; Martinez-Gonzalez, M.A.; Gea, A.; Mendonca, R.D.; Lahortiga-Ramos, F.; Bes-Rastrollo, M. Ultra-processed food consumption and the incidence of depression in a Mediterranean cohort: The SUN Project. Eur. J. Nutr. 2019, 1-11. [CrossRef]

6. Fiolet, T.; Srour, B.; Sellem, L.; Kesse-Guyot, E.; Alles, B.; Mejean, C.; Deschasaux, M.; Fassier, P.; Latino-Martel, P.; Beslay, M.; et al. Consumption of ultra-processed foods and cancer risk: Results from NutriNet-Sante prospective cohort. BMJ 2018, 360, k322. [CrossRef]

7. Schnabel, L.; Buscail, C.; Sabate, J.M.; Bouchoucha, M.; Kesse-Guyot, E.; Alles, B.; Touvier, M.; Monteiro, C.A.; Hercberg, S.; Benamouzig, R.; et al. Association between ultra-processed food consumption and functional gastrointestinal disorders: Results from the french nutrinet-sante cohort. Am. J. Gastroenterol. 2018, 113, 1217-1228. [CrossRef] [PubMed]

8. Zallot, C.; Quilliot, D.; Chevaux, J.B.; Peyrin-Biroulet, C.; Gueant-Rodriguez, R.M.; Freling, E.; Collet-Fenetrier, B.; Williet, N.; Ziegler, O.; Bigard, M.A.; et al. Dietary beliefs and behavior among inflammatory bowel disease patients. Inflamm. Bowel Dis. 2013, 19, 66-72. [CrossRef] [PubMed]

9. de Vries, J.H.M.; Dijkhuizen, M.; Tap, P.; Witteman, B.J.M. Patient's dietary beliefs and behaviours in inflammatory bowel disease. Dig. Dis. (Basel Switz.) 2019, 37, 131-139. [CrossRef]

10. Limdi, J.K.; Aggarwal, D.; McLaughlin, J.T. Dietary practices and beliefs in patients with inflammatory bowel disease. Inflamm. Bowel Dis. 2016, 22, 164-170. [CrossRef] [PubMed]

11. Casanova, M.J.; Chaparro, M.; Molina, B.; Merino, O.; Batanero, R.; Duenas-Sadornil, C.; Robledo, P.; Garcia-Albert, A.M.; Gomez-Sanchez, M.B.; Calvet, X.; et al. Prevalence of malnutrition and nutritional characteristics of patients with inflammatory bowel disease. J. Crohn's Colitis 2017, 11, 1430-1439. [CrossRef] 
12. Diederen, K.; Krom, H.; Koole, J.C.D.; Benninga, M.A.; Kindermann, A. Diet and anthropometrics of children with inflammatory bowel disease: A comparison with the general population. Inflamm. Bowel Dis. 2018, 24, 1632-1640. [CrossRef]

13. Tubbs, A.L.; Liu, B.; Rogers, T.D.; Sartor, R.B.; Miao, E.A. Dietary salt exacerbates experimental colitis. J. Immunol. 2017, 199, 1051-1059. [CrossRef]

14. Monteleone, I.; Marafini, I.; Dinallo, V.; Di Fusco, D.; Troncone, E.; Zorzi, F.; Laudisi, F.; Monteleone, G. Sodium chloride-enriched diet enhanced inflammatory cytokine production and exacerbated experimental colitis in mice. J. Crohn's Colitis 2017, 11, 237-245. [CrossRef] [PubMed]

15. Aguiar, S.L.F.; Miranda, M.C.G.; Guimaraes, M.A.F.; Santiago, H.C.; Queiroz, C.P.; Cunha, P.D.S.; Cara, D.C.; Foureaux, G.; Ferreira, A.J.; Cardoso, V.N.; et al. High-salt diet induces IL-17-Dependent gut inflammation and exacerbates colitis in mice. Front. Immunol. 2017, 8, 1969. [CrossRef] [PubMed]

16. Miranda, P.M.; De Palma, G.; Serkis, V.; Lu, J.; Louis-Auguste, M.P.; McCarville, J.L.; Verdu, E.F.; Collins, S.M.; Bercik, P. High salt diet exacerbates colitis in mice by decreasing Lactobacillus levels and butyrate production. Microbiome 2018, 6, 57. [CrossRef]

17. Calvo, M.S.; Moshfegh, A.J.; Tucker, K.L. Assessing the health impact of phosphorus in the food supply: Issues and considerations. Adv. Nutr. 2014, 5, 104-113. [CrossRef]

18. Sugihara, K.; Masuda, M.; Nakao, M.; Abuduli, M.; Imi, Y.; Oda, N.; Okahisa, T.; Yamamoto, H.; Takeda, E.; Taketani, Y. Dietary phosphate exacerbates intestinal inflammation in experimental colitis. J. Clin. Biochem. Nutr. 2017, 61, 91-99. [CrossRef]

19. Khalili, H.; Malik, S.; Ananthakrishnan, A.N.; Garber, J.J.; Higuchi, L.M.; Joshi, A.; Peloquin, J.; Richter, J.M.; Stewart, K.O.; Curhan, G.C.; et al. Identification and characterization of a novel association between dietary potassium and risk of Crohn's disease and ulcerative colitis. Front. Immunol. 2016, 7, 554. [CrossRef]

20. Chan, S.S.; Luben, R.; van Schaik, F.; Oldenburg, B.; Bueno-de-Mesquita, H.B.; Hallmans, G.; Karling, P.; Lindgren, S.; Grip, O.; Key, T.; et al. Carbohydrate intake in the etiology of Crohn's disease and ulcerative colitis. Inflamm. Bowel Dis. 2014, 20, 2013-2021. [CrossRef]

21. Racine, A.; Carbonnel, F.; Chan, S.S.; Hart, A.R.; Bueno-de-Mesquita, H.B.; Oldenburg, B.; van Schaik, F.D.; Tjonneland, A.; Olsen, A.; Dahm, C.C.; et al. Dietary patterns and risk of inflammatory bowel disease in Europe: Results from the EPIC study. Inflamm. Bowel Dis. 2016, 22, 345-354. [CrossRef]

22. Nickerson, K.P.; McDonald, C. Crohn's disease-associated adherent-invasive Escherichia coli adhesion is enhanced by exposure to the ubiquitous dietary polysaccharide maltodextrin. PLoS ONE 2012, 7, e52132. [CrossRef]

23. Nickerson, K.P.; Homer, C.R.; Kessler, S.P.; Dixon, L.J.; Kabi, A.; Gordon, I.O.; Johnson, E.E.; de la Motte, C.A.; McDonald, C. The dietary polysaccharide maltodextrin promotes Salmonella survival and mucosal colonization in mice. PLoS ONE 2014, 9, e101789. [CrossRef]

24. Nickerson, K.P.; Chanin, R.; McDonald, C. Deregulation of intestinal anti-microbial defense by the dietary additive, maltodextrin. Gut Microbes 2015, 6, 78-83. [CrossRef]

25. Laudisi, F.; Di Fusco, D.; Dinallo, V.; Stolfi, C.; Di Grazia, A.; Marafini, I.; Colantoni, A.; Ortenzi, A.; Alteri, C.; Guerrieri, F.; et al. The food additive maltodextrin promotes endoplasmic reticulum stress-driven mucus depletion and exacerbates intestinal inflammation. Cell. Mol. Gastroenterol. Hepatol. 2019, 7, 457-473. [CrossRef]

26. Sylvetsky, A.C.; Jin, Y.; Clark, E.J.; Welsh, J.A.; Rother, K.I.; Talegawkar, S.A. Consumption of low-calorie sweeteners among children and adults in the United States. J. Acad. Nutr. Diet. 2017, 117, 441-448. [CrossRef]

27. Khalili, H.; Hakansson, N.; Chan, S.S.; Ludvigsson, J.F.; Olen, O.; Chan, A.T.; Hart, A.R.; Wolk, A. No association between consumption of sweetened beverages and risk of later-onset Crohn's disease or ulcerative colitis. Clin. Gastroenterol. Hepatol. Off. Clin. Pract. J. Am. Gastroenterol. Assoc. 2019, 17, 123-129. [CrossRef]

28. Ahn, H.S. Increased incidence of inflammatory bowel disease in Korea may not be explained by food additives. Inflamm. Bowel Dis. 2015, 21, E17. [CrossRef]

29. Rodriguez-Palacios, A.; Harding, A.; Menghini, P.; Himmelman, C.; Retuerto, M.; Nickerson, K.P.; Lam, M.; Croniger, C.M.; McLean, M.H.; Durum, S.K.; et al. The artificial sweetener splenda promotes gut proteobacteria, dysbiosis, and myeloperoxidase reactivity in Crohn's disease-like ileitis. Inflamm. Bowel Dis. 2018, 24, 1005-1020. [CrossRef] 
30. Shang, Q.; Sun, W.; Shan, X.; Jiang, H.; Cai, C.; Hao, J.; Li, G.; Yu, G. Carrageenan-induced colitis is associated with decreased population of anti-inflammatory bacterium, Akkermansia muciniphila, in the gut microbiota of C57BL/6J mice. Toxicol. Lett. 2017, 279, 87-95. [CrossRef]

31. Fahoum, L.; Moscovici, A.; David, S.; Shaoul, R.; Rozen, G.; Meyron-Holtz, E.G.; Lesmes, U. Digestive fate of dietary carrageenan: Evidence of interference with digestive proteolysis and disruption of gut epithelial function. Mol. Nutr. Food Res. 2017, 61. [CrossRef] [PubMed]

32. Bhattacharyya, S.; Shumard, T.; Xie, H.; Dodda, A.; Varady, K.A.; Feferman, L.; Halline, A.G.; Goldstein, J.L.; Hanauer, S.B.; Tobacman, J.K. A randomized trial of the effects of the no-carrageenan diet on ulcerative colitis disease activity. Nutr. Healthy Aging 2017, 4, 181-192. [CrossRef] [PubMed]

33. Munyaka, P.M.; Sepehri, S.; Ghia, J.-E.; Khafipour, E. Carrageenan gum and adherent invasive escherichia coli in a piglet model of inflammatory bowel disease: Impact on Intestinal mucosa-associated microbiota. Front. Microbiol. 2016, 7, 462. [CrossRef]

34. Viennois, E.; Merlin, D.; Gewirtz, A.T.; Chassaing, B. Dietary emulsifier-induced low-grade inflammation promotes colon carcinogenesis. Cancer Res. 2017, 77, 27-40. [CrossRef]

35. Chassaing, B.; Koren, O.; Goodrich, J.K.; Poole, A.C.; Srinivasan, S.; Ley, R.E.; Gewirtz, A.T. Dietary emulsifiers impact the mouse gut microbiota promoting colitis and metabolic syndrome. Nature 2015, 519, 92-96. [CrossRef]

36. Chassaing, B.; Van de Wiele, T.; De Bodt, J.; Marzorati, M.; Gewirtz, A.T. Dietary emulsifiers directly alter human microbiota composition and gene expression ex vivo potentiating intestinal inflammation. Gut 2017, 66, 1414-1427. [CrossRef] [PubMed]

37. Holder, M.K.; Peters, N.V.; Whylings, J.; Fields, C.T.; Gewirtz, A.T.; Chassaing, B.; de Vries, G.J. Dietary emulsifiers consumption alters anxiety-like and social-related behaviors in mice in a sex-dependent manner. Sci. Rep. 2019, 9, 172. [CrossRef]

38. Salameh, E.; Meleine, M.; Gourcerol, G.; do Rego, J.C.; do Rego, J.L.; Legrand, R.; Breton, J.; Aziz, M.; Guerin, C.; Coeffier, M.; et al. Chronic colitis-induced visceral pain is associated with increased anxiety during quiescent phase. Am. J. Physiol. Gastrointest. Liver Physiol. 2019. [CrossRef]

39. Vivinus-Nebot, M.; Frin-Mathy, G.; Bzioueche, H.; Dainese, R.; Bernard, G.; Anty, R.; Filippi, J.; Saint-Paul, M.C.; Tulic, M.K.; Verhasselt, V.; et al. Functional bowel symptoms in quiescent inflammatory bowel diseases: Role of epithelial barrier disruption and low-grade inflammation. Gut 2014, 63, 744-752. [CrossRef]

40. Zhan, Y.L.; Zhan, Y.A.; Dai, S.X. Is a low FODMAP diet beneficial for patients with inflammatory bowel disease? A meta-analysis and systematic review. Clin. Nutr. (Edinb. Scotl.) 2018, 37, 123-129. [CrossRef]

41. Sloan, T.J.; Jalanka, J.; Major, G.A.D.; Krishnasamy, S.; Pritchard, S.; Abdelrazig, S.; Korpela, K.; Singh, G.; Mulvenna, C.; Hoad, C.L.; et al. A low FODMAP diet is associated with changes in the microbiota and reduction in breath hydrogen but not colonic volume in healthy subjects. PLOS ONE 2018, 13, e0201410. [CrossRef]

42. Greger, J.L.; Sutherland, J.E. Aluminum exposure and metabolism. Crit. Rev. Clin. Lab. Sci. 1997, 34, 439-474. [CrossRef] [PubMed]

43. de Chambrun, G.P.; Body-Malapel, M.; Frey-Wagner, I.; Djouina, M.; Deknuydt, F.; Atrott, K.; Esquerre, N.; Altare, F.; Neut, C.; Arrieta, M.C.; et al. Aluminum enhances inflammation and decreases mucosal healing in experimental colitis in mice. Mucosal Immunol. 2014, 7, 589-601. [CrossRef]

44. Esquerre, N.; Basso, L.; Dubuquoy, C.; Djouina, M.; Chappard, D.; Blanpied, C.; Desreumaux, P.; Vergnolle, N.; Vignal, C.; Body-Malapel, M. Aluminum ingestion promotes colorectal hypersensitivity in rodents. Cell. Mol. Gastroenterol. Hepatol. 2018, 7, 185-196. [CrossRef] [PubMed]

45. Winkler, H.C.; Notter, T.; Meyer, U.; Naegeli, H. Critical review of the safety assessment of titanium dioxide additives in food. J. Nanobiotechn. 2018, 16, 51. [CrossRef] [PubMed]

46. Butler, M.; Boyle, J.J.; Powell, J.J.; Playford, R.J.; Ghosh, S. Dietary microparticles implicated in Crohn's disease can impair macrophage phagocytic activity and act as adjuvants in the presence of bacterial stimuli. Inflamm. Res. Off. J. Eur. Histamine Res. Soc. 2007, 56, 353-361. [CrossRef] [PubMed]

47. Bettini, S.; Boutet-Robinet, E.; Cartier, C.; Comera, C.; Gaultier, E.; Dupuy, J.; Naud, N.; Tache, S.; Grysan, P.; Reguer, S.; et al. Food-grade TiO2 impairs intestinal and systemic immune homeostasis, initiates preneoplastic lesions and promotes aberrant crypt development in the rat colon. Sci. Rep. 2017, 7, 40373. [CrossRef] [PubMed] 
48. Weir, A.; Westerhoff, P.; Fabricius, L.; Hristovski, K.; von Goetz, N. Titanium dioxide nanoparticles in food and personal care products. Environ. Sci. Technol. 2012, 46, 2242-2250. [CrossRef]

49. Ruiz, P.A.; Moron, B.; Becker, H.M.; Lang, S.; Atrott, K.; Spalinger, M.R.; Scharl, M.; Wojtal, K.A.; Fischbeck-Terhalle, A.; Frey-Wagner, I.; et al. Titanium dioxide nanoparticles exacerbate DSS-induced colitis: Role of the NLRP3 inflammasome. Gut 2017, 66, 1216-1224. [CrossRef] [PubMed]

50. Hong, F.; Wu, N.; Ge, Y.; Zhou, Y.; Shen, T.; Qiang, Q.; Zhang, Q.; Chen, M.; Wang, Y.; Wang, L.; et al. Nanosized titanium dioxide resulted in the activation of TGF-beta/Smads/p38MAPK pathway in renal inflammation and fibration of mice. J. Biomed. Mater. Res. Part A 2016, 104, 1452-1461. [CrossRef]

51. Borghi, S.M.; Mizokami, S.S.; Pinho-Ribeiro, F.A.; Fattori, V.; Crespigio, J.; Clemente-Napimoga, J.T.; Napimoga, M.H.; Pitol, D.L.; Issa, J.P.M.; Fukada, S.Y.; et al. The flavonoid quercetin inhibits titanium dioxide (TiO2)-induced chronic arthritis in mice. J. Nutr. Biochem. 2018, 53, 81-95. [CrossRef]

52. Manchope, M.F.; Artero, N.A.; Fattori, V.; Mizokami, S.S.; Pitol, D.L.; Issa, J.P.M.; Fukada, S.Y.; Cunha, T.M.; Alves-Filho, J.C.; Cunha, F.Q.; et al. Naringenin mitigates titanium dioxide (TiO2)-induced chronic arthritis in mice: Role of oxidative stress, cytokines, and NFkappaB. Inflamm. Res. Off. J. Eur. Histamine Res. Soc. 2018, 67, 997-1012. [CrossRef]

53. Braniste, V.; Jouault, A.; Gaultier, E.; Polizzi, A.; Buisson-Brenac, C.; Leveque, M.; Martin, P.G.; Theodorou, V.; Fioramonti, J.; Houdeau, E. Impact of oral bisphenol A at reference doses on intestinal barrier function and sex differences after perinatal exposure in rats. Proc. Natl. Acad. Sci. USA 2010, 107, 448-453. [CrossRef] [PubMed]

54. DeLuca, J.A.; Allred, K.F.; Menon, R.; Riordan, R.; Weeks, B.R.; Jayaraman, A.; Allred, C.D. Bisphenol-A alters microbiota metabolites derived from aromatic amino acids and worsens disease activity during colitis. Exp. Biol. Med. 2018, 243, 864-875. [CrossRef]

55. Al Amir, I.; Dubayle, D.; Heron, A.; Delayre-Orthez, C.; Anton, P.M. Maillard reaction products from highly heated food prevent mast cell number increase and inflammation in a mouse model of colitis. Nutr. Res. 2017, 48, 26-32. [CrossRef] [PubMed]

56. Jahdali, N.A.L.; Gadonna-Widehem, P.; Delayre-Orthez, C.; Marier, D.; Garnier, B.; Carbonero, F.; Anton, P.M. Repeated oral exposure to $\mathrm{N}$ (epsilon)-Carboxymethyllysine, a maillard reaction product, alleviates gut microbiota dysbiosis in colitic mice. Dig. Dis. Sci. 2017, 62, 3370-3384. [CrossRef]

57. Limketkai, B.N.; Iheozor-Ejiofor, Z.; Gjuladin-Hellon, T.; Parian, A.; Matarese, L.E.; Bracewell, K.; MacDonald, J.K.; Gordon, M.; Mullin, G.E. Dietary interventions for induction and maintenance of remission in inflammatory bowel disease. Cochrane Database Syst. Rev. 2019, 2, CD012839. [CrossRef]

58. Forbes, A.; Escher, J.; Hebuterne, X.; Klek, S.; Krznaric, Z.; Schneider, S.; Shamir, R.; Stardelova, K.; Wierdsma, N.; Wiskin, A.E.; et al. ESPEN guideline: Clinical nutrition in inflammatory bowel disease. Clin. Nutr. (Edinb. Scotl.) 2017, 36, 321-347. [CrossRef]

59. Altomare, R.; Damiano, G.; Abruzzo, A.; Palumbo, V.D.; Tomasello, G.; Buscemi, S.; Lo Monte, A.I. Enteral nutrition support to treat malnutrition in inflammatory bowel disease. Nutrients 2015, 7, 2125-2133. [CrossRef]

60. Arcella, D.; Boobis, A.; Cressey, P.; Erdely, H.; Fattori, V.; Leblanc, J.C.; Lipp, M.; Reuss, R.; Scheid, S.; Tritscher, A.; et al. Harmonized methodology to assess chronic dietary exposure to residues from compounds used as pesticide and veterinary drug. Crit. Rev. Toxicol. 2019, 1-10. [CrossRef]

61. Baudry, J.; Assmann, K.E.; Touvier, M.; Alles, B.; Seconda, L.; Latino-Martel, P.; Ezzedine, K.; Galan, P.; Hercberg, S.; Lairon, D.; et al. Association of frequency of organic food consumption with cancer risk: Findings from the nutrinet-sante prospective cohort study. JAMA Intern. Med. 2018, 178, 1597-1606. [CrossRef] [PubMed]

62. Lomer, M.C.; Harvey, R.S.; Evans, S.M.; Thompson, R.P.; Powell, J.J. Efficacy and tolerability of a low microparticle diet in a double blind, randomized, pilot study in Crohn's disease. Eur. J. Gastroenterol. Hepatol. 2001, 13, 101-106. [CrossRef]

63. Lomer, M.C.; Grainger, S.L.; Ede, R.; Catterall, A.P.; Greenfield, S.M.; Cowan, R.E.; Vicary, F.R.; Jenkins, A.P.; Fidler, H.; Harvey, R.S.; et al. Lack of efficacy of a reduced microparticle diet in a multi-centred trial of patients with active Crohn's disease. Eur. J. Gastroenterol. Hepatol. 2005, 17, 377-384. [CrossRef]

64. Vidarsdottir, J.B.; Johannsdottir, S.E.; Thorsdottir, I.; Bjornsson, E.; Ramel, A. A cross-sectional study on nutrient intake and -status in inflammatory bowel disease patients. Nutr. J. 2016, 15, 61. [CrossRef] [PubMed] 
65. Wright, R.; Truelove, S.C. A controlled therapeutic trial of various diets in ulcerative colitis. Br. Med. J. 1965, 2, 138-141. [CrossRef]

66. Strisciuglio, C.; Giannetti, E.; Martinelli, M.; Sciorio, E.; Staiano, A.; Miele, E. Does cow's milk protein elimination diet have a role on induction and maintenance of remission in children with ulcerative colitis? Acta Paediatr. 2013, 102, e273-e278. [CrossRef]

67. Tóth, C.; Dabóczi, A.; Howard, M.; Miller, N.J.; Clemens, Z. Crohn's disease successfully treated with the paleolithic ketogenic diet. Int. J. Case Rep. Images 2016, 7, 570-578. [CrossRef]

68. Komperod, M.J.; Sommer, C.; Mellin-Olsen, T.; Iversen, P.O.; Roseth, A.G.; Valeur, J. Persistent symptoms in patients with Crohn's disease in remission: An exploratory study on the role of diet. Scand. J. Gastroenterol. 2018, 53, 573-578. [CrossRef] [PubMed]

69. Pedersen, N.; Ankersen, D.V.; Felding, M.; Wachmann, H.; Vegh, Z.; Molzen, L.; Burisch, J.; Andersen, J.R.; Munkholm, P. Low-FODMAP diet reduces irritable bowel symptoms in patients with inflammatory bowel disease. World J. Gastroenterol. 2017, 23, 3356-3366. [CrossRef]

70. Suarez, F.L.; Savaiano, D.A.; Levitt, M.D. A comparison of symptoms after the consumption of milk or lactose-hydrolyzed milk by people with self-reported severe lactose intolerance. N. Engl. J. Med. 1995, 333, 1-4. [CrossRef]

71. Lim, H.S.; Kim, S.K.; Hong, S.J. Food elimination diet and nutritional deficiency in patients with inflammatory bowel disease. Clin. Nutr. Res. 2018, 7, 48-55. [CrossRef] [PubMed]

72. Rocha, R.; Sousa, U.H.; Reis, T.L.M.; Santana, G.O. Nutritional status as a predictor of hospitalization in inflammatory bowel disease: A review. World J. Gastrointest. Pharmacol. Ther. 2019, 10, 50-56. [CrossRef]

73. Taylor, L.; Almutairdi, A.; Shommu, N.; Fedorak, R.; Ghosh, S.; Reimer, R.A.; Panaccione, R.; Raman, M. Cross-sectional analysis of overall dietary intake and mediterranean dietary pattern in patients with Crohn's disease. Nutrients 2018, 10, 1761. [CrossRef] [PubMed]

74. Moran, G.W.; Dubeau, M.F.; Kaplan, G.G.; Panaccione, R.; Ghosh, S. The increasing weight of Crohn's disease subjects in clinical trials: A hypothesis-generatings time-trend analysis. Inflamm. Bowel Dis. 2013, 19, 2949-2956. [CrossRef] [PubMed]

75. Mejean, C.; Lampure, A.; Si Hassen, W.; Gojard, S.; Peneau, S.; Hercberg, S.; Castetbon, K. Influence of food preparation behaviors on 5-year weight change and obesity risk in a French prospective cohort. Int. J. Behav. Nutr. Phys. Act. 2018, 15, 120. [CrossRef] [PubMed]

76. Lam, M.C.L.; Adams, J. Association between home food preparation skills and behaviour, and consumption of ultra-processed foods: Cross-sectional analysis of the UK National Diet and nutrition survey (2008-2009). Int. J. Behav. Nutr. Phys. Act. 2017, 14, 68. [CrossRef] [PubMed] 


\title{
Towards a Food Pharmacy: Immunologic Modulation through Diet
}

\author{
Ilse Molendijk ${ }^{1}$, Sander van der Marel ${ }^{2}$ and P.W. Jeroen Maljaars ${ }^{1, *}$ \\ 1 Department of Gastroenterology and Hepatology, Leiden University Medical Center, 2313 ZA Leiden, \\ The Netherlands; i.molendijk@lumc.nl \\ 2 Department of Gastroenterology and Hepatology, Haaglanden Medical Center, 2512 VA The Hague, \\ The Netherlands; s.van.der.marel@haaglandenmc.nl \\ * Correspondence: p.w.j.maljaars@lumc.nl; Tel.: +31-71-526-5217; Fax: +31-71-524-8115
}

Received: 22 April 2019; Accepted: 27 May 2019; Published: 31 May 2019

\begin{abstract}
Patients frequently wonder whether their dietary pattern influences the course of inflammatory bowel disease (IBD). Many patients even avoid certain foods that aggravate their symptoms. Although interest in nutritional interventions is rising among physicians, the current application of nutritional interventions in the IBD population is limited due to the lack of scientific evidence from clinical trials. Several studies, however, have identified associations between diet, gut microbiota, intestinal epithelial integrity, and mucosal immune responses. In patients consuming predominantly a Western diet high in n-6 poly-unsaturated fatty acids (PUFAs), sugars, and meat, and low in fruits and vegetables, an impaired gut epithelial barrier and disturbances in the intestinal microbiota have been observed, resulting in a chronic mucosal inflammation. An anti-inflammatory diet may restore this disbalance. In this review, we discuss the effects of diet on the composition of the microbiota, the gut epithelial barrier function, and the mucosal immune system.
\end{abstract}

Keywords: inflammatory bowel disease; dietary modification; exclusive enteral nutrition; lifestyle modification; Mediterranean diet

\section{Introduction}

Inflammatory bowel diseases (IBDs), consisting of Crohn's disease (CD) and ulcerative colitis (UC), are chronic immune-mediated disorders that can have a large impact on the wellbeing and quality of life of patients worldwide [1,2]. More than 1.5 million Americans are affected by IBDs, as are over 2.2 million Europeans [3], and the incidence of IBDs is increasing in newly industrialized countries in Africa, Asia, and South America [4]. Both diseases are characterized by a relapsing-remitting disease course, necessitating aggressive medical therapies in some cases [1,2].

Diet is important for patients with IBD. Patients often ask their physician whether the food they eat can influence the course of their inflammatory bowel disease. Very little data is available to answer this question. Data from studies in oncology show that eating or avoiding certain foods can increase or decrease the risk for developing colorectal cancer and other digestive cancers, and that this is probably related to the pro-inflammatory aspects of certain foods. This would suggest that nutrition may also affect both the pathogenesis and the disease course of IBD.

Additionally, it appears that dietary patterns in the decades before the onset of CD have been associated with the development of this disease. Patients who followed a healthy diet characterized by high fruit, vegetable, and fish intake during high school were $50 \%$ less likely to develop CD [5]. High intake of sugar and soft drinks combined with a low intake of vegetables was associated with UC risk [6].

These observations appear to be corroborated by observations in developing countries and among immigrants from developing countries: adaptation to a Western lifestyle with a Western diet increases 
both the incidence and prevalence of IBD in these populations [7,8]. The Western diet is high in fat (animal and milk fats, $\mathrm{n}-6$ polyunsaturated fatty acids (PUFAs)) and refined sugars, and contains little fiber and healthy fats (n-3 PUFA). Apart from increasing the risk of developing IBD, the disease course may also be influenced by dietary patterns. Intake of a diet with a high fat content or a low ratio of pro-inflammatory n-6 to anti-inflammatory n-3 fats was associated with a higher level of disease activity [9-11].

This suggests that a Western diet is an environmental risk factor for developing IBD.

\section{Diet, Symptoms, and Food Avoidance}

A number of studies have assessed CD patients' perception of the role of food and nutrients in gastrointestinal symptoms. Kinsey and Burden [12] found that $42 \%$ of IBD patients reported that food affected their symptoms "a lot" or "severely", and 51\% of the respondents indicated that diet was important or extremely important as a means of controlling symptoms. Another study found that an even higher percentage, around $70 \%$ of patients, thought that diet could trigger a disease flare [13]. Spicy foods are often considered a potential culprit: in a study by Limdi et al., $80 \%$ of the patients thought that eating food that was too spicy could result in a disease [14]. Specific items in the diet may aggravate symptoms such as abdominal pain, bloating, and diarrhea, whereas other dietary products alleviate these complaints. For instance, eating products with a high fat content, raw vegetables, and fruits and drinking carbonated beverages were perceived to increase the risk of a relapse by $40-50 \%$ of the patients [14]. The perceived relationship between specific foods and IBD complaints and disease activity leads to a high level of food avoidance. Casanova et al. [13] found that during remission, $77 \%$ of patients avoid certain foods, and this increased to $86 \%$ in patients with active disease. The avoidance of certain products increases the risk of vitamin and other biochemical deficiencies, and also increases the risk of malnutrition, which was estimated to occur in around $14 \%$ of the IBD population [13]. Currently, only a diet low in fermentable oligosaccharides, disaccharides, monosaccharides, and polyols (FODMAPS) has been shown to reduce complaints in a substantial part of the IBD population [15].

\section{Exclusive Enteral Nutrition}

Although certain dietary patterns may be related to a higher risk of developing IBD or a higher risk of disease activity, this does not show that dietary adjustments can influence the disease course or induce remission in patients with active disease. High quality studies in this area are lacking, and this is reflected in the results of a recent meta-analysis on the efficacy of the most studied dietary intervention, exclusive enteral nutrition (EEN), in which all conclusions were based on "very low quality evidence" [16]. EEN is a completely liquid formula containing all macro- and micronutrients a patient needs. EEN formulas can be classified as elemental (monomeric-free amino acids, glucose, oligosaccharides, and small amounts of essential fatty acids), semi-elemental (oligomeric-dipeptides, tripeptides, disaccharides, and medium chain triglycerides) or polymeric (whole protein, oligosaccharides, and long-chain triglycerides). Interventional studies have shown that clinical remission and mucosal healing can be achieved when a patient's diet is replaced by exclusive enteral nutrition. However, the most successful studies have been performed in pediatric patients (Table 1). In a prospective trial, 34 children with newly diagnosed CD received EEN. Twenty-six patients completed at least 6 weeks of EEN. Early clinical remission at week 8 was achieved in $84 \%$ of the patients and $76 \%$ of the patients had early biochemical remission [17]. Early complete or nearly complete endoscopic mucosal healing was associated with a reduction in endoscopic relapse, anti-TNF (tumor necrosis factor) use, and hospitalization 1 year after EEN. In a 10-week prospective open label study on 37 children who had recently been diagnosed with active $C D, 19$ children received polymeric EEN to induce remission while 18 children received corticosteroids to induce remission. [18]. At week 10, clinical remission was comparable between both groups, but the number of patients with mucosal healing was significantly higher in the polymeric EEN group than the corticosteroid group 
$(p<0.05)$. A recent meta-analysis of studies in pediatric patients (consisting of the Borrelli [18] and Terrin [19] papers—see Table 1) found that $83 \%$ of pediatric patients achieved remission with EEN, compared to $61 \%$ on steroids, but this reached statistical significance only in the per-protocol analysis (Relative risk (RR) 1.43, 95\% CI 1.03 to 1.97) [16]. EEN is currently the first-line treatment to induce remission in children with active $\mathrm{CD}[20,21]$. No placebo-controlled trials have been performed in adults with active CD. However, in the controlled studies performed in adults comparing EEN with steroids, steroids appeared to be more effective in inducing remission than EEN [22-24], and a recent meta-analysis confirmed this: steroids were more effective in inducing remission than placebo (Table 1; RR $0.65,95 \%$ CI 0.52 to $0.82 ; p<0.05$ ) [16]. There was no difference in efficacy between elemental, semi-elemental, and polymeric diets; however, a non-significant trend favoring very low fat and low long-chain triglycerides was observed. The lack of efficacy in adults may be due to non-compliance. Adults more often stop the EEN due to intolerance, and this may be related to the taste or to the method of delivery (a nasogastric tube) or unpalatability when the EEN is ingested orally [16]. Based on these studies, European Crohn and Colitis Organisation (ECCO) guidelines state that enteral therapy is only appropriate as an add-on treatment to support nutrition and not as a primary therapy in adults with active CD [25].

Table 1. Details of papers on EEN referenced in the manuscript.

\begin{tabular}{|c|c|c|c|c|c|}
\hline Reference & Population & Intervention & Control & Duration & Outcome \\
\hline Narula [16] & $\begin{array}{l}\text { Meta-analysis } \\
\text { of six studies, } \\
352 \text { adults }\end{array}$ & $\begin{array}{l}\text { Enteral nutrition } \\
\qquad(\mathrm{EN})\end{array}$ & $\begin{array}{l}\text { Corticosteroids } \\
\text { (CS) }\end{array}$ & & $\begin{array}{c}\text { Clinical remission EN: } 45 \% \\
\text { vs. CS: } 73 \%, p<0.05\end{array}$ \\
\hline Grover [17] & 34 children & Nutrison & No control & 8 weeks & $\begin{array}{c}\text { Clinical remission } 84 \% \\
\text { mucosal healing } 42 \%\end{array}$ \\
\hline Borrelli [18] & 37 children & $\begin{array}{l}\text { Polymeric diet } \\
\text { (PD) }\end{array}$ & $\begin{array}{l}\text { Corticosteroids } \\
\text { (CS) }\end{array}$ & 10 weeks & $\begin{array}{c}\text { Clinical remission PD: } 79 \% \\
\text { vs. CS: } 67 \%, p=\text { ns. } \\
\text { Mucosal healing PD: } 74 \% \\
\text { vs. CS: } 33 \%, p<0.05\end{array}$ \\
\hline Terrin [19] & 20 children & $\begin{array}{l}\text { Semi-elemental } \\
\text { diet (SED) }\end{array}$ & $\begin{array}{l}\text { Corticosteroids } \\
\text { (CS) }\end{array}$ & 8 weeks & $\begin{array}{l}\text { Clinical remission SED: } \\
90 \% \text { vs. CS: } 50 \%, p<0.01\end{array}$ \\
\hline \multicolumn{6}{|c|}{ Maintenance of remission } \\
\hline Hanai [27] & 95 adults & Elemental diet (ED) & $\begin{array}{l}\text { 6-Mercaptopurin } \\
(6-\mathrm{MP}), \text { no } \\
\text { intervention }\end{array}$ & 2 years & $\begin{array}{c}\text { Relapse rate: ED 53\%, } \\
\text { 6-MP } 40 \% \text {, control 73\% } \\
\text { ( } p<0.05 \text { ED vs. control; } \\
\text { 6-MP vs. control) }\end{array}$ \\
\hline \multicolumn{6}{|c|}{ EEN as co-medication } \\
\hline Nguyen [28] & $\begin{array}{l}\text { Meta-analysis } \\
\text { of four studies, } \\
342 \text { adults }\end{array}$ & $\begin{array}{l}\text { Specialized enteral } \\
\text { nutrition combined } \\
\text { with infliximab } \\
\text { (combo) }\end{array}$ & $\begin{array}{l}\text { Infliximab } \\
\text { monotherapy }\end{array}$ & 1 year & $\begin{array}{c}\text { Induction: } 69.4 \text { in combo } \\
\text { vs. } 45.4 \text { in mono }(p<0.01) \text {; } \\
\text { Maintenance: Combo } \\
74.5 \% \text {, mono } 49.4 \% \\
(p<0.01)\end{array}$ \\
\hline
\end{tabular}

CD: Crohn's disease; EEN: exclusive enteral nutrition. For a full review of the efficacy of EEN in inducing remission in active Crohn's disease, please see Narula et al. [16].

EEN has also been successful in preventing relapse in children with quiescent disease [29]. In adults, a prospective study randomly assigning 26 patients to a "half elemental" diet and 25 patients to a free diet resulted in a significantly lower relapse rate in the "half elemental" diet group after a mean follow-up of nearly 1 year compared to the patients who continued their own diet [26]. The compliance to the assigned intervention was similar in the two groups. In a study on 95 patients with $\mathrm{CD}$ in remission, 6-mercaptopurine (6-MP) was compared to an elemental diet and to a control group [27]. After 24 months, both the elemental diet and the 6-MP were significantly more effective in 
maintaining quiescent disease compared to patients in the control group $(p<0.05)$. No significant difference in the number of patients in remission was observed between the 6-MP and elemental diet group, suggesting that both interventions were equally able to maintain remission, although this may also be related to the relatively small sample size. A meta-analysis on EEN as an add-on treatment to anti-tumor necrosis factor (TNF) therapy found that the combination improved outcomes compared to anti-TNF without a dietary prescription [28]. Both the number of patients that reached remission as well as the number of patients that were in remission after a year increased significantly when the anti-TNF treatment was combined with the enteral nutrition $(p<0.01)$.

Together, this shows that enteral nutrition can induce remission, maintain remission, and improve the efficacy of other treatments. However, limited palatability and tolerability limit the applicability of this intervention.

\section{Anti-Inflammatory Diet}

Although EEN seems to reduce patients' abdominal symptoms and inflammation [16-18], it is not completely clear how treatment with EEN results in these beneficial effects. However, in general, three mechanisms have been identified through which a diet could affect an inflamed bowel, although these mechanisms will undoubtedly interact. The current understanding of $\mathrm{CD}$ pathogenesis points to a continuum of interactions between the intestinal microbiota, the immune system, and the epithelial barrier function, and all three are amenable to specific environmental factors, such as diet [30,31]. We will discuss these three factors below.

\section{Intestinal Barrier Function}

Patients with active Crohn's disease (CD) have an increased intestinal permeability. Even when little inflammation is present, intestinal integrity may still be reduced. This is associated with an elevated risk of a disease flare [32] and gastrointestinal complaints [33]. Intestinal permeability is controlled via tight junctions (TJs). TJs seal the paracellular space between the epithelial cells, thereby separating luminal content such as bacterial products and food antigens from the interstitium. TJs are selectively permeable for water, ions, and small molecules, however, a small proportion of luminal pathogens can cross the TJs. Patients that are susceptible to this translocated content develop an exaggerated mucosal immune response, resulting in an increased gut permeability-a "leaky gut" $[34,35]$. An increased intestinal permeability is also linked to the development of several diseases, including cardiovascular disease, diabetes, and non-alcoholic fatty liver disease (NAFLD) [35-38]. Improving intestinal barrier function may reduce gastrointestinal complaints and decrease the risk of a flare and the rise of metabolic diseases. The gut is continuously exposed to antigens in the food we consume. These dietary antigens may interact with the gut microbiota, changing its composition. This can result in an increased intestinal permeability, leading to an exaggerated immune response. Pathogens such as Escherichia coli O127:H6 (EPEC) and O157:H7 (EHEC), and bacterial toxins from, for instance, Clostridium difficile interfere with TJs between the epithelial cells, leading to an increased permeability. Also, the non-pathogenic E. coli strain C25 induces defects in the TJ complexes [39]. On the other hand, in a mouse model of necrotizing enterocolitis, Bifidobacteria preserved TJs and thereby intestinal barrier function [40]. The non-pathogenic yeast Saccharomyces boulardii supports barrier integrity and stimulates regeneration of damaged intestinal tissue in different diseases [41]. Dietary interventions such as EEN may be able to influence intestinal permeability [42,43]. In vitro, TNF-alpha decreased the TJ integrity of a monolayer culture. A polymeric formula completely restored the intestinal integrity of these monolayers [44]. Interleukin-10 knockout mice with colitis treated with EEN had an increased expression of TJs at a correct location and reduced epithelial apoptosis [45]. However, as discussed above, the lack of efficacy of EEN in adults may be due to the fact that EEN is unpalatable and/or that EEN is administered via a nasogastric tube. More palatable oral diets may be a solution for this problem. The Mediterranean diet (MD), rich in fresh vegetables, fruits, legumes, 
minimally processed whole-grains, nuts, fish, and olive oil, reduces systemic inflammation and is associated with increased diversity in gut microbiota and intestinal integrity $[37,46,47]$.

\section{Gut Microbiota}

Our intestines are colonized with large numbers of bacteria, archaea, viruses, and eukaryotic microbes that form the gut microbiota [48]. The microbiota protects us against pathogens, regulates local immune responses, and has an important impact on host metabolic functions. In the first three years of life, the microbiota is shaped [49]. Next to antibiotic therapy and severe illness in these years, diet influences the composition of the microbiota. The composition of the microbiota depends on our dietary patterns, which can have both short-term and long-term effects on the variety of the gut microbiota [50]. There is a wide variation in the gut microbiota composition in the elderly [51]. These variations are associated with specific food patterns of these people and separate healthy elderly from those in long-term residential care [50]. The microbiota of the latter has been shown to be significantly less diverse than that of healthy elderly, resulting in increased frailty [50]. Changes in the diversity of the microbiota have been correlated with an increased incidence of chronic inflammatory diseases such as obesity [52], type 2 diabetes [53], cardiovascular disease [54,55], asthma [56], rheumatoid arthritis [57], colorectal cancer [58], and IBD [59,60].

There is growing evidence that a predominantly Western diet, high in fat and refined sugars, influences the composition of the microbiota. Consumption of high-fat milk and sugar-sweetened soda is linked to a lower diversity, whereas buttermilk and high polyphenol content beverages are associated with high microbiota diversity [61]. De Filippo et al., compared the gut microbiota of children aged 1-6 years living in Florence (Italy), where they consume a typical diet of the developed world, with children in Boulpon, a village in Burkina Faso, where they eat a traditional rural African diet high in fibers [62]. The microbiota of the children from Boulpon comprised less Firmicutes and Enterobacteriaceae (Shigella and Escherichia) compared to the children from Florence. Prevotella, Xylanibacter (Bacteroidetes), and Treponema (Spirochaetes) were exclusively present in the microbiota of the children from Boulpon, possibly as a result of high fiber intake. These bacteria can ferment xylan and cellulose, resulting in the production of short-chain fatty acids (SCFAs). SCFAs are an important source of energy for the mucosa, regulate homeostasis in the colon, and maintain an anti-inflammatory state [63]. Marchesi et al. [64] demonstrated that CD patients have decreased levels of butyrate, an abundant bioactive SCFA, and reduced levels of SCFAs in general in comparison to healthy subjects. A plant-based diet rich in polysaccharides and low in fat and sugars may select for SCFAs-producing bacteria and may be used in the maintenance of remission in patients with inflammatory diseases such as IBD. Microbial structures can rapidly be altered by a change in diet. In a two-week food exchange trial, African Americans consumed a high-fiber/low-fat African diet and rural Africans consumed a high-fat/low-fiber Western diet [65]. "Africanization" of the diet led to a 2.5-fold increase of butyrate in the colon as a result of fermentation of fibers by changes in microbiota, whereas in Africans consuming a Western diet, the amounts of butyrate were reduced by $50 \%$. In concordance, a study among 14 obese men showed that diet-driven changes occurred within 3-4 days but were also reversed within the same time frame [66].

\section{Immune Responses}

The consumption of processed foods in developed countries has led to an excess of salt intake. Increased dietary salt intake is a risk factor for the development of cardiovascular diseases and autoimmune diseases through the induction of pathogenic T helper 17 (Th17) cells [67], a cell type which plays a pivotal role in the pathogenesis of CD [30]. Mutations in the nucleotide-binding oligomerization domain-containing protein 2 (NOD2) gene is associated with CD. NOD-like receptors (NLRs) sense muramyl dipeptide (MDP), present in all Gram-negative and -positive bacteria, leading to bacterial clearance. Mutations in the NOD2 gene, as seen in patients with IBD, are linked to less clearance of bacteria and suppression of the anti-inflammatory cytokine interleukin (IL)-10 [68]. Another family of innate immune receptors associated with IBD are the Toll-like receptors (TLRs). TLRs are expressed 
by dendritic cells, and TLR2 and TLR4 are upregulated under inflammatory conditions [2]. TLR2 and TLR4 are increased in the mucosa of patients with IBD compared to healthy controls, resulting in the loss of mucosal homeostasis [68]. Specific dietary patterns can influence the expression of both families of innate immune receptors. For instance, a diet high in fat and carbohydrates increased the expression of TLR2 and TLR4 [69]. Plasma concentrations of lipopolysaccharide (LPS) were also increased [69]. The consumption of orange juice during this meal, however, prevented the enhanced expression of both TLRs and LPS. This was probably due to the reactive oxygen species (ROS)-suppressive effect of flavonoids (naringenin and hesperidin) in the orange juice. A meal high in fibers did not result in an increased concentration of LPS and the induction of TLR2 and TLR4. The authors concluded that, although the content of LPS in a high-fiber meal is comparable to a meal high in fat and carbohydrates, the inflammatory nature of the latter possibly reduced the integrity of the mucosal barrier that normally prevents the translocation of bacterial products from the gut. In a mouse model of $C D$, a Western diet induced gene transcription of NOD2 and increased gut permeability, allowing the adherent-invasive E. coli to colonize, which resulted in an inflammatory state [70]. Together, these data suggest that a Western diet, high in salt, fat, and carbohydrates, may contribute to the development of IBD in predisposed patients, and to the loss of remission in patients with quiescent disease.

\section{A Mediterranean Diet as an Anti-Inflammatory Diet}

A Mediterranean diet (MD) is able to promote a healthy microbiota, reduce systemic inflammation, and reduce intestinal permeability. Two clinical trials evaluating the effect of a MD have been performed in patients with rheumatoid arthritis (RA) and were systematically reviewed by Forsyth et al. [71]. McKellar et al. [72] assigned patients with RA aged 30-70 years to either a 6-week hands-on cooking workshop ( $2 \mathrm{~h}$ per week delivered by nutritionists and teaching staff from local colleges), whereas the control group received a hand out with the general principles of a healthy diet. In this study, the MD reduced symptoms and improved physical functioning. The second study was a randomized controlled trial by Sköldstam et al. [73]. In this study, patients were randomized between a Cretan diet versus an ordinary Western diet. Symptoms, physical functioning, and biomarkers of inflammation (i.e., C-reactive protein (CRP)) improved in the intervention arm. The authors of the systematic review concluded that joint pain could be reduced and joint function could be improved using a MD. In addition, the risk of cardiovascular disease, which is substantially elevated in RA patients, could also be reduced by a MD. Our group recently performed a trial in 38 IBD patients using a group-based Mediterranean diet intervention (Molendijk et al., ECCO 2019) [74]. Patients with quiescent IBD that were willing to change their lifestyle, including dietary habits, were enrolled. Support was provided during three instruction and feedback days, and patients were able to use an online peer support platform. In this study, we found that $70 \%$ of patients were able to alter their dietary habits toward a more Mediterranean pattern. Second, we found that quality of life, as assessed with the short-IBD questionnaire, was increased and CRP was decreased in the active group. The magnitude of the changes in CRP and quality of life was correlated to the magnitude of the dietary change.

Together, these data suggest that a MD can increase the wellbeing of patients with inflammatory disorders. Whether a MD can alter the disease course or reduce inflammation is uncertain. However, the fact that the CRP was reduced in the Mediterranean arm is in this respect encouraging.

\section{Conclusions}

Recent studies have shown promising results of dietary alterations, such as the introduction of a MD, on the three most important players in the pathogenesis of IBD: intestinal permeability, gut microbiota composition, and inflammatory responses. Although patients are interested in lifestyle advice and a subset of physicians is eager to employ lifestyle-related interventions, the current application of lifestyle interventions in clinical practice is limited. This has a number of causes, such as lack of evidence-based interventions (which intervention works) and evidence-based practices (how to apply the intervention in clinical practice). What is clear is that networks have to be established, with physicians of 
different backgrounds, dieticians, physical therapists, and (physician-)scientists as participants. These collaborations will facilitate the development and implementation of these interventions.

Author Contributions: I.M. performed literature search and wrote the manuscript. S.v.d.M. critically reviewed the manuscript. P.W.J.M. and I.M. designed the study. P.W.J.M. critically reviewed the manuscript. All authors approved the final version of the manuscript.

Funding: This research received no external funding.

Conflicts of Interest: The authors declare no conflicts of interest.

\section{References}

1. Ungaro, R.; Mehandru, S.; Allen, P.B.; Peyrin-Biroulet, L.; Colombel, J.F. Ulcerative colitis. Lancet 2017, 389, 1756-1770. [CrossRef]

2. Torres, J.; Mehandru, S.; Colombel, J.F.; Peyrin-Biroulet, L. Crohn's disease. Lancet 2017, 389, $1741-1755$. [CrossRef]

3. Ananthakrishnan, A.N. Epidemiology and risk factors for IBD. Nat. Rev. Gastroenterol. Hepatol. 2015, 12, 205-217. [CrossRef] [PubMed]

4. Ng, S.C.; Shi, H.Y.; Hamidi, N.; Underwood, F.E.; Tang, W.; Benchimol, E.I.; Panaccione, R.; Ghosh, S.; Wu, J.C.Y.; Chan, F.K.L.; et al. Worldwide incidence and prevalence of inflammatory bowel disease in the 21st century: A systematic review of population-based studies. Lancet 2018, 390, 2769-2778. [CrossRef]

5. Ananthakrishnan, A.N.; Khalili, H.; Song, M.; Higuchi, L.M.; Richter, J.M.; Nimptsch, K.; Kana Wu, K.; Chan, A. High school diet and risk of crohn's disease and ulcerative colitis. Inflamm. Bowel Dis. 2015, 21, 2311-2319.

6. Racine, A.; Carbonnel, F.; Chan, S.S.; Hart, A.R.; Bueno-de-Mesquita, H.B.; Oldenburg, B.; van Schaik, F.D.M.; Tjonneland, A.; Olsen, A.; Dahm, C.C.; et al. Dietary patterns and risk of inflammatory bowel disease in Europe: Results from the EPIC study. Inflamm. Bowel Dis. 2016, 22, 345-354. [CrossRef] [PubMed]

7. Molodecky, N.A.; Soon, I.S.; Rabi, D.M.; Ghali, W.A.; Ferris, M.; Chernoff, G.; Benchimol, E.I.; Panaccione, R.; Ghosh, S.; Barkema, H.W.; et al. Increasing incidence and prevalence of the inflammatory bowel diseases with time, based on systematic review. Gastroenterology 2012, 142, 46-54.e42, quiz e30. [CrossRef] [PubMed]

8. Lewis, J.D.; Abreu, M.T. Diet as a trigger or therapy for inflammatory bowel diseases. Gastroenterology 2017, 152, 398-414 e6. [CrossRef]

9. Guerreiro, C.S.; Ferreira, P.; Tavares, L.; Santos, P.M.; Neves, M.; Brito, M.; Cravo, M. Fatty acids, IL6, and TNFalpha polymorphisms: An example of nutrigenetics in Crohn's disease. Am. J. Gastroenterol. 2009, 104, 2241-2249. [CrossRef]

10. Tanaka, M.; Iwao, Y.; Sasaki, S.; Okamoto, S.; Ogata, H.; Hibi, T.; Kazuma, K. Moderate dietary temperance effectively prevents relapse of Crohn disease: A prospective study of patients in remission. Gastroenterol. Nurs. 2007, 30, 202-210. [CrossRef]

11. Calder, P.C. n-3 polyunsaturated fatty acids, inflammation, and inflammatory diseases. Am. J. Clin. Nutr. 2006, 83 (Suppl. 6), 1505s-1519s. [CrossRef]

12. Kinsey, L.; Burden, S. A survey of people with inflammatory bowel disease to investigate their views of food and nutritional issues. Eur. J. Clin. Nutr. 2016, 70, 852-854. [CrossRef]

13. Casanova, M.J.; Chaparro, M.; Molina, B.; Merino, O.; Batanero, R.; Duenas-Sadornil, C.; Robledo, P.; Garcia-Albert, A.M.; Gómez-Sánchez, M.B.; Calvet, X.; et al. Prevalence of malnutrition and nutritional characteristics of patients with inflammatory bowel disease. J. Crohns Colitis 2017, 11, 1430-1439. [CrossRef]

14. Limdi, J.K.; Aggarwal, D.; McLaughlin, J.T. Dietary practices and beliefs in patients with inflammatory bowel disease. Inflamm. Bowel Dis. 2016, 22, 164-170. [CrossRef]

15. Zhan, Y.L.; Zhan, Y.A.; Dai, S.X. Is a low FODMAP diet beneficial for patients with inflammatory bowel disease? A meta-analysis and systematic review. Clin. Nutr. 2018, 37, 123-129. [CrossRef] [PubMed]

16. Narula, N.; Dhillon, A.; Zhang, D.; Sherlock, M.E.; Tondeur, M.; Zachos, M. Enteral nutritional therapy for induction of remission in Crohn's disease. Cochrane Database Syst. Rev. 2018, 4, CD000542. [CrossRef] [PubMed]

17. Grover, Z.; Muir, R.; Lewindon, P. Exclusive enteral nutrition induces early clinical, mucosal and transmural remission in paediatric Crohn's disease. J. Gastroenterol. 2014, 49, 638-645. [CrossRef] 
18. Borrelli, O.; Cordischi, L.; Cirulli, M.; Paganelli, M.; Labalestra, V.; Uccini, S.; Russo, P.M.; Cucchiara, S. Polymeric diet alone versus corticosteroids in the treatment of active pediatric Crohn's disease: A randomized controlled open-label trial. Clin. Gastroenterol. Hepatol. 2006, 4, 744-753. [CrossRef]

19. Terrin, G.; Berni Canani, R.; Ambrosini, A.; Viola, F.; Bueno de Mesquita, M.; Di Nardo, G.; Cucchiara, S A semielemental diet (Pregomin) as primary therapy for inducing remission in children with active Crohn's disease. Ital. J. Pediatr. 2002, 28, 401-405.

20. Forbes, A.; Escher, J.; Hebuterne, X.; Klek, S.; Krznaric, Z.; Schneider, S.; Shamir, R.; Stardelova, K.; Wierdsma, N.; Wiskin, A.E.; et al. ESPEN guideline: Clinical nutrition in inflammatory bowel disease. Clin. Nutr. 2017, 36, 321-347. [CrossRef]

21. Ruemmele, F.M.; Veres, G.; Kolho, K.L.; Griffiths, A.; Levine, A.; Escher, J.C.; Amil Dias, J.; Barabino, A.; Braegger, C.P.; Bronsky, J.; et al. Consensus guidelines of ECCO/ESPGHAN on the medical management of pediatric Crohn's disease. J. Crohns Colitis 2014, 8, 1179-1207. [CrossRef]

22. Griffiths, A.M.; Ohlsson, A.; Sherman, P.M.; Sutherland, L.R. Meta-analysis of enteral nutrition as a primary treatment of active Crohn's disease. Gastroenterology 1995, 108, 1056-1067. [CrossRef]

23. Messori, A.; Trallori, G.; D’Albasio, G.; Milla, M.; Vannozzi, G.; Pacini, F. Defined-formula diets versus steroids in the treatment of active Crohn's disease: A meta-analysis. Scand. J. Gastroenterol. 1996, 31, 267-272. [CrossRef]

24. Fernandez-Banares, F.; Cabre, E.; Esteve-Comas, M.; Gassull, M.A. How effective is enteral nutrition in inducing clinical remission in active Crohn's disease? A meta-analysis of the randomized clinical trials. JPEN J. Parenter. Enteral. Nutr. 1995, 19, 356-364. [CrossRef]

25. Gomollon, F.; Dignass, A.; Annese, V.; Tilg, H.; Van Assche, G.; Lindsay, J.O.; Peyrin-Biroulet, L.; Cullen, G.J.; Daperno, M.; Rieder, T.K.F.; et al. 3rd European Evidence-based Consensus on the Diagnosis and Management of Crohn's Disease 2016: Part 1: Diagnosis and Medical Management. J. Crohns Colitis 2017, 11, 3-25. [CrossRef]

26. Takagi, S.; Utsunomiya, K.; Kuriyama, S.; Yokoyama, H.; Takahashi, S.; Iwabuchi, M.; Takahashi, H.; Takahashi, S.; Kinouchi, Y.; Hiwatashi, Y.; et al. Effectiveness of an 'half elemental diet' as maintenance therapy for Crohn's disease: A randomized-controlled trial. Aliment. Pharmacol. Ther. 2006, 24, 1333-1340. [CrossRef]

27. Hanai, H.; Iida, T.; Takeuchi, K.; Arai, H.; Arai, O.; Abe, J.; Tanaka, T.; Maruyama, Y.; Ikeya, K.; Sugimoto, K.; et al. Nutritional therapy versus 6-mercaptopurine as maintenance therapy in patients with Crohn's disease. Dig. Liver Dis. 2012, 44, 649-654. [CrossRef]

28. Nguyen, D.L.; Palmer, L.B.; Nguyen, E.T.; McClave, S.A.; Martindale, R.G.; Bechtold, M.L. Specialized enteral nutrition therapy in Crohn's disease patients on maintenance infliximab therapy: A meta-analysis. Ther. Adv. Gastroenterol. 2015, 8, 168-175. [CrossRef]

29. Cabre, E.; Gassull, M.A. Nutrition in inflammatory bowel disease: Impact on disease and therapy. Curr. Opin. Gastroenterol. 2001, 17, 342-349. [CrossRef]

30. Kaser, A.; Zeissig, S.; Blumberg, R.S. Inflammatory bowel disease. Annu. Rev. Immunol. 2010, $28,573-621$. [CrossRef]

31. Zeissig, S.; Burgel, N.; Gunzel, D.; Richter, J.; Mankertz, J.; Wahnschaffe, U.; Kroesen, A.; Zeitz, M.; Fromm, M.; Schulzke, J.D. Changes in expression and distribution of claudin 2, 5 and 8 lead to discontinuous tight junctions and barrier dysfunction in active Crohn's disease. Gut 2007, 56, 61-72. [CrossRef]

32. Gutierrez, A.; Zapater, P.; Juanola, O.; Sempere, L.; Garcia, M.; Laveda, R.; Martínez, A.; Scharl, M.; González-Navajas, J.M.; Such, J.; et al. Gut bacterial DNA translocation is an independent risk factor of flare at short term in patients with Crohn's disease. Am. J. Gastroenterol. 2016, 111, 529-540. [CrossRef]

33. Chang, J.; Leong, R.W.; Wasinger, V.C.; Ip, M.; Yang, M.; Phan, T.G. Impaired intestinal permeability contributes to ongoing bowel symptoms in patients with inflammatory bowel disease and mucosal healing. Gastroenterology 2017, 153, 723-731 e1. [CrossRef]

34. Landy, J.; Ronde, E.; English, N.; Clark, S.K.; Hart, A.L.; Knight, S.C.; Ciclitira, P.J.; Al-Hassi, H.O. Tight junctions in inflammatory bowel diseases and inflammatory bowel disease associated colorectal cancer. World J. Gastroenterol. 2016, 22, 3117-3126. [CrossRef]

35. Odenwald, M.A.; Turner, J.R. Intestinal permeability defects: Is it time to treat? Clin. Gastroenterol. Hepatol. 2013, 11, 1075-1083. [CrossRef] 
36. Odenwald, M.A.; Turner, J.R. The intestinal epithelial barrier: A therapeutic target? Nat. Rev. Gastroenterol. Hepatol. 2017, 14, 9-21. [CrossRef]

37. Casas, R.; Sacanella, E.; Urpi-Sarda, M.; Corella, D.; Castaner, O.; Lamuela-Raventos, R.M.; Salas-Salvadó, J.; Martínez-González, M.-A.; Ros, E.; Estruch, R. Long-term immunomodulatory effects of a mediterranean diet in adults at high risk of cardiovascular disease in the PREvencion con DIeta MEDiterranea (PREDIMED) randomized controlled trial. J. Nutr. 2016, 146, 1684-1693. [CrossRef]

38. Mu, Q.; Kirby, J.; Reilly, C.M.; Luo, X.M. Leaky gut as a danger signal for autoimmune diseases. Front. Immunol. 2017, 8, 598. [CrossRef]

39. Zareie, M.; Riff, J.; Donato, K.; McKay, D.M.; Perdue, M.H.; Soderholm, J.D.; Karmali, M.; Cohen, M.B.; Hawkins, J.; Sherman, P.M. Novel effects of the prototype translocating Escherichia coli, strain C25 on intestinal epithelial structure and barrier function. Cell. Microbiol. 2005, 7, 1782-1797. [CrossRef]

40. Bergmann, K.R.; Liu, S.X.L.; Tian, R.L.; Kushnir, A.; Turner, J.R.; Li, H.L.; Chou, P.M.; Weber, C.R.; De Plaen, I.G. Bifidobacteria stabilize claudins at tight junctions and prevent intestinal barrier dysfunction in mouse necrotizing enterocolitis. Am. J. Pathol. 2013, 182, 1595-1606. [CrossRef]

41. Terciolo, C.; Dapoigny, M.; Andre, F. Beneficial effects of Saccharomyces boulardii CNCM I-745 on clinical disorders associated with intestinal barrier disruption. Clin. Exp. Gastroenterol. 2019, 12, 67-82. [CrossRef]

42. Araujo, J.R.; Tomas, J.; Brenner, C.; Sansonetti, P.J. Impact of high-fat diet on the intestinal microbiota and small intestinal physiology before and after the onset of obesity. Biochimie 2017, 141, 97-106. [CrossRef] [PubMed]

43. Ma, N.; Tian, Y.; Wu, Y.; Ma, X. Contributions of the Interaction Between Dietary Protein and Gut Microbiota to Intestinal Health. Curr. Protein Pept. Sci. 2017, 18, 795-808. [CrossRef]

44. Nahidi, L.; Day, A.S.; Lemberg, D.A.; Leach, S.T. Differential effects of nutritional and non-nutritional therapies on intestinal barrier function in an in vitro model. J. Gastroenterol. 2012, 47, 107-117. [CrossRef]

45. Wang, H.; Shi, P.; Zuo, L.; Dong, J.; Zhao, J.; Liu, Q.; Zhu, W. Dietary non-digestible polysaccharides ameliorate intestinal epithelial barrier dysfunction in IL-10 knockout mice. J. Crohns Colitis 2016, 10, 1076-1086. [CrossRef]

46. Casas, R.; Sacanella, E.; Estruch, R. The immune protective effect of the Mediterranean diet against chronic low-grade inflammatory diseases. Endocr. Metab. Immune Disord. Drug Targets 2014, 14, 245-254. [CrossRef]

47. Mitsou, E.K.; Kakali, A.; Antonopoulou, S.; Mountzouris, K.C.; Yannakoulia, M.; Panagiotakos, D.B.; Kyriacou, A. Adherence to the Mediterranean diet is associated with the gut microbiota pattern and gastrointestinal characteristics in an adult population. Br. J. Nutr. 2017, 117, 1645-1655. [CrossRef]

48. Shreiner, A.B.; Kao, J.Y.; Young, V.B. The gut microbiome in health and in disease. Curr. Opin. Gastroen. 2015, 31, 69-75. [CrossRef]

49. Yatsunenko, T.; Rey, F.E.; Manary, M.J.; Trehan, I.; Dominguez-Bello, M.G.; Contreras, M.; Magris, M.; Hidalgo, G.; Baldassano, R.N.; Anokhin, A.P.; et al. Human gut microbiome viewed across age and geography. Nature 2012, 486, 222-227. [CrossRef]

50. Claesson, M.J.; Jeffery, I.B.; Conde, S.; Power, S.E.; O’Connor, E.M.; Cusack, S.; Harris, H.M.B.; Coakley, M.; Lakshminarayanan, B.; O'Sullivan, O.; et al. Gut microbiota composition correlates with diet and health in the elderly. Nature 2012, 488, 178-184. [CrossRef]

51. Claesson, M.J.; Cusack, S.; O'Sullivan, O.; Greene-Diniz, R.; de Weerd, H.; Flannery, E.; Marchesi, J.R.; Falush, D.; Dinan, T.; Fitzgerald, G.; et al. Composition, variability, and temporal stability of the intestinal microbiota of the elderly. Proc. Natl. Acad. Sci. USA 2011, 108 (Suppl. 1), 4586-4591. [CrossRef]

52. Le Chatelier, E.; Nielsen, T.; Qin, J.; Prifti, E.; Hildebrand, F.; Falony, G.; Almeida, M.; Arumugam, M.; Batto, J.-M.; Kennedy, S.; et al. Richness of human gut microbiome correlates with metabolic markers. Nature 2013, 500, 541-546. [CrossRef]

53. Karlsson, F.H.; Tremaroli, V.; Nookaew, I.; Bergstrom, G.; Behre, C.J.; Fagerberg, B.; Nielsen, J.; Bäckhed, F. Gut metagenome in European women with normal, impaired and diabetic glucose control. Nature 2013, 498, 99-103. [CrossRef] 
54. Wang, Z.; Klipfell, E.; Bennett, B.J.; Koeth, R.; Levison, B.S.; Dugar, B.; Feldstein, A.E.; Britt, E.B.; Fu, X.; Chung, Y.-M.; et al. Gut flora metabolism of phosphatidylcholine promotes cardiovascular disease. Nature 2011, 472, 57-63. [CrossRef]

55. Koeth, R.A.; Wang, Z.; Levison, B.S.; Buffa, J.A.; Org, E.; Sheehy, B.T.; Britt, E.B.; Fu, X.; Wu, Y.; Li, L.; et al. Intestinal microbiota metabolism of L-carnitine, a nutrient in red meat, promotes atherosclerosis. Nat. Med. 2013, 19, 576-585. [CrossRef]

56. Devereux, G. The increase in the prevalence of asthma and allergy: Food for thought. Nat. Rev. Immunol. 2006, 6, 869-874. [CrossRef]

57. Scher, J.U.; Sczesnak, A.; Longman, R.S.; Segata, N.; Ubeda, C.; Bielski, C.; Rostron, T.; Cerundolo, V.; Pamer, E.G.; Abramson, S.B.; et al. Expansion of intestinal Prevotella copri correlates with enhanced susceptibility to arthritis. Elife 2013, 2, e01202. [CrossRef]

58. Kostic, A.D.; Chun, E.; Robertson, L.; Glickman, J.N.; Gallini, C.A.; Michaud, M.; Clancy, T.E.; Chung, D.C.; Lochhead, P.; Hold, G.L.; et al. Fusobacterium nucleatum potentiates intestinal tumorigenesis and modulates the tumor-immune microenvironment. Cell Host Microbe 2013, 14, 207-215. [CrossRef]

59. Gevers, D.; Kugathasan, S.; Denson, L.A.; Vazquez-Baeza, Y.; Van Treuren, W.; Ren, B.; Schwager, E.; Knights, D.; Song, S.J.; Yassour, M.; et al. The treatment-naive microbiome in new-onset Crohn's disease. Cell Host Microbe 2014, 15, 382-392. [CrossRef]

60. Kostic, A.D.; Xavier, R.J.; Gevers, D. The microbiome in inflammatory bowel disease: Current status and the future ahead. Gastroenterology 2014, 146, 1489-1499. [CrossRef]

61. Zhernakova, A.; Kurilshikov, A.; Bonder, M.J.; Tigchelaar, E.F.; Schirmer, M.; Vatanen, T.; Mujagic, Z.; Vila, A.V.; Falony, G.; Vieira-Silva, S.; et al. Population-based metagenomics analysis reveals markers for gut microbiome composition and diversity. Science 2016, 352, 565-569. [CrossRef]

62. De Filippo, C.; Cavalieri, D.; Di Paola, M.; Ramazzotti, M.; Poullet, J.B.; Massart, S.; Collini, S.; Pieraccini, G.; Lionetti, P. Impact of diet in shaping gut microbiota revealed by a comparative study in children from Europe and rural Africa. Proc. Natl. Acad. Sci. USA 2010, 107, 14691-14696. [CrossRef]

63. Scheppach, W.; Weiler, F. The butyrate story: Old wine in new bottles? Curr. Opin. Clin. Nutr. Metab. Care 2004, 7, 563-567. [CrossRef]

64. Marchesi, J.R.; Holmes, E.; Khan, F.; Kochhar, S.; Scanlan, P.; Shanahan, F.; Shanahan, F.; Wilson, I.D.; Wang, Y Rapid and noninvasive metabonomic characterization of inflammatory bowel disease. J. Proteome Res. 2007, 6, 546-551. [CrossRef]

65. O'Keefe, S.J.; Li, J.V.; Lahti, L.; Ou, J.; Carbonero, F.; Mohammed, K.; Posma, J.M.; Kinross, J.; Wahl, E.; Ruder, E.; et al. Fat, fibre and cancer risk in African Americans and rural Africans. Nat. Commun. 2015, 6, 6342. [CrossRef]

66. Walker, A.W.; Ince, J.; Duncan, S.H.; Webster, L.M.; Holtrop, G.; Ze, X.; Brown, D.; Stares, M.D.; Scott, P.; Bergerat, A.; et al. Dominant and diet-responsive groups of bacteria within the human colonic microbiota. ISME J. 2011, 5, 220-230. [CrossRef]

67. Kleinewietfeld, M.; Manzel, A.; Titze, J.; Kvakan, H.; Yosef, N.; Linker, R.A.; Muller, A.N.; Hafler, D.A. Sodium chloride drives autoimmune disease by the induction of pathogenic TH17 cells. Nature 2013, 496, 518-522. [CrossRef]

68. De Souza, H.S.; Fiocchi, C. Immunopathogenesis of IBD: Current state of the art. Nat. Rev. Gastroenterol. Hepatol. 2016, 13, 13-27. [CrossRef]

69. Ghanim, H.; Sia, C.L.; Upadhyay, M.; Korzeniewski, K.; Viswanathan, P.; Abuaysheh, S.; Mohanty, P.; Dandona, P. Orange juice neutralizes the proinflammatory effect of a high-fat, high-carbohydrate meal and prevents endotoxin increase and Toll-like receptor expression. Am. J. Clin. Nutr. 2010, 91, 940-949. [CrossRef]

70. Martinez-Medina, M.; Denizot, J.; Dreux, N.; Robin, F.; Billard, E.; Bonnet, R.; Darfeuille-Michaud, A.; Barnich, N. Western diet induces dysbiosis with increased E coli in CEABAC10 mice, alters host barrier function favouring AIEC colonisation. Gut 2014, 63, 116-124. [CrossRef]

71. Forsyth, C.; Kouvari, M.; D'Cunha, N.M.; Georgousopoulou, E.N.; Panagiotakos, D.B.; Mellor, D.D.; Kellett, J.; Naumovski, N. The effects of the Mediterranean diet on rheumatoid arthritis prevention and treatment: A systematic review of human prospective studies. Rheumatol. Int. 2018, 38, 737-747. [CrossRef] 
72. McKellar, G.; Morrison, E.; McEntegart, A.; Hampson, R.; Tierney, A.; Mackle, G.; Scoular, J.; Scott, J.A.; Capell, H.A. A pilot study of a Mediterranean-type diet intervention in female patients with rheumatoid arthritis living in areas of social deprivation in Glasgow. Ann. Rheum. Dis. 2007, 66, 1239-1243. [CrossRef]

73. Sköldstam, L.; Hagfors, L.; Johansson, G. An experimental study of a Mediterranean diet intervention for patients with rheumatoid arthritis. Ann. Rheum. Dis. 2003, 62, 208-214. [CrossRef] [PubMed]

74. Molendijk, I.; Martens, J.E.; van Lingen, E.; van der Marel, S.; van Veen-Lievaart, M.E.; van der Meulen, A.E.; Barnhoorn, M.C.; Ernst-Stegeman, A.M.; Maljaars, J. P592 Towards a Food Pharmacy: increased dietary quality reduces CRP and improves quality of life in IBD patients in remission. J. Crohn's Colitis 2019, 13 (Suppl. 1), S411. [CrossRef]

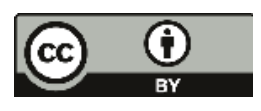

(C) 2019 by the authors. Licensee MDPI, Basel, Switzerland. This article is an open access article distributed under the terms and conditions of the Creative Commons Attribution (CC BY) license (http://creativecommons.org/licenses/by/4.0/). 
Review

\title{
Chemopreventive Effects of Strawberry and Black Raspberry on Colorectal Cancer in Inflammatory Bowel Disease
}

\author{
Tong Chen ${ }^{1,2, *}$, Ni Shi ${ }^{1,2}$ and Anita Afzali ${ }^{3,4}$ \\ 1 Division of Medical Oncology, Department of Internal Medicine, The Ohio State University, Columbus, \\ OH 43210, USA; ni.shi@osumc.edu \\ 2 Comprehensive Cancer Center, The Ohio State University, Columbus, OH 43210, USA \\ 3 Division of Gastroenterology, Hepatology and Nutrition, The Ohio State University, Columbus, OH 43210, \\ USA; Anita.Afzali@osumc.edu \\ 4 Inflammatory Bowel Disease Center, Wexner Medical Center, The Ohio State University, Columbus, \\ OH 43210, USA \\ * Correspondence: tong.chen@osumc.edu; Tel.: +1-(614)-685-9119
}

Received: 18 March 2019; Accepted: 27 May 2019; Published: 3 June 2019

\begin{abstract}
Colorectal cancer (CRC) remains the third most common cause of cancer-related death in the United States and the fourth globally with a rising incidence. Inflammatory bowel disease (IBD) is a chronic immunologically mediated disease that imposes a significant associated health burden, including the increased risk for colonic dysplasia and CRC. Carcinogenesis has been attributed to chronic inflammation and associated with oxidative stress, genomic instability, and immune effectors as well as the cytokine dysregulation and activation of the nuclear factor kappa B (NFkB) signaling pathway. Current anti-inflammation therapies used for IBD treatment have shown limited effects on CRC chemoprevention, and their long-term toxicity has limited their clinical application. However, natural food-based prevention approaches may offer significant cancer prevention effects with very low toxicity profiles. In particular, in preclinical and clinical pilot studies, strawberry and black raspberry have been widely selected as food-based interventions because of their potent preventive activities. In this review, we summarize the roles of strawberry, black raspberry, and their polyphenol components on CRC chemoprevention in IBD.
\end{abstract}

Keywords: colorectal cancer; inflammatory bowel disease; colorectal cancer; dysplasia; berries; chemoprevention

\section{Introduction}

Inflammatory bowel disease (IBD) (e.g., Crohn's disease and ulcerative colitis) is a chronic immunologically mediated disease that develops in interactions between genetics, immunology, the environment, and the microbiome [1,2]. IBD is highly prevalent in North America and Europe, affecting about 1.3 million people in the United States and 2.2 million people in Europe [1]. Moreover, the rising prevalence of IBD has been reported across other countries worldwide. Notably, patients with IBD are at the increased risk of developing colorectal cancer (CRC), which further increases the urgency of understanding and treating this disease [3].

In IBD, chronic inflammation induces oncogenic mutations, genomic instability, immune microenvironment changes, early tumor promotion, and angiogenesis. These factors are likely related to the increased risk of CRC in IBD [4]. Importantly, supporting the association between chronic inflammation in IBD and CRC risk, the results of studies employing 5-aminosalicylate (5-ASAs) to target and reduce inflammation identified some chemopreventive effects in IBD [5]. In population-based 
cohorts, the incidence of CRC in patients with ulcerative colitis was 2.4-fold higher than in the controls [6]. Furthermore, some results suggested that at 10 years after the initial onset of ulcerative colitis around $2 \%$ of patients with ulcerative colitis developed CRC: $8 \%$ after 20 years and $18 \%$ after 30 years of disease duration [7]. Previous data also showed that $2.9 \%$ of Crohn's disease patients developed CRC within 10 years after the initial onset of the disease, and that percentage increased to $5.6 \%$ after 20 years and $8.3 \%$ after 30 years. Due to multifocal tumors and cell histopathology, CRC that develops in IBD patients tends to have a worse prognosis and survival rate compared to sporadic CRC in the advanced stage [8]. Additionally, the mean age for developing CRC in IBD patients (40-50 years) tends to be lower than the mean age for sporadic CRC (60 years) [4].

Due to the increased risk and poorer prognosis associated with CRC in individuals with IBD, its early detection and prevention are essential. In adults aged 50-75 years with an average risk of CRC, the US Preventive Services Task Force recommends yearly CRC screening by a fecal occult blood test (FOBT), sigmoidoscopy every five years, and colonoscopy every 10 years. In patients with IBD, a colonoscopy is recommended every one to two years beginning at 8-10 years from the diagnosis of the disease as well as multiple colonic biopsy specimens for dysplasia detection to screen for dysplasia or CRC [9]. Although chemoprevention is another important goal for IBD-related CRC management, the current therapies used in IBD have shown little to no effect, and the long-term toxicity associated with these treatments limits their clinical application for this purpose.

Diets that are high in animal fat and low in fruit and vegetables can be important triggers of both IBD and cancers [10,11]. Based on the associations between diet and cancers, natural food-based prevention approaches may represent a promising strategy. Notably, food-based approaches involving components of fruits and vegetables, vitamins, minerals, probiotics, and herbal medicines have shown potential cancer prevention effects with low toxicity profiles. Flavonoids, which are a major class of polyphenols that occur naturally in fruit and vegetables, have shown promising in vitro anti-colon cancer effects. Additionally, several case-control and cohort studies have verified the inverse association between flavonoid intake and CRC [12-14]. In particular, berries are a good source of polyphenols, especially anthocyanins, micronutrients, and fiber, which suggests that these foods may be associated with benefits for cardiovascular and immune health as well as chemoprevention [15]. Moreover, strawberry and black raspberry have been widely selected for food-based interventions in preclinical and pilot clinical studies because of their potent activity in the prevention of oral, esophageal, colon, skin, and prostate cancer [16-20]. Strawberry is the most commonly consumed berry type on the current market. Our previous study showed the significant chemoprevention effects of strawberry in CRC mouse model and its role in inflammation inhibition [18]. We also demonstrate that black raspberry inhibits oxidative stress, the inflammation mediators, cyclooxygenase-2 (COX-2) and inducible nitric oxide synthase (iNOS) and exhibits superior anti-cancer effects than inhibitors of COX-2 and iNOS in an animal model [21,22]. In this review, we summarize the roles of strawberry, black raspberry, and their polyphenol components on CRC chemoprevention in IBD.

\section{Animal Models of Inflammatory Bowel Disease and IBD-Related Colorectal Cancer}

Genetic engineering models can produce IBD in animals, such as muc-2 deficiency, muc-2/C3GnT dual deletion, muc-2/Core 1-derived O-glycans deficiency, P-gp deficiency, T-bet knockout, IKK- $\gamma$ or IKK- $\beta$ deletion, STAT3 deficiency, STAT3/IL-22 dual deficiency, XBP1 deletion, IL-10 deficiency, and IL-7 overexpression $[2,11]$. These targeted genes are responsible for epithelial barrier function, intestinal permeability, oxidative stress, cytokine, inflammation and immune effector regulation signaling pathway, and gut microflora etc. Additionally, colonic epithelium injures, ectogenic immunogenic response, adoptive transfer of naïve $\mathrm{CD} 4^{+} \mathrm{T}$ cells to immune deficiency animals are strategies to induce IBD in animals. In Dextran sulfate sodium (DSS) model, DSS in drinking water is toxic in the epithelial lining of the colon and produce severe colitis. DSS treatment combined with gene deficiency in immune effectors regulation, cytokine response and immune response signaling pathways can enhance the inflammation in animals. Intrarectal injection of the haptenating agent 2,4,6-trinitrobenzene sulfonic 
acid (TNBS) or 4-ethoxymethylene-2-phenyl-2-oxazolin-5-one (oxazolone) can cause ulcerative colitis with different features. The inflammation in the TNBS model is regulated by TH1-mediated immune response with $\mathrm{CD}^{+} \mathrm{T}$ cells, neutrophils and macrophages infiltration showing symptoms including severe diarrhea, weight loss, and rectal prolapse similar to Crohn's disease. TNBS model is used for the immune-related studies for Crohn's disease. The oxazolone model driven by NKT cells and IL-13 is considered as a valuable model for ulcerative colitis. The regulatory $\mathrm{T}$ cell deficiency in naïve $\mathrm{CD} 4^{+} \mathrm{T}$ cells is a critical factor for inflammation in the transfer model. This model is ideal for immunoregulation and regulatory $\mathrm{T}$ cell research.

In studies on colon carcinogenesis and chemopreventive screening interventions for CRC in IBD, gene engineering mouse model such as apc mutation, apc deletion, smad mutation, smad 3 knockout, rag2-deficiency, k-ras mutation have been used [23]. In chemically induced preclinical models, murine treated with azoxymethane (AOM)/DSS has been a commonly used model for IBD-related CRC research for over a decade. AOM is a metabolite of 1,2-dimethylhydrazine (DMH) and the carcinogen to induce CRC in murine. It is important to note that AOM-induced tumors showed histopathological characteristics similar to human CRC, such as frequent $\mathrm{K}$-Ras, $\beta$-catenin mutation, and microsatellite instability [24]. When combined with AOM, DSS works as a promoter of colorectal carcinogenesis through an initial acute inflammation phase. To induce colon carcinogenesis in murine, a single intraperitoneal injection of $\mathrm{AOM}$ at $10 \mathrm{mg} / \mathrm{kg}$ body weight or less can be given, followed by one to three cycles of $2 \%$ or $3 \%$ DSS in drinking water. In this method, tumors typically form 14 weeks after the AOM injection. Multistep tumor histogenesis can be efficiently reproduced in the AOM/DSS murine model. As found in our former studies, intraepithelial neoplasia, including changes ranging from dysplasia to adenoma and adenocarcinoma, were observed (Figure 1) [18]. T lymphocytes and other immune cells typically found in CRC patients are also frequently observed in tumors developed in AOM/DSS murine models. Therefore, AOM/DSS murine models are important for the investigation of inflammation-induced colon carcinogenesis and chemoprevention strategy screening.

A

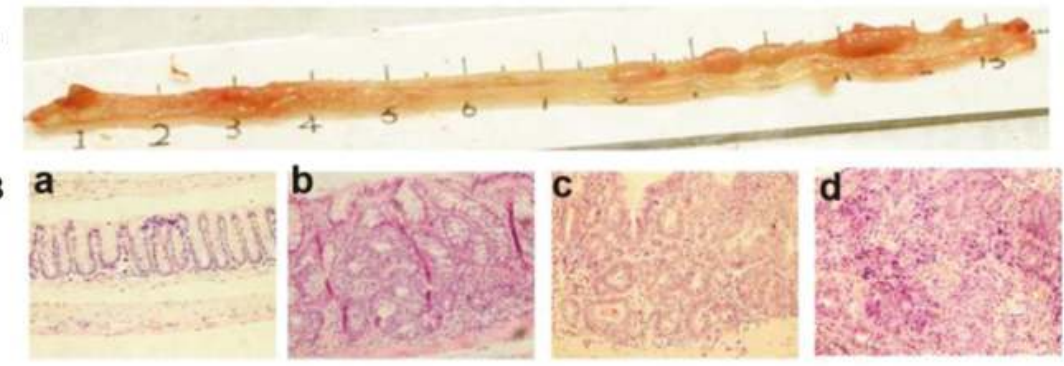

Figure 1. Histopathology of azoxymethane (AOM)/dextran sodium sulfate (DSS)-induced colorectal cancer (CRC) in mice. (A) The representative macroscopic appearance of a mouse colon treated with AOM/DSS; (B) The representative hematoxylin and eosin stained sections showing different histopathology of mouse colon, including normal (a), dysplasia (b), adenoma (c), and adenocarcinoma (d).

\section{Molecular Mechanisms Associated with Chronic Inflammation and Colorectal Cancer}

\subsection{Inflammation-Dependent Oxidative Stress}

Previous studies identified that carcinogenesis in IBD was related to reactive nitrogen intermediates (RNI) and reactive oxygen species (ROS) $[3,25,26]$. RNI and ROS are produced by neutrophils and macrophages, and they may cause oxidative damage to DNA, proteins, and lipids in the surrounding mucosal cells $[3,23,24]$. RNI, an indicator of nitrosative stress, is associated with nitrotyrosine, which is formed when peroxynitrite interacts with protein tyrosine. Hence, nitrotyrosine can serve as an important biomarker. In murine treated with AOM/DSS, more numerous and concentrated 
nitrotyrosine positive cells were observed as well as the greater expression of iNOS [18]. ROS are small oxygen-derived molecules produced by various biochemical and physiological oxidative processes, including superoxide $\left(\mathrm{O}_{2}^{-}\right)$, hydroxyl $(\mathrm{OH})$, peroxyl $\left(\mathrm{RO}_{2}\right)$, and alkoxyl $(\mathrm{RO})$, hypochlorous acid $(\mathrm{HOCl})$, ozone $\left(\mathrm{O}_{3}\right)$, singlet oxygen $\left(\mathrm{O}_{2}\right)$, and hydrogen peroxide $\left(\mathrm{H}_{2} \mathrm{O}_{2}\right)$ [21]. The imbalance in the generation and elimination of ROS and RNI was found to lead to oxidative stress. Hepatic malondialdehyde (MDA) levels, which are indicators of oxidative stress, were increased in murine treated with AOM/DSS [27]. Enzymatic and nonenzymatic antioxidants, such as superoxide dismutase, catalase, glutathione peroxidase and glutathione reductase, were decreased in an AOM/DSS mouse model [27]. Deficiencies in antioxidant genes, such as transcription factor NF-E2-related factor 2 (Nrf-2) and glutathione peroxidase 3 (Gpx3), were associated with increased numbers of aberrant crypt foci and tumors with higher dysplasia $[28,29]$.

In patients with IBD-associated CRC, increased RNI and ROS concentrations were related to oxidative damage and active DNA damage response at cancer sites [30,31]. In AOM/DSS mouse models, knocking down genes that code for DNA damage repair-related enzymes, e.g., Alkyladenine DNA glycosylase (Aag), mutY DNA glycosylase (Mutyh), 8-oxoguanine DNA glycosylase 1 (Ogg1), may increase CRC incidence $[9,30,32]$. Overall, there is sufficient evidence from animal studies to support the carcinogenic role of inflammation-dependent oxidative stress in CRC developed from IBD.

\subsection{Genomic Instability}

Genomic instability includes base pair mutation, microsatellite instability, chromosome abnormality, gene fusion, gene copy number change, and other small or large structural variations. Inflammation-dependent oxidative stress is also related to high genomic instability in IBD. Genomic instability profiles were found significantly different between IBD-related CRC and sporadic CRC [33]. For example, mutations in IDH1 R132, BRAF V600E; the amplification of FGFR1, FGFR2, ERBB2; and the fusion protein EML4-ALK are more commonly found in IBD related CRC compared to sporadic CRC. Microsatellite instability formed by the malfunction of genes coding for DNA repair mismatch enzymes was found to be a clinical feature of Lynch syndrome and sporadic CRC, but not IBD-related CRC [34]. Little overlap between microsatellite instability in IBD-related CRC and sporadic CRC has been found [35,36]. Aneuploidy is higher in IBD-related CRC than in sporadic CRC, and it could function as an independent factor for the prediction of dysplasia and CRC developed in IBD patients [25,37]. Due to the higher inflammation-dependent oxidative stress and genomic instability in IBD, the critical molecular events in CRC development in sporadic CRC differ from those in IBD-related CRC. For example, the loss of heterozygosity in chromosome 17p, the locus of TP53 (a well-known tumor suppressor) and mutations of TP53 occurred earlier in IBD-related CRC than in sporadic CRC [3,38]. Other study found that the frequency of TP53 mutation in inflamed, non-dysplastic colonic epithelia was correlated with increased oxidative stress [39]. TP53 mutation may be caused by oxidative stress and inflammation in IBD, which promote carcinogenesis of IBD-related CRC. Inflammation-dependent oxidative stress and genomic instability play critical roles in IBD-related carcinogenesis.

\subsection{Cytokines}

A pro-inflammatory microenvironment also contributes to carcinogenesis in IBD. Studies on IBD-associated CRC patients and AOM/DSS mouse models showed that activated neutrophils, fibroblasts, dendritic cells, macrophages, and T cells were present in IBD related CRC tissue [40-43]. The cytokines released by these effectors in a pro-inflammatory microenvironment also showed distinct roles in carcinogenesis in IBD. For instance, the tumor necrosis factor (TNF), which is released by monocyte/macrophage lineages and plays an important role in cell proliferation, differentiation and death, was shown to be increased in an AOM/DSS mouse model [44]. Hence, TNF- $\alpha$ antagonists could significantly reduce the number and size of tumors in this model [18,45]. Interleukin 1 (IL-1)/interleukin 6 (IL-6) axis activation was found to be involved in IBD related CRC carcinogenesis. In IBD, IL-6 is mainly released by monocytes, macrophages, and by T and B lymphocytes. IL-1 produced by 
neutrophils has also been shown to promote the development of CRC in IBD by inducing IL-6 production in intestine-resident mononuclear phagocytes [43]. High expressions of IL- $1 \beta$ and IL-6 were found in an AOM/DSS mouse model [18]. Knocking down IL-6 in AOM/DSS mouse models reduced the number of tumors developed [46,47]. The IL-23/IL-17 axis, including IL-17A, IL-17F, IL-22, and IL-23, are also important cytokines in IBD. The elevated IL-17A and IL-17F in IBD patients are released by Th17 cells. The successful development of Th17 cells to induce chronic inflammation was found to be dependent on the high levels of IL-21, IL-23, IL-6, and TGF $[48,49]$. IL-23 was found to be essential for the expression of TNF $\alpha$, IL-6, and TNF $\gamma$ in the pro-inflammatory microenvironment. These cytokines have the potential to bind to receptors or transcriptional factors in order to activate related signaling pathways to promote carcinogenesis.

\section{4. $N F \kappa B$}

A key molecular component in the pro-inflammation molecular network is nuclear factor kappa $\mathrm{B}(\mathrm{NF} \kappa \mathrm{B})$. Under normal circumstances, NFKB dimers remain in the cytoplasm by binding to the

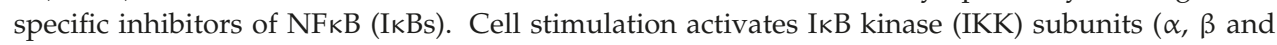
$\gamma)$ to induce the degradation of IKB proteins. The unbound NFKB then translocates to the nucleus and initiates transcription of several hundred target genes to promote the carcinogenesis of CRC. Lipopolysaccharide (LPS), and pro-inflammatory cytokines TNF $\alpha$, IL-1, and CD40 ligand can activate the NFKB pathway. In inflammatory cells, NFKB is generally activated by reacting to bacteria, virus, and necrotic cell production as well as some inflammatory cytokines. The activation of NFKB could induce its downstream pathways including growth and survival signals as well as angiogenic factors. In malignant cells, NFKB reacts to inflammatory cytokine, growth, and survival signals and angiogenic factors released from inflammatory cells and regulate cell cycle related genes, thus, induce apoptosis inhibition effects and promote invasion and metastasis. Specifically, in AOM/DSS mouse models, studies found that in myeloid cells, IKK- $\beta$-driven NFKB promoted the production of cytokines that acted as growth factors in pre-malignant enterocytes. In enterocytes, IKK- $\beta$-driven NFKB was found to activate anti-apoptotic genes, thereby suppressing the apoptotic elimination of pre-neoplastic cells.

\subsection{Microbiome}

The alteration in composition and function of bacterial microbiota and fungal microbiota are considered as significant factors for IBD development [50,51]. The gut microbiome is linked to chronic inflammation in IBD [52]. The cytokines and increased ROS production induced by inflammation favors the outgrowth of some bacteria or kill others, and shape the gut microbiome. The gut microbiome plays an important role in the host immune system. Gut microbiome regulates the function of $\mathrm{T}$ help cell profile, CD4+ Treg cell, the production of several interleukins, such as (IL)-17A, IL-17F, IL-21, and IL-22. Compared to healthy people, IBD patients have more bacteria with inflammatory function and fewer bacteria with anti-inflammatory function [50,51,53,54]. Bacteria with anti-inflammatory effects such as Faecalibacterium prausnitzii, Blautia faecis, Roseburia inulinivorans, Ruminococcus torques, and Clostridium lavalense decreased and adhesion-invasive Escherichia coli with pro-inflammatory effects increased in IBD patients.

\section{Chemoprevention of Colorectal Cancer in Inflammatory Bowel Disease with Anti-Inflammation Pharmaceuticals}

One of the first therapies used to treat IBD is 5-ASA, which inhibits the production of cyclo-oxygenase and prostaglandin, thromboxane synthetase, platelet activating factor synthetase, and IL-1 to reduce the acute inflammatory response [5,52]. However, a systematic review and meta-analysis conducted by Velayos and colleagues that assessed 5-ASA for CRC chemoprevention showed only a slight preventative role of 5-ASA in CRC development [52]. The chemopreventive effects of corticosteroid are not clear, as toxicities from long-term use have limited its implementation as a chemopreventive agent [5]. A major trial that was conducted to assess the roles of immunomodulators, 
such as azathioprine, 6-mercaptopurine, and methotrexate, showed no significant protective effects on CRC [5]. Both azathioprine and 6-mercaptopurine can inhibit purine nucleotide synthesis, metabolism and the DNA/RNA production in immune effectors, and methotrexate can inhibit DNA/RNA production by competitively inhibiting dihydrofolate reductase during tetrahydrofolate synthesis. Thus, these drugs may inhibit DNA/RNA productions in other somatic cells and increase the risk of gene structure change and genomic instability. The side effects of immunomodulators have been associated with increased risks of lymphoma and non-melanomatous skin cancers, thus, limit the use of these medications in IBD patients in the clinical setting. NSAIDs, which inhibit the conversion of arachidonic acid to prostaglandins in the cyclo-oxygenase-2 pathway, have some chemoprevention effects on CRC. The U.S. Preventive Services Task Force (USPSTF) recommends aspirin for adults aged 50-59 to prevent CRC. However, most of the IBD patients are diagnosed in their $20 \mathrm{~s}$ and $30 \mathrm{~s}$ [55], and USPSTF didn't recommend aspirin for adults younger than 50 to take aspirin for colon cancer prevention. The role of aspirin in chemoprevention of IBD related CRC is limited. Moreover, several pieces of evidence showed that NSAIDs promote IBD development [2]. Overall, agents that target inflammation have shown modest results in preventing CRC development in IBD patients.

The complexity of the inflammatory pathways and multiple processes involved in CRC carcinogenesis indicates that a multitude of targets exist; hence, new chemoprevention agents are needed to address this issue. The additive and synergistic activities of multiple bioactive phytochemicals in natural food products may have greater overall efficacy and safety in cancer prevention. Hence, in the following section, we discuss the chemoprevention effects of black raspberry and strawberry on CRC.

\section{Chemoprevention of Colorectal Cancer in Inflammatory Bowel Disease with Berries}

\subsection{Efficiency of Strawberry and Black Raspberry in Cell Lines and Animal Models}

The major investigational models and findings on the chemoprevention effects associated with strawberry and black raspberry are summarized in Table 1. The black raspberry extract showed anti-cancer activity in HT-29 colon cancer cells by regulating the cell cycle and apoptosis signaling pathways [56,57]. In the DSS-treated ulcerative colitis mouse model, the short-term black raspberry intervention reduced the degree of mucosal ulceration, suppressed the levels of pro-inflammation cytokines TNF- $\alpha$ and IL-1 $\beta$, and inhibited the COX-2 and NFKB signaling pathways [58]. When the berry intervention was extended for a longer period of time, the number of macrophages and neutrophils infiltrating the colon tissue decreased and the hypermethylation of tumor suppressor genes increased [59]. In another model of ulcerative colitis using IL-10 knockout mice, a diet of 5\% black raspberry was shown to significantly reduce ulceration in colonic mucosa and submucosa, which are associated with aberrant epigenetic dysregulation in the Wingless/Integrated (Wnt) signaling pathway [60].

Table 1. Preventive effects of black raspberry and strawberry on Inflammatory Bowel Disease (IBD) and CRC.

\begin{tabular}{|c|c|c|}
\hline Systems & Major Findings & Ref. \\
\hline \multicolumn{3}{|l|}{ Black Raspberry } \\
\hline $\begin{array}{l}\text { 1. CRC Cell Lines } \\
\text { HT-29/HT-116 cell lines }\end{array}$ & Regulating cell cycle and apoptosis & {$[56,57]$} \\
\hline \multicolumn{3}{|l|}{ 2. Animal Models of IBD } \\
\hline DSS treated mouse & $\begin{array}{l}\text { Colonic epithelium acute injury } \downarrow \text {, ulceration } \downarrow \text {, } \\
\text { TNF- } \alpha \text { and IL- } 1 \beta \downarrow \text {, COX2 and NFkB } \downarrow \\
\text { Ulceration } \downarrow \text {, macrophages and neutrophils } \\
\text { infiltrated the colon tissue } \downarrow, \text { NFkB } \downarrow \text {, Dkk3 } 3 \uparrow \text {, } \\
\beta \text {-Catenin nuclear localization } \downarrow, \text { c-Myc, DNMT3B, } \\
\text { HDAC } 1, \text { HDAC2, MBD2 } \downarrow\end{array}$ & [58] \\
\hline
\end{tabular}


Table 1. Cont.

\begin{tabular}{|c|c|c|}
\hline Systems & Major Findings & Ref. \\
\hline IL-10 knockout mice & $\begin{array}{l}\text { Ulceration } \downarrow \text {, Wnt pathway } \downarrow \text {, wif1, sox } 17 \text {, and } \\
\text { qki } \uparrow, \text { dnmt } 3 b \text {, hdac1, hdac2, and mbd2 }\end{array}$ & {$[60]$} \\
\hline \multicolumn{3}{|l|}{ 3. Animal Models of IBD-Related CRC } \\
\hline Mouse epidermal JB6Cl41 cells & 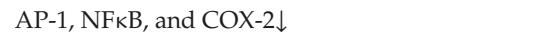 & {$[61,62]$} \\
\hline AOM induced rat model & $\begin{array}{l}\text { ACF multiplicity } \downarrow \text {, total tumor multiplicity } \downarrow \text {, } \\
\text { urinary 8-OHdG } \downarrow\end{array}$ & {$[63]$} \\
\hline Muc2-/- mice & COX-2, TNF- $\alpha$, IL-1, IL-6, and IL-10 $\downarrow$ & [64] \\
\hline \multicolumn{3}{|l|}{ 4. Clinical Studies of CRC } \\
\hline CRC patients & 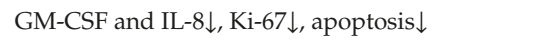 & [65] \\
\hline CRC patients & $\begin{array}{l}\text { Wnt pathway } \downarrow \text { (SFRP2, WIF1, } \beta \text {-catenin, } \\
\text { E-cadherin), DNMT1 } \downarrow\end{array}$ & {$[66]$} \\
\hline \multicolumn{3}{|l|}{ Strawberry } \\
\hline \multicolumn{3}{|l|}{ 1. CRC Cell Lines } \\
\hline HT-29 cell lines & Proliferation $\downarrow$, cell apoptosis and p21WAF1 $\uparrow$ & {$[57,67]$} \\
\hline CaCo-2 & Proliferation $\downarrow$ & {$[68]$} \\
\hline HCT-116 & Proliferation $\downarrow$ & [69] \\
\hline \multicolumn{3}{|l|}{ 2. Animal Models of IBD } \\
\hline Gum acacia induced IBD rats & $\begin{array}{l}\text { Disease activity index } \downarrow \text {, lesion scores } \downarrow \text {, } \\
\text { antioxidant enzymes myeloperoxidase } \uparrow \text {, tissue } \\
\text { catalase } \uparrow \text {, superoxide dismutase } \uparrow\end{array}$ & [70] \\
\hline \multicolumn{3}{|l|}{ 3. Animal Models of IBD-Related CRC } \\
\hline AOM/DSS mouse & $\begin{array}{l}\text { Tumor incidence } \downarrow \text {, nitrosative stress } \downarrow \text {, TNF- } \alpha \text {, } \\
\text { IL- } 1 \beta \text {, IL-6, COX- } 2 \text { and iNOS } \downarrow \text {, PI3K, Akt, ERK } \\
\text { and NFKB } \downarrow\end{array}$ & [18] \\
\hline
\end{tabular}

In mouse epidermal JB6Cl41 cells, carcinogen BaP diol-epoxide (BPDE) treatment activated the activator protein 1 (AP-1), NFkB, and COX-2, which were then inhibited by the methanol extract of black raspberry [61,62]. In an AOM-induced aberrant crypt foci rat model, black raspberry diets significantly reduced the aberrant crypt foci multiplicity, total tumor multiplicity, and urinary 8-hydroxy-2'-deoxyguanosine (8-OHdG) levels [63]. A black raspberry diet was also significantly associated with reduced expression of COX-2 and pro-inflammatory cytokines TNF- $\alpha$, IL-1, IL-6, and IL-10. Interestingly, when it was compared to the mechanism associated with tumor development in Apc1638+/- mice, the inhibition of $\beta$-catenin was found only in Apc1638+/- mice [64].

Strawberry extracts were shown to inhibit HT-29 proliferation by stimulating cell apoptosis and p21WAF1 $[57,67]$. Strawberries also showed significant anti-tumor effects on CRC cells CaCo-2 and HCT-116 [68,69]. In a gum acacia-induced IBD rat model, ethanolic extract of Fragaria vesca, a wild strawberry, decreased the disease activity index and lesion scores and increased antioxidant enzymes including myeloperoxidase, tissue catalase and superoxide dismutase [70]. In the AOM/DSS mouse model, $2.5 \%, 5 \%$, or $10 \%$ (wt $\%$ ) lyophilized strawberry significantly decreased tumor incidence, suppressed nitrosative stress, decreased the inflammation mediators TNF- $\alpha$, IL- $1 \beta$, IL-6, COX-2 and iNOS, and inhibited the phosphorylation of phosphatidylinositol 3-kinase (PI3K), Akt (Protein Kinase B), extracellular signal-regulated kinase (ERK), and NFkB [18].

In comparison to other berries, black raspberry and strawberry showed the potential for superior effects in CRC cell lines. In a study that compared the pro-apoptosis effects of six popularly consumed berries (blackberry, black raspberry, blueberry, cranberry, red raspberry, and strawberry), black raspberry and strawberry showed the most significant pro-apoptotic effects in CRC cell lines [57]. In another study that compared different berry extracts, strawberries showed the best anti-proliferation effects with an EC50 value of $20 \mathrm{ug} / \mathrm{mL}$ [68]. 


\subsection{Efficiency of Black Raspberry in Clinical Studies}

It is reported that a phase I study with black raspberry has been conducted in patients with CRC. The overall objective of this study is to identify measurable genetic and epigenetic biomarkers modulated by black raspberry in patients with CRC. This study demonstrates that $60 \mathrm{~g}$ of black raspberry powder per day for nine weeks significantly modulate the Wnt pathway through demethylating tumor suppressor genes SFRP2 and WIF1, and decrease the expression of $\beta$-catenin and E-cadherin. Black raspberry also decreases the expression of DNMT1, GM-CSF and IL-8 as well as the proliferation of biomarker Ki-67 and apoptosis $[65,66]$. The reports on the effects of strawberry on CRC or IBD are not available.

\subsection{Major Bioactive Components in Berries}

Anthocyanins are flavonoid compounds that are responsible for the colors of most fruits and vegetables, and they contribute to the protective effects of fruits and vegetables against chronic diseases and cancer (Figure 2). According to our previous studies, anthocyanins are the main components in strawberry and black raspberry (Table 2) $[18,22]$. Among the anthocyanins, cyanidin rutinoside is the most abundant in black raspberry, whereas pelargonidin glucoside is the most abundant in strawberry. Pelargonidin rutinoside is the only anthocyanin that has been identified in both black raspberry and strawberry. Following anthocyanins, ellagic acid and derivatives are the second most abundant components of strawberry although they are rare in black raspberry. Among ellagic acid and its derivatives, agrimoniin is the main component of strawberry. Additionally, ellagitannins account for about $10 \%$ of the phytochemical components in black raspberry and strawberry. Flavonols have also been identified in both black raspberry and strawberry, but in much lower concentrations than anthocyanins.

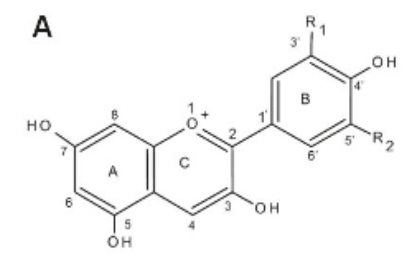

\begin{tabular}{ccc}
\hline Anthocyanins & R1 & R2 \\
\hline Pelargonidin $(\mathrm{Pg})$ & $\mathrm{H}$ & $\mathrm{H}$ \\
Cyanidin $(\mathrm{Cy})$ & $\mathrm{OH}$ & $\mathrm{H}$ \\
Delphinidin (De) & $\mathrm{OH}$ & $\mathrm{OH}$ \\
Peonidin (Pn) & $\mathrm{OCH}_{3}$ & $\mathrm{H}$ \\
Petunidin (Pt) & $\mathrm{OCH}_{3}$ & $\mathrm{OH}$ \\
Malvidin (Ma) & $\mathrm{OCH}_{3}$ & $\mathrm{OCH}_{3}$ \\
\hline
\end{tabular}

B<smiles></smiles>

Cyanidin-3-O-glucoside<smiles>COc1cc2c(O)cc(O)cc2[o+]c1-c1ccc(O)cc1</smiles>

Pelargonidin-3-O-glucoside<smiles>CCOc1cc2c(O)cc(O)cc2[o+]c1-c1ccc(O)c(O)c1</smiles>

Cyanidin-3-O-rutinoside<smiles>COc1cc2c(O)cc(O)cc2[o+]c1-c1ccc(O)cc1</smiles>

Pelargonidin-3-O-rutinoside

Figure 2. Structure of anthocyanins. (A) The structural classification of the six most common anthocyanins; (B) The structural classification of the four most common anthocyanins identified in strawberry and black raspberry. 
Table 2. Major components in strawberry and black raspberry.

\begin{tabular}{|c|c|c|c|c|c|}
\hline \multicolumn{3}{|c|}{ Strawberry } & \multicolumn{3}{|c|}{ Black Raspberry } \\
\hline & $\begin{array}{c}\mathrm{mg} / \\
100 \mathrm{mg}\end{array}$ & $\begin{array}{c}\text { \% by } \\
\text { mg }\end{array}$ & & $\begin{array}{c}\mathrm{mg} / \\
100 \mathrm{mg}\end{array}$ & $\begin{array}{c}\% \text { by } \\
\text { mg }\end{array}$ \\
\hline Anthocyanins & & & Anthocyanins & & \\
\hline pelargonidin glucoside & 367.7 & 41.1 & cyanidin rutinoside & 2924.7 & 58.2 \\
\hline pelargonidin malonyl glucoside & 83.9 & 9.4 & cyanidin xylorutinoside & 916.3 & 18.2 \\
\hline pelargonidin rutinoside & 55.3 & 6.2 & cyanidin glucoside & 245.1 & 4.9 \\
\hline \multirow[t]{3}{*}{ cyanidin glucoside } & 14.9 & 1.7 & cyanidin sambubioside & 103.5 & 2.1 \\
\hline & & & pelargonidin rutinoside & 38.3 & 0.8 \\
\hline & 130.4 & $58.4 \%$ & & 4227.9 & $84.2 \%$ \\
\hline Ellagitannins & & & Ellagitannins & & \\
\hline ellagitannin & 64.1 & 7.2 & sanguiin $\mathrm{H} 6$ & 173.2 & 3.4 \\
\hline ellagitannin & 11.4 & 1.3 & ellagitannin 783-1 & 101.1 & 2.0 \\
\hline ellagitannin & 23.1 & 2.6 & ellagitannin 933-2 & 75.8 & 1.5 \\
\hline \multirow[t]{6}{*}{ Lambertianin } & 20.3 & 2.3 & elagitannin 783-2 & 75.8 & 1.5 \\
\hline & & & ellagitannin 935-1 & 62.8 & 1.3 \\
\hline & & & ellagitannin 933-1 & 50.5 & 1.0 \\
\hline & & & Lambertiannin & 31.3 & 0.6 \\
\hline & & & ellagitannin 935-2 & 7.9 & 0.2 \\
\hline & 118.9 & $13.3 \%$ & & 578.5 & $11.5 \%$ \\
\hline Ellagic acid and derivatives & & & Ellagic acid and derivatives & & \\
\hline Agrimoniin & 144.5 & 16.2 & methyl ellagic acid pentoside & 16.0 & 0.3 \\
\hline ellagic acid rhamnoside & 23.1 & 2.6 & ellagic acid & 9.3 & 0.2 \\
\hline \multirow[t]{3}{*}{ ellagic acid } & 7.3 & 0.8 & ellagic acid rhamnoside & 5.8 & 0.1 \\
\hline & & & $\begin{array}{l}\text { myricetin hexoside, EA } \\
\text { derivative (coelution) }\end{array}$ & 3.8 & 0.1 \\
\hline & 174.9 & $19.5 \%$ & & 34.8 & $0.7 \%$ \\
\hline Flavonols & & & Flavonols & & \\
\hline quercetin hexuronide & 58.8 & 6.6 & quercetin hexuronide & 82.2 & 1.6 \\
\hline kaempferol glucoside/hexuronide & 14.5 & 1.6 & rutin (quercetin rutinoside) & 75.4 & 1.5 \\
\hline \multirow[t]{2}{*}{ kaempferol malonyl hexoside } & 5.1 & 0.6 & quercetin xylorutinoside & 24.2 & 0.5 \\
\hline & 78.4 & $8.8 \%$ & & 181.8 & $3.6 \%$ \\
\hline
\end{tabular}

Berry extracts and anthocyanin compounds have been studied both in vivo and in vitro. Table 4 provides a summary of the role of anthocyanins in CRC cell lines or animal models. Anthocyanin-enriched fractions from black raspberry inhibited cell growth in HT-29 and HCT-119 cells $[57,67,69,71-93]$. In CRC, an anthocyanin-enriched extract of black raspberry showed effects similar to black raspberry powder on CRC cell lines in suppressing cell proliferation, inducing apoptosis, decreasing the activity of DNMT1 and DNMT3B and of demethylate CDKN2A, SFRP2, SFRP5 and WIF1 in the Wnt pathway $[60,71]$. Cyanidin-3-glycoside, which is the anthocyanin found in black raspberry, decreased DNA strand breakage in human colon epithelial cells (HCEC), and it reduced cytotoxicity induced by peroxyl radicals by suppressing apoptosis and decreasing the sub-G1 phase of the cell population in Caco-2 CRC cells [72,73]. Cyanidin-3-O-beta glucopyranoside and its aglycon form, cyanidin chloride, was shown to have the potential to function in inhibiting cell growth and proliferation and in decreasing the ROS level in Caco-2 cells [74]. Strawberry extracts also showed cell growth and proliferation inhibition effects, antioxidative effects, and p21WAF1 suppression effects in HT-29, HCT-116 cells $[57,66,70]$. The anthocyanins identified in other fruit and vegetables that showed anti-cancer effects in CRC cells lines included the anthocyanins found in black raspberry and strawberry. 
Table 3. Effects of anthocyanins in IBD and IBD-related CRC.

\begin{tabular}{|c|c|c|c|c|}
\hline Original Sources & Models & Major Findings & Anthocyanin Profiles & Ref. \\
\hline \multicolumn{5}{|l|}{ Cell Lines } \\
\hline \multicolumn{5}{|l|}{ 1. Black Raspberry } \\
\hline Black raspberry extract & $\begin{array}{l}\text { Cell line } \\
\text { HT-29 } \\
\text { HCT-116 }\end{array}$ & $\begin{array}{l}\text { Inhibited cell growth } \\
\text { HT-29 IC50 = 89.11, } \\
\text { HCT-116, IC50 = 89.00 }\end{array}$ & $\begin{array}{l}\text { cyanidin-3-sophoroside rhamnoside, } \\
\text { cyanidin-3-sambubioside } \\
\text { rhamnoside, cyanidin-3-rutinoside }\end{array}$ & [57] \\
\hline $\begin{array}{l}\text { Black raspberry } \\
\text { anthocyanin-enriched } \\
\text { extract }\end{array}$ & $\begin{array}{l}\text { Cell line } \\
\text { HCT116 }\end{array}$ & $\begin{array}{l}\text { Decreased DNMT activity; } \\
\text { decreased methylation of } \\
\text { CDKN2A, SFRP5, SFRP2 and } \\
\text { WIF1; suppressed cell } \\
\text { proliferation; induced apoptosis }\end{array}$ & $\begin{array}{l}\text { cyanidin-3-O-glucoside, cyanidin- } \\
\text { 3-O-rutinoside, } \\
\text { cyanidin-3-O-xylosylrutinoside, } \\
\text { cyanidin-3-O-sambubioside }\end{array}$ & [58] \\
\hline \multicolumn{5}{|l|}{ 2. Strawberry } \\
\hline Strawberry extract & $\begin{array}{l}\text { Cell line } \\
\text { HT-29 } \\
\text { HCT-116 }\end{array}$ & $\begin{array}{l}\text { Inhibited cell growth } \\
\text { HT-29 } \mathrm{IC}_{50}=114.30 \\
\text { HCT-116, IC } \\
50=62.00\end{array}$ & $\begin{array}{l}\text { cyanidin-3-glucoside, } \\
\text { pelargonidin-3-glucoside, } \\
\text { pelargonidin-3-rutinoside }\end{array}$ & [57] \\
\hline Strawberry extract & $\begin{array}{l}\text { Cell line } \\
\text { HT29 } \\
\text { HCT-116 }\end{array}$ & Antioxidative effects. & $\begin{array}{l}\text { cyanidin-3-glucoside, pelargonidin, } \\
\text { cyanidin-3-glucoside, pelargonidin, } \\
\text { pelargonidin-3-rutinoside }\end{array}$ & [66] \\
\hline Strawberry extract & $\begin{array}{l}\text { Cell line } \\
\text { HT29 }\end{array}$ & $\begin{array}{l}\text { Inhibited proliferation; reduced } \\
\text { expression of p21WAF1 }\end{array}$ & $\begin{array}{l}\text { cyanidin derivative; pelargonidin } \\
\text { derivative }\end{array}$ & [67] \\
\hline \multicolumn{5}{|l|}{ 3. Anthocyanins } \\
\hline cyanidin-3-glycoside & $\begin{array}{l}\text { Cell line } \\
\text { HCEC }\end{array}$ & Decreased DNA strand breakage & cyanidin-3-glycoside & [72] \\
\hline Cyanidin-3-glycoside & $\begin{array}{l}\text { Cell line } \\
\text { Caoco-2 }\end{array}$ & $\begin{array}{l}\text { Reduced cytotoxicity induced by } \\
\text { AAPH; suppressed apoptosis; } \\
\text { decreased sub-G1 phase cell } \\
\text { population }\end{array}$ & cyanidin-3-glycoside & [73] \\
\hline $\begin{array}{l}\text { Cyanidin-3-O-beta } \\
\text { glucopyranoside, } \\
\text { cyanidin chloride }\end{array}$ & $\begin{array}{l}\text { Cell line } \\
\text { Caoco-2 }\end{array}$ & $\begin{array}{l}\text { Inhibited cell growth and } \\
\text { proliferation; decreased reactive } \\
\text { oxygen species (ROS) level }\end{array}$ & $\begin{array}{l}\text { cyanidin-3-O-beta glucopyranoside, } \\
\text { cyanidin chloride }\end{array}$ & [74] \\
\hline \multicolumn{5}{|c|}{ 4. Protocatechuic Acid (PCA) } \\
\hline $\begin{array}{l}\text { Brown rice extracted } \\
\text { PCA }\end{array}$ & $\begin{array}{l}\text { Cell line } \\
\text { SW480 }\end{array}$ & $\begin{array}{l}\text { Inhibited cell growth and colony } \\
\text { formation }\end{array}$ & PCA & [75] \\
\hline \multicolumn{5}{|c|}{ 5. Other Fruit and Vegetables } \\
\hline Blueberry & $\begin{array}{l}\text { Cell line } \\
\text { Caoco-2 }\end{array}$ & $\mathrm{IC}_{50} 0.53 \pm 0.04$ & $\begin{array}{l}\text { delphinidin 3-galactoside, delphinidin } \\
\text { 3-glucoside, cyanidin 3-galactoside, } \\
\text { delphinidin 3-arabinoside, cyanidin } \\
\text { 3-glucoside, petunidin 3-galactoside, } \\
\text { cyanidin 3-arabinoside, petunidin } \\
\text { 3-glucoside, peonidin 3-galactoside, } \\
\text { petunidin 3-arabinoside, peonidin } \\
\text { 3-glucoside, malvidin 3-galactoside, } \\
\text { peonidin 3-arabinoside, malvidin } \\
\text { 3-glucoside, malvidin 3-arabinoside }\end{array}$ & [76] \\
\hline Blueberry extract & $\begin{array}{l}\text { Cell lines } \\
\text { HT-29 }\end{array}$ & $\begin{array}{l}\text { Inhibited cell growth; induced } \\
\text { apoptosis }\end{array}$ & $\begin{array}{l}\text { delphinidin } 3 \text { - } O \text { - } \beta \text {-glucopyranoside; } \\
\text { cyanidin } 3-O-\beta \text {-galactopyranoside; } \\
\text { cyanidin } 3-O \text { - } \beta \text {-glucopyranoside; } \\
\text { petunidin } 3-O-\beta \text {-glucopyranoside; } \\
\text { peonidin } 3-O-\beta \text {-galactopyranoside; } \\
\text { peonidin } 3-O-\beta \text {-glucopyranoside; } \\
\text { malvidin } 3-O-\beta \text {-glucopyranoside. }\end{array}$ & [77] \\
\hline $\begin{array}{l}\text { Bilberry purified } \\
\text { anthocyanins }\end{array}$ & $\begin{array}{l}\text { Cell line } \\
\text { HCT-116 }\end{array}$ & Decreased cell viability & $\begin{array}{l}\text { pelargonidin, cyanidin, peonidin, } \\
\text { delphinidin, and malvidin }\end{array}$ & [78] \\
\hline $\begin{array}{l}\text { Cocoplum anthocyanins } \\
\text { exert }\end{array}$ & $\begin{array}{l}\text { Cell line } \\
\text { HT-29 }\end{array}$ & $\begin{array}{l}\text { Cell proliferation was suppressed; } \\
\text { increased intracellular ROS } \\
\text { production; increased intracellular } \\
\text { ROS production }\end{array}$ & $\begin{array}{l}\text { delphinidin-3-glucoside, cyanidin } \\
\text { 3-glucoside, petunidin 3-glucoside, } \\
\text { delphinidin 3-(6-acetoyl) galactoside, } \\
\text { delphinidin 3-(6-oxaloyl) arabinoside, } \\
\text { peonidin 3-glucoside, petunidin } \\
\text { 3-(6-acetoyl) galactoside, petunidin } \\
\text { 3-(6-oxaloyl) arabinoside, peonidin } \\
\text { 3-(6-acetoyl) glucoside, peonidin } \\
\text { 3-(6-oxaloyl) arabinoside }\end{array}$ & [79] \\
\hline $\begin{array}{l}\text { Eugenia jambolana (Java } \\
\text { Plum) fruit extract }\end{array}$ & $\begin{array}{l}\text { Cell lines } \\
\text { HCT-116 } \\
\text { colon cancer } \\
\text { stem cells }\end{array}$ & $\begin{array}{l}\text { Inhibited proliferation; induced } \\
\text { apoptosis }\end{array}$ & $\begin{array}{l}\text { delphinidin-3,5-diglucoside, } \\
\text { cyanidin-3,5-diglucoside, } \\
\text { ptunidin-3,5-diglucosid, } \\
\text { dtunidin-3,5-diglucosid, } \\
\text { peonidin-3,5-diglucoside, } \\
\text { monidin-3,5-diglucosid, } \\
\text { cyanidin-3-glucoside, } \\
\text { petunidin-3-glucoside, } \\
\text { etunidin-3-glucosi }\end{array}$ & [80] \\
\hline
\end{tabular}


Table 4. Effects of anthocyanins in IBD and IBD-related CRC.

\begin{tabular}{|c|c|c|c|c|}
\hline Original Sources & Models & Major Findings & Anthocyanin Profiles & Ref. \\
\hline \multicolumn{5}{|l|}{ Cell Lines } \\
\hline $\begin{array}{l}\text { Anthocyanin-containing } \\
\text { baked purple-fleshed } \\
\text { potato extract }\end{array}$ & $\begin{array}{l}\text { Colon cancer } \\
\text { stem cells }\end{array}$ & $\begin{array}{l}\text { Suppressed proliferation; elevated } \\
\text { apoptosis; decreased } \beta \text {-catenin, } \\
\text { c-Myc and Cyclin D1 }\end{array}$ & $\begin{array}{l}\text { pet-3-rut-5-glc, mal-3-rut-5-glc, } \\
\text { cya-3-O(6-O-malonyl- } \\
\beta \text {-D-glc), peo-3-(p-coum)-isophoro-5-glc, } \\
\text { peo-3-rut-5-glc, } \\
\text { pet-3-(p-coum)-rut-5-glc, } \\
\text { peo-3-caffeyl-rut-5-glc, } \\
\text { pel-3-(p-coum)-rut-5-glc, } \\
\text { pel-3-(4-ferul-rut)-5-glc, } \\
\text { peo-3-(p-coum)-rut-5-glc, } \\
\text { mal-3-(p-coum)-rut-5-glc }\end{array}$ & [81] \\
\hline Blue maize extract & $\begin{array}{l}\text { Cell line } \\
\text { Caco2 and } \\
\text { HT29 }\end{array}$ & Suppressed proliferation & $\begin{array}{l}\text { cyanidin 3-glucoside, cyanidin } \\
\text { 3-glucoside, cyanidin } \\
\text { malonyl-glucoside, cyanidin } \\
\text { succinyl-glucoside, pelargonidin } \\
\text { 3-glucoside, pelargonidin } \\
\text { malonyl-glucoside }\end{array}$ & [82] \\
\hline Eggplant extract & $\begin{array}{l}\text { Cell lines } \\
\text { HT-29 }\end{array}$ & Decreased DNA damage & $\begin{array}{l}\text { delphinidin-3-rhamnosyl-glucoside-5- } \\
\text { glucoside, delphinidin-3-rutinoside-5- } \\
\text { glucoside }\end{array}$ & [83] \\
\hline $\begin{array}{l}\text { anthocyanin-enriched } \\
\text { purple-fleshed sweet } \\
\text { potato }\end{array}$ & Cell line SW480 & $\begin{array}{l}\text { Decreased cell number, G1 phase } \\
\text { arrest }\end{array}$ & peonidin-3-glucoside & [84] \\
\hline $\begin{array}{l}\text { Vitis coignetiae Pullia } \\
\text { extract }\end{array}$ & & $\begin{array}{l}\text { Inhibited cell invasion; } \\
\text { suppressed MMP-2, MMP-9, } \\
\text { NFkB }\end{array}$ & $\begin{array}{l}\text { delphinidin-3,5-diglucoside, } \\
\text { cyanidin-3,5-diglucoside, } \\
\text { petunidin-3,5-diglucoside, } \\
\text { delphinidin-3-glucoside, } \\
\text { malvdin-3,5-diglucoside, } \\
\text { peonidin-3,5-diglucoside, } \\
\text { cyanidin-3-glucoside, } \\
\text { petunidin-3-glucoside, } \\
\text { peonidin-3-glucoside, } \\
\text { malvidin-3-glucoside }\end{array}$ & [85] \\
\hline Blackberry extract & $\begin{array}{l}\text { Cell lines } \\
\text { HT-29 }\end{array}$ & $\begin{array}{l}\text { Inhibited cell growth; inhibited } \\
\text { IL-12 release }\end{array}$ & $\begin{array}{l}\text { cyanidin-3-glucoside, } \\
\text { cyanidin-3-arabinoside, } \\
\text { delphinidin-3-xyloside, } \\
\text { cyanidin-3-xyloside, } \\
\text { cyanidin-3-malonylglucoside, } \\
\text { cyanidin-3-dioxalylglucoside }\end{array}$ & [86] \\
\hline Tart cherry anthocyanin & $\begin{array}{l}\text { Cell line } \\
\text { HT 29, HCT16 }\end{array}$ & Inhibited cell growth & $\begin{array}{l}\text { 3-cyanidin } 2^{\prime \prime}-O-\beta \text {-D-glucopyranosyl- } \\
6^{\prime \prime}-O-\alpha \text {-L-rhamnopyransyl- } \beta \text {-D- } \\
\text { glucopyranoside }\end{array}$ & [87] \\
\hline \multicolumn{5}{|l|}{ Animal Models } \\
\hline \multicolumn{5}{|l|}{ 1. PCA } \\
\hline PCA & $\begin{array}{l}\text { AOM-treated } \\
\text { rat }\end{array}$ & $\begin{array}{l}\text { Decreased the number of aberrant } \\
\text { crypt foci, ornithine decarboxylase } \\
\text { activity and AgNOR }\end{array}$ & PCA & {$[88,89]$} \\
\hline PCA & DSS-treated rat & $\begin{array}{l}\text { Prevented diarrhea and bleeding; } \\
\text { decreased pro-inflammatory } \\
\text { cytokines; nitric oxide } \\
\text { concentration, oxidative damage, } \\
\text { and expression of COX-2 and } \\
\text { iNOS }\end{array}$ & PCA & [90] \\
\hline \multicolumn{5}{|c|}{ 2. Other fruit and vegetables } \\
\hline $\begin{array}{l}\text { Anthocyanin-rich } \\
\text { extracts from bilberry, } \\
\text { chokeberry, and grape }\end{array}$ & $\begin{array}{l}\text { AOM-treated } \\
\text { rat }\end{array}$ & $\begin{array}{l}\text { Reduced total ACF and the } \\
\text { number of large ACF } \\
\text { Suppressed cell proliferation } \\
\text { reduces COX-2 }\end{array}$ & & [91] \\
\hline $\begin{array}{l}\text { Anthocyanin derived } \\
\text { from purple sweet potato } \\
\text { color in their basal diet }\end{array}$ & $\begin{array}{l}\text { AOM/DSS } \\
\text { rats }\end{array}$ & $\begin{array}{l}\text { Decreased MDF, colon weight, } \\
\text { low-grade dysplasia and total } \\
\text { histopathology changes; } \\
\text { decreased the expression of } \\
\beta \text {-catenin, Ki67and Cyclin D1 }\end{array}$ & & [92] \\
\hline $\begin{array}{l}\text { anthocyanin-enriched } \\
\text { purple-fleshed sweet } \\
\text { potato }\end{array}$ & $\begin{array}{l}\text { Animal } \\
\text { AOM mice }\end{array}$ & $\begin{array}{l}\text { Suppressed formation of aberrant } \\
\text { crypt foci; decreased PCNA; } \\
\text { increased caspase- } 3\end{array}$ & peonidin-3-glucoside & [84] \\
\hline Tart cherry anthocyanin & $\mathrm{Apc}^{\mathrm{Min}}$ mice & $\begin{array}{l}\text { Decreased the number and } \\
\text { volume of adenomas }\end{array}$ & $\begin{array}{l}\text { 3-cyanidin 6"-O- } \alpha \text {-L-rhamnopyranosyl- } \\
\beta \text {-D-glucopyranoside }\end{array}$ & [87] \\
\hline Tomato & DSS mice & $\begin{array}{l}\text { Increased bacterial Parabacteroides } \\
\text { and Lactobacilli }\end{array}$ & & [93] \\
\hline
\end{tabular}


The distribution of anthocyanins has been found in almost all tissues. The black raspberry phytochemicals, cyanidin-3-rutinoside and cyanidin-3-xylosylrutinoside, have been detected in oral cancer tissues in patients after the administration of oral troches containing freeze-dried black raspberry powder as well as in prostate tissue in mice models after a diet of black raspberry powder [94,95] Importantly, bioavailability and pharmacokinetic studies of strawberry anthocyanins showed that only modest amounts of ingested anthocyanins were absorbed from the upper small intestine. Most phytochemicals enter the colon where the substantial microbial metabolism and interaction with the colonic epithelium take place [18]. Protocatechuic acid (PCA) is one of the main metabolites of anthocyanins that can be absorbed by animals and humans. PCA significantly inhibited cell proliferation and colony formation in colon cancer SW 480 cells [75]. PCA also prevented diarrhea and bleeding in DSS-treated rats, and it decreased pro-inflammatory cytokines, nitric oxide concentration, and oxidative damage as well as the expression of COX-2 and iNOS [90]. Additionally, PCA diets may decrease the number of aberrant crypt foci, ornithine decarboxylase activity and the expression of AgNOR in AOM-induced CRC rat models [88,89]. In strawberries, 4-hydroxybenzoic acid (4HBA) was considered a metabolite of pelargonidin-3-glucoside, but it did not show anti-cancer activity in colon cancer in a limited number of studies [96,97].

The growth and spread of cancer depended not only on the biological characteristics of the tumor cells but also on the host immunology response. Black raspberry extracts and the single anthocyanins component cyanidin-3-rutinoside and quercitin-3-rutinoside were shown to inhibit T cell proliferation, limit myeloid-derived suppressor cells (MDSC) expansion, and suppress MDSC capacity [98]. Cyanidin-3-glucoside and cyanidin-3-rutinoside also reduced the inflammation cytokines TNF- $\alpha$, IL-6, IL-1 $\beta$ in lipopolysaccharide (LPS)-treated murine macrophage RAW264.7 cells [99].

In contrast to the consistent effects of anthocyanins in black raspberry and strawberry on chemoprevention, the results of studies that assessed the ellagitannin components of berries in chemoprevention have varied. Some studies found that ellagitannins were the most responsible for the anti-cancer activity of berry extracts $[56,67]$. However, the findings of other studies suggested that berry ellagitannins may not be sufficient for the prevention of many cancers, such as esophageal squamous cell carcinoma, because various concentrations of ellagitannins showed no differences in chemoprevention [100].

A recent study that used a high-resolution $1 \mathrm{H}$ NMR-based multivariate statistical model showed that anthocyanins, cyanidin 3-rutinoside, and cyanidin 3-xylosylrutinoside were the predominant contributors to the anti-cancer effects of black raspberry. However, in the same study, salicylic acid derivatives (salicylic acid glucosyl ester), quercetin 3-glucoside, quercetin 3-rutinoside, p-coumaric acid, epicatechin, methyl ellagic acid derivatives (methyl ellagic acetyl pentose), and citric acid derivatives were also shown to contribute significantly to anti-cancer effects [101].

Anthocyanins have brought the research community's attention in the field of IBD [102,103]. In our opinion, anthocyanins are the major components responsible for the chemoprevention effects of black raspberry and strawberry. This opinion is based on five main lines of evidence: (1) anthoycanins are the most abundant components in black raspberry and strawberry; (2) the anti-cancer effects of anthocyanins and the role of anthocyanins in immune modification are well-documented; (3) PCA, the metabolites of anthocyanins in black raspberry, show chemoprevention effects similar to those of black raspberry; (4) anthocyanins are found in cancer tissues; (5) the colon is the major site of the metabolism of anthocyanins. However, because other components also significantly contribute to anti-cancer effects, it is clear that further studies are needed to assess the chemopreventive compounds in black raspberry and strawberry.

\subsection{Mechanisms Associated with Preventative Effects of Berries on Colon Cancer}

The benefits of berries in reducing antioxidative stress are well known. In a study that compared the antioxidant role of 10 phenolic compounds in strawberry extracts, the most potent antioxidants identified by a trolox equivalent antioxidant capacity (TEAC) assay were cyanidin-3-glucoside, 
cyanidin-3-pelargonidin, and kaempferol [66]. Moreover, urinary 8-OHdG levels and tissue nitrosative stress are important biomarkers of oxidative stress, which were reduced by the intake of black raspberry and strawberry $[18,63]$. Overall, several studies using cell lines, animal models, and human clinical trials of strawberry and black raspberry reported significantly decreased oxidative and inflammatory signals, such as COX-2 and NFKB. However, not all related signals were shown to be regulated by either black raspberry or strawberry, including the following: DNA repair-related enzymes, such as Aag, Mutyh, Ogg1; enzymatic and nonenzymatic antioxidants, such as SOD; and the antioxidant genes Nrf-2, Gpx3. Therefore, these molecular processes should be examined in future studies.

One form of genomic instability is the loss of heterozygosity, which in previous studies was reduced by the topical application of the black raspberry gel on oral intraepithelial neoplasia lesions (17p13 location containing TP53 genes are included in the loss of heterozygosity). Hypermethylation, or the increased number of methyl groups added to the promoter region, were shown to function to inhibit the transcription of target genes. Similar to the loss of heterozygosity, hypermethylation was shown to be an important approach in deactivating tumor suppressor genes during carcinogenesis. As previously mentioned in this review, black raspberry powder and anthocyanin-enriched extract of black raspberry worked to demethylate CDKN2A, SFRP2, SFRP5, and WIF1 in the Wnt pathway through suppressing DNMT1 and DNMT3B $[60,71]$. In a phase Ib study on the effects of black raspberries on rectal polyps in patients with familial adenomatous polyposis, a greater number of the demethylated transcription start sites and the increased expression of DNMT1 was found in patients who received a black raspberry intervention compared to those that did not [104]. Combined with the demonstrated antioxidant effects of black raspberry and strawberry, the above evidence suggests the potential of black raspberry and strawberry to prevent inflammation-dependent oxidative stress and genomic instability in colon epithelial cells.

In a DSS-treated ulcerative colitis mouse model, an intervention employing a long-term black raspberry diet resulted in the decreased infiltration by macrophages and neutrophils in colon tissue [61]. Black raspberry and strawberry were both associated with the reduced expression of COX-2 and the pro-inflammatory mediators, COX-2, iNOS, TNF- $\alpha$, IL-1, IL-6, and IL-10 in cell studies, animal models, and clinical trials [17-22]. Cytokine GM-CSF and IL-8 were also found to be decreased by black raspberry powder in untreated colon cancer patients [67]. Black raspberry also altered innate immune cell trafficking in NMBA-induced esophageal squamous cell carcinoma in a rat model [105]. Single anthocyanins cyanidin-3-rutinoside and quercitin-3-rutinoside inhibited MDSC expansion and modulated T lymphocyte proliferation [98]. Hence, another interesting area for further research is the regulation of immune effectors by black raspberry and strawberry.

$\mathrm{NFKB}$ is present in almost all cell types, and it is involved in inflammation, cell differentiation, and carcinogenesis. The cyclin-dependent kinase inhibitor, p21WAF1/Cip1, was induced by black raspberry in a Muc2-/- mice CRC model [64]. In addition, the cell proliferation markers Ki-67, c-Jun, p27, Erk1/2, MAPK, and AKT were regulated by treatment with black raspberry or strawberry $[18,103$, 104,106]. Oxidative stress activates MAPK family members, including p38 MAPK and JNK, which subsequently activated genes involved in cellular proliferation. MAPK is activated by a range of stimuli, and it mediates several physiological processes, which are observed during carcinogenesis. AKT signaling plays an important role in multiple cellular processes, including cell proliferation, survival, motility, and angiogenesis. The induction of cell survival by AKT is mediated through NFKB signaling. The activation of the AKT and ERK pathways acts synergistically to promote the mechanistic target of rapamycin signaling, which controls NFKB activity $[18,20]$. NFKB was also found to regulate cell apoptosis during carcinogenesis. Black raspberry and strawberry increased apoptosis markers, TUNNEL staining, and the Bcl-2/Bax ratio in both animal models and human patients [16,105-108]. $\mathrm{NF} \kappa \mathrm{B}$ is required for the stabilization of snail, which is the transcription factor involved in regulating the expression of E-cadherin to induce cell migration and invasion induced by inflammatory cytokines. The inhibition of NFKB was found to potentially induce the degradation of Snail, which increased the expression of E-cadherin and hence inhibited cell migration and invasion. NFkB signaling was 
an important molecular event associated with the chemoprevention effects of black raspberry and strawberry on IBD-induced CRC. The mechanisms associated with the chemoprevention effects of black raspberry and strawberry are summarized in Figure 3.

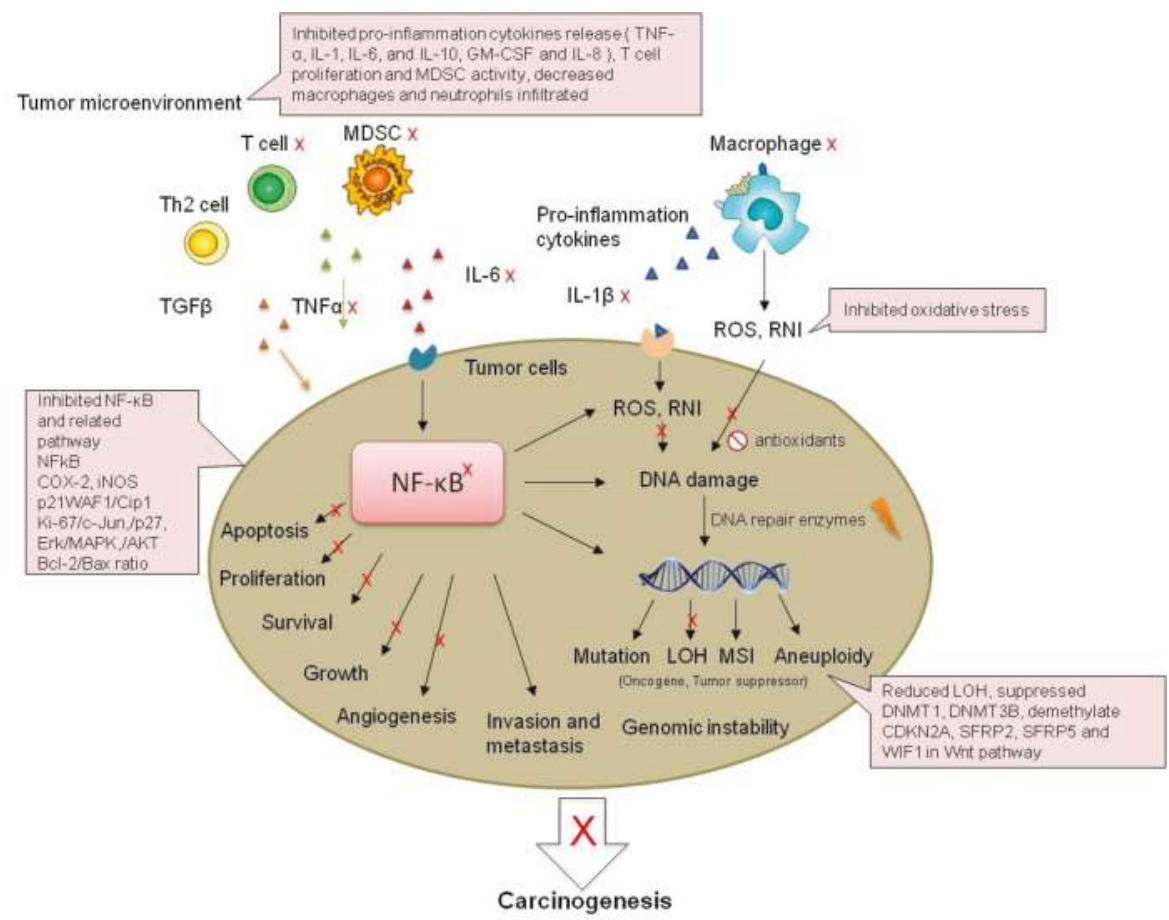

Figure 3. Possible mechanisms of the inhibition of IBD-related CRC by strawberry and black raspberry. MDSC: myeloid-derived suppressor cells; RNI: reactive nitrogen intermediates; LOH: Loss of heterozygosity; MSI: microsatellite instability.

\section{Conclusions}

Strawberry and black raspberry have been shown to have potential cancer prevention effects with low toxicity profiles in IBD-related CRC. Inflammation-induced carcinogenesis has been associated with oxidative stress, genomic instability, immune effectors, cytokine dysregulation, and the NFKB signaling pathway. In contrast to anti-inflammation pharmaceuticals, strawberry and black raspberry interventions have shown to have a synergistic role in multiple molecular events, including suppressing cytokines release, decreasing oxidative stress, reducing genomic instability, and inhibiting NFKB and related signaling pathways. The chemopreventive activity of strawberry and black raspberry is likely due to multiple nutrients and bioactives, especially anthocyanins. The clinical translational application of berries in IBD patients for CRC prevention is limited, which may suggest that this "low-hanging fruit" should be assessed in future clinical trials on colon cancer prevention in IBD. The evidence to date is a step toward the development of specific phytochemicals and metabolites as chemopreventive agents based on the principles of pharmacognosy.

Author Contributions: Conceptualization, T.C. and N.S.; writing-original draft preparation, T.C. and N.S.; writing-review and editing, T.C., N.S. and A.A.; supervision, T.C.; project administration, T.C.; funding acquisition, T.C.

Funding: This research was funded by the California Strawberry Commission and the internal fund from the Department of Internal Medicine, The Ohio State University. 
Acknowledgments: The authors would like to thank Stephanie Fortier, MSc (The Ohio State University, Division of Medical Oncology) for her help in editing this manuscript.

Conflicts of Interest: Chen $\mathrm{T}$ and Shi $\mathrm{N}$ have no potential conflicts of interest to disclose. Afzali A is a consultant for AbbVie, UCB, Takeda, Pfizer, and Janssen.

\section{References}

1. Ananthakrishnan, A.N. Epidemiology and risk factors for ibd. Nat. Rev. Gastroenterol. Hepatol. 2015, 12, 205-217. [CrossRef] [PubMed]

2. Ananthakrishnan, A.N.; Bernstein, C.N.; Iliopoulos, D.; Macpherson, A.; Neurath, M.F.; Ali, R.A.R.; Vavricka, S.R.; Fiocchi, C. Environmental triggers in IBD: A review of progress and evidence. Nat. Rev. Gastroenterol. Hepatol. 2018, 15, 39-49. [CrossRef] [PubMed]

3. Feagins, L.A.; Souza, R.F.; Spechler, S.J. Carcinogenesis in ibd: Potential targets for the prevention of colorectal cancer. Nat. Rev. Gastroenterol. Hepatol. 2009, 6, 297-305. [CrossRef] [PubMed]

4. Mattar, M.C.; Lough, D.; Pishvaian, M.J.; Charabaty, A. Current management of inflammatory bowel disease and colorectal cancer. Gastrointest. Cancer Res. 2011, 4, 53-61.

5. Subramanian, V.; Logan, R.F. Chemoprevention of colorectal cancer in inflammatory bowel disease. Best Pract. Res. Clin. Gastroenterol. 2011, 25, 593-606. [CrossRef]

6. Jess, T.; Rungoe, C.; Peyrin-Biroulet, L. Risk of colorectal cancer in patients with ulcerative colitis: A meta-analysis of population-based cohort studies. Clin. Gastroenterol. Hepatol. 2012, 10, 639-645. [CrossRef]

7. Eaden, J.A.; Abrams, K.R.; Mayberry, J.F. The risk of colorectal cancer in ulcerative colitis: A meta-analysis. Gut 2001, 48, 526-535. [CrossRef]

8. Liao, J.; Seril, D.N.; Lu, G.G.; Zhang, M.; Toyokuni, S.; Yang, A.L.; Yang, G.Y. Increased susceptibility of chronic ulcerative colitis-induced carcinoma development in DNA repair enzyme ogg1 deficient mice. Mol. Carcinog. 2008, 47, 638-646. [CrossRef]

9. Kornbluth, A.; Sachar, D.B. Ulcerative colitis practice guidelines in adults (update): American college of gastroenterology, practice parameters committee. Am. J. Gastroenterol. 2004, 99, 1371-1385. [CrossRef]

10. Lewis, J.D.; Abreu, M.T. Diet as a trigger or therapy for inflammatory bowel diseases. Gastroenterology 2017, 152, 398-414.e6. [CrossRef]

11. Khalili, H.; Chan, S.S.M.; Lochhead, P.; Ananthakrishnan, A.N.; Hart, A.R.; Chan, A.T. The role of diet in the aetiopathogenesis of inflammatory bowel disease. Nat. Rev. Gastroenterol. Hepatol. 2018, 15, 525-535. [CrossRef] [PubMed]

12. Woo, H.D.; Kim, J. Dietary flavonoid intake and risk of stomach and colorectal cancer. World J. Gastroenterol. 2013, 19, 1011-1019. [CrossRef]

13. Arts, I.C.; Jacobs, D.R., Jr.; Gross, M.; Harnack, L.J.; Folsom, A.R. Dietary catechins and cancer incidence among postmenopausal women: The iowa women's health study (united states). Cancer Causes Control 2002, 13, 373-382. [CrossRef] [PubMed]

14. Simons, C.C.; Hughes, L.A.; Arts, I.C.; Goldbohm, R.A.; van den Brandt, P.A.; Weijenberg, M.P. Dietary flavonol, flavone and catechin intake and risk of colorectal cancer in the netherlands cohort study. Int. J. Cancer 2009, 125, 2945-2952. [CrossRef] [PubMed]

15. Stoner, G.D. Foodstuffs for preventing cancer: The preclinical and clinical development of berries. Cancer Prev. Res. 2009, 2, 187-194. [CrossRef]

16. Zhu, X.R.; Xiong, L.F.; Zhang, X.Y.; Shi, N.; Zhang, Y.T.; Ke, J.; Sun, Z.; Chen, T. Lyophilized strawberries prevent 7,12-dimethylbenz[alpha]anthracene (dmba)-induced oral squamous cell carcinogenesis in hamsters. J. Funct. Foods 2015, 15, 476-486. [CrossRef]

17. Chen, T.; Yan, F.; Qian, J.; Guo, M.; Zhang, H.; Tang, X.; Chen, F.; Stoner, G.D.; Wang, X. Randomized phase ii trial of lyophilized strawberries in patients with dysplastic precancerous lesions of the esophagus. Cancer Prev. Res. 2012, 5, 41-50. [CrossRef]

18. Shi, N.; Clinton, S.K.; Liu, Z.; Wang, Y.; Riedl, K.M.; Schwartz, S.J.; Zhang, X.; Pan, Z.; Chen, T. Strawberry phytochemicals inhibit azoxymethane/dextran sodium sulfate-induced colorectal carcinogenesis in crj: Cd-1 mice. Nutrients 2015, 7, 1696-1715. [CrossRef] 
19. Duncan, F.J.; Martin, J.R.; Wulff, B.C.; Stoner, G.D.; Tober, K.L.; Oberyszyn, T.M.; Kusewitt, D.F.; Van Buskirk, A.M. Topical treatment with black raspberry extract reduces cutaneous uvb-induced carcinogenesis and inflammation. Cancer Prev. Res. 2009, 2, 665-672. [CrossRef]

20. Mallery, S.R.; Tong, M.; Shumway, B.S.; Curran, A.E.; Larsen, P.E.; Ness, G.M.; Kennedy, K.S.; Blakey, G.H.; Kushner, G.M.; Vickers, A.M.; et al. Topical application of a mucoadhesive freeze-dried black raspberry gel induces clinical and histologic regression and reduces loss of heterozygosity events in premalignant oral intraepithelial lesions: Results from a multicentered, placebo-controlled clinical trial. Clin. Cancer Res. 2014, 20, 1910-1924.

21. Shi, N.; Chen, F.; Zhang, X.; Clinton, S.K.; Tang, X.; Sun, Z.; Chen, T. Suppression of oxidative stress and nfkappab/mapk signaling by lyophilized black raspberries for esophageal cancer prevention in rats. Nutrients 2017, 9, 413. [CrossRef]

22. Shi, N.; Riedl, K.M.; Schwartz, S.J.; Zhang, X.L.; Clinton, S.K.; Chen, T. Efficacy comparison of lyophilised black raspberries and combination of celecoxib and pbit in prevention of carcinogen-induced oesophageal cancer in rats. J. Funct. Foods 2016, 27, 84-94. [CrossRef]

23. Fu, S.K.; Lawrance, I.C. Anima model of IBD-associated CRC and colorectal cancer tumorigenesis. Clin. Med. Insight 2015, 7, 1-9.

24. De Robertis, M.; Massi, E.; Poeta, M.L.; Carotti, S.; Morini, S.; Cecchetelli, L.; Signori, E.; Fazio, V.M. The aom/dss murine model for the study of colon carcinogenesis: From pathways to diagnosis and therapy studies. J. Carcinog. 2011, 10, 9.

25. Pozza, A.; Scarpa, M.; Ruffolo, C.; Polese, L.; Erroi, F.; Bridda, A.; Norberto, L.; Frego, M. Colonic carcinogenesis in ibd: Molecular events. Ann. Ital. Chir. 2011, 82, 19-28.

26. Grivennikov, S.I.; Greten, F.R.; Karin, M. Immunity, inflammation, and cancer. Cell 2010, 140, 883-899. [CrossRef]

27. Pandurangan, A.K.; Saadatdoust, Z.; Esa, N.M.; Hamzah, H.; Ismail, A. Dietary cocoa protects against colitis-associated cancer by activating the nrf2/keap1 pathway. Biofactors 2015, 41, 1-14. [CrossRef]

28. Osburn, W.O.; Karim, B.; Dolan, P.M.; Liu, G.; Yamamoto, M.; Huso, D.L.; Kensler, T.W. Increased colonic inflammatory injury and formation of aberrant crypt foci in nrf2-deficient mice upon dextran sulfate treatment. Int. J. Cancer 2007, 121, 1883-1891. [CrossRef]

29. Barrett, C.W.; Ning, W.; Chen, X.; Smith, J.J.; Washington, M.K.; Hill, K.E.; Coburn, L.A.; Peek, R.M.; Chaturvedi, R.; Wilson, K.T.; et al. Tumor suppressor function of the plasma glutathione peroxidase gpx3 in colitis-associated carcinoma. Cancer Res. 2013, 73, 1245-1255. [CrossRef]

30. Kawanishi, S.; Hiraku, Y.; Pinlaor, S.; Ma, N. Oxidative and nitrative DNA damage in animals and patients with inflammatory diseases in relation to inflammation-related carcinogenesis. Biol. Chem. 2006, 387, 365-372. [CrossRef]

31. Sohn, J.J.; Schetter, A.J.; Yfantis, H.G.; Ridnour, L.A.; Horikawa, I.; Khan, M.A.; Robles, A.I.; Hussain, S.P.; Goto, A.; Bowman, E.D.; et al. Macrophages, nitric oxide and micrornas are associated with DNA damage response pathway and senescence in inflammatory bowel disease. PLoS ONE 2012, 7, e44156. [CrossRef]

32. Grasso, F.; Di Meo, S.; De Luca, G.; Pasquini, L.; Rossi, S.; Boirivant, M.; Biffoni, M.; Bignami, M.; Di Carlo, E. The mutyh base excision repair gene protects against inflammation-associated colorectal carcinogenesis. Oncotarget 2015, 6, 19671-19684. [CrossRef]

33. Yaeger, R.; Shah, M.A.; Miller, V.A.; Kelsen, J.R.; Wang, K.; Heins, Z.J.; Ross, J.S.; He, Y.; Sanford, E.; Yantiss, R.K.; et al. Genomic alterations observed in colitis-associated cancers are distinct from those found in sporadic colorectal cancers and vary by type of inflammatory bowel disease. Gastroenterology 2016, 151, 278-287. [CrossRef]

34. Boland, C.R.; Goel, A. Microsatellite instability in colorectal cancer. Gastroenterology 2010, 138, 2073-2087 e2073. [CrossRef]

35. van Dieren, J.M.; Wink, J.C.; Vissers, K.J.; van Marion, R.; Hoogmans, M.M.; Dinjens, W.N.; Schouten, W.R.; Tanke, H.J.; Szuhai, K.; Kuipers, E.J.; et al. Chromosomal and microsatellite instability of adenocarcinomas and dysplastic lesions (dalm) in ulcerative colitis. Diagn. Mol. Pathol. 2006, 15, 216-222. [CrossRef]

36. Willenbucher, R.F.; Aust, D.E.; Chang, C.G.; Zelman, S.J.; Ferrell, L.D.; Moore, D.H., 2nd; Waldman, F.M. Genomic instability is an early event during the progression pathway of ulcerative-colitis-related neoplasia. Am. J. Pathol. 1999, 154, 1825-1830. [CrossRef] 
37. Tsai, J.H.; Rabinovitch, P.S.; Huang, D.; Small, T.; Mattis, A.N.; Kakar, S.; Choi, W.T. Association of aneuploidy and flat dysplasia with development of high-grade dysplasia or colorectal cancer in patients with inflammatory bowel disease. Gastroenterology 2017, 153, 1492-1495. [CrossRef]

38. Itzkowitz, S.H. Molecular biology of dysplasia and cancer in inflammatory bowel disease. Gastroenterol. Clin. N. Am. 2006, 35, 553-571. [CrossRef]

39. Hussain, S.P.; Amstad, P.; Raja, K.; Ambs, S.; Nagashima, M.; Bennett, W.P.; Shields, P.G.; Ham, A.J.; Swenberg, J.A.; Marrogi, A.J.; et al. Increased p53 mutation load in noncancerous colon tissue from ulcerative colitis: A cancer-prone chronic inflammatory disease. Cancer Res. 2000, 60, 3333-3337.

40. Garrett, W.S.; Punit, S.; Gallini, C.A.; Michaud, M.; Zhang, D.; Sigrist, K.S.; Lord, G.M.; Glickman, J.N.; Glimcher, L.H. Colitis-associated colorectal cancer driven by t-bet deficiency in dendritic cells. Cancer Cell 2009, 16, 208-219. [CrossRef]

41. Neufert, C.; Becker, C.; Tureci, O.; Waldner, M.J.; Backert, I.; Floh, K.; Atreya, I.; Leppkes, M.; Jefremow, A.; Vieth, M.; et al. Tumor fibroblast-derived epiregulin promotes growth of colitis-associated neoplasms through erk. J. Clin. Investig. 2013, 123, 1428-1443. [CrossRef]

42. Neurath, M.F. Cytokines in inflammatory bowel disease. Nat. Rev. Immunol. 2014, 14, 329-342. [CrossRef]

43. Wang, Y.; Wang, K.; Han, G.C.; Wang, R.X.; Xiao, H.; Hou, C.M.; Guo, R.F.; Dou, Y.; Shen, B.F.; Li, Y.; et al. Neutrophil infiltration favors colitis-associated tumorigenesis by activating the interleukin-1 (il-1)/il-6 axis. Mucosal. Immunol. 2014, 7, 1106-1115. [CrossRef]

44. Francescone, R.; Hou, V.; Grivennikov, S.I. Cytokines, ibd, and colitis-associated cancer. Inflamm. Bowel. Dis. 2015, 21, 409-418. [CrossRef]

45. Popivanova, B.K.; Kitamura, K.; Wu, Y.; Kondo, T.; Kagaya, T.; Kaneko, S.; Oshima, M.; Fujii, C.; Mukaida, N. Blocking tnf-alpha in mice reduces colorectal carcinogenesis associated with chronic colitis. J. Clin. Investig. 2008, 118, 560-570.

46. Grivennikov, S.; Karin, E.; Terzic, J.; Mucida, D.; Yu, G.Y.; Vallabhapurapu, S.; Scheller, J.; Rose-John, S.; Cheroutre, H.; Eckmann, L.; et al. Il-6 and stat3 are required for survival of intestinal epithelial cells and development of colitis-associated cancer. Cancer Cell 2009, 15, 103-113. [CrossRef]

47. Matsumoto, S.; Hara, T.; Mitsuyama, K.; Yamamoto, M.; Tsuruta, O.; Sata, M.; Scheller, J.; Rose-John, S.; Kado, S.; Takada, T. Essential roles of il-6 trans-signaling in colonic epithelial cells, induced by the il-6/soluble-il-6 receptor derived from lamina propria macrophages, on the development of colitis-associated premalignant cancer in a murine model. J. Immunol. 2010, 184, 1543-1551. [CrossRef]

48. McGovern, D.P.; Rotter, J.I.; Mei, L.; Haritunians, T.; Landers, C.; Derkowski, C.; Dutridge, D.; Dubinsky, M.; Ippoliti, A.; Vasiliauskas, E.; et al. Genetic epistasis of il23/il17 pathway genes in crohn's disease. Inflamm. Bowel. Dis. 2009, 15, 883-889. [CrossRef]

49. Zenewicz, L.A.; Antov, A.; Flavell, R.A. Cd4 t-cell differentiation and inflammatory bowel disease. Trends Mol. Med. 2009, 15, 199-207. [CrossRef]

50. Ni, J.; Wu, G.D.; Albenberg, L.; Tomov, V.T. Gut microbiota and IBD: Causation or correlation? Nat. Rev. Gastroenterol. Hepatol. 2017, 14, 573-584. [CrossRef]

51. Zhou, M.; He, J.; Shen, Y.; Zhang, C.; Wang, J.; Chen, Y. New Frontiers in Genetics, Gut Microbiota, and Immunity: A Rosetta Stone for the Pathogenesis of Inflammatory Bowel Disease. Biomed. Res. Int. 2017, 2017, 8201672. [CrossRef]

52. Velayos, F.S.; Terdiman, J.P.; Walsh, J.M. Effect of 5-aminosalicylate use on colorectal cancer and dysplasia risk: A systematic review and metaanalysis of observational studies. Am. J. Gastroenterol. 2005, 100, 1345-1353. [CrossRef]

53. Nishida, A.; Inoue, R.; Inatomi, O.; Bamba, S.; Naito, Y.; Andoh, A. Gut microbiota in the pathogenesis of inflammatory bowel disease. Clin. J. Gastroenterol. 2018, 11, 1-10. [CrossRef]

54. Santino, A.; Scarano, A.; De Santis, S.; De Benedictis, M.; Giovinazzo, G.; Chieppa, M. Gut Microbiota Modulation and Anti-Inflammatory Properties of Dietary Polyphenolsin IBD: New and Consolidated Perspectives. Curr. Pharm. Des. 2017, 23, 2344-2351. [CrossRef]

55. Duricova, D.; Burisch, J.; Jess, T.; Gower-Rousseau, C.; Lakatos, P.L.; ECCO-EpiCom. Age-related differences in presentation and course of inflammatory bowel disease: An update on the population-based literature. J. Crohns Colitis 2014, 8, 1351-1361. [CrossRef] 
56. Cho, H.; Jung, H.; Lee, H.; Yi, H.C.; Kwak, H.K.; Hwang, K.T. Chemopreventive activity of ellagitannins and their derivatives from black raspberry seeds on ht-29 colon cancer cells. Food Funct. 2015, 6, 1675-1683. [CrossRef]

57. Seeram, N.P.; Adams, L.S.; Zhang, Y.; Lee, R.; Sand, D.; Scheuller, H.S.; Heber, D. Blackberry, black raspberry, blueberry, cranberry, red raspberry, and strawberry extracts inhibit growth and stimulate apoptosis of human cancer cells in vitro. J. Agric. Food Chem. 2006, 54, 9329-9339. [CrossRef]

58. Montrose, D.C.; Horelik, N.A.; Madigan, J.P.; Stoner, G.D.; Wang, L.S.; Bruno, R.S.; Park, H.J.; Giardina, C.; Rosenberg, D.W. Anti-inflammatory effects of freeze-dried black raspberry powder in ulcerative colitis. Carcinogenesis 2011, 32, 343-350. [CrossRef]

59. Wang, L.S.; Kuo, C.T.; Stoner, K.; Yearsley, M.; Oshima, K.; Yu, J.; Huang, T.H.; Rosenberg, D.; Peiffer, D.; Stoner, G.; et al. Dietary black raspberries modulate DNA methylation in dextran sodium sulfate (dss)-induced ulcerative colitis. Carcinogenesis 2013, 34, 2842-2850. [CrossRef]

60. Wang, L.S.; Kuo, C.T.; Huang, T.H.; Yearsley, M.; Oshima, K.; Stoner, G.D.; Yu, J.; Lechner, J.F.; Huang, Y.W. Black raspberries protectively regulate methylation of wnt pathway genes in precancerous colon tissue. Cancer Prev. Res. 2013, 6, 1317-1327. [CrossRef]

61. Huang, C.; Huang, Y.; Li, J.; Hu, W.; Aziz, R.; Tang, M.S.; Sun, N.; Cassady, J.; Stoner, G.D. Inhibition of benzo(a)pyrene diol-epoxide-induced transactivation of activated protein 1 and nuclear factor kappab by black raspberry extracts. Cancer Res. 2002, 62, 6857-6863.

62. Lu, H.; Li, J.; Zhang, D.; Stoner, G.D.; Huang, C. Molecular mechanisms involved in chemoprevention of black raspberry extracts: From transcription factors to their target genes. Nutr. Cancer 2006, 54, 69-78. [CrossRef]

63. Harris, G.K.; Gupta, A.; Nines, R.G.; Kresty, L.A.; Habib, S.G.; Frankel, W.L.; LaPerle, K.; Gallaher, D.D.; Schwartz, S.J.; Stoner, G.D. Effects of lyophilized black raspberries on azoxymethane-induced colon cancer and 8-hydroxy-2'-deoxyguanosine levels in the fischer 344 rat. Nutr. Cancer 2001, 40, 125-133. [CrossRef]

64. Bi, X.; Fang, W.; Wang, L.S.; Stoner, G.D.; Yang, W. Black raspberries inhibit intestinal tumorigenesis in apc1638+/- and muc2-/- mouse models of colorectal cancer. Cancer Prev. Res. 2010, 3, 1443-1450. [CrossRef]

65. Wang, L.S.; Arnold, M.; Huang, Y.W.; Sardo, C.; Seguin, C.; Martin, E.; Huang, T.H.; Riedl, K.; Schwartz, S.; Frankel, W.; et al. Modulation of genetic and epigenetic biomarkers of colorectal cancer in humans by black raspberries: A phase i pilot study. Clin. Cancer Res. 2011, 17, 598-610. [CrossRef]

66. Wang, L.S.; Kuo, C.T.; Cho, S.J.; Seguin, C.; Siddiqui, J.; Stoner, K.; Weng, Y.I.; Huang, T.H.; Tichelaar, J.; Yearsley, M.; et al. Black raspberry-derived anthocyanins demethylate tumor suppressor genes through the inhibition of dnmt1 and dnmt3b in colon cancer cells. Nutr. Cancer 2013, 65, 118-125. [CrossRef]

67. Wu, Q.K.; Koponen, J.M.; Mykkanen, H.M.; Torronen, A.R. Berry phenolic extracts modulate the expression of p21(waf1) and bax but not bcl-2 in ht-29 colon cancer cells. J. Agric. Food Chem. 2007, 55, 1156-1163. [CrossRef]

68. McDougall, G.J.; Ross, H.A.; Ikeji, M.; Stewart, D. Berry extracts exert different antiproliferative effects against cervical and colon cancer cells grown in vitro. J. Agric. Food Chem. 2008, 56, 3016-3023. [CrossRef]

69. Zhang, Y.; Seeram, N.P.; Lee, R.; Feng, L.; Heber, D. Isolation and identification of strawberry phenolics with antioxidant and human cancer cell antiproliferative properties. J. Agric. Food Chem. 2008, 56, 670-675. [CrossRef]

70. Kanodia, L.; Borgohain, M.; Das, S. Effect of fruit extract of Fragaria vesca L. on experimentally induced inflammatory bowel disease in albino rats. Indian J. Pharm. 2011, 43, 18-21. [CrossRef]

71. Mentor-Marcel, R.A.; Bobe, G.; Sardo, C.; Wang, L.S.; Kuo, C.T.; Stoner, G.; Colburn, N.H. Plasma cytokines as potential response indicators to dietary freeze-dried black raspberries in colorectal cancer patients. Nutr. Cancer 2012, 64, 820-825. [CrossRef]

72. Duthie, S.J.; Gardner, P.T.; Morrice, P.C.; Wood, S.G.; Pirie, L.; Bestwick, C.C.; Milne, L.; Duthie, G.G. DNA stability and lipid peroxidation in vitamin e-deficient rats in vivo and colon cells in vitro-modulation by the dietary anthocyanin, cyanidin-3-glycoside. Eur. J. Nutr. 2005, 44, 195-203. [CrossRef]

73. Elisia, I.; Kitts, D.D. Anthocyanins inhibit peroxyl radical-induced apoptosis in caco-2 cells. Mol. Cell. Biochem. 2008, 312, 139-145. [CrossRef]

74. Renis, M.; Calandra, L.; Scifo, C.; Tomasello, B.; Cardile, V.; Vanella, L.; Bei, R.; La Fauci, L.; Galvano, F. Response of cell cycle/stress-related protein expression and DNA damage upon treatment of $\mathrm{CaCO}_{2}$ cells with anthocyanins. Br. J. Nutr. 2008, 100, 27-35. [CrossRef] 
75. Hudson, E.A.; Dinh, P.A.; Kokubun, T.; Simmonds, M.S.; Gescher, A. Characterization of potentially chemopreventive phenols in extracts of brown rice that inhibit the growth of human breast and colon cancer cells. Cancer Epidemiol. Biomark. Prev. 2000, 9, 1163-1170.

76. Bornsek, S.M.; Ziberna, L.; Polak, T.; Vanzo, A.; Ulrih, N.P.; Abram, V.; Tramer, F.; Passamonti, S. Bilberry and blueberry anthocyanins act as powerful intracellular antioxidants in mammalian cells. Food Chem. 2012, 134, 1878-1884. [CrossRef]

77. Katsube, N.; Iwashita, K.; Tsushida, T.; Yamaki, K.; Kobori, M. Induction of apoptosis in cancer cells by bilberry (vaccinium myrtillus) and the anthocyanins. J. Agric. Food Chem. 2003, 51, 68-75. [CrossRef]

78. Lala, G.; Malik, M.; Zhao, C.; He, J.; Kwon, Y.; Giusti, M.M.; Magnuson, B.A. Anthocyanin-rich extracts inhibit multiple biomarkers of colon cancer in rats. Nutr. Cancer 2006, 54, 84-93. [CrossRef]

79. Kangawa, Y.; Yoshida, T.; Maruyama, K.; Okamoto, M.; Kihara, T.; Nakamura, M.; Ochiai, M.; Hippo, Y.; Hayashi, S.M.; Shibutani, M. Cilostazol and enzymatically modified isoquercitrin attenuate experimental colitis and colon cancer in mice by inhibiting cell proliferation and inflammation. Food Chem. Toxicol. 2017, 100, 103-114. [CrossRef]

80. Charepalli, V.; Reddivari, L.; Radhakrishnan, S.; Vadde, R.; Agarwal, R.; Vanamala, J.K. Anthocyanin-containing purple-fleshed potatoes suppress colon tumorigenesis via elimination of colon cancer stem cells. J. Nutr. Biochem. 2015, 26, 1641-1649. [CrossRef]

81. Urias-Lugo, D.A.; Heredia, J.B.; Muy-Rangel, M.D.; Valdez-Torres, J.B.; Serna-Saldivar, S.O.; Gutierrez-Uribe, J.A. Anthocyanins and phenolic acids of hybrid and native blue maize (Zea mays L.) extracts and their antiproliferative activity in mammary (MCF7), liver (HepG2), colon (Caco2 and HT29) and prostate (PC3) cancer cells. Plant Foods Hum. Nutr. 2015, 70, 193-199. [CrossRef] [PubMed]

82. Jing, P.; Qian, B.; Zhao, S.; Qi, X.; Ye, L.; Monica Giusti, M.; Wang, X. Effect of glycosylation patterns of chinese eggplant anthocyanins and other derivatives on antioxidant effectiveness in human colon cell lines. Food Chem. 2015, 172, 183-189. [CrossRef] [PubMed]

83. Lim, S.; Xu, J.; Kim, J.; Chen, T.Y.; Su, X.; Standard, J.; Carey, E.; Griffin, J.; Herndon, B.; Katz, B.; et al. Role of anthocyanin-enriched purple-fleshed sweet potato p40 in colorectal cancer prevention. Mol. Nutr. Food Res. 2013, 57, 1908-1917. [CrossRef] [PubMed]

84. Yun, J.W.; Lee, W.S.; Kim, M.J.; Lu, J.N.; Kang, M.H.; Kim, H.G.; Kim, D.C.; Choi, E.J.; Choi, J.Y.; Lee, Y.K.; et al Characterization of a profile of the anthocyanins isolated from vitis coignetiae pulliat and their anti-invasive activity on ht-29 human colon cancer cells. Food Chem. Toxicol. 2010, 48, 903-909. [CrossRef]

85. Dai, J.; Patel, J.D.; Mumper, R.J. Characterization of blackberry extract and its antiproliferative and anti-inflammatory properties. J. Med. Food 2007, 10, 258-265. [CrossRef]

86. Yi, W.; Fischer, J.; Krewer, G.; Akoh, C.C. Phenolic compounds from blueberries can inhibit colon cancer cell proliferation and induce apoptosis. J. Agric. Food Chem. 2005, 53, 7320-7329. [CrossRef]

87. Kang, S.Y.; Seeram, N.P.; Nair, M.G.; Bourquin, L.D. Tart cherry anthocyanins inhibit tumor development in apc(min) mice and reduce proliferation of human colon cancer cells. Cancer Lett. 2003, 194, 13-19. [CrossRef]

88. Kawamori, T.; Tanaka, T.; Kojima, T.; Suzui, M.; Ohnishi, M.; Mori, H. Suppression of azoxymethane-induced rat colon aberrant crypt foci by dietary protocatechuic acid. Jpn. J. Cancer Res. 1994, 85, 686-691. [CrossRef]

89. Tanaka, T.; Kojima, T.; Suzui, M.; Mori, H. Chemoprevention of colon carcinogenesis by the natural product of a simple phenolic compound protocatechuic acid: Suppressing effects on tumor development and biomarkers expression of colon tumorigenesis. Cancer Res. 1993, 53, 3908-3913.

90. Farombi, E.O.; Adedara, I.A.; Awoyemi, O.V.; Njoku, C.R.; Micah, G.O.; Esogwa, C.U.; Owumi, S.E.; Olopade, J.O. Dietary protocatechuic acid ameliorates dextran sulphate sodium-induced ulcerative colitis and hepatotoxicity in rats. Food Funct. 2016, 7, 913-921. [CrossRef]

91. Venancio, V.P.; Cipriano, P.A.; Kim, H.; Antunes, L.M.; Talcott, S.T.; Mertens-Talcott, S.U. Cocoplum (chrysobalanus icaco 1.) anthocyanins exert anti-inflammatory activity in human colon cancer and non-malignant colon cells. Food Funct. 2017, 8, 307-314. [CrossRef]

92. Charepalli, V.; Reddivari, L.; Vadde, R.; Walia, S.; Radhakrishnan, S.; Vanamala, J.K. Eugenia jambolana (java plum) fruit extract exhibits anti-cancer activity against early stage human hct-116 colon cancer cells and colon cancer stem cells. Cancers (Basel) 2016, 8, 29. [CrossRef] [PubMed]

93. Scarano, A.; Butelli, E.; De Santis, S.; Cavalcanti, E.; Hill, L.; De Angelis, M.; Giovinazzo, G.; Chieppa, M.; Martin, C.; Santino, A. Combined Dietary Anthocyanins, Flavonols, and Stilbenoids Alleviate Inflammatory Bowel Disease Symptoms in Mice. Front. Nutr. 2018, 4, 75. [CrossRef] [PubMed] 
94. Pojer, E.; Mattivi, F.; Johnson, D.; Stockley, C.S. The case for anthocyanin consumption to promote human health. Compr. Rev. Food Sci. Food Saf. 2013, 12, 24.

95. Knobloch, T.J.; Uhrig, L.K.; Pearl, D.K.; Casto, B.C.; Warner, B.M.; Clinton, S.K.; Sardo-Molmenti, C.L.; Ferguson, J.M.; Daly, B.T.; Riedl, K.; et al. Suppression of proinflammatory and prosurvival biomarkers in oral cancer patients consuming a black raspberry phytochemical-rich troche. Cancer Prev. Res. 2016, 9 , 159-171. [CrossRef]

96. Teoh, W.Y.; Tan, H.P.; Ling, S.K.; Abdul Wahab, N.; Sim, K.S. Phytochemical investigation of gynura bicolor leaves and cytotoxicity evaluation of the chemical constituents against het 116 cells. Nat. Prod. Res. 2016, 30, 448-451. [CrossRef] [PubMed]

97. Femia, A.P.; Caderni, G.; Buzzigoli, C.; Cocca, E.; Salvadori, M.; Dolara, P. Effect of simple phenolic compounds on azoxymethane-induced aberrant crypt foci in rat colon. Nutr. Cancer 2001,41, 107-110.

98. Mace, T.A.; King, S.A.; Ameen, Z.; Elnaggar, O.; Young, G.; Riedl, K.M.; Schwartz, S.J.; Clinton, S.K.; Knobloch, T.J.; Weghorst, C.M.; et al. Bioactive compounds or metabolites from black raspberries modulate $t$ lymphocyte proliferation, myeloid cell differentiation and jak/stat signaling. Cancer Immunol. Immunother. 2014, 63, 889-900. [CrossRef] [PubMed]

99. Jo, Y.H.; Park, H.C.; Choi, S.; Kim, S.; Bao, C.; Kim, H.W.; Choi, H.K.; Lee, H.J.; Auh, J.H. Metabolomic analysis reveals cyanidins in black raspberry as candidates for suppression of lipopolysaccharide-induced inflammation in murine macrophages. J. Agric. Food Chem. 2015, 63, 5449-5458. [CrossRef]

100. Wang, L.S.; Hecht, S.; Carmella, S.; Seguin, C.; Rocha, C.; Yu, N.; Stoner, K.; Chiu, S.; Stoner, G. Berry ellagitannins may not be sufficient for prevention of tumors in the rodent esophagus. J. Agric. Food Chem. 2010, 58, 3992-3995. [CrossRef] [PubMed]

101. Paudel, L.; Wyzgoski, F.J.; Giusti, M.M.; Johnson, J.L.; Rinaldi, P.L.; Scheerens, J.C.; Chanon, A.M.; Bomser, J.A.; Miller, A.R.; Hardy, J.K.; et al. Nmr-based metabolomic investigation of bioactivity of chemical constituents in black raspberry (rubus occidentalis 1.) fruit extracts. J. Agric. Food Chem. 2014, 62, 1989-1998. [CrossRef]

102. Farzaei, M.H.; El-Senduny, F.F.; Momtaz, S.; Parvizi, F.; Iranpanah, A.; Tewari, D.; Naseri, R.; Abdolghaffari, A.H.; Rezaei, N. An update on dietary consideration in inflammatory bowel disease: Anthocyanins and more. Expert Rev. Gastroenterol. Hepatol. 2018, 10, 1007-1024. [CrossRef] [PubMed]

103. Sodagari, H.R.; Farzaei, M.H.; Bahramsoltani, R.; Abdolghaffari, A.H.; Mahmoudi, M.; Rezaei, N. Dietary anthocyanins as a complementary medicinal approach for management of inflammatory bowel disease. Expert Rev. Gastroenterol. Hepatol. 2015, 9, 807-820. [CrossRef] [PubMed]

104. Wang, L.S.; Burke, C.A.; Hasson, H.; Kuo, C.T.; Molmenti, C.L.; Seguin, C.; Liu, P.; Huang, T.H.; Frankel, W.L.; Stoner, G.D. A phase ib study of the effects of black raspberries on rectal polyps in patients with familial adenomatous polyposis. Cancer Prev. Res. 2014, 7, 666-674. [CrossRef] [PubMed]

105. Wang, L.S.; Hecht, S.S.; Carmella, S.G.; Yu, N.; Larue, B.; Henry, C.; McIntyre, C.; Rocha, C.; Lechner, J.F.; Stoner, G.D. Anthocyanins in black raspberries prevent esophageal tumors in rats. Cancer Prev. Res. 2009, 2, 84-93. [CrossRef]

106. Peiffer, D.S.; Wang, L.S.; Zimmerman, N.P.; Ransom, B.W.; Carmella, S.G.; Kuo, C.T.; Chen, J.H.; Oshima, K.; Huang, Y.W.; Hecht, S.S.; et al. Dietary consumption of black raspberries or their anthocyanin constituents alters innate immune cell trafficking in esophageal cancer. Cancer Immunol. Res. 2016, 4, 72-82. [CrossRef]

107. Peiffer, D.S.; Zimmerman, N.P.; Wang, L.S.; Ransom, B.W.; Carmella, S.G.; Kuo, C.T.; Siddiqui, J.; Chen, J.H.; Oshima, K.; Huang, Y.W.; et al. Chemoprevention of esophageal cancer with black raspberries, their component anthocyanins, and a major anthocyanin metabolite, protocatechuic acid. Cancer Prev. Res. 2014, 7, 574-584. [CrossRef]

108. Chen, T.; Rose, M.E.; Hwang, H.; Nines, R.G.; Stoner, G.D. Black raspberries inhibit n-nitrosomethylbenzylamine (nmba)-induced angiogenesis in rat esophagus parallel to the suppression of cox-2 and inos. Carcinogenesis 2006, 27, 2301-2307. [CrossRef]

(C) 2019 by the authors. Licensee MDPI, Basel, Switzerland. This article is an open access article distributed under the terms and conditions of the Creative Commons Attribution (CC BY) license (http://creativecommons.org/licenses/by/4.0/). 


\title{
Micronutrient Absorption and Related Outcomes in People with Inflammatory Bowel Disease: A Review
}

\author{
Kyle Kilby ${ }^{1}$, Holly Mathias ${ }^{2}$, Lindsay Boisvenue ${ }^{3}$, Courtney Heisler ${ }^{4}$ and Jennifer L. Jones ${ }^{4, *}$ \\ 1 Faculty of Medicine, Dalhousie University, 1459 Oxford Street, Halifax, NS B3J 4R2, Canada; \\ Kyle.Kilby@dal.ca \\ 2 School of Health and Human Performance, Dalhousie University, 6230 South Street, Halifax, NS B3H 1T8, \\ Canada; Holly.Mathias@dal.ca \\ 3 Seaway Valley Community Health Care, 353 Pitt Street, Cornwall, ON K6J 3R1, Canada; \\ BoisvenueL@seawayvalleychc.ca \\ 4 Nova Scotia Collaborative Inflammatory Bowel Disease Program, Division of Digestive Care and Endoscopy, \\ QEII Health Science Centre, Room 932, Victoria Building, 1276 South Park Street, Halifax, NS B3H 2Y9, \\ Canada; courtney.heisler@nshealth.ca \\ * Correspondence: jljones@dal.ca; Tel.: +1-902-473-6456
}

Received: 8 April 2019; Accepted: 8 June 2019; Published: 20 June 2019

\begin{abstract}
Inflammatory Bowel Disease (IBD) is a chronic disorder associated with immune dysregulation and chronic inflammation of the digestive tract. While it is poorly understood, the role of nutrition and nutrient status in the etiology of IBD and its associated outcomes has led to increased research relating to micronutrient deficiency. This review offers an overview of recent literature related to micronutrient absorption and outcomes in adults with IBD. Although the absorption and IBD-related outcomes of some micronutrients (e.g., vitamin D and iron) are well understood, other micronutrients (e.g., vitamin A) require further research. Increased research and clinician knowledge of the relationship between micronutrients and IBD may manifest in improved nutrient screening, monitoring, treatment, and outcomes for people living with IBD.
\end{abstract}

Keywords: Inflammatory Bowel Disease; micronutrients; vitamin; mineral; deficiency

\section{Introduction}

Inflammatory Bowel Diseases (IBD) (including Crohn's Disease (CD) and Ulcerative Colitis (UC)) are complex disorders involving immune dysregulation, leading to infiltration and destruction of the gastrointestinal tract. At its core, IBD results from an imbalance between pro-inflammatory and anti-inflammatory signaling, however the etiology of this imbalance, and of IBD itself, remains poorly understood [1]. An expanding body of literature implicates a shared role of genetics and the environment in the development of disease. The contributing role of environmental factors in the pathogenesis of IBD is further substantiated by observed rising IBD incidence rates in populations that have immigrated from geographic regions of low disease prevalence to regions where the disease is more prevalent [2]. Population-based studies highlight an association between increased intake of total fat, processed meat, and protein (common components of 'Western' diets), and the incidence of IBD [3,4].

In turn, IBD itself affects nutrition intrinsically by disrupting the absorption of nutrients in the gut, and extrinsically through reduced total food intake due to pain and fatigue, as well as the avoidance of food groups that exacerbate their symptoms [5-11]. Given differences in disease location between CD (commonly involving the ileocecal junction but can affect any part of the gastrointestinal tract) and UC (localized to the rectum and colon), and the distribution of nutrient absorption sites within the gut, the spectrum of the association between IBD and nutrient status varies in both magnitude and selectivity $[12,13]$. Micronutrient status is further impacted by increased gastrointestinal losses and 
the surgical treatment of IBD itself, which decreases the amount of mucosal surface area available for absorption [14]. The effect of selected micronutrient deficiencies on patient outcomes has been difficult to characterize. The severity of deficiency often moves lockstep with disease severity, making it difficult to draw conclusions about the specific impact of micronutrient levels on patient outcomes without experimental interventional studies to eliminate known or residual confounding effect. This review seeks to highlight many of the common micronutrient deficiencies associated with IBD and examine the reciprocal relationship between micronutrients and IBD with respect to absorption and outcomes. Furthermore, this review aims to examine current recommendations for micronutrient supplementation and screening for patients with IBD.

\section{Literature Review}

\subsection{Materials \& Methods}

An initial literature search was performed in order to develop a set of well-studied and clinically relevant micronutrients. Medline, Pubmed, Embase and CINAHL databases were searched for English language peer-reviewed articles published during 2009-2019 using the keywords 'inflammatory bowel disease' and, 'micronutrients' alongside, 'deficiency', 'outcomes', 'screening', and/or 'treatment'. This search strategy resulted in 1334 articles. Articles were excluded if they did not focus on the adult IBD patients or patient outcomes. From this, a subset of 160 papers were used to determine which micronutrient deficiencies warranted further investigation.

Individual literature searches for each micronutrient were performed, with no exclusion criteria. Each search followed the same formula of keywords, with 'inflammatory bowel disease' and the vitamin in question, as well as the modifiers, 'outcomes', 'deficiency', 'supplementation', or 'therapy'. From these subsearches, all relevant results were included. Finally, snowballing was used to find additional sources that were not returned by the search.

\subsection{Micronutrients}

Micronutrients are categorized as having four main roles in supporting major body function: cofactors in metabolism, coenzymes in metabolism, genetic controls, and antioxidants [15]. Therefore, deficiency of essential micronutrients leads to deleterious downstream effects involving metabolism, gene expression and oxidative stress [15]. In the context of IBD, the role of micronutrients in genetic control and antioxidation are of particular importance [16]. Vitamin A, B, and E have been implicated in regulating immune responses and homeostasis within the gut, inhibiting the release of inflammatory cytokines and favoring the differentiation of regulatory $\left(\mathrm{T}_{\mathrm{REG}}\right)$ lymphocytes over proinflammatory $\mathrm{T}_{\mathrm{H}} 17$ lymphocytes [16]. In healthy patients, production of reactive oxygen species (ROS) is balanced by the activity of antioxidative micronutrients such as vitamins A and E, as well as enzymes requiring trace metals (zinc and selenium as examples) as cofactors [16]. In IBD, immune dysregulation and inflammation result in the increased production of ROS, further underlying the importance of antioxidative micronutrients in mitigating IBD pathogenesis [17]. This relationship creates a chicken and the egg situation: Is increasing disease severity responsible for micronutrient deficiencies by impairing absorption? Or are dwindling levels of micronutrients, which act as antioxidants and immune regulators, responsible for progression of disease?

\subsection{Vitamins}

\subsubsection{Vitamin A}

Vitamin A, and its active metabolite retinoic acid, have widespread effects through the body. In the context of the immune response, retinoic acid signaling results in expression of Foxp3, a transcription factor that leads to the differentiation of naïve $\mathrm{T}$ cells to $\mathrm{T}_{\mathrm{REG}}$ cells [18-21]. These $\mathrm{T}_{\text {REG }}$ cells attenuate the immune response through the release of anti-inflammatory cytokines [22,23]. Conversely, retinoic 
acid inhibits the expression of IL-6 receptors, preventing the differentiation of naive cells into proinflammatory $\mathrm{T}_{\mathrm{H}} 17$ cells [20]. Furthermore, retinoic acid induces the expression of $\alpha 4 \beta 7$ and CCR9 on $\mathrm{T}$ cells [24]. The expression of these molecules causes $\mathrm{T}$ cells to preferentially migrate into the gut wall following exposure to antigen [25].

Vitamin A absorption chiefly occurs in the proximal intestine and can be directly converted into retinoic acid in the gut by enterocytes [26]. Given that vitamin A stores in the liver buffer decreasing levels in the blood, measuring serum vitamin A does not accurately assess the total amount present in the body [27]. Soares-Mota et al., 2015 used relative dose response testing to indirectly assess liver stores of vitamin A in 38 Brazilian patients with CD and 34 healthy subjects. They found that deficiencies in vitamin A stores were significantly more common in those suffering from $C D$ $(37 \%$ vs. $12 \%, p<0.005)$. Interestingly, they did not find any association between vitamin A deficiency, disease location, or disease activity [27].

Provitamins of vitamin A have also been examined in the context of IBD. One such provitamin, $\beta$-carotene, has been well studied [28]. Compared to controls, $\beta$-carotene levels in IBD patients have been shown to be significantly decreased, with further decrease in those with active disease [29]. As well, supplementation with $\beta$-carotene lead to disease amelioration in a dextran sodium sulfate (DSS) murine model of colitis [30]. Conclusions are much harder to draw from results of human trials. Firstly, in the articles reviewed by Rezaie et al., 2007, each study uses $\beta$-carotene alongside other antioxidative micronutrients, making it difficult to judge its effect [31]. Furthermore, the review notes that the studies used small doses of $\beta$-carotene and did not see normalization of $\beta$-carotene serum levels. The one study reviewed that used high dose antioxidants, including $\beta$-carotene, saw decreases in the required corticosteroid dose, while all other studies were negative [31,32]. Again, without elucidating the effect of $\beta$-carotene supplementation in isolation, it is impossible to determine its clinical utility. It is also difficult to distinguish whether any effects are due to its conversion to vitamin A or its own antioxidative ability.

The role of non-provitamin A carotenoids in IBD will only be described briefly, as their role in the context of IBD requires further elucidation. Presently, deficiencies of these carotenoids, namely lycopene and lutein, have been described in IBD patients $[30,33]$. However, the clinical significance of these deficiencies in IBD is unclear. While the effect of dietary supplementation has not been determined, a single retrospective study using the self-reported dietary records of 56 UC patients in remission associated higher lycopene and lutein intake with decreased fecal blood and mucus, with no change in abdominal pain [34]. Lutein was also able to attenuate ulceration of the GI tract in DSS murine models [35]. Given the exceeding rarity of clinical serum testing for these compounds, and the lack of data surrounding their supplementation, significant further research is required before any recommendation can be made.

Given the ability of vitamin A to attenuate intestinal inflammation in murine models and the prevalence of deficiency in the Brazilian CD population, further research into the role of vitamin A in IBD is warranted [27,36]. At this point, research regarding vitamin A supplementation in IBD is limited to two small studies published in 1985, (Wright et al., 1985: $n=86$; Norrby et al., 1985, $n=8$ ) [37,38]. Neither of these papers found a significant benefit of supplementation. However, drastic changes in the management and treatment of IBD over the past 30 years, combined with small sample sizes in both studies make this an attractive topic to revisit using well-designed clinical trials [39]. 


\subsubsection{Folate}

While folate (also known as vitamin B9) deficiency has classically been associated with anemia in IBD patients, it's role in IBD is likely more complex than previously thought. Not only is folate essential in the synthesis of nucleotides, but it is implicated in the regulation of $\mathrm{T}_{\text {REG }}$ cells [40]. In vitro, folate acts as a pro-survival signal for $\mathrm{T}_{\mathrm{REG}}$ cells $[41,42]$. Similarly, in murine models, deficiency in folate leads to the reduction of $\mathrm{T}_{\mathrm{REG}}$ cells in the intestine [40]. Murine models have also demonstrated a predisposition towards intestinal inflammation in mice being fed diets deficient in folate [42,43]. Furthermore, folate deficiency (as well as B12 deficiency) inhibits the conversion of homocysteine to methionine, which results in increased levels of homocysteine in the blood and intestinal mucosa of a significant portion of IBD patients [44]. Homocysteine acts as a pro-oxidant amino acid, increasing oxidative stress in the body [45]. Homocysteine also promotes the secretion of pro-inflammatory chemokines, and the differentiation of naïve $\mathrm{CD} 4+\mathrm{T}$ cells into pro-inflammatory $\mathrm{T}_{\mathrm{H}} 17$ cells [46]. Homocysteine levels are correlated with thrombotic events, and interestingly, IBD patients have an increased prevalence of thrombosis compared to those without IBD (5-year prevalence, $7.5 \%$ vs. $4.5 \%$, $p<0.001$ ) [47]. Furthermore, the patients are at increased risk of mesenteric ischemia (HR 11.2), and female IBD patients have significantly higher risk of MI (HR 1.6) and stroke (HR 2.1) [48].

Folate is absorbed in the jejunum following its conversion from folate polyglutamate to monoglutamate in the gut [49]. Further conversion in the liver results in the production of the metabolically active tetrahydrofolate (THF) [49]. Folate levels are significantly lower in patients with both CD and UC, with one prospective study of 180 IBD patients suggesting that $22.2 \%$ of CD patients and $4.3 \%$ of UC patients are deficient [50]. While the mechanism behind folate deficiency has not been well categorized, jejunal dysfunction and reduced intake of folate-containing foods are likely contributors. Furthermore, the use of methotrexate or sulfasalazine in IBD treatment leads to errors of folate metabolism and absorption [51,52].

While anemia is common in IBD patients, the authors were unable to find a study that assessed the proportion of patients with megaloblastic anemias in the setting of folate deficiency. Furthermore, folate supplementation remains a relatively unexplored topic. At this point, only the effect of supplementation on colorectal cancer has been described. A meta-analysis of 10 such articles determined that folate supplementation significantly lowers the risk of colorectal cancer in IBD patients (HR 0.58; 95\% CI: 0.36-0.66) [53]. However, the studies analyzed used varying doses of folate, making recommendations difficult. The effect of folate supplementation on homocysteine levels in the intestinal mucosa, and the risk of thrombotic event, are logical targets of future research.

\subsubsection{Vitamin B12}

Unlike the micronutrients discussed above, no direct connection between vitamin B12 (cobalamin) and immune regulation has been described. Vitamin B12 has been included in this review due to the clinical consequences of its deficiency, as well as the impact of $\mathrm{CD}$ on the site of its intestinal absorption. Vitamin B12 is essential in the metabolism of macronutrients, synthesis of DNA, and nerve function [54]. Deficiency is associated with macrocytic anemia via impaired folate metabolism, neurologic damage, and hyperhomocysteinemia [54]. While serious, these complications are uncommonly reported in IBD patients. In order to be absorbed, vitamin B12 requires activation by intrinsic factor produced in the stomach. Following activation, vitamin B12 is only absorbed via specific receptors present in the terminal ileum [55]. This location makes its deficiency a particular concern in CD, as a significant proportion of CD patients develop ileal disease [56].

Numerous studies exist that assess B12 status in IBD patients and the results vary [50,57-60]. In recent years, both a systemic review and meta-analysis of the literature have concluded that IBD is not associated with significantly lower serum B12 levels [61]. However, surgical resection of the ileum is associated with B12 deficiency [61]. Meta-analysis by Pan et al., 2017 suggests that patients with ileal resections were disproportionately underrepresented in the literature surrounding B12 deficiency [61]. Considering that ileal disease is common in $\mathrm{CD}$, and upwards of $50 \%$ of $\mathrm{CD}$ patients require surgery 
within 10 years of diagnosis, patients with ileal resection, and therefore B12 deficiency, likely represent a larger group than described [62].

\subsubsection{Vitamin D}

The relationship of IBD and vitamin D is likely the most complicated of the IBD-micronutrient relationships. While normally associated with calcium metabolism and bone health, the role of vitamin $\mathrm{D}$ in the regulation of the immune response has been the topic of much research in recent years [16]. In the gut, vitamin D's active form 1 $\alpha, 25$-dihydroxyvitamin D3 (calcitriol) alters the T cell response, favoring $\mathrm{T}_{\mathrm{REG}}$ cells and inhibiting production of proinflammatory cytokines [63]. Calcitriol also impairs the function of interstitial dendritic cells, limiting their ability to activate $\mathrm{T}$ cells within the gut [63] Furthermore, direct signaling via vitamin $\mathrm{D}$ receptors on $\mathrm{T}$ cells leads to upregulation of programmed death receptors and the downregulation of the activating receptor CD69 [64]. These properties provide a rational physiological means by which vitamin D deficiency affects IBD pathogenesis. This is further substantiated by murine models of IBD in which vitamin D supplementation improves symptoms, and deficiency increases symptoms $[65,66]$.

Vitamin D is acquired through the diet, however the majority of vitamin D is synthesized endogenously in the skin [67]. Once acquired, vitamin D must then be metabolized step-wise in the liver and kidney into its active form 1 $\alpha, 25$-dihydroxyvitamin D3 [67]. Vitamin D deficiency is common in IBD patients, with a meta-analysis identifying deficient vitamin D levels in $57.7 \%$ of CD patients [68]. The exact reason for vitamin D deficiency in IBD is not well understood. Considering that few foods naturally contain vitamin D, deficiency is likely a combination of reduced intake to diet restrictions and aversions, as well as impairment of the absorption of fat-soluble vitamins [69].

Literature correlating vitamin D deficiency with IBD disease activity is abundant and relatively homogenous [70-75]. However, there exists a limited amount of data in patients whose vitamin D levels have been restored through supplementation. Broadly, vitamin D levels are inversely associated with inflammatory markers, while positively associated with bone mineral density (BMD), anti-inflammatory cytokine production, and response to anti-tumor necrosis factor (TNF) therapy [70,76-80]. In both CD and UC, vitamin D deficiency is also associated with more frequent relapses of disease, disease severity, risk of colorectal cancer [81-83]. Similarly, a landmark longitudinal study of 965 American IBD patients saw a significant association between low vitamin $\mathrm{D}$ concentration and increased utilization of healthcare, increased pain, and decreased quality of life [84]. This study also found that vitamin D normalization significantly reduced healthcare utilization in the same population [84]. Ananthakrishnan et al., 2013 have corroborated this finding, showing that normalization of vitamin D levels reduces risk of surgery in $C D$ patients [85]. In terms of BMD, concurrent supplementation with calcium and vitamin D led to significantly increased BMD in the hip and lumbar spine [86,87].

Dosing of vitamin D for effect in IBD is not well-established [88]. Doses of vitamin D3 at $4000 \mathrm{IU} /$ day are adequate in correcting deficiency but given the conflicting evidence around the effect of supplementation on disease course in the first place, the most appropriate therapeutic dose has not been determined $[88,89]$.

\subsubsection{Calcium}

Calcium homeostasis is a complex, multifaceted phenomenon. Bone, which is $60 \%$ comprised of calcium hydroxyapatite $\left(\mathrm{Ca}_{10}\left(\mathrm{PO}_{4}\right)_{6}(\mathrm{OH})_{2}\right.$, undergoes constant remodeling throughout life. Therefore, the pathways responsible for the control of calcium homeostasis are critically important. The supply of calcium involves paracellular and transcellular intestinal and renal absorption and reabsorption [90]. Chronic intestinal inflammation of IBD, as well as dietary and lifestyle alterations and medications used to treat IBD, impact these physiologic homeostatic pathways [7,9,87,90,91]. Intestinal malabsorption, as a result of decreased dairy product intake, leads to low luminal ionic calcium $\left(\mathrm{Ca}^{2+}\right)$ concentrations resulting in increased transcellular active transport (versus more non-active paracellular transport when luminal $\mathrm{Ca}^{2+}$ concentrations are high via decreased duodenal transit time 
and down regulation of protein responsible for transcellular $\mathrm{Ca}^{2+}$ transport proteins). $\mathrm{Ca}^{2+}$ is absorbed through facilitated diffusion mediated by transient receptor potential villanoid channels (TRPV6 and 5), which are regulated by vitamin D3, parathyroid hormone, estrogens, $\mathrm{pH}$ - and $\mathrm{Ca}^{2+}$ dependent regulatory mechanisms, and bidirectional $\mathrm{Na}+/ \mathrm{Ca}^{2+}$ exchange [90].

Beyond the known negative influence of vitamin D insufficiency and malnutrition, there is a relative paucity of knowledge relating to the impact of acute and chronic intestinal inflammation itself on $\mathrm{Ca}^{2+}$ homeostasis in the kidneys and intestine. A study by Huybers et al. demonstrated in a TNF- $\alpha$ overexpressing mouse model of Crohn's ileitis that multiple epithelial proteins involved in $\mathrm{Ca}^{2+}$ absorption and transport, including TRPV6, were down regulated [92]. As a result of changes in the expression of $\mathrm{Ca}^{2+}$ transport genes, bone resorption increased. When these same mice were treated with methylprednisone, similar inhibitory effects were seen in TRPV6 and calbindin D9K mRNA and protein. Although limited data exists in IBD, a very small $(n=4)$ study of CD patients in remission revealed intestinal absorption of $\mathrm{Ca}^{2+}$, as well as response to $1,25(\mathrm{OH})_{2} \mathrm{D}_{3}$, to be similar to that of controls [92].

In addition to calcium, phosphate $\left(\mathrm{P}_{\mathrm{i}}\right)$ homeostasis is important in the maintenance of BMD. Prolonged phosphate deficiency may lead to bone demineralization (osteomalacia and rickets). Although little is known about the impact of intestinal inflammation on $P_{i}$ absorption, as with serum $\mathrm{Ca}^{2+}$ it is known that serum levels of $P_{\mathrm{i}}$ do not reflect minor alterations in active mineral transport. Therefore, it is very uncommon to observe changes in serum $\mathrm{Ca}^{2+}$ or $\mathrm{P}_{\mathrm{i}}$ in IBD patients. The Klotho gene, a multifunctional protein, has been implicated in the regulation of both $\mathrm{Ca}^{2+}$ and $\mathrm{P}_{\mathrm{i}}$ metabolism through its influence on transepithelial $\mathrm{Ca}^{2+}$ transport via promotion of transport channel recruitment and retention. Thurston et al., have demonstrated renal Klotho to be significantly down-regulated in various murine models of colitis via TNF and IFN-gamma [93].

Through complex absorptive, transport, and hydroxylation mechanisms, vitamin D3 is converted to the hormonally active form of vitamin $\mathrm{D}, 1,25(\mathrm{OH})_{2} \mathrm{D}_{3}$, which is responsible for most, if not all, of the biological effects of vitamin D. $1,25(\mathrm{OH})_{2} \mathrm{D}_{3}$ has a pleiotropic role and is a key regulator of bone turn-over, with the potential to facilitate both osteoclastic and osteoblastic pathways. Numerous studies have demonstrated the association between vitamin D deficiency or insufficiency and IBD when compared with control or healthy individuals $[94,95]$. The mechanisms for this observed associations in IBD may include intestinal malabsorption, decreased dietary intake, disrupted enterohepatic circulation, renal insufficiency and inadequate sunshine exposure. Given the association between IBD and decreased vitamin D status, numerous interventional studies have been conducted in an attempt to determine the impact of vitamin D supplementation on IBD-related clinical outcomes. The data related to BMD loss or accrual have been mixed $[90,96,97]$. In fact, there have been several studies that suggest, in the setting of active inflammation, that high concentrations of $1,25(\mathrm{OH})_{2} \mathrm{D}_{3}$ may promote the development of osteoporosis in CD [98]. Likewise, the outcomes of studies relating to bone formation and resorption in IBD patients have been conflicting, likely due to flaws in study design, power, confounding factors, IBD complexity, and challenges related to understanding and measuring bone turn-over rates in both adults and children with IBD. More well designed and adequately powered clinical studies are required to definitely answer these questions relating to bone formation and resorption in IBD [90].

The presumption that the BMD effects observed in IBD are simply as a result of vitamin D3 and calcium deficiency has more recently been recognized to be overly simplistic. The complex interplay between the inflammatory disease process itself and the mediators of mineral homeostasis and bone (re)absorption complicates efforts to clearly elucidate the role that these nutrients and hormones play in the pathogenesis of metabolic bone disease in patients with IBD, as well as the role that acute and chronic inflammation may play in alterations of mineral and hormonal homeostasis.

\subsubsection{Vitamin K}

The mechanism of vitamin $\mathrm{K}$ absorption is two-fold. Vitamin $\mathrm{K}$ is both directly absorbed from the diet and produced in the gut by commensal GI flora. As a cofactor, vitamin $\mathrm{K}$ is crucial to the production of coagulation factors and in the maintenance of bone [99]. As well, much like 
many of the other micronutrients discussed, vitamin $\mathrm{K}$ is implicated in immune signaling and inflammation [16]. Poor vitamin K levels are associated with increasing levels of IL-6 in the blood, a pro-inflammatory cytokine that is implicated in IBD pathology $[100,101]$. Similarly, in murine models, vitamin K supplementation reduced intestinal inflammation in the presence of the bacterial antigen lipopolysaccharide (LPS), while deficiency aggravated disease in DSS models of colitis in mice [102,103]. This provides a dual rationale for vitamin K supplementation in IBD: avoid complications such as bleeding and bone loss, while hopefully pushing the body away from pro-inflammatory immune pathways.

Vitamin K deficiency is found in $31 \%$ of IBD patients, with dysregulation of the gut biota and bile salt malabsorption proposed as mechanisms [69]. In these patients, low levels of vitamin K are associated with low BMD, as well as clinical activity and duration of disease [104-106]. In long-standing IBD, the prevalence of osteoporosis can be as high as $41 \%$ [107]. Considering this, and the adverse outcomes associated with fractures, BMD is of critical importance in IBD management. Daily supplementation of vitamin K over 12 months was unable to alter the indices of bone health in IBD patients with vitamin $\mathrm{K}$ deficiencies [108]. Furthermore, in rheumatoid arthritis, which shares some common inflammatory pathophysiology with IBD, vitamin K supplementation did not significantly alter the level of IL-6 or the clinical activity of disease in patients [109]. Given the link of vitamin K deficiency to low BMD and high IL-6, these results are disappointing.

\subsection{Metals}

\subsubsection{Iron}

Aside from vitamin D, iron is likely the micronutrient that has been most heavily studied in the context of IBD. Iron has attracted attention due to the high portion of IBD patients who suffer from anemia [110]. The mean prevalence of anemia calculated by meta-analysis is $16 \%$ in outpatients, increasing to $68 \%$ in inpatients [99]. As well, the overall portion of IBD patients with iron deficiency is $45 \%$ [111]. The physiological means by which IBD causes iron-deficiency is straight forward. Mucosal bleeding leads to iron loss in the stool, meanwhile inflammatory changes altering hepcidin and iron metabolism impact iron absorption [112].

Anemia, and the associated fatigue, can be one of the most crippling symptoms of IBD, with quality of life scores mirroring hemoglobin $(\mathrm{Hb})$ levels [113]. On this basis alone, the treatment of iron-deficiency anemia in IBD is paramount. Current recommendations suggest the end goal of iron supplementation is the normalization of iron store levels, as well as $\mathrm{Hb}$ [114]. Oral and intravenous iron products are available for use in IBD. Clinically, and in meta-analysis, intravenous iron supplementation is associated with significantly fewer GI side effects, as well as slightly greater increases in $\mathrm{Hb}$ [115]. However, the patient's quality of life did not significantly differ between intravenous and oral iron [116]. Importantly, anemia reoccurs rapidly in IBD, further necessitating the use of iron maintenance therapies [117]. In line with this, European Crohn's and Colitis Organization (ECCO) guidelines suggest that patients who have been treated for iron-deficiency anemia be monitored every 3 months for the first year, and every 6-12 months for the recurrence of anemia [114]. 


\subsubsection{Zinc}

As a cofactor, zinc is intimately involved in the regulation of both the innate and adaptive arms of the immune system [118]. Importantly, zinc downregulates NF- $\mathrm{kB}$ signaling through the zinc-finger protein A20, leading to decreased expression of its downstream products (namely IL-6 and TNF- $\alpha$ ) [119-121]. It is worth noting that certain mutations in the gene that encodes A20 (TNFAIP3) are positively associated with the risk of developing numerous inflammatory conditions, including IBD [121]. As well, deletion of TNFAIP3 in mice intestinal cells leads to sensitivity to DSS-induced colitis. Conversely, zinc deficiency results in increased production of pro-inflammatory molecules in murine models [122]. Finally, zinc also exerts an anti-oxidative effect through its inhibition of NADPH oxidase, and its role as a cofactor for superoxide dismutase $[123,124]$. The relationship between zinc and IBD on both a theoretical and genetic level, make it a micronutrient of interest to explore further.

Zinc deficiency is present in a significant portion of IBD patients $(15.2-65 \%)[8,125]$. Zinc levels are balanced through absorption in the proximal small intestine, and excretion in intestinal and pancreatic secretions [126]. Losses are increased in association with chronic diarrhea, high output ostomies, and high output fistulas, which often pose a problem for IBD patients. These increased losses combined with the malabsorptive state of intestinal inflammation are likely responsible for zinc deficiency in IBD patients.

Clinically, an association has also been observed between zinc deficiency and IBD. A prospective study of 170,756 women over 26 years found that increasing zinc intake was inversely corelated with CD risk [127]. Furthermore, an American cohort study of 773 CD and 233 UC patients highlighted the relationship between zinc deficiency and adverse patient outcomes. They found that zinc deficiency was associated with a significant increase in hospitalizations (CD: OR 1.44. 95\% CI [1.02-2.04]; UC: OR $2.14,95 \%$ CI [1.07-4.29]), surgery (CD: OR 2.05, 95\% CI [1.38-3.05]; UC: OR 1.64, 95\% CI [0.59-4.52]), and complications (CD: OR 1.50, 95\% CI [1.04-2.15]; UC: OR 1.97, 95\% CI [0.94-4.11]) [128]. In patients who were able to achieve normal zinc levels within 12 months, the risk of adverse outcomes returned to baseline [128]. Although zinc deficiency is associated with disease activity and worse outcomes, a single small cross-over trial $(n=14)$ that explored the effect of daily zinc supplementation did not show changes in the level of pro-inflammatory cytokines, or disease activity [129]. Given the positive results seen by Siva et al., 2017, larger trials following zinc supplementation may be a reasonable step forward [128].

\subsubsection{Selenium}

Much like zinc, selenium is integral to the immune function. Selenoproteins, through which selenium exerts its effect, encompass a group of proteins with diverse pro- and anti-inflammatory effects that are beyond the scope of this review. Relevant to IBD, glutathione peroxidases (GPx) utilize selenium to mitigate the effect of ROS [130]. Whereas loss of the genes encoding GPx proteins results in increased severity of disease in DSS murine models [131-133].

Selenium deficiency is common in IBD patients with a recent study reporting $30.9 \%$ of patients as deficient, and numerous others observing levels that were significantly decreased compared to control [134-139]. In DSS-treated mice, selenium deficiency leads to increased severity of disease and upregulation of pro-inflammatory TH1 cytokines [140,141]. In humans, selenium deficiency has been investigated for its possible role in cardiovascular disease, however a recent review by Benstoem et al., 2015 describes the literature as "inconclusive" [142].

Selenium supplementation in the context of IBD has not been documented in the literature outside of murine models. In these models, supplementation ameliorated cox-dependent inflammation by altering the PGE2 pathway [143,144]. Given the abundance of animal data, and the strong physiological link between selenium and immune function, the study of selenium therapy should be a future focus. Table 1 presents the immune implications of micronutrient deficiencies, including selenium, in adult IBD patients. 
Table 1. Immune implications of micronutrient deficiencies in IBD.

\begin{tabular}{|c|c|c|}
\hline Micronutrient & Level in IBD & Purported Pathogenic Role of Deficiency \\
\hline Vitamin A & $\downarrow[27]$ & $\begin{array}{c}\downarrow T_{\text {REG }} \text { cell differentiation }[18-21] \\
\uparrow T_{H 17} \text { cell differentiation }[20] \\
\downarrow T \text { cell migration into the gut [25] }\end{array}$ \\
\hline Folate & $\downarrow[43]$ & $\begin{array}{c}\downarrow T_{\text {REG }} \text { cell survival }[31,32] \\
\uparrow \text { Proinflammatory signaling }[37,39] \\
\uparrow \text { Oxidative stress }[37,38]\end{array}$ \\
\hline Vitamin B12 & $=\downarrow$ in patients with ileal resection [54] & - \\
\hline Vitamin D & $\downarrow[61]$ & $\begin{array}{c}\uparrow \mathrm{T} \text { cell activation [56] } \\
\uparrow \text { Proinflammatory signaling [56] } \\
\downarrow \text { Decreased T cell turnover [57] }\end{array}$ \\
\hline Calcium & $\downarrow[87,90,91]$ & - \\
\hline Vitamin K & $\downarrow[62]$ & $\uparrow$ Proinflammatory signaling $[84,85]$ \\
\hline Iron & $\downarrow[104]$ & - \\
\hline Zinc & $\downarrow[8,118]$ & $\begin{array}{l}\uparrow \text { Proinflammatory signaling [114] } \\
\uparrow \text { Oxidative stress }[116,117]\end{array}$ \\
\hline Selenium & $\downarrow[134-139]$ & $\begin{array}{c}\uparrow \text { Proinflammatory signaling [141] } \\
\uparrow \text { Oxidative stress [130] }\end{array}$ \\
\hline
\end{tabular}

\section{Recommendations and Conclusions}

The understanding of the role of micronutrients in immune system function has changed greatly in recent years, as has our understanding of the pathophysiologic mechanism(s) of Inflammatory Bowel Disease (IBD). However, the etiology of IBD and the role that micronutrients play in the development of IBD is still unclear. There are clear physiologic links between immune-regulating micronutrients and the balance of pro- and anti-inflammatory processes in the gut (see Table 1) [16]. However, experimental models often oversimplify what is experienced clinically (see Table 2). The same is true of micronutrients with anti-oxidative effects [16,17]. In addition, the bidirectional relationship of deficiency and disease makes the exact role of each micronutrient difficult to characterize, as IBD impacts nutritional status through both decreased intake (impaired absorption, avoidance of trigger foods, decreased appetite, decreased surface area secondary to resection, etc.), and increased gastric losses (chronic diarrhea, intestinal blood loss, mucus losses, etc.). The effect of micronutrients on outcomes is most easily understood in the context of iron, in which IBD leads to iron deficiency, and deficiency leads to anemia. However, for the other micronutrients, the relationship between IBD, deficiency, and clinical outcomes is much less clear. Table 2 presents the clinical outcomes associated with level of micronutrients discussed in this paper, in adult IBD patients. 
Table 2. Clinical outcomes associated with micronutrient levels in IBD.

\begin{tabular}{|c|c|c|}
\hline Micronutrient & Effect of Deficiency & Effect of Supplementation \\
\hline Vitamin A & - & - \\
\hline Folate & $\begin{array}{c}\text { Macrocytic anemia } \\
\uparrow \text { Homocysteinemia [44] }\end{array}$ & $\downarrow$ Risk of colorectal cancer [53] \\
\hline Vitamin B12 & $\begin{array}{c}\text { Macrocytic anemia } \\
\uparrow \text { Homocysteinemia } \\
\text { Neurologic damage [54] }\end{array}$ & - \\
\hline Vitamin D & $\begin{array}{c}\uparrow \text { Inflammatory markers [70,77] } \\
\uparrow \text { Healthcare utilization [84] } \\
\uparrow \text { Disease activity [70-75] } \\
\uparrow \text { Risk of colorectal cancer [83] } \\
\downarrow \text { Quality of life }[72,85]\end{array}$ & $\begin{array}{c}\uparrow \text { Bone mineral density } \\
\downarrow \text { Risk of surgery }\end{array}$ \\
\hline Vitamin K & $\begin{array}{c}\downarrow \text { Bone mineral density [104-106] } \\
\uparrow \text { Bleeding risk [99] }\end{array}$ & $\begin{aligned} & \leftrightarrow \text { Disease activity } \\
\leftrightarrow & \text { Bone mineral density }\end{aligned}$ \\
\hline Iron & Iron-deficiency anemia & $\begin{array}{l}\text { Resolution of anemia } \\
\uparrow \text { Quality of life [113] }\end{array}$ \\
\hline Zinc & $\begin{array}{c}\uparrow \text { CD risk [127] } \\
\uparrow \text { Hospitalizations } \\
\uparrow \text { Surgery } \\
\uparrow \text { Complications [126] }\end{array}$ & $\leftrightarrow$ Disease activity \\
\hline Selenium & ? Cardiovascular disease [142] & - \\
\hline
\end{tabular}

hile association studies suggest the benefit of treating deficiency of iron, zinc and vitamin D, similar studies have not been performed for other micronutrients. Similarly, literature regarding the use of supplementation as therapy is limited to a few, small scale studies that only targeted specific subgroups of patients $[37,38,129]$. These factors make specific recommendations on dosage for supplementation difficult. At this point, current European Society for Clinical Nutrition and Metabolism (ESPEN) recommendations suggest that micronutrient levels be assessed annually in IBD patients, with the correction of any deficit with supplementation [145]. Guidelines for iron supplementation are more specific, suggesting iron repletion therapy in all IBD patients with iron-deficiency anemia, with the end goal of therapy being the normalization of both hemoglobin and iron stores [114]. Intravenous iron should be considered in patients with clinically active disease and those who are intolerant of oral iron, while oral iron should be considered in those with inactive disease or milder anemia [114]. The dose of iron is dependent on the route and formulation chosen, however a response of $2 \mathrm{~g} / \mathrm{dL}$ within 4 weeks is appropriate [114]. As for zinc, a 3-month course of daily supplementation with $200 \mathrm{mg}$ of $\mathrm{ZnSO}_{4}$ normalizes zinc serum concentrations in deficient IBD patients [146]. Given that zinc citrate has similar bioavailability and is more palatable to patients its use may be preferred [147]. In addition, one author suggests increasing zinc supplementation in patients with diarrhea in order to offset increased GI loses, at a rate of 30-40 mg elemental zinc for every liter of stool output [148].

For the other nutrients discussed in this paper, evidence-based recommendations for supplementation in the context of IBD do not exist. Rather, clinicians should focus on the correction of any micronutrient deficiencies that are found using appropriate oral therapy. Previous authors have posited varying suggestions on dosage with regards to reversing deficit, with high dose oral therapy as the common theme among them. A 2003 review by Eiden highlights such suggestions in IBD patients and is a valuable reference for clinicians with deficient IBD patients [149]. 
The development of specific recommendations for supplementation and micronutrient levels to targets is a priority. In order to do so, large, well-designed, controlled studies focused on supplementation are required. Given the consistency of the observed association between zinc and vitamin D and IBD clinical disease activity and inflammation, further study of supplementation of vitamin $\mathrm{D}$ and zinc as an adjunct to therapy is appropriate [16,36]. Given their link to immunity and oxidation, further characterization of the role of vitamin $\mathrm{A}, \mathrm{B} 9$, and $\mathrm{K}$ in IBD pathogenesis is also warranted.

Author Contributions: Conceptualization, J.L.J. and L.B.; Methodology, J.L.J. and H.M.; Formal Analysis, K.K.; Data Curation, H.M.; Writing-Original Draft Preparation, K.K.; Writing-Review \& Editing, K.K., L.B., H.M., C.C., J.L.J.; Supervision, J.L.J.; Project Administration, H.M.

Funding: This research received no external funding.

Conflicts of Interest: The authors declare no conflict of interest.

\section{References}

1. Lee, S.H.; Kwon, J.e.; Cho, M.-L. Immunological pathogenesis of inflammatory bowel disease. Intest. Res. 2018, 16, 26-42. [CrossRef] [PubMed]

2. Benchimol, E.I.; Mack, D.R.; Guttmann, A.; Nguyen, G.C.; To, T.; Mojaverian, N.; Quach, P.; Manuel, D.G. Inflammatory bowel disease in immigrants to Canada and their children: A population-based cohort study. Am. J. Gastroenterol. 2015, 110, 553-563. [CrossRef] [PubMed]

3. Hou, J.K.; Abraham, B.; El-Serag, H. Dietary intake and risk of developing inflammatory bowel disease: A systematic review of the literature. Am. J. Gastroenterol. 2011, 106, 563-573. [CrossRef] [PubMed]

4. Racine, A.; Carbonnel, F.; Chan, S.S.M.; Hart, A.R.; Bueno-De-Mesquita, H.B.; Oldenburg, B.; Van Schaik, F.D.M.; Tjønneland, A.; Olsen, A.; Dahm, C.C.; et al. Dietary patterns and risk of inflammatory bowel disease in Europe: Results from the EPIC study. Inflamm. Bowel Dis. 2016, 22, 345-354. [CrossRef] [PubMed]

5. Limdi, J.K. Dietary practices and inflammatory bowel disease. Indian J. Gastroenterol. 2018, 37, 284-292. [CrossRef] [PubMed]

6. Taylor, L.; Almutairdi, A.; Shommu, N.; Fedorak, R.; Ghosh, S.; Reimer, R.A.; Panaccione, R.; Raman, M. Cross-sectional analysis of overall dietary intake and mediterranean dietary pattern in patients with Crohn's disease. Nutrients 2018, 10, 1761. [CrossRef] [PubMed]

7. Urbano, A.P.S.; Sassaki, L.Y.; Dorna, M.S.; de Barros Leite Carvalhaes, M.A.; Martini, L.A.; Ferreira, A.L.A. Nutritional intake according to injury extent in ulcerative colitis patients. J. Hum. Nutr. Diet. 2013, 26, 445-451. [CrossRef] [PubMed]

8. Vagianos, K.; Clara, I.; Carr, R.; Graff, L.A.; Walker, J.R.; Targownik, L.E.; Lix, L.M.; Rogala, L.; Miller, N.; Bernstein, C.N. What are adults with inflammatory bowel disease (IBD) eating? A closer look at the dietary habits of a population-based Canadian IBD cohort. J. Parenter. Enter. Nutr. 2016, 40, 405-411. [CrossRef]

9. Vidarsdottir, J.B.; Johannsdottir, S.E.; Thorsdottir, I.; Bjornsson, E.; Ramel, A. A cross-sectional study on nutrient intake and -status in inflammatory bowel disease patients. Nutr. J. 2016, 15, 61. [CrossRef] [PubMed]

10. Zallot, C.; Quilliot, D.; Chevaux, J.B.; Peyrin-Biroulet, C.; Guéant-Rodriguez, R.M.; Freling, E.; Collet-Fenetrier, B.; Williet, N.; Ziegler, O.; Bigard, M.A.; et al. Dietary beliefs and behavior among inflammatory bowel disease patients. Inflamm. Bowel Dis. 2013, 19, 66-72. [CrossRef]

11. Walton, M.; Alaunyte, I. Do patients living with ulcerative colitis adhere to healthy eating guidelines? A cross-sectional study. Br. J. Nutr. 2014, 112, 1628-1635. [CrossRef] [PubMed]

12. Tontini, G.E.; Vecchi, M.; Pastorelli, L.; Neurath, M.F.; Neumann, H. Differential diagnosis in inflammatory bowel disease colitis: State of the art and future perspectives. World J. Gastroenterol. 2015, 21, 21-46. [CrossRef] [PubMed]

13. Basu, T.K.; Donaldson, D. Intestinal absorption in health and disease: Micronutrients. Best Pract. Res. Clin. Gastroenterol. 2003, 17, 957-979. [CrossRef]

14. Lucendo, A.J.; De Rezende, L.C. Importance of nutrition in inflammatory bowel disease. World J. Gastroenterol. 2009, 15, 2081-2088. [CrossRef] [PubMed]

15. Shenkin, A. Micronutrients in health and disease. Postgrad. Med. J. 2006, 82, 559-567. [CrossRef] [PubMed] 
16. Hosomi, K.; Kunisawa, J. The specific roles of vitamins in the regulation of immunosurveillance and maintenance of immunologic homeostasis in the gut. Immune Netw. 2017, 17, 13-19. [CrossRef] [PubMed]

17. Tian, T.; Wang, Z.; Zhang, J. Pathomechanisms of oxidative stress in inflammatory bowel disease and potential antioxidant therapies. Oxid. Med. Cell. Longev. 2017, 2017, 4535194. [CrossRef]

18. Elias, K.M.; Laurence, A.; Davidson, T.S.; Stephens, G.; Kanno, Y.; Shevach, E.M.; O'Shea, J.J. Retinoic acid inhibits TH17 polarization and enhances FoxP3 expression through a Stat-3/Stat-5 independent signaling pathway. Blood 2008, 111, 1013-1020. [CrossRef]

19. Hill, J.A.; Hall, J.A.; Sun, C.M.; Cai, Q.; Ghyselinck, N.; Chambon, P.; Belkaid, Y.; Mathis, D.; Benoist, C. Retinoic acid enhances Foxp3 induction indirectly by relieving inhibition from CD4 ${ }^{+} \mathrm{CD} 44$ hi cells. Immunity 2008, 29, 758-770. [CrossRef]

20. Mucida, D.; Park, Y.; Kim, G.; Turovskaya, O.; Scott, I.; Cheroutre, H. Reciprocal TH17 and regulatory T Cell differentiation mediated by retinoic acid. Science 2007, 317, 256-260. [CrossRef]

21. Sun, C.-M.; Hall, J.A.; Blank, R.B.; Bouladoux, N.; Oukka, M.; Mora, J.R.; Belkaid, Y. Small intestine lamina propria dendritic cells promote de novo generation of Foxp3 T REG cells via retinoic acid. J. Exp. Med. 2007, 204, 1775-1785. [CrossRef] [PubMed]

22. Lewkowicz, N.; Klink, M.; Mycko, M.P.; Lewkowicz, P. Neutrophil-CD4 ${ }^{+} \mathrm{CD} 25^{+} \mathrm{T}$ regulatory cell interactions: A possible new mechanism of infectious tolerance. Immunobiology 2013, 218, 455-464. [CrossRef] [PubMed]

23. Owczarczyk-Saczonek, A.; Czerwińska, J.; Placek, W. The role of regulatory T cells and anti-inflammatory cytokines in psoriasis. Acta Dermatovenerol. Alp. Pannonica Adriat. 2018, 27, 17-23. [CrossRef] [PubMed]

24. Iwata, M.; Hirakiyama, A.; Eshima, Y.; Kagechika, H.; Kato, C.; Song, S.Y. Retinoic acid imprints gut-homing specificity on T cells. Immunity 2004, 21, 527-538. [CrossRef] [PubMed]

25. Iwata, M. Retinoic acid production by intestinal dendritic cells and its role in T-cell trafficking. Semin. Immunol. 2009, 21, 8-13. [CrossRef] [PubMed]

26. Reboul, E. Absorption of vitamin A and carotenoids by the enterocyte: Focus on transport proteins. Nutrients 2013, 5, 3563-3581. [CrossRef] [PubMed]

27. Soares-Mota, M.; Silva, T.A.; Gomes, L.M.; Pinto, M.A.S.; Mendonça, L.M.C.; Farias, M.L.F.; Nunes, T.; Ramalho, A.; Zaltman, C. High prevalence of vitamin A deficiency in Crohn's disease patients according to serum retinol levels and the relative dose-response test. World J. Gastroenterol. 2015, 21, 1614-1620. [CrossRef] [PubMed]

28. Biasi, F.; Leonarduzzi, G.; Oteiza, P.I.; Giuseppe, P. Inflammatory bowel disease: Mechanisms, redox considerations, and therapeutic targets. Antioxid. Redox Signal. 2013, 19, 1711-1747. [CrossRef]

29. Trivedi, P.P.; Jena, G.B. Mechanistic insight into beta-carotene-mediated protection against ulcerative colitis-associated local and systemic damage in mice. Eur. J. Nutr. 2015, 54, 639-652. [CrossRef] [PubMed]

30. Hengstermann, S.; Valentini, L.; Schaper, L.; Buning, C.; Koernicke, T.; Maritchnegg, M.; Buhner, S.; Tillinger, W.; Regano, N.; Guglielmi, F.; et al. Altered status of antioxidant vitamins and fatty acids in patients with inactive inflammatory bowel disease. Clin. Nutr. 2008, 27, 571-578. [CrossRef] [PubMed]

31. Rezaie, A.; Parker, R.D.; Abdollahi, M. Oxidative stress and pathogenesis of inflammatory bowel disease: An epiphenomenon or the cause? Dig. Dis. Sci. 2007, 52, 2015-2021. [CrossRef] [PubMed]

32. Seidner, D.L.; Lashner, B.A.; Brzezinski, A.; Banks, P.L.C.; Goldblum, J.; Fiocchi, C.; Katz, J.; Lichtenstein, G.R.; Anton, P.A.; Kam, L.Y.; et al. An oral supplement enriched with fish oil, soluble fiber, and antioxidants for corticosteroid sparing in ulcerative colitis: A randomized, controlled trial. Clin. Gastroenterol. Hepatol. 2005, 3, 358-369. [CrossRef]

33. Wendland, B.E.; Aghdassi, E.; Tam, C.; Carrier, J.; Steinhart, A.H.; Wolman, S.L.; Baron, D.; Allard, J.P. Lipid peroxidation and plasma antioxidant micronutrients in Crohn disease. Am. J. Clin. Nutr. 2001, 74, 259-264. [CrossRef] [PubMed]

34. Glabska, D.; Guzek, D.; Zakrzemska, P.; Wiodarek, D.; Lech, G. Lycopene, lutein and zeaxanthin may reduce faecal blood, mucus and pus but not abdominal pain in individuals with ulcerative colitis. Nutrients 2016, 8, 613 . [CrossRef] [PubMed]

35. Liu, C.; Bronson, R.; Wang, X.-D. Effects of lutein supplementation on the prevention of colonic inflammation and neoplasia in mice. FASED J. 2015, 29. [CrossRef] 
36. Kang, S.G.; Wang, C.; Matsumoto, S.; Kim, C.H. High and low vitamin A therapies induce distinct FoxP3+ T cell subsets and effectively control intestinal inflammation. Gastroenterology 2009, 137, 1391-1402. [CrossRef] [PubMed]

37. Wright, J.P.; Mee, A.S.; Parfitt, A.; Marks, I.N.; Burns, D.G.; Sherman, M.; Tigler-Wybrandi, N.; Isaacs, S. Vitamin A therapy in patients with Crohn's disease. Gastroenterology 1985, 88, 512-514. [CrossRef]

38. Norrby, S.; Sjödahl, R.; Tagesson, C. Ineffectiveness of vitamin A therapy in severe Crohn's disease. Acta Chir. Scand. 1985, 151, 465-468. [PubMed]

39. Ghishan, F.K.; Kiela, P.R. Vitamins and minerals in inflammatory bowel disease. Gastroenterol. Clin. N. Am. 2017, 46, 797-808. [CrossRef]

40. Yamaguchi, T.; Hirota, K.; Nagahama, K.; Ohkawa, K.; Takahashi, T.; Nomura, T.; Sakaguchi, S. Control of immune responses by antigen-specific regulatory T cells expressing the folate receptor. Immunity 2007, 27, 145-159. [CrossRef]

41. Kunisawa, J.; Hashimoto, E.; Ishikawa, I.; Kiyono, H. A pivotal role of vitamin B9 in the maintenance of regulatory T cells in vitro and in vivo. PLoS ONE 2012, 7, e32094. [CrossRef]

42. Kinoshita, M.; Kayama, H.; Kusu, T.; Yamaguchi, T.; Kunisawa, J.; Kiyono, H.; Sakaguchi, S.; Takeda, K. Dietary folic acid promotes survival of Foxp3+ regulatory T cells in the Colon. J. Immunol. 2012, 189, 2869-2878. [CrossRef] [PubMed]

43. Zhu, S.; Li, J.; Bing, Y.; Yan, W.; Zhu, Y.; Xia, B.; Chen, M. Diet-induced hyperhomocysteinaemia increases intestinal inflammation in an animal model of colitis. J. Crohn's Colitis 2015, 9, 708-719. [CrossRef] [PubMed]

44. Oussalah, A.; Guéant, J.L.; Peyrin-Biroulet, L. Meta-analysis: Hyperhomocysteinaemia in inflammatory bowel diseases. Aliment. Pharmacol. Ther. 2011, 34, 1173-1184. [CrossRef] [PubMed]

45. Tyagi, N.; Sedoris, K.C.; Steed, M.; Ovechkin, A.V.; Moshal, K.S.; Tyagi, S.C. Mechanisms of homocysteine-induced oxidative stress. Am. J. Physiol. Heart Circ. Physiol. 2005, 289, H2649-H2656. [CrossRef]

46. Gao, X.; Li, J.; Chen, M. Effect of homocysteine on the differentiation of CD4 + T cells into TH17 cells. Dig. Dis. Sci. 2018, 63, 3339-3347. [CrossRef]

47. Cohen, J.; Ragni, M.V.; Comer, D.M.; Yabes, J.G. Inflammatory bowel disease and thrombosis: A national inpatient sample (NIS) study. Blood 2017, 130, 3372.

48. Ha, C.; Magowan, S.; Accortt, N.A.; Chen, J.; Stone, C.D. Risk of arterial thrombotic events in inflammatory bowel disease. Am. J. Gastroenterol. 2009, 104, 1445-1451. [CrossRef]

49. Milman, N. Intestinal absorption of folic acid-New physiologic \& molecular aspects. Indian J. Med. Res. 2012, 136, 725-728.

50. Bermejo, F.; Algaba, A.; Guerra, I.; Chaparro, M.; De-La-Poza, G.; Valer, P.; Piqueras, B.; Bermejo, A.; García-Alonso, J.; Pérez, M.J.; et al. Should we monitor vitamin B12 and folate levels in Crohn's disease patients? Scand. J. Gastroenterol. 2013, 48, 1272-1277. [CrossRef]

51. Herfarth, H.H.; Kappelman, M.D.; Long, M.D.; Isaacs, K.L. Use of methotrexate in the treatment of inflammatory bowel diseases (IBD). Inflamm. Bowel Dis. 2016, 22, 224-233. [CrossRef] [PubMed]

52. Halstead, C.; Gandhi, G.; Tamura, T. Sulfasalazine inhibits the absorption of folates in ulcerative colitis. N. Engl. J. Med. 1981, 305, 1513-1517. [CrossRef] [PubMed]

53. Burr, N.E.; Hull, M.A.; Subramanian, V. Folic acid supplementation may reduce colorectal cancer risk in patients with inflammatory bowel disease: A systematic review and meta-analysis. J. Clin. Gastroenterol. 2017, 51, 247-253. [CrossRef] [PubMed]

54. O'Leary, F.; Samman, S. Vitamin B12 in health and disease. Nutrients 2010, 2, 299-316. [CrossRef] [PubMed]

55. Herbert, Y. Progress in gastroenterology: Absorption of vitamin B12 and folic acid. Gastroenterology 1968, 54, 110-115. [CrossRef]

56. Gasche, C.; Scholmerich, J.; Brynskov, J.; D’Haens, G.; Hanauer, S.B.; Irvine, E.J.; Jewell, D.P.; Rachmilewitz, D.; Sachar, D.B.; Sandborn, W.J.; et al. A simple classification of Crohn's disease: Report of the working party for the world congresses of gastroenterology, Vienna 1998. Inflamm. Bowel Dis. 2000, 6, 8-15. [CrossRef] [PubMed]

57. Battat, R.; Kopylov, U.; Szilagyi, A.; Saxena, A.; Rosenblatt, D.S.; Warner, M.; Bessissow, T.; Seidman, E.; Bitton, A. vitamin B12 deficiency in inflammatory bowel disease: Prevalence, risk factors, evaluation, and management. Inflamm. Bowel Dis. 2014, 20, 1120-1128. [CrossRef] [PubMed] 
58. Battat, R.; Kopylov, U.; Byer, J.; Sewitch, M.J.; Rahme, E.; Nedjar, H.; Zelikovic, E.; Dionne, S.; Bessissow, T.; Afif, W.; et al. Vitamin B12 deficiency in inflammatory bowel disease: A prospective observational pilot study. Eur. J. Gastroenterol. Hepatol. 2017, 29, 1361-1367. [CrossRef]

59. Ward, M.G.; Kariyawasam, V.C.; Mogan, S.B.; Patel, K.V.; Pantelidou, M.; Sobczyńska-Malefora, A.; Porté, F.; Griffin, N.; Anderson, S.H.C.; Sanderson, J.D.; et al. Prevalence and risk factors for functional vitamin B12 deficiency in patients with Crohn's disease. Inflamm. Bowel Dis. 2015, 21, 2839-2847. [CrossRef]

60. Yakut, M.; Üstün, Y.; Kabaçam, G.; Soykan, I. Serum vitamin B12 and folate status in patients with inflammatory bowel diseases. Eur. J. Int. Med. 2010, 21, 320-323. [CrossRef]

61. Pan, Y.; Liu, Y.; Guo, H.; Jabir, M.S.; Liu, X.; Cui, W.; Li, D. Associations between folate and vitamin B12 levels and inflammatory bowel disease: A meta-analysis. Nutrients 2017, 9, 382. [CrossRef] [PubMed]

62. Frolkis, A.D.; Dykeman, J.; Negrón, M.E.; Debruyn, J.; Jette, N.; Fiest, K.M.; Frolkis, T.; Barkema, H.W.; Rioux, K.P.; Panaccione, R.; et al. Risk of surgery for inflammatory bowel diseases has decreased over time: A systematic review and meta-analysis of population-based studies. Gastroenterology 2013, 145, 996-1006. [CrossRef] [PubMed]

63. Jeffery, L.E.; Burke, F.; Mura, M.; Zheng, Y.; Qureshi, O.S.; Walker, L.S.K.; Lammas, D.A.; Raza, K.; Sansom, D.M. 1,25-dihydroxyvitamin D3 and interleukin-2 combine to inhibit T cell production of inflammatory cytokines and promote development of regulatory T cells expressing CTLA-4 and FoxP3. J. Immunol. 2009, 183, 5458-5467. [CrossRef] [PubMed]

64. Bendix, M.; Greisen, S.; Dige, A.; Hvas, C.L.; Bak, N.; Jørgensen, S.P.; Dahlerup, J.F.; Deleuran, B.; Agnholt, J. Vitamin D increases programmed death receptor-1 expression in Crohn's disease. Oncotarget 2017, 8, 24177-24186. [CrossRef] [PubMed]

65. Cantorna, M.T.; Munsick, C.; Bemiss, C.; Mahon, B.D. 1,25-dihydroxycholecalciferol prevents and ameliorates symptoms of experimental murine inflammatory bowel disease. J. Nutr. 2018, 130, 2648-2652. [CrossRef] [PubMed]

66. Lagishetty, V.; Misharin, A.V.; Liu, N.Q.; Lisse, T.S.; Chun, R.F.; Ouyang, Y.; McLachlan, S.M.; Adams, J.S.; Hewison, M. Vitamin D deficiency in mice impairs colonic antibacterial activity and predisposes to colitis. Endocrinology 2010, 151, 2423-2432. [CrossRef] [PubMed]

67. Jones, G.; Strugnell, S.A.; Deluca, H.F. Current understanding of the molecular actions of vitamin D. Physiol. Rev. 1998, 78, 1193-1231. [CrossRef]

68. Sadeghian, M.; Saneei, P.; Siassi, F.; Esmaillzadeh, A. Vitamin D status in relation to Crohn's disease: Meta-analysis of observational studies. Nutrition 2016, 32, 505-514. [CrossRef]

69. Fabisiak, N.; Fabisiak, A.; Watala, C.; Fichna, J. Fat-soluble vitamin deficiencies and inflammatory bowel disease: Systematic review and meta-analysis. J. Clin. Gastroenterol. 2017, 51, 878-889. [CrossRef]

70. Alrefai, D.; Jones, J.; El-Matary, W.; Whiting, S.J.; Aljebreen, A.; Mirhosseini, N.; Vatanparast, H. The association of vitamin d status with disease activity in a cohort of Crohn's disease patients in Canada. Nutrients 2017, 9, 1112. [CrossRef]

71. Blanck, S.; Aberra, F. Vitamin D deficiency is associated with ulcerative colitis disease activity. Dig. Dis. Sci. 2013, 58, 1698-1702. [CrossRef] [PubMed]

72. De Castro, F.D.; Magalhães, J.; Carvalho, P.B.; Moreira, M.J.; Mota, P.; Cotter, J. Lower levels of vitamin D correlate with clinical disease activity and quality of life in inflammatory bowel disease. Arq. Gastroenterol. 2016, 52, 260-265. [CrossRef] [PubMed]

73. Dolatshahi, S.; Pishgar, E.; Jamali, R. Does serum 25 hydroxy vitamin D level predict disease activity in ulcerative colitis patients? Acta Clin. Belg. Int. J. Clin. Lab. Med. 2016, 71, 46-50. [CrossRef] [PubMed]

74. Torki, M.; Gholamrezaei, A.; Mirbagher, L.; Danesh, M.; Kheiri, S.; Emami, M.H. Vitamin D deficiency associated with disease activity in patients with inflammatory bowel diseases. Dig. Dis. Sci. 2015, 60, 3085-3091. [CrossRef] [PubMed]

75. Ulitsky, A.; Ananthakrishnan, A.N.; Naik, A.; Skaros, S.; Zadvornova, Y.; Binion, D.G.; Issa, M. Vitamin D deficiency in patients with inflammatory bowel disease: Association with disease activity and quality of life. J. Parenter. Enter. Nutr. 2011, 35, 308-316. [CrossRef] [PubMed]

76. Abraham, B.P.; Prasad, P.; Malaty, H.M. Vitamin D deficiency and corticosteroid use are risk factors for low bone mineral density in inflammatory bowel disease patients. Dig. Dis. Sci. 2014, 59, 1878-1884. [CrossRef] 
77. Garg, M.; Rosella, O.; Lubel, J.S.; Gibson, P.R. Association of circulating vitamin D concentrations with intestinal but not systemic inflammation in inflammatory bowel disease. Inflamm. Bowel Dis. 2013, 19, 2634-2643. [CrossRef] [PubMed]

78. Garg, M.; Hendy, P.; Ding, J.; Shaw, S.; Hold, G.; Hart, H. The effect of vitamin D on intestinal inflammation and faecal microbiota in patients with ulcerative colitis. J. Crohn's Colitis 2018, 12, 963-972. [CrossRef] [PubMed]

79. Gubatan, J.; Mitsuhashi, S.; Longhi, M.S.; Zenlea, T.; Rosenberg, L.; Robson, S.; Moss, A.C. Higher serum vitamin $\mathrm{D}$ levels are associated with protective serum cytokine profiles in patients with ulcerative colitis. Cytokine 2018, 103, 38-45. [CrossRef] [PubMed]

80. Winter, R.W.; Collins, E.; Cao, B.; Carrellas, M.; Crowell, A.M.; Korzenik, J.R. Higher 25-hydroxyvitamin D levels are associated with a greater odds of remission with anti-tumor necrosis factor-a medications among patients with inflammatory bowel disease. Aliment. Pharmacol. Ther. 2018, 45, 653-659. [CrossRef]

81. Ananthakrishnan, A.N.; CHeng, S.-C.; Cai, T.; Cagan, A.; Gainer, V.S.; Szolovits, P.; Shaw, S.Y.; Churchill, S.; Karlson, E.W.; Murphy, S.N.; et al. Association between reduced plasma 25-hydroxy vitamin D and increased risk of cancer in patients with inflammatory bowel diseases. Clin. Gastroenterol. Hepatol. 2014, 13, 821-827. [CrossRef]

82. Gubatan, J.; Mitsuhasi, S.; Zenlea, T.; Rosenberg, L.; Robson, S.; Moss, A.C. Low serum vitamin D during remission increases risk of clinical relapse in patients with ulcerative colitis. Clin. Gastroenterol. Hepatol. 2017, 15, 240-246. [CrossRef] [PubMed]

83. Meckel, K.; Li, Y.C.; Lim, J.; Kocherginsky, M.; Weber, C.; Almoghrabi, A.; Chen, X.; Kaboff, A.; Sadiq, F.; Hanauer, S.B.; et al. Serum 25-hydroxyvitamin D concentration is inversely associated with mucosal inflammation in patients with ulcerative colitis. Am. J. Clin. Nutr. 2016, 104, 113-120. [CrossRef] [PubMed]

84. Kabbani, T.A.; Koutroubakis, I.E.; Schoen, R.E.; Ramos-Rivers, C.; Shah, N.; Swoger, J.; Regueiro, M.; Barrie, A.; Schwartz, M.; Hashash, J.G.; et al. Association of vitamin D level with clinical status in inflammatory bowel disease: A 5-year longitudinal study. Am. J. Gastroenterol. 2016, 111, 712-719. [CrossRef]

85. Ananthakrishnan, A.N.; Cagan, A.; Gainer, V.S.; Cai, T.; Cheng, S.C.; Savova, G.; Chen, P.; Szolovits, P.; Xia, Z.; De Jager, P.L.; et al. Normalization of plasma 25-hydroxy vitamin D is associated with reduced risk of surgery in Crohn's disease. Inflamm. Bowel Dis. 2013, 19, 1921-1927. [CrossRef]

86. Bakker, S.F.; Dik, V.K.; Witte, B.I.; Lips, P.; Roos, J.C.; Van Bodegraven, A.A. Increase in bone mineral density in strictly treated Crohn's disease patients with concomitant calcium and vitamin D supplementation. J. Crohn's Colitis 2013, 7, 377-384. [CrossRef] [PubMed]

87. Casals-Seoane, F.; Chaparro, M.; Maté, J.; Gisbert, J.P. Clinical course of bone metabolism disorders in patients with inflammatory bowel disease: A 5-year prospective study. Inflamm. Bowel Dis. 2016, 22, 1929-1936. [CrossRef] [PubMed]

88. Mathur, J.; Naing, S.; Mills, P.; Limsui, D. A randomized clinical trial of vitamin D3 (Cholecalciferol) in ulcerative colitis patients with hypovitaminosis D3. Peer] 2017, 5, e3654. [CrossRef] [PubMed]

89. Narula, N.; Cooray, M.; Anglin, R.; Muqtadir, Z.; Narula, A.; Marshall, J.K. Impact of high-dose vitamin D3 supplementation in patients with Crohn's disease in remission: A pilot randomized double-blind controlled study. Dig. Dis. Sci. 2017, 62, 448-455. [CrossRef] [PubMed]

90. Ghishan, F.; Kiela, P. Advances in the understanding of mineral and bone metabolism in inflammatory bowel diseases. Am. J. Physiol. Gastrointest. Liver Physiol. 2010, 300, G191-G201. [CrossRef]

91. Krela-Kazmierczak, I.; Szymczak, A.; Tomczak, M.; Lykowska-Szuber, L.; Linke, K.; Eder, P. Calcium and phosphate metabolism in patients with inflammatory bowel diseases. Pol. Arch. Med. Wewn. 2015, 125, 588-590. [CrossRef] [PubMed]

92. Huybers, S.; Apostolaki, M.; van der Eerden, B.C.; Kollias, G.; Naber, T.H.; Bindels, R.J.; Hoenderop, J.G. Murine $\mathrm{TNF}^{\triangle \mathrm{ARE}}$ Crohn's disease model displays diminished expression of intestinal $\mathrm{Ca}^{2+}$ transporters. Inflamm. Bowel Dis. 2008, 14, 803-811. [CrossRef] [PubMed]

93. Thurston, R.D.; Larmonier, C.B.; Majewski, P.M.; Ramalingam, R.; Mi-dura-Kiela, M.; Laubitz, D.; Vandewalle, A.; Besselsen, D.G.; Muhlbauer, M.; Jobin, C.; et al. Tumor necrosis factor and interferon-gamma down-regulate Klotho in mice with colitis. Gastroenterology 2010, 138, 1384-1394. [CrossRef] [PubMed]

94. El-Matary, W.; Sikora, S.; Spady, D. Bone mineral density, vitamin D, and disease activity in children newly diagnosed with inflammatory bowel disease. Dig. Dis. Sci. 2011, 56, 825-829. [CrossRef] [PubMed] 
95. Leslie, W.D.; Miller, N.; Rogala, L.; Bernstein, C.N. Vitamin D status and bone density in recently diagnosed inflammatory bowel disease: The manitoba IBD cohort study. Am. J. Gastroenterol. 2008, 103, 1451-1459. [CrossRef] [PubMed]

96. Benchimol, E.I.; Ward, L.M.; Gallagher, J.C.; Rauch, F.; Barrowman, N.; Warren, J.; Beedle, S.; Mack, D.R. Effect of calcium and vitamin D supplementation on bone mineral density in children with inflammatory bowel disease. J. Pediatr. Gastroenterol. Nutr. 2007, 45, 538-545. [CrossRef] [PubMed]

97. Bernstein, C.N.; Sargent, M.; Leslie, W.D. Serum osteoprotegerin is in-creased in Crohn's disease: A population-based case control study. Inflamm. Bowel Dis. 2005, 11, 325-330. [CrossRef] [PubMed]

98. Abreu, M.T.; Kantorovich, V.; Vasiliauskas, E.A.; Gruntmanis, U.; Matuk, R.; Daigle, K.; Chen, S.; Zehnder, D.; Lin, Y.C.; Yang, H.; et al. Measurement of vitamin D levels in inflammatory bowel disease patients reveals a subset of Crohn's disease patients with elevated 1,25-dihydroxyvitamin D and low bone mineral density. Gut 2004, 53, 1129-1136. [CrossRef]

99. Grober, U.; Reichrath, J.; Holick, M.; Kisters, K. Vitamin K: An old vitamin in a new. Dermato-Endocrinology 2014, 6, e968490. [CrossRef]

100. Shea, M.K.; Dallal, G.E.; Dawson-Hughes, B.; Ordovas, J.M.; O’Donnell, C.J.; Gundberg, C.M.; Peterson, J.W.; Booth, S.L. Vitamin K, circulating cytokines, and bone mineral density in older men and women. Am. J. Clin. Nutr. 2008, 46, 220-231. [CrossRef]

101. Yen, D.; Cheung, J.; Scheerens, H.; Poulet, F.; McClanahan, T.; Mckenzie, B.; Kleinschek, M.A.; Owyang, A.; Mattson, J.; Blumenschein, W.; et al. IL-23 is essential for T cell-mediated colitis and promotes inflammation via Il-17 and IL-6. J. Clin. Investig. 2006, 116, 1310-1316. [CrossRef] [PubMed]

102. Ohsaki, Y.; Shirakawa, H.; Hiwatashi, K.; Furukawa, Y.; Mizutani, T.; Komai, M. Vitamin K suppresses lipopolysaccharide-induced inflammation in the rat. Biosci. Biotechnol. Biochem. 2006, 70, 926-932. [CrossRef]

103. Shiraishi, E.; Iijima, H.; Shinzaki, S.; Nakajima, S.; Inoue, T.; Hiyama, S.; Kawai, S.; Araki, M.; Yamaguchi, T.; Hayashi, Y.; et al. Vitamin K deficiency leads to exacerbation of murine dextran sulfate sodium-induced colitis. J. Gastroenterol. 2016, 51, 346-356. [CrossRef] [PubMed]

104. Kuwabara, A.; Tanaka, K.; Tsugawa, N.; Nakase, H.; Tsuji, H.; Shide, K.; Kamao, M.; Chiba, T.; Inagaki, N.; Okano, T.; et al. High prevalence of vitamin K and D deficiency and decreased BMD in inflammatory bowel disease. Osteoporos. Int. 2009, 20, 935-942. [CrossRef] [PubMed]

105. Nakajima, S.; Iijima, H.; Egawa, S.; Shinzaki, S.; Kondo, J.; Inoue, T.; Hayashi, Y.; Ying, J.; Mukai, A.; Akasaka, T.; et al. Association of vitamin K deficiency with bone metabolism and clinical disease activity in inflammatory bowel disease. Nutrition 2011, 27, 1023-1028. [CrossRef] [PubMed]

106. Schoon, E.J.; Müller, M.C.A.; Vermeer, C.; Schurgers, L.J.; Brummer, R.-J.M.; Stockbrügger, R.W. Low serum and bone vitamin K status in patients with longstanding Crohn's disease: Another pathogenetic factor of osteoporosis in Crohn's disease? Gut 2001, 48, 473-477. [CrossRef] [PubMed]

107. Ali, T.; Lam, D.; Bronze, M.S.; Humphrey, M.B. Osteoporosis in inflammatory bowel disease. Am. J. Med. 2009, 122, 599-604. [CrossRef] [PubMed]

108. O'Connor, E.M.; Grealy, G.; McCarthy, J.; Desmond, A.; Craig, O.; Shanahan, F.; Cashman, K.D. Effect of phylloquinone (vitamin K1) supplementation for 12 months on the indices of vitamin K status and bone health in adult patients with Crohn's disease. Br. J. Nutr. 2014, 112, 1163-1174. [CrossRef]

109. Shishavan, N.G.; Gargari, B.P.; Jafarabadi, M.A.; Kolahi, S.; Haggifar, S.; Noroozi, S. Vitamin K1 supplementation did not alter inflammatory markers and clinical status in patients with rheumatoid arthritis. Int. J. Vitam. Nutr. Res. 2019. [CrossRef]

110. Niepel, D.; Klag, T.; Malek, N.P.; Wehkamp, J. Practical guidance for the management of iron deficiency in patients with inflammatory bowel disease. Ther. Adv. Gastroenterol. 2018, 11. [CrossRef]

111. Gisbert, J.; Gomollon, F. Common misconceptions in the diagnosis and management of anemia in inflammatory bowel disease. Am. J. Gastroenterol. 2008, 103, 1299-1307. [CrossRef]

112. Cavallaro, F.; Duca, L.; Pisani, L.F.; Rigolini, R.; Spina, L.; Tontini, G.E.; Munizio, N.; Costa, E.; Cappellini, M.D.; Vecchi, M.; et al. Anti-TNF-mediated modulation of prohepcidin improves iron availability in inflammatory bowel disease, in an IL-6-mediated fashion. Can. J. Gastroenterol. Hepatol. 2017, 2017, 6843976. [CrossRef] [PubMed]

113. Sobrado, C.W.; Cançado, R.D.; Sobrado, L.F.; Frugis, M.O.; Sobrado, M.F. Treatment of anemia and improvement of quality of life among patients with Crohn's disease: Experience using ferric carboxymaltose. Arq. Gastroenterol. 2016, 52, 255-259. [CrossRef] [PubMed] 
114. Dignass, A.U.; Gasche, C.; Bettenworth, D.; Birgegård, G.; Danese, S.; Gisbert, J.P.; Gomollon, F.; Iqbal, T.; Katsanos, K.; Koutroubakis, I.; et al. European consensus on the diagnosis and management of iron deficiency and anaemia in inflammatory bowel diseases. J. Crohn's Colitis 2015, 9, 211-222. [CrossRef] [PubMed]

115. Bonovas, S.; Fiorino, G.; Allocca, M.; Lytras, T.; Tsantes, A.; Peyrin-Biroulet, L.; Danese, S. Intravenous versus oral iron for the treatment of anemia in inflammatory bowel disease. Medicine (Baltimore) 2016, 95, e2308. [CrossRef]

116. Lee, T.W.; Kolber, M.R.; Fedorak, R.N.; Van Zanten, S.V. Iron replacement therapy in inflammatory bowel disease patients with iron deficiency anemia: A systematic review and meta-analysis. J. Crohn's Colitis 2012, 6, 267-275. [CrossRef]

117. Kulnigg, S.; Teischinger, L.; Dejaco, C.; Waldhör, T.; Gasche, C. Rapid recurrence of IBD-associated anemia and iron deficiency after intravenous iron sucrose and erythropoietin treatment. Am. J. Gastroenterol. 2009, 104, 1460-1467. [CrossRef]

118. Shankar, A.H.; Prasad, A.S. Zinc and immune function: The biological basis of altered resistance to infection. Am. J. Clin. Nutr. 1998, 68, 447-463. [CrossRef]

119. Gammoh, N.Z.; Rink, L. Zinc in infection and inflammation. Nutrients 2017, 9, 624. [CrossRef] [PubMed]

120. Jarosz, M.; Olbert, M.; Wyszogrodzka, G.; Młyniec, K.; Librowski, T. Antioxidant and anti-inflammatory effects of zinc. Zinc-dependent NF-KB signaling. Inflammopharmacology 2017, 25, 11-24. [CrossRef]

121. Ma, A.; Malynn, B.A. A20: Linking a complex regulator of ubiquitylation to immunity and human disease. Nat. Rev. Immunol. 2013, 12, 774-785. [CrossRef] [PubMed]

122. Wong, C.P.; Rinaldi, N.A.; Ho, E. Zinc deficiency enhanced inflammatory response by increasing immune cell activation and inducing IL6 promoter demethylation. Mol. Nutr. Food Res. 2015, 59, 991-999. [CrossRef]

123. Kloubert, V.; Rink, L. Zinc as a micronutrient and its preventive role of oxidative damage in cells. Food Funct. 2015, 6, 3195-3204. [CrossRef] [PubMed]

124. Mohammadi, E.; Qujeq, D.; Taheri, H.; Hajian-Tilaki, K. Evaluation of serum trace element levels and superoxide dismutase activity in patients with inflammatory bowel disease: Translating basic research into clinical application. Biol. Trace Elem. Res. 2017, 177, 235-240. [CrossRef] [PubMed]

125. Filippi, J.; Al-Jaouni, R.; Wiroth, J.B.; Hébuterne, X.; Schneider, S.M. Nutritional deficiencies in patients with Crohn's disease in remission. Inflamm. Bowel Dis. 2006, 12, 185-191. [CrossRef]

126. Lee, H.H.; Prasad, A.S.; Brewer, G.J.; Owyang, C. Zinc absorption in human small intestine. Am. J. Physiol. Liver Physiol. 1989, 256, G87-G91. [CrossRef] [PubMed]

127. Ananthakrishnan, A.N.; Khalili, H.; Song, M.; Higuchi, L.M.; Richter, J.M.; Chan, A.T. Zinc intake and risk of Crohn's disease and ulcerative colitis: A prospective cohort study. Int. J. Epidemiol. 2015, 44, 1995-2005. [CrossRef]

128. Siva, S.; Rubin, D.T.; Gulotta, G.; Wroblewski, K.; Pekow, J. Zinc deficiency is associated with poor clinical outcomes in patients with inflammatory bowel disease. Inflamm. Bowel Dis. 2017, 23, 152-157. [CrossRef]

129. Mulder, T.P.J.; Veer, A.V.D.S.; Verspaget, H.W.; Griffioen, G.; Peña, A.S.; Janssens, A.R.; Lamers, C.B.H.W. Effect of oral zinc supplementation on metallothionein and superoxide dismutase concentrations in patients with inflammatory bowel disease. J. Gastroenterol. Hepatol. 1994, 9, 472-477. [CrossRef]

130. Kudva, A.K.; Shay, A.E.; Prabhu, K.S. Selenium and inflammatory bowel disease. Am. J. Physiol. Gastrointest. Liver Physiol. 2015, 309, 71-77. [CrossRef]

131. Barrett, C.W.; Ning, W.; Chen, X.; Smith, J.J.; Washington, M.K.; Hill, K.E.; Coburn, L.A.; Peek, R.M.; Chaturvedi, R.; Wilson, K.T.; et al. Tumor suppressor function of the plasma glutathione peroxidase gpx3 in colitis-associated carcinoma. Cancer Res. 2013, 73, 1245-1255. [CrossRef] [PubMed]

132. Esworthy, R.S.; Arand, R.; Martin, M.G.; Doroshow, J.H.; Binder, S.W.; Chu, F.F. Mice with combined disruption of Gpx1 and Gpx2 genes have colitis. Am. J. Physiol. Gastrointest. Liver Physiol. 2001, 281, 848-855. [CrossRef]

133. Esworthy, R.S.; Kim, B.; Larson, G.P.; Yip, M.L.R.; Smith, D.D.; Li, M.; Chu, F. A colitis locus on chromosome 2 impacting the severity of early-onset disease in mice deficient in GPX1 and GPX2. Inflamm. Bowel Dis. 2011, 17, 1373-1386. [CrossRef] [PubMed]

134. Han, Y.M.; Yoon, H.; Lim, S.; Sung, M.K.; Shin, C.M.; Park, Y.S.; Kim, N.; Lee, D.H.; Kim, J.S. Risk factors for vitamin D, zinc, and selenium deficiencies in Korean patients with inflammatory bowel disease. Gut Liver 2017, 11, 363-369. [CrossRef] [PubMed] 
135. Rannem, T.; Ladefoged, K.; Hylander, E.; Hegnhoj, J.; Jarnum, S. Selenium status in patients with Crohn's disease. Am. J. Clin. Nutr. 1992, 56, 933-937. [CrossRef] [PubMed]

136. Hinks, L.J.; Inwards, K.D.; Lloyd, B.; Clayton, B. Reduced concentrations of selenium in mild Crohn's disease. J. Clin. Pathol. 1988, 41, 198-201. [CrossRef] [PubMed]

137. Gentschew, L.; Bishop, K.S.; Han, D.Y.; Morgan, A.R.; Fraser, A.G.; Lam, W.J.; Karunasinghe, N.; Campbell, B.; Ferguson, L.R. Selenium, selenoprotein genes and Crohn's disease in a case-control population from Auckland, New Zealand. Nutrients 2012, 4, 1247-1259. [CrossRef] [PubMed]

138. Geerlin, B.J.; Badart-Smook, A.; Stockbrugger, R.W.; Brummer, R.-J.M. Comprehensive nutritional status in recently diagnosed patients with inflammatory bowel disease compared with population controls. Eur. J. Clin. Nutr. 2000, 54, 514-521. [CrossRef]

139. Aguilar-Tablada, T.C.; Navarro-Alarcon, M.; Granados, J.Q.; Sanchez, C.S.; Rufian-Henares, J.A.; Nogueras-Lopez, F. Ulcerative colitis and Crohn's disease are associated with decreased serum selenium concentrations and increased cardiovascular risk. Nutrients 2016, 8, 780. [CrossRef]

140. Barrett, C.W.; Singh, K.; Motley, A.K.; Lintel, M.K.; Matafonova, E.; Bradley, A.M.; Ning, W.; Poindexter, S.V.; Parang, B.; Reddy, V.K.; et al. Dietary selenium deficiency exacerbates DSS-induced epithelial injury and AOM/DSS-induced tumorigenesis. PLoS ONE 2013, 8, e67845. [CrossRef]

141. Sun, Z.; Xu, Z.; Wang, D.; Yao, H.; Li, S. Selenium deficiency inhibits differentiation and immune function and imbalances the TH1/TH2 of dendritic cells. Metabolomics 2018, 10, 759-767. [CrossRef] [PubMed]

142. Benstoem, C.; Goetzenich, A.; Kraemer, S.; Borosch, S.; Manzanares, W.; Hardy, G.; Stoppe, C. Selenium and its supplementation in cardiovascular disease-What do we know? Nutrients 2015, 7, 3094-3118. [CrossRef] [PubMed]

143. Kaur, R.; Thakur, S.; Rastogi, R.; Kaushal, N. Resolution of cox mediated inflammation by Se supplementation in mouse experimental model of colitis. PLoS ONE 2018, 13, e0201356. [CrossRef] [PubMed]

144. Gandhi, U.H.; Kaushal, N.; Ravindra, K.C.; Hegde, S.; Nelson, S.M.; Narayan, V.; Vunta, H.; Prabhu, K.S Selenoprotein-dependent up-regulation of hematopoietic prostaglandin D2 synthase in macrophages is mediated through the activation of peroxisome proliferator-activated receptor (PPAR) gamma. J. Biol. Chem. 2011, 286, 27471-27482. [CrossRef] [PubMed]

145. Forbes, A.; Escher, J.; Hébuterne, X.; Kłęk, S.; Krznaric, Z.; Schneider, S.; Shamir, R.; Stardelova, K.; Wierdsma, N.; Wiskin, A.E.; et al. ESPEN guideline: Clinical nutrition in inflammatory bowel disease. Clin. Nutr. 2017, 36, 321-347. [CrossRef] [PubMed]

146. Brignola, C.; Belloli, C.; De Simone, C.; Evangelisti, A.; Parente, R.; Mancini, R.; Iannone, P.; Mocheggiani, E.; Fabris, N.; Morini, M.C.; et al. Zinc supplementation restores plasma concentrations of zinc and thymulin in patients with Crohn's disease. Aliment. Pharmacol. Ther. 1993, 7, 275-280. [CrossRef] [PubMed]

147. Wegmüller, R.; Tay, F.; Zeder, C.; Brnic, M.; Hurrell, R.F. Zinc absorption by young adults from supplemental zinc citrate is comparable with that from zinc gluconate and higher than from zinc oxide. J. Nutr. 2014, 144, 132-136. [CrossRef]

148. Jeejeebhoy, K.N. Short bowel syndrome: A nutritional and medical approach. CMAJ 2002, 166, 1297-1302. [CrossRef]

149. Eiden, K.A. Nutritional considerations in inflammatory bowel disease. Pract. Gastroenterol. 2003, 5, 33-54.

(C) 2019 by the authors. Licensee MDPI, Basel, Switzerland. This article is an open access article distributed under the terms and conditions of the Creative Commons Attribution (CC BY) license (http://creativecommons.org/licenses/by/4.0/). 
Review

\title{
Dietary Composition and Effects in Inflammatory Bowel Disease
}

\author{
Fernando Castro ${ }^{1}$ and Heitor S. P. de Souza ${ }^{1,2, *}$ \\ 1 Department of Internal Medicine, School of Medicine, Federal University of Rio de Janeiro, \\ 21941-913 Rio de Janeiro, Brazil; fernandommcastro@gmail.com \\ 2 Department of Internal Medicine, D'Or Institute for Research and Education (IDOR), \\ 22281-100 Rio de Janeiro, Brazil \\ * Correspondence: heitor.souza@gmail.com or hsouza@hucff.ufrj.br; Tel.: +55-21-3938-2669
}

Received: 26 May 2019; Accepted: 19 June 2019; Published: 21 June 2019

\begin{abstract}
Dramatic changes in the environment and human lifestyle have been associated with the rise of various chronic complex diseases, such as inflammatory bowel disease (IBD). A dysbiotic gut microbiota has been proposed as a crucial pathogenic element, contributing to immune imbalances and fostering a proinflammatory milieu, which may be associated with disease relapses or even the initiation of IBD. In addition to representing important regulators of the mucosal immunity and the composition of the gut microbiota, food components have been shown to be potential environmental triggers of epigenetic modifications. In the context of chronic intestinal inflammation, dietary habits and specific food components have been implicated as important modulators of epigenetic mechanisms, including DNA methylation, which may predispose a person to the increased risk of the initiation and evolution of IBD. This review provides novel insights about how dietary factors may interact with the intestinal mucosa and modulate immune homeostasis by shaping the intestinal ecosystem, as well as the potential influence of diet in the etiopathogenesis and management of IBD.
\end{abstract}

Keywords: inflammatory bowel disease; dietary habits; food components; gut microbiota; immune homeostasis; epigenetic changes

\section{Introduction}

Inflammatory bowel disease (IBD), including Crohn's disease (CD) and ulcerative colitis (UC), is regarded as a heterogeneous disease of unknown etiology, with an unpredictable clinical course and usually labeled as a chronic disabling disorder [1,2]. Although the mechanisms leading to the development of IBD are rather complex and have not yet been completely elucidated, the current information points to a multifactorial origin. The chronic inflammatory process underlying the pathogenesis of IBD reflects a dysregulated immune response, stemming from a conflicting interaction involving several environmental factors, an altered innate immunity, and a predisposing genetic background [3-6].

Information derived from epidemiological studies has revealed consistent associations between the increase in the occurrence in IBD and other autoimmune and chronic inflammatory disorders, and the decline in infectious diseases, accompanied by the use of antibiotics, immunizations, and overall improvements in sanitary conditions and the quality of water [7]. IBD has progressively become a global disease, affecting newly industrialized nations in Asia, South America, and the Middle East [8-10]. Although the prevalence of IBD in newly industrialized countries is still lower than in developed countries, the incidence rate is increasing rapidly [11]. Among all the environmental changes usually associated with IBD, dietary habits are one element, both from the patients' perspective, including personal observations and common practical concerns, and due to scientific evidence implicating diet as a triggering factor in disease relapses [12] In this article, we discuss the effects of diet on IBD, especially considering the basic physiological processes that underlie these associations. 


\section{Diet and Microbiota}

Although the influence of dietary habits on the intestinal microbiota has long been speculated, only after the introduction of new technology, including next-generation DNA sequencing and metabolic profiling, was reliable information able to be obtained [13]. For example, the microbiota composition is currently thought of as a dynamic process that changes with age and fluctuates in consonance with environmental exposures, including diet, among other factors [14].

The results of studies analyzing the microbial communities of the gut obtained from mammalian species have endorsed the assumption that diet can influence microbial diversity. Such diversity has been shown to increase from carnivores to omnivores and then to herbivores [15]. Regarding humans, the intestinal microbiota reflects predominant omnivorous habits, but substantial inconsistencies have been reported. For example, opposing patterns of the intestinal microbiota composition were reported when comparing samples from African children living in rural communities with children from a European urban center, a phenomenon that has been attributed to dietary differences [16]. In this case, in terms of health issues, individuals from the European urban center are expected to be at increased risk for the development of several immune-mediated disorders. Such diseases, likely including IBD, are probably associated with the microbiome structure, often resulting from dietary incompatibilities between the current environments and those in which humans evolved.

The Western style of diet, defined by high caloric content due to large amounts of fat and carbohydrates, has been associated with a markedly reduced microbiome diversity [17]. In addition to the plethora of fat, Western diets usually have high concentrations of dietary omega- 6 fatty acids, from vegetable oils, resulting in a high omega- 6 to omega- 3 ratio. Whereas omega- 3 fatty acids, such as a-linolenic acid from vegetables and eicosapentaenoic acid and docosahexaenoic acid from fish, have anti-inflammatory properties, omega- 6 fatty acids, particularly arachidonic acid, are considered pro-inflammatory. This may explain why the Western diet has been regarded as a key factor for producing intestinal inflammation [18]. Other relevant studies focusing on diet potentially affecting the intestinal microbiota are summarized in Table 1.

Previous studies have analyzed the gut microbiota of infants and children within one population, but few have attempted to compare the microbial composition considering distinct socio-economic, geographic, and cultural aspects [16]. To analyze the potential effect of environmental factors on defining how and from where a gut microbiota may be acquired, investigators characterized bacterial species and gene contents of fecal samples from a cohort of healthy Amerindians from Venezuela, rural Malawian communities, and metropolitan USA inhabitants. The investigators found that bacterial diversity increases with age, and the degree of similarity among members of a family is consistent in all three populations. The fecal microbiota of USA adults was the least diverse compared to the others [19]. Therefore, it appears that differences in social structures and cultural traditions known to impact food and many other environmental factors may also condition the magnitude of the circulation of microbes among members of a family or community. 
Table 1. Relationship between gut microbiota alterations and dietary patterns.

\begin{tabular}{|c|c|c|c|}
\hline Genus & Characteristics & Dietary Component & Gastrointestinal (GI) Effect \\
\hline $\begin{array}{l}\text { Prevotella } \\
\text { Bacteroides }\end{array}$ & Gram-negative anaerobic & Diets rich in fat and carbohydrates & Involved in colitis $[17,20-23]$ \\
\hline Clostridium & Gram-positive anaerobic & Decrease in high-fat but not high-sugar diets & $\begin{array}{l}\text { Affects the clinical course of inflammatory } \\
\text { bowel disease (IBD) [21-24] }\end{array}$ \\
\hline Ruminococcus & Gram-positive anaerobic & $\begin{array}{l}\text { Fermentation of dietary fiber and decrease in } \\
\text { high-fat but not high-sugar diets }\end{array}$ & {$[21,25,26]$} \\
\hline $\begin{array}{l}\text { Streptococcus } \\
\text { Lactobacillus }\end{array}$ & Gram-positive & Constituents of the normal animal microbiota & $\begin{array}{l}\text { Induces remission in ulcerative colitis (UC) } \\
\text { patients [21] }\end{array}$ \\
\hline Escherichia & Gram-negative anaerobic & High-fat and high-sugar diets & $\begin{array}{l}\text { Overgrowth of Escherichia coli and involved in } \\
\text { colitis }[16,22,27]\end{array}$ \\
\hline Bifidobacterium & Gram-positive anaerobic & High-fiber diet & Induces remission in UC patients $[28,29]$ \\
\hline Fusobacterium & Gram-negative anaerobic & - & Involved in colitis and colon cancer [30] \\
\hline- & - & Alcohol & $\begin{array}{c}\text { Decreased butyrate/total short chain fatty acids } \\
\text { (SCFA) ratio in stool [31] }\end{array}$ \\
\hline
\end{tabular}

Source: adapted from Levine et al. [5] and Rapozo et al. [21].

\section{Diet and Immune Response}

Western lifestyle factors have been associated with the progressive increase in various metabolic, autoimmune, and chronic inflammatory disorders, including IBD [32-34]. In addition to industrialization, urbanization, the improvement of sanitary conditions, antibiotic usage, and other factors, abundant evidence supports the involvement of dietary habits in shaping the gut microbiota and influencing the interaction with the immune system [35-37].

Dietary fiber has been shown to interfere in host immunity via multiple pathways. Fibers and starches present in fruits and vegetables represent a substrate for the production of butyrate and other short chain fatty acids (SCFAs) by intestinal microbiota. Butyrate, for example, is well-recognized as a critical element in epithelial homeostasis, but also plays an important role in downregulating the immune response by inhibiting the transcription of inflammatory cytokines and promoting the differentiation of lamina propria Tregs $[5,38,39]$. Low dietary fiber may precipitate the catabolism of the mucous layer, leading to increased permeability and compromising the epithelial barrier against potential pathogenic luminal bacteria [40].

Investigations based on experimental models have provided results regarding the effects of specific diets on the immune response. For instance, a high-fat (HF) and high-sugar diet generated a proinflammatory intestinal environment, associated with an overgrowth of Proteobacteria, reduction in butyrate production, and expression of the butyrate GPR43 receptor, also underexpressed in CD patients [41]. Results from another experimental model showed that HF diets led to the upregulation of tumor necrosis factor- $\alpha$ (TNF- $\alpha$ ) and interferon- $\gamma$ expression, and to the decrease in the densities of colonic Tregs [42]. A high intake of fat has been shown to have several effects on the mucosal immune system, revealing the complex interaction between the lamina propria immune cells and the intestinal microbiota. For example, the ingestion of high-fat meals increases the expression of interlukein-1 $\beta$ (IL-1 $\beta)$, TNF- $\alpha$, IL-6, and necrosis factor- $\mathrm{KB}(\mathrm{NF}-\mathrm{\kappa B})$ in the colon $[43,44]$. Likewise, mice fed with cholesterol-enriched meals displayed acute inflammasome-dependent intestinal inflammation, increasing the production of IL- $1 \beta$, and driving the accumulation of CD11b+ and CD11c+ cells in the lamina propria [45].

The increasing knowledge about the influence of the gut microbiome on the mucosal and systemic immune response has contributed to the understanding of the mechanisms underlying the pathogenesis of IBD and other chronic inflammatory conditions. Dysbiosis has been confirmed in samples from patients with CD, displaying an increase in Enterobacteriales such as Escherichia coli, and a decrease in Clostridiales such as Faecalibacterium prausnitzii [46-48]. In a study involving UC, the results supported a role for reduced microbial diversity, although not as prominent as the dysbiosis seen in CD [49]. A large cohort study analyzing fecal samples from four different European countries revealed that $\mathrm{CD}$ and $\mathrm{UC}$ are distinct in terms of the microbiome [50]. Notably, some specific intestinal 
microbes can shape the immune response. For example, Listeria monocytogenes, Clostridium difficile, and Toxoplasma gondii have been shown to elicit innate lymphoid cells-1 (ILC1) and Th1 cells, which orchestrate the secretion of cytokines, such as interferon-gamma and TNF- $\alpha$, critically important in the immunity against those intestinal pathogens [51]. Clostridia strains have been shown to promote the accumulation of Foxp3 Treg cells in the gut [52], which in turn down-regulate inflammatory responses, including those in experimental colitis [53]. Some microorganisms induce the activation of subsets of immune cells responsible for promoting intestinal inflammation, such as Th17 cells [54]. For instance, segmented filamentous bacteria can activate Th17 cells, whereas a decrease in Th17 cell-inducing bacteria can attenuate the severity of colitis in mice [55]. In previous studies, adherent invasive E. coli (AIEC) and Bifidobacteria adolescentis [56,57], in addition to E. coli 0157 and Citrobacter rodentium [54], have been shown to induce populations of Th17 cells.

Dietary habits can affect human health either directly or via changes in the gut microbiota. The gut microbiota is a highly complex and dynamic system that can be shaped by environmental factors, including the diet [58]. Regarding the dietary factors directly or indirectly associated with the immune response and intestinal homeostasis, diets with high concentrations of fat and sugar have been associated with intestinal colonization with potential IBD-related pathobionts. For instance, adherent invasive E. coli (AIEC) strains, induced by high-fat high-sugar diets, may become pathogenic when the ligand CEACAM 6 is presented by the intestinal epithelium. Under such circumstances, AIEC may form biofilms, adhere to, and translocate via $\mathrm{M}$ cells and the associated epithelium [59]. These findings reinforce the idea that changes in the local environment exert a selective pressure on several species, which in turn shape the microbiota according to the most adaptable microorganisms to the intestinal milieu [60]. A selection of dietary factors that may affect the host immunity is listed in Table 2.

Table 2. Dietary patterns potentially affecting microbiota and immunity.

\begin{tabular}{|c|c|}
\hline Dietary Component & GI and Immunological Effect \\
\hline High-fat, high-sugar diet & $\begin{array}{ll}\text { - } & \text { Intestinal mucosa dysbiosis [41] } \\
\text { - } & \text { Higher degree of crypt abscesses } \\
\text { - } & \text { Decreased butyrate production } \\
\text { - } & \text { Overgrowth of E. coli [41] }\end{array}$ \\
\hline Milk fat (saturated fat) & $\begin{array}{ll} & \text { Induced pro-inflammatory Th1 immune response [61] } \\
\text { - } & \text { Increased incidence of colitis [61] } \\
\text { - } & \text { Dysbiosis }\end{array}$ \\
\hline High-fiber diet & $\begin{array}{ll}\text { - } & \text { Protected from acute colitis [62] } \\
\text { - } & \text { Did not increase SCFAs production in patients with UC [62] }\end{array}$ \\
\hline Animal versus plant diet & $\begin{array}{l}\text { Animal-based food increased the abundance of bile-tolerant microorganisms } \\
\text { and decreased the proportions of Firmicutes [27] }\end{array}$ \\
\hline Alcohol & - $\quad$ Decreased butyrate/total SCFA ratio in stool [31] \\
\hline Maltodextrin & $\begin{array}{ll}\text { - } & \text { Biofilm formation of adherent invasive E. coli }[63,64] \\
\text { - } & \text { Enhanced E. coli adhesion independent of cellular receptor CEACAM } 6[63,64]\end{array}$ \\
\hline
\end{tabular}

\section{Diet and IBD}

Advances resulting from clinical and experimental investigations have demonstrated that the microbiome might play a critical role in the pathogenesis of IBD $[65,66]$. In parallel, results from other studies have supported a link between dietary patterns and the structure of the intestinal microbiome in healthy individuals and in patients with gastrointestinal disorders, including IBD $[17,27,67]$. Given the potential effects that diet can have on the composition and function of the intestinal microbiome, diet may also be involved in the pathogenesis of IBD, either directly or indirectly. As a consequence, 
due to the associations with the microbiome and IBD, specific dietary interventions are promising novel therapies.

Data have confirmed that food plays a crucial role in the gut microenvironment, and modifications of dietary habits may have an important impact on the microbial composition and function, but also on the gut barrier and immunity [5]. Previous studies demonstrated that modifications in a single food group might produce various outcomes. For instance, changing the dietary composition from a predominant plant- to animal-based diet results in a change in the intestinal microbial environment and functionality, determining alterations in bile acids and sulfide metabolism, which have been implicated in IBD pathogenesis $[27,68]$. In an experimental study, investigators demonstrated that a low-fiber diet induces the expansion and activity of colonic mucous-degrading microorganisms, potentially favoring the development of severe colitis by enteric pathogens [40]. In another experimental study, mice fed a Western-style diet (rich in saturated fats and simple carbohydrates, but depleted in dietary fiber), developed an alteration in the colonic microbiota, resulting in increased permeability and a reduced inner mucus layer. Both defects could be prevented by transplanting microbiota from chow-fed mice, whereas the administration of Bifidobacterium longum was sufficient to restore the mucus layer [69]. These findings suggest that specific bacteria are crucial for normal mucus and barrier function and, in humans, this information could help us to understand important mechanisms underlying IBD pathogenesis.

Unveiling the association between diet and the development of IBD would ideally require knowledge on dietary details before the onset of the disease. In this regard, large longitudinal studies have been performed that have started to provide information to support specific dietary patterns as risk factors for IBD [17]. For instance, the metabolic activity of the microbiota may present important abnormalities in IBD. A reduced production of SCFAs, which are promoted by the presence of dietary fiber, has been shown in UC and CD. Particularly in patients with UC, a deficient production of SCFAs has been shown, and could not be corrected by increasing the intake of wheat bran-associated fiber and high amylose-associated-resistant starch $[5,70]$.

Among the dietary factors potentially implicated in the development of IBD, dietary fiber has received special attention due to evidence showing associated protective mechanisms, including the conversion to SCFAs [71,72], interference in the structure of the gut microbiota [16], and maintenance of the barrier function [73]. However, a large prospective cohort study evaluating the impact of the intake of dietary fiber from several sources failed to identify any protective effect against the development of IBD [74].

Regarding the presumably harmful Western diet, in addition to its general composition characterized by high animal or dairy fat and animal protein, it is important to draw attention to the ubiquitous presence of emulsifiers, thickeners, and artificial sweeteners, many of which are associated with abnormal intestinal permeability, dysbiosis, and intestinal inflammation in animal models $[75,76]$. As an integral part of this type of diet, the common use of processed foods, usually rich in phosphate [77], but poor in micronutrients such zinc and other nutrients including omega-3 fatty acids, and vitamins D and E [78-80], may also be involved in the development or the predisposition to chronic intestinal inflammation.

\section{Environmental Factors and IBD}

The rise of IBD in the developing world has usually been attributed to the industrialization and urbanization of societies [11,81]. The observation of the coincident increase in immune-mediated and inflammatory diseases in the more developed and industrialized nations has long been associated with the hygiene hypothesis [82]. This hypothesis appears to be relatively reductionist, as it does not explain the dynamics of IBD evolution in long-modernized Asian countries, for example. Whereas improvements in sanitation and hygiene practices are usually related to the urban compared to the rural environment, several other concomitant factors affecting the rise of immune-mediated disorders need to be pondered. For example, areas with a higher prevalence of IBD also have fewer 
infections and are characterized by the widespread use of vaccines and antibiotics, in addition to the availability of clean food and water [83-85]. Other considerations associated with modern urban life in developed and developing nations include, for instance, population density, lifestyle modifications, level of education, and exposure to pollutants [86]. A positive association between ambient air pollution and IBD has been previously suggested [81], and the residential exposures to nitrogen dioxide and sulfur dioxide were shown to increase the risk of early-onset IBD [87].

The increased risk for developing IBD has also been associated with the exposure to different medications. Among them, the use of antibiotics has been shown to increase the risk of IBD, especially CD $[88,89]$. In a nested case-control analysis of the population-based database of prescription drugs, investigators found that patients diagnosed with IBD in childhood were more likely to have used antibiotics during the first year of life [90]. This positive association was further corroborated, for example, in a prospective study in which the use of antibiotics was strongly associated with the development of CD in childhood [91]. Although causality cannot be firmly established to date, exposure to antibiotics in childhood has been proposed to affect the normal development of tolerance to enteric bacteria, increasing the risk of IBD [92].

Other medications have been linked to IBD. For example, oral contraceptives have been shown to have a positive association with the development of IBD, in particular with CD [93]. In a case-control study, investigators found a positive association between nonsteroidal anti-inflammatory drugs (NSAIDs) and IBD [94]. In a cohort study, investigators found that the regular use of NSAIDs may increase CD activity [95]. However, a systematic review did not find a reliable association between the use of NSAIDs and risk of CD and UC exacerbation [96]. NSAIDs are capable of disrupting the intestinal epithelial barrier, and this may predispose the individual to the invasion of pathogens and may affect the intestinal homeostasis. The conflicting results regarding the association between NSAIDs and IBD may reflect different methodologies but also the participation of other uncontrolled environmental factors generating unanticipated and confounding effects.

Smoking is a well-established modifying factor in IBD, with opposing effects in CD and UC [97], but the mechanism by which it regulates intestinal inflammation is still unclear. The composition of cigarettes is heterogeneous and complex with several chemical elements that may affect different targets in the intestinal cells and modulate the microbiota $[98,99]$. Other evidence suggests that chemicals released from cigarette smoking can interfere with functions related to the immune response, such as the production of cytokines [100], and significantly impact the microbiota [101].

The functionality of vitamin D, an important regulator of mucosal immunity, is critically related to the exposure to sun with natural UV light [102]. Therefore, low sunlight exposure has been proposed to be a risk factor for the development of IBD, especially CD $[103,104]$. This is in line with the incidence and prevalence rates of IBD being greater in the Northern Hemisphere, where UV light is markedly lower [105]. However, the proposed association between IBD, particularly CD, and the northern latitudes should be interpreted not only in terms of geography, but in a wider context, where several other variables interact in a complex and dynamic concert, with largely unpredictable outcomes.

\section{Epidemiological Associations between Diet and IBD}

Although a consistent correlation between environmental factors and the pathogenesis of several disorders is usually difficult to prove due to the intervening effect of potentially confounding variables and the uncertain dynamics of exposures, associations between IBD and diet have been reported. The realization that the increase in the worldwide incidence of IBD and other immune-mediated diseases began in more developed and industrialized countries, particularly in North America and Northern Europe, has led investigators to hypothesize the global spread of a "Westernized" lifestyle [106-108].

The observation that the risk of developing IBD increases when people move from a low-risk to high-risk area appears to further support the role of environmental factors in the detriment of genetic predisposition, and reinforces the utility of studies examining immigration. For example, distinct investigations showed that immigrants to Israel had a higher prevalence of IBD than populations born 
in Israel $[107,109]$. Another population-based study demonstrated that in Sweden, a high incidence region, second-generation but not first-generation immigrants developed IBD at rates comparable to the native Swedish population. These results appear to underscore the importance of early-life exposures in determining disease initiation [110].

Epidemiological studies investigating the South Asian population indicate that the incidence and prevalence of adult IBD are significantly lower than in North America or the U.K. [111-114]. However, higher incidences of IBD in patients from South Asia have been reported in adult individuals who subsequently migrated to the U.K. [115], and in children migrating to British Columbia, Canada, at rates higher than in other ethnicities, including children of Western European descent [116]. In another similar study analyzing a migrant group to investigate the role of environmental factors in the development of IBD, Middle Eastern migrants to Australia had a significantly higher risk compared with the Caucasian population. The results, including the analysis of additional exposures such as smoking, antibiotic usage, and hygiene markers, suggested that migrants might be more sensitive to environmental challenges influencing the gut microbiome [117]. This thought is further supported by the results of a population-based study in the Asia-Pacific area in which the investigators suggested that early childhood factors and markers of altered intestinal microbiota may modulate the risk of IBD later in life [118].

Another study analyzing an Asian population combined a series of Chinese patients with IBD with the sequences from RISK and PRISM cohorts of IBD patients from the United States for a meta-analysis comparing gut microbiome profiles across ethnic groups. The investigators found that the gut dysbiosis observed in IBD is similar among Chinese and Western populations [119]. This may lead to the question of whether the gut microbiota provides universal biomarkers in IBD regardless of ethnicity. Results from another study suggest the existence of diverse gut microbial taxa with differential patterns of abundance common to various immune-mediated inflammatory diseases (IMIDs), including IBD [120]. These findings appear to corroborate the possible use of microbial taxa patterns as biomarkers for the detection and diagnosis of IBD, but also support the idea that the gut microbiota may constitute a common component of the etiology of IMIDs.

Several observational studies have attempted to characterize dietary patterns that could contribute to a higher risk of IBD development. These studies pointed to an increased risk of IBD among people consuming larger proportions of meat and fats, and a lower risk among people with diets rich in fiber, fruits, and vegetables [121,122]. However, as in other complex diseases, it may be difficult to interpret the role of individual risk factors, and an analysis of dietary patterns should consider exposures to groups of foods [5], distinct food components, and cultural habits, including specificities in food preparation.

\section{Diet and Epigenetic Modifications in IBD}

The epigenome, an intersection between the environment and the genome, has been implicated in the regulation of gene expression and cellular functions, and plays an essential role in the delineation of phenotypes and their preservation [21]. Novel information has contributed to an improved understanding of the role of epigenetic modifications in defining the molecular basis of IBD [123,124]. Regarding IBD, the first epigenetic modifications associated with disease pathogenesis were based on DNA methylation studies [125].

Currently, among the environmental factors, dietary factors were found to be powerful stimuli, being associated with peculiar patterns of gene expression and epigenetic signatures [126]. For instance, the diet supplies substrates for DNA methylation and can regulate the enzymatic activity required in the one-carbon cycle. Consequently, elements such as folate, choline, and water-soluble B vitamins have been implicated in methylation patterns $[123,127]$, which are essential elements for the synthesis and repair of DNA that modulate gene expression [128]. Experimental evidence obtained also supports the idea that dietary nutraceuticals possess potential as epigenetic modulators. For example, data from studies involving DNA methylation and chromatin repair indicate that polyphenols can regulate gene 
expression [129]. Polyphenols are secondary plant metabolites, common components of vegetables, fruits, green tea, and red wine, and have been recognized for their antioxidant properties. In addition, studies have shown beneficial effects of polyphenols through the modulation of NF-kB expression, and chromatin remodeling via the modulation of histone deacetylases and DNA methyltransferase activities, reversing altered gene expression [130]. In experimental IBD, resveratrol, a natural phenolic compound, was shown to decrease inflammatory cytokines and profibrotic factors, supporting a potential therapeutic benefit [131].

Curcumin, another natural product commonly used in cooking, has been investigated in experimental models of IBD, demonstrating beneficial effects mediated by the suppression of proinflammatory mediators [132]. In a randomized, multicenter, double-blind, placebo-controlled trial, curcumin has shown beneficial results in maintenance therapy for patients with UC [133]. Although the exact mechanisms by which curcumin may exert a wide range of biological actions have not been established, effects have been attributed to the regulation of histone acetylation/deacetylation and the expression of various microRNAs [134].

Several nutrients have been shown to modulate immune responses, potentially counteracting inflammatory processes [135], in actions also mediated through epigenetic regulation [123,136]. Processed foods, deficient in micronutrients including selenium and folate, have been implicated in the progression of diseases, such as colorectal cancer and possibly IBD [21,137-139]. In an experimental model, a selenium-deficient diet was shown to result in markedly hypomethylated colon DNA [140]. Selenium supplementation was able to prevent tissue damage by modulating the expression of key genes responsible for inflammation in experimental IBD [141]. Although low levels of selenium have been detected in the serum of patients with IBD [142], studies in human IBD involving potential effects of selenium supplementation are still limited [21].

To investigate the mechanisms underlying the potential beneficial effects of the Mediterranean diet, an intervention with two different diets, one rich in nuts and the other one in extra-virgin olive oil, was conducted. The evaluation of the methylation status of peripheral white blood cells genes showed that both diets influenced methylation, characteristically of genes such as CPT1B and GNAS involved in intermediate metabolism, inflammation, and the signal transduction process. Therefore, the investigators concluded that the expected beneficial effects of the Mediterranean diet, supplemented with nuts or extra-virgin olive oil, could be mediated, at least in part, through epigenetic modifications $[16,143,144]$.

In addition to the direct effects of dietary constituents on epigenetic modifications in human intestinal tissues, the resident microbiota has been shown to alter host histone acetylation and methylation. In this regard, particularly short-chain fatty acids such as acetate, butyrate, and propionate, mostly produced by the microbial fermentation of fiber, appear to play a critical role in the epigenetic control of the inflammatory response. In a diet poor in fiber, the inhibition of SCFA production was correlated with disturbed chromatin effects, which were restored following supplementation with SCFA [145]. In human IBD, SCFA-producing bacteria (Roseburia) and butyrate-producing bacteria (Faecalibacterium) are reduced [146]. However, the application of butyrate-producing bacteria as nutraceuticals in humans has been questioned and needs further investigation $[123,147]$. Figure 1 summarizes the potential interactions between diet and the microbiota and other environmental factors, resulting in epigenetic modifications, exemplifying the effects of different diets with their respective major characteristics. 


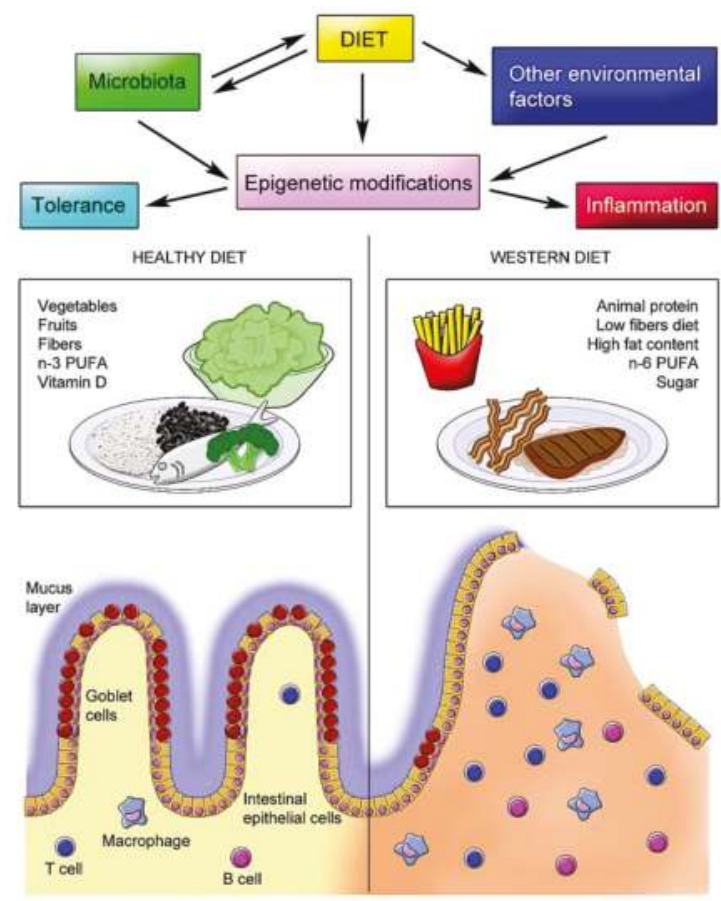

Figure 1. Schematic model of diet-host interactions in the intestine. The interaction between dietary elements and the intestinal mucosa is highly complex and, in normal conditions, results in a tolerogenic response. However, in genetically predisposed individuals, the interplay of specific dietary constituents with the resident microbiota and other environmental factors induces epigenetic modifications that affect the immune response, further compromising the epithelial barrier and defense mechanisms, leading to chronic inflammation, as observed in inflammatory bowel disease (IBD).

\section{Clinical Effects of Dietary Factors}

Clinical experience shows that patients usually associate their symptoms or disease relapses with the ingestion of certain foods and, as a result, they often change their diet empirically, with variable and, at most, temporary effects. In practice, decisions on dietary modifications usually do not follow professional nutritional advice and may have detrimental consequences [17,148-150]. In a prospective study investigating the dietary influence in the course of UC, researchers observed that a large ingestion of meat, more so of red and processed meat, in addition to ethanol, protein, sulfur, and sulfate, enhanced the chances of triggering a flare [151,152]. In the case of patients with CD, a diet rich in total fat, saturated fat, monounsaturated fatty acids, and a higher ratio of omega-6:omega-3 polyunsaturated fatty acids (PUFAs) was related to disease relapses [153,154]. Of note, the oral administration of iron sulfate or heme in dietary iron, which is present in meat, was shown to increase the severity of chemically-induced colitis in rodents $[155,156]$. However, although a substantial number of iron-deficient patients tolerate oral iron poorly, only a small percentage of patients with IBD have disease relapses attributed to oral iron [157]. Anemia is a common complication of IBD, mostly multifactorial, but frequently related to iron deficiency due to loss from bleeding and decreased iron absorption or ingestion [158]. Although many physicians have concerns regarding the management of iron deficiency in IBD, current data suggest that oral iron therapy should be preferred in mild cases or during clinical remission, unless patients are intolerant or have an inadequate response [159]. The aggravation of experimental colitis following dietary iron has been associated with a marked reduction in the fecal abundance of 
Firmicutes and Bacteroidetes, and an increase in Proteobacteria [160]. Hence, poor outcomes following oral iron replacement in patients with IBD could be related to exacerbation of the intestinal dysbiosis.

\section{Dietary Interventions in IBD}

Simple dietary interventions usually improve gastrointestinal symptoms in patients with IBD, including a reduction in the ingestion of dairy products and also fermentable oligosaccharides, disaccharides, monosaccharides, and polyols (FODMAPS) [161]. More recent data have demonstrated the possible roles of specific dietary therapy in IBD. For example, dietary therapies, such as exclusive enteral nutrition (EEN), have been successfully used to induce remission in early or new-onset CD [162]. Theoretically, EEN involves the isolated use of medical formulas in a liquid form without exposure to other foods, usually for six to eight weeks [5]. This strategy is apparently more feasible in the pediatric population, in contrast to adults. Investigations have demonstrated that EEN may induce remission in more than $60 \%$ of children with CD followed by a significant reduction in inflammatory markers, including the erythrocyte sedimentation rate (ESR), C-reactive protein (CRP), and fecal calprotectin [5,163-165]. Grover et al. conducted a prospective open label study to assess mucosal healing before and after eight weeks of EEN, and concluded that the therapy is effective for inducing early clinical, biochemical, mucosal, and transmural remission, and was associated with improved outcomes at one year [166].

Given practical concerns and clinical limitations, other research groups have employed a different strategy based on the combination of partial enteral nutrition (PEN) with a specific exclusion diet granting access to whole foods. The diet was evaluated in a retrospective cohort of children and young adults with mild-to-moderate $\mathrm{CD}$, and remission was achieved in 70\% of patients. Clinical improvement was followed by the reduction in ESR and CRP and was associated with mucosal healing [167]. Afterward, this diet-low in animal fat, rich in complex carbohydrates, and with a moderate exposure to soluble fiber-was evaluated for the induction of remission in children and adults failing biologics. More than $60 \%$ of the patients achieved clinical remission, with a marked decrease in inflammatory markers [168].

Other important theoretical dietary elements, fiber, and prebiotics supplements have been evaluated as interventions in CD. However, a systematic review of randomized controlled trials found no evidence for the use of fiber or prebiotics to induce or maintain remission in CD [169]. No evidence for the restriction of fiber in the absence of obstructive symptoms or stricturing disease has been established for CD. In another study, a semi-vegetarian diet with considerable amounts of dietary fiber was shown to induce a high remission rate with no negative effects [170]. The same group investigated the effect of infliximab combined with a plant-based diet as first-line therapy for patients with CD naïve to biologics and found that the combined therapy can induce remission in most cases [171].

Although the most consistent studies regarding dietary interventions in IBD usually refer to $\mathrm{CD}$, previous systematic evaluations performed by independent groups have supported the potential beneficial effects of diet in both CD and UC according to patients' perceptions $[149,172]$. In an observational prospective cohort study, patients with UC in clinical remission were followed for one year to investigate the effect of diet on disease relapse. The investigators identified an increased risk of UC relapses associated with the consumption of meat (particularly red and processed meat), eggs, and alcoholic beverages, which have been attributed to the high dietary content of sulfur [151]. In addition to red meat and eggs, milk and dairy products contain high concentrations of cysteine, which can be used by sulfate-reducing bacteria (SRB) to produce hydrogen sulfide $\left(\mathrm{H}_{2} \mathrm{~S}\right)[173,174]$. $\mathrm{H}_{2} \mathrm{~S}$, in turn, has been shown to induce proinflammatory effects in the colon and intestinal epithelial cells [175-177]. Some studies have demonstrated the increased presence of SRB and $\mathrm{H}_{2} \mathrm{~S}$ production in the colon of patients with UC [178-180]. However, despite all evidence suggesting a link between sulfur or sulfate-containing foods and UC, current data do not allow a causal association. While more controlled studies regarding $\mathrm{SRB}$ and $\mathrm{H}_{2} \mathrm{~S}$ in $\mathrm{UC}$ are awaited, patients should be aware of the potential detrimental effects of high-sulfur-containing dietary products. 
The nutritional theories of IBD pathogenesis involving carbohydrates, for example, have been based on the increasing evidence of the beneficial effects of the Specific Carbohydrate Diet in the treatment of both CD and UC, resulting in improvement in clinical parameters and inflammatory biomarkers [181-183]. The hypothesis that carbohydrate variation increases the predisposition to IBD has been supported by the evidence of resultant immune dysfunction, mucosal barrier defects, and dysbiosis, which are represented by the Westernized dietary features $[1,184,185]$. Carbohydrate monotony could be beneficial in IBD [186]. This has been corroborated by a successful report using the Paleo diet for a small cohort of patients with IBD [187]. Paleo is also an exclusion diet, supposedly representing habits of the Paleolithic era, consisting of a non-cereal, plant-based diet, including non-domesticated meats, and a low intake of carbohydrates, which are taken from a monotonous source. The rational for the use of carbohydrate monotony derives from the usually lower prevalence of IBD in rural or less developed communities consuming locally-produced seasonal products [16], in contrast to the modern Westernized diet, for which the digestive tract would theoretically not have had time to adapt [188].

In terms of dietary supplements, vitamin D appears to have a role in IBD. Vitamin D3 (animal sources and skin exposure to ultraviolet light) and vitamin D2 (plant sources) are first hydroxylated in the liver, and then in the kidney and extrarenal tissues, including intestinal macrophages, into the active form 1,25-dihydroxyvitamin D. The active vitamin D binds to its specific receptor in different tissues, including immune cells, modulating gene expression. Vitamin D deficiency has been frequently seen in patients with IBD; therefore, it has been regarded as a protective factor against disease development $[189,190]$. Particularly in patients with CD, vitamin D deficiency has been consistently associated with disease activity [79,191,192]. Vitamin D3 supplementation for patients with CD has been suggested to alleviate clinical manifestations [193]. In another clinical investigation, a randomized double-blind placebo-controlled study, dietary supplementation with 1200 IU vitamin D3 daily for 12 months significantly increased the vitamin D levels, but only modestly reduced the risk of disease relapse [194].

Currently, the exact mechanisms by which vitamin D may reduce the severity of $\mathrm{CD}$ are still unclear. In a study using peripheral blood mononuclear cells, investigators found that vitamin $\mathrm{D}$ was able to reduce the expression of pro-inflammatory M1 cytokines but did not induce the anti-inflammatory M2 phenotype [195]. In other experimental studies, vitamin D deficiency, or even its impaired signaling, were shown to worsen different models of experimental colitis through multiple effects, including epithelial barrier disruption [196,197], reduced mucosal immunity [198], and an association with gut dysbiosis $[199,200]$.

\section{Future Directions}

Among the environmental changes associated with the progressive and global expansion of IBD, diet has emerged as an essential regulatory element of the gut microbiome, which in turn has been strongly associated with the pathogenesis of several complex chronic inflammatory and autoimmune disorders. In addition to the involvement in dysbiosis, dietary components have been shown to interfere in intestinal homeostasis, modulating the barrier function and innate immunity mechanisms. Therefore, the accumulated scientific evidence appears to position diet as a central element both in disease pathogenesis as a critical risk factor and also as a potential opportunity for therapeutic interventions. Currently, several clinical trials are being conducted in different institutions and, hopefully, novel approaches to dietary therapy will be available in the near future.

Considering the progressive burden due to IBD anticipated in the next decades, dietary interventions appear particularly interesting, presumably due to the expected irrelevant side effects, lower costs, and potential application in the prevention of disease initiation or relapses. For the moment, however, the simplest approach includes the avoidance of foods that patients self-identify as worsening their symptoms, in addition to high-fat, high-carbohydrate meals and processed foods. Notably, dietary measures should be considered within the wider context of the patient's routine, not only in terms of food quality, but also changes in lifestyle, including dietary habits. For this 
purpose, while the results of large prospective controlled studies are awaited to provide more specific dietary guidance to patients, it is important to reinforce the role of multidisciplinary teams, including nutritionists, for offering better attention to and individualized support for patients with IBD.

Author Contributions: F.C. participated in the investigation and writing-original draft preparation; H.S.P.d.S. participated in the conceptualization, investigation, data curation, writing-review and editing, supervision, and funding acquisition.

Funding: This research was funded by Brazilian Research Council (CNPq), grant number 302401/2016-4, and FAPERJ (Fundação Carlos Chagas Filho de Amparo a Pesquisa do Estado do Rio de Janeiro), grant number E26/202.781/2017. The APC was funded by Fundação CAPES/PROEX, grant number 8882.331795/2010-01.

Acknowledgments: This research was funded by Brazilian Research Council (CNPq), grant number 302401/2016-4, and FAPERJ (Fundação Carlos Chagas Filho de Amparo a Pesquisa do Estado do Rio de Janeiro), grant number E26/202.781/2017. The APC was funded by Fundação CAPES/PROEX, grant number 8882.331795/2010-01. We thank Claudio Bernardazzi for his assistance with the figure.

Conflicts of Interest: The authors declare no conflict of interest.

\section{References}

1. deSouza, H.S.; Fiocchi, C. Immunopathogenesis of ibd: Current state of the art. Nat. Rev. Gastroenterol. Hepatol. 2016, 13, 13-27. [CrossRef]

2. Siebert, U.; Wurm, J.; Gothe, R.M.; Arvandi, M.; Vavricka, S.R.; von Kanel, R.; Begre, S.; Sulz, M.C.; Meyenberger, C.; Sagmeister, M. Swiss IBDCSG: Predictors of temporary and permanent work disability in patients with inflammatory bowel disease: Results of the swiss inflammatory bowel disease cohort study. Inflamm. Bowel Dis. 2013, 19, 847-855. [CrossRef]

3. Knights, D.; Lassen, K.G.; Xavier, R.J. Advances in inflammatory bowel disease pathogenesis: Linking host genetics and the microbiome. Gut 2013, 62, 1505-1510. [CrossRef]

4. de Souza, H.S.P.; Fiocchi, C.; Iliopoulos, D. The ibd interactome: An integrated view of aetiology, pathogenesis and therapy. Nat. Rev. Gastroenterol. Hepatol. 2017, 14, 739-749. [CrossRef]

5. Levine, A.; Sigall Boneh, R.; Wine, E. Evolving role of diet in the pathogenesis and treatment of inflammatory bowel diseases. Gut 2018, 67, 1726-1738. [CrossRef]

6. Uniken Venema, W.T.; Voskuil, M.D.; Dijkstra, G.; Weersma, R.K.; Festen, E.A. The genetic background of inflammatory bowel disease: From correlation to causality. J. Pathol. 2017, 241, 146-158. [CrossRef]

7. Rook, G.A. Hygiene hypothesis and autoimmune diseases. Clin. Rev. Allergy Immunol. 2012, 42, 5-15. [CrossRef]

8. Park, S.J.; Kim, W.H.; Cheon, J.H. Clinical characteristics and treatment of inflammatory bowel disease: A comparison of eastern and western perspectives. World J. Gastroenterol. 2014, 20, 11525-11537. [CrossRef]

9. Ng, S.C.; Tang, W.; Ching, J.Y.; Wong, M.; Chow, C.M.; Hui, A.J.; Wong, T.C.; Leung, V.K.; Tsang, S.W.; $\mathrm{Yu}$, H.H.; et al. Incidence and phenotype of inflammatory bowel disease based on results from the Asia-pacific Crohn's and colitis epidemiology study. Gastroenterology 2013, 145, 158-165. [CrossRef]

10. Kaplan, G.G. The global burden of ibd: From 2015 to 2025. Nat. Rev. Gastroenterol. Hepatol. 2015, 12, $720-727$. [CrossRef]

11. Bernstein, C.N. Review article: Changes in the epidemiology of inflammatory bowel disease-clues for aetiology. Aliment. Pharmacol. Ther. 2017, 46, 911-919. [CrossRef]

12. Marion-Letellier, R.; Savoye, G.; Ghosh, S. Ibd: In food we trust. J. Crohn's Colitis 2016, 10, 1351-1361. [CrossRef]

13. Duffy, L.C.; Raiten, D.J.; Hubbard, V.S.; Starke-Reed, P. Progress and challenges in developing metabolic footprints from diet in human gut microbial cometabolism. J. Nutr. 2015, 145, 1123S-1130S. [CrossRef]

14. Clemente, J.C.; Ursell, L.K.; Parfrey, L.W.; Knight, R. The impact of the gut microbiota on human health: An integrative view. Cell 2012, 148, 1258-1270. [CrossRef]

15. Ley, R.E.; Hamady, M.; Lozupone, C.; Turnbaugh, P.J.; Ramey, R.R.; Bircher, J.S.; Schlegel, M.L.; Tucker, T.A.; Schrenzel, M.D.; Knight, R.; et al. Evolution of mammals and their gut microbes. Science 2008, 320, 1647-1651. [CrossRef] 
16. De Filippo, C.; Cavalieri, D.; Di Paola, M.; Ramazzotti, M.; Poullet, J.B.; Massart, S.; Collini, S.; Pieraccini, G.; Lionetti, P. Impact of diet in shaping gut microbiota revealed by a comparative study in children from Europe and rural Africa. Proc. Natl. Acad. Sci. USA 2010, 107, 14691-14696. [CrossRef]

17. Lewis, J.D.; Abreu, M.T. Diet as a trigger or therapy for inflammatory bowel diseases. Gastroenterology 2017, 152, 398-414.e6. [CrossRef]

18. Calder, P.C. Polyunsaturated fatty acids and inflammation. Biochem. Soc. Trans. 2005, 33, 423-427. [CrossRef]

19. Yatsunenko, T.; Rey, F.E.; Manary, M.J.; Trehan, I.; Dominguez-Bello, M.G.; Contreras, M.; Magris, M.; Hidalgo, G.; Baldassano, R.N.; Anokhin, A.P.; et al. Human gut microbiome viewed across age and geography. Nature 2012, 486, 222-227. [CrossRef]

20. Barcenilla, A.; Pryde, S.E.; Martin, J.C.; Duncan, S.H.; Stewart, C.S.; Henderson, C.; Flint, H.J. Phylogenetic relationships of butyrate-producing bacteria from the human gut. Appl. Environ. Microbiol. 2000, 66, 1654-1661. [CrossRef]

21. Rapozo, D.C.; Bernardazzi, C.; de Souza, H.S. Diet and microbiota in inflammatory bowel disease: The gut in disharmony. World J. Gastroenterol. 2017, 23, 2124-2140. [CrossRef]

22. Man, S.M.; Kaakoush, N.O.; Mitchell, H.M. The role of bacteria and pattern-recognition receptors in Crohn's disease. Nat. Rev. Gastroenterol. Hepatol. 2011, 8, 152-168. [CrossRef]

23. Takahashi, K.; Nishida, A.; Fujimoto, T.; Fujii, M.; Shioya, M.; Imaeda, H.; Inatomi, O.; Bamba, S.; Sugimoto, M.; Andoh, A. Reduced abundance of butyrate-producing bacteria species in the fecal microbial community in Crohn's disease. Digestion 2016, 93, 59-65. [CrossRef]

24. Zhu, B.; Wang, X.; Li, L. Human gut microbiome: The second genome of human body. Protein Cell 2010, 1 , 718-725. [CrossRef]

25. Harris, R.A.; Shah, R.; Hollister, E.B.; Tronstad, R.R.; Hovdenak, N.; Szigeti, R.; Versalovic, J.; Kellermayer, R. Colonic mucosal epigenome and microbiome development in children and adolescents. J. Immunol. Res. 2016, 2016, 9170162. [CrossRef]

26. Guslandi, M. Role of probiotics in Crohn's disease and in pouchitis. J. Clin. Gastroenterol. 2015, 49 (Suppl. 1), S46-S49. [CrossRef]

27. David, L.A.; Maurice, C.F.; Carmody, R.N.; Gootenberg, D.B.; Button, J.E.; Wolfe, B.E.; Ling, A.V.; Devlin, A.S.; Varma, Y.; Fischbach, M.A.; et al. Diet rapidly and reproducibly alters the human gut microbiome. Nature 2014, 505, 559-563. [CrossRef]

28. Sokol, H. Probiotics and antibiotics in ibd. Dig. Dis. 2014, 32 (Suppl. 1), 10-17. [CrossRef]

29. Ishikawa, H.; Akedo, I.; Umesaki, Y.; Tanaka, R.; Imaoka, A.; Otani, T. Randomized controlled trial of the effect of bifidobacteria-fermented milk on ulcerative colitis. J. Am. Coll. Nutr. 2003, 22, 56-63. [CrossRef]

30. Gevers, D.; Kugathasan, S.; Denson, L.A.; Vazquez-Baeza, Y.; Van Treuren, W.; Ren, B.; Schwager, E.; Knights, D.; Song, S.J.; Yassour, M.; et al. The treatment-naive microbiome in new-onset Crohn's disease. Cell Host Microbe 2014, 15, 382-392. [CrossRef]

31. Forsyth, C.B.; Shaikh, M.; Bishehsari, F.; Swanson, G.; Voigt, R.M.; Dodiya, H.; Wilkinson, P.; Samelco, B.; Song, S.; Keshavarzian, A. Alcohol feeding in mice promotes colonic hyperpermeability and changes in colonic organoid stem cell fate. Alcohol. Clin. Exp. Res. 2017, 41, 2100-2113. [CrossRef]

32. Cani, P.D.; Bibiloni, R.; Knauf, C.; Waget, A.; Neyrinck, A.M.; Delzenne, N.M.; Burcelin, R. Changes in gut microbiota control metabolic endotoxemia-induced inflammation in high-fat diet-induced obesity and diabetes in mice. Diabetes 2008, 57, 1470-1481. [CrossRef]

33. Turnbaugh, P.J.; Ley, R.E.; Mahowald, M.A.; Magrini, V.; Mardis, E.R.; Gordon, J.I. An obesity-associated gut microbiome with increased capacity for energy harvest. Nature 2006, 444, 1027-1031. [CrossRef]

34. Cox, A.J.; West, N.P.; Cripps, A.W. Obesity, inflammation, and the gut microbiota. Lancet Diabetes Endocrinol. 2015, 3, 207-215. [CrossRef]

35. Frank, D.N.; Robertson, C.E.; Hamm, C.M.; Kpadeh, Z.; Zhang, T.; Chen, H.; Zhu, W.; Sartor, R.B.; Boedeker, E.C.; Harpaz, N.; et al. Disease phenotype and genotype are associated with shifts in intestinal-associated microbiota in inflammatory bowel diseases. Inflamm. Bowel Dis. 2011, 17, 179-184. [CrossRef]

36. Maeda, Y.; Kurakawa, T.; Umemoto, E.; Motooka, D.; Ito, Y.; Gotoh, K.; Hirota, K.; Matsushita, M.; Furuta, Y.; Narazaki, M.; et al. Dysbiosis contributes to arthritis development via activation of autoreactive $t$ cells in the intestine. Arthr. Rheumatol. 2016, 68, 2646-2661. [CrossRef] 
37. Ott, S.J.; Musfeldt, M.; Wenderoth, D.F.; Hampe, J.; Brant, O.; Folsch, U.R.; Timmis, K.N.; Schreiber, S. Reduction in diversity of the colonic mucosa associated bacterial microflora in patients with active inflammatory bowel disease. Gut 2004, 53, 685-693. [CrossRef]

38. Wang, H.; Shi, P.; Zuo, L.; Dong, J.; Zhao, J.; Liu, Q.; Zhu, W. Dietary non-digestible polysaccharides ameliorate intestinal epithelial barrier dysfunction in il-10 knockout mice. J. Crohn's Colitis 2016, 10, 1076-1086. [CrossRef]

39. Furusawa, Y.; Obata, Y.; Fukuda, S.; Endo, T.A.; Nakato, G.; Takahashi, D.; Nakanishi, Y.; Uetake, C.; Kato, K.; Kato, T.; et al. Commensal microbe-derived butyrate induces the differentiation of colonic regulatory t cells. Nature 2013, 504, 446-450. [CrossRef]

40. Desai, M.S.; Seekatz, A.M.; Koropatkin, N.M.; Kamada, N.; Hickey, C.A.; Wolter, M.; Pudlo, N.A.; Kitamoto, S.; Terrapon, N.; Muller, A.; et al. A dietary fiber-deprived gut microbiota degrades the colonic mucus barrier and enhances pathogen susceptibility. Cell 2016, 167, 1339-1353.e21. [CrossRef]

41. Agus, A.; Denizot, J.; Thevenot, J.; Martinez-Medina, M.; Massier, S.; Sauvanet, P.; Bernalier-Donadille, A.; Denis, S.; Hofman, P.; Bonnet, R.; et al. Western diet induces a shift in microbiota composition enhancing susceptibility to adherent-invasive E. coli infection and intestinal inflammation. Sci. Rep. 2016, 6, 19032. [CrossRef]

42. Ma, X.; Torbenson, M.; Hamad, A.R.; Soloski, M.J.; Li, Z. High-fat diet modulates non-cd1d-restricted natural killer $\mathrm{t}$ cells and regulatory $\mathrm{t}$ cells in mouse colon and exacerbates experimental colitis. Clin. Exp. Immunol. 2008, 151, 130-138. [CrossRef]

43. Kim, K.A.; Gu, W.; Lee, I.A.; Joh, E.H.; Kim, D.H. High fat diet-induced gut microbiota exacerbates inflammation and obesity in mice via the tlr4 signaling pathway. PLoS ONE 2012, 7, e47713. [CrossRef]

44. Liu, Z.; Brooks, R.S.; Ciappio, E.D.; Kim, S.J.; Crott, J.W.; Bennett, G.; Greenberg, A.S.; Mason, J.B. Diet-induced obesity elevates colonic tnf-alpha in mice and is accompanied by an activation of wnt signaling: A mechanism for obesity-associated colorectal cancer. J. Nutr. Biochem. 2012, 23, 1207-1213. [CrossRef]

45. Progatzky, F.; Sangha, N.J.; Yoshida, N.; McBrien, M.; Cheung, J.; Shia, A.; Scott, J.; Marchesi, J.R.; Lamb, J.R.; Bugeon, L.; et al. Dietary cholesterol directly induces acute inflammasome-dependent intestinal inflammation. Nat. Commun. 2014, 5, 5864. [CrossRef]

46. Joossens, M.; Huys, G.; Cnockaert, M.; De Preter, V.; Verbeke, K.; Rutgeerts, P.; Vandamme, P.; Vermeire, S. Dysbiosis of the faecal microbiota in patients with Crohn's disease and their unaffected relatives. Gut 2011, 60, 631-637. [CrossRef]

47. Varela, E.; Manichanh, C.; Gallart, M.; Torrejon, A.; Borruel, N.; Casellas, F.; Guarner, F.; Antolin, M. Colonisation by Faecalibacterium prausnitzii and maintenance of clinical remission in patients with ulcerative colitis. Aliment. Pharmacol. Ther. 2013, 38, 151-161. [CrossRef]

48. Wright, E.K.; Kamm, M.A.; Teo, S.M.; Inouye, M.; Wagner, J.; Kirkwood, C.D. Recent advances in characterizing the gastrointestinal microbiome in Crohn's disease: A systematic review. Inflamm. Bowel Dis. 2015, 21, 1219-1228.

49. Sokol, H.; Leducq, V.; Aschard, H.; Pham, H.P.; Jegou, S.; Landman, C.; Cohen, D.; Liguori, G.; Bourrier, A.; Nion-Larmurier, I.; et al. Fungal microbiota dysbiosis in ibd. Gut 2017, 66, 1039-1048. [CrossRef]

50. Pascal, V.; Pozuelo, M.; Borruel, N.; Casellas, F.; Campos, D.; Santiago, A.; Martinez, X.; Varela, E.; Sarrabayrouse, G.; Machiels, K.; et al. A microbial signature for Crohn's disease. Gut 2017, 66, 813-822. [CrossRef]

51. Powell, N.; MacDonald, T.T. Recent advances in gut immunology. Parasite Immunol. 2017, 39. [CrossRef]

52. Atarashi, K.; Tanoue, T.; Oshima, K.; Suda, W.; Nagano, Y.; Nishikawa, H.; Fukuda, S.; Saito, T.; Narushima, S.; Hase, K.; et al. Treg induction by a rationally selected mixture of clostridia strains from the human microbiota. Nature 2013, 500, 232-236. [CrossRef]

53. Sefik, E.; Geva-Zatorsky, N.; Oh, S.; Konnikova, L.; Zemmour, D.; McGuire, A.M.; Burzyn, D.; Ortiz-Lopez, A.; Lobera, M.; Yang, J.; et al. Mucosal immunology. Individual intestinal symbionts induce a distinct population of rorgamma(+) regulatory t cells. Science 2015, 349, 993-997. [CrossRef]

54. Atarashi, K.; Tanoue, T.; Ando, M.; Kamada, N.; Nagano, Y.; Narushima, S.; Suda, W.; Imaoka, A.; Setoyama, H.; Nagamori, T.; et al. Th17 cell induction by adhesion of microbes to intestinal epithelial cells. Cell 2015, 163, 367-380. [CrossRef] 
55. Tan, T.G.; Sefik, E.; Geva-Zatorsky, N.; Kua, L.; Naskar, D.; Teng, F.; Pasman, L.; Ortiz-Lopez, A.; Jupp, R.; $\mathrm{Wu}, \mathrm{H} . J . ;$ et al. Identifying species of symbiont bacteria from the human gut that, alone, can induce intestinal th17 cells in mice. Proc. Natl. Acad. Sci. USA 2016, 113, E8141-E8150. [CrossRef]

56. Viladomiu, M.; Kivolowitz, C.; Abdulhamid, A.; Dogan, B.; Victorio, D.; Castellanos, J.G.; Woo, V.; Teng, F.; Tran, N.L.; Sczesnak, A.; et al. Iga-coated E. coli enriched in Crohn's disease spondyloarthritis promote th17-dependent inflammation. Sci. Transl. Med. 2017, 9, eaaf9655. [CrossRef]

57. Ivanov, I.I.; Atarashi, K.; Manel, N.; Brodie, E.L.; Shima, T.; Karaoz, U.; Wei, D.; Goldfarb, K.C.; Santee, C.A.; Lynch, S.V.; et al. Induction of intestinal th17 cells by segmented filamentous bacteria. Cell 2009, 139, 485-498. [CrossRef]

58. Statovci, D.; Aguilera, M.; MacSharry, J.; Melgar, S. The impact of western diet and nutrients on the microbiota and immune response at mucosal interfaces. Front. Immunol. 2017, 8, 838. [CrossRef]

59. Martinez-Medina, M.; Denizot, J.; Dreux, N.; Robin, F.; Billard, E.; Bonnet, R.; Darfeuille-Michaud, A.; Barnich, N. Western diet induces dysbiosis with increased e coli in ceabac10 mice, alters host barrier function favouring AIEC colonisation. Gut 2014, 63, 116-124. [CrossRef]

60. Tilg, H.; Moschen, A.R. Food, immunity, and the microbiome. Gastroenterology 2015, 148, 1107-1119. [CrossRef]

61. Devkota, S.; Wang, Y.; Musch, M.W.; Leone, V.; Fehlner-Peach, H.; Nadimpalli, A.; Antonopoulos, D.A.; Jabri, B.; Chang, E.B. Dietary-fat-induced taurocholic acid promotes pathobiont expansion and colitis in il10-/- mice. Nature 2012, 487, 104-108. [CrossRef]

62. Silveira, A.L.M.; Ferreira, A.V.M.; de Oliveira, M.C.; Rachid, M.A.; da Cunha Sousa, L.F.; Dos Santos Martins, F.; Gomes-Santos, A.C.; Vieira, A.T.; Teixeira, M.M. Preventive rather than therapeutic treatment with high fiber diet attenuates clinical and inflammatory markers of acute and chronic dss-induced colitis in mice. Eur. J. Nutr. 2017, 56, 179-191. [CrossRef]

63. Nickerson, K.P.; McDonald, C. Crohn's disease-associated adherent-invasive escherichia coli adhesion is enhanced by exposure to the ubiquitous dietary polysaccharide maltodextrin. PLoS ONE 2012, 7, e52132. [CrossRef]

64. Nickerson, K.P.; Chanin, R.; McDonald, C. Deregulation of intestinal anti-microbial defense by the dietary additive, maltodextrin. Gut Microbes 2015, 6, 78-83. [CrossRef]

65. Hold, G.L.; Smith, M.; Grange, C.; Watt, E.R.; El-Omar, E.M.; Mukhopadhya, I. Role of the gut microbiota in inflammatory bowel disease pathogenesis: What have we learnt in the past 10 years? World J. Gastroenterol. 2014, 20, 1192-1210. [CrossRef]

66. Colombel, J.F. Decade in review-ibd: Ibd-genes, bacteria and new therapeutic strategies. Nat. Rev. Gastroenterol. Hepatol. 2014, 11, 652-654. [CrossRef]

67. Zhernakova, A.; Kurilshikov, A.; Bonder, M.J.; Tigchelaar, E.F.; Schirmer, M.; Vatanen, T.; Mujagic, Z.; Vila, A.V.; Falony, G.; Vieira-Silva, S.; et al. Population-based metagenomics analysis reveals markers for gut microbiome composition and diversity. Science 2016, 352, 565-569. [CrossRef]

68. Figliuolo, V.R.; Dos Santos, L.M.; Abalo, A.; Nanini, H.; Santos, A.; Brittes, N.M.; Bernardazzi, C.; de Souza, H.S.P.; Vieira, L.Q.; Coutinho-Silva, R.; et al. Sulfate-reducing bacteria stimulate gut immune responses and contribute to inflammation in experimental colitis. Life Sci. 2017, 189, 29-38. [CrossRef]

69. Schroeder, B.O.; Birchenough, G.M.H.; Stahlman, M.; Arike, L.; Johansson, M.E.V.; Hansson, G.C.; Backhed, F. Bifidobacteria or fiber protects against diet-induced microbiota-mediated colonic mucus deterioration. Cell Host Microbe 2018, 23, 27-40.e7. [CrossRef]

70. James, S.L.; Christophersen, C.T.; Bird, A.R.; Conlon, M.A.; Rosella, O.; Gibson, P.R.; Muir, J.G. Abnormal fibre usage in uc in remission. Gut 2015, 64, 562-570. [CrossRef]

71. Vinolo, M.A.; Rodrigues, H.G.; Nachbar, R.T.; Curi, R. Regulation of inflammation by short chain fatty acids. Nutrients 2011, 3, 858-876. [CrossRef]

72. Hartog, A.; Belle, F.N.; Bastiaans, J.; de Graaff, P.; Garssen, J.; Harthoorn, L.F.; Vos, A.P. A potential role for regulatory t-cells in the amelioration of dss induced colitis by dietary non-digestible polysaccharides. J. Nutr. Biochem. 2015, 26, 227-233. [CrossRef]

73. Roberts, C.L.; Keita, A.V.; Duncan, S.H.; O’Kennedy, N.; Soderholm, J.D.; Rhodes, J.M.; Campbell, B.J Translocation of Crohn's disease escherichia coli across m-cells: Contrasting effects of soluble plant fibres and emulsifiers. Gut 2010, 59, 1331-1339. [CrossRef] 
74. Andersen, V.; Chan, S.; Luben, R.; Khaw, K.T.; Olsen, A.; Tjonneland, A.; Kaaks, R.; Grip, O.; Bergmann, M.M.; Boeing, H.; et al. Fibre intake and the development of inflammatory bowel disease: A european prospective multi-centre cohort study (epic-ibd). J. Crohn's Colitis 2018, 12, 129-136. [CrossRef]

75. Chassaing, B.; Koren, O.; Goodrich, J.K.; Poole, A.C.; Srinivasan, S.; Ley, R.E.; Gewirtz, A.T. Dietary emulsifiers impact the mouse gut microbiota promoting colitis and metabolic syndrome. Nature 2015, 519, $92-96$. [CrossRef]

76. Chassaing, B.; Van de Wiele, T.; De Bodt, J.; Marzorati, M.; Gewirtz, A.T. Dietary emulsifiers directly alter human microbiota composition and gene expression ex vivo potentiating intestinal inflammation. Gut 2017, 66, 1414-1427. [CrossRef]

77. Khalili, H.; Malik, S.; Ananthakrishnan, A.N.; Garber, J.J.; Higuchi, L.M.; Joshi, A.; Peloquin, J.; Richter, J.M.; Stewart, K.O.; Curhan, G.C.; et al. Identification and characterization of a novel association between dietary potassium and risk of Crohn's disease and ulcerative colitis. Front. Immunol. 2016, 7, 554. [CrossRef]

78. Mayneris-Perxachs, J.; Bolick, D.T.; Leng, J.; Medlock, G.L.; Kolling, G.L.; Papin, J.A.; Swann, J.R.; Guerrant, R.L. Protein- and zinc-deficient diets modulate the murine microbiome and metabolic phenotype. Am. J. Clin. Nutr. 2016, 104, 1253-1262. [CrossRef]

79. Del Pinto, R.; Pietropaoli, D.; Chandar, A.K.; Ferri, C.; Cominelli, F. Association between inflammatory bowel disease and vitamin d deficiency: A systematic review and meta-analysis. Inflamm. Bowel Dis. 2015, 21, 2708-2717. [CrossRef]

80. de Bruyn, J.R.; van Heeckeren, R.; Ponsioen, C.Y.; van den Brink, G.R.; Lowenberg, M.; Bredenoord, A.J.; Frijstein, G.; D'Haens, G.R. Vitamin d deficiency in Crohn's disease and healthy controls: A prospective case-control study in the netherlands. J. Crohn's Colitis 2014, 8, 1267-1273. [CrossRef]

81. Maaser, C.; Langholz, E.; Gordon, H.; Burisch, J.; Ellul, P.; Ramirez, V.H.; Karakan, T.; Katsanos, K.H.; Krustins, E.; Levine, A.; et al. European Crohn's and colitis organisation topical review on environmental factors in ibd. J. Crohn's Colitis 2017, 11, 905-920. [CrossRef]

82. Strachan, D.P. Hay fever, hygiene, and household size. BMJ 1989, 299, 1259-1260. [CrossRef]

83. Yazdanbakhsh, M.; Matricardi, P.M. Parasites and the hygiene hypothesis: Regulating the immune system? Clin. Rev. Allergy Immunol. 2004, 26, 15-24. [CrossRef]

84. Feillet, H.; Bach, J.F. Increased incidence of inflammatory bowel disease: The price of the decline of infectious burden? Curr. Opin. Gastroenterol. 2004, 20, 560-564. [CrossRef]

85. Foster, A.; Jacobson, K. Changing incidence of inflammatory bowel disease: Environmental influences and lessons learnt from the south Asian population. Front. Pediatr. 2013, 1, 34. [CrossRef]

86. Ponder, A.; Long, M.D. A clinical review of recent findings in the epidemiology of inflammatory bowel disease. Clin. Epidemiol. 2013, 5, 237-247.

87. Kaplan, G.G.; Hubbard, J.; Korzenik, J.; Sands, B.E.; Panaccione, R.; Ghosh, S.; Wheeler, A.J.; Villeneuve, P.J. The inflammatory bowel diseases and ambient air pollution: A novel association. Am. J. Gastroenterol. 2010, 105, 2412-2419. [CrossRef]

88. Ungaro, R.; Bernstein, C.N.; Gearry, R.; Hviid, A.; Kolho, K.L.; Kronman, M.P.; Shaw, S.; Van Kruiningen, H.; Colombel, J.F.; Atreja, A. Antibiotics associated with increased risk of new-onset Crohn's disease but not ulcerative colitis: A meta-analysis. Am. J. Gastroenterol. 2014, 109, 1728-1738. [CrossRef]

89. Aniwan, S.; Tremaine, W.J.; Raffals, L.E.; Kane, S.V.; Loftus, E.V., Jr. Antibiotic use and new-onset inflammatory bowel disease in Olmsted county, Minnesota: A population-based case-control study. J. Crohn's Colitis 2018, 12, 137-144. [CrossRef]

90. Shaw, S.Y.; Blanchard, J.F.; Bernstein, C.N. Association between the use of antibiotics in the first year of life and pediatric inflammatory bowel disease. Am. J. Gastroenterol. 2010, 105, 2687-2692. [CrossRef]

91. Hviid, A.; Svanstrom, H.; Frisch, M. Antibiotic use and inflammatory bowel diseases in childhood. Gut 2011, 60, 49-54. [CrossRef]

92. Hildebrand, H.; Malmborg, P.; Askling, J.; Ekbom, A.; Montgomery, S.M. Early-life exposures associated with antibiotic use and risk of subsequent Crohn's disease. Scand. J. Gastroenterol. 2008, 43, 961-966. [CrossRef]

93. Cornish, J.A.; Tan, E.; Simillis, C.; Clark, S.K.; Teare, J.; Tekkis, P.P. The risk of oral contraceptives in the etiology of inflammatory bowel disease: A meta-analysis. Am. J. Gastroenterol. 2008, 103, 2394-2400. [CrossRef] 
94. Felder, J.B.; Korelitz, B.I.; Rajapakse, R.; Schwarz, S.; Horatagis, A.P.; Gleim, G. Effects of nonsteroidal antiinflammatory drugs on inflammatory bowel disease: A case-control study. Am. J. Gastroenterol. 2000, 95, 1949-1954. [CrossRef]

95. Long, M.D.; Kappelman, M.D.; Martin, C.F.; Chen, W.; Anton, K.; Sandler, R.S. Role of nonsteroidal anti-inflammatory drugs in exacerbations of inflammatory bowel disease. J. Clin. Gastroenterol. 2016, 50, 152-156. [CrossRef]

96. Moninuola, O.O.; Milligan, W.; Lochhead, P.; Khalili, H. Systematic review with meta-analysis: Association between acetaminophen and nonsteroidal anti-inflammatory drugs (nsaids) and risk of Crohn's disease and ulcerative colitis exacerbation. Aliment. Pharmacol. Ther. 2018, 47, 1428-1439. [CrossRef]

97. Cosnes, J. Tobacco and ibd: Relevance in the understanding of disease mechanisms and clinical practice. Best Pract. Res. Clin. Gastroenterol. 2004, 18, 481-496. [CrossRef]

98. Parkes, G.C.; Whelan, K.; Lindsay, J.O. Smoking in inflammatory bowel disease: Impact on disease course and insights into the aetiology of its effect. J. Crohn's Colitis 2014, 8, 717-725. [CrossRef]

99. Biedermann, L.; Fournier, N.; Misselwitz, B.; Frei, P.; Zeitz, J.; Manser, C.N.; Pittet, V.; Juillerat, P.; von Kanel, R.; Fried, M.; et al. High rates of smoking especially in female Crohn's disease patients and low use of supportive measures to achieve smoking cessation-Data from the swiss ibd cohort study. J. Crohn's Colitis 2015, 9, 819-829. [CrossRef]

100. Sher, M.E.; Bank, S.; Greenberg, R.; Sardinha, T.C.; Weissman, S.; Bailey, B.; Gilliland, R.; Wexner, S.D. The influence of cigarette smoking on cytokine levels in patients with inflammatory bowel disease. Inflamm. Bowel Dis. 1999, 5, 73-78. [CrossRef]

101. Biedermann, L.; Brulisauer, K.; Zeitz, J.; Frei, P.; Scharl, M.; Vavricka, S.R.; Fried, M.; Loessner, M.J.; Rogler, G.; Schuppler, M. Smoking cessation alters intestinal microbiota: Insights from quantitative investigations on human fecal samples using fish. Inflamm. Bowel Dis. 2014, 20, 1496-1501. [CrossRef]

102. Adorini, L.; Penna, G. Dendritic cell tolerogenicity: A key mechanism in immunomodulation by vitamin $d$ receptor agonists. Hum. Immunol. 2009, 70, 345-352. [CrossRef]

103. Jorgensen, S.P.; Hvas, C.L.; Agnholt, J.; Christensen, L.A.; Heickendorff, L.; Dahlerup, J.F. Active Crohn's disease is associated with low vitamin d levels. J. Crohn's Colitis 2013, 7, e407-e413. [CrossRef]

104. Limketkai, B.N.; Bayless, T.M.; Brant, S.R.; Hutfless, S.M. Lower regional and temporal ultraviolet exposure is associated with increased rates and severity of inflammatory bowel disease hospitalisation. Aliment. Pharmacol. Ther. 2014, 40, 508-517. [CrossRef]

105. Holmes, E.A.; Xiang, F.; Lucas, R.M. Variation in incidence of pediatric Crohn's disease in relation to latitude and ambient ultraviolet radiation: A systematic review and analysis. Inflamm. Bowel Dis. 2015, 21, 809-817. [CrossRef]

106. Magnus, P.; Jaakkola, J.J. Secular trend in the occurrence of asthma among children and young adults: Critical appraisal of repeated cross sectional surveys. BMJ 1997, 314, 1795-1799. [CrossRef]

107. Lee, D.; Albenberg, L.; Compher, C.; Baldassano, R.; Piccoli, D.; Lewis, J.D.; Wu, G.D. Diet in the pathogenesis and treatment of inflammatory bowel diseases. Gastroenterology 2015, 148, 1087-1106. [CrossRef]

108. Alcalde-Cabero, E.; Almazan-Isla, J.; Garcia-Merino, A.; de Sa, J.; de Pedro-Cuesta, J. Incidence of multiple sclerosis among european economic area populations, 1985-2009: The framework for monitoring. BMC Neurol. 2013, 13, 58. [CrossRef]

109. Krawiec, J.; Odes, H.S.; Lasry, Y.; Krugliak, P.; Weitzman, S. Aspects of the epidemiology of Crohn's disease in the Jewish population in Beer Sheva, Israel. Isr. J. Med. Sci. 1984, 20, 16-21.

110. Li, X.; Sundquist, J.; Hemminki, K.; Sundquist, K. Risk of inflammatory bowel disease in first- and second-generation immigrants in Sweden: A nationwide follow-up study. Inflamm. Bowel Dis. 2011, 17, 1784-1791. [CrossRef]

111. Sood, A.; Midha, V.; Sood, N.; Bhatia, A.S.; Avasthi, G. Incidence and prevalence of ulcerative colitis in Punjab, north India. Gut 2003, 52, 1587-1590. [CrossRef]

112. Khosla, S.N.; Girdhar, N.K.; Lal, S.; Mishra, D.S. Epidemiology of ulcerative colitis in hospital and select general population of northern India. J. Assoc. Phys. India 1986, 34, 405-407.

113. Loftus, C.G.; Loftus, E.V., Jr.; Harmsen, W.S.; Zinsmeister, A.R.; Tremaine, W.J.; Melton, L.J., 3rd; Sandborn, W.J. Update on the incidence and prevalence of Crohn's disease and ulcerative colitis in Olmsted county, Minnesota, 1940-2000. Inflamm. Bowel Dis. 2007, 13, 254-261. [CrossRef] 
114. Bernstein, C.N.; Wajda, A.; Svenson, L.W.; MacKenzie, A.; Koehoorn, M.; Jackson, M.; Fedorak, R.; Israel, D.; Blanchard, J.F. The epidemiology of inflammatory bowel disease in Canada: A population-based study. Am. J. Gastroenterol. 2006, 101, 1559-1568. [CrossRef]

115. Fellows, I.W.; Freeman, J.G.; Holmes, G.K. Crohn's disease in the city of derby, 1951-1985. Gut 1990, 31, 1262-1265. [CrossRef]

116. Pinsk, V.; Lemberg, D.A.; Grewal, K.; Barker, C.C.; Schreiber, R.A.; Jacobson, K. Inflammatory bowel disease in the south Asian pediatric population of British Columbia. Am. J. Gastroenterol. 2007, 102, 1077-1083. [CrossRef]

117. Ko, Y.; Butcher, R.; Leong, R.W. Epidemiological studies of migration and environmental risk factors in the inflammatory bowel diseases. World J. Gastroenterol. 2014, 20, 1238-1247. [CrossRef]

118. Ng, S.C.; Tang, W.; Leong, R.W.; Chen, M.; Ko, Y.; Studd, C.; Niewiadomski, O.; Bell, S.; Kamm, M.A.; de Silva, H.J.; et al. Environmental risk factors in inflammatory bowel disease: A population-based case-control study in Asia-Pacific. Gut 2015, 64, 1063-1071. [CrossRef]

119. Zhou, Y.; Xu, Z.Z.; He, Y.; Yang, Y.; Liu, L.; Lin, Q.; Nie, Y.; Li, M.; Zhi, F.; Liu, S.; et al. Gut microbiota offers universal biomarkers across ethnicity in inflammatory bowel disease diagnosis and infliximab response prediction. MSystems 2018, 3, e00188-17. [CrossRef]

120. Forbes, J.D.; Chen, C.Y.; Knox, N.C.; Marrie, R.A.; El-Gabalawy, H.; de Kievit, T.; Alfa, M.; Bernstein, C.N.; Van Domselaar, G. A comparative study of the gut microbiota in immune-mediated inflammatory diseases-does a common dysbiosis exist? Microbiome 2018, 6, 221. [CrossRef]

121. Hou, J.K.; Abraham, B.; El-Serag, H. Dietary intake and risk of developing inflammatory bowel disease: A systematic review of the literature. Am. J. Gastroenterol. 2011, 106, 563-573. [CrossRef]

122. Ananthakrishnan, A.N. Epidemiology and risk factors for ibd. Nat. Rev. Gastroenterol. Hepatol. 2015, 12, 205-217. [CrossRef]

123. Aleksandrova, K.; Romero-Mosquera, B.; Hernandez, V. Diet, gut microbiome and epigenetics: Emerging links with inflammatory bowel diseases and prospects for management and prevention. Nutrients 2017, 9, 962. [CrossRef]

124. Dabritz, J.; Menheniott, T.R. Linking immunity, epigenetics, and cancer in inflammatory bowel disease. Inflamm. Bowel Dis. 2014, 20, 1638-1654. [CrossRef]

125. Barnett, M.; Bermingham, E.; McNabb, W.; Bassett, S.; Armstrong, K.; Rounce, J.; Roy, N. Investigating micronutrients and epigenetic mechanisms in relation to inflammatory bowel disease. Mutat. Res. 2010, 690, 71-80. [CrossRef]

126. Gallou-Kabani, C.; Vige, A.; Gross, M.S.; Junien, C. Nutri-epigenomics: Lifelong remodelling of our epigenomes by nutritional and metabolic factors and beyond. Clin. Chem. Lab. Med. 2007, 45, 321-327. [CrossRef]

127. Anderson, O.S.; Sant, K.E.; Dolinoy, D.C. Nutrition and epigenetics: An interplay of dietary methyl donors, one-carbon metabolism and DNA methylation. J. Nutr. Biochem. 2012, 23, 853-859. [CrossRef]

128. Lu, Q.; Qiu, X.; Hu, N.; Wen, H.; Su, Y.; Richardson, B.C. Epigenetics, disease, and therapeutic interventions. Ageing Res. Rev. 2006, 5, 449-467. [CrossRef]

129. Remely, M.; Lovrecic, L.; de la Garza, A.L.; Migliore, L.; Peterlin, B.; Milagro, F.I.; Martinez, A.J.; Haslberger, A.G. Therapeutic perspectives of epigenetically active nutrients. Br. J. Pharmacol. 2015, 172, 2756-2768. [CrossRef]

130. Russell, W.R.; Duncan, S.H.; Scobbie, L.; Duncan, G.; Cantlay, L.; Calder, A.G.; Anderson, S.E.; Flint, H.J. Major phenylpropanoid-derived metabolites in the human gut can arise from microbial fermentation of protein. Mol. Nutr. Food Res. 2013, 57, 523-535. [CrossRef]

131. Rahal, K.; Schmiedlin-Ren, P.; Adler, J.; Dhanani, M.; Sultani, V.; Rittershaus, A.C.; Reingold, L.; Zhu, J.; McKenna, B.J.; Christman, G.M.; et al. Resveratrol has antiinflammatory and antifibrotic effects in the peptidoglycan-polysaccharide rat model of Crohn's disease. Inflamm. Bowel Dis. 2012, 18, 613-623. [CrossRef]

132. Hanai, H.; Sugimoto, K. Curcumin has bright prospects for the treatment of inflammatory bowel disease. Curr. Pharm. Des. 2009, 15, 2087-2094. [CrossRef]

133. Hanai, H.; Iida, T.; Takeuchi, K.; Watanabe, F.; Maruyama, Y.; Andoh, A.; Tsujikawa, T.; Fujiyama, Y.; Mitsuyama, K.; Sata, M.; et al. Curcumin maintenance therapy for ulcerative colitis: Randomized, multicenter, double-blind, placebo-controlled trial. Clin. Gastroenterol. Hepatol. Off. Clin. Prac. J. Am. Gastroenterol. Assoc. 2006, 4, 1502-1506. 
134. Reuter, S.; Gupta, S.C.; Park, B.; Goel, A.; Aggarwal, B.B. Epigenetic changes induced by curcumin and other natural compounds. Genes Nutr. 2011, 6, 93-108. [CrossRef]

135. Burdge, G.C.; Hoile, S.P.; Lillycrop, K.A. Epigenetics: Are there implications for personalised nutrition? Curr. Opin. Clin. Nutr. Metab. Care 2012, 15, 442-447. [CrossRef]

136. Beaudet, A.L. Epigenetics and complex human disease: Is there a role in ibd? J. Pediatr. Gastroenterol. Nutr. 2008, 46 (Suppl. 1), E2.

137. Garfinkel, M.D.; Ruden, D.M. Chromatin effects in nutrition, cancer, and obesity. Nutrition 2004, 20, 56-62. [CrossRef]

138. Wani, N.A.; Hamid, A.; Kaur, J. Folate status in various pathophysiological conditions. IUBMB life 2008, 60, 834-842. [CrossRef]

139. McKay, J.A.; Williams, E.A.; Mathers, J.C. Gender-specific modulation of tumorigenesis by folic acid supply in the APC mouse during early neonatal life. Br. J. Nutr. 2008, 99, 550-558. [CrossRef]

140. Davis, C.D.; Uthus, E.O.; Finley, J.W. Dietary selenium and arsenic affect DNA methylation in vitro in caco-2 cells and in vivo in rat liver and colon. J. Nutr. 2000, 130, 2903-2909. [CrossRef]

141. Tirosh, O.; Levy, E.; Reifen, R. High selenium diet protects against tnbs-induced acute inflammation, mitochondrial dysfunction, and secondary necrosis in rat colon. Nutrition 2007, 23, 878-886. [CrossRef]

142. Castro Aguilar-Tablada, T.; Navarro-Alarcon, M.; Quesada Granados, J.; Samaniego Sanchez, C.; Rufian-Henares, J.A.; Nogueras-Lopez, F. Ulcerative colitis and Crohn's disease are associated with decreased serum selenium concentrations and increased cardiovascular risk. Nutrients 2016, 8, 780. [CrossRef]

143. Arpon, A.; Milagro, F.I.; Razquin, C.; Corella, D.; Estruch, R.; Fito, M.; Marti, A.; Martinez-Gonzalez, M.A.; Ros, E.; Salas-Salvado, J.; et al. Impact of consuming extra-virgin olive oil or nuts within a mediterranean diet on DNA methylation in peripheral white blood cells within the predimed-navarra randomized controlled trial: A role for dietary lipids. Nutrients 2017, 10, 15. [CrossRef]

144. Kau, A.L.; Ahern, P.P.; Griffin, N.W.; Goodman, A.L.; Gordon, J.I. Human nutrition, the gut microbiome and the immune system. Nature 2011, 474, 327-336. [CrossRef]

145. Krautkramer, K.A.; Kreznar, J.H.; Romano, K.A.; Vivas, E.I.; Barrett-Wilt, G.A.; Rabaglia, M.E.; Keller, M.P.; Attie, A.D.; Rey, F.E.; Denu, J.M. Diet-microbiota interactions mediate global epigenetic programming in multiple host tissues. Mol. Cell 2016, 64, 982-992. [CrossRef]

146. Kostic, A.D.; Xavier, R.J.; Gevers, D. The microbiome in inflammatory bowel disease: Current status and the future ahead. Gastroenterology 2014, 146, 1489-1499. [CrossRef]

147. Eeckhaut, V.; Ducatelle, R.; Sas, B.; Vermeire, S.; Van Immerseel, F. Progress towards butyrate-producing pharmabiotics: Butyricicoccus pullicaecorum capsule and efficacy in tnbs models in comparison with therapeutics. Gut 2014, 63, 367. [CrossRef]

148. Limdi, J.K.; Aggarwal, D.; McLaughlin, J.T. Dietary practices and beliefs in patients with inflammatory bowel disease. Inflamm. Bowel Dis. 2016, 22, 164-170. [CrossRef]

149. Zallot, C.; Quilliot, D.; Chevaux, J.B.; Peyrin-Biroulet, C.; Gueant-Rodriguez, R.M.; Freling, E.; Collet-Fenetrier, B.; Williet, N.; Ziegler, O.; Bigard, M.A.; et al. Dietary beliefs and behavior among inflammatory bowel disease patients. Inflamm. Bowel Dis. 2013, 19, 66-72. [CrossRef]

150. Tanaka, M.; Kawakami, A.; Iwao, Y.; Fukushima, T.; Yamamoto-Mitani, N. Coping strategies for possible flare-ups and their perceived effectiveness in patients with inflammatory bowel disease. Gastroenterology nursing. Off. J. Soc. Gastroenterol. Nur. Assoc. 2016, 39, 42-47. [CrossRef]

151. Jowett, S.L.; Seal, C.J.; Pearce, M.S.; Phillips, E.; Gregory, W.; Barton, J.R.; Welfare, M.R. Influence of dietary factors on the clinical course of ulcerative colitis: A prospective cohort study. Gut 2004, 53, 1479-1484. [CrossRef]

152. Magee, E.A.; Edmond, L.M.; Tasker, S.M.; Kong, S.C.; Curno, R.; Cummings, J.H. Associations between diet and disease activity in ulcerative colitis patients using a novel method of data analysis. Nutr. J. 2005, 4, 7. [CrossRef]

153. Guerreiro, C.S.; Ferreira, P.; Tavares, L.; Santos, P.M.; Neves, M.; Brito, M.; Cravo, M. Fatty acids, il6, and tnfalpha polymorphisms: An example of nutrigenetics in Crohn's disease. Am. J. Gastroenterol. 2009, 104, 2241-2249. [CrossRef]

154. Ferreira, P.; Cravo, M.; Guerreiro, C.S.; Tavares, L.; Santos, P.M.; Brito, M. Fat intake interacts with polymorphisms of caspase9, fasligand and ppargamma apoptotic genes in modulating Crohn's disease activity. Clin. Nutr. 2010, 29, 819-823. [CrossRef] 
155. Reifen, R.; Matas, Z.; Zeidel, L.; Berkovitch, Z.; Bujanover, Y. Iron supplementation may aggravate inflammatory status of colitis in a rat model. Dig. Dis. Sci. 2000, 45, 394-397. [CrossRef]

156. Carrier, J.; Aghdassi, E.; Cullen, J.; Allard, J.P. Iron supplementation increases disease activity and vitamin e ameliorates the effect in rats with dextran sulfate sodium-induced colitis. J. Nutr. 2002, 132, 3146-3150. [CrossRef]

157. de Silva, A.D.; Tsironi, E.; Feakins, R.M.; Rampton, D.S. Efficacy and tolerability of oral iron therapy in inflammatory bowel disease: A prospective, comparative trial. Aliment. Pharmacol. Ther. 2005, 22, 1097-1105. [CrossRef]

158. Goldberg, N.D. Iron deficiency anemia in patients with inflammatory bowel disease. Clin. Exp. Gastroenterol. 2013, 6, 61-70. [CrossRef]

159. Nielsen, O.H.; Ainsworth, M.; Coskun, M.; Weiss, G. Management of iron-deficiency anemia in inflammatory bowel disease: A systematic review. Medicine 2015, 94, e963. [CrossRef]

160. Mahalhal, A.; Williams, J.M.; Johnson, S.; Ellaby, N.; Duckworth, C.A.; Burkitt, M.D.; Liu, X.; Hold, G.L.; Campbell, B.J.; Pritchard, D.M.; et al. Oral iron exacerbates colitis and influences the intestinal microbiome. PLoS ONE 2018, 13, e0202460. [CrossRef]

161. Colombel, J.F.; Shin, A.; Gibson, P.R. AGA clinical practice update on functional gastrointestinal symptoms in patients with inflammatory bowel disease: Expert review. Clin. Gastroenterol. Hepatol. Off. Clin. Prac. J. Am. Gastroenterol. Assoc. 2019, 17, 380-390.e1.

162. Ruemmele, F.M.; Veres, G.; Kolho, K.L.; Griffiths, A.; Levine, A.; Escher, J.C.; Amil Dias, J.; Barabino, A.; Braegger, C.P.; Bronsky, J.; et al. Consensus guidelines of ecco/espghan on the medical management of pediatric Crohn's disease. J. Crohn's Colitis 2014, 8, 1179-1207. [CrossRef]

163. Lee, D.; Baldassano, R.N.; Otley, A.R.; Albenberg, L.; Griffiths, A.M.; Compher, C.; Chen, E.Z.; Li, H.; Gilroy, E.; Nessel, L.; et al. Comparative effectiveness of nutritional and biological therapy in North American children with active Crohn's disease. Inflamm. Bowel Dis. 2015, 21, 1786-1793. [CrossRef]

164. Connors, J.; Basseri, S.; Grant, A.; Giffin, N.; Mahdi, G.; Noble, A.; Rashid, M.; Otley, A.; Van Limbergen, J. Exclusive enteral nutrition therapy in paediatric Crohn's disease results in long-term avoidance of corticosteroids: Results of a propensity-score matched cohort analysis. J. Crohn's Colitis 2017, 11, 1063-1070. [CrossRef]

165. Cohen-Dolev, N.; Sladek, M.; Hussey, S.; Turner, D.; Veres, G.; Koletzko, S.; Martin de Carpi, J.; Staiano, A.; Shaoul, R.; Lionetti, P.; et al. Differences in outcomes over time with exclusive enteral nutrition compared with steroids in children with mild to moderate Crohn's disease: Results from the growth cd study. J. Crohn's Colitis 2018, 12, 306-312. [CrossRef]

166. Grover, Z.; Burgess, C.; Muir, R.; Reilly, C.; Lewindon, P.J. Early mucosal healing with exclusive enteral nutrition is associated with improved outcomes in newly diagnosed children with luminal Crohn's disease. J. Crohn's Colitis 2016, 10, 1159-1164. [CrossRef]

167. Sigall-Boneh, R.; Pfeffer-Gik, T.; Segal, I.; Zangen, T.; Boaz, M.; Levine, A. Partial enteral nutrition with a Crohn's disease exclusion diet is effective for induction of remission in children and young adults with Crohn's disease. Inflamm. Bowel Dis. 2014, 20, 1353-1360. [CrossRef]

168. Sigall Boneh, R.; Sarbagili Shabat, C.; Yanai, H.; Chermesh, I.; Ben Avraham, S.; Boaz, M.; Levine, A. Dietary therapy with the Crohn's disease exclusion diet is a successful strategy for induction of remission in children and adults failing biological therapy. J. Crohn's Colitis 2017, 11, 1205-1212. [CrossRef]

169. Wedlake, L.; Slack, N.; Andreyev, H.J.; Whelan, K. Fiber in the treatment and maintenance of inflammatory bowel disease: A systematic review of randomized controlled trials. Inflamm. Bowel Dis. 2014, 20, 576-586. [CrossRef]

170. Chiba, M.; Tsuji, T.; Nakane, K.; Komatsu, M. High amount of dietary fiber not harmful but favorable for Crohn disease. Perm. J. 2015, 19, 58-61. [CrossRef]

171. Chiba, M.; Tsuji, T.; Nakane, K.; Tsuda, S.; Ishii, H.; Ohno, H.; Watanabe, K.; Ito, M.; Komatsu, M.; Sugawara, T. Induction with infliximab and a plant-based diet as first-line (ipf) therapy for Crohn disease: A single-group trial. Perm. J. 2017, 21, 17-009. [CrossRef] [PubMed]

172. Cohen, A.B.; Lee, D.; Long, M.D.; Kappelman, M.D.; Martin, C.F.; Sandler, R.S.; Lewis, J.D. Dietary patterns and self-reported associations of diet with symptoms of inflammatory bowel disease. Dig. Dis. Sci. 2013, 58, 1322-1328. [CrossRef] [PubMed] 
173. Levine, J.; Ellis, C.J.; Furne, J.K.; Springfield, J.; Levitt, M.D. Fecal hydrogen sulfide production in ulcerative colitis. Am. J. Gastroenterol. 1998, 93, 83-87. [CrossRef] [PubMed]

174. Magee, E.A.; Richardson, C.J.; Hughes, R.; Cummings, J.H. Contribution of dietary protein to sulfide production in the large intestine: An in vitro and a controlled feeding study in humans. Am. J. Clin. Nutr. 2000, 72, 1488-1494. [CrossRef] [PubMed]

175. Medani, M.; Collins, D.; Docherty, N.G.; Baird, A.W.; O'Connell, P.R.; Winter, D.C. Emerging role of hydrogen sulfide in colonic physiology and pathophysiology. Inflamm. Bowel Dis. 2011, 17, 1620-1625. [CrossRef] [PubMed]

176. Attene-Ramos, M.S.; Nava, G.M.; Muellner, M.G.; Wagner, E.D.; Plewa, M.J.; Gaskins, H.R. DNA damage and toxicogenomic analyses of hydrogen sulfide in human intestinal epithelial FHS 74 int cells. Environ. Mol. Mutagen. 2010, 51, 304-314. [CrossRef] [PubMed]

177. Babidge, W.; Millard, S.; Roediger, W. Sulfides impair short chain fatty acid beta-oxidation at acyl-coa dehydrogenase level in colonocytes: Implications for ulcerative colitis. Mol. Cell. Biochem. 1998, 181, 117-124. [CrossRef] [PubMed]

178. Pitcher, M.C.; Beatty, E.R.; Cummings, J.H. The contribution of sulphate reducing bacteria and 5-aminosalicylic acid to faecal sulphide in patients with ulcerative colitis. Gut 2000, 46, 64-72. [CrossRef] [PubMed]

179. Loubinoux, J.; Bronowicki, J.P.; Pereira, I.A.; Mougenel, J.L.; Faou, A.E. Sulfate-reducing bacteria in human feces and their association with inflammatory bowel diseases. FEMS Microbiol. Ecol. 2002, 40, 107-112. [CrossRef]

180. Khalil, N.A.; Walton, G.E.; Gibson, G.R.; Tuohy, K.M.; Andrews, S.C. In vitro batch cultures of gut microbiota from healthy and ulcerative colitis (uc) subjects suggest that sulphate-reducing bacteria levels are raised in uc and by a protein-rich diet. Int. j. Food Sci. Nutr. 2014, 65, 79-88. [CrossRef]

181. Suskind, D.L.; Wahbeh, G.; Cohen, S.A.; Damman, C.J.; Klein, J.; Braly, K.; Shaffer, M.; Lee, D. Patients perceive clinical benefit with the specific carbohydrate diet for inflammatory bowel disease. Dig. Dis. Sci. 2016, 61, 3255-3260. [CrossRef] [PubMed]

182. Burgis, J.C.; Nguyen, K.; Park, K.T.; Cox, K. Response to strict and liberalized specific carbohydrate diet in pediatric Crohn's disease. World J. Gastroenterol. 2016, 22, 2111-2117. [CrossRef] [PubMed]

183. Obih, C.; Wahbeh, G.; Lee, D.; Braly, K.; Giefer, M.; Shaffer, M.L.; Nielson, H.; Suskind, D.L. Specific carbohydrate diet for pediatric inflammatory bowel disease in clinical practice within an academic ibd center. Nutrition 2016, 32, 418-425. [CrossRef] [PubMed]

184. Britto, S.; Kellermayer, R. Carbohydrate monotony as protection and treatment for inflammatory bowel disease. J. Crohn's Colitis 2019. [CrossRef] [PubMed]

185. Fiocchi, C. Tailoring treatment to the individual patient-Will inflammatory bowel disease medicine be personalized? Dig. Dis. 2015, 33 (Suppl. 1), 82-89. [CrossRef]

186. Nagy-Szakal, D.; Mir, S.A.; Ross, M.C.; Tatevian, N.; Petrosino, J.F.; Kellermayer, R. Monotonous diets protect against acute colitis in mice: Epidemiologic and therapeutic implications. J. Pediatr. Gastroenterol. Nutr. 2013, 56, 544-550. [CrossRef]

187. Konijeti, G.G.; Kim, N.; Lewis, J.D.; Groven, S.; Chandrasekaran, A.; Grandhe, S.; Diamant, C.; Singh, E.; Oliveira, G.; Wang, X.; et al. Efficacy of the autoimmune protocol diet for inflammatory bowel disease. Inflamm. Bowel Dis. 2017, 23, 2054-2060. [CrossRef]

188. Knight-Sepulveda, K.; Kais, S.; Santaolalla, R.; Abreu, M.T. Diet and inflammatory bowel disease. Gastroenterol. Hepatol. 2015, 11, 511-520.

189. van der Sloot, K.W.J.; Amini, M.; Peters, V.; Dijkstra, G.; Alizadeh, B.Z. Inflammatory bowel diseases: Review of known environmental protective and risk factors involved. Inflamm. Bowel Dis. 2017, 23, 1499-1509. [CrossRef]

190. Schaffler, H.; Schmidt, M.; Huth, A.; Reiner, J.; Glass, A.; Lamprecht, G. Clinical factors are associated with vitamin d levels in ibd patients: A retrospective analysis. J. Dig. Dis. 2018, 19, 24-32. [CrossRef]

191. Suibhne, T.N.; Cox, G.; Healy, M.; O’Morain, C.; O'Sullivan, M. Vitamin d deficiency in Crohn's disease: Prevalence, risk factors and supplement use in an outpatient setting. J. Crohn's Colitis 2012, 6, 182-188. [CrossRef] [PubMed]

192. Alrefai, D.; Jones, J.; El-Matary, W.; Whiting, S.J.; Aljebreen, A.; Mirhosseini, N.; Vatanparast, H. The association of vitamin d status with disease activity in a cohort of Crohn's disease patients in Canada. Nutrients 2017, 9, 1112. [CrossRef] [PubMed] 
193. Narula, N.; Cooray, M.; Anglin, R.; Muqtadir, Z.; Narula, A.; Marshall, J.K. Impact of high-dose vitamin d3 supplementation in patients with Crohn's disease in remission: A pilot randomized double-blind controlled study. Dig. Dis. Sci. 2017, 62, 448-455. [CrossRef] [PubMed]

194. Jorgensen, S.P.; Agnholt, J.; Glerup, H.; Lyhne, S.; Villadsen, G.E.; Hvas, C.L.; Bartels, L.E.; Kelsen, J.; Christensen, L.A.; Dahlerup, J.F. Clinical trial: Vitamin d3 treatment in Crohn's disease-A randomized double-blind placebo-controlled study. Aliment. Pharmacol. Ther. 2010, 32, 377-383. [CrossRef] [PubMed]

195. Dionne, S.; Duchatelier, C.F.; Seidman, E.G. The influence of vitamin d on $\mathrm{m} 1$ and $\mathrm{m} 2$ macrophages in patients with Crohn's disease. Innate Immun. 2017, 23, 557-565. [CrossRef] [PubMed]

196. Liu, W.; Chen, Y.; Golan, M.A.; Annunziata, M.L.; Du, J.; Dougherty, U.; Kong, J.; Musch, M.; Huang, Y.; Pekow, J.; et al. Intestinal epithelial vitamin d receptor signaling inhibits experimental colitis. J. Clin. Investig. 2013, 123, 3983-3996. [CrossRef]

197. Wu, S.; Zhang, Y.G.; Lu, R.; Xia, Y.; Zhou, D.; Petrof, E.O.; Claud, E.C.; Chen, D.; Chang, E.B.; Carmeliet, G.; et al. Intestinal epithelial vitamin d receptor deletion leads to defective autophagy in colitis. Gut 2015, 64, 1082-1094. [CrossRef]

198. Ryz, N.R.; Lochner, A.; Bhullar, K.; Ma, C.; Huang, T.; Bhinder, G.; Bosman, E.; Wu, X.; Innis, S.M.; Jacobson, K.; et al. Dietary vitamin $\mathrm{d} 3$ deficiency alters intestinal mucosal defense and increases susceptibility to Citrobacter rodentium-induced colitis. Am. J. Ophysiol. Gastrointest. Liver physiol. 2015, 309, G730-G742. [CrossRef]

199. Ooi, J.H.; Li, Y.; Rogers, C.J.; Cantorna, M.T. Vitamin d regulates the gut microbiome and protects mice from dextran sodium sulfate-induced colitis. J. Nutr. 2013, 143, 1679-1686. [CrossRef]

200. Jin, D.; Wu, S.; Zhang, Y.G.; Lu, R.; Xia, Y.; Dong, H.; Sun, J. Lack of vitamin d receptor causes dysbiosis and changes the functions of the murine intestinal microbiome. Clin. Ther. 2015, 37, 996-1009.e7. [CrossRef]

(C) 2019 by the authors. Licensee MDPI, Basel, Switzerland. This article is an open access article distributed under the terms and conditions of the Creative Commons Attribution (CC BY) license (http://creativecommons.org/licenses/by/4.0/). 


\title{
Communication
}

\section{Dietary Support in Elderly Patients with Inflammatory Bowel Disease}

\author{
Piotr Eder *, ${ }^{*}$, Alina Niezgódka ${ }^{\dagger}$, Iwona Krela-Kaźmierczak, Kamila Stawczyk-Eder, \\ Estera Banasik and Agnieszka Dobrowolska \\ Department of Gastroenterology, Dietetics and Internal Medicine, Poznan University of Medical Sciences, \\ Heliodor Święcicki Hospital, 60-355 Poznań, Poland; niezgodkaalina@gmail.com (A.N.); krela@op.pl (I.K.-K.); \\ kamilastawczyk@wp.pl (K.S.-E.); esta717@gmail.com (E.B.); agdob@ump.edu.pl (A.D.) \\ * Correspondence: piotr.eder@op.pl; Tel.: +4-869-805-0797 or +4-861-869-1343 \\ + These two authors contribute equally to this paper.
}

Received: 30 April 2019; Accepted: 21 June 2019; Published: 24 June 2019

\begin{abstract}
Ageing of the human population has become a big challenge for health care systems worldwide. On the other hand, the number of elderly patients with inflammatory bowel disease (IBD) is also increasing. Considering the unique clinical characteristics of this subpopulation, including many comorbidities and polypharmacy, the current therapeutic guidelines for the management of IBD should be individualized and applied with caution. This is why the role of non-pharmacological treatments is of special significance. Since both IBD and older age are independent risk factors of nutritional deficiencies, appropriate dietary support should be an important part of the therapeutic approach. In this review paper we discuss the interrelations between IBD, older age, and malnutrition. We also present the current knowledge on the utility of different diets in the management of IBD. Considering the limited data on how to support IBD therapy by nutritional intervention, we focus on the Mediterranean and Dietary Approaches to Stop Hypertension diets, which seem to be the most beneficial in this patient group. We also discuss some new findings on their hypothetical anti-inflammatory influence on the course of IBD.
\end{abstract}

Keywords: inflammatory bowel disease; malnutrition; Mediterranean diet; older age

\section{Introduction}

The frequency of Crohn's disease (CD) and ulcerative colitis (UC), two forms of inflammatory bowel disease (IBD), is increasing worldwide [1-3]. Simultaneously with the demographic ageing of the human population observed in recent decades, especially in developed countries, the number of elderly IBD patients is also increasing [4-8]. Considering the definition of elderly as aged 60 years and above, it is estimated that $25-35 \%$ of CD and UC patients meet this criterion. These data encompass both those who were diagnosed before reaching 60 and those who were diagnosed when over 60 (elderly onset). The latter group, representing $10-15 \%$ of all IBD patients, reflects the second peak of CD and UC morbidity [9]. UC is the more frequent IBD subtype in this age range, since one in eight UC patients is older than 60, compared to one in $20 \mathrm{CD}$ cases [9]. A population-based cohort study by Charpentier et al. revealed that, among elderly people suffering from IBD, $65 \%$ are between 60 and 70 years old, $25 \%$ between 70 and 80 years old, and $10 \%$ are older than 80 [10].

There is an increasing body of evidence showing several differences in the clinical course and management of elderly IBD patients, compared with those suffering from UC or CD at a younger age. One of the most important characteristics is a tendency for less aggressive therapeutic regimens [11-13]. On the other hand, the significance of non-pharmacological and non-surgical interventions is higher. Since both IBD and older age are independent risk factors of nutritional deficiencies, appropriate dietary support is needed, especially in the cases of patients older than 60 with UC or CD $[12,14,15]$. In this 
review, we discuss the influence of older age on the physiology of the gastrointestinal tract and the mechanisms leading to malnutrition, especially in the context of IBD in the elderly. We summarize the main differences in the clinical course and management of IBD in the elderly with special emphasis on the role of diet. We also present some recommendations for nutritional support for this unique population.

In order to analyze the current literature on dietary support among elderly IBD patients, we searched the PubMed and Web of Science databases using the key words "diet and IBD", "elderly IBD", "diet in elderly people", and "nutrition in elderly IBD patients". We identified mainly review papers, meta-analyses, and guidelines published after 2010. Moreover, we analyzed conference abstracts from the Congresses of the European Crohn's and Colitis Organisation (ECCO) in 2018 and 2019.

\subsection{Elderly IBD Patient_Differences in Clinical Course and Management}

There are several characteristic features of elderly IBD. The delay in the diagnosis of CD or UC is longer than that in younger patients. This is related to less specific symptoms and to a frequent co-existence of other comorbidities and polypharmacy. Differential diagnosis encompasses many entities like ischemic colitis, infectious diseases, drug adverse reactions (non-steroidal anti-inflammatory drugs, anticoagulation, anti-platelet drugs, chemotherapy, etc.), diverticulitis, radiation colitis, and microscopic colitis. Performing invasive diagnostic investigations (like colonoscopy) can also be challenging, since older age, other comorbidities and drugs used are risk factors for severe complications $[5,6,16,17]$.

The clinical course of elderly IBD seems to be less aggressive $[6,8]$. In $C D$, there is a higher frequency of colonic location $[16,18]$. On the other hand, complications like strictures or perianal involvement and extraintestinal manifestations are less common. As a result, the clinical presentation of elderly CD can be similar to UC, with rectal bleeding as a main symptom. Abdominal pain, weight loss, and diarrhea are less typical [16,18-20]. In the case of elderly UC, the predominant location is E2 or E3 according to the Montreal classification, whereas isolated proctitis is rare [20,21]. The disease is more stable over time and there is a low frequency of proximal disease colonic extension $[19,20]$. The need for a colectomy is also relatively low. A French population-based registry (EPIMAD) showed that only $16 \%$ of elderly onset UC patients underwent a colectomy in a ten-year follow-up period [6,8,22-24].

Despite a milder clinical course in long-term observation, the first IBD episode can be paradoxically more severe than in younger patients [18]. Older age also seems to be related to a more frequent hospitalization rate in IBD $[18,20]$. A study by Ananthakrishnan et al. revealed that hospitalized IBD patients older than 65 are at a higher risk of significant malnutrition, anaemia, and hypovolemia [25]. The frequency of thromboembolic complications is increased due to hypercoagulability, dehydration, prolonged bed rest, and immobilization. This is why the hospitalization of elderly IBD patients seems to be connected with higher fatality [18,26-29].

Although there are no randomized, controlled trials assessing the therapeutic strategies for IBD in the elderly, medical and surgical management is often different from patients of a younger age [30-34]. The usage of many medications is limited due to their higher toxicity (e.g., corticosteroids), the risk of interactions (e.g., thiopurines with allopurinol, mesalamine with anticoagulants), contraindications (e.g., renal insufficiency in the case of mesalamine, severe congestive heart failure in the case of anti-tumor necrosis factor alpha antibodies), and higher rates of adverse events (e.g., serious infections, diabetes, arterial hypertension, mental disorders in the case of corticosteroids or neoplastic complications in the case of thiopurines) [7,35-53]. The safety of newly registered immunosuppressive molecules and biological agents (tofacitinib, anti-integrins-vedolizumab, or anti-IL-12/23 antibodies-ustekinumab) in elderly IBD patients has not been studied at all [54].

General indications for surgery in IBD patients aged $>60$ are similar, when compared with the younger subgroup, however, a decision to use surgical intervention should be taken with caution since there is a higher risk of post-operative complications and mortality $[5,11]$. Nevertheless, in many cases surgery is inevitable, which is why, in order to improve therapeutic outcomes, optimal treatment 
should be applied preoperatively, with minimization of corticosteroid use and extensive nutritional support [5,11,55-57].

The rules for disease monitoring in elderly IBD patients should be also adjusted for age and concomitant morbidities. Since repeated endoscopic assessment is often impossible, the importance of non-invasive markers of inflammatory activity, like fecal calprotectin or C-reactive protein, is high $[58,59]$. In terms of cross-sectional imaging methods, repeated computed tomography or magnetic resonance (MR) imaging can be difficult and, in many cases, contraindicated [60]. This is due to the fact that a significant proportion of older patients suffer from renal insufficiency or are at a high risk of this complication, which makes the administration of an intravenous contrast agent impossible. Another limitation for MR imaging is the high frequency of metallic implants (e.g., after a total hip or knee replacement or after the implantation of a cardiac rhythm control device) in elderly people. This is why more common use of an abdominal ultrasound should be advised for the objective assessment of morphological abnormalities in the gastrointestinal tract [60].

\subsection{Ageing, IBD, and Malnutrition-What Are the Connections?}

As discussed above, there are many limitations for the routine application of classical therapeutic approaches in the case of IBD in elderly patients. Thus, non-pharmacological and non-surgical interventions are of great importance. The role of dietary support is especially high, since ageing by itself increases the risk of malnutrition. Epidemiological analyses show that $5 \%-20 \%$ of European citizens aged 60 and older suffer from malnutrition, while for hospitalized patients or those in long-term care, these numbers are even higher [61]. Thus, obligatory assessment of the nutritional status of all older patients is recommended by both the American Society for Parenteral and Enteral Nutrition (ASPEN) and the European Society for Parenteral and Enteral Nutrition (ESPEN). A Mini Nutritional Assessment (MNA) is believed to be the most appropriate tool for this purpose [62-64].

The etiology of malnutrition in older people is multifactorial. There are multiple medical conditions associated with a high risk of weight loss and nutritional deficiencies, like cancer, pulmonary disorders (chronic obstructive pulmonary disease), diabetes, cerebrovascular and neurological diseases, and gastrointestinal disorders. Many of those conditions are characterized by an increased catabolism, loss of appetite, and dysphagia. Multimorbidity and polypharmacy-typical phenomena among elderly people-are also connected with higher hospitalization rates, and increased probability of significant drug interactions [65]. These factors can independently promote malnutrition [65-67]. Another important problem is poor oral health and dental status leading to chewing difficulties and mouth dryness, which can cause lower food intake [68,69]. Depression, anxiety, dementia, and many other neuropsychological factors can result in unintentional weight loss and nutritional deficiencies [70-72]. There are also many social determinants of malnutrition risk, like poverty, loneliness and isolation, an inability to shop or cook, secondary to cognitive disorders and/or physical disability $[73,74]$. Interestingly, although ageing per se is not always associated with malnutrition, there are several physiological phenomena increasing the risk of weight loss. Decreasing appetite among elderly and otherwise healthy people can be explained by a reduction in stomach capacity and impairment of gastric relaxation, accompanied by lower gastric emptying. One of the etiological hypotheses for these processes in older people is fluctuation in the production and secretion of several enterohormones. There are data suggesting that higher levels of cholecystokinin and lower concentration of ghrelin can contribute to early satiation after food consumption. Moreover, degenerative processes in the gastrointestinal tract can result in the reduction in the number of taste buds, which can be accompanied by a deterioration in the sense of smell. These phenomena can additionally demotivate the patients to consume regularly [75-77].

Since the mechanisms underlying malnutrition in elderly people are complex, three types of weight loss proposed by Roubenoff can coexist: wasting, cachexia, and sarcopenia [78,79]. Wasting is associated with inadequate dietary intake and results in involuntary weight loss [80]. Cachexia is caused by induced catabolic processes, with pro-inflammatory cytokines like interleukin-1 (IL-1), tumor necrosis factor-alpha (TNF-alpha), IL-6, and others having a predominant role. The main 
consequence of cachexia is a decrease in fat-free mass and body cell mass [81]. Sarcopenia is defined as a loss of muscle mass $[78,79,82]$. The etiology of this phenomenon is poorly understood, however, a dominant role is hypothetically played by a lack of physical activity, induction of the pro-inflammatory response, and dysregulation of anabolic hormones, like testosterone or growth hormone $[83,84]$.

IBD is independently associated with an increased risk of malnutrition. Epidemiological data shows that $65 \%-75 \%$ of CD patients and $18 \%-62 \%$ of UC patients have nutritional deficiencies [85]. The discrepancies in these numbers are a consequence of the different definitions of malnutrition. Body mass index (BMI) is among the most frequently used criteria, but it has been widely criticized recently, since it does not take into account several qualitative and quantitative parameters like the relation between fat and muscle mass, the concentration of micro- and macronutrients, recent changes in body mass or disease activity [86]. There are many data showing that low body mass is only one of the dimensions reflecting malnutrition in IBD. Among other parameters, which have been frequently reported, are deficiencies in iron, calcium, selenium, vitamin $\mathrm{D}$ and/or vitamin $\mathrm{K}$ [85].

The etiology of malnutrition in IBD is complex. It encompasses disease-related and treatment-related factors [85]. In the first group, a decrease in food intake seems to be the most important. This phenomenon can be related to IBD symptoms, like nausea, vomiting, abdominal pain, diarrhea, fever or fatigue. Also, it has been shown that hospitalization is associated with a higher risk of inappropriate food intake due to a frequent need to fast in preparation for different investigations or due to an inadequate hospital diet [85,87]. Moreover, disease activity by itself, with the production of multiple pro-inflammatory cytokines, induces catabolic processes, and promotes increased energy expenditure, contributing to malnutrition. The absorptive functions of the gastrointestinal tract are also impaired due to bowel wall damage with a loss of epithelial integrity, bacterial overgrowth, and increased intestinal motility. The same factors contribute to enhanced nutrient loss $[85,88]$. Considering the treatment-related causes of malnutrition, there are data on the negative impact of steroids on body composition. Moreover, nitroimidazoles or immunosuppressive drugs (thiopurines, methotrexate) can also alter the appetite, leading to reduced food intake [85]. On the other hand, multiple surgical resections limit the absorptive gastrointestinal surface, in spite of the high compensatory potential of the remaining parts of the intestines [85].

Taking into consideration the complex etiology and high frequency of malnutrition among elderly people and IBD patients analyzed separately, the significance of this phenomenon among CD and UC patients aged 60 and older becomes especially challenging. In order to prevent and/or adequately treat this unique subpopulation, proper dietary support is needed.

\subsection{The Role of Diet in IBD and the Elderly}

There are no strict dietitian recommendations for patients with IBD, since there are insufficient data for promoting any special diet. The general rule is that patients should cover their energy demand by eating well-balanced meals containing complex carbohydrates, proteins and fats, mainly of plant origin, rich in vegetables and fruits, with the elimination of highly processed foods [89]. Special attention should be paid to appropriate iron and vitamin D consumption. In each case, however, a highly individualized recommendation should be defined in order to adjust the nutritional needs to a concrete, clinical scenario, especially in older people.

According to current knowledge, in the case of physiological ageing special recommendations should be given for protein, vitamin D, and water consumption. In order to maintain muscle mass, the PROT-AGE study group defined the daily protein requirement, which is $1.0-1.2 \mathrm{~g}$ protein $/ \mathrm{kg}$ body weight [90]. Moreover, all individuals should supplement vitamin D3 in a dose of 800-2000 IU per day [91]. Adults should drink $30-35 \mathrm{~mL} / \mathrm{kg}$ body weight (at least $1500 \mathrm{~mL} /$ day or $1-1.5 \mathrm{~mL} / 1 \mathrm{kcal}$ ) of water (preferably medium-carbonized and still water). Since there is an increased risk of dehydration among older people, the recommendations for daily water consumption in this subpopulation (similar to the pediatric population) are more precisely defined as $100 \mathrm{~mL}$ of water for the first $10 \mathrm{~kg}$, then $50 \mathrm{~mL}$ for second $10 \mathrm{~kg}$, and 15-20 mL for each additional kilogram of body weight [92,93]. In addition, 
older adults are in the groups at risk of vitamin B12 deficiency. The usual dietary sources of vitamin B12 are animal products, including fish, meat, poultry, eggs, milk, and milk products. Vitamin B12 is generally not present in plant foods, but a lot of these products are fortified. The vitamin B12 recommended dietary allowance for older adults is $2.4 \mu \mathrm{g} /$ day $[94,95]$.

In the case of high IBD activity, especially in patients with severe diarrhea and abdominal pain due to stricturing CD, it is advisable to avoid a high intake of fiber and lactose, in order to prevent bacterial overgrowth and reduce the number of bowel movements [96]. The daily protein requirement is $1.2-1.5 \mathrm{~g}$ protein $/ \mathrm{kg}$ body weight [96]. Resting energy expenditure during a flare is $25-30 \mathrm{kcal} / \mathrm{kg}$ standard body weight [96-98]. Moreover, according to the ESPEN recommendations, oral nutrition supplements (ONS) should be considered in addition to a normal diet for the treatment of nutritional deficiencies in the case of IBD exacerbation [96]. ONS contain high amounts of all (complete) or selected (incomplete) macro- and microelements in relatively small volume products. ONS can be also divided into two categories: standard ONS which contain different nutritional compounds in proportions characteristic for a normal oral diet and specific ONS which is composed adequately for some particular patient populations (e.g., Parkinson's disease, Alzheimer's disease, etc.). ONS can be used together with meals; they contain no lactose, gluten, purines or cholesterol and should be considered in each case of increased malnutrition risk or diagnosed malnutrition [96,99].

Recent years, however, have brought plenty of data about the crucial role of impaired microbiota in the pathogenesis of intestinal inflammation. Moreover, there is a growing body of evidence that also ageing is associated with changes in intestinal microbiota composition. In 2007 the ELDERMET consortium was established to investigate this topic [100]. They found (by using the pyrosequencing of $16 \mathrm{~S}$ rRNA method) that there was an increase in Bacteroidetes and a concomitant decrease in Firmicutes species among older people, however there was a significant inter-individual variability in the composition of elderly gut microbiota [101]. One of the reasons was the health status of the investigated subjects. The statistical analysis indicated a clear separation between community-dwelling subjects and long-stay home residents [101]. Another observation was that health status and the diversity of the intestinal microbiota in the ELDERMET study correlated with the patients' nutritional habits. It was shown that the diversity index of the fecal microbiota was significantly associated with a low-fat and high-fiber diet [101]. It is, however, still not known whether changes in gut microbiota are a result of dietary intervention or are more related to unhealthy ageing by itself.

Nevertheless, the possibility of shaping the intestinal microbiota by nutritional interventions would be very attractive. The hypothetical promotion of a "healthy" in-vironment (microbiota) by environmental factors (diet) seems to be an interesting concept for therapeutic intervention also in IBD. This is why dietary intervention is currently considered not only in the context of sufficient nutritional support, but also as a potential modulator of intestinal inflammation [102,103]. Our understanding of the link between nutrition, intestinal microbiota, and inflammatory response is still poor, however, due to the development of new technologies such as metabolic profiling and next-generation DNA sequencing, we know that microbiota composition changes after exposure to different modifying factors [104]. For example, there are data showing that a high-fat and low-fiber diet, as well as an animal-based diet, increase the abundance of Bacteroidetes and Prevotella, which are believed to participate in the development of chronic inflammation in the gastrointestinal tract [105]. On the other hand, dietary fiber can promote short-chain fatty acids synthesis by colonic microbiota, which can lead to the suppression of pro-inflammatory cytokines from dendritic cells and macrophages [104,106,107]. Another hypothetical association between the diet and inflammation is the epigenetic regulation of gene expression by different nutritional components. There is some evidence that the typical Western diet, deficient in micronutrients, like selenium and folate, can influence DNA methylation, which promotes pro-inflammatory phenomena and seems to increase colorectal cancer susceptibility [104]. What is more, in an experimental model of IBD it was shown that selenium supplementation prevented tissue damage through interfering with the expression of the key genes responsible for inflammation [104,108]. Nevertheless, although these concepts of the associations between diet, microbiota, and inflammatory 
response are very promising, we are still not able to translate this knowledge into clinical practice. We hope that it will be possible in the future to modulate our microbiota by changing the in-vironmental milieu via nutritional intervention, but we still need more data.

Among different diets already studied in the context of IBD, main attention is being paid to the low-fermentable oligosaccharide, disaccharide, monosaccharide, and polyol (FODMAP) and anti-inflammatory diet (IBD-AID), although supporting scientific evidence is relatively poor [104]. Recently, the advantages of the Mediterranean or the Dietary Approach to Stop Hypertension (DASH) diets in the context of chronic inflammation have also been discussed $[105,106]$.

The main rule of the low-FODMAP diet is to exclude highly fermentable and poorly absorbed carbohydrates and polyols. In this diet, consumption of different food types is strongly discouraged, such as many fruits (e.g., apple, blackberry, grapefruit, mango, nectarine, peach, plum, watermelon), vegetables (e.g., artichoke, asparagus, avocado, onion, cabbage, garlic, leek, pea), dairy (e.g., cow, goat, sheep, condensed and evaporated milk), beverages (e.g., green tea, soft drinks, white tea, coconut water) and many nuts, seeds and legumes. Moreover, breaded meat or meat made with high fructose corn syrup should be avoided. This is not a long-term diet and the dietary limitations should last for only 6-8 weeks. Then patients should gradually restart foods high in FODMAPs in order to establish an individual tolerance to specific oligosaccharides, disaccharides, monosaccharides, and polyols. The utility of the low-FODMAP diet has been shown mainly for patients with irritable bowel syndrome (IBS), since there is a hypothesis that high fermentation in the gastrointestinal lumen can lead to increased intestinal permeability and provoke intestinal hypersensitivity in a genetically susceptible host $[107,108]$. Data on the usefulness of this diet in IBD are limited and mainly come from retrospective cohorts. It is advised that a low-FODMAP diet can be used in selected IBD patients with IBS-like symptoms in addition to conventional therapy, but only under strict dietitian supervision [104-115].

IBD-AID is a multistep and highly individualized dietary intervention, limiting some specific carbohydrates (e.g., refined sugar, gluten-based grains, certain starches). Olendzki et al., who developed IBD-AID, hypothesized that this can decrease the growth of several pro-inflammatory bacteria in the gastrointestinal tract, preventing dysbiosis [116]. In the next step, the patient should ingest prebiotics and probiotics (e.g., leek, onion, fermented food) to promote restoration of the microbiota. Moreover, the consumption of total and saturated fat, and hydrogenated oils should be avoided, together with the individual identification of dietary intolerances and nutritional deficiencies. The rules of IBD-AID were first published in 2017 and until now there were no randomized, controlled trials conducted in order to confirm the initial, promising reports on the use of this diet as an adjunct therapy for the treatment of IBD [104,116].

Lack of sufficient data for the usefulness of the low-FODMAP diet and IBD-AID in IBD result in a high skepticism of clinicians to promote this kind of dietary intervention. In the case of elderly IBD patients, another important limitation for the use of these diets is their complexity. Moreover, there is a high risk of several nutritional deficiencies due to the restriction and avoidance of different foods, especially when the dietary intervention is conducted without professional support. This can have serious negative consequences, considering the general increased risk of malnutrition and the presence of serious comorbidities in older people. This is why it seems to be more reasonable to promote safer diets, with more robust data in the context of elderly patients.

Considering the nutritional requirements and characteristics of elderly patients with IBD discussed above, as well as the most common disorders among older people (arterial hypertension and other cardiovascular diseases, type 2 diabetes, hypercholesterolemia), the DASH or Mediterranean diet could be recommended for this unique population. The main restrictions in the DASH diet concern carbohydrates and fats, in particular by limiting simple carbohydrates (glucose, fructose, saccharose) and reducing the intake of saturated fats. This means a significant reduction in the consumption of sweets, sugar confectionery, sweeteners, fruit preservatives (less than five portions per week), as well as red meat and highly processed food. Vegetables and fruits should be eaten $4-5$ times/day, and whole grain products 6-8 times/day. The DASH diet also includes medium-fat dairy products 
(2-3 portions/day), however, this needs to be accompanied with regular consumption of vegetable oils (preferably raw, inter alia, to enable the absorption of fat-soluble vitamins). The recommended frequency for eating fatty saltwater fish (herring, salmon, mackerel, halibut, sardine, codfish, flounder) is 2-4 times/week. Different seeds, nuts, legumes are also an important part of the DASH diet, since they contain (similar to vegetable oils—linseed, soybean or rapeseed oil- and fatty saltwater fish) high amounts of omega-3 polyunsaturated fatty acids (PUFA). Another main rule of this type of diet is a significant reduction in salt (sodium) consumption [117].

Although there are no data on the utility of the DASH diet in IBD, its beneficial effect on general health status and cardiovascular risk is well known [118-121]. Moreover, Nilsson et al. showed that adherence to a DASH-style diet was significantly associated with a lower clustered metabolic risk among older women, and it promoted a systemic anti-inflammatory environment, independently of physical activity [122]. The authors concluded that the DASH diet should be considered as a key target for nutritional intervention among elderly people to prevent age-related metabolic abnormalities.

The general recommendations of the Mediterranean diet are very similar to DASH. It emphasizes eating primarily plant-based foods (fruits, vegetables, whole grains, legumes, nuts, seeds, olive oil), which should be consumed several times per day, together with dairy products (mainly different types of cheese, yogurts). Low or moderate alcohol drinking (preferably red wine with a meal) is also advised. Fish, eggs, and poultry can be consumed several times per week. In contrast, consumption of sweets and red meat should be significantly reduced (a few times per month). The details of the Mediterranean diet can vary depending on the region of the Mediterranean Basin; however, the general rule is to eat foods coming from this geographic area [123,124].

In contrast to the DASH diet, there are some data on the usefulness of Mediterranean diet in IBD. Marlow et al. demonstrated that even a short-term (six weeks) nutritional intervention is beneficial for patients with $\mathrm{CD}$, decreasing the concentration of several pro-inflammatory markers with a trend to normalize the composition of intestinal microbiota. Transcriptomics analyses confirmed small changes in many genes, providing a cumulative anti-inflammatory effect of the diet [125]. In another study, Godny and colleagues showed that the Mediterranean diet is associated with decreased fecal calprotectin in patients after pouch surgery in UC, which is accompanied by an improvement in gut microbiota composition [126]. Moreover, Molendijk et al. demonstrated a beneficial effect of long-term nutritional intervention in IBD. In this study, six months of the Mediterranean diet improved the quality of life and reduced CRP levels. The level of improvement was associated with adherence to the rules of this type of diet [127].

The question remains, which hypothetical mechanisms could be related to the anti-inflammatory properties of the Mediterranean diet in IBD. As discussed above, this diet is characterized by a low intake of omega-6 PUFA, high intake of omega-3 PUFA, and dietary fiber, which seems to be important in the context of IBD. In line with that, the most recent epidemiological data indicate that a higher ratio of omega-6/omega-3 PUFA in the diet can be associated with an increased UC incidence [128]. Moreover, Hou et al. noted that a high intake of omega-6 PUFA, saturated fats, and meat is correlated with an increased risk of developing UC and CD [129]. It was also shown in a murine dextran sulfate sodium (DSS)-induced colitis model that omega-6/omega-3 PUFA ratio in the diet can influence the inflammatory processes in the gastrointestinal tract [130]. The authors observed that an $\alpha$-linolenic acid (ALA)-enriched diet with a decreased uptake of linoleic acid (LA) resulted in less severe colitis in mice, with a markedly alleviated intestinal inflammation [130]. This was supported by Pearl et al. who showed the association between severity of intestinal inflammation and increased content of omega-6 PUFA in inflamed mucosa in UC patients [131]. Furthermore, Uchiyama et al. investigated the influence of a diet therapy involving the use of an "omega-3 PUFA food exchange table". The authors showed that omega-3 PUFA significantly increased the erythrocyte membrane omega-3/omega-6 PUFA ratio in IBD patients, what was associated with clinical remission of the disease [132]. Recently, another experimental study on the protective role of omega-3 PUFA has been published. Charpentier et al. showed that supplementation of omega-3 PUFA significantly decreased 
colon inducible nitric oxide synthase (iNOS) and cyclooxygenase-2 (COX-2) expression, as well as IL-6 and leukotriene B4 production in 2,4,6-trinitrobenzene sulfonic acid (TNBS)-induced colitis [133].

Dietary fiber is also believed to have a protective effect on the development of inflammation in the gastrointestinal tract. Moreover, the short-chain fatty acids, regarded as one of the major microbial metabolites of dietary fiber, have the potential to improve intestinal mucosal immunity and maintain homeostasis [134]. There are several experimental and clinical data supporting these hypotheses. Liu et al. showed in a murine DSS-induced colitis model that supplementation of $\beta$-glucans at a dose of $500 \mathrm{mg} / \mathrm{kg}$ per day reduced the severity of clinical activity of the disease. $\beta$-glucans-enriched diet resulted in a smaller weight loss, improvement in the number of bowel movements, and amelioration of the inflammatory response assessed microscopically. It has been also shown that $\beta$-glucans supplementation inhibited the expression of pro-inflammatory proteins, such as TNF- $\alpha$, IL-1, IL-6 or NOS [135]. On the other hand, based on the data from the Nurses' Health Study, it was suggested in a prospective study that a long-term intake of dietary fiber was associated with lower risk of CD, but not UC [136]. A meta-analysis, performed by Liu and colleagues, indicated that the intake of dietary fiber was related to a decreased risk of developing IBD [137]. In a recent study by Andersen et al. an inverse association between the consumption of cereal fiber and $\mathrm{CD}$ in non-smokers was confirmed [138].

The only theoretical limitation of the DASH or Mediterranean diet in IBD is the high amount of whole grain cereal products, nuts, and seeds of leguminous plants which can stimulate intestinal peristalsis and increase the frequency of bowel movements. This is why it is advised to reduce the consumption of these particular foods during an IBD flare, whereas in patients in remission the individually tolerated amount of these products should be established.

The main rules of DASH and the Mediterranean diet are presented in Tables 1 and 2.

Table 1. The rules of Dietary Approaches to Stop Hypertension (DASH) diet [117].

\begin{tabular}{|c|c|c|c|}
\hline \multicolumn{4}{|c|}{ The DASH Diet (Dietary Approaches to Stop Hypertension) } \\
\hline Dietary Product & $\begin{array}{l}\text { The Frequency of } \\
\text { Consumption }\end{array}$ & Indicated & Contraindicated \\
\hline Cereal products & 6-8/day & whole grain & refined \\
\hline Vegetables & 4-5/day & all & - \\
\hline Fruits & 4-5/day & all & - \\
\hline Protein & 6 or less/day & $\begin{array}{l}\text { fatty saltwater fish, lean meat, } \\
\text { seeds of leguminous plants }\end{array}$ & fatty, red meat \\
\hline Nuts and seeds & $4-5 /$ week & all & - \\
\hline Fats & 2-3/day & $\begin{array}{l}\text { vegetable oils rich in } \\
\text { unsaturated fatty acids }\end{array}$ & animal fat, coconut oil, palm oil \\
\hline Dairy products & 2-3/day & low-fat or fat-free & full-fat \\
\hline Drinks & several times a day & unspecified & $\begin{array}{c}\text { drinks containing } \\
\text { simple carbohydrates }\end{array}$ \\
\hline \multicolumn{4}{|c|}{ Other } \\
\hline $\begin{array}{l}\text { Sweets, confectionery } \\
\text { products }\end{array}$ & 5 or less/week & - & - \\
\hline Sodium & Max. 2300 mg/day & - & - \\
\hline
\end{tabular}


Table 2. The rules of the Mediterranean diet [124].

\begin{tabular}{|c|c|c|c|}
\hline \multicolumn{4}{|c|}{ The Mediterranean Diet } \\
\hline Dietary Product & $\begin{array}{l}\text { The Frequency of } \\
\text { Consumption }\end{array}$ & Indicated & Contraindicated \\
\hline Cereal products & several times a day & whole grains & refined \\
\hline Vegetables & several times a day & all & - \\
\hline Fruits & several times a day & all & - \\
\hline Fish and seafood & $\begin{array}{l}\text { several times a week } \\
\text { (at least } 2 \text { times a week) }\end{array}$ & $\begin{array}{l}\text { fatty saltwater fish (tuna, salmon, } \\
\text { sardines, herring) and mussels, } \\
\text { oysters and shrimps }\end{array}$ & - \\
\hline Poultry and eggs & several times a week & all & - \\
\hline Red meat & a few times a month & - & - \\
\hline $\begin{array}{l}\text { Nuts and seeds of } \\
\text { leguminous plants }\end{array}$ & several times a day & all & - \\
\hline Fats & several times a day & olive oil & $\begin{array}{l}\text { animal fats such as lard, } \\
\text { butter, fatty beef, fatty pork, } \\
\text { poultry with skin }\end{array}$ \\
\hline Dairy products & several times a day & all & - \\
\hline Drinks & several times a day & still water & sugary drinks \\
\hline $\begin{array}{l}\text { Sweets, confectionery } \\
\text { products }\end{array}$ & few times a week & - & - \\
\hline Red wine & $\begin{array}{l}\text { every day; women max. } 1, \\
\text { men max. } 2 \text { glasses/day }\end{array}$ & - & - \\
\hline
\end{tabular}

\section{Conclusions}

The ageing of the human population has become a big challenge for health care systems worldwide. The increasing proportion of elderly people is a result of significant improvements in medical care, successful prophylaxis of infectious diseases, and declining birth rates in developed countries. On the other hand, the number of elderly IBD patients is also increasing and we have to face the problem of managing this unique population. Since there are several important differences in the clinical characteristics of older IBD patients, appropriate nutritional intervention and counseling should become a crucial element of the therapy. Although there are no data on the definite therapeutic influence of any diet on the course of IBD, it seems to be reasonable, considering data presented in this paper, to actively promote a healthy diet among elderly patients with IBD with special emphasis on the DASH or Mediterranean-style diet. Patients with IBD aged $>60$ are also at increased risk of cardiovascular diseases, type 2 diabetes, and arterial hypertension. This is why these two similar types of nutrition can cover not only the dietary requirements characteristic of a chronic inflammatory condition, but also due to its anti-inflammatory properties, they can improve the metabolic abnormalities typical in older age. Of course, it seems rational to advocate these types of diets only in parallel with classical treatment of IBD and even regardless of subsequent gastrointestinal disorders or any other disease. Nevertheless, since application of the current, aggressive therapeutic approaches in a significant proportion of elderly IBD patients is limited, the use of the Mediterranean and DASH diets is reasonable, especially in this unique population.

Author Contributions: Conceptualization, P.E. and A.N.; formal analysis, P.E. and I.K.-K.; resources, P.E., A.N., K.S.-E., and E.B.; writing-original draft preparation, P.E. and A.N.; writing-review and editing, I.K.-K., K.S.-E., and E.B.; visualization, P.E. and. A.N.; supervision, P.E. and A.D.; project administration, P.E. and A.N.; funding acquisition, P.E. and. A.D.

Funding: This research received no external funding.

Conflicts of Interest: P.E. received lecture fees from Abbvie Poland and travel grants from Astellas, Abbvie Poland, Janssen and Ferring; K.S.-E. received travel grants from Alvogen and Janssen; I.K.-K. received travel grants from Alvogen, Abbvie and Astellas; A.D. received travel grants from Alvogen, Abbvie and Astellas and lecture fees from Abbvie Poland and Alvogen. A.N. and E.B. declare no conflicts of interest. 


\section{References}

1. Andersen, V.; Chan, S.; Luben, R.; Khaw, K.T.; Olsen, A.; Tjonneland, A.; Kaaks, R.; Grip, O.; Bergmann, M.M.; Boeing, H.; et al. Fiber intake and the development of inflammatory bowel disease: A European prospective multi-centre cohort study (EPIC-IBD). J. Crohns Colitis 2018, 12, 129-136. [CrossRef] [PubMed]

2. Nimmons, D.; Limdi, J.K. Elderly patients and inflammatory bowel disease. World J. Gastrointest. Pharmacol. Ther. 2016, 7, 51-65. [CrossRef] [PubMed]

3. Cosnes, J.; Gower-Rousseau, C.; Seksik, P.; Cortot, A. Epidemiology and natural history of inflammatory bowel diseases. Gastroenterology 2011, 140, 1785-1794. [CrossRef] [PubMed]

4. Molinié, F.; Gower-Rousseau, C.; Yzet, T.; Merle, V.; Grandbastien, B.; Marti, R.; Lerebours, E.; Dupas, J.L.; Colombel, J.F.; Salomez, J.L.; et al. Opposite evolution in incidence of Crohn's disease and ulcerative colitis in Northern France (1988-1999). Gut 2004, 53, 843-848. [CrossRef] [PubMed]

5. Molodecky, N.A.; Soon, I.S.; Rabi, D.M.; Ghali, W.A.; Ferris, M.; Chernoff, G.; Benchimol, E.; Panaccione, R.; Ghosh, S.; Barkema, H.W.; et al. Increasing incidence and prevalence of the inflammatory bowel diseases with time, based on systematic review. Gastroenterology 2012, 142, 46-54. [CrossRef] [PubMed]

6. Sturm, A.; Maaser, C.; Mendall, M.; Karagiannis, D.; Karatzas, P.; Ipenburg, N.; Sebastian, S.; Rizzello, F.; Limdi, J.; Katsanos, K.; et al. European Crohn's and colitis organisation topical review on IBD in the elderly. J. Crohns Colitis 2017, 11, 263-273. [CrossRef] [PubMed]

7. Katz, S.; Pardi, D.S. Inflammatory bowel disease of the elderly: Frequently asked questions (FAQs). Am. J. Gastroenterol. 2011, 106, 1889-1897. [CrossRef]

8. Taleban, S.; Colombel, J.F.; Mohler, M.J.; Fain, M.J. Inflammatory bowel disease and the elderly: A review. J. Crohns Colitis 2015, 9, 507-515. [CrossRef] [PubMed]

9. Del Val, J.H. Old-age inflammatory bowel disease onset: A different problem? World J. Gastroenterol. 2011, 17, 2734-2739. [CrossRef] [PubMed]

10. Hussain, S.W.; Pardi, D.S. Inflammatory bowel disease in the elderly. Drugs Aging 2010, 27, 617-624. [CrossRef]

11. Charpentier, C.; Salleron, J.; Savoye, G.; Fumery, M.; Merle, V.; Laberenne, J.E.; Vasseur, F.; Dupas, J.L.; Cortot, A.; Dauchet, L.; et al. Natural history of elderly-onset inflammatory bowel disease: A population based cohort study. Gut 2014, 63, 423-432. [CrossRef] [PubMed]

12. Arnott, I.; Rogler, G.; Halfvarson, J. The Management of Inflammatory Bowel Disease in Elderly: Current Evidence and Future Perspectives. Inflamm. Intest. Dis. 2018, 2, 189-199. [CrossRef] [PubMed]

13. Kedia, S.; Limdi, J.K.; Ahuja, V. Management of inflammatory bowel disease in older persons: Evolving paradigms. Intest. Res. 2018, 16, 194-208. [CrossRef]

14. Lobatón, T.; Ferrante, M.; Rutgeerts, P.; Ballet, V.; Van Assche, G.; Vermeire, S. Efficacy and safety of anti-TNF therapy in elderly patients with inflammatory bowel disease. Aliment. Pharmacol. Ther. 2015, 42, 441-445. [CrossRef] [PubMed]

15. Ha, C.Y.; Katz, S. Clinical implications of ageing for the management of IBD. Nat. Rev. Gastroenterol. Hepatol. 2014, 11, 128-138. [CrossRef] [PubMed]

16. Wagtmans, M.J.; Verspaget, H.W.; Lamers, C.B.; van Hogezand, R.A. Crohn's disease in the elderly: A comparison with young adults. J. Clin. Gastroenterol. 1998, 27, 129-133. [CrossRef] [PubMed]

17. Cohen, S.H.; Gerding, D.N.; Johnson, S.; Kelly, C.P.; Loo, V.G.; McDonald, L.C.; Pepin, J.; Wilcox, M.H. Clinical practice guidelines for Clostridium difficile infection in adults: 2010 update by the Society for Healthcare Epidemiology of America (SHEA) and the Infectious Diseases Society of America (IDSA). Infect. Control Hosp. Epidemiol. 2010, 31, 431-455. [CrossRef]

18. Harper, P.C.; McAuliffe, T.L.; Beeken, W.L. Crohn's disease in the elderly. A statistical comparison with younger patients matched for sex and duration of disease. Arch Intern. Med. 1986, 146, 753-755. [CrossRef]

19. Greth, J.; Török, H.P.; Koenig, A.; Folwaczny, C. Comparison of inflammatory bowel disease at younger and older age. Eur. J. Med. Res. 2004, 9, 552-554.

20. Polito, J.M.; Childs, B.; Mellits, E.D.; Tokayer, A.Z.; Harris, M.L.; Bayless, T.M. Crohn's disease: Influence of age at diagnosis on site and clinical type of disease. Gastroenterology 1996, 111, 580-586. [CrossRef]

21. Silverberg, M.S.; Satsangi, J.; Ahmad, T.; Arnott, I.D.; Bernstein, C.N.; Brant, S.; Caprilli, R.; Colombel, J.F.; Gasche, C.; Geboes, K.; et al. Toward an integrated clinical, molecular and serological classification 
of inflammatory bowel disease: Report of a Working Party of the 2005 Montreal World Congress of Gastroenterology. Can. J. Gastroenterol. Hepatol. 2005, 19, 5-36. [CrossRef] [PubMed]

22. Han, S.W.; McColl, E.; Barton, J.R.; James, P.; Steen, I.N.; Welfare, M.R. Predictors of quality of life in ulcerative colitis: The importance of symptoms and illness representations. Inflamm. Bowel Dis. 2005, 11, 24-34. [CrossRef] [PubMed]

23. Lakatos, P.L.; David, G.; Pandur, T.; Erdelyi, Z.; Mester, G.; Balogh, M.; Szipocs, I.; Molnar, C.; Komaromi, E.; Kiss, L.S.; et al. IBD in the elderly population: Results from a population-based study in Western Hungary, 1977-2008. J. Crohns Colitis 2011, 5, 5-13. [CrossRef] [PubMed]

24. Gower-Rousseau, C.; Vasseur, F.; Fumery, M.; Savoye, G.; Salleron, J.; Dauchet, L.; Turck, D.; Cortot, A.; Peyrin-Biroulet, L.; Colombel, J.F. Epidemiology of inflammatory bowel diseases: New insights from a French population-based registry (EPIMAD). Dig. Liver Dis. 2013, 45, 89-94. [CrossRef] [PubMed]

25. Ananthakrishnan, A.N.; Binion, D.G. Treatment of ulcerative colitis in the elderly. Dig. Dis. 2009, 27, 327-334. [CrossRef]

26. Loftus, E.V. A matter of life or death: Mortality in Crohn's disease. Inflamm. Bowel Dis. 2002, 8, 428-429. [CrossRef]

27. Wolters, F.L.; Russel, M.G.; Sijbrandij, J.; Ambergen, T.; Odes, S.; Riis, L.; Langholz, E.; Politi, P.; Qasim, A.; Koutroubakis, I.; et al. Phenotype at diagnosis predicts recurrence rates in Crohn's disease. Gut 2006, 55, 1124-1130. [CrossRef]

28. Foxworthy, D.M.; Wilson, J.A. Crohn's disease in the elderly. Prolonged delay in diagnosis. J. Am. Geriatr. Soc. 1985, 33, 492-495. [CrossRef]

29. Farrokhyar, F.; Swarbrick, E.T.; Irvine, E.J. A critical review of epidemiological studies in inflammatory bowel disease. Scand. J. Gastroenterol. 2001, 36, 2-15. [CrossRef]

30. Stepaniuk, P.; Bernstein, C.N.; Targownik, L.E.; Singh, H. Characterization of inflammatory bowel disease in elderly patients: A review of epidemiology, current practices and outcomes of current management strategies. Can. J. Gastroenterol. Hepatol. 2015, 29, 327-333. [CrossRef]

31. MacLaughlin, E.J.; Raehl, C.L.; Treadway, A.K.; Sterling, T.L.; Zoller, D.P.; Bond, C.A. Assessing medication adherence in the elderly: Which tools to use in clinical practice? Drugs Aging 2005, 22, 231-255. [CrossRef] [PubMed]

32. Hanauer, S.B. Clinical perspectives in Crohn's disease. Turning traditional treatment strategies on their heads: Current evidence for "step-up" versus "top-down". Rev. Gastroenterol. Disord. 2007, 7, 17-22.

33. Prelipcean, C.C.; Mihai, C.; Gogalniceanu, P.; Mihai, B. What is the impact of age on adult patients with inflammatory bowel disease? Clujul. Med. 2013, 86, 3-9. [PubMed]

34. Greenwald, D.A.; Brandt, L.J. Inflammatory Bowel Disease After Age 60. Curr. Treat Options Gastroenterol. 2003, 6, 213-225. [CrossRef] [PubMed]

35. De Boer, N.K.; Wong, D.R.; Jharap, B.; de Graaf, P.; Hooymans, P.M.; Mulder, C.J.; Rijmen, F.; Engels, L.G.; van Bodegraven, A.A. Dose-dependent influence of 5-aminosalicylates on thiopurine metabolism. Am. J. Gastroenterol. 2007, 102, 2747-2753. [CrossRef] [PubMed]

36. Gisbert, J.P.; Gomollón, F. Thiopurine-induced myelotoxicity in patients with inflammatory bowel disease: A review. Am. J. Gastroenterol. 2008, 103, 1783-1800. [CrossRef]

37. Wells, P.S.; Holbrook, A.M.; Crowther, N.R.; Hirsh, J. Interactions of warfarin with drugs and food. Ann. Intern. Med. 1994, 121, 676-683. [CrossRef] [PubMed]

38. Thomas, T.P. The complications of systemic corticosteroid therapy in the elderly. A retrospective study. Gerontology 1984, 30, 60-65. [CrossRef] [PubMed]

39. Akerkar, G.A.; Peppercorn, M.A.; Hamel, M.B.; Parker, R.A. Corticosteroid-associated complications in elderly Crohn's disease patients. Am. J. Gastroenterol. 1997, 92, 461-464.

40. Stallmach, A.; Hagel, S.; Gharbi, A.; Settmacher, U.; Hartmann, M.; Schmidt, C.; Bruns, T. Medical and surgical therapy of inflammatory bowel disease in the elderly-prospects and complications. J. Crohns Colitis 2011, 5, 177-188. [CrossRef]

41. Frey, B.M.; Frey, F.J. Clinical pharmacokinetics of prednisone and prednisolone. Clin. Pharmacokinet. 1990, 19, 126-146. [CrossRef] [PubMed]

42. Govani, S.M.; Higgins, P.D. Combination of thiopurines and allopurinol: Adverse events and clinical benefit in IBD. J. Crohns Colitis 2010, 4, 444-449. [CrossRef] [PubMed] 
43. Present, D.H.; Meltzer, S.J.; Krumholz, M.P.; Wolke, A.; Korelitz, B.I. 6-Mercaptopurine in the management of inflammatory bowel disease: Short- and long-term toxicity. Ann. Intern. Med. 1989, 111, 641-649. [CrossRef] [PubMed]

44. Black, A.J.; McLeod, H.L.; Capell, H.A.; Powrie, R.H.; Matowe, L.K.; Pritchard, S.C.; Collie-Duguid, E.S.; Reid, D.M. Thiopurine methyltransferase genotype predicts therapy-limiting severe toxicity from azathioprine. Ann. Intern. Med. 1998, 129, 716-718. [CrossRef]

45. Cuffari, C.; Dassopoulos, T.; Turnbough, L.; Thompson, R.E.; Bayless, T.M. Thiopurine methyltransferase activity influences clinical response to azathioprine in inflammatory bowel disease. Clin. Gastroenterol. Hepatol. 2004, 2, $410-417$. [CrossRef]

46. Siegel, C.A.; Marden, S.M.; Persing, S.M.; Larson, R.J.; Sands, B.E. Risk of lymphoma associated with combination anti-tumor necrosis factor and immunomodulator therapy for the treatment of Crohn's disease: A meta-analysis. Clin. Gastroenterol. Hepatol. 2009, 7, 874-881. [CrossRef]

47. Khan, N.; Abbas, A.M.; Lichtenstein, G.R.; Loftus, E.V.; Bazzano, L.A. Risk of lymphoma in patients with ulcerative colitis treated with thiopurines: A nationwide retrospective cohort study. Gastroenterology 2013, 145, 1007-1015. [CrossRef]

48. Beaugerie, L.; Brousse, N.; Bouvier, A.M.; Colombel, J.F.; Lémann, M.; Cosnes, J.; Hébuterne, X.; Cortot, A.; Bouhnik, Y.; Gendre, J.P.; et al. Lymphoproliferative disorders in patients receiving thiopurines for inflammatory bowel disease: A prospective observational cohort study. Lancet 2009, 374, 1617-1625. [CrossRef]

49. Cottone, M.; Kohn, A.; Daperno, M.; Armuzzi, A.; Guidi, L.; D’Inca, R.; Bossa, F.; Angelucci, E.; Biancone, L.; Gionchetti, P.; et al. Advanced age is an independent risk factor for severe infections and mortality in patients given anti-tumor necrosis factor therapy for inflammatory bowel disease. Clin. Gastroenterol. Hepatol. 2011, 9, 30-35. [CrossRef]

50. Colombel, J.F.; Loftus, E.V.; Tremaine, W.J.; Egan, L.J.; Harmsen, W.S.; Schleck, C.D.; Zinsmeister, A.R.; Sandborn, W.J. The safety profile of infliximab in patients with Crohn's disease: The Mayo clinic experience in 500 patients. Gastroenterology 2004, 126, 19-31. [CrossRef]

51. Fidder, H.; Schnitzler, F.; Ferrante, M.; Noman, M.; Katsanos, K.; Segaert, S.; Henckaerts, L.; Van Assche, G.; Vermeire, S.; Rutgeerts, P. Long-term safety of infliximab for the treatment of inflammatory bowel disease: A single-centre cohort study. Gut 2009, 58, 501-508. [CrossRef] [PubMed]

52. O'Meara, S.; Nanda, K.S.; Moss, A.C. Antibodies to infliximab and risk of infusion reactions in patients with inflammatory bowel disease: A systematic review and meta-analysis. Inflamm. Bowel Dis. 2014, 20, 1-6. [CrossRef] [PubMed]

53. Denadai, R.; Teixeira, F.V.; Saad-Hossne, R. Management of psoriatic lesions associated with anti-TNF therapy in patients with IBD. Nat. Rev. Gastroenterol. Hepatol. 2012, 9, 744. [CrossRef] [PubMed]

54. Singh, J.A.; Wells, G.A.; Christensen, R.; Tanjong Ghogomu, E.; Maxwell, L.; Macdonald, J.K.; Filippini, G.; Skoetz, N.; Francis, D.; Lopes, L.C.; et al. Adverse effects of biologics: A network meta-analysis and Cochrane overview. Cochrane Database Syst. Rev. 2011, 2, CD008794. [CrossRef] [PubMed]

55. Kaplan, G.G.; Hubbard, J.; Panaccione, R.; Shaheen, A.A.; Quan, H.; Nguyen, G.C.; Dixon, E.; Ghosh, S.; Myers, R.P. Risk of comorbidities on postoperative outcomes in patients with inflammatory bowel disease. Arch Surg. 2011, 146, 959-964. [CrossRef]

56. Bollegala, N.; Jackson, T.D.; Nguyen, G.C. Increased postoperative mortality and complications among elderly patients with inflammatory bowel diseases: An analysis of the national surgical quality improvement program cohort. Clin. Gastroenterol. Hepatol. 2016, 14, 1274-1281. [CrossRef] [PubMed]

57. Nguyen, G.C.; Bernstein, C.N.; Benchimol, E.I. Risk of Surgery and Mortality in Elderly-onset Inflammatory Bowel Disease: A Population-based Cohort Study. Inflamm. Bowel Dis. 2017, 23, 218-223. [CrossRef]

58. Cintolo, M.; Costantino, G.; Pallio, S.; Fries, W. Mucosal healing in inflammatory bowel disease: Maintain or de-escaleta therapy. World J. Gastrointest. Pathophysiol. 2016, 7, 1-16. [CrossRef]

59. Vrabie, R.; Kane, S. Noninvasive markers of disease activity in inflammatory bowel disease. Gastroenterol. Hepatol. 2014, 10, 576-584.

60. Kucharzik, T.; Kannengiesser, K.; Petersen, F. The use of ultrasound in inflammatory bowel disease. Ann. Gastroenterol. 2017, 30, 135-144. [CrossRef]

61. De La Montana, J.; Miguez, M. Suitability of the short-form Mini Nutritional Assessment in free-living elderly people in the northwest of Spain. J. Nutr. Health Aging 2011, 15, 187-191. [CrossRef] [PubMed] 
62. Guigoz, Y.; Vellas, B.; Garry, P.J. Mini Nutritional Assessment: A practical assessment tool for grading the nutritional state of elderly patients. Facts Res. Gerontol. 1994, 2, 15-59.

63. Kozakova, R.; Jarosova, D.; Zelenikova, R. Comparison of three screening tools for nutritional status assessment of the elderly in their homes. Biomed. Pap. Med. Fac. Univ. Palacky Olomouc. Czech Repub. 2012, 156, 371-376. [CrossRef] [PubMed]

64. Cederholm, T.; Barazzoni, R.; Austin, P.; Ballmer, P.; Biolo, G.; Bischoff, S.C.; Compher, C.; Correia, I.; Higashiguchi, T.; Kolst, M.; et al. ESPEN guidelines on definitions and terminology of clinical nutrition. Clin. Nutr. 2017, 36, 49-64. [CrossRef] [PubMed]

65. Fávaro-Moreira, N.C.; Krausch-Hofmann, S.; Matthys, C.; Vereecken, C.; Vanhauwaert, E.; Declercq, A.; Bekkering, G.E.; Duyck, J. Risk Factors for Malnutrition in Older Adults: A Systematic Review of the Literature Based on Longitudinal Data. Adv. Nutr. 2016, 7, 507-522. [CrossRef] [PubMed]

66. Burks, C.E.; Jones, C.W.; Braz, V.A.; Swor, R.A.; Richmond, N.L.; Hwang, K.S.; Hollowell, A.G.; Weaver, M.A.; Platts-Mills, T.F. Risk Factors for Malnutrition among Older Adults in the Emergency Department: A Multicenter Study. J. Am. Geriatr. Soc. 2017, 65, 1741-1747. [CrossRef] [PubMed]

67. Mangels, A.R. Malnutrition in Older Adults. Am. J. Nurs. 2018, 118, 34-41. [CrossRef] [PubMed]

68. Kossioni, A.E. The Association of Poor Oral Health Parameters with Malnutrition in Older Adults: A Review Considering the Potential Implications for Cognitive Impairment. Nutrients 2018, 10, 1709. [CrossRef]

69. Kazemi, S.; Savabi, G.; Khazaei, S.; Savabi, O.; Esmaillzadeh, A.; Hassanzadeh Keshteli, A.; Adibi, P. Association between food intake and oral health in elderly: SEPAHAN systematic review no. 8. Dent. Res. J. 2011, 8, 15-20.

70. Prell, T.; Perner, C. Disease Specific Aspects of Malnutrition in Neurogeriatric Patients. Front Aging Neurosci. 2018, 10, 80. [CrossRef]

71. Al-Rasheed, R.; Alrasheedi, R.; Johani, R.; Alrashidi, H.; Almaimany, B.; Alshalawi, B.; Kelantan, A.; Banjar, G.; Alzaher, A.; Alqadheb, A. Malnutrition in elderly and its relation to depression. Int. J. Community Med. Public Health 2018, 5, 2156-2160. [CrossRef]

72. Miranda, D.; Cardoso, R.; Gomes, R.; Guimarães, I.; de Abreu, D.; Godinho, C.; Pereira, P.; Domingos, J.; Pona, N.; Ferreira, J.J. Undernutrition in institutionalized elderly patients with neurological diseases: Comparison between different diagnostic criteria. J. Nursing Home Res. 2016, 2, 76-82.

73. Corish, C.A.; Bardon, L.A. Malnutrition in older adults: Screening and determinants. Proc. Nutr. Soc. 2018, 3, 1-8. [CrossRef] [PubMed]

74. Jain, M.; Khushboo, G. Physiological Determinants of Malnutrition in Elderly. Nov. Tech. Nutri. Food Sci. 2018, 2, 1-4. [CrossRef]

75. Artemissia-Phoebe, N. Appetite, Metabolism and Hormonal Regulation in Normal Ageing and Dementia. Diseases 2018, 6, 66.

76. Morley, J.E. Undernutrition in older adults. Family Practice 2012, 29, 89-93. [CrossRef] [PubMed]

77. Rizzi, M.; Mazzuoli, S.; Regano, N.; Inguaggiato, R.; Bianco, M.; Leandro, G.; Bugianesi, E.; Noè, D.; Orzes, N.; Pallini, P.; et al. Undernutrition, risk of malnutrition and obesity in gastroenterological patients: A multicenter study. World J. Gastrointest. Oncol. 2016, 8, 563-572. [CrossRef]

78. Roubenoff, R.; Heymsfield, S.B.; Kehayias, J.J.; Cannon, J.G.; Rosenberg, I.H. Standardization of nomenclature of body composition in weight loss. Am. J. Clin. Nutr. 1997, 66, 192-196. [CrossRef]

79. Roubenoff, R. Sarcopenia: Effects on Body Composition and Function. J. Geront. 2003, 58, $1012-1017$. [CrossRef]

80. Roubenoff, R. The Pathophysiology of Wasting in the Elderly. J. Nutr. 1999, 129, 256-259. [CrossRef]

81. Roubenoff, R. Inflammatory and hormonal mediators of cachexia. J. Nutr. 1997, 127, 1014-1016. [CrossRef] [PubMed]

82. Roubenoff, R. Hormones, cytokines and body composition: Can lessons from illness be applied to aging? J. Nutr. 1993, 123, 469-473. [CrossRef] [PubMed]

83. Morley, J.E. Hormones and Sarcopenia. Curr. Pharm. Des. 2017, 23, 4484-4492. [CrossRef] [PubMed]

84. Myung, J.S.; Yun Kyung, J.; In Joo, K. Testosterone and Sarcopenia. World J. Mens Health 2018, 36, $192-198$.

85. Scaldaferri, F.; Pizzoferrato, M.; Lopetuso, L.; Musca, T.; Ingravalle, F.; Sicignano, L.; Mentella, M.; Miggiano, G.; Mele, M.; Gaetani, E.; et al. Nutrition and IBD: Malnutrition and/or sarcopenia? A practical guide. Gastroenterol. Res. Pract. 2017, 8646495. 
86. Bryant, R.; Trott, M.; Bartholomeusz, F.; Andrews, J.M. Systematic review: Body composition in adults with inflammatory bowel disease. Aliment. Pharmacol. Ther. 2013, 38, 213-225. [PubMed]

87. Lucendo, A.; De Rezende, L. Importance of nutrition in inflammatory bowel disease. World J. Gastroenterol. 2009, 15, 2081-2088.

88. Hwang, C.; Ross, V.; Mahadevan, U. Micronutrient deficiencies in inflammatory bowel disease. Inflamm. Bowel Dis. 2012, 18, 1961-1981. [CrossRef]

89. Chen, X.; Maguire, B.; Brodaty, H.; O'Leary, F. Dietary Patterns and Cognitive Health in Older Adults: A Systematic Review. J. Alzheimers Dis. 2019, 67, 583-619. [CrossRef]

90. Bauer, J.; Biolo, G.; Cederholm, T.; Cesari, M.; Cruz-Jentoft, A.J.; Morley, J.E.; Phillips, S.; Sieber, C.; Stehle, P.; Teta, D.; et al. Evidence-based recommendations for optimal dietary protein intake in older people: A position paper from the PROT-AGE Study Group. J. Am. Med. Dir. Assoc. 2013, 14, 542-559. [CrossRef]

91. Pludowski, P.; Holick, M.F.; Grant, W.B.; Konstantynowicz, J.; Mascarenhas, M.; Hag, A.; Povoroznyuk, V.; Balatska, N.; Barbosa, A.P.; Karonova, T.; et al. Vitamin D supplementation guidelines. J. Steroid. Biochem. Mol. Biol. 2018, 175, 125-135. [CrossRef] [PubMed]

92. EFSA Panel on Dietetic Products, Nutrition, and Allergies (NDA), Scientific Opinion on Dietary reference values for water. EFSA J. 2010, 8, 1459.

93. Manz, F. Hydration and disease. J. Am. Coll. Nutr. 2007, 26, 535-541. [CrossRef]

94. Stover, J.P. Vitamin B12 and older adults. Curr. Opin. Clin. Nutr. Metab. Care 2010, 13, 24-27. [CrossRef] [PubMed]

95. Hughes, C.F.; Ward, W.; Hoey, L.; McNulty, H. Vitamin B12 and ageing: Current issues and interaction with folate. Ann. Clin. Biochem. 2013, 50, 315-329. [CrossRef] [PubMed]

96. Owczarek, D.; Rodacki, T.; Domagala-Rodacka, R.; Cibor, D.; Mach, T. Diet and nutritional factors in inflammatory bowel diseases. World J. Gastroenterol. 2016, 22, 895-905. [CrossRef]

97. Van Gossum, A.; Cabre, E. ESPEN Guidelines on Parenetral Nutrition: Gastroenterology. Clin. Nutr. 2009, 28, 415-427. [CrossRef]

98. Sasaki, M.; Johtatsu, T. Energy metabolism in Japanese Patients with Crohn's disease. J. Clin. Biochem. Nutr. 2010, 46, 68-72. [CrossRef]

99. Kłęk, S.; Jankowski, M.; Kruszewski, W.J.; Fijuth, J.; Kapała, A.; Kabata, P.; Wysocki, P.; Krzakowski, M.; Rutkowski, P. Clinical nutrition in oncology: Polish recommendations. Oncol. Clin. Pract. 2015, 11, 172-188.

100. Claesson, M.J.; Jeffery, I.B.; Conde, S.; Power, S.E.; O'Connor, E.M.; Cusack, S.; Harris, H.M.B.; Coakley, M.; Lakshminarayanan, B.; O'Sullivan, O.; et al. Gut microbiota composition correlates with diet and health in the elderly. Nature 2012, 488, 178-184. [CrossRef]

101. Brussow, H. Microbiota and healthy ageing: Observational and nutritional intervantion studies. Microb. Biotechnol. 2013, 6, 326-334. [CrossRef] [PubMed]

102. Lee, D.; Albenberg, L.; Compher, C.; Baldassano, R.; Piccoli, D.; Lewis, J.D.; Wu, G.D. Diet in the Pathogenesis and Treatment of Inflammatory Bowel Diseases. Gastroenterology 2015, 148, 1087-1106. [CrossRef] [PubMed]

103. Wẹdrychowicz, A.; Zając, A.; Tomasik, P. Advances in nutritional therapy in inflammatory bowel diseases: Review. World J. Gastroenterol. 2016, 21, 1045-1066. [CrossRef] [PubMed]

104. Knight-Sepulveda, K.; Kais, S.; Santaolalla, R.; Abreu, M.T. Diet and Inflammatory Bowel Disease. Gastroenterol. Hepatol. 2015, 11, 511-520.

105. Sanches Machado d'Almeida, K.; Ronchi Spillere, S.; Zuchinali, P.; Corrêa Souza, G. Mediterranean Diet and Other Dietary Patterns in Primary Prevention of Heart Failure and Changes in Cardiac Function Markers: A Systematic Review. Nutrients 2018, 10, 58. [CrossRef]

106. Casas, R.; Sacanella, E.; Estruch, R. The Immune Protective Effect of the Mediterranean Diet against Chronic Low-grade Inflammatory Diseases. Endocr. Metab. Immune Disord. Drug Targets 2016, 14, 245-254. [CrossRef]

107. Bischoff, S.C.; Barbara, G.; Buurman, W.; Ockhuizen, T.; Schulzke, J.D.; Serino, M.; Tilg, H.; Watson, A.; Wells, J.M. Intestinal permeability-a new target for disease prevention and therapy. BMC Gastroenterol. 2014, 14, 189. [CrossRef]

108. Harper, A.; Naghibi, M.M.; Garcha, D. The Role of Bacteria, Probiotics and Diet in Irritable Bowel Syndrome. Foods 2018, 7, 13. [CrossRef]

109. Shepherd, S.J.; Gibson, P.R. Fructose malabsorption and symptoms of irritable bowel syndrome: Guidelines for effective dietary management. J. Am. Diet. Assoc 2006, 106, 1631-1639. [CrossRef] 
110. Ong, D.K.; Mitchell, S.B.; Barrett, J.S.; Shepherd, S.; Irving, P.; Biesiekierski, J.; Smith, S.; Gibson, P.; Muir, J.G. Manipulation of dietary short chain carbohydrates alters the pattern of gas production and genesis of symptoms in irritable bowel syndrome. J. Gastroenterol. Hepatol. 2010, 25, 1366-1373. [CrossRef]

111. Biesiekierski, J.R.; Peters, S.L.; Newnham, E.D.; Rosella, O.; Muir, J.; Gibson, P. No effects of gluten in patients with self-reported non-celiac gluten sensitivity after dietary reduction of fermentable, poorly absorbed, short-chain carbohydrates. Gastroenterology 2013, 145, 320-328. [CrossRef] [PubMed]

112. Gibson, P.R.; Newnham, E.; Barrett, J.S.; Shepherd, S.J.; Muir, J.G. Review article: Fructose malabsorption and the bigger picture. Aliment. Pharmacol. Ther. 2007, 25, 349-363. [CrossRef] [PubMed]

113. Gibson, P.R.; Shepherd, S.J. Personal view: Food for thought-Western lifestyle and susceptibility to Crohn's disease. The FODMAP hypothesis. Aliment. Pharmacol. Ther. 2005, 21, 1399-1409. [CrossRef] [PubMed]

114. Gibson, P.R.; Shepherd, S.J. Evidence-based dietary management of functional gastrointestinal symptoms: The FODMAP approach. J. Gastroenterol. Hepatol. 2010, 25, 252-258. [CrossRef] [PubMed]

115. Gearry, R.B.; Irving, P.M.; Barrett, J.S.; Nathan, D.M.; Shepherd, S.J.; Gibson, P.R. Reduction of dietary poorly absorbed short-chain carbohydrates (FODMAPs) improves abdominal symptoms in patients with inflammatory bowel disease-a pilot study. J. Crohns Colitis 2009, 3, 8-14. [CrossRef] [PubMed]

116. Olendzki, B.C.; Silverstein, T.D.; Persuitte, G.M.; Ma, Y.; Baldwin, K.R.; Cave, D. An anti-inflammatory diet as treatment for inflammatory bowel disease: A case series report. Nutr. J. 2014, 13, 5. [CrossRef] [PubMed]

117. Lowering Your BloodPressure With DASH. Available online: www.nhlbi.nih.gov/files/docs/public/heart/ new_dash.pdf (accessed on 10 December 2018).

118. Whelton, P.K.; Carey, R.M.; Aronow, W.S.; Casey, D.E.; Collins, K.J.; Dennison Himmelfarm, C.; DePalma, S.M.; Gidding, S.; Jamerson, K.A.; Jones, D.W.; et al. 2017 ACC/AHA/AAPA/ABC/ACPM/AGS/APhA/ASH/ASPC/NMA/PCNA Guideline for the Prevention, Detection, Evaluation, and Management of High Blood Pressure in Adults. Hypertension 2018, 71, 1269-1324. [CrossRef] [PubMed]

119. Chiavaroli, L.; Viguiliouk, E.; Nishi, S.K.; Blanco Mejia, S.; Rahelić, D.; Kahleová, H.; Salas-Salvadó, J.; Kendall, C.W.; Sievenpiper, J.L. DASH Dietary Pattern and Cardiometabolic Outcomes: An Umbrella Review of Systematic Reviews and Meta-Analyses. Nutrients 2019, 11, 338. [CrossRef] [PubMed]

120. Hummel, S.L.; Mitchell Seymour, E.; Brook, R.D.; Sheth, S.S.; Ghosh, E.; Zhu, S.; Weder, A.B.; Kovacs, S.J.; Kolias, T.J. Low-Sodium DASH Diet Improves Diastolic Function and Ventricular-Arterial Coupling in Hypertensive Heart Failure with Preserved Ejection Fraction. Circ. Heart Fail. 2013, 6, 1165-1171. [CrossRef] [PubMed]

121. Azadbakht, L.; Rashidi Pour Fard, N.; Karimi, M.; Hassan Baghaei, M.; Surkan, P.J.; Rahimi, M.; Esmaillzadeh, A.; Willett, W.C. Effects of the Dietary Approaches to Stop Hypertension (DASH) Eating Plan on Cardiovascular Risks Among Type 2 Diabetic Patients. Diabetes Care 2011, 34, 55-57. [CrossRef]

122. Nilsson, A.; Halvardsson, P.; Kadi, F. Adherence to DASH-Style Dietary Pattern Impacts on Adiponectin and Clustered Metabolic Risk in Older Women. Nutrients 2019, 11, 805. [CrossRef] [PubMed]

123. Sofi, F.; Abbate, R.; Gensini, G.F.; Casini, A. Accruing evidence on benefits of adherence to the Mediterranean diet on health: An updated systematic review and meta-analysis. Am. J. Clin. Nutr. 2010, 92, 1189-1196. [CrossRef] [PubMed]

124. Make Each Day Mediterranean. Available online: https://oldwayspt.org/system/files/atoms/files/ NewMedKit_0.pdf (accessed on 6 December 2018).

125. Marlow, G.; Ellett, S.; Ferguson, I.R.; Zhu, S.; Karunasinghe, N.; Jesuthasan, A.C.; Yeo Han, D.; Fraser, A.G.; Ferguson, L.R. Transcriptomics to study the effect of a Mediterranean-inspired diet on inflammation in Crohn's disease patients. Hum. Genomics 2013, 7, 24. [CrossRef] [PubMed]

126. Godny, L.; Pfeffer-Gik, T.; Goren, I.; Tulchinsky, H.; Dotan, I. Adherence to Mediterranean diet is associated with decreased faecal calprotectin in patients after pouch surgery. In Proceedings of the 13th Congress of the European Crohn's and Colitis Organisation, Vienna, Austria, 14-17 February 2018.

127. Molendijk, I.; Martens, J.; van Lingen, E.; van der Marel, S.; van Veen-Lievaart, M.; van der Meulen, A.; Barnhoorn, M.; Ernst-Stegeman, A.; Maljaars, J. Towards a Food Pharmacy: Increased dietary quality reduces CRP and improves quality of life in IBD patients in remission. In Proceedings of the 14th Congress of the European Crohn's and Colitis Organisation, Copenhagen, Denmark, 6-9 March 2019. 
128. Ananthakrishnan, A.N.; Khalili, H.; Konijeti, G.G.; Higuchi, L.M.; de Silva, P.; Fuchs, C.S.; Willett, W.C.; Richter, J.M.; Chan, A.T. Long-term intake of dietary fat and risk of ulcerative colitis and Crohn's disease. Gut 2014, 63, 776-784. [CrossRef] [PubMed]

129. Hou, J.K.; Abraham, B.; El-Serag, H. Dietary intake and risk of developing inflammatory bowel disease: A systematic review of the literature. Am. J. Gastroenterol. 2011, 106, 563-573. [CrossRef] [PubMed]

130. Tyagi, A.; Kumar, U.; Reddy, S.; Santosh, V.S.; Mohammed, S.B.; Ehtesham, N.Z.; Ibrahim, A. Attenuation of colonic inflammation by partial replacement of dietary linoleic acid with $\alpha$-linolenic acid in a rat model of inflammatory bowel disease. Br. J. Nutr. 2012, 108, 1612-1622. [CrossRef] [PubMed]

131. Pearl, D.S.; Masoodi, M.; Eiden, M.; Brümmer, J.; Gullick, D.; McKeever, T.M.; Whittaker, M.A.; Nitch-Smith,H.; Brown, J.F.; Shute, J.K.; et al. Altered colonic mucosal availability of n-3 and n-6 polyunsaturated fatty acids in ulcerative colitis and the relationship to disease activity. J. Crohns Colitis 2014, 8, 70-79. [CrossRef]

132. Uchiyama, K.; Nakamura, M.; Odahara, S.; Koido, S.; Katahira, K.; Shiraishi, H.; Shiraishi, H.; Ohkusa, T.; Fujise, K.; Tajiri, H. N-3 polyunsaturated fatty acid diet therapy for patients with inflammatory bowel disease. Inflamm. Bowel Dis. 2010, 16, 1696-1707. [CrossRef]

133. Charpentier, C.; Chan, R.; Salameh, E.; Mbodji, K.; Ueno, A.; Guérin, C.; Ghosh, S.; Savoye, G.; Marion-Letellier, R. Dietary n-3 PUFA may attenuate experimental colitis. Mediators Inflamm. 2018, 8430614. [CrossRef]

134. Han, M.; Wang, C.; Liu, P.; Li, D.; Li, Y.; Ma, X. Dietary Fiber Gap and Host Gut Microbiota. Protein Pept. Lett. 2017, 24, 388-396. [CrossRef]

135. Liu, B.; Lin, Q.; Yang, T.; Zeng, L.; Shi, L.; Chen, Y.; Luo, F. Oat $\beta$-glucan ameliorates dextran sulfate sodium (DSS)-Induced ulcerative colitis in mice. Food Funct. 2015, 6, 3454-3463. [CrossRef] [PubMed]

136. Ananthakrishnan, A.N.; Khalili, H.; Konijeti, G.G.; Higuchi, L.M.; de Silva, P.; Korzenik, J.R.; Fuchs, C.S.; Willett, W.C.; Richter, J.M.; Chan, A.T. A prospective study of long-term intake of dietary fiber and risk of Crohn's disease and ulcerative colitis. Gastroenterology 2013, 145, 970-977. [CrossRef] [PubMed]

137. Liu, X.; Wu, Y.; Li, F.; Zhang, D. Dietary fiber intake reduces risk of inflammatory bowel disease: Result from a meta-analysis. Nutr. Res. 2015, 35, 753-758. [CrossRef] [PubMed]

138. Juneja, M.; Baidoo, L.; Marc, B.; Schwartz, A.B.; Regueiro, M.; Dunn, M.; Binion, D.G. Geriatric inflammatory bowel disease: Phenotypic presentation, treatment patterns, nutritional status, outcomes, and comorbidity. Dig. Dis. Sci. 2012, 57, 2408-2415. [CrossRef] [PubMed]

(C) 2019 by the authors. Licensee MDPI, Basel, Switzerland. This article is an open access article distributed under the terms and conditions of the Creative Commons Attribution (CC BY) license (http://creativecommons.org/licenses/by/4.0/). 
Review

\title{
Diet in the Pathogenesis and Management of Ulcerative Colitis; A Review of Randomized Controlled Dietary Interventions
}

\author{
Ammar Hassanzadeh Keshteli ${ }^{1,2}$, Karen L. Madsen ${ }^{1,2}$ and Levinus A. Dieleman ${ }^{1,2, *}$ \\ 1 Department of Medicine, University of Alberta, Edmonton, AB T6G 2P5, Canada \\ 2 Centre of Excellence for Gastrointestinal Inflammation and Immunity Research (CEGIIR), Edmonton, \\ AB T6G 2X8, Canada \\ * Correspondence: 1.dieleman@ualberta.ca; Tel.: +780-492-8691; Fax: +780-492-8121
}

Received: 30 April 2019; Accepted: 28 June 2019; Published: 30 June 2019

\begin{abstract}
Epidemiological and experimental studies have suggested that diet is one of the environmental factors that contributes to the onset and pathophysiology of ulcerative colitis. Although many patients suffering from ulcerative colitis attribute their symptoms or disease relapse to dietary factors, only a few well-designed randomized controlled trials have been done to investigate the role of diet in the management of ulcerative colitis. Here, we review the potential mechanisms of the relationship between diet and pathogenesis of ulcerative colitis and summarize randomized controlled dietary interventions that have been conducted in ulcerative colitis patients.
\end{abstract}

Keywords: diet; inflammatory bowel disease; ulcerative colitis

\section{Introduction}

Ulcerative colitis (UC) - a subtype of inflammatory bowel disease (IBD)—is a chronic, idiopathic inflammatory disease that affects the colon and is characterized by relapsing and remitting mucosal inflammation [1]. UC patients mostly present blood in the stool and diarrhea [1]. UC is associated with major morbidity in Western countries, and its incidence is increasing in developing countries [2]. The multifactorial pathophysiology of UC includes genetic predisposition, epithelial barrier defects, dysregulated immune responses, microbial dysbiosis, and environmental factors [1,2].

It has been suggested that environmental factors play a major role in the pathogenesis of IBD. Early-life events such as mode of birth, breastfeeding, and exposure to antibiotics and other factors such as air pollution, smoking, psychological state, exercise, and diet are among the potential environmental contributors of IBD development or disease activity [3].

Significant changes in dietary intake during the past decades have been associated with the increase in incidence of UC. The relationship between diet and UC development has been indicated in several epidemiological studies [4]. Two recent meta-analysis studies showed that soft drink consumption and sucrose intake were associated with $69 \%$ and $10 \%$ increased risk of UC development, respectively [5,6]. Consumption of fruits (odds ratio: 0.57 ) and vegetables (odds ratio: 0.71 ) was related to decreased odds of UC development in another meta-analysis study [7]. A significant association between meat intake (red meat in particular) and UC risk was found in a meta-analysis of seven epidemiological studies (summary relative risk: 1.47) [8]. Furthermore, whereas $n-3$ polyunsaturated fatty acids (PUFAs) content of diet was related to decreased odds of UC development (odds ratio: 0.56) [9], dietary arachidonic acid (an $n-6$ PUFA) as measured in adipose tissue increased risk of UC development (relative risk: 4.16) in a large prospective cohort study among Danish adults [10]. Although the exact pathophysiological mechanisms in which diet plays a role in IBD development remain unknown, several plausible explanations including its effects on composition of gut microbiota, 
production of microbial metabolites, alterations in mucosal immunity, and mucosal barrier function have been proposed [11] (Figure 1).
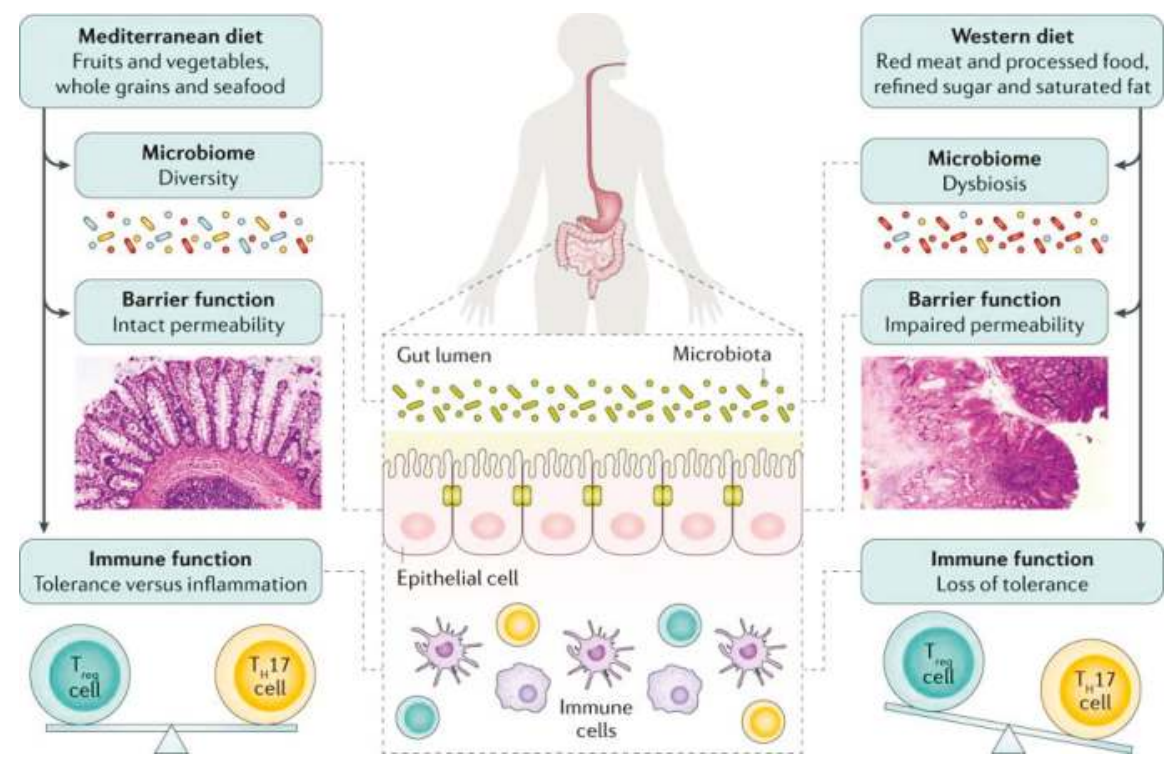

Figure 1. Although the exact mechanisms responsible for the association between diet and development of inflammatory bowel disease is unknown, several mechanisms have been suggested. An unhealthy dietary pattern such as a Western diet has been linked to changes in the gut microbiome and epithelial barrier function and seems to have a direct influence on immune function, triggering a pro-inflammatory environment characterized by an imbalance in the T helper $17\left(\mathrm{~T}_{\mathrm{H}} 17\right)$ cell to regulatory $\mathrm{T}\left(\mathrm{T}_{\text {reg }}\right)$ cell ratio [Adapted with permission [11]].

Abnormalities in the intestinal microbiota have been reported in some, but not all UC patients [1,12-14]. In some studies, UC patients have been shown to have decreased bacterial diversity, characterized by a decreased Firmicutes and increased Gammaproteobacteria and Enterobacteriaceae [15]. However, it is not clear if bacterial dysbiosis is the cause or effect of mucosal inflammation in UC [1,13] (Figure 2). 


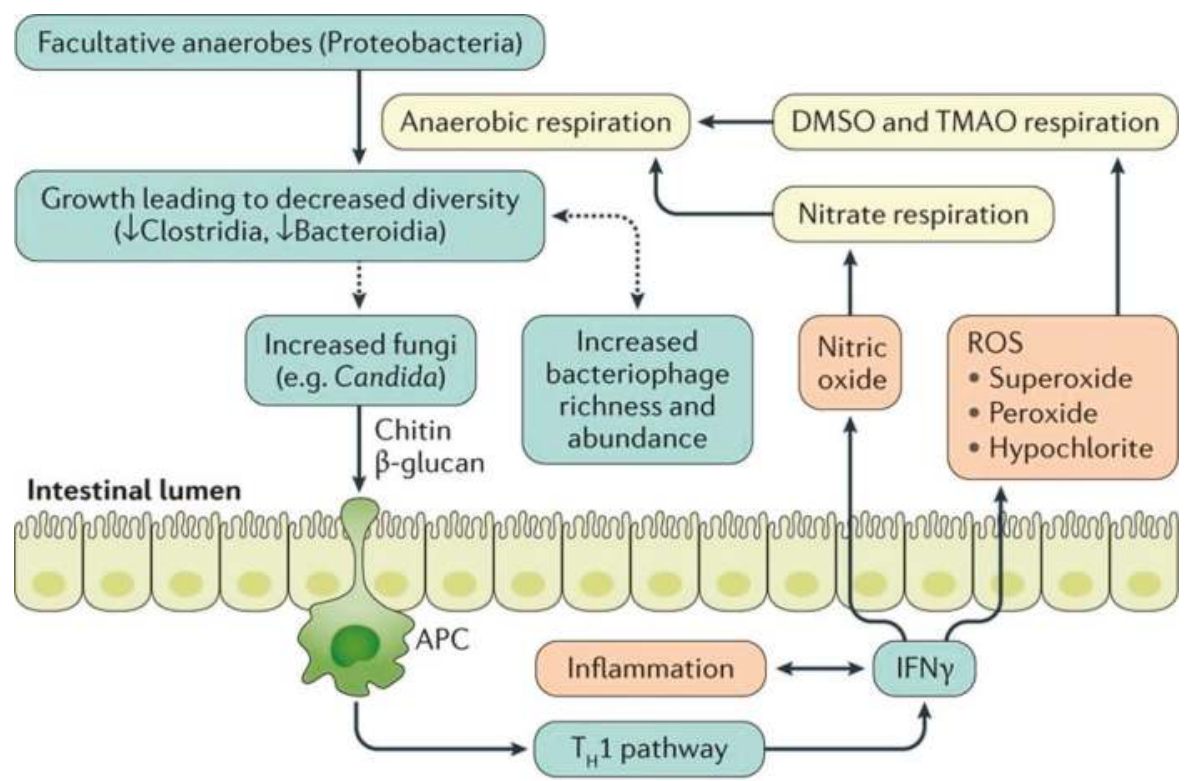

Figure 2. The relationship between gut microbiota and colonic inflammation in inflammatory bowel disease. Inflammation in colon stimulates production of Interferon gamma (IFN- $\gamma$ ) that eventually generates reactive oxygen species (ROS). ROS make products for anaerobic respiration. These products can be used by facultative anaerobes to outgrow, which leads to decreased bacterial diversity. The dysbiotic microbiota may further stimulate the growth of fungi that can worsen inflammation via chitin and $\beta$ -glucan antigen-presenting cell (APC) activation of the type $1 \mathrm{~T}$ helper $\left(\mathrm{T}_{\mathrm{H}} 1\right)$ pathway. In addition, the microbial dysbiosis is associated with increased bacteriophage richness and abundance, which can affect the bacterial microbiota via gene transfer. DMSO, dimethyl sulfoxide; TMAO, trimethylamine $\mathrm{N}$-oxide. [Adapted with permission [13]].

Dietary factors can be related to UC pathogenesis or disease course through direct effects on the host or indirect effects through modulations of composition or function of gut microbiota. Diet has a major role in shaping gut microbial composition [16]. For instance, increased Bacteroidetes and decreased Firmicutes and Enterobacteriaceae in rural African children in comparison to European children were mainly attributed to differences in dietary patterns between the two populations [17]. Therefore, it has been suggested that diet-induced changes in microbiota may transform healthy gut microbiota into a disease-inducing entity that could either initiate or perpetuate inflammation in patients with IBD [16]. Agus et al. [18] indicated that a high fat/high sugar diet resulted in intestinal mucosal dysbiosis characterized by an overgrowth of pro-inflammatory proteobacteria and a decrease in protective bacteria. In addition, they showed that the transplantation of feces from high fat/high sugar fed mice to germ-free mice increased susceptibility to adherent-invasive Escherichia coli infection.

In addition to their significant effects on microbial composition, dietary factors can also affect the metabolic functions of gut microbiota. Short chain fatty acids (SCFA), which are defined as the groups of fatty acids with fewer than six carbons including formic acid (C1), acetic acid (C2), propionic acid (C3), butyric acid (C4), and valeric acid (C5), are derived from commensal bacterial fermentation of indigestible dietary fibers in both the small and large intestines $[19,20]$. Acetate, propionate and butyrate account for more than $95 \%$ of all the SCFA content in the gut [19]. Acetate and butyrate in particular have an essential role in maintaining mucosal barrier function and modulating immune function [21,22] (Figure 3). SCFA regulate the functions of epithelial and/or immune cells through altering gene expression, cellular differentiation, chemotaxis, proliferation, and apoptosis [19]. 
The number of SCFA-producing bacteria such as Faecalibacterium prausnitzii is decreased in some UC patients, and these are inversely correlated with disease activity [23]. Moreover, a western diet characterized by high intake of sugar $[18,24]$ and fat [18] and decreased amount of dietary fiber was associated with decreased SCFAs and increased susceptibility to colitis in experimental studies.

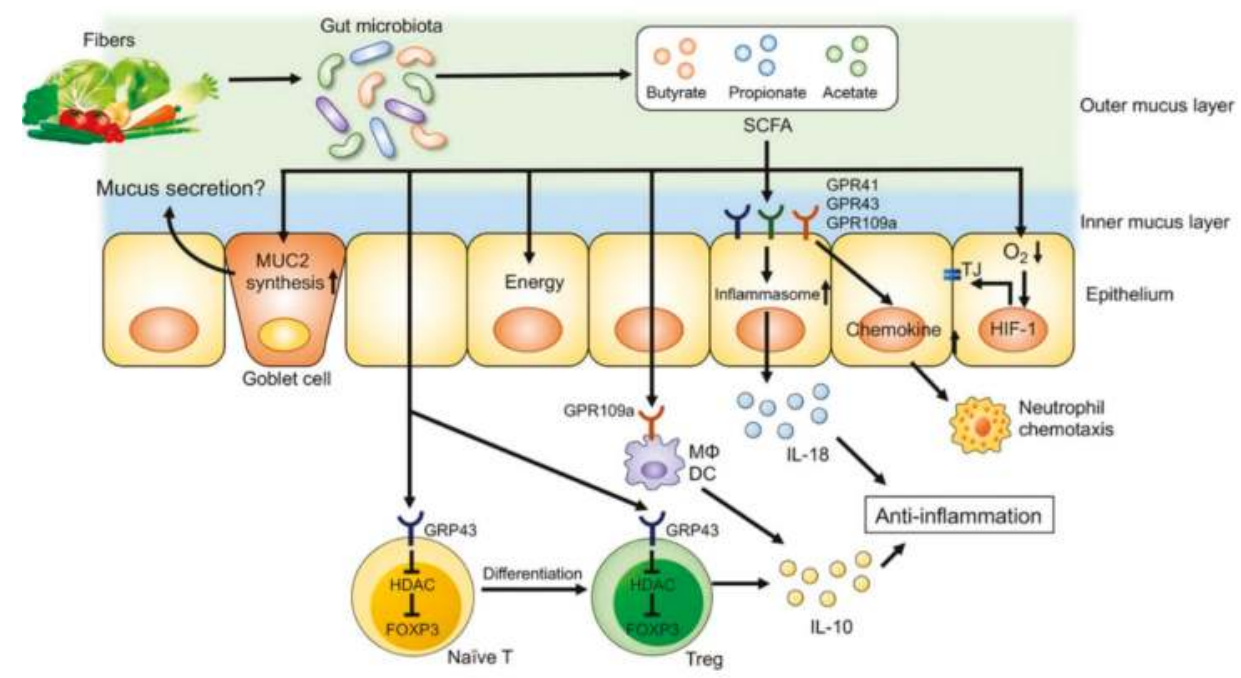

Figure 3. The role of fiber-derived short chain fatty acids (SCFAs) in regulation of intestinal homeostasis. SCFAs serve as energy substrates for colonocytes. In addition, SCFAs regulate intestinal barrier function and immune system through G-protein-coupled receptors (GPRs) signaling. SCFAs promote the differentiation of regulatory T (Treg) cells and the production of interleukin (IL)-10 through GPR43. Furthermore, SCFA facilitate inflammasome activation in colonic epithelial cells through GPR43, stimulating IL-18 production that is critical for anti-inflammation and epithelial repair. SCFAs also regulate intestinal barrier function via enhancing the expression of tight junction proteins and the synthesis of mucin (MUC)2. DC, dendritic cells; FOXP3, forkhead box P3; HDAC, histone deacetylases; Mф, macrophages; TJ, tight junctions. [Adapted with permission [22]].

The mucus layer and intestinal epithelium are the first physical and chemical barriers against intestinal bacteria, pathogens, and food antigens. A dysregulated mucosal immune response characterized by alterations in the innate immune system, activation of effector T-cells, increased presence of B-cells and antibody production, and increased production of pro-inflammatory mediators have a major role in the pathogenesis of IBD [1,25]. Dietary factors can have direct effects on host cells. For instance, it was shown that luminal iron may directly affect function of intestinal epithelial cells and T cells in addition to triggering epithelial cell stress-associated apoptosis [26]. Zinc is an important cofactor for various intestinal metalloproteinases, and zinc deficiency has been associated with reduced barrier integrity and increased permeability in IBD patients [27]. There is also increasing evidence for a role of vitamin $\mathrm{D}$ in strengthening the innate immune system and reducing inflammation in experimental and human IBD [28]. Relationships between PUFAs content of diet and inflammatory processes in IBD have also been shown $[29,30]$. Eicosapentaenoic acid and docosahexaenoic acid that are long chain dietary $n-3$ PUFAs inhibit genes that are involved in inflammatory process and alter the composition of cell membranes by displacing $n-6$ PUFAs, influencing lipid raft formation in cell signaling [30]. Dietary amino acids act as key regulatory factors in cellular and microbial metabolic pathways and play important roles in gut homeostasis. Intestinal inflammation as seen in IBD affects several metabolic pathways related to metabolism of amino acids [22]. It has been reported that several food additives, such as maltodextrin, emulsifying agents, or thickeners such as carboxymethyl 
cellulose, carrageenan, and xanthan gum, may also have detrimental effects on intestinal homeostasis as well [31]. A complete review of all dietary factors and host interactions is beyond the scope of this review; however, those interactions have been described comprehensively in other recent review articles [11,21,22,31-34].

Currently, dietary recommendations for management of IBD-related symptoms are scarce and non-evidence-based, mainly due to the limited number of dietary interventions in this population. In the present review article, we summarize findings from previously conducted dietary interventions in UC patients.

\section{Methods}

An electronic search in MEDLINE (Ovid) from inception to April 1, 2019, was conducted in order to identify any dietary intervention studies on UC subjects). Reference lists of included studies were also checked to identify relevant studies that might have been missed during initial search in MEDLINE. Studies that only focused on nutritional supplements, enteral or parenteral nutrition, or were published in languages other than English were not included. A comprehensive full-text review of identified studies was conducted after the title screening and abstract screening of potentially relevant articles. Collected data included journal name, publication year, design of the study, age, sex, sample size, disease condition, intervention and comparator(s) of interest, outcome(s), outcome measures, and main findings. The MEDLINE search strategy was as follows:

1. randomized controlled trial/

2. clinical trial.pt.

3. randomi?ed.ti,ab.

4. placebo.ti,ab.

5. randomly.ti,ab.

6. trial.ti,ab.

7. 1 or 2 or 3 or 4 or 5 or 6

8. Inflammatory Bowel Diseases/

9. inflammatory bowel disease.tw.

10. ibd.tw.

11. ulcerative colitis.tw.

12. colitis.tw.

13. 8 or 9 or 10 or 11 or 12

14. Diet/

15. diet*.tw.

16. food.tw.

17. 14 or 15 or 16

18. 7 and 13 and 17

\section{Results}

Our primary electronic search yielded 424 unique references. After title and abstract screening, nine studies were selected for full-text review. Following the full-text review, seven randomized controlled trials that met the inclusion and exclusion criteria were selected for this review [35-41]. The general characteristics of the included studies are summarized in Table 1. 


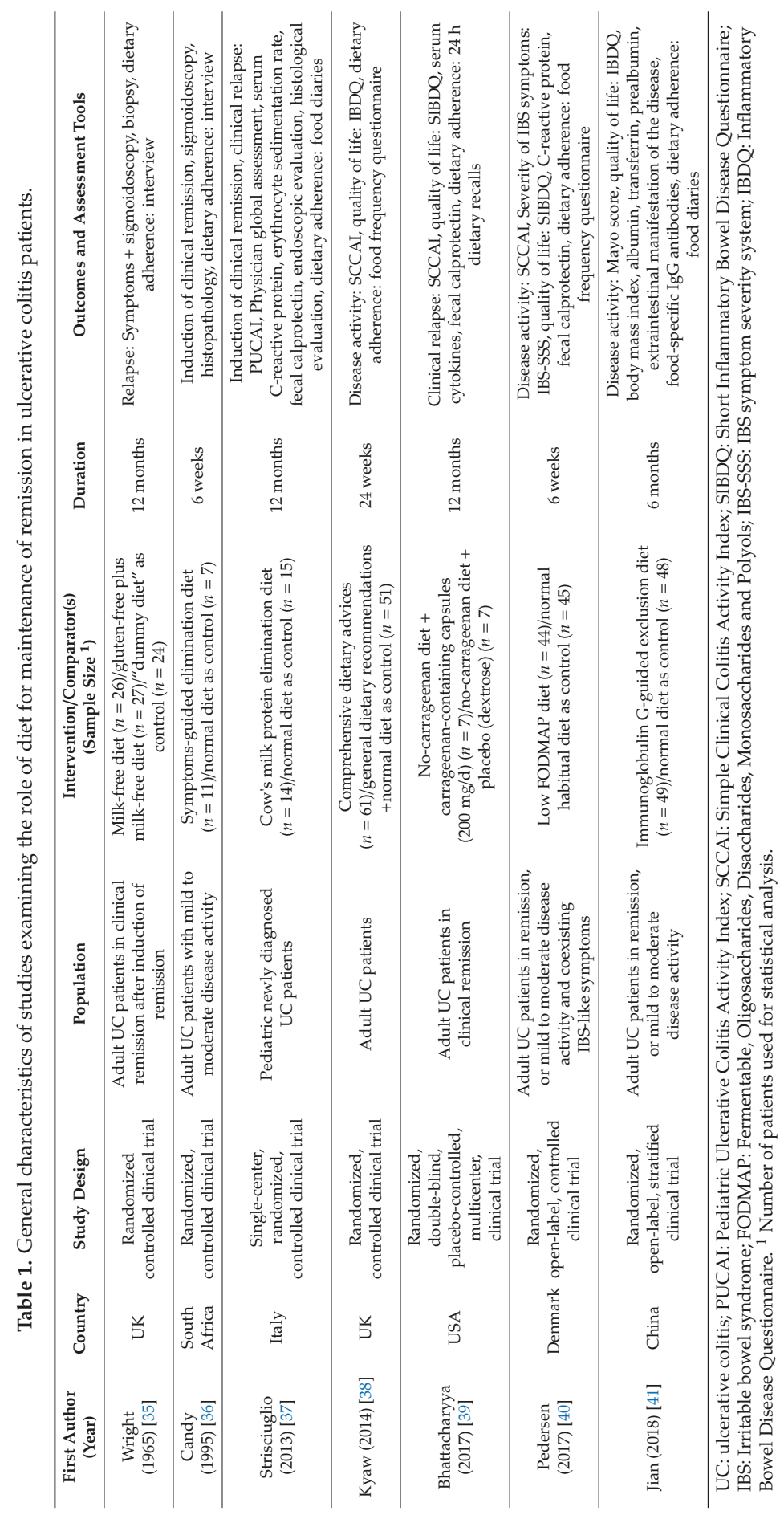


Wright et al. [35] randomly allocated UC patients with disease relapse into a milk-free diet $(n=26)$ (all milk and milk products, whether in the form of dairy products such as fresh milk and cheese or as powdered milk, were excluded, and butter was permitted), a gluten-free plus milk-free diet $(n=27)$, or a control group $(n=24)$. Patients were asked to follow the diets for one year after the induction of remission, and they were followed monthly to assess if they experienced disease relapse, which was defined as diarrhea with an average of four or more stools a day for at least a week and with macroscopic blood present, together with sigmoidoscopic evidence of inflammation. Although the relapse rate was higher in patients randomized to the control group in comparison to those on a milk-free diet $(79.2 \%$ vs. $61.5 \%)$, it did not reach statistical significance $(p=0.2)$. In addition, the relapse rate in the gluten-free plus milk-free diet was $70.4 \%$, which was comparable to that in the other two groups.

In a small randomized controlled trial for 6 weeks [36], 18 adult UC patients with mild to moderate disease activity were randomized to a symptoms-guided elimination diet $(n=11)$ or a control group $(n=7)$. Patients in the control group were asked to document but not alter their dietary intake. However, patients in the experimental group were instructed to exclude foods that appeared to provoke their symptoms. Fried foods were prohibited. In addition, refined sugars, additives and preservatives, all condiments and spices other than salt, and beverages other than boiled water were prohibited during the 6-week trial for patients randomized to the elimination diet group. In the first week, dairy products were excluded from the diet, but were introduced over the next weeks in the following order: Skim milk, yogurt, skim-milk cheese, full-cream milk, cream, and full-cream cheese. Each week, subjects in the intervention group were interviewed in person, and their symptoms were reviewed in relation to the foods eaten during the previous week. The food menu was expanded over the 6-week trial to include as more variety of foods that each participant could tolerate. The induction of clinical remission rate (the passage of normal stools with absence of rectal bleeding) 6 weeks after the baseline visit was significantly higher in patients who received the symptoms-guided diet ( $36.3 \% \mathrm{vs} .0 .0 \%)$. However, the endoscopic and histologic improvement was comparable between the two groups.

In another study [37], children with newly diagnosed UC were randomly assigned to a cow's milk protein (CMP) elimination diet $(n=14)$ or a normal diet as the control group $(n=15)$. The study aimed to compare the clinical remission rate between the two groups following the IBD induction therapy and the rate of clinical relapse (defined as the occurrence or worsening of symptoms accompanied by an increase of Pediatric Ulcerative Colitis Activity Index $>10$ points which required treatment with corticosteroids, immunosuppressive agents, or surgery) between the two groups during the one-year trial. The authors reported that the clinical response rate four weeks after the initiation of the induction therapy was not different between the two groups ( $92.8 \%$ in CMP elimination diet vs. $80.0 \%$ in the control group, $p=0.6)$. In addition, clinical relapse rate was comparable between the two groups $(53.8 \%$ in CMP elimination diet group vs. $53.3 \%$ in the control group). In addition, they found no significant changes in serum C-reactive protein (CRP), erythrocyte sedimentation rate or fecal calprotectin (FCP) in the two diet groups from baseline to the last visit.

Kyaw et al. [38] recruited 112 adult UC patients and randomly assigned them to a dietary intervention and a control group. Patients in the intervention group were given an educational booklet that contained dietary recommendations to eat little and often (four to six times a day), drink adequate fluids, decrease excess intake of fat, decrease simple carbohydrates, and decrease high-fiber foods during flare. Patients were also advised to increase intake of "good-quality protein" during flare and eliminate dairy products if they were lactose intolerant. Patients randomized to the control group were provided with a booklet that included general recommendations on healthy eating (e.g., to choose higher fiber or whole grain carbohydrates, to eat lots of fruits and vegetables) and were assigned to follow their usual diet. At 24 weeks, there was a significant reduction in the Simple Clinical Colitis Activity Index (SCCAI) score in the intervention group compared with an increase in the score in the control group. However, there was no statistically significant change in quality of life scores from baseline to week 24 in the two groups. 
Bhattacharyya et al. [39] conducted a small, randomized, double-blind, placebo-controlled, multicenter, clinical trial on UC patients to investigate the effect of the common food additive carrageenan on clinical relapse rates. The authors recruited UC patients over the age of 18 in clinical remission (SCCAI $\leq 2)$. Patients randomized to the carrageenan group $(n=5)$ received the carrageenan-containing capsules (200 $\mathrm{mg} /$ day). Patients randomized to the placebo group received similar-appearing dextrose-containing capsules $(n=7)$. The study duration was 12 months, and participants were instructed to follow a carrageenan-free diet during that period. The primary outcome measure was occurrence of clinical relapse, which was defined as an increase of two (or more) points on the SCCAI in association with an increase in treatment. The Short Inflammatory Bowel Disease Questionnaire was used to assess changes in quality of life. In addition, blood and stool samples were collected to measure inflammatory markers. They found that UC patients who were on a carrageenan-free diet plus placebo had a lower relapse rate in comparison to patients who were on a similar diet plus two oral capsules of carrageenan per day $(0.0 \% \mathrm{vs}$. $60.0 \%, p=0.05)$. In addition, they reported that carrageenan consumption aggravated disease activity as indicated by increase in FCP $(p=0.06)$ and interleukin-6 $(p=0.02)$. However, there was no statistically significant difference between the two groups in terms of changes in quality of life scores.

Pedersen et al. [40] conducted an open-label trial of patients with IBD (61 UC and 28 CD) in remission or with mild-to-moderate disease and coexisting IBS-like symptoms. Patients were randomly assigned to a low Fermentable, Oligosaccharides, Disaccharides, Monosaccharides and Polyols (FODMAP) diet $(n=44)$ or a normal diet $(n=45)$ for 6 weeks. In UC patients, there was a significant decrease in severity of IBS-related symptoms (assessed by IBS symptom Severity System) in both diet groups, and this response was not different between the two groups. However, the authors reported a significant decrease in disease activity assessed by SCCAI but only in patients randomized to the low-FODMAP diet. In addition, low-FODMAP diet increased quality of life of IBD patients (assessed by SIBDQ). However, low-FODMAP diet did not change CRP and FCP levels significantly.

In a recent open-label, stratified study, Jian et al. [41] randomly allocated 97 UC patients who were in remission or had mild to moderate disease activity to a food exclusion group versus a sham diet group. At baseline, the presence of blood IgG antibodies specific to egg, wheat, milk, corn, tomato, crab, rice, soybean, cod, shrimp, mushrooms, beef, chicken, and pork antigens were tested. Based on IgG antibody titers, patients randomized to the exclusion diet group were instructed to stop or reduce taking specific food items. Patients in the control group were asked to follow their routine diet. The duration of the trial was 6 months. They reported that in comparison to the control diet, following the exclusion diet was associated with a significant decrease in Mayo scores and improvement in quality of life.

\section{Discussion}

It has been suggested that environmental factors including diet play an important role in the pathophysiology of IBD and especially in UC, a chronic colonic inflammation. In the present article, after a brief overview of potential mechanisms in which diet plays a role in the pathogenesis of IBD, we then reviewed dietary intervention studies in UC patients.

The three randomized controlled trials that have been performed to assess the efficacy of dietary interventions for maintenance of remission in UC [35,37,39] were all focused on complete exclusion of one or more food items that were hypothesized to trigger IBD symptoms. Two of these studies $[35,37]$ aimed to eliminate milk or dairy products; however, they failed to show a significant decrease in relapse rate in patients randomized to the elimination diet group in comparison to those randomized to the control diet. This finding is important as unnecessary dietary restrictions that lack supporting scientific evidence may result in several nutritional deficiencies (e.g., calcium due to exclusion of milk and dairy products) in IBD patients [42]. Therefore, patients should be informed by their health care team about the possible harmful effects of food elimination diets.

In the present review, the only elimination diet that was associated with a reduction in clinical relapse rate in UC patients who were in remission at baseline was a carrageenan-free diet [39]. Carrageenan belongs to a family of sulfated polysaccharides and are extracted from seaweeds. It is 
approved as "generally recognized as safe" by the United States Food and Drug Administration and is used in the food industry for its gelling, thickening, and stabilizing properties. It has been suggested that carrageenan may reduce protein and peptide bioaccessibility, disrupt normal epithelial function, and promote intestinal inflammation [43]. However, others have been skeptical about these findings, which are mainly derived from experimental animal studies [44]. The results from the randomized clinical trial in which a carrageenan-free diet was found to be related to lower relapse rate and decreased inflammation (as assessed by decreased serum interleukin-6 and FCP) should be interpreted with caution as the sample size of this multi-center trial was very small $(n=12)$ and the reported $p$-values obtained from parametric tests were marginally significant. Therefore, these interesting findings need to be confirmed in future well-powered randomized controlled studies.

We identified only two studies $[36,37]$ that tested the efficacy of diet for induction of remission in UC patients. In the first study, exclusion of food items that were found to trigger UC-related symptoms was associated with higher clinical remission rate in comparison to a normal diet [36]. Although the elimination of foods was based on each participant's self-reported food intolerance, there were some general recommendations regarding specific food groups/items such as dairy products, refined sugar, and beverages. However, the study was performed on a small number of patients $(n=18)$, and the duration of follow-up was short (6 weeks). In addition, the intervention did not result in endoscopic or histologic improvement in that time period. Furthermore, patients in the intervention group experienced a mean weight loss of $2.5 \mathrm{~kg}$ that was not explained in the study. The authors also reported that there was no food that triggered symptoms in all patients. However, spicy and curried foods and fruits (specially grapes, melon, and citruses) were commonly reported to provoke symptoms. In the second study, which was performed in pediatric UC patients with active disease, elimination of cow milk protein from diet was not beneficial neither for induction or for maintenance of remission during a one-year follow-up in comparison to a control diet [37]. As mentioned by the authors, the dietary restrictions that many IBD patients follow often are not supported by scientific evidence. These inappropriate diets reduce caloric intake and may contribute to malnutrition and micronutrient deficiencies, especially in pediatric patients. Whether a subgroup of patients with UC (e.g., patients with lactose intolerance or atopy) will benefit from elimination diets or not needs to be explored in future clinical trials.

In this review, we also included three other studies that recruited patients with active disease and UC remission concurrently. They reported the effectiveness of comprehensive dietary advices [38], low FODMAP [40] or IgG-guided exclusion diets [41] in reduction of disease activity in UC patients. Although these findings are encouraging, one of the major limitations of these studies is that they did not report their findings for patients with active disease versus patients in UC remission separately to allow meaningful interpretations [45]. Therefore, we suggest that in future studies the dietary interventions be focused on clearly specified groups of patients (e.g., active disease or in remission) or study outcomes to be reported for different groups of participants separately.

Diet is of major interest for IBD patients, and they use a variety of dietary strategies to manage their underlying disease and related symptoms [34]. Despite the significant role of diet in the development of IBD or management of gastrointestinal symptoms in these patients, we could identify only a few randomized controlled trials that assessed the efficacy of diet for induction of remission, maintenance of remission, or improvement of gastrointestinal symptoms in UC patients. In addition, in the previous studies, the underlying mechanisms in which diet may prevent increases in colonic or systemic inflammation and ultimately help patients to maintain remission have not been investigated. There are many omics fields involved in the study of pathogenesis of IBD such as genomics, metagenomics, transcriptomics, proteomics, and metabolomics [32]. As dietary factors have a significant impact on some of these key players of IBD development, investigating the changes in this multi-omic network of IBD during a controlled dietary intervention has the potential to elucidate the underlying mechanisms of diet-IBD interactions. High quality, well-powered human dietary intervention studies for management of IBD may include the following: Quantification of baseline habitual diet using 
appropriate tools such as food frequency questionnaires, monitoring of adherence to the diet using food recalls/records, large long-term controlled trials, use of a control diet to determine the specificity of observed effects to the intervention, use of a variety of subjective and objective endpoints (e.g., symptoms, quality of life, clinical biomarkers, endoscopic and histological evaluations) to monitor response to dietary interventions [34], and consider the use of omic-based assessments of serum, urine, stool, and/or intestinal biopsies to investigate underlying protective mechanisms. Considering findings from previous observational studies and clinical trials, investigating the potential benefits of following a healthy dietary pattern, such as experimental anti-inflammatory diets that incorporate several dietary recommendations, is of great value in the management of UC-related symptoms and inflammation. Furthermore, as indicated in elimination diet studies, food intolerances are individual-based, and not all patients will benefit from excluding certain food items/groups. Therefore, personalized dietary recommendations that take into account each patient's food intolerances and food preferences should be the subject of future well-designed dietary trials in IBD patients.

\section{Conclusions}

In conclusion, we found that there have been few well-designed and/or adequately powered randomized clinical trials to investigate the role of diet in maintenance of remission in UC patients. As suggested in a recent Cochrane systematic review [45], consensus on the composition of evidence-based dietary interventions in IBD patients is required and there is a need for more high-quality, well-powered, randomized, controlled trials to assess the efficacy of these interventions.

Funding: A.H.K. was supported by a graduate studentship from Alberta Innovates-Health Solutions. K.L.M. and L.A.D. took part in the IMAGINE study funded by the Canadian Institute of Health and Research (CIHR). L.A.D. and K.L.M. were funded by Alberta Innovates-Bio Solution. K.L.M. and L.A.D. were funded through a CIHR operating grant.

Conflicts of Interest: The authors declare no conflict of interest.

\section{References}

1. Ungaro, R.; Mehandru, S.; Allen, P.B.; Peyrin-Biroulet, L.; Colombel, J.F. Ulcerative colitis. Lancet 2017, 389, 1756-1770. [CrossRef]

2. Ramos, G.P.; Papadakis, K.A. Mechanisms of Disease: Inflammatory Bowel Diseases. Mayo Clin. Proc. 2019, 94, 155-165. [CrossRef] [PubMed]

3. Ananthakrishnan, A.N. Debate session: So what causes inflammatory bowel disease? It's all in the environment. J. Gastroenterol. Hepatol. 2018, 33, 24. [CrossRef] [PubMed]

4. Hou, J.K.; Abraham, B.; El-Serag, H. Dietary Intake and Risk of Developing Inflammatory Bowel Disease: A Systematic Review of the Literature. Am. J. Gastroenterol. 2011, 106, 563-573. [CrossRef] [PubMed]

5. Nie, J.Y.; Zhao, Q. Beverage consumption and risk of ulcerative colitis: Systematic review and meta-analysis of epidemiological studies. Medicine (Baltimore) 2017, 96, e9070. [CrossRef] [PubMed]

6. Wang, F.; Feng, J.; Gao, Q.; Ma, M.; Lin, X.; Liu, J.; Li, J.; Zhao, Q. Carbohydrate and protein intake and risk of ulcerative colitis: Systematic review and dose-response meta-analysis of epidemiological studies. Clin. Nutr. 2017, 36, 1259-1265. [CrossRef]

7. Li, F.; Liu, X.; Wang, W.; Zhang, D. Consumption of vegetables and fruit and the risk of inflammatory bowel disease: a meta-analysis. Eur. J. Gastroenterol. Hepatol. 2015, 27, 623-630. [CrossRef] [PubMed]

8. Ge, J.; Han, T.-J.; Liu, J.; Li, J.-S.; Zhang, X.-H.; Wang, Y.; Li, Q.-Y.; Zhu, Q.; Yang, C.-M. Meat intake and risk of inflammatory bowel disease: A meta-analysis. Turk. J. Gastroenterol. 2015, 26, 492-497. [CrossRef] [PubMed]

9. John, S.; Luben, R.; Shrestha, S.S.; Welch, A.; Khaw, K.-T.; Hart, A.R. Dietary n-3 polyunsaturated fatty acids and the aetiology of ulcerative colitis: A UK prospective cohort study. Eur. J. Gastroenterol. Hepatol. 2010, 22, 602-606. [CrossRef]

10. De Silva, P.S.; Olsen, A.; Christensen, J.; Schmidt, E.B.; Overvaad, K.; Tjonneland, A.; Hart, A.R. An association between dietary arachidonic acid, measured in adipose tissue, and ulcerative colitis. Gastroenterology 2010, 139, 1912-1917. [CrossRef] [PubMed] 
11. Khalili, H.; Chan, S.S.M.; Lochhead, P.; Ananthakrishnan, A.N.; Hart, A.R.; Chan, A.T. The role of diet in the aetiopathogenesis of inflammatory bowel disease. Nat. Rev. Gastroenterol. Hepatol. 2018, 15, 525-535. [CrossRef] [PubMed]

12. McIlroy, J.; Ianiro, G.; Mukhopadhya, I.; Hansen, R.; Hold, G.L. Review article: the gut microbiome in inflammatory bowel disease-avenues for microbial management. Aliment. Pharmacol. Ther. 2018, 47, $26-42$. [CrossRef] [PubMed]

13. Ni, J.; Wu, G.D.; Albenberg, L.; Tomov, V.T. Gut microbiota and IBD: causation or correlation? Nat. Rev. Gastroenterol. Hepatol. 2017, 14, 573-584. [CrossRef] [PubMed]

14. Nishida, A.; Inoue, R.; Inatomi, O.; Bamba, S.; Naito, Y.; Andoh, A. Gut microbiota in the pathogenesis of inflammatory bowel disease. Clin. J. Gastroenterol. 2018, 11, 1-10. [CrossRef] [PubMed]

15. Frank, D.N.; Amand, A.L.S.; Feldman, R.A.; Boedeker, E.C.; Harpaz, N.; Pace, N.R. Molecular-phylogenetic characterization of microbial community imbalances in human inflammatory bowel diseases. Proc. Natl. Acad. Sci. USA 2007, 104, 13780-13785. [CrossRef] [PubMed]

16. Brown, K.; DeCoffe, D.; Molcan, E.; Gibson, D.L. Diet-Induced Dysbiosis of the Intestinal Microbiota and the Effects on Immunity and Disease. Nutrients 2012, 4, 1095-1119. [CrossRef] [PubMed]

17. De Filippo, C.; Cavalieri, D.; Di Paola, M.; Ramazzotti, M.; Poullet, J.B.; Massart, S.; Collini, S.; Pieraccini, G.; Lionetti, P. Impact of diet in shaping gut microbiota revealed by a comparative study in children from Europe and rural Africa. Proc. Natl. Acad. Sci. USA 2010, 107, 14691-14696. [CrossRef]

18. Agus, A.; Denizot, J.; Thévenot, J.; Martinez-Medina, M.; Massier, S.; Sauvanet, P.; Bernalier-Donadille, A.; Denis, S.; Hofman, P.; Bonnet, R.; et al. Western diet induces a shift in microbiota composition enhancing susceptibility to Adherent-Invasive E. coli infection and intestinal inflammation. Sci. Rep. 2016, 6, 19032. [CrossRef] [PubMed]

19. Sun, M.; Wu, W.; Liu, Z.; Cong, Y. Microbiota metabolite short chain fatty acids, GPCR, and inflammatory bowel diseases. J. Gastroenterol. 2017, 52, 1-8. [CrossRef]

20. Neis, E.P.; van Eijk, H.M.; Lenaerts, K.; Olde Damink, S.W.; Blaak, E.E.; Dejong, C.H.; Rensen, S.S. Distal versus proximal intestinal short-chain fatty acid release in man. Gut 2019, 68, 764-765. [CrossRef]

21. Reddavide, R.; Rotolo, O.; Caruso, M.G.; Stasi, E.; Notarnicola, M.; Miraglia, C.; Nouvenne, A.; Meschi, T.; De' Angelis, G.L.; Di Mario, F.; et al. The role of diet in the prevention and treatment of Inflammatory Bowel Diseases. Acta Biomed. 2018, 89, 60-75. [PubMed]

22. Sugihara, K.; Morhardt, T.L.; Kamada, N. The Role of Dietary Nutrients in Inflammatory Bowel Disease. Front. Immunol. 2019, 9, 3183. [CrossRef] [PubMed]

23. Machiels, K.; Joossens, M.; Sabino, J.; De Preter, V.; Arijs, I.; Eeckhaut, V.; Ballet, V.; Claes, K.; Van Immerseel, F.; Verbeke, K.; et al. A decrease of the butyrate-producing species Roseburia hominis and Faecalibacterium prausnitzii defines dysbiosis in patients with ulcerative colitis. Gut 2014, 63, 1275-1283. [CrossRef] [PubMed]

24. Koleva, P.; Ketabi, A.; Valcheva, R.; Gänzle, M.G.; Dieleman, L.A. Chemically defined diet alters the protective properties of fructo-oligosaccharides and isomalto-oligosaccharides in HLA-B27 transgenic rats. PLoS ONE 2014, 9, e111717. [CrossRef] [PubMed]

25. Ahluwalia, B.; Moraes, L.; Magnusson, M.K.; Ohman, L. Immunopathogenesis of inflammatory bowel disease and mechanisms of biological therapies. Scand. J. Gastroenterol. 2018, 53, 379-389. [CrossRef] [PubMed]

26. Werner, T.; Wagner, S.J.; Martínez, I.; Walter, J.; Chang, J.S.; Clavel, T.; Kisling, S.; Schuemann, K.; Haller, D. Depletion of luminal iron alters the gut microbiota and prevents Crohn's disease-like ileitis. Gut 2011, 60, 325-333. [CrossRef]

27. Sturniolo, G.C.; Di Leo, V.; Ferronato, A.; D’Odorico, A.; D’Incà, R. Zinc Supplementation Tightens “Leaky Gut" in Crohn's Disease. Inflamm. Bowel Dis. 2001, 7, 94-98. [CrossRef]

28. Reich, K.M.; Fedorak, R.N.; Madsen, K.; I Kroeker, K. Vitamin D improves inflammatory bowel disease outcomes: Basic science and clinical review. World J. Gastroenterol. 2014, 20, 4934-4947. [CrossRef]

29. Scoville, E.A.; Allaman, M.M.; Adams, D.W.; Motley, A.K.; Peyton, S.C.; Ferguson, S.L.; Horst, S.N.; Williams, C.S.; Beaulieu, D.B.; Schwartz, D.A.; et al. Serum Polyunsaturated Fatty Acids Correlate with Serum Cytokines and Clinical Disease Activity in Crohn's Disease. Sci. Rep. 2019, 9, 2882. [CrossRef]

30. Scaioli, E.; Liverani, E.; Belluzzi, A. The Imbalance between n-6/n-3 Polyunsaturated Fatty Acids and Inflammatory Bowel Disease: A Comprehensive Review and Future Therapeutic Perspectives. Int. J. Mol. Sci. 2017, 18, 2619. [CrossRef] 
31. Ruemmele, F.M. Role of Diet in Inflammatory Bowel Disease. Ann. Nutr. Metab. 2016, 68, 33-41. [CrossRef] [PubMed]

32. Ananthakrishnan, A.N.; Bernstein, C.N.; Iliopoulos, D.; Macpherson, A.; Neurath, M.F.; Ali, R.A.R.; Vavricka, S.R.; Fiocchi, C. Environmental triggers in IBD: A review of progress and evidence. Nat. Rev. Gastroenterol. Hepatol. 2018, 15, 39-49. [CrossRef] [PubMed]

33. Levine, A.; Boneh, R.S.; Wine, E. Evolving role of diet in the pathogenesis and treatment of inflammatory bowel diseases. Gut 2018, 67, 1726-1738. [CrossRef] [PubMed]

34. Haskey, N.; Gibson, D.L. An Examination of Diet for the Maintenance of Remission in Inflammatory Bowel Disease. Nutrients 2017, 9, 259. [CrossRef] [PubMed]

35. Wright, R.; Truelove, S.C. A Controlled Therapeutic Trial of Various Diets in Ulcerative Colitis. BMJ 1965, 2, 138-141. [CrossRef] [PubMed]

36. Candy, S.; Borok, G.; Wright, J.P.; Boniface, V.; Goodman, R. The value of an elimination diet in the management of patients with ulcerative colitis. South Afr. Med. J. 1995, 85, 1176-1179.

37. Strisciuglio, C.; Giannetti, E.; Martinelli, M.; Sciorio, E.; Staiano, A.; Miele, E. Does cow's milk protein elimination diet have a role on induction and maintenance of remission in children with ulcerative colitis? Acta Paediatr. 2013, 102, e273-e278. [CrossRef]

38. Kyaw, M.H.; Moshkovska, T.; Mayberry, J. A prospective, randomized, controlled, exploratory study of comprehensive dietary advice in ulcerative colitis: Impact on disease activity and quality of life. Eur. J. Gastroenterol. Hepatol. 2014, 26, 910-917. [CrossRef]

39. Bhattacharyya, S.; Shumard, T.; Xie, H.; Dodda, A.; Varady, K.A.; Feferman, L.; Halline, A.G.; Goldstein, J.L.; Hanauer, S.B.; Tobacman, J.K. A randomized trial of the effects of the no-carrageenan diet on ulcerative colitis disease activity. Nutr. Healthy Aging 2017, 4, 181-192. [CrossRef]

40. Pedersen, N.; Ankersen, D.V.; Felding, M.; Wachmann, H.; Végh, Z.; Molzen, L.; Burisch, J.; Andersen, J.R.; Munkholm, P. Low-FODMAP diet reduces irritable bowel symptoms in patients with inflammatory bowel disease. World J. Gastroenterol. 2017, 23, 3356-3366. [CrossRef]

41. Jian, L.; Anqi, H.; Gang, L.; Litian, W.; Yanyan, X.; Mengdi, W.; Tong, L. Food Exclusion Based on IgG Antibodies Alleviates Symptoms in Ulcerative Colitis: A Prospective Study. Inflamm. Bowel Dis. 2018, 24, 1918-1925. [CrossRef] [PubMed]

42. Lim, H.-S.; Kim, S.-K.; Hong, S.-J. Food Elimination Diet and Nutritional Deficiency in Patients with Inflammatory Bowel Disease. Clin. Nutr. Res. 2018, 7, 48-55. [CrossRef] [PubMed]

43. Fahoum, L.; (Moscovici), A.J.; David, S.; Shaoul, R.; Rozen, G.; Meyron-Holtz, E.G.; Lesmes, U.; Meyron-Holtz, E.G. Digestive fate of dietary carrageenan: Evidence of interference with digestive proteolysis and disruption of gut epithelial function. Mol. Nutr. Food Res. 2017, 61, 1600545. [CrossRef] [PubMed]

44. Weiner, M.L.; McKim, J.M. Comment on "Revisiting the carrageenan controversy: Do we really understand the digestive fate and safety of carrageenan in our foods?". Food Funct. 2019, 10, 1760-1762. [CrossRef] [PubMed]

45. Limketkai, B.N.; Iheozor-Ejiofor, Z.; Gjuladin-Hellon, T.; Parian, A.; Matarese, L.E.; Bracewell, K.; Macdonald, J.K.; Gordon, M.; Mullin, G.E. Dietary interventions for induction and maintenance of remission in inflammatory bowel disease. Cochrane Database Syst. Rev. 2019, 2, CD012. [CrossRef]

(C) 2019 by the authors. Licensee MDPI, Basel, Switzerland. This article is an open access article distributed under the terms and conditions of the Creative Commons Attribution (CC BY) license (http://creativecommons.org/licenses/by/4.0/). 


\title{
A Personalised Dietary Approach-A Way Forward to Manage Nutrient Deficiency, Effects of the Western Diet, and Food Intolerances in Inflammatory Bowel Disease
}

\author{
Bobbi B Laing ${ }^{1,2}$, Anecita Gigi Lim ${ }^{1}$ and Lynnette R Ferguson ${ }^{1, *}$ \\ 1 Faculty of Medical and Health Sciences, University of Auckland, Auckland 1023, New Zealand \\ 2 Nutrition Society of New Zealand, Palmerston North 4444, New Zealand \\ * Correspondence: 1.ferguson@auckland.ac.nz
}

Received: 7 May 2019; Accepted: 2 July 2019; Published: 5 July 2019

\begin{abstract}
This review discusses the personalised dietary approach with respect to inflammatory bowel disease (IBD). It identifies gene-nutrient interactions associated with the nutritional deficiencies that people with IBD commonly experience, and the role of the Western diet in influencing these. It also discusses food intolerances and how particular genotypes can affect these. It is well established that with respect to food there is no "one size fits all" diet for those with IBD. Gene-nutrient interactions may help explain this variability in response to food that is associated with IBD. Nutrigenomic research, which examines the effects of food and its constituents on gene expression, shows that-like a number of pharmaceutical products—food can have beneficial effects or have adverse (side) effects depending on a person's genotype. Pharmacogenetic research is identifying gene variants with adverse reactions to drugs, and this is modifying clinical practice and allowing individualised treatment. Nutrigenomic research could enable individualised treatment in persons with IBD and enable more accurate tailoring of food intake, to avoid exacerbating malnutrition and to counter some of the adverse effects of the Western diet. It may also help to establish the dietary pattern that is most protective against IBD.
\end{abstract}

Keywords: inflammatory bowel disease; Westernisation; genotypes; nutrient deficiency; food intolerance; FODMAPs; gluten; fructose; lactose; brassica; mushrooms

\section{Introduction}

The personalised dietary approach in combination with the knowledge of the genotype and genetic variants of an individual who has nutrient deficiencies, follows the Western diet, or may have food intolerances may be helpful for people who have inflammatory bowel disease (IBD). Gene-nutrient interactions could help explain the variability in response to food that is associated with IBD, and using this knowledge with a personalised dietary approach may offer a way forward. Identification of individuals' genotypes is a first step to building up data sets which combine this information with data on individuals and their gut microbiome, dietary patterns, exercise routines, blood parameters and anthropometric measurements. This information can be used to build machine-learning algorithms to predict what foods/meals reduce inflammation and abdominal symptoms and maintain a healthy gut flora. Zeevi et al. have pioneered this approach in their work with diabetes [1]. They used this combination of information to successfully predict which foods/meals lowered postprandial blood glucose. Identifying genotypes associated with nutrient deficiencies or low nutrient absorption, the negative effects of the Western diet and the tolerance or intolerance of particular foods may also advise people on how to eat a greater variety of food. A wider range of food available to the gut microbiota enables their greater diversity, and this is associated with a healthy gut [2]. 
Pharmacogenetic research (the study of the variability in drug response due to genotype) is identifying gene variants with adverse reactions to drugs, and this is modifying clinical practice and allowing individualised treatment. The aim is to provide the right drug, at the right dose, at the right time, to the right patient [3]. Individual responses can be dependent on an individual's genotype. Genetic variants can alter the metabolism of drugs as well as responsiveness, and this may necessitate changes to standardised drug regimes. The standard dose may not maintain the required therapeutic level if individuals metabolise the drug more quickly; conversely, if they are slow metabolisers the standard dose maybe toxic. Knowledge of pharmacogenetics and its application to precision medicine to enhance treatment responses and minimize drug-related adverse events is one approach that has been suggested as the way forward for IBD [4]. Pharmacogenetics information facilitates the identification of responders and non-responders to drugs and guides the decision about optimal treatment [5]. Similarly, further nutrigenomic research could identify nutrient-gene interactions in people with IBD. This would enable the more accurate tailoring of food intake and could suggest dietary patterns which avoid exacerbating malnutrition, curtail the adverse effects of the Western diet and explain intolerance to particular foods. This could lead to the minimization of abdominal symptoms and disease activity in those who experience IBD.

In an extensive study of 366,351 European individuals in 2016 aiming to identify dietary patterns and risk of IBD, no dietary pattern was identified with the risk of either of the two main expressions of IBD (i.e., ulcerative colitis (UC) or Crohn's disease (CD)) [6]. However, when cases occurring within the first two years after dietary assessment were excluded, there was a positive association between a "high sugar and soft drinks" pattern and UC risk if they also had a low vegetable intake [6]. This study shows there are many challenges in determining the best dietary pattern for individuals with a particular chronic disease like IBD, given the genetic differences between people and their environments [7]. The current consensus is that no single nutrition recommendation can be made to modify this situation with respect to IBD—-that is, there is no "one size fits all" solution for people with IBD [8]. However, using the personalised dietary approach combined with information on people's genotypes may offer a way to resolve this conundrum.

The aim of this narrative review (based on searching two data bases, PubMed and Google Scholar) is to discuss the personalised dietary approach with respect to IBD. First it will identify nutritional deficiencies which individuals with IBD commonly experience, and give an example of a key nutrient whose absorption is affected by genotype. Secondly it will review the role of the Western diet in contributing to nutrient deficiencies and IBD, and give examples of some of the genes involved. Thirdly it will review the various food intolerances and food avoidances associated with IBD which may also contribute to nutrient deficiencies, and how particular genotypes can contribute to these.

\section{Nutrient Deficiencies Associated with IBD}

\subsection{Nutritional Status of People with Inflammatory Bowel Disease}

Three studies which have assessed the nutritional status of people with IBD are illustrated in Table 1. Assessment of people with IBD often shows they are under weight, with specific vitamin and mineral imbalances, and this contributes to their ill health [9-14]. The three studies in this table illustrate the common nutritional findings. Vagianos and colleagues collected dietary information by questionnaires (including dietary supplements) and observed multiple and clinically significant vitamin and mineral deficiencies in adults with $\mathrm{CD}$ who attended a gastroenterology outpatient clinic [9]. Inadequate reported intake was defined as $<66 \%$ of the dietary reference intake (RFI) for each micronutrient. These authors reported that when comparisons were made by disease type or disease activity, there were no significant differences in the percentage of subjects consuming inadequate amounts of micronutrients. Stein and Bott (2008) reported on nutrient deficiencies in hospitalised adults with CD [10]. Hartman et al. in 2016 [15] investigated the nutritional status of children and adolescents who were ambulatory outpatients at a gastroenterology clinic. They reported on the 
percentage median nutrient intake compared with the required daily allowance (RDA) and dietary intake of healthy children. The intake was considered low if it was less than $80 \%$ of the RDA. During this study, two thirds of participants had active disease. However, there was no significant difference in nutrient intake between those with active disease and those in remission, and no association of nutrient levels with disease activity.

Table 1. Nutrient deficiencies in adults and adolescents with inflammatory bowel disease (IBD).

\begin{tabular}{cccc}
\hline Reference & Vagianos et al. [9] & Stein and Bott [10] & Hartman et al. [15] \\
\cline { 2 - 4 } & Outpatient & Inpatient & Outpatient \\
\hline Year of Investigation & 2007 & 2008 & 2016 \\
Number of Participants & 71 & na & 68 \\
Gender (Male, Female) & 32,52 & na & na \\
Age (years) & $37.6 \pm 14.3$ & Adult & $13.9 \pm 3.2$ \\
Country & Canada & Germany & Israel \\
Dietary Measurement & FFQ, 4-day records, & na & 3-day records, \\
Laboratory Measurement & Anthropometric & na & Anthropometric \\
Dietary Deficiency & Biomarkers for nutrients (CD) & & Biomarkers for nutrients \\
\hline Measures & & \% Inadequate Intake & \% \\
\hline Vitamin B6 of RDA \\
Vitamin B12 & \% $66 \%$ RFI & na & 10 \\
Folate & 4.2 & 48 & 12 \\
Vitamin C & 5.6 & $56-62$ & 34 \\
Vitamin A & 20 & na & 31 \\
Vitamin D & 9.9 & $11-50$ & 57 \\
Vitamin E & 30 & $23-75$ & 25 \\
Calcium & 38 & na & 65 \\
Iron & 59 & 13 & 79 \\
Magnesium & 25 & $25-50$ & 22 \\
Zinc & 14 & $14-33$ & 69 \\
\hline
\end{tabular}

CD: Crohn's disease; FFQ: food frequency questionnaire; na: not available; RFI: dietary reference intake; RDA: required daily allowance.

All three studies showed low levels of vitamin A and D and folate in people with CD. Stein and Bott [10] and Hartman et al. [15] both found substantial risk for deficiencies of magnesium, zinc and iron (Table 1). Stein and Bott [10] also showed lower $B_{12}$ concentrations for inpatients and Vagianos et al. commented that in those with CD (compared to those with UC, the other main expression of IBD) had lower $B_{12}$ concentrations with their serum biomarkers. Also, among men with $C D$ there was a significantly higher proportion who had haemoglobin below the normal range compared to men with UC. Hartman et al. reported that anaemia was the most common sign of nutrient deficiency in their adolescents (51\%). These studies illustrate that people with different expressions of IBD (i.e., CD or UC) have different nutrient needs. It also illustrates that it is important to assess nutritional status (through laboratory measurement of nutrient biomarkers) to avoid exacerbating malnutrition if particular foods are removed from the diet.

For people with $\mathrm{CD}$ in particular, malnutrition may also occur because of the severity of oral lesions experienced, which results in difficulties with tasting, chewing and swallowing. This means that some foods are avoided (e.g., tomatoes because of their perceived acidity). Oral involvement in CD has been identified in $8 \%-29 \%$ of people, irrespective of disease activity [16]. Examples of oral symptoms include labial swelling (which also may include gingiva and mucosa), mucosal polyps, cracking at the edges of the lips (angular cheilitis), ulcers, fissures and granulomas [17,18]. The mucous membranes in the mouth normally have a rapid cell turnover of 3-7 days, in contrast to the skin which is up to 28 days [19]. Loss of cells without replacement can lead to the mouth showing early signs of systemic disease and early predisposition to nutritional deficiency. This is another reason why optimal nutrition is important for people with IBD. 
Many vitamins and minerals are considered necessary for healthy mucous membranes (Table 2) [20]. A number of these essential nutrients are low in people with people with IBD (Table $1[9,10,15])$. These nutrient deficiencies, and a higher presence of bacteria associated with dental caries, undoubtedly contribute to the oral symptoms experienced by people with CD. Sufficient levels of these nutrients would need to be included in any food regime suggested for IBD.

Table 2. Nutrients associated with healthy mucous membranes [20].

\begin{tabular}{ccc}
\hline Water Soluble Vitamins & Fat-Soluble Vitamins & Minerals \\
\hline Vitamin $\mathrm{B}_{2}$ (riboflavin) & $\mathrm{A}$ & Calcium \\
Vitamin $\mathrm{B}_{3}$ (niacin) & $\mathrm{D}$ & Fluoride \\
Vitamin $\mathrm{B}_{6}$ family & $\mathrm{E}$ & Zinc \\
Vitamin $\mathrm{B}_{12}$ (cobalamin) & & Iron \\
Folic acid (folate) & & \\
Vitamin $C$ (ascorbic acid) & & \\
\hline
\end{tabular}

\subsection{Nutrient Absorption Impacted by Genotype}

One example of a nutrient whose absorption can be impacted by genotype is beta-carotene. The gene that affects its absorption is the beta-carotene $15,15^{\prime}$-monooxygenase gene (BCMO1). This gene encodes a protein which is an important enzyme in the metabolism of the nutrient beta-carotene to vitamin A [21]. It is expressed in a number of tissues (e.g., liver, lungs, skin, small intestine). Within the enterocytes of the mucous membranes, the enzyme cleaves beta-carotene into two retinal molecules. These are packaged with other dietary lipids into chylomicrons and then secreted into the lymphatic system. Fat in the diet enables optimal chylomicron formation [21,22]. According to the study by Leung et al., carrying one of the two polymorphisms (R267S: rs12934922 or A379V: rs7501331) of the $B C M O 1$ gene impedes the conversion of beta-carotene to retinol [23]. This study was based on a healthy population from the UK. Up to $45 \%$ of people in this study carried one of these two polymorphisms. Having one of the two polymorphisms of the BCMO1 gene in conjunction with IBD could thus have an impact on the health status of the carrier. New Zealand (NZ) is a country with a high incidence of IBD (26/100,000 in 2016) [24], and in their most recent National Nutrition Survey (2008/9) reported that the NZ population sourced $58 \%$ of their vitamin A intake from carotenoids. The NZ population as a whole was also recorded as $22.7 \%$ and $12.1 \%$ deficient in vitamin A for males and females, respectively. For those aged 15-18 years, these percentages increased to $27.4 \%$ and $37.5 \%$ [25].

Adequate vitamin A intake is necessary for optimal activity of the innate and adaptive immune systems. Vitamin A is needed for the appropriate innate immune defence response. It affects neutrophil reactions to microbes, macrophage phagocytosis, and natural killer cell activity. Cultured human macrophages enriched with retinoic acid have been shown to have increased resistance to $M$. tuberculosis by down-regulating tryptophan-aspartate-containing coat protein (TACO) [26]. Vitamin A is also needed for the optimal formation of $\mathrm{T}$ and $\mathrm{B}$ cells in the adaptive immune system and the balanced type 1 and type 2 cytokine responses. Impairment of these functions through vitamin A deficiency leads to epithelial barrier dysfunction and inflammation—hallmarks of IBD [27]. Vitamin A deficiency in children increases the risk of signs and symptoms often associated with CD (i.e., impairment of iron use that aggravates anaemia, respiratory infections and diminished growth rates) [28]. If individuals with IBD also carry one of these at-risk alleles, they are at risk of being vitamin A deficient.

This may be a contributing factor to this nutrient deficiency reported in people with IBD. Using the personalised dietary approach and identifying if a person with IBD carries these variants linked to poor absorption of vitamin A would enable the tailoring of nutrient advice to ameliorate this. The Western diet may also contribute to nutrient deficiencies. 


\section{The Western Diet}

The incidence of gastro-intestinal disorders like CD and UC has been increasing [29-32]. This has often been related to the current Western diet, which has changed substantially in the past seven decades [33-39]. This is because of changes in food production and technology, which provides easy access of urban based populations to cheap ultra-processed foods and foods high in refined grains, oil and sugar [40]. In Bangladesh, for example, the incidence of diagnosed IBD is very low. However, when Bangladeshis move to the United Kingdom where they are more highly exposed to Western culture and food choices, the incidence of IBD increases substantially within a generation [41]. This may also be associated with autoimmune disease developing as a result of increasing latitude, decreased exposure to sunlight and lower vitamin D intake [42]. They may also receive better health care which identifies their disease pathology more accurately. However, modern Western food is also becoming more available in the developing world. In the review of the global epidemiology of IBD by M'Koma, it was noted that there has been an increase in the incidence and prevalence of IBD in parts of Asia and Africa-particularly in urban areas and in higher socio-economic classes [43]. This Westernisation of the diet has resulted in lower intakes of dietary fibre, increased consumption of saturated fatty acids, and sugar. Westernisation has also introduced other factors such as changes in birthing methods, increased use of artificial formula instead of breast feeding, and the use of sugar substitutes, food additives and antibiotics. Many of these changes have been associated with a reduction in gut microbial diversity, which is a feature of IBD $[44,45]$.

\subsection{Lower Intakes of Dietary Fibre}

As the diet becomes more Westernised and processed, "Western" food often contains less fibre. Current intakes of fibre associated with the Western diet are assessed to be about $20 \mathrm{~g} /$ day, compared to a fibre content of 70-120 g/day when diets rich in fruit, vegetables and grains are consumed, as is estimated for hunter-gathers and agriculturalists in human history [46]. The low consumption of fruit, vegetables and dietary fibre and the increased intake of animal fat and refined sugars have been observed in people with disorders like IBD [47], as are exclusion diets for IBD that are made popular in the media [48].

The risk of IBD has been negatively associated with the intake of fibre $[34,49,50]$. Fibre can be defined in a number of ways, as illustrated by the different definitions by the American Association of Cereal Chemists (AACC) [51] and The Codex Alimentarius Commission [52]. A recent AACC definition describes fibre as being a component of "edible plants or analogous carbohydrates that are resistant to digestion and absorption in the human small intestine, with complete or partial fermentation in the large intestine" [53]. They include in their definition polysaccharides, oligosaccharides, lignin and associated plant substances. Fibre has been further subdivided into rapidly digested starch, slowly digested starch and resistant starch (RS), and the latter is classified into five categories (RS1-RS5). High levels of resistant starch are found in unripe bananas, raw potatoes, maize, pulse grains and legumes. However, depending on their processing, the RS content can vary, and in legumes this can range from a few percent to 80\% [54]. The RS3 category (retrograded starch) has been shown in wild type interleukin-10-deficient IBD murine studies to decrease ileal and colonic inflammatory lesions compared to a low-fat diet $[55,56]$. The authors suggested that soluble fibre and resistant starch suppress gut inflammation. The inclusion of soluble fibre and resistant starch in the diet of individuals with IBD may be helpful for this reason.

Fibre can also be described as soluble and insoluble [57]. The removal of insoluble fibre, a component of the skins of fruit, vegetables, whole grains, and seeds, is a current recommendation by the Crohn's and Colitis Foundation of America [58]. When people are experiencing disease activity (sometimes described as a flare or relapse), insoluble fibre may intensify bloating, pain, diarrhoea and gas. However, the consumption of soluble fibre in vegetables (e.g., the brassica family, dried peas and beans, parsnips, carrots, potatoes, spinach, squash, zucchini) and fruit (e.g., pip and stone fruit, berries, bananas, dates, cantaloupe, grapes and pineapple), oat cereals and barley is recommended. Soluble 
fibre promotes water absorption, enabling the gut contents to form a gel-like consistency, which delays emptying of the gut and reduces diarrhoea [58]. Soluble fibre also provides a substrate for bacterial fermentation in the colon [59]. The soluble plant fibres in plantain and broccoli reduce the movement of E. coli across M cells in Peyer's patches in CD patients [60]. In CD, E. coli is found in increased numbers in the mucosa $[61,62]$. This means it is important that appropriate fibre types are chosen if people with IBD are removing food or food groups (e.g., selecting gluten-free diets or foods low in FODMAPs (fermentable oligo-, di- and mono-saccharides and polyols)) so they do not inadvertently contribute to an increase in disease activity later in their health journey [63].

There are also identified gene-nutrient interactions associated with the role of fibre. One of these is the free fatty acid receptor 2 gene (FFAR2; previously known as G protein-coupled receptor 43-GPR43). This gene is a member of the largest gene family - the seven-transmembrane receptor group known as G-protein-coupled receptors (GPCRs) [64]. These are involved as mediators of cellular responses to many neurotransmitters and hormones [65]. FFAR2 is highly expressed in neutrophils [66]. One pathological marker of $\mathrm{CD}$ is the migration of neutrophilic granulocytes into the mucosa of the intestine [67]. FFAR2 also activates pathways involved in intracellular $\mathrm{Ca}^{2+}$ release and inhibits the build-up of cAMP [68].

The FFAR2 receptors are present in fat tissues, inflammatory cells and the large intestine [69]. In the large intestine, the receptors are stimulated by short-chain fatty acids (SCFAs), which are produced by bacteria fermenting dietary fibre [21,70]. Lower intakes of dietary fibre decrease the production of SCFAs, which stimulates FFAR2 signalling. The study by Sivaprakasam et al. showed the critical role of FFAR2 in the maintenance of healthy gut microbiota, leading to the suppression of intestinal carcinogenesis [71].

The interaction of SCFAs and FFAR2 greatly affects inflammatory processes. SCFAs play an important role in colon homeostasis, as they promote pathways involved in cell differentiation, growth regulation, immune regulation and apoptosis [72]. SCFAs and butyrate in particular seem to be the favoured fuel source of the bacteria in the colon as well as of colonocytes, which are required for the functional integrity of the epithelium [73-76]. The source of SCFAs is the intake of highly fermentable fibres, the residues of which are converted by anaerobic bacteria into SCFAs upon reaching the large bowel [77]. SCFAs have been observed to increase the beneficial populations of Bifidobacterium and Lactobacillus, which have a role in anti-inflammatory outcomes and immune functions $[38,78,79]$. SCFAs appear to be important in epithelial barrier maintenance and immune system regulation. Maslowski and Mackay consider the SCFAs produced in the colon to be very important for immunoregulation. They propose that the lower dietary fibre intake associated with the modern Western diet is the driver behind the increased incidence of inflammatory diseases associated with this lifestyle factor [70].

However, there is currently no published literature about the significance of this gene with respect to people with IBD and their intake of fibre. People with IBD (whether with disease activity or in remission) have been observed to have a low intake of fruit, vegetables and dietary fibre $[49,80]$. Perhaps particular variants in the FFAR2 gene may affect a person's response to fibre, and this in turn contributes to changes in microbiota composition. This may explain how those who follow a strict FODMAP diet experience a reduction in beneficial bacteria [81,82].

\subsection{Increased Consumption of Saturated Fatty Acids}

Some authors believe that the Western diet has also become pro-inflammatory as a result of the increased consumption of saturated fatty acids and decreased consumption of polyunsaturated fatty acids (PUFAs), especially long-chain PUFAs (LC-PUFAs) [83-87]. There has also been a decrease in the ratio of the omega- 3 and omega-6 PUFAs ingested, partly because of a lower consumption of fish. Fish intolerance and allergy is well-recognised in childhood, being the third most frequent allergen after cow's milk and egg in European children [88-90]. There is less evidence implicating fish intolerance or allergy in $\mathrm{CD}$. Untersmayr et al. observed that antacid medication plus fish consumption impeded the digestion of dietary proteins in their BALB/c mouse fish allergy model [91]. 
In earlier times, people consumed only small amounts of these PUFAs, and in about the same proportion. Today it is estimated that this ratio is now about 1:10-1:20-25 omega-3:omega-6. This has occurred from the increased intake of vegetable oils (e.g., soy, safflower, corn and sunflower) [83,92,93]. The genes and their variants linked to differences in fatty acid absorption include fatty acid desaturase genes (FADS1, FADS2), the peroxisome proliferator-activated receptor genes (PPARA, PPARG), the X-ray repair cross-complementing protein 1 gene $(X R C C 1)$ and stearoyl-CoA desaturase (SCD1). A number of studies have discussed the role that these genes play in the serum levels of LC-PUFA-omega-3 and omega- 6 fatty acids, as well as the effects of various variants on metabolic pathways and how this impacts inflammation and cancer risk [94-104]. These studies show that the impact of these fatty acids—especially on inflammation—is also influenced by the person's genotype. This may have deleterious effects on individuals with IBD who exclude fish and fish oils from their diet and have a high omega-3:omega-6 PUFA ratio.

\subsection{Fructose and Sugar Consumption}

There is much debate in the literature about the effects of fructose consumption [105-116]. The rise in obesity, diabetes, metabolic syndrome, IBD, coronary heart disease, and whether the increased consumption of fructose or fructans has contributed to these disorders, has been widely discussed [105-116]. Over recent decades, increasing amounts of fructose have been consumed as part of the Western diet. This has been particularly associated with the addition of sugars to processed foods, particularly high-fructose corn syrup (HFCS, comprising $~ 60 \%$ fructose) in the USA or with cane sugar (50\% fructose) in NZ. Both are cheap and ubiquitous sweeteners, especially in soft drinks and cereal products [117]. The effect of fructose in infant formulas on vascular inflammation has also been investigated in full-term babies. The study found that the fructose formula had a higher inflammatory response than the fructose-free formula, but the difference was not significant [118]. The authors commented that the result may become significant in a preterm infant [118]. According to recent USDA figures, the estimated calories of HFCS consumed daily has risen from $0.4 \mathrm{~g} /$ day in 1970, peaked at $44.3 \mathrm{~g} /$ day in 1997 , and has gradually declined (31.0 g/day in 2015) $[25,119,120]$. However, total "sweetener" consumption has increased compared to the 1970 figures (from $87.3 \mathrm{~g} /$ day in 1970 to $94.1 \mathrm{~g} /$ day in 2015) [119]. In NZ, the Adult Nutrition surveys of 1997 and 2008-2009 report that the mean fructose intake and the mean total sugar consumption for European males and females has declined from 1997 ( $26 \mathrm{~g} /$ day and $139 \mathrm{~g} /$ day, respectively), to 2008 (23.4 g/day and $100.3 \mathrm{~g} /$ day respectively) [121]. Unfortunately, there are no recent national consumption figures to see whether that national trend has continued. A small regional study $(n=227)$ in 2014 on a Canterbury (NZ) population aged 50 years reported that the mean intake for fructose for men was $25.5 \mathrm{~g}$ and $20.6 \mathrm{~g}$ for females [122]. This suggests that the downward trend has not continued.

Once ingested, fructose is transported into the blood stream through sugar-transporting proteins GLUT2 and GLUT5, via the small intestine in limited amounts to the liver. The liver removes some of the fructose from circulation to maintain the correct proportion of glucose to fructose (10 times lower) [123-126], and converts fructose into other metabolites including triglycerides and uric acid. This latter function is thought to be a contributor to the rise in obesity, metabolic syndrome and diabetes $[106,110,127,128]$. Fructose intake also stimulates the thioredoxin-interacting protein gene (TXNIP), which can lead to higher fructose intake [129]. The TXNIP gene has been associated with promoting inflammation in endothelial cells, mediating hepatic inflammation, and regulating NF- KB [130-134]. TXNIP has been shown to be involved in the differentiation of epithelial cells in the intestine [135]. TXNIP mRNA, which negatively regulates the TXNIP gene, shows decreased expression in the inflamed mucosa of the colon in people with UC [136]. Interestingly, thioredoxin-1 produced by the gene TXNIP is also inhibited by the production of vitamin D3 up-regulated protein 1 (VDUP1) when vitamin $\mathrm{D}_{3}$ is given $[137,138]$.

The relationship between fructose uptake and the TXNIP gene may explain why fructose intake has been increasingly linked to intestinal symptoms of bloating, abdominal pain, diarrhoea and fructose 
intolerance in individuals with IBD [126,139-142]. This underlies the need to consider fructose intake when managing these symptoms. A recent NZ study by Spencer et al. on people with irritable bowel syndrome (IBS) $(n=277)$ based in Canterbury, NZ found that the participants' IBS score, constipation and diarrhoea were not associated with fructose intake. However, they found that greater mean daily fructose intakes $(p=0.05)$ were associated with a lower prevalence of IBS pain [122].

In adults, fructose intolerance is most commonly assessed by breath testing after swallowing $25 \mathrm{~g}$ of fructose dissolved in water. Breath measurements are made on a 30-min schedule for up to three hours. A value of $\geq 20$ ppm $\mathrm{H}_{2}$, accompanied by symptoms (e.g., bloating, diarrhoea), is an affirmation of fructose intolerance $[139,143]$. This is because the hydrogen produced by gut bacteria is reacting with the fructose which has not been absorbed in the small intestine. Breath testing is a common method of testing for fructose, and there are a variety of methodologies in its application, as well as questions about its reliability [144]. A North American consensus statement has been drawn up for hydrogen and methane-based breath testing in gastrointestinal disorders to address these issues [145].

When it is suggested that high-fructose-containing foods be eliminated in the diet, care must be taken in deciding which foods are excluded, as the assessment of what constitutes a high-fructose food varies [146]. If people stay on a low-fructose diet for too long, they may be in danger of nutrient deficiencies due to lack of fruit and vegetables in the diet. It also needs to be remembered that fructose taken as a bolus (e.g., after swallowing $25 \mathrm{~g}$ of fructose in a fructose intolerance test) is metabolized differently to when it is ingested in the presence of glucose. Many people absorb fructose easily along with sucrose or with glucose that is at similar levels [147].

\subsection{Other Western Diet Factors}

Westernisation has also introduced other factors into the diet, such as changes in birthing methods, using artificial infant formula instead of breast feeding, and the use of sugar substitutes, food additives and antibiotics (Table 3). Many of these changes have been associated with a reduction in gut microbial diversity as well as dysbiosis (i.e., microbial imbalances in the gut)—a feature of IBD [44,45]. This path to dysbiosis may start early, with lack of exposure to appropriate gut microbiota as an infant. The composition of an infant's gut bacteria is affected by the delivery method (i.e., whether infants are born by caesarean (C-section) or vaginally, and whether breast-fed or given infant formula. C-section babies have microbiota that resembles that on the mother's skin, and those born vaginally are seeded by the microbiota in the mother's vagina $[148,149]$. The microbiota seeded from the mother's vagina is thought to contribute to a normal infant microbiome, whereas having a C-section and all the factors associated with the reason for having a C-section does not [150].

The type of first foods also contributes to the composition of the microbiota. Breast feeding promotes immune development and protects against many diseases. In a small study on 3-month-old babies 1214 probe sets for epithelial cells were significantly differently expressed in exclusively breast-fed $(n=12)$ and formula-fed babies $(n=10)$ [151]. This may explain some of the differences that have been noted in clinical and epidemiological observations of these two groups. The two groups have different gut development, with the breast-fed babies being described as being "less leaky". Bacteroidetes species, which are important players in the commensal bacteria, were not found in the stools of the formula-fed infants [151]. Breast milk also contains human milk oligosaccharides (HMOs) which stimulate the growth of the intestinal flora, acting as prebiotics for beneficial bacteria [152]. Human bioactive milk proteins modulate the immune system, and this too would contribute to the enhancement of gut barrier function $[153,154]$. Formula feeding is becoming an increasingly common pattern of infant feeding in Western culture.

The increasing use of artificial sweeteners (ASs) may also contribute to dysbiosis [155]. A number of studies have been conducted on the effects of ASs on the gut. These have looked at their effects on secretion, absorption, gut motility, the microbiome and gastrointestinal symptoms [156-160]. Some studies imply that ASs have measurable effects on the oral/gut microbiota $[155,161,162]$. In one example, male rats were randomised into control and AS groups and were exposed to doses ranging 
from 1.1 to $11 \mathrm{mg} / \mathrm{kg} /$ day for 12 weeks (the USA Federal Drug Administration (FDA) acceptable daily intake for humans is $5 \mathrm{mg} / \mathrm{kg} /$ day). At the low dose of $1.1 \mathrm{mg} / \mathrm{kg}$, the researchers reported that total anaerobes were reduced by approximately $50 \%$ in all sucralose plus maltodextrin groups. However, when this study was reviewed by an expert panel [163], it was criticised for not having an isocaloric carbohydrate control, and not correcting for the water content of the stools. Both these factors can affect the interpretation of the bacterial concentrations. This report also commented that the difference between control and treatment groups for bacterial counts given were also less than 10 -fold. They concluded that this meant that the results could not be interpreted as indicative of adverse change, but reflected the normal range of variation in bacteria over this period of time. However, further research in healthy human subjects continues to suggest that microbiota are adversely affected by ASs. Suez and colleagues showed that with 11 weeks of exposure to three common ASs, saccharin altered gut microbiota and induced glucose intolerance [161]. This indicates the need for more studies-particularly in humans—-to elucidate the effects of ASs on microbiota [164-166].

Table 3. Western diet food factors, effects related to IBD and associated genes. LC-PUFA: long-chain polyunsaturated fatty acid; SCFA: short-chain fatty acid.

\begin{tabular}{|c|c|c|}
\hline Western Diet Food Factors & Effects Related to IBD & Associated Genes \\
\hline $\begin{array}{l}\text { Foods low in resistant starch and } \\
\text { soluble dietary fibre [47] }\end{array}$ & $\begin{array}{l}\text { - Increased risk of IBD }[34,36,49,50] \\
\text { Increased risk of E. coli in the } \\
\text { mucosa [60] } \\
\text { Decreased production of SCFAs } \\
\text { (important in epithelial barrier } \\
\text { maintenance and immune system } \\
\text { regulation) [69] }\end{array}$ & $\begin{array}{c}\text { FFAR2 } \\
\text { (The interaction of SCFAs and } \\
\text { FFAR2 greatly affects } \\
\text { inflammatory processes) }[21,70]\end{array}$ \\
\hline $\begin{array}{l}\text { Increased consumption of } \\
\text { saturated fatty acids with } \\
\text { decreased consumption of } \\
\text { LC-PUFAs from the increased } \\
\text { intake of soy, safflower, corn and } \\
\text { sunflower oils and reduced } \\
\text { consumption of fish }[83,92,93]\end{array}$ & $\begin{array}{l}\text { - Inflammation and cancer risk } \\
\text { impacted by gene variant influence on } \\
\text { metabolic pathways [85-87] }\end{array}$ & $\begin{array}{l}\text { FADS1 and FADS2 } \\
\text { PPARA, PPARG } \\
\text { XRCC1, SCD1 [94-104] }\end{array}$ \\
\hline $\begin{array}{l}\text { Increased fructose consumption } \\
\qquad[25,119,120]\end{array}$ & $\begin{array}{l}\text { Associated with the rise in obesity, } \\
\text { diabetes, metabolic syndrome, IBD, } \\
\text { coronary heart disease [105-116] } \\
\text { Linked to intestinal symptoms of } \\
\text { bloating, abdominal pain, diarrhoea } \\
\text { and fructose intolerance in IBD [112] } \\
\text { Possible effect on vascular } \\
\text { inflammation from infant formulas } \\
\text { containing fructose [118] }\end{array}$ & $\begin{array}{l}\text { TXNIP [128] } \\
\text { (associated with promoting } \\
\text { inflammation in endothelial cells, } \\
\text { mediating hepatic inflammation, } \\
\text { and regulating NF-kB) [129-133] }\end{array}$ \\
\hline $\begin{array}{l}\text { Increased use of infant formula, } \\
\text { artificial sweeteners, food } \\
\text { emulsifiers and antibiotics } \\
{[167,168]}\end{array}$ & $\begin{array}{ll}\text { - } & \text { Reduction in gut microbiotal } \\
\text { diversity }[44,45] \\
\text { - } & \text { Increase in antibiotic resistance [168] }\end{array}$ & Genes in the microbiome [168] \\
\hline
\end{tabular}

Two other recent additions to the Western diet are emulsifiers in food to improve mouth feel in low-fat products, and antibiotics in animal feed $[167,168]$. Emulsifiers have been shown in murine studies to have an impact on the mouse gut microbiota that promotes colitis and decrease in (alpha) diversity [167]. Antibiotics are used to reduce disease in livestock kept in confined quarters. Repeated courses of antibiotics can lead to the development of what is termed "the resistome" - the collection of genes in bacteria that confers resistance to antibiotics [169]. Increased antibiotic exposure in humans through food sourced from animals that have had antibiotics may affect the human biome. When these products from these animals are consumed, they contribute to antibiotic resistance. The US Food and Drug Administration (2009) indicated that $80 \%$ of antimicrobial products in the USA were used in 
animal feed as growth stimulants, not for veterinary use [170]. In the highly pastoral country New Zealand it is reported that about $60 \%$ of antibiotics are administered to animals [171]. The Ministry for Primary Industries (NZ) states the use of antibiotics in cattle and sheep is low compared to the intensive rearing pig and poultry industries [172].

The issue for people with IBD is its effect on the genetics of their microbiome when they incorporate these products into their diet. For example, with antibiotics, antibiotic resistance genes are built up in the microbial community both in pathogenic and non-pathogenic bacteria, and this builds the resistome [169]. Genes in the resistome are readily transferred not only from one bacterial cell to another, but also by cassettes of multiple genes $[173,174]$. This has been shown to result in a build-up of multidrug resistance to $E$. coli in domestic animals $[175,176]$. These bacteria have also been identified as being in increased numbers in those with CD [177-179]. Collectively, all these Western diet factors impinge on the genetics of the biome and reduce microbiota diversity. Personalising the dietary approach for people with IBD and identifying the effects of genes associated with these changes (Table 3) would help reduce this adverse impact on the genes of the microbiome. It would help maintain microbiotal diversity and positively modulate disease activity.

\section{Food Intolerances}

IBD has been linked with a number of food intolerances, especially with foods containing gluten, dairy products/lactose or fructose (discussed earlier), or food high in FODMAPs. Many of these intolerances can also be linked to specific genes or genotypes. Other food intolerances linked with genotypes and IBD are brassica vegetables, mustard, wasabi, raw and cooked tomatoes, sweet potatoes, mushrooms, sulphur dioxide, sulphites and sulphur compounds. The specific genes or genotypes associated with these intolerances will be discussed further. This section begins with a discussion of the FODMAP approach, as this is the newest dietary strategy suggested as being helpful for IBD. It also links to a number of food intolerances associated with particular genotypes and/or gene variants.

\subsection{FODMAPS}

FODMAPs are fermentable oligo-, di- and mono-saccharides and polyols which, if lowered in the diet, have been shown to be effective in decreasing the abdominal symptoms associated with IBS. This approach is being proposed as being helpful also for people with IBD [2]. Foods that are identified as FODMAPs have been discussed extensively in the literature [111,180-184]. Table 4 gives examples of food sources of FODMAPs [185-191] and genes that are associated with them.

Table 4. Sources of foods high in fermentable oligo-, di- and mono-saccharides and polyols (FODMAPs) [185-191].

\begin{tabular}{|c|c|c|}
\hline FODMAPs & Examples of Food Sources & Examples of Associated Genes \\
\hline Fructose & $\begin{array}{c}\text { Honey, mangoes, watermelon, } \\
\text { grapes, fruit juices, high-fructose } \\
\text { corn syrup }\end{array}$ & $\begin{array}{l}\text { TXNIP with fructose that is linked } \\
\text { to intestinal symptoms of bloating, } \\
\text { abdominal pain, diarrhoea in IBD } \\
\text { [125,138-141] }\end{array}$ \\
\hline Lactose & $\begin{array}{l}\text { Dairy products (e.g., milk, } \\
\text { ice-cream, yoghurt, soft cheeses) }\end{array}$ & $\begin{array}{l}\text { Lactose intolerance associated } \\
\text { with variants of the LCT gene } \\
{[191,192]}\end{array}$ \\
\hline $\begin{array}{l}\text { Fermentable } \\
\text { oligosaccharides }\end{array}$ & $\begin{array}{l}\text { Brussels sprouts, broccoli, cabbage, } \\
\text { peas, beetroot, garlic, leeks, onions, } \\
\text { wheat or rye bread, pasta, } \\
\text { couscous, chickpeas, lentils }\end{array}$ & $\begin{array}{c}\text { GSTMl and GSTTl, and DIO1 } \\
\text { variants, with tolerance of brassica } \\
\text { vegetables [193-196]; HLA-DQA1 } \\
\text { and } H L A-D Q B 1 \text { variants with } \\
\text { gluten intolerance [197] }\end{array}$ \\
\hline Polyols & $\begin{array}{l}\text { Stone fruits, apples, pears, prunes, } \\
\text { avocados, mushrooms; sweeteners: } \\
\text { mannitol, sorbitol, xylitol, } \\
\text { erythritol and isomalt. }\end{array}$ & OCTN1 with mushrooms [198] \\
\hline
\end{tabular}


Foods high in FODMAPs have been characterized in a healthy population (i.e., those who do not experience inflammation) as not being well absorbed in the small intestine, having a laxative effect if taken in higher doses and fermented rapidly by bacteria [2]. Inflammation of the gut wall-a characteristic of IBD—also interrupts enzyme production by the brush border cells, and this also contributes to the sugars not being absorbed. Unabsorbed sugars go to the large intestine, where bacteria interact with them. When some metabolites (e.g., butan 2,3 diol, ketones, acids, aldehydes) are formed, they can result in an alteration of the signalling mechanisms of the bacteria and this causes gas, pain and diarrhoea [192]. Diarrhoea has a negative impact on water and electrolyte balance, and may lead to dehydration. Gas, pain and diarrhoea commonly occur in non-IBD gastro-intestinal tracts (i.e., healthy populations) if doses of oral fructose or polyols over $50 \mathrm{~g}$ are taken $[113,193,194]$.

This physiological reaction is the rationale for using the FODMAP dietary regime in gastrointestinal disorders like IBD [111,195]. This is based on studies with irritable bowel syndrome (IBS), where the decreased consumption of these fermentable carbohydrates has decreased abdominal symptoms (i.e., pain, flatulence, bloating, constipation, diarrhoea). There is no question that the by-products of FODMAP digestion and absorption may be especially unpleasant, exacerbating the symptoms of individuals with gastrointestinal disorders. Thus, it might appear as self-evident that avoidance of this group of compounds could be therapeutic for individuals with IBD—especially if they also have IBS [111,195]. A number of trials with IBS have suggested that this approach shows potential for improving symptoms in IBD. However, to avoid compromising people's nutritional status, care must be taken so that any changes instigated in sources of fruits and vegetables do not reduce the total recommended intake of fruits, vegetables or fibres [196].

How does the FODMAP approach relate to genotypes? As will be discussed further on, intolerance to some vegetables (e.g., brassica, mushrooms, cooked tomatoes) whole grains and dairy products may be linked to specific genotypes and gene variants (Table 5). Recent research has also indicated that following a strict FODMAP diet can also have an effect on the genome of the microbiome and encourage harmful gut microbiota and a decrease in Bifidobacterium $[183,184]$. In people with disorders like IBD, their microbiotal composition is different from those found in healthy people [197-199]. Individuals with IBD have reduced numbers of microbiota, fewer divergent species with lower numbers of Firmicutes, and increased number of Proteobacteria species compared to healthy people [200-203]. This means that strict adherence to a low-FODMAP diet could contribute to this dysbiosis.

In addition, people with IBD may have a loss of function of the fucosyltransferase 2 (FUT2) gene, which identifies them as a "non-secretor". A non-secretor has a significant reduction in microbiotal diversity, richness and abundance [204]. The FUT2 gene has been identified as increasing the susceptibility to CD [205-207]. Non-secretor individuals are also infrequently colonised by Bifidobacterium bifidum (important in the infant flora), B. adolescentis and B. catenulatum/pseudocatenulatum (important in adult intestinal flora) [204]. Bifidobacteria are an important part of the human intestinal microbiome. They are crucial to the development of a healthy infant microbiota, encouraging appropriate gut maturity and gut integrity, modulating immunity and negating the effects of pathogens $[208,209]$. This would suggest that people with IBD who are non-secretors have an increased risk of having a less-effective defence against a number of pathogens and are consequently more vulnerable to having an inflamed gut. Following a low-FODMAP diet may exacerbate this. Further research is needed on those who are a non-secretors, using the low-FODMAP approach to see how this affects their microbiota.

\subsection{Gluten}

Intolerance to foods containing gluten is associated with the autoimmune disease coeliac disease and with non-coeliac gluten sensitivity. These two have symptoms in common (e.g., bloating, diarrhoea, constipation, abdominal pain and weight loss). Unlike coeliac disease, gluten sensitivity is not associated with antibody formation and damage to the epithelium of the gut. Coeliac disease has genetic links. Gluten intolerance is associated with gene variants of the major histocompatibility 
complex, class II, DQ alpha 1 (HLA-DQA1) gene and the major histocompatibility complex, class II, DQ beta 1 (HLA-DQB1) gene [210]. Festen and colleagues in their meta-analysis of two genome-wide association studies GWAS also identified the genes interleukin 18 receptor accessory protein (IL18RAP); phosphatase, non-receptor type 2 (PTPN2); T cell activation RhoGTPase activating protein (TAGAP) and pseudouridine synthase 10 (PUS10) as having shared risk loci for CD and coeliac disease [211]. The gene PTPN2 is involved in the regulation of the epithelial barrier function and is modulated by the vitamin D receptor gene (VDR) [212]. Current nutrition advice for people with IBD includes checking whether they have the autoimmune coeliac disease or if they have non-coeliac gluten sensitivity. The blood test to detect the presence of anti-tissue transglutaminase (tTG) antibodies can be taken. These antibodies are produced in the small intestine when inflammation occurs in response to gluten. Another test that has a high specificity for coeliac disease is the anti-endomysial antibody (EMA) test which detects villus atrophy—a feature of coeliac disease [213]. If IBD patients prove to have an adverse response to gluten from these tests, then avoiding these foods and products containing them is advised. These foods include wheat, oats, barley (which includes malt and malt vinegar) and rye. There is no definitive test for non-coeliac gluten sensitivity except for eliminating gluten from the diet to see if symptoms subside. The role of non-coeliac gluten or wheat sensitivity in the general population as well as in IBD has been debated extensively. A review of the topic suggests that it may be linked in IBD with those who also have IBS.

\subsection{Dairy Products and Lactose}

The sensitivity to dairy foods in people with IBD is about $10 \%-20 \%$ [214-216]. Some studies have also linked increased incidence of IBD with the increased intake of dairy foods [217], particularly cheese [218]. This may be because of the fat content of cheese [219]. Nolan-Clark and associates noted that in their study on CD, sensitivity to dairy products was not related to disease activity status [219]. In a year-long trial on UC conducted by Wright et al. it was noted that patients had fewer relapses on a milk-free diet [216].

This sensitivity to dairy products is often associated with the reduction of lactase enzyme in adulthood. Lactase is an enzyme located in the villus enterocytes that is required for the digestion of lactose in milk. Lactase catalyses lactose, the disaccharide in milk, to galactose and glucose (i.e., monosaccharides which can be absorbed into the blood stream). In most mammals, its activity declines shortly after weaning. In humans, the enzyme may persist with its activity into adulthood. This is known as lactase persistence, and it is a genetically determined trait. In Europe, a single allele-T-13910 of the lactase phlorizin hydrolase (LCT) gene-is the main one. However, in Africa and the Middle East many more mutations are associated with lactase persistence $[220,221]$.

This lactase persistence has associations with CD. In an NZ-based study [222], lactase persistence in the Caucasian population was associated with an increased risk of CD for those with the lactase (LCT) gene who were homozygous for the T allele of rs4988235 as compared with those homozygous for the $\mathrm{C}$ allele $(\mathrm{OR}=1.61,95 \% \mathrm{CI}$ : 1.03-2.51). Increasing lactase non-persistence in populations has also been linked to a decreased risk of CD [223]. In a study encompassing populations from 26 countries around the world, CD decreased as lactase non-persistence increased $(p<0.01)$, [223].

Not consuming dairy products raises the risk of having insufficient intakes of calcium in the diet, especially if few other sources of calcium are consumed. Calcium is not only essential for bone health, but $\mathrm{Ca}^{2+}$ is extensively used in biological messenger systems and cell functions [224]. Low calcium intake of people with Crohn's disease increases their risk of bone demineralisation and osteoporosis. These are well-known complications of this condition. Bernstein reported for people with IBD that "The overall relative risk of fractures is $40 \%$ greater than that in the general population and increases with age" [225]. Osteoporosis can also be exacerbated by the use of steroid medications (a common treatment in IBD), as they have a detrimental effect on calcium and phosphate metabolism [226]. Calcium intake is important with respect to IBD because, as discussed earlier, Vagianos et al. observed that $25 \%$ of their adult outpatients consumed less than the recommended intake of calcium. Further, 
the study of Hartman et al. showed that $79 \%$ of the adolescent outpatients consumed less than $80 \%$ of RDA $[9,15]$. If people are not tolerant to dairy foods, non-dairy sources of calcium are available, such as fortified soy, almond and rice milks, tofu, sardines, almonds, sesame seeds, broccoli and fortified breakfast cereals and juices [227].

\subsection{Brassica}

Vegetables such as broccoli, cabbage, Brussels sprouts and cauliflower are members of the brassica family of vegetables. These are known to be a good source of various micronutrients (e.g., selenium, iodine) [228] and various phytochemicals (e.g., glucosinolates and quercetin) [229]. Common members from this group of plants are recommended to be excluded from the low-FODMAP diet (Brussels sprouts, broccoli and cabbage) [195]. However, two randomized controlled diet studies conducted by Brauer et al. found different responses in individuals depending on their glutathione S-transferase Mu 1 (GSTMl) genotype with supplements of specific brassica vegetables (broccoli, cabbage, cauliflower and radish sprouts) [230]. Their study found that this combination of brassicas altered the circulating peptides transthyretin (TTR) and zinc alpha 2-glycoprotein (ZAG) fragments in a sensitive and identifiable manner. TTR declines in serum with early signs of inflammation. ZAG (an adipokine) is mainly involved in lipid mobilisation. In GSTMl+ individuals, ZAG and TTR declined with brassica intake. However, this did not occur in those with the GSTMl-nul1 genotype.

GSTMl and genotypes of GSTTl are also significant with respect to individuals and their intakes of brassica. There have been over 500 studies on GST enzymes showing that genotypes of these GSTMl and GSTTl have an influence on cancer outcomes, contingent on the expression or non-expression of these genes [231]. GSTT1 and GSTMl belong to the GST gene super family, which is an extensive group of enzymes. The GST family contains several classes (e.g., alpha, kappa, mu, omega, pi, sigma, theta and zeta) $[232,233]$. GST enzymes have significant roles in the metabolism of electrophilic compounds (e.g., drugs and carcinogens) [234].

At the GSTMl locus, four allelic variants have been discovered in humans, and the null allele is present in about $50 \%$ of individuals [235]. Where the GSTMl gene deletions or the null genotype exist, there is a total lack of the protein encoded by the gene [236]. People with deletions are thought to be more vulnerable to toxic xenobiotics (e.g., antibiotics and drugs) [237]. There is also a variation between different cultural groups in the frequency of the GSTMl deletion. In Caucasian and Asian populations, the frequency of the GSTMl null genotype genetic polymorphism is estimated to be about $53 \%$. However, the frequency is lower in African- American populations, at 27\% [231].

GST enzymes can also be acted on by isothiocyanates (found in brassicas) either as inducers or as substrates in liver detoxification pathways, so a deletion or lowered activity of a GST gene can result in a persistent or elevated level of isothiocyanates in the body [238]. Studies based in Asia suggest that the null or less-active genotypes for GSTT1 and GSTM1 are protective and lower the risk of colorectal cancer [239]. However, the opposite occurred in the USA-based studies. The active or expressed genotypes, not the null genotypes, were associated with higher risk reduction [240]. The disparity of these results may reflect the different glucosinolate content of the brassica consumed. In Asia, Chinese cabbage (bok choy) is the predominately eaten brassica, and its main glucosinolate is glucobrassicin, whereas in the USA where raw broccoli is the more favoured brassica, the glucosinolate contents differ [241]. The main glucosinolate in this vegetable is glucoraphanin [241]. Glucobrassicin is an indole, whereas glucoraphanin is a sulforaphane. Their distinct chemical structures as shown in Table 5 (adapted from Higdon et al.) may mean that they are metabolised differently as they pass through the intestine, and this would suggest that their influence on the GST genes could also be dissimilar. 
Table 5. Differences in glucosinolate structures.

\begin{tabular}{cc}
\hline Glucobrassicin (in bok choy) & Glucoraphanin (in broccoli) \\
\hline Indole-3- & Sulforaphane \\
\hline
\end{tabular}

A pilot study by Campbell et al. [242] was conducted on the GSTT1 (-/-) deletion in people with CD, in a New Zealand cohort from the Auckland IBD study [243]. This also showed those consuming broccoli, cauliflower and Chinese greens were more likely to report experiencing a beneficial effect if they had the GSTT1 deletion. This may well apply to people with other gastrointestinal disorders such as IBS [244]. Responses to another brassica, rocket (arugula), have also been found to be dependent on a variant in the single-nucleotide polymorphism (SNP) rs9469220 of the human leucocyte antigen gene (HLA) in people with CD [245]. An inflammatory colonic phenotype has also been associated with variants in the HLA gene region [246-248]. This polymorphism has been linked to total IgE levels, which have a key role in sensitivity type 1 reactions in allergies linked with rhinitis, dermatitis, asthma and urticaria [249-251]. This adverse reaction to rocket in people with CD suggests that consumption of this food initiates the immune response in a type I sensitivity reaction. Another variant in the SNP rs7515322 in the iodothyronine deiodinase 1 gene (DIO1) has also been associated with an adverse response to broccoli. DIO1 is part of the selenoenzyme family, which are important as signalling molecules for thyroid hormones and pivotal to thyroid metabolism. Both selenium and iodine deficiencies exist in NZ, and this may heighten individuals' adverse response to broccoli if they have this variant [245]. This influence of micronutrients may also explain inter-country variation in responses to broccoli.

\subsection{Mustard, Wasabi, Raw and Cooked Tomatoes and Sweet Potatoes}

In another study by Marlow et al. [252], tolerance of mustard, wasabi, raw and cooked tomatoes and sweet potatoes was dependent on the G variant of the SNP rs12212067 in the FOX03 gene. Seventeen foods were identified with beneficial effects, and four foods with detrimental effects. Sweet potatoes had the highest reported frequency of beneficial effects, and mustard, wasabi, raw and cooked tomatoes were detrimental for over $25 \%$ of the study population with the G allele (SNP rs12212067) associated with the FOX03 gene. FODMAP guidelines suggest that tomato products such as canned tomatoes are not appropriate in the low-FODMAP diet, although plum tomatoes are [139]. This guidance could perhaps be tailored more for people using the low-FODMAP diet if their gene variants in the FOX03 gene were identified.

\subsection{Mushrooms}

In a study on $\mathrm{CD}$ in an $\mathrm{NZ}$ population, a statistically significant association with a variant (the $\mathrm{T}$ allele) of the SNP rs1050152 of the organic cation transporter gene OCTN1 was found. This variant is related to mushroom intolerance in those with $\mathrm{CD}$, when compared with the general population. This indicates that $\mathrm{NZ}$ people with $\mathrm{CD}$ who have this variant have an increased risk of adverse symptoms associated with the intake of mushrooms [244]. 


\subsection{Sulphur Dioxide, Sulphites and Sulphur Compounds}

Sulphur dioxide and sulphites (at concentrations greater than $10 \mathrm{mg} / \mathrm{kg}$ or $10 \mathrm{mg} / \mathrm{L}$ ) are now listed by the EU as a common cause of food intolerances and allergens [253]. Additive sulphites are often used in the food industry, as they have multiple functions. They are used as food preservatives, as they stop microbial growth and reduce the spoilage of food. They can act as antioxidants, inhibiting browning actions. They can modify texture in doughs. They are found in wine, soft drinks, sausages and burgers [254]. There is some evidence that they may aggravate IBD symptoms. Magee et al. report in their novel method of diet analysis that in UC, sulphites adversely affect symptoms through their anti-thiamine activity. Thiamine has a critical role in energy metabolism and cell function [255]. Low levels have been implicated in fatigue and IBD in those in remission (UC) or quiescent (CD) [256]. Those with very active disease who ate large quantities of food containing sulphites also had high food sigmoidoscopy scores [257].

Earlier studies linking sulphur compounds with IBD have described hydrogen sulphide as a bacterial toxin in people with UC [258]. This description was based on the experimental work where sulphated polysaccharides were ingested by guinea pigs and rabbits $[259,260]$. These sulphur products impaired butyrate oxidation - a characteristic of UC [261]. Butyrate, as a SCFA (as mentioned earlier), plays an important part in colon homeostasis by promoting the development of the intestinal barrier through enhancing tight-junction formation, which is impaired in people with IBD [262]. More recent work by Carbonero et al. has summarised the significance of microbiota in colonic sulphur metabolism and identified a number of gut microbiota taxa that participate in it [263]. These authors suggest that common variants in host genes involved in the sulphide oxidation pathways may be implicated in ineffective epithelial sulphide detoxification. This makes people with these variants predisposed to sulphide intolerance and consequent inflammation. The literature suggests that sulphur dioxide sulphites, and other ingested sulphur-containing products, are another example of where nutrient-gene interaction occurs. Particular variants are associated with their intolerance. Using the personalised nutrition approach and identifying these gene variants in people with IBD would identify those who are intolerant to sulphur dioxide, sulphites and sulphur compounds, and so remove foods containing these substances and reduce inflammation.

\section{Ethical Issues}

The willingness of individuals to engage in genetic testing that would enable a personalised diet/nutrition approach will depend on how they understand the benefits this would bring compared to their perceived privacy risk [264]. Genetic testing for individuals can now be readily accessed with a number of commercial applications available (e.g., Fitgenes, 23andMe) $[265,266]$. The availability of genetic testing also raises a number of questions: Who should hold and have access to this information? How safely is the information kept? How informed is the consent for consumers? [266]. The protection of an individual's genetic information and the validation of genetic tests needs to be guaranteed to ensure consumer safety. Health practitioners also need to be able to understand the role of genetic testing in their clinical practice so that they can integrate this information appropriately and offer a personalised nutrition approach.

\section{Conclusions}

This review gives examples of genotypes and genetic variants that have been associated with nutrient deficiencies, the Western diet, and food intolerances. Variation in genotypes could help explain the variability in response to food that is associated with IBD. We need to identify the variants associated with adverse reactions to food, as is currently being done in pharmacogenetic research with respect to drugs. Identification of gene variants would allow more precise tailoring of diets to avoid exacerbating malnutrition in vulnerable IBD groups. Use of the personalised nutrition approach can also more accurately identify individual food intolerances, help ameliorate abdominal symptoms 
and improve the quality of life for people with IBD. Further nutrigenomic research can continue to inform and improve the personalised nutrition approach in IBD. In the future, this information could be combined with data on individuals such as their gut microbiome, dietary patterns, exercise routines, blood parameters and anthropometric measurements. This combination of information could be used to build machine-learning algorithms to predict which foods/meals reduce inflammation and abdominal symptoms. This may also help to establish the dietary patterns and lifestyles that are most protective against IBD in those genetically susceptible to IBD. This combination of data may also help individuals with established IBD to tailor their diet and lifestyle to avoid exacerbating their signs and symptoms, and promote longer periods of remission. The protection of an individual's genetic information and the validation of genetic tests needs to be guaranteed to ensure consumers' safety when using this information with a personalised nutrition approach.

Author Contributions: Writing—original draft preparation, review and editing, B.B.L.; draft preparation, review and editing, A.G.L.; conceptualization, draft preparation, review and editing, L.R.F.

Conflicts of Interest: There are no conflicts of interest to be declared.

\section{References}

1. Zeevi, D.; Korem, T.; Zmora, N.; Israeli, D.; Rothschild, D.; Weinberger, A.; Ben-Yacov, O.; Lador, D.; Avnit-Sagi, T.; Lotan-Pompan, M. Personalized nutrition by prediction of glycemic responses. Cell 2015, 163, 1079-1094. [CrossRef]

2. Ercolini, D.; Fogliano, V. Food design to feed the human gut microbiota. J. Agric. Food Chem. 2018, 66, 3754-3758. [CrossRef] [PubMed]

3. Grissinger, M. The five rights: A destination without a map. Pharm. Ther. 2010, 35, 542.

4. Halawi, H.; Camilleri, M. Pharmacogenetics and the treatment of functional gastrointestinal disorders. Pharmacogenomics 2017, 18, 1085-1094. [CrossRef] [PubMed]

5. Zhou, J.; Shen, J.; Seifer, B.J.; Jiang, S.; Wang, J.; Xiong, H.; Xie, L.; Wang, L.; Sui, X. Approaches and genetic determinants in predicting response to neoadjuvant chemotherapy in locally advanced gastric cancer. Oncotarget 2017, 8, 30477-30494. [CrossRef] [PubMed]

6. Racine, A.; Carbonnel, F.; Chan, S.S.; Hart, A.R.; Bueno-de-Mesquita, H.B.; Oldenburg, B.; van Schaik, F.D.; Tjonneland, A.; Olsen, A.; Dahm, C.C.; et al. Dietary patterns and risk of inflammatory bowel disease in europe: Results from the EPIC Study. Inflamm. Bowel Dis. 2016, 22, 345-354. [CrossRef] [PubMed]

7. Frazier-Wood, A.C. Dietary patterns, genes, and health: Challenges and obstacles to be overcome. Curr. Nutr. Rep. 2015, 4, 82-87. [CrossRef] [PubMed]

8. Ferguson, L.R. Nutrigenetics, nutrigenomics and inflammatory bowel diseases. Expert Rev. Clin. Immunol. 2013, 9, 717-726. [CrossRef] [PubMed]

9. Vagianos, K.; Bector, S.; McConnell, J.; Bernstein, C.N. Nutrition assessment of patients with inflammatory bowel disease. J. Parenter. Enter. Nutr. 2007, 31, 311-319. [CrossRef] [PubMed]

10. Stein, J.; Bott, C. Diet and Nutrition in Crohn's Disease and Ulcerative Colitis 20 Questions 20 Answers; Falk Foundation e.V.: Freiburg, Germany, 2008.

11. Basson, A. Nutrition management in the adult patient with Crohn's disease. S. Afr. J. Clin. Nutr. 2012, 25, 164-172. [CrossRef]

12. Harries, A.; Rhodes, J. Undernutrition in Crohn's disease: An anthropometric assessment. Clin. Nutr. 1985, 4, 87-89. [CrossRef]

13. Gassull, M.A.; Cabré, E. Nutrition in inflammatory Bowel disease. Curr. Opin. Clin. Nutr. Metab. Care 2001, 4, 561-569. [CrossRef] [PubMed]

14. Lomer, M.C.; Kodjabashia, K.; Hutchinson, C.; Greenfield, S.M.; Thompson, R.P.; Powell, J.J. Intake of dietary iron is low in patients with Crohn's disease: A case-control study. Br. J. Nutr. 2004, 91, 141-148. [CrossRef] [PubMed]

15. Hartman, C.; Marderfeld, L.; Davidson, K.; Mozer-Glassberg, Y.; Poraz, I.; Silbermintz, A.; Zevit, N.; Shamir, R. Food intake adequacy in children and adolescents with inflammatory Bowel disease. J. Pediatr. Gastroenterol. Nutr. 2016, 63, 437-444. [CrossRef] [PubMed] 
16. Lourenço, S.; Hussein, T.; Bologna, S.; Sipahi, A.; Nico, M. Oral manifestations of inflammatory Bowel Disease: A review based on the observation of six cases. J. Eur. Acad. Dermatol. Venereol. 2010, 24, $204-207$. [CrossRef]

17. Malins, T.; Wilson, A.; Ward-Booth, R. Recurrent buccal space abscesses: A complication of Crohn's disease. Oral Surg. Oral Med. Oral Pathol. 1991, 72, 19-21. [CrossRef]

18. Plauth, M.; Jenss, H.; Meyle, J. Oral manifestations of Crohn's Disease: An analysis of 79 cases. J. Clin. Gastroenterol. 1991, 13, 29-37. [CrossRef]

19. Schlosser, B.J.; Pirigyi, M.; Mirowski, G.W. Oral manifestations of hematologic and nutritional diseases. Otolaryngol. Clin. N. Am. 2011, 44, 183-203. [CrossRef]

20. Thomas, D.M.; Mirowski, G.W. Nutrition and oral mucosal diseases. Clin. Dermatol. 2010, $28,426-431$. [CrossRef]

21. Rebhan, M.; Chalifa-Caspi, V.; Prilusky, J.; Lancet, D. GeneCards: A novel functional genomics compendium with automated data mining and query reformulation support. Bioinformatics 1998, 14, 656-664. [CrossRef]

22. D'Ambrosio, D.N.; Clugston, R.D.; Blaner, W.S. Vitamin A metabolism: An update. Nutrients 2011, 3, 63-103. [CrossRef] [PubMed]

23. Leung, W.; Hessel, S.; Meplan, C.; Flint, J.; Oberhauser, V.; Tourniaire, F.; Hesketh, J.; Von Lintig, J.; Lietz, G. Two common single nucleotide polymorphisms in the gene encoding B-Carotene 15, 15' -monoxygenase alter B-carotene metabolism in female volunteers. FASEB J. 2009, 23, 1041-1053. [CrossRef] [PubMed]

24. Su, H.Y.; Gupta, V.; Day, A.S.; Gearry, R.B. Rising incidence of inflammatory Bowel Disease in Canterbury, New Zealand. Inflamm. Bowel Dis. 2016, 22, 2238-2244. [CrossRef] [PubMed]

25. University of Otago; Ministry of health New Zealand. A Focus on Nutrition: Key Findings of the 2008/09 New Zealand Adult Nutrition Survey; Ministry of Health: Wellington, New Zealand, 2011.

26. Anand, P.K.; Kaul, D. Downregulation of TACO gene transcription restricts mycobacterial entry/survival within human macrophages. FEMS Microbiol. Lett. 2005, 250, 137-144. [CrossRef] [PubMed]

27. Stephensen, C.B. Vitamin A, infection, and immune function. Annu. Rev. Nutr. 2001, 21, 167-192. [CrossRef] [PubMed]

28. Sommer, A. Vitamin A Deficiency; Wiley Online Library: Hoboken, NJ, USA, 2001.

29. Thia, K.T.; Loftus, E.V.; Sandborn, W.J.; Yang, S. An update on the epidemiology of inflammatory Bowel Disease in Asia. Am. J. Gastroenterol. 2008, 103, 3167-3182. [CrossRef] [PubMed]

30. Baumgart, D.C.; Bernstein, C.N.; Abbas, Z.; Colombel, J.F.; Day, A.S.; D’Haens, G.; Dotan, I.; Goh, K.L.; Hibi, T.; Kozarek, R.A. IBD around the world: Comparing the epidemiology, diagnosis, and treatment: Proceedings of the World Digestive Health Day 2010-Inflammatory bowel disease task force meeting. Inflamm. Bowel Dis. 2011, 17, 639-644. [CrossRef] [PubMed]

31. Gismera, C.S.; Aladren, B.S. Inflammatory Bowel diseases: A disease (s) of modern times? is incidence still increasing? World J. Gastroenterol. 2008, 14, 5491-5498. [CrossRef] [PubMed]

32. Logan, I.; Bowlus, C.L. The geoepidemiology of autoimmune intestinal diseases. Autoimmun. Rev. 2010, 9 , A372-A378. [CrossRef] [PubMed]

33. Lomer, M.C.; Thompson, R.P.; Powell, J.J. Fine and ultrafine particles of the diet: Influence on the mucosal immune response and association with Crohn's disease. In Proceedings-Nutrition Society of London; Cambridge University Press: Cambridge, UK, 2002.

34. Persson, P.; Ahlbom, A.; Hellers, G. Diet and inflammatory bowel disease: A case-control study. Epidemiology 1992, 3, 47-52. [CrossRef] [PubMed]

35. Hou, J.K.; Abraham, B.; El-Serag, H. Dietary intake and risk of developing inflammatory bowel disease: A systematic review of the literature. Am. J. Gastroenterol. 2011, 106, 563-573. [CrossRef]

36. Chapman-Kiddell, C.A.; Davies, P.S.; Gillen, L.; Radford-Smith, G.L. Role of diet in the development of inflammatory bowel disease. Inflamm. Bowel Dis. 2010, 16, 137-151. [CrossRef] [PubMed]

37. Sartor, R.B. Mechanisms of disease: Pathogenesis of Crohn's disease and ulcerative colitis. Nat. Rev. Gastroenterol. Hepatol. 2006, 3, 390-407. [CrossRef] [PubMed]

38. Conterno, L.; Fava, F.; Viola, R.; Tuohy, K.M. Obesity and the gut microbiota: Does up-regulating colonic fermentation protect against obesity and metabolic disease? Genes Nutr. 2011, 6, 241-260. [CrossRef] [PubMed]

39. Myles, I.A. Fast food fever: Reviewing the impacts of the western diet on immunity. Nutr. J. 2014, 13, 1. [CrossRef] [PubMed] 
40. Cordain, L.; Eaton, S.B.; Sebastian, A.; Mann, N.; Lindeberg, S.; Watkins, B.A.; O'Keefe, J.H.; Brand-Miller, J. Origins and evolution of the Western diet: Health implications for the 21st century. Am. J. Clin. Nutr. 2005, 81, 341-354. [CrossRef] [PubMed]

41. Tsironi, E.; Feakins, R.M.; Roberts, C.S.; Rampton, D.S. Incidence of inflammatory bowel disease is rising and abdominal tuberculosis is falling in bangladeshis in East London, United Kingdom. Am. J. Gastroenterol. 2004, 99, 1749-1755. [CrossRef] [PubMed]

42. Dankers, W.; Colin, E.M.; Van Hamburg, J.P.; Lubberts, E. Vitamin D in autoimmunity: Molecular mechanisms and therapeutic potential. Front. Immunol. 2017, 7, 697. [CrossRef] [PubMed]

43. M'koma, A.E. Inflammatory bowel disease: An expanding global health problem. Clin. Med. Insights Gastroenterol. 2013, 6, S12731. [CrossRef] [PubMed]

44. Segata, N. Gut microbiome: Westernization and the disappearance of intestinal diversity. Curr. Biolog. 2015, 25, R611-R613. [CrossRef] [PubMed]

45. Broussard, J.L.; Devkota, S. The changing microbial landscape of Western society: Diet, dwellings and discordance. Mol. Metab. 2016, 5, 737-742. [CrossRef] [PubMed]

46. Tuohy, K.M.; Conterno, L.; Gasperotti, M.; Viola, R. Up-regulating the human intestinal microbiome using whole plant foods, polyphenols, and/or fiber. J. Agric. Food Chem. 2012, 60, 8776-8782. [CrossRef] [PubMed]

47. Amre, D.K.; D'Souza, S.; Morgan, K.; Seidman, G.; Lambrette, P.; Grimard, G.; Israel, D.; Mack, D.; Ghadirian, P.; Deslandres, C. Imbalances in dietary consumption of fatty acids, vegetables, and fruits are associated with risk for Crohn's Disease in children. Am. J. Gastroenterol. 2007, 102, 2016-2025. [CrossRef] [PubMed]

48. Hou, J.K.; Lee, D.; Lewis, J. Diet and inflammatory bowel disease: Review of patient-targeted recommendations. Clin. Gastroenterol. Hepatol. 2014, 12, 1592-1600. [CrossRef] [PubMed]

49. Kelly, D.G.; Fleming, C.R. Nutritional considerations in inflammatory bowel diseases. Gastroenterol. Clin. N. Am. 1995, 24, 597-611.

50. Reif, S.; Klein, I.; Lubin, F.; Farbstein, M.; Hallak, A.; Gilat, T. Pre-illness dietary factors in inflammatory bowel disease. Gut 1997, 40, 754-760. [CrossRef] [PubMed]

51. DeVries, J.W. On defining dietary fibre. Proc. Nutr. Soc. 2003, 62, 37-43. [CrossRef]

52. Jones, J.M. CODEX-aligned dietary fiber definitions help to bridge the 'fiber gap'. Nutr. J. 2014, 13, 34 [CrossRef]

53. ACCC International Cereals \& Grains association. Dietary Fiber. 2019. Available online: https://www. aaccnet.org/initiatives/definitions/Pages/DietaryFiber.aspx (accessed on 16 March 2019).

54. Ashwar, B.A.; Gani, A.; Shah, A.; Wani, I.A.; Masoodi, F.A. Preparation, health benefits and applications of resistant starch-A review. Starch-Stärke 2016, 68, 287-301. [CrossRef]

55. Higgins, J.A.; Brown, I.L. Resistant starch: A promising dietary agent for the prevention/treatment of inflammatory bowel disease and bowel cancer. Curr. Opin. Gastroenterol. 2013, 29, 190-194. [CrossRef]

56. Bassaganya-Riera, J.; DiGuardo, M.; Viladomiu, M.; De Horna, A.; Sanchez, S.; Einerhand, A.W.; Sanders, L.; Hontecillas, R. Soluble fibers and resistant starch ameliorate disease activity in interleukin-10-deficient mice with inflammatory bowel disease. J. Nutr. 2011, 141, 1318-1325. [CrossRef] [PubMed]

57. Pituch-Zdanowska, A.; Banaszkiewicz, A.; Albrecht, P. The role of dietary fibre in inflammatory bowel disease. Prz Gastroenterol. 2015, 10, 135-141. [CrossRef] [PubMed]

58. Crohn's \& Colitis Foundation of America. Diet, Nutrition, and Inflammatory Bowel Diseases; Crohn's \& Colitis Foundation of America: New York, NY, USA, 2013.

59. The role of vegetables in the cause remission and regression of Crohn's disease in an Auckland cohort. Available online: https://researchspace.auckland.ac.nz/handle/2292/19961 (accessed on 17 September 2018).

60. Roberts, C.L.; Keita, Å.V.; Duncan, S.H.; O’Kennedy, N.; Söderholm, J.D.; Rhodes, J.M.; Campbell, B.J. Translocation of Crohn's disease Escherichia coli across M-cells: Contrasting effects of soluble plant fibres and emulsifiers. Gut 2010, 59, 1331-1339. [CrossRef] [PubMed]

61. Miller, H.; Zhang, J.; Kuolee, R.; Patel, G.B.; Chen, W. Intestinal M cells: The fallible sentinels? World J. Gastroenterol. 2007, 13, 1477-1486. [CrossRef] [PubMed]

62. Siebers, A.; Finlay, B.B. M cells and the pathogenesis of mucosal and systemic infections. Trends Microbiol. 1996, 4, 22-29. [CrossRef]

63. Staudacher, H.M.; Kurien, M.; Whelan, K. Nutritional implications of dietary interventions for managing gastrointestinal disorders. Curr. Opin. Gastroenterol. 2018, 34, 105-111. [CrossRef] [PubMed] 
64. Bockaert, J.; Pin, J.P. Molecular tinkering of G protein-coupled receptors: An evolutionary success. EMBO J. 1999, 18, 1723-1729. [CrossRef]

65. Stoddart, L.A.; Smith, N.J.; Milligan, G. International union of pharmacology. LXXI. free fatty acid receptors FFA1,-2, and-3: pharmacology and pathophysiological functions. Pharmacol. Rev. 2008, 60, 405-417. [CrossRef]

66. Senga, T.; Iwamoto, S.; Yoshida, T.; Yokota, T.; Adachi, K.; Azuma, E.; Hamaguchi, M.; Iwamoto, T. LSSIG is a novel murine leukocyte-specific GPCR that is induced by the activation of STAT3. Blood 2003, 101, 1185-1187. [CrossRef]

67. Fournier, B.; Parkos, C. The role of neutrophils during intestinal inflammation. Mucosal Immunol. 2012, 5 , 354-366. [CrossRef]

68. Le Poul, E.; Loison, C.; Struyf, S.; Springael, J.; Lannoy, V.; Decobecq, M.; Brezillon, S.; Dupriez, V.; Vassart, G.; Van Damme, J. Functional characterization of human receptors for short chain fatty acids and their Role in polymorphonuclear cell activation. J. Biol. Chem. 2003, 278, 25481-25489. [CrossRef] [PubMed]

69. Bindels, L.B.; Dewulf, E.M.; Delzenne, N.M. GPR43/FFA2: physiopathological relevance and therapeutic prospects. Trends. Pharmacol. Sci. 2013. [CrossRef] [PubMed]

70. Maslowski, K.M.; Vieira, A.T.; Ng, A.; Kranich, J.; Sierro, F.; Yu, D.; Schilter, H.C.; Rolph, M.S.; Mackay, F.; Artis, D. Regulation of inflammatory responses by gut microbiota and chemoattractant receptor. GPR Nat. 2009, 461, 1282-1286. [CrossRef] [PubMed]

71. Sivaprakasam, S.; Gurav, A.; Paschall, A.; Coe, G.; Chaudhary, K.; Cai, Y.; Kolhe, R.; Martin, P.; Browning, D.; Huang, L. An essential role of Ffar2 (Gpr43) in dietary fibre-mediated promotion of healthy composition of gut microbiota and suppression of intestinal carcinogenesis. Oncogenesis 2016, 5, e238. [CrossRef] [PubMed]

72. Bordonaro, M.; Mariadason, J.M.; Aslam, F.; Heerdt, B.G.; Augenlicht, L.H. Butyrate-induced apoptotic cascade in colonic carcinoma cells: modulation of the beta-catenin-Tcf pathway and concordance with effects of sulindac and trichostatin A but not curcumin. Cell Growth Differ. 1999, 10, 713-720. [PubMed]

73. Evans, W. Pharmacogenomics: Marshalling the human genome to individualise drug therapy. Gut 2003, 52, ii10-ii18. [CrossRef] [PubMed]

74. Eastwood, M.; Kritchevsky, D. Dietary fiber: How did we get where we are? Annu. Rev. Nutr. 2005, 25, 1-8. [CrossRef] [PubMed]

75. Maslowski, K.M.; Mackay, C.R. Diet, gut microbiota and immune responses. Nat. Immunol. 2010, 12, 5-9. [CrossRef] [PubMed]

76. Schneeman, B.O.; Gallaher, D. Effects of dietary fiber on digestive enzyme activity and bile acids in the small intestine. In Proceedings of the Society for Experimental Biology and Medicine; Society for Experimental Biology and Medicine: New York, NY, USA, 1985; pp. 409-414.

77. Lupton, J.R. Microbial degradation products influence colon cancer risk: The butyrate controversy. J. Nutr. 2004, 134, 479-482. [CrossRef] [PubMed]

78. Blaut, M.; Clavel, T. Metabolic diversity of the intestinal microbiota: Implications for health and disease. J. Nutr. 2007, 137, 751S-755S. [CrossRef]

79. National Academy Press. Dietary, Functional and Total Fibre. Available online: https://www.nap.edu/read/ 10490/chapter/9Accessed (accessed on 9 August 2018).

80. Silva, A.F.D.; Schieferdecker, M.E.M.; Amarante, H.M.B.d.S. Food intake in patients with inflammatory bowel disease. ABCD Arq. Bras. Cir. Dig. 2011, 24, 204-209. [CrossRef]

81. Staudacher, H.M.; Irving, P.M.; Lomer, M.C.; Whelan, K. Mechanisms and efficacy of dietary FODMAP Restriction in IBS. Nat. Rev. Gastroenterol. Hepatol. 2014, 11, 256. [CrossRef] [PubMed]

82. Halmos, E.P.; Christophersen, C.T.; Bird, A.R.; Shepherd, S.J.; Gibson, P.R.; Muir, J.G. Diets that differ in their FODMAP content alter the colonic luminal microenvironment. Gut 2015, 64, 93-100. [CrossRef] [PubMed]

83. Simopoulos, A.P. Omega-3 fatty acids in health and disease and in growth and development. Am. J. Clin. Nutr. 1991, 54, 438-463. [CrossRef] [PubMed]

84. Razack, R.; Seidner, D.L. Nutrition in inflammatory bowel disease. Curr. Opin. Gastroenterol. 2007, 23, 400-405. [CrossRef]

85. Patterson, E.; Wall, R.; Fitzgerald, G.; Ross, R.; Stanton, C. Health implications of high dietary omega-6 polyunsaturated fatty acids. J. Nutr. Metab. 2012, 2012, 1-16. [CrossRef] [PubMed]

86. Park, J.; Kwon, S.; Han, Y.; Hahm, K.; Kim, E. Omega-3 polyunsaturated fatty acids as potential chemopreventive agent for gastrointestinal cancer. J. Cancer. Prev. 2013, 18, 201. [CrossRef] [PubMed] 
87. Thomas, J.; Thomas, C.; Radcliffe, J.; Itsiopoulos, C. Omega-3 fatty acids in early prevention of inflammatory neurodegenerative disease: A focus on Alzheimer's disease. BioMed Res. Int. 2015, 2015, 1-13. [CrossRef] [PubMed]

88. Pascual, C.Y.; Reche, M.; Fiandor, A.; Valbuena, T.; Cuevas, T.; Esteban, M.M. Fish allergy in childhood. Pediatr. Allergy. Immunol. 2008, 19, 573-579. [CrossRef] [PubMed]

89. Vitoria, J.C.; Camarero, C.; Sojo, A.; Ruiz, A.; Rodriguez-Soriano, J. Enteropathy related to fish, rice, and chicken. Arch. Dis. Child. 1982, 57, 44-48. [PubMed]

90. Dannaeus, A.; Johansson, S.; Foucard, T.; Öhman, S. Clinical and immunological aspects of food allergy in childhood I. Estimation of IgG, IgA and IgE antibodies to food antigens in children with food allergy and atopic dermatitis. Acta Paediatrica 1977, 66, 31-37. [CrossRef]

91. Untersmayr, E.; Schöll, I.; Swoboda, I.; Beil, W.J.; Förster-Waldl, E.; Walter, F.; Riemer, A.; Kraml, G.; Kinaciyan, T.; Spitzauer, S. Antacid medication inhibits digestion of dietary proteins and causes food allergy: A fish allergy model in BALB/C mice. J. Allergy Clin. Immunol. 2003, 112, 616-623. [CrossRef]

92. Leaf, A.; Weber, P.C. A new era for science in nutrition. Am. J. Clin. Nutr. 1987. [CrossRef] [PubMed]

93. Eaton, S.B.; Konner, M. Paleolithic nutrition: A consideration of its nature and current implications. N. Engl. J. Med. 1985, 312, 283-289. [CrossRef] [PubMed]

94. Schaeffer, L.; Gohlke, H.; Muller, M.; Heid, I.M.; Palmer, L.J.; Kompauer, I.; Demmelmair, H.; Illig, T.; Koletzko, B.; Heinrich, J. Common genetic variants of the FADS1 FADS2 gene cluster and their reconstructed haplotypes are associated with the fatty acid composition in phospholipids. Hum. Mol. Genet. 2006, 15, 1745-1756. [CrossRef] [PubMed]

95. Fajas, L.; Auboeuf, D.; Raspé, E.; Schoonjans, K.; Lefebvre, A.; Saladin, R.; Najib, J.; Laville, M.; Fruchart, J.; Deeb, S. The organization, promoter analysis, and expression of the human PPAR $\gamma$ gene. J. Biol. Chem. 1997, 272, 18779-18789. [CrossRef]

96. Tai, E.S.; Corella, D.; Demissie, S.; Cupples, L.A.; Coltell, O.; Schaefer, E.J.; Tucker, K.L.; Ordovas, J.M. Polyunsaturated fatty acids interact with the PPARA-L162V polymorphism to affect plasma triglyceride and apolipoprotein C-III concentrations in the Framingham Heart Study. J. Nutr. 2005, 135, 397-403. [CrossRef]

97. De Keyser, C.E.; Becker, M.L.; Uitterlinden, A.G.; Hofman, A.; Lous, J.J.; Elens, L.; Visser, L.E.; Van Schaik, R.H.; Stricker, B.H. Genetic variation in the PPARA gene is associated with simvastatin-mediated cholesterol reduction in the Rotterdam Study. Pharmacogenomics 2013, 14, 1295-1304. [CrossRef]

98. Chen, M.; Wang, Y.; Zhang, W.; Han, X. Abstract PR428: Association of PparA Rs4253728 G> A gene polymorphisms with Cyp3A4 enzyme activity and fentanyl post-operative intravenous analgesic effect. Anesth. Analg. 2016, 123, 109-110. [CrossRef]

99. Ferreira, P.; Cravo, M.; Guerreiro, C.S.; Tavares, L.; Santos, P.M.; Brito, M. Fat intake interacts with polymorphisms of Caspase9, FasLigand and PPARgamma apoptotic genes in modulating Crohn's Disease activity. Clin. Nutr. 2010, 29, 819-823. [CrossRef]

100. Jiang, Z.; Li, C.; Xu, Y.; Cai, S. A meta-analysis on XRCC1 and XRCC3 polymorphisms and colorectal cancer risk. Int. J. Colorectal Dis. 2010, 25, 169-180. [CrossRef] [PubMed]

101. Stern, M.C.; Butler, L.M.; Corral, R.; Joshi, A.D.; Yuan, J.M.; Koh, W.P.; Yu, M.C. Polyunsaturated fatty acids, DNA repair single nucleotide polymorphisms and colorectal cancer in the Singapore Chinese Health Study. J. Nutrigenet. Nutrigenomics 2009, 2, 273-279. [CrossRef] [PubMed]

102. Stryjecki, C.; Roke, K.; Clarke, S.; Nielsen, D.; Badawi, A.; El-Sohemy, A.; Ma, D.W.; Mutch, D.M. Enzymatic activity and genetic variation in SCD1 modulate the relationship between fatty acids and inflammation. Mol. Genet. Metab. 2012, 105, 421-427. [CrossRef] [PubMed]

103. Larsson, S.C.; Kumlin, M.; Ingelman-Sundberg, M.; Wolk, A. dietary long-chain N-3 fatty acids for the prevention of cancer: A review of potential mechanisms. Am. J. Clin. Nutr. 2004, 79, 935-945. [CrossRef] [PubMed]

104. Nicholson, M.L.; Neoptolemos, J.P.; Clayton, H.A.; Talbot, I.C.; Bell, P.R. Inhibition of experimental colorectal carcinogenesis by dietary N-6 polyunsaturated fats. Carcinogenesis 1990, 11, 2191-2197. [CrossRef]

105. Bray, G.; Popkin, B.M. Calorie-sweetened beverages and fructose: What have we learned 10 years later. Pediatr. Obes. 2013, 8, 242-248. [CrossRef]

106. Elliott, S.S.; Keim, N.L.; Stern, J.S.; Teff, K.; Havel, P.J. Fructose, weight gain, and the insulin resistance syndrome1-3. Am. J. Clin. Nutr. 2002, 76, 911-922. [CrossRef] 
107. Basu, S.; Yoffe, P.; Hills, N.; Lustig, R.H. The relationship of sugar to population-level diabetes prevalence: An econometric analysis of repeated cross-sectional data. PloS ONE 2013, 8, e57873. [CrossRef]

108. Goran, M.I.; Ulijaszek, S.J.; Ventura, E.E. High fructose corn syrup and diabetes prevalence: A global perspective. Glob. Public Health 2013, 8, 55-64. [CrossRef]

109. DiNicolantonio, J.J.; O'Keefe, J.H.; Lucan, S.C. Added fructose: A principal driver of type 2 diabetes mellitus and its consequences. Mayo Clin. Proc. 2015, 90, 372-381. [CrossRef]

110. Malik, V.S.; Schulze, M.B.; Hu, F.B. Intake of sugar-sweetened beverages and weight gain: A systematic review. Am. J. Clin. Nutr. 2006, 84, 274-288. [CrossRef] [PubMed]

111. Shepherd, S.J.; Gibson, P.R. Fructose malabsorption and symptoms of irritable bowel syndrome: Guidelines for effective dietary management. J. Am. Diet. Assoc. 2006, 106, 1631-1639. [CrossRef] [PubMed]

112. Gibson, P.; Shepherd, S. Personal view: Food for thought-western lifestyle and susceptibility to Crohn's Disease. the FODMAP hypothesis. Aliment. Pharmacol. Ther. 2005, 21, 1399-1409. [CrossRef] [PubMed]

113. Rumessen, J.J. Fructose and related food carbohydrates: Sources, intake, absorption, and clinical implications. Scand. J. Gastroenterol. 1992, 27, 819-828. [CrossRef] [PubMed]

114. Nguyen, S.; Choi, H.K.; Lustig, R.H.; Hsu, C. Sugar-sweetened beverages, serum uric acid, and blood pressure in adolescents. J. Pediatr. 2009, 154, 807-813. [CrossRef] [PubMed]

115. Rippe, J.M.; Angelopoulos, T.J. Fructose-containing sugars and cardiovascular disease. Adv. Nutr. 2015, 6, 430-439. [CrossRef] [PubMed]

116. DiNicolantonio, J.J.; Berger, A. Added sugars drive nutrient and energy deficit in obesity: A new paradigm Open Heart 2016, 3, e000469. [CrossRef] [PubMed]

117. Brinton, E.A. The time has come to flag and reduce excess fructose intake. Atherosclerosis 2016, 253, $262-264$ [CrossRef] [PubMed]

118. Schroeder, V.A.; Mattioli, L.F.; Kilkenny, T.A.; Belmont, J.M. Effects of lactose-containing vs lactose-free infant formula on postprandial superior mesenteric artery flow in term infants. JPEN J. Parenter. Enteral Nutr. 2014, 38, 236-242. [CrossRef] [PubMed]

119. USDA ERS. Table High Fructose Corn Syrup: Estimated Number of Per Capita Calories Consumed Daily by Calendar Year; USDA ERS: Washington, DC, USA, 2016.

120. Russell, D.; Parnell, W.; Wilson, N.; Faed, J.; Ferguson, E.; Herbison, P.; Horwath, C.; Nye, T.; Reid, P.; Walker, R. NZ Food: NZ People. Key Results of the 1997 National Nutrition Survey; Ministry of Health: Wellington, New Zealand, 1999; p. 71.

121. Spencer, R.; Gearry, R.; Pearson, J.; Skidmore, P. Relationship between fructose and lactose intakes and functional gastrointestinal symptoms in a sample of 50-year-old cantabrians in New Zealand. NZ Med. J. 2014, 127, 39.

122. Gould, G.W.; Thomas, H.M.; Jess, T.J.; Bell, G.I. Expression of human glucose transporters in xenopus oocytes: Kinetic characterization and substrate specificities of the erythrocyte, liver, and brain isoforms. Biochemistry 1991, 30, 5139-5145. [CrossRef] [PubMed]

123. Burant, C.F.; Takeda, J.; Brot-Laroche, E.; Bell, G.I.; Davidson, N.O. Fructose transporter in human spermatozoa and small intestine is GLUT5. J. Biol. Chem. 1992, 267, 14523-14526. [PubMed]

124. Douard, V.; Ferraris, R.P. Regulation of the Fructose Transporter GLUT5 in Health and Disease. Am. J. Physiol. Endocrinol. Metab. 2008, 295, E227-E237. [CrossRef] [PubMed]

125. Berni Canani, R.; Pezzella, V.; Amoroso, A.; Cozzolino, T.; Di Scala, C.; Passariello, A. Diagnosing and treating intolerance to carbohydrates in children. Nutrients 2016, 8, 157. [CrossRef] [PubMed]

126. Kolderup, A.; Svihus, B. Fructose metabolism and relation to atherosclerosis, type 2 diabetes, and obesity. J. Nutr. Metab. 2015, 2015, 823081. [CrossRef] [PubMed]

127. Stanhope, K.L.; Schwarz, J.M.; Keim, N.L.; Griffen, S.C.; Bremer, A.A.; Graham, J.L.; Hatcher, B.; Cox, C.L.; Dyachenko, A.; Zhang, W.; et al. Consuming fructose-sweetened, not glucose-sweetened, beverages increases visceral adiposity and lipids and decreases insulin sensitivity in overweight/obese humans. J. Clin. Invest. 2009, 119, 1322-1334. [CrossRef]

128. Dotimas, J.R.; Lee, A.W.; Schmider, A.B.; Carroll, S.H.; Shah, A.; Bilen, J.; Elliott, K.R.; Myers, R.B.; Soberman, R.J.; Yoshioka, J. Diabetes Regulates Fructose Absorption through Thioredoxin-Interacting Protein. eLife 2016, 5, e18313. [CrossRef] 
129. Wang, X.Q.; Nigro, P.; World, C.; Fujiwara, K.; Yan, C.; Berk, B.C. Thioredoxin Interacting Protein Promotes Endothelial Cell Inflammation in Response to Disturbed Flow by Increasing Leukocyte Adhesion and Repressing Kruppel-Like Factor. Circ. Res. 2012, 110, 560-568. [CrossRef]

130. Liu, Y.; Lian, K.; Zhang, L.; Wang, R.; Yi, F.; Gao, C.; Xin, C.; Zhu, D.; Li, Y.; Yan, W. TXNIP Mediates NLRP3 Inflammasome Activation in Cardiac Microvascular Endothelial Cells as a Novel Mechanism in Myocardial Ischemia/Reperfusion Injury. Basic Res. Cardiol. 2014, 109, 1-14. [CrossRef]

131. Park, M.; Kim, D.; Lim, S.; Choi, J.; Kim, J.; Yoon, K.; Lee, J.; Lee, J.; Han, H.; Choi, I. Thioredoxin-Interacting Protein Mediates Hepatic Lipogenesis and Inflammation Via PRMT1 and PGC-1 $\alpha$ Regulation in Vitro and in Vivo. J. Hepatol. 2014, 61, 1151-1157. [CrossRef]

132. Zhang, X.; Zhang, J.; Chen, X.; Hu, Q.; Wang, M.; Jin, R.; Zhang, Q.; Wang, W.; Wang, R.; Kang, L. Reactive Oxygen Species-Induced TXNIP Drives Fructose-Mediated Hepatic Inflammation and Lipid Accumulation through NLRP3 Inflammasome Activation. Antioxid. Redox Signal 2015, 22, 848-870. [CrossRef] [PubMed]

133. Kelleher, Z.T.; Sha, Y.; Foster, M.W.; Foster, W.M.; Forrester, M.T.; Marshall, H.E. Thioredoxin-Mediated Denitrosylation Regulates Cytokine-Induced Nuclear Factor kappaB (NF-kappaB) Activation. J. Biol. Chem. 2014, 289, 3066-3072. [CrossRef] [PubMed]

134. Takahashi, Y.; Ishii, Y.; Murata, A.; Nagata, T.; Asai, S. Localization of Thioredoxin-Interacting Protein (TXNIP) mRNA in Epithelium of Human Gastrointestinal Tract. J. Histochem. Cytochem. 2003, 51, 973-976. [CrossRef] [PubMed]

135. Takahashi, Y.; Masuda, H.; Ishii, Y.; Nishida, Y.; Kobayashi, M.; Asai, S. Decreased Expression of Thioredoxin Interacting Protein mRNA in Inflamed Colonic Mucosa in Patients with Ulcerative Colitis. Oncol. Rep. 2007, 18, 531-536. [CrossRef] [PubMed]

136. Billiet, L.; Furman, C.; Cuaz-Pérolin, C.; Paumelle, R.; Raymondjean, M.; Simmet, T.; Rouis, M. Thioredoxin-1 and its Natural Inhibitor, Vitamin D 3 Up-Regulated Protein 1, are Differentially Regulated by PPAR $\alpha$ in Human Macrophages. J. Mol. Biol. 2008, 384, 564-576. [CrossRef] [PubMed]

137. Chung, J.W.; JEON, J.; YOON, S.; Choi, I. Vitamin D3 Upregulated Protein 1 (VDUP1) is a Regulator for Redox Signaling and Stress-mediated Diseases. J. Dermatol. 2006, 33, 662-669. [CrossRef]

138. Fedewa, A.; Rao, S.S. Dietary Fructose Intolerance, Fructan Intolerance and FODMAPs. Curr. Gastroenterol. Rep. 2014, 16, 1-8. [CrossRef]

139. Choi, Y.K.; Johlin, F.C.; Summers, R.W.; Jackson, M.; Rao, S.S. Fructose Intolerance: An Under-Recognized Problem. Am. J. Gastroenterol. 2003, 98, 1348-1353. [CrossRef]

140. Choi, Y.K.; Kraft, N.; Zimmerman, B.; Jackson, M.; Rao, S.S. Fructose Intolerance in IBS and Utility of Fructose-Restricted Diet. J. Clin. Gastroenterol. 2008, 42, 233-238. [CrossRef]

141. Buzas, G.M. Fructose and Fructose Intolerance. Orv. Hetil. 2016, 157, 1708-1716.

142. Rao, S.S.; Attaluri, A.; Anderson, L.; Stumbo, P. Ability of the Normal Human Small Intestine to Absorb Fructose: Evaluation by Breath Testing. Clin. Gastroenterol. Hepatol. 2007, 5, 959-963. [CrossRef] [PubMed]

143. Yao, C.K.; Tuck, C.J.; Barrett, J.S.; Canale, K.E.; Philpott, H.L.; Gibson, P.R. Poor Reproducibility of Breath Hydrogen Testing: Implications for its Application in Functional Bowel Disorders. United Eur. Gastroenterol. J. 2017, 5, 284-292. [CrossRef] [PubMed]

144. Rezaie, A.; Buresi, M.; Lembo, A.; Lin, H.; McCallum, R.; Rao, S.; Schmulson, M.; Valdovinos, M.; Zakko, S.; Pimentel, M. Hydrogen and Methane-Based Breath Testing in Gastrointestinal Disorders: The North American Consensus. Am. J. Gastroenterol. 2017, 112, 775. [CrossRef] [PubMed]

145. Campbell, B.; Han, D.Y.; Triggs, C.M.; Fraser, A.G.; Ferguson, L.R. Brassicaceae: Nutrient Analysis and Investigation of Tolerability in People with Crohn's Disease in a New Zealand Study. Funct. Foods Health Dis. 2012, 2, 460-486. [CrossRef]

146. Laughlin, M. Normal Roles for Dietary Fructose in Carbohydrate Metabolism. Nutrients 2014, 6, 3117-3129. [CrossRef] [PubMed]

147. Neu, J.; Rushing, J. Cesarean Versus Vaginal Delivery: Long-Term Infant Outcomes and the Hygiene Hypothesis. Clin. Perinatol. 2011, 38, 321-331. [CrossRef] [PubMed]

148. Dominguez-Bello, M.G.; Costello, E.K.; Contreras, M.; Magris, M.; Hidalgo, G.; Fierer, N.; Knight, R. Delivery Mode Shapes the Acquisition and Structure of the Initial Microbiota Across Multiple Body Habitats in Newborns. Proc. Natl. Acad. Sci. USA 2010, 107, 11971-11975. [CrossRef]

149. Stinson, L.F.; Payne, M.S.; Keelan, J.A. A Critical Review of the Bacterial Baptism Hypothesis and the Impact of Caesarean Delivery on the Infant Microbiome. Front. Med. 2018, 5, 135. [CrossRef] 
150. Donovan, S.M.; Wang, M.; Li, M.; Friedberg, I.; Schwartz, S.L.; Chapkin, R.S. Host-Microbe Interactions in the Neonatal Intestine: Role of Human Milk Oligosaccharides. Adv. Nutr. 2012, 3, 450S-455S. [CrossRef]

151. Wu, S.; Tao, N.; German, J.B.; Grimm, R.; Lebrilla, C.B. Development of an Annotated Library of Neutral Human Milk Oligosaccharides. J. Proteome Res. 2010, 9, 4138-4151. [CrossRef]

152. Lönnerdal, B. Bioactive Proteins in Breast Milk. J. Paediatr. Child Health 2013, 49, 1-7. [CrossRef] [PubMed]

153. Andreas, N.J.; Kampmann, B.; Le-Doare, K.M. Human Breast Milk: A Review on its Composition and Bioactivity. Early Hum. Dev. 2015, 91, 629-635. [CrossRef]

154. Abou-Donia, M.B.; El-Masry, E.M.; Abdel-Rahman, A.A.; McLendon, R.E.; Schiffman, S.S. Splenda Alters Gut Microflora and Increases Intestinal P-Glycoprotein and Cytochrome P-450 in Male Rats. J. Toxicol. Environ. Health A 2008, 71, 1415-1429. [CrossRef] [PubMed]

155. Mattes, R.D.; Popkin, B.M. Nonnutritive Sweetener Consumption in Humans: Effects on Appetite and Food Intake and their Putative Mechanisms. Am. J. Clin. Nutr. 2009, 89, 1-14. [CrossRef] [PubMed]

156. Sylvetsky, A.; Rother, K.I.; Brown, R. Artificial Sweetener use among Children: Epidemiology, Recommendations, Metabolic Outcomes, and Future Directions. Pediatr. Clin. North Am. 2011, 58, 1467-1480. [CrossRef]

157. Sylvetsky, A.C.; Welsh, J.A.; Brown, R.J.; Vos, M.B. Low-Calorie Sweetener Consumption is Increasing in the United States. Am. J. Clin. Nutr. 2012, 96, 640-646. [CrossRef] [PubMed]

158. Bianchi, R.G.; Muir, E.T.; Cook, D.L.; Nutting, E.F. The Biological Properties of Aspartame. II. Actions Involving the Gastrointestinal System. J. Environ. Pathol. Toxicol. 1980, 3, 355-362. [PubMed]

159. Spencer, M.; Gupta, A.; Dam, L.V.; Shannon, C.; Menees, S.; Chey, W.D. Artificial Sweeteners: A Systematic Review and Primer for Gastroenterologists. J. Neurogastroenterol. Motil. 2016, 22, 168-180. [CrossRef]

160. Suez, J.; Korem, T.; Zeevi, D.; Zilberman-Schapira, G.; Thaiss, C.A.; Maza, O.; Israeli, D.; Zmora, N.; Gilad, S.; Weinberger, A. Artificial Sweeteners Induce Glucose Intolerance by Altering the Gut Microbiota. Nature 2014, 514, 181-186. [CrossRef]

161. Prashant, G.; Patil, R.B.; Nagaraj, T.; Patel, V.B. The Antimicrobial Activity of the Three Commercially Available Intense Sweeteners Against Common Periodontal Pathogens: An in Vitro Study. J. Contemp. Dent. Pract. 2012, 13, 749-752. [CrossRef]

162. Brusick, D.; Borzelleca, J.F.; Gallo, M.; Williams, G.; Kille, J.; Hayes, A.W.; Pi-Sunyer, F.X.; Williams, C.; Burks, W. Expert Panel Report on a Study of Splenda in Male Rats. Regul. Toxicol. Pharmacol. 2009, 55, 6-12. [CrossRef] [PubMed]

163. Schiffman, S.S. Rationale for further Medical and Health Research on High-Potency Sweeteners. Chem. Senses 2012, 37, 671-679. [CrossRef] [PubMed]

164. Suez, J.; Korem, T.; Zilberman-Schapira, G.; Segal, E.; Elinav, E. Non-Caloric Artificial Sweeteners and the Microbiome: Findings and Challenges. Gut Microbes 2015, 6, 149-155. [CrossRef] [PubMed]

165. Bian, X.; Chi, L.; Gao, B.; Tu, P.; Ru, H.; Lu, K. The Artificial Sweetener Acesulfame Potassium Affects the Gut Microbiome and Body Weight Gain in CD-1 Mice. PLoS ONE 2017, 12, e0178426. [CrossRef] [PubMed]

166. Chassaing, B.; Koren, O.; Goodrich, J.K.; Poole, A.C.; Srinivasan, S.; Ley, R.E.; Gewirtz, A.T. Dietary Emulsifiers Impact the Mouse Gut Microbiota Promoting Colitis and Metabolic Syndrome. Nature 2015, 519, 92. [CrossRef]

167. Zinöcker, M.; Lindseth, I. The Western Diet-microbiome-Host Interaction and its Role in Metabolic Disease. Nutrients 2018, 10, 365. [CrossRef] [PubMed]

168. Wright, G.D. The Antibiotic Resistome: The Nexus of Chemical and Genetic Diversity. Nat. Rev. Microbiol. 2007, 5, 175-186. [CrossRef]

169. United States Food and Drug Administration. Summary Report on Antimicrobials Sold or Distributed for Use in Food-Producing Animals; United States Food and Drug Administration: Silver Spring, MD, USA, 2009.

170. Kedgley, S. Parliament, press release. Scoop Independent News, 11 April 2011.

171. Ministry for Primary Industries NZ. Antbiotic Resistance and Food; Ministry for Primary Industries NZ: Wellington, New Zealand, 2013.

172. Ruiz-Garbajosa, P.; Bonten, M.J.; Robinson, D.A.; Top, J.; Nallapareddy, S.R.; Torres, C.; Coque, T.M.; Cantón, R.; Baquero, F.; Murray, B.E. Multilocus Sequence Typing Scheme for Enterococcus Faecalis Reveals Hospital-Adapted Genetic Complexes in a Background of High Rates of Recombination. J. Clin. Microbiol. 2006, 44, 2220-2228. [CrossRef] 
173. Pray, L.; Pillsbury, L.; Tomayko, E. The Human Microbiome, Diet and Health Workshop Summary; The National Academies Press: Washington, DC, USA, 2012; pp. 5-7.

174. Silbergeld, E.K.; Graham, J.; Price, L.B. Industrial Food Animal Production, Antimicrobial Resistance, and Human Health. Annu. Rev. Public Health 2008, 29, 151-169. [CrossRef]

175. Lindsey, R.L.; Frye, J.G.; Thitaram, S.N.; Meinersmann, R.J.; Fedorka-Cray, P.J.; Englen, M.D. Characterization of Multidrug-Resistant Escherichia Coli by Antimicrobial Resistance Profiles, Plasmid Replicon Typing, and Pulsed-Field Gel Electrophoresis. Microb. Drug Resist. 2011, 17, 157-163. [CrossRef]

176. Knight, P.; Campbell, B.J.; Rhodes, J.M. Host-Bacteria Interaction in Inflammatory Bowel Disease. Br. Med. Bull. 2008, 88, 95-113. [CrossRef] [PubMed]

177. Martin, H.M.; Campbell, B.J.; Hart, C.A.; Mpofu, C.; Nayar, M.; Singh, R.; Englyst, H.; Williams, H.F.; Rhodes, J.M. Enhanced Escherichia Coli Adherence and Invasion in Crohn's Disease and Colon Cancer. Gastroenterology 2004, 127, 80-93. [CrossRef] [PubMed]

178. Darfeuille-Michaud, A.; Boudeau, J.; Bulois, P.; Neut, C.; Glasser, A.; Barnich, N.; Bringer, M.; Swidsinski, A.; Beaugerie, L.; Colombel, J. High Prevalence of Adherent-Invasive Escherichia Coli Associated with Ileal Mucosa in Crohn's Disease. Gastroenterology 2004, 127, 412-421. [CrossRef] [PubMed]

179. Gearry, R.B.; Irving, P.M.; Barrett, J.S.; Nathan, D.M.; Shepherd, S.J.; Gibson, P.R. Reduction of Dietary Poorly Absorbed Short-Chain Carbohydrates (FODMAPs) Improves Abdominal Symptoms in Patients with Inflammatory Bowel Disease-A Pilot Study. J. Crohns Colitis 2009, 3, 8-14. [CrossRef] [PubMed]

180. Barbalho, S.M.; Goulart, R.d.A. Aranão, Ana Luíza de Carvalho; de Oliveira, Pamela Grazielle Correa. Inflammatory Bowel Diseases and Fermentable Oligosaccharides, Disaccharides, Monosaccharides, and Polyols: An Overview. J. Med. Food 2018, 21, 633-640. [CrossRef] [PubMed]

181. Smith, M.A.; Smith, T.; Trebble, T.M. Nutritional Management of Adults with Inflammatory Bowel Disease: Practical Lessons from the Available Evidence. Frontline Gastroenterol. 2012, 3, 172-179. [CrossRef] [PubMed]

182. Hill, P.; Muir, J.G.; Gibson, P.R. Controversies and Recent Developments of the Low-FODMAP Diet. Gastroenterol. Hepatol. 2017, 13, 36-45.

183. Sloan, T.J.; Jalanka, J.; Major, G.A.; Krishnasamy, S.; Pritchard, S.; Abdelrazig, S.; Korpela, K.; Singh, G.; Mulvenna, C.; Hoad, C.L. A Low FODMAP Diet is Associated with Changes in the Microbiota and Reduction in Breath Hydrogen but Not Colonic Volume in Healthy Subjects. PloS ONE 2018, 13, e0201410. [CrossRef]

184. Mital, B.; Steinkraus, K. Utilization of Oligosaccharides by Lactic Acid Bacteria during Fermentation of Soy Milk. J. Food Sci. 1975, 40, 114-118. [CrossRef]

185. Naessens, M.; Cerdobbel, A.; Soetaert, W.; Vandamme, E.J. Leuconostoc Dextransucrase and Dextran: Production, Properties and Applications. J. Chem. Technol. Biotechnol. 2005, 80, 845-860. [CrossRef]

186. Van den Ende, W. Multifunctional Fructans and Raffinose Family Oligosaccharides. Front. Plant Sci. 2013, 4 , 247. [PubMed]

187. Kuo, T.M.; VanMiddlesworth, J.F.; Wolf, W.J. Content of Raffinose Oligosaccharides and Sucrose in various Plant Seeds. J. Agric. Food Chem. 1988, 36, 32-36. [CrossRef]

188. Wang, Y.; Wang, L. Structures and Properties of Commercial Maltodextrins from Corn, Potato, and Rice Starches. Starch-Stärke 2000, 52, 296-304. [CrossRef]

189. Plaza-Díaz, J.; Martinez Augustin, O.; Gil Hernández, A. Foods as Sources of Mono and Disaccharides: Biochemical and Metabolic Aspects. Nutr. Hosp. 2013, 28, 5-16. [PubMed]

190. Calorie Control Council. Polyols. 2018. Available online: https://caloriecontrol.org/category/polyols/ (accessed on 5 November 2018).

191. Ingram, C.J.; Mulcare, C.A.; Itan, Y.; Thomas, M.G.; Swallow, D.M. Lactose Digestion and the Evolutionary Genetics of Lactase Persistence. Hum. Genet. 2009, 124, 579-591. [CrossRef] [PubMed]

192. Gerbault, P.; Liebert, A.; Itan, Y.; Powell, A.; Currat, M.; Burger, J.; Swallow, D.M.; Thomas, M.G. Evolution of Lactase Persistence: An Example of Human Niche Construction. Philos. Trans. R. Soc. Lond. B. Biol. Sci. 2011, 366, 863-877. [CrossRef] [PubMed]

193. Brauer, H.A.; Libby, T.E.; Mitchell, B.L.; Li, L.; Chen, C.; Randolph, T.W.; Yasui, Y.Y.; Lampe, J.W.; Lampe, P.D Cruciferous Vegetable Supplementation in a Controlled Diet Study Alters the Serum Peptidome in a GSTM1-Genotype Dependent Manner. Nutr. J. 2011, 10, 11. [CrossRef]

194. Kristal, A.R.; Lampe, J.W. Brassica Vegetables and Prostate Cancer Risk: A Review of the Epidemiological Evidence. Nutr. Cancer 2002, 42, 1-9. [CrossRef] 
195. Laing, B.; Han, D.Y.; Ferguson, L.R. Candidate Genes Involved in Beneficial or Adverse Responses to Commonly Eaten Brassica Vegetables in a New Zealand Crohn's Disease Cohort. Nutrients 2013, 5, 5046-5064. [CrossRef]

196. Higdon, J.V.; Delage, B.; Williams, D.E.; Dashwood, R.H. Cruciferous Vegetables and Human Cancer Risk: Epidemiologic Evidence and Mechanistic Basis. Pharmacol. Res. 2007, 55, 224-236. [CrossRef]

197. NIH. Celiac Disease. In Genetics Home Reference; NIH: Stapleton, NY, USA, 2013.

198. Petermann, I.; Triggs, C.M.; Huebner, C.; Han, D.Y.; Gearry, R.B.; Barclay, M.L.; Demmers, P.S.; McCulloch, A.; Ferguson, L.R. Mushroom Intolerance: A Novel Diet-Gene Interaction in Crohn's Disease. Br. J. Nutr. 2009, 102, 506. [CrossRef] [PubMed]

199. Campbell, A.K.; Matthews, S.B.; Vassel, N.; Cox, C.D.; Naseem, R.; Chaich, i.J.; Holland, I.B.; Green, J.; Wann, K.T. Bacterial Metabolic 'Toxins': A New Mechanism for Lactose and Food Intolerance, and Irritable Bowel Syndrome. Toxicology 2010, 278, 268-276. [CrossRef] [PubMed]

200. FAO/WHO. Carbohydrates in Human Nutrition; Report of a Joint FAO/WHO Expert Consultation; FAO Food and Nutrition Paper; FAO: Rome, Italy, 1998.

201. Hyams, J.S. Sorbitol Intolerance: An Unappreciated Cause of Functional Gastrointestinal Complaints. Gastroenterology 1983, 84, 30-33. [PubMed]

202. Gibson, P.R.; Shepherd, S.J. Evidence-based Dietary Management of Functional Gastrointestinal Symptoms: The FODMAP Approach. J. Gastroenterol. Hepatol. 2010, 25, 252-258. [CrossRef] [PubMed]

203. Catassi, G.; Lionetti, E.; Gatti, S.; Catassi, C. The Low FODMAP Diet: Many Question Marks for a Catchy Acronym. Nutrients 2017, 9, 292. [CrossRef] [PubMed]

204. Qin, J.; Li, R.; Raes, J.; Arumugam, M.; Burgdorf, K.S.; Manichanh, C.; Nielsen, T.; Pons, N.; Levenez, F.; Yamada, T. A Human Gut Microbial Gene Catalogue Established by Metagenomic Sequencing. Nature 2010, 464, 59-65. [CrossRef] [PubMed]

205. Ley, R.E.; Peterson, D.A.; Gordon, J.I. Ecological and Evolutionary Forces Shaping Microbial Diversity in the Human Intestine. Cell 2006, 124, 837-848. [CrossRef]

206. Turnbaugh, P.J.; Ley, R.E.; Mahowald, M.A.; Magrini, V.; Mardis, E.R.; Gordon, J.I. An Obesity-Associated Gut Microbiome with Increased Capacity for Energy Harvest. Nature 2006, 444, 1027-1131. [CrossRef]

207. Frank, D.N.; Robertson, C.E.; Hamm, C.M.; Kpadeh, Z.; Zhang, T.; Chen, H.; Zhu, W.; Sartor, R.B.; Boedeker, E.C.; Harpaz, N. Disease Phenotype and Genotype are Associated with Shifts in Intestinal-Associated Microbiota in Inflammatory Bowel Diseases. Inflamm. Bowel Dis. 2010, 17, 179-184. [CrossRef]

208. Frank, D.N.; St Amand, A.L.; Feldman, R.A.; Boedeker, E.C.; Harpaz, N.; Pace, N.R. Molecular-Phylogenetic Characterization of Microbial Community Imbalances in Human Inflammatory Bowel Diseases. Proc. Natl. Acad. Sci. USA 2007, 104, 13780-13785. [CrossRef]

209. Walker, A.W.; Sanderson, J.D.; Churcher, C.; Parkes, G.C.; Hudspith, B.N.; Rayment, N.; Brostoff, J.; Parkhill, J.; Dougan, G.; Petrovska, L. High-Throughput Clone Library Analysis of the Mucosa-Associated Microbiota Reveals Dysbiosis and Differences between Inflamed and Non-Inflamed Regions of the Intestine in Inflammatory Bowel Disease. BMC Microbiol. 2011, 11, 7. [CrossRef] [PubMed]

210. Nagalingam, N.A.; Lynch, S.V. Role of the Microbiota in Inflammatory Bowel Diseases. Inflamm. Bowel Dis. 2012, 18, 968-984. [CrossRef] [PubMed]

211. Wacklin, P.; Mäkivuokko, H.; Alakulppi, N.; Nikkilä, J.; Tenkanen, H.; Räbinä, J.; Partanen, J.; Aranko, K.; Mättö, J. Secretor Genotype (FUT2 Gene) is Strongly Associated with the Composition of Bifidobacteria in the Human Intestine. PLoS ONE 2011, 6, e20113. [CrossRef] [PubMed]

212. McGovern, D.P.; Jones, M.R.; Taylor, K.D.; Marciante, K.; Yan, X.; Dubinsky, M.; Ippoliti, A.; Vasiliauskas, E.; Berel, D.; Derkowski, C. Fucosyltransferase 2 (FUT2) Non-Secretor Status is Associated with Crohn's Disease. Hum. Mol. Genet. 2010, 19, 3468-3476. [CrossRef] [PubMed]

213. Miyoshi, J.; Yajima, T.; Okamoto, S.; Matsuoka, K.; Inoue, N.; Hisamatsu, T.; Shimamura, K.; Nakazawa, A.; Kanai, T.; Ogata, H. Ectopic Expression of Blood Type Antigens in Inflamed Mucosa with Higher Incidence of FUT2 Secretor Status in Colonic Crohn's Disease. J. Gastroenterol. 2011, 46, 1056-1063. [CrossRef]

214. Rausch, P.; Rehman, A.; Künzel, S.; Häsler, R.; Ott, S.J.; Schreiber, S.; Rosenstiel, P.; Franke, A.; Baines, J.F. Colonic Mucosa-Associated Microbiota is Influenced by an Interaction of Crohn Disease and FUT2 (Secretor) Genotype. Proc. Natl. Acad. Sci. USA 2011, 108, 19030-19035. [CrossRef] [PubMed] 
215. Salminen, S.; Gueimonde, M. Gut Microbiota in Infants between 6 and 24 Months of Age. Nestle Nutr. Workshop Ser. Pediatr. Program. 2005, 56, 43-56.

216. Ebaid, H.; Hassanein, K.A. Comparative Immunomudulating Effects of Five Orally Administrated Bifidobacteria Species in Male Albino Rats. Egypt. J. Biol. 2007, 9, 14-23.

217. Festen, E.A.; Goyette, P.; Green, T.; Boucher, G.; Beauchamp, C.; Trynka, G.; Dubois, P.C.; Lagacé, C.; Stokkers, P.C.; Hommes, D.W. A Meta-Analysis of Genome-Wide Association Scans Identifies IL18RAP, PTPN2, TAGAP, and PUS10 as Shared Risk Loci for Crohn's Disease and Celiac Disease. PLoS Genet. 2011, 7, e1001283. [CrossRef]

218. Visser, R.; Barrett, K.; McCole, D. Regulation of Epithelial Barrier Function by Crohn's Disease Associated Gene PTPN. BMC Proc. 2012, 6, O41. [CrossRef]

219. Armstrong, D.; Don-Wauchope, A.C.; Verdu, E.F. Testing for Gluten-Related Disorders in Clinical Practice: The Role of Serology in Managing the Spectrum of Gluten Sensitivity. Can. J. Gastroenterol. 2011, 25, $193-197$. [CrossRef] [PubMed]

220. Mishkin, S. Controversies regarding the Role of Dairy-Products in Inflammatory Bowel Disease. Can. J. Gastroenterol. 1994, 8, 205-212. [CrossRef]

221. Truelove, S.C. Ulcerative Colitis Provoked by Milk. Br. Med. J. 1961, 1, 154-160. [CrossRef] [PubMed]

222. Wright, R.; Truelove, S.C. A Controlled Therapeutic Trial of various Diets in Ulcerative Colitis. Br. Med. J. 1965, 2, 138-141. [CrossRef] [PubMed]

223. Mishkin, S. Dairy Sensitivity, Lactose Malabsorption, and Elimination Diets in Inflammatory Bowel Disease. Am. J. Clin. Nutr. 1997, 65, 564-567. [CrossRef]

224. Maconi, G.; Ardizzone, S.; Cucino, C.; Bezzio, C.; Russo, A.G.; Bianchi Porro, G. Pre-Illness Changes in Dietary Habits and Diet as a Risk Factor for Inflammatory Bowel Disease: A Case-Control Study. World J. Gastroenterol. 2010, 16, 4297-4304. [CrossRef] [PubMed]

225. Nolan-Clark, D.; Tapsell, L.C.; Hu, R.; Han, D.Y.; Ferguson, L.R. Effects of Dairy Products on Crohn's Disease Symptoms are Influenced by Fat Content and Disease Location but Not Lactose Content or Disease Activity Status in a New Zealand Population. J. Am. Diet. Assoc. 2011, 111, 1165-1172. [CrossRef]

226. Nolan, D.; Han, D.; Lam, W.; Morgan, A.; Fraser, A.; Tapsell, L.; Ferguson, L. Genetic Adult Lactase Persistence is Associated with Risk of Crohn's Disease in a New Zealand Population. BMC Res. Notes 2010, 3, 339. [CrossRef]

227. Shrier, I.; Szilagyi, A.; Correa, J.A. Impact of Lactose Containing Foods and the Genetics of Lactase on Diseases: An Analytical Review of Population Data. Nutr. Cancer 2008, 60, 292-300. [CrossRef]

228. Barbado, M.; Fablet, K.; Ronjat, M.; De Waard, M. Gene Regulation by Voltage-Dependent Calcium Channels. Biochim. Biophys. Acta 2009, 1793, 1096-1104. [CrossRef]

229. Bernstein, C.N.; Leslie, W.D.; Leboff, M.S. AGA Technical Review on Osteoporosis in Gastrointestinal Diseases. Gastroenterology 2003, 124, 795-841. [CrossRef] [PubMed]

230. Caniggia, A.; Nuti, R.; Lore, F.; Vattimo, A. Pathophysiology of the Adverse Effects of Glucoactive Corticosteroids on Calcium Metabolism in Man. J. Steroid Biochem. 1981, 15, 153-161. [CrossRef]

231. New Zealand Nutrition Foundation. Calcium. 2018. Available online: https://nutritionfoundation.org.nz/ nutrients-vitamins-and-minerals/calcium (accessed on 15 June 2019).

232. Burlingame, B.A.; Milligan, G.; Apimerika, D.; Arthur, J. The Concise New Zealand Food Composition Tables; New Zealand Institute for Crop \& Food Research: Auckland, New Zealand, 2009.

233. Duke, J.; Beckstrom-Sternberg, S. Phytochemical Database USDA-ARS-NGRL; Beltsbille Agricultural Research Center: Beltsbille, MD, USA, 1998.

234. Parl, F.F. Glutathione S-Transferase Genotypes and Cancer Risk. Cancer Lett. 2005, 221, 123-129. [CrossRef] [PubMed]

235. Beckett, G.J.; Hayes, J.D. Glutathione S-Transferases: Biomedical Applications. Adv. Clin. Chem. 1993, 30, 282.

236. Wilce, M.C.; Parker, M.W. Structure and Function of Glutathione S-Transferases. Biochim. Biophys. Acta 1994, 1205, 1-18. [CrossRef]

237. Webb, G.; Vaska, V.; Coggan, M.; Board, P. Chromosomal Localization of the Gene for the Human Theta Class Glutathione Transferase (GSTT1). Genomics 1996, 33, 121-123. [CrossRef] 
238. Sheehan, D.; Meade, G.; Foley, V.; Dowd, C. Structure, Function and Evolution of Glutathione Transferases: Implications for Classification of Non-Mammalian Members of an Ancient Enzyme Superfamily. Biochem. J. 2001, 360, 1-16. [CrossRef]

239. Hayes, J.D.; Strange, R.C. Glutathione S-Transferase Polymorphisms and their Biological Consequences. Pharmacology 2000, 61, 154-166. [CrossRef]

240. Inskip, A.; Elexperu-Camiruaga, J.; Buxton, N.; Dias, P.; MacIntosh, J.; Campbell, D.; Jones, P.; Yengi, L.; Talbot, J.; Strange, R. Identification of Polymorphism at the Glutathione S-Transferase, GSTM3 Locus: Evidence for Linkage with GSTM1* A. Biochem. J. 1995, 312, 713. [CrossRef]

241. Seow, A.; Yuan, J.; Sun, C.; Van Den Berg, D.; Lee, H.; Mimi, C.Y. Dietary Isothiocyanates, Glutathione S-Transferase Polymorphisms and Colorectal Cancer Risk in the Singapore Chinese Health Study. Carcinogenesis 2002, 23, 2055-2061. [CrossRef]

242. Steck, S.E.; Hebert, J.R. GST Polymorphism and Excretion of Heterocyclic Aromatic Amine and Isothiocyanate Metabolites After Brassica Consumption. Environ. Mol. Mutagen. 2009, 50, 238-246. [CrossRef]

243. Campbell, B.; Han, D.; Jiun, W.; Morgan, A.; Triggs, C.; Fraser, A.C.; Ferguson, L.R. Deletion of the GSTT1 Genotype Linked to Tolerance of Brassicaceae in People with Crohn's Disease in a New Zealand Cohort. In Proceedings of the Annual Scientific Meeting of the Nutrition Society of New Zealand Auckland, Auckland, New Zealand, 21-22 November 2012.

244. Gentschew, L.; Bishop, K.S.; Han, D.Y.; Morgan, A.R.; Fraser, A.G.; Lam, W.J.; Karunasinghe, N.; Campbell, B.; Ferguson, L.R. Selenium, Selenoprotein Genes and Crohn's Disease in a Case-Control Population from Auckland, New Zealand. Nutrients 2012, 4, 1247-1259. [CrossRef] [PubMed]

245. Ahmad, T.; Marshall, S.E.; Jewell, D. Genetics of Inflammatory Bowel Disease: The Role of the HLA Complex. World J. Gastroenterol. 2006, 12, 3628. [CrossRef] [PubMed]

246. Lees, C.W.; Satsangi, J. Genetics of Inflammatory Bowel Disease: Implications for Disease Pathogenesis and Natural History. Expert Rev. Gastroenterol. Hepatol. 2009, 3, 513-534. [CrossRef] [PubMed]

247. Annese, V.; Piepoli, A.; Latiano, A.; Lombardi, G.; Napolitano, G.; Caruso, N.; Cocchiara, E.; Accadia, L.; Perri, F.; Andriulli, A. HLA-DRB1 Alleles may Influence Disease Phenotype in Patients with Inflammatory Bowel Disease: A Critical Reappraisal with Review of the Literature. Dis. Colon. Rectum. 2005, 48, 57-65. [CrossRef] [PubMed]

248. Moffatt, M.F.; Gut, I.G.; Demenais, F.; Strachan, D.P.; Bouzigon, E.; Heath, S.; Von Mutius, E.; Farrall, M.; Lathrop, M.; Cookson, W.O. A Large-Scale, Consortium-Based Genomewide Association Study of Asthma. N. Engl. J. Med. 2010, 363, 1211-1221. [CrossRef] [PubMed]

249. Levin, A.; Mathias, R.; Huang, L.R.; Roth, L.A.; Daley, D.; Myers, R.A.; Himes, B.E.; Romieu, I.; Yang, M.; Eng, C.; et al. A Meta-Analysis of Genome Wide Association Studies for Serum Total IgE in Diverse Populations Groups; American Academy of Allergy, Asthma and Immunology: Milwaukee, WI, USA, 2013; p. 1176.

250. Gould, H.J.; Sutton, B.J.; Beavil, A.J.; Beavil, R.L.; McCloskey, N.; Coker, H.A.; Fear, D.; Smurthwaite, L. The Biology of IgE and the Basis of Allergic Disease. Annu. Rev. Immunol. 2003, 21, 579-628. [CrossRef]

251. Marlow, G.; Han, D.Y.; Triggs, C.M.; Ferguson, L.R. Food Intolerance: Associations with the rs 12212067 Polymorphism of FOXO3 in Crohn's Disease Patients in New Zealand. J. Nutrigen. Nutrigen. 2015, 8, 70-80. [CrossRef]

252. Food Safety Authority of Ireland. Food Ingredients that must be Declared as Allergens in the EU. Available online: https://www.fsai.ie/legislation/food_legislation/food_information/14_allergens.html (accessed on 2 February 2019).

253. Wedzicha, B.L. Chemistry of Sulphur Dioxide in Foods; Elsevier Applied Science Publishers Ltd.: Amsterdam, The Netherlands, 1984.

254. Coates, P.M.; Blackman, M.; Betz, J.; Cragg, G.M.; Levine, M.; Moss, J.; White, J.D. Encyclopedia of Dietary Supplements; Informa Healthcare: London, UK, 2010.

255. Costantini, A.; Pala, M.I. Thiamine and Fatigue in Inflammatory Bowel Diseases: An Open-Label Pilot Study. J. Altern. Complement. Med. 2013, 19, 704-708. [CrossRef]

256. Magee, E.A.; Edmond, L.M.; Tasker, S.M.; Kong, S.C.; Curno, R.; Cummings, J.H. Associations between Diet and Disease Activity in Ulcerative Colitis Patients using a Novel Method of Data Analysis. Nutr. J. 2005, 4, 7. [CrossRef]

257. Pitcher, M.C.; Cummings, J.H. Hydrogen Sulphide: A Bacterial Toxin in Ulcerative Colitis? Gut 1996, 39, 1-4 [CrossRef] [PubMed] 
258. Marcus, R.; Watt, J. Seaweeds and Ulcerative Colitis in Laboratory Animals. Lancet 1969, 2, 489-490. [CrossRef]

259. Marcus, R.; Watt, J. Ulcerative Disease of the Colon in Laboratory Animals Induced by Pepsin Inhibitors. Gastroenterology 1974, 67, 473-483. [CrossRef]

260. Roediger, W. The Colonic Epithelium in Ulcerative Colitis: An Energy-Deficiency Disease? Lancet 1980, 316, 712-715. [CrossRef]

261. Peng, L.; Li, Z.; Green, R.S.; Holzman, I.R.; Lin, J. Butyrate Enhances the Intestinal Barrier by Facilitating Tight Junction Assembly Via Activation of AMP-Activated Protein Kinase in Caco-2 Cell Monolayers. J. Nutr. 2009, 139, 1619-1625. [CrossRef] [PubMed]

262. Carbonero, F.; Benefiel, A.C.; Alizadeh-Ghamsari, A.H.; Gaskins, H.R. Microbial Pathways in Colonic Sulfur Metabolism and Links with Health and Disease. Front. Physiol. 2012, 3, 448. [CrossRef] [PubMed]

263. Berezowska, A.; Fischer, A.R.; Ronteltap, A.; Lans, I.A.; Trijp, H.C. Consumer Adoption of Personalised Nutrition Services from the Perspective of a Risk-benefit Trade-Off. Genes Nutr. 2015, 10, 42. [CrossRef]

264. Nutrisearch. Fitgenes (DNA Testing). 2018. Available online: https://www.nutrisearch.co.nz/lab-diagnostics/ fitgenes-dna-testing/ (accessed on 13 June 2018).

265. Zettler, P.J.; Sherkow, J.S.; Greely, H.T. 23 and Me, the Food and Drug Administration, and the Future of Genetic Testing. JAMA Intern. Med. 2014, 174, 493-494. [CrossRef]

266. Annas, G.J.; Elias, S. 23andMe and the FDA. N. Engl. J. Med. 2014, 370, 985-988. [CrossRef]

(C) 2019 by the authors. Licensee MDPI, Basel, Switzerland. This article is an open access article distributed under the terms and conditions of the Creative Commons Attribution (CC BY) license (http://creativecommons.org/licenses/by/4.0/). 

MDPI

St. Alban-Anlage 66

4052 Basel

Switzerland

Tel. +41 616837734

Fax +41 613028918

www.mdpi.com

Nutrients Editorial Office

E-mail: nutrients@mdpi.com www.mdpi.com/journal/nutrients

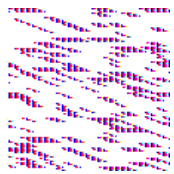



MDPI

St. Alban-Anlage 66

4052 Basel

Switzerland

Tel: +41 616837734

Fax: +41 613028918

www.mdpi.com 